Peter Ruhwedel

\title{
Aufsichtsrats- planungssysteme
}




\section{Peter Ruhwedel}

\section{Aufsichtsratsplanungssysteme}

Ausgelöst durch zahlreiche Unternehmenskrisen wird seit Beginn der 90er Jahre in der Corporate Governance-Diskussion dieFunktionsfähigkeit des deutschen Aufsichtsratssystems in Fragegestellt. Der Gesetzgeber reagierte hierauf zunächst mit dem KonTraG und zuletzt mit dem TransPuG. Der Autor entwickelt aus betriebswirtschaftlicher Perspektive Gestaltungsvorschläge, die zu einer Funktionsverbesserung des Aufsichtsrats beitragen. Ausgehend von einer Analyse der juristischen Rahmenbedingungen zeigt der Autor, daß ein wesentlicher Teil der Aufsichtsratsaufgaben zukunftsbezogen ist. Zur Erfüllung dieser Aufgaben sollten Aktiengesellschaften daher über ein Aufsichtsratsplanungssystem verfügen, das die Aufsichtsratsmitglieder umfassend unterstützt. Es wird ein theoretisch fundiertes Gesamtsystem entwikkelt, das sich durch hohe praktische Umsetzbarkeit auszeichnet. Zur Förderung der notwendigen Professionalisierung des Aufsichtsrats wird außerdem ein wertorientiertes Anreizsystem gestaltet, das das Planungssystem ergänzen muß.

Peter Ruhwedel, geboren 1969 in Recklinghausen, studierte nach einer Ausbildung zum Industriekaufmann Wirtschaftswissenschaft an der Ruhr-Universität in Bochum. Nach seinem Abschluß als Diplom-Ökonom und einer Tätigkeit als Unternehmensberater arbeitete er von 1997 bis zu seiner Promotion 2001 als wissenschaftlicher Mitarbeiter am Lehrstuhl für Planung und Organisation an der Ruhr-Universität Bochum. Seit seiner Dissertation ist er als Unternehmensberater tätig. 
Aufsichtsratsplanungssysteme 


\section{BOCHUMER BEITRÄGE ZUR UNTERNEHMUNGSFÜHRUNG UND UNTERNEHMENSFORSCHUNG}

Herausgegeben von Prof. Dr. Michael Abramovici, Prof. Dr. Dr. h.c. mult. Walther Busse von Colbe, Prof. Dr. Dr. h.c. Werner H. Engelhardt, Prof. Dr. Roland Gabriel, Prof. Dr. Amo Jaeger, Prof. Dr. Gert Laßmann, Prof. Dr. Wolfgang Maßberg, Prof. Dr. Bernhard Pellens, Prof. Dr. Marion Steven, Prof. Dr. Rolf Wartmann, Prof. Dr. Brigitte Werners

Band 64

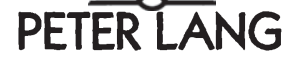

Frankfurt am Main - Berlin - Bern - Bruxelles - New York · Oxford - Wien 


\section{Peter Ruhwedel}

\section{Aufsichtsratsplanungssysteme}

Theoretische Grundlagen und praktische Ausgestaltung

in Publikumsaktiengesellschaften

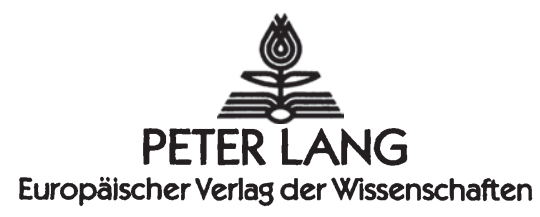


Die Deutsche Bibliothek - CIP-Einheitsaufnahme

Ruhwedel, Peter:

Aufsichtsratsplanungssysteme : theoretische Grundlagen und praktische Ausgestaltung in Publikumsaktiengesellschaften / Peter Ruhwedel. - Frankfurt am Main ; Berlin ; Bern ; Bruxelles ; New York ; Oxford ; Wien : Lang, 2002

(Bochumer Beitrăge zur Unternehmungsfuhrung und Unternehmensforschung ; Bd. 64)

Open Access: The online version of this publication is published on www. peterlang.com and www.econstor.eu under the international Creative Commons License CC-BY 4.0. Learn more on how you can use and share this work: http:// creativecommons.org/licenses/by/4.0.

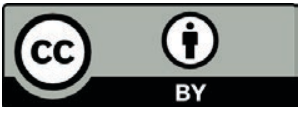

This book is available Open Access thanks to the kind support of ZBW - Leibniz-Informationszentrum Wirtschaft.

Zugl.: Bochum, Univ., Diss., 2001

ISBN3-631-39023-8

Gedruckt auf alterungsbestăndigem, săurefreiem Papier.

\author{
D294 \\ ISSN 0175-7105 \\ ISBN3-631-39023-8 \\ ISBN 978-3-631-75491-7 (eBook) \\ (C) Peter Lang GmbH \\ Europäischer Verlag der Wissenschaften \\ Frankfurt am Main 2002 \\ Alle Rechte vorbehalten.
}

Das Werk einschließlich aller seiner Teile ist urheberrechtlich geschutzt. Jede Verwertung außerhalb der engen Grenzen des

Urheberrechtsgesetzes ist ohne Zustimmung des Verlages unzulässig und strafbar. Das gilt insbesondere fur Vervielfältigungen, Übersetzungen, Mikroverfilmungen und die Einspeicherung und Verarbeitung in elektronischen Systemen.

Printed in Germany 123467 www.peterlang.de 


\section{„Aufsichtsräte glauben nicht an Wunder, sie verlassen sich darauf."}

(Hakelmacher, Falken-Parabel, 1999, S. 8.) 
Peter Ruhwedel - 978-3-631-75491-7 Downloaded from PubFactory at 01/11/2019 04:17:06AM 


\section{Geleitwort}

Der Aufsichtsrat stand seit seiner gesetzlichen Einführung immer wieder in der Kritik. Die Kritik reichte von seiner fehlerbehafteten institutionellen Ausgestaltung über die durch unzureichende Kompetenz gekennzeichnete personelle $\mathrm{Zu}$ sammensetzung bis hin zu der Mandatswahrnehmung durch die Delegierten der Anteilseigner, denen häufig eher die Verfolgung persönlicher Ziele als der Interessen der Aktionäre vorgeworfen wurde. Mit der Institutionalisierung der heftig bekämpften unternehmerischen Mitbestimmung hat sich diese Kritik verfestigt und sogar noch erweitert, so daß im Ergebnis den Aufsichtsräten in Publikumsaktiengesellschaften eine faktische Funktionsunfähigkeit vorgeworfen wurde.

Mit der in Theorie und Praxis geäußerten Kritik am Überwachungsorgan deutscher Aktiengesellschaften sind üblicherweise auch Verbesserungsvorschläge zum Abbau der identifizierten Defizite verbunden. Doch während bei der Problemanalyse weitgehende Einigkeit besteht, sind die Reformansätze häufig durch persönliche oder Gruppeninteressen geprägt. Noch deutlicher wird diese Uneinigkeit schließlich bei der Durchsetzung möglicher Reformen, die beispielsweise bei dem KonTraG auf dem kleinsten gemeinsamen Nenner verharrte. Daneben orientieren sich potentielle und tatsächliche Reformen eher an juristischen und weniger an betriebswirtschaftlichen Denkkategorien, so daß es zumindest zweifelhaft erscheint, daß dem durch die zunehmende Internationalisierung des Kapitalmarktes aufgebauten Reformdruck auf die Spitzenverfassung deutscher Publikumsaktiengesellschaften hinreichend Rechnung getragen wird.

Herr Dr. Ruhwedel hat dies zum Anlaß genommen, um in seiner Arbeit einen konsequent betriebswirtschaftlich ausgerichteten Beitrag für eine wirksame Verbesserung der Funktionsfähigkeit des Aufsichtsrats in Publikumsaktiengesellschaften zu leisten, ohne dabei jedoch das juristisch Machbare aus dem Auge zu verlieren. Ausgehend von einer Analyse der Ausgangssituation, die zu einem klaren Verständnis der Aufgaben des Aufsichtsrats im Zusammenspiel mit dem Vorstand beiträgt, wird deutlich, daß der Aufsichtsrat nicht nur das oberste Überwachungsorgan der Aktiengesellschaft darstellt, sondern ihm vielmehr umfangreiche und wesentliche Aufgaben im Rahmen der zukunftsorientierten Gestaltung der Erfolgspotentiale der Gesellschaft zukommen. Herr Dr. Ruhwedel zeigt, dass eine Beschränkung der Tätigkeit des Aufsichtsrats auf reine Kontrolltätigkeiten weder juristisch intendiert noch betriebswirtschaftlich sinnvoll ist und er vielmehr zusätzlich aktiv tätig werden kann und muß. 
Ausgehend von der klaren Aufgabenanalyse des Aufsichtsrats wird in der vorliegenden Arbeit ein Aufsichtsratsplanungssystem entwickelt, als dessen Element die Aufsichtsratsmitglieder in die Lage versetzt werden, ihren Beitrag für eine wertorientierte Unternehmensentwicklung zu leisten. Die umfassende Ausgestaltung des Planungssystems umfasst neben personellen auch organisatorische, objektbezogene, prozessurale sowie instrumentelle Aspekte, so daß mit dem vorliegenden holistischen Ansatz die bisher dominierende Partialbetrachtung überwunden und ein überaus gelungener Vorschlag entwickelt wird, der alle relevanten betriebswirtschaftlichen Problembereiche beinhaltet. Ergänzt wird das Aufsichtratsplanungssystem durch Vorschläge für ein wertorientiertes Anreizsystem für die Aufsichtsratsmitglieder, das ihrer wachsenden Bedeutung innerhalb einer wertorientierten Unternehmensführung entspricht.

Es ist zu hoffen, dass die von Herrn Dr. Ruhwedel entwickelten Vorschläge aufgegriffen und in der Praxis umgesetzt werden, da sie zu einer auch im internationalen Kontext wettbewerbsfähigen Corporate Governance beitragen können. Wie wichtig dies für die Kapitalmarktfähigkeit deutscher Publikumsaktiengesellschaften ist, zeigen die gegenwärtig zu entwickelnden Vorschläge der Kodex-Kommission sehr eindrucksvoll, die die internationale Akzeptanz des deutschen Zweikammermodells (endlich) erreichen wollen.

Prof. Dr. Wolfgang Mag 


\section{Vorwort}

Die hier vorliegende Arbeit wurde im Juli 2001 von der wirtschaftswissenschaftlichen Fakultät der Ruhr-Universität Bochum, an der ich am Lehrstuhl für Theoretische Betriebswirtschaftslehre, insbesondere Planung und Organisation, von Juni 1997 bis August 2001 als wissenschaftlicher Mitarbeiter beschäftigt war, als Dissertation angenommen. Ihre Entstehungsgeschichte reicht jedoch in meine Tätigkeit bei der C\&L Unternehmensberatung GmbH zurück. Nachdem ich mich dazu entschieden hatte, den Weg zurück aus dem „Licht des Beraterlebens" in die „Einöde der Hochschule“ zu wagen, wies mich Werner Große, Partner bei C\&L, auf eine zu verabschiedende Gesetzesnovelle hin, die zu einer nachhaltigen Verbesserung der Überwachung von und in deutschen Aktiengesellschaften führen sollte: das Gesetz zur Kontrolle und Transparenz im Unternehmensbereich (KonTraG). Ihm kommt damit gleichsam der Verdienst zu, diese Forschungsarbeit angeregt zu haben. Ich glaube jedoch nicht, daß ihm zum damaligen Zeitpunkt bewußt war, was er damit anrichten würde.

Von den während der Diskussion der Gesetzesnovelle aufgezeigten facettenreichen Defiziten und Problemlösungsvorschlägen faszinierten (und erschreckten) mich insbesondere diejenigen zur Aufsichtsratstätigkeit. Meine nicht auf jeder Entwicklungsstufe ausgereiften Gedanken zu diesem Problemfeld konnten dabei erst durch den Freiraum, den mir mein Doktorvater Prof. Dr. Wolfgang Mag einräumte, den hier vorliegenden Reifegrad erreichen. Dafür und für seine stete Diskussionsbereitschaft danke ich ihm. Herrn Prof. Dr. Roland Gabriel danke ich für die Bereitschaft zur Übernahme des Zweitgutachtens und insbesondere für seine Hinweise zum informationstechnologischen Teil dieser Arbeit. Ich danke außerdem dem Institut für Unternehmungsführung und Unternehmensforschung an der Ruhr-Universität Bochum für die Möglichkeit, eine einjährige Praxisphase im Rahmen der Kooperation des Instituts mit der Infracor GmbH zu absolvieren. Darüber hinaus danke ich dem Institut sowie dem Peter Lang Verlag für die Aufnahme dieser Arbeit in die Bochumer Schriftenreihe zur Unternehmungsführung und Unternehmensforschung.

Der vierjährige Entstehungsprozeß dieser Arbeit hat Freude bereitet, oft aber auch Mühe und Kraft gekostet - nicht nur meine. Da sind zunächst die ehemaligen und jetzigen Mitarbeiter des Lehrstuhls für Planung und Organisation zu nennen: Dr. Hilmar Henselek, der mir stets ein freundschaftlicher und hilfsbereiter Begleiter war und darüber hinaus jeden Tag für den erforderlichen Koffeinspiegel sorgte, Dr. Ulrike Ufer und Dr. Harald Meinhövel, die sich der dankenswerten Aufgabe stellten, verschiedene Fassungen der Arbeit auf ihren pro- 
motionswürdigen Gehalt zu prüfen, sowie Dr. Gabi Schulte-Florian, Thomas Thurm und mein in München verschollener Freund Stephan Bülle. Außerhalb des Lehrstuhls haben sich Dr. Dirk Beier, der fast waschechte Rechnungsleger Dr. Joachim Gassen, Dr. Susanne Klimpel, meine Schwester Ulrike Schürmann sowie meine langjährigen Freunde Andreas Leclaire, Christof Nickel und Jörg Schäfer, die auch in schwierigen Zeiten immer für mich da waren, der Mühe unterzogen, Teile bzw. die gesamte Arbeit zu lesen. Ihnen allen danke ich sehr!

Darüber hinaus bin ich in der glücklichen Lage, mich auch an einem zweiten Lehrstuhl fast wie zu Hause zu fühlen. Dies verdanke ich den Mitarbeiterinnen und Mitarbeitern des Lehrstuhls für Internationale Unternehmensrechnung, die mich sehr freundschaftlich in ihrem Kreis aufgenommen haben. Besonderen Dank schulde ich meinem Freund Dr. Ralf Schremper, der mir an unzähligen Dissertationsabenden eine größere Hilfe war, als ich ihm jemals sagen konnte. Die Entstehung einer solchen Arbeit erfordert jedoch nicht nur kollegiale und freundschaftliche Unterstützung, sondern auch familiäre Hilfe. Mein Dank gilt daher allen kleinen und großen, alten und neuen Familienmitgliedern, insbesondere meinen lieben Eltern Renate und Wilhelm Ruhwedel.

$\mathrm{Zu}$ meinem großen Bedauern habe ich während der Entstehung dieser Arbeit aufgrund privater Veränderungen Freunde verloren. Aber ich habe auch etwas gewonnen, daß mein Leben vollkommen verändert hat: Die Liebe meiner Freundin Franca Hillebrandt. Über jedwede fachliche und persönliche Unterstützung hinaus hat sie mir in ihrer liebevollen Art gezeigt, daß es neben der wissenschaftlichen Arbeit weit wichtigere Dinge gibt, die es zu entdecken gilt! Ihr und meinen Eltern widme ich diese Arbeit. 


\section{Inhaltsverzeichnis}

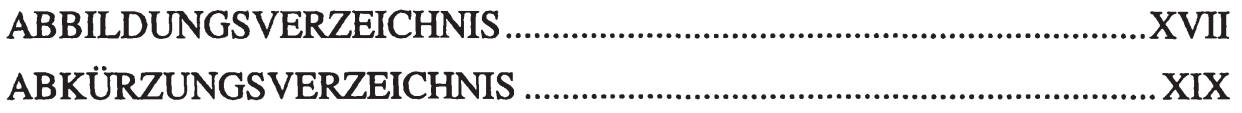

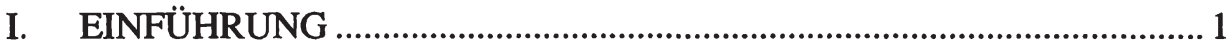

A. PROBLEMSTELLUNG UND ZIELSETZUNG................................................. 1

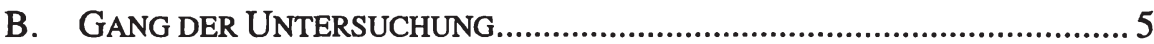

II. UNTERNEHMUNGSFÜHRUNG IN DER AKTIENGESELLSCHAFT .. 8

A. WESEN DER UNTERNEHMUNGSFÜHRUNG........................................... 8

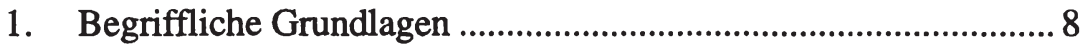

a) Begriff der Unternehmungsführung ....................................... 8

b) Relevante Führungsfunktionen ............................................ 11

(1) Planung ........................................................................ 11

(2) Entscheidung .............................................................. 12

(3) Kontrolle ....................................................................13

c) Hierarchische Vermaschung von Führungsprozessen ......... 16

2. Ziele als Handlungsmaßstäbe einer Unternehmung .................... 18

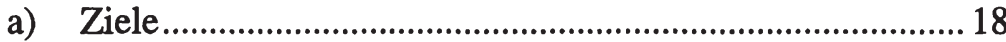

b) Zielbeziehungen ................................................................. 19

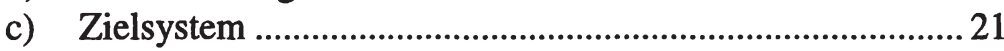

d) Anspruchsgruppen der Unternehmung ................................ 22

B. DIE SPITZENVERFASSUNG DER AKTIENGESELLSCHAFT .........................26

1. Grundlagen der Aktiengesellschaft...............................................26

a) Geschichtliche Entwicklung des Rechts der

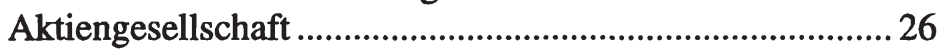

b) Konkretisierung des Untersuchungsobjekts.......................... 29

(1) Bedeutung und Charakteristika der

Publikumsaktiengesellschaft ......................................2 29

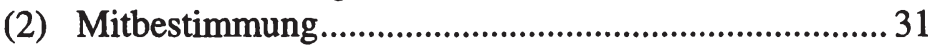

2. Ausgestaltung der Spitzenverfassung ......................................... 32

a) Die Verfassung der Aktiengesellschaft............................... 32

(1) Begriffliche Eingrenzung ............................................ 32

(2) Organe der Aktiengesellschaft .....................................34

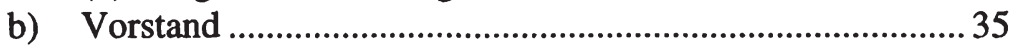

(1) Bildung und Zusammensetzung .................................... 35

(2) Aufgaben ..................................................................... 37

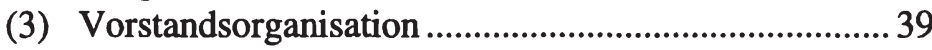

(a) Aufbauorganisation................................................39

(b) Ablauforganisation............................................... 42

(4) Verantwortlichkeit und Haftung der

Vorstandsmitglieder 


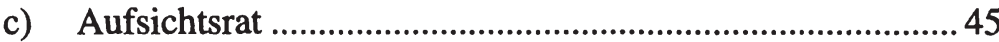

(1) Persönliche Anforderungen an

Aufsichtsratsmitglieder.

(2) Bildung und Zusammensetzung ...................................46

(3) Die Überwachungsaufgabe des Aufsichtsrats .............. 49

(a) Begriffliche Einordnung ......................................49

(b) Kontrolle .............................................................5 50

(c) Kontrollmaßstäbe.................................................52

(d) Einwirkungsmöglichkeiten ..................................53

(4) Aufsichtsratsorganisation .............................................5 57

(a) Überblick ..........................................................5

(b) Aufbauorganisation...............................................58

(i) Aufsichtsratsvorsitzender.............................58

(ii) Ausschüsse ..................................................5

(c) Ablauforganisation................................................62

(5) Verantwortlichkeit und Haftung der

Aufsichtsratsmitglieder....................................................63

C. ZIELERREICHUNG IN PUBLIKUMSAKTIENGESELLSCHAFTEN ..................66

1. Ökonomische Analyse der Spitzenverfassung.............................66

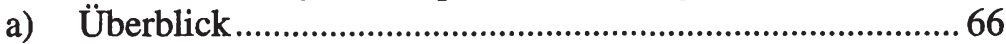

b) Verfügungsrechtsdelegation in der

Publikumsaktiengesellschaft ................................................68

(1) Verfügungsrechte........................................................68

(2) Externe Effekte ............................................................. 70

c) Principal Agent-Beziehungen als Folge der

Verfügungsrechtsdelegation..................................................71

d) Probleme in Principal Agent-Beziehungen............................73

e) Lösungsansätze zum Abbau der Principal Agent-

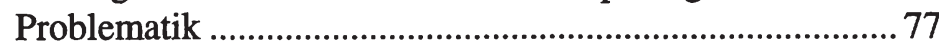

2. Der Shareholder Value-Ansatz als unternehmungspolitisches

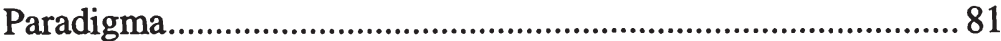

a) Überprüfung der Zweckmäßigkeit als unternehmerische

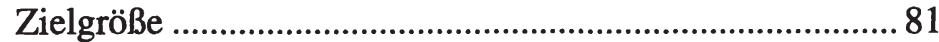

b) Verringerung der Principal Agent-Problematik ................... 87

c) Überprüfung der Rechtmäßigkeit als unternehmerische Zielgröße

D. FUNKTIONSFELDER DES AUFSICHTSRATS IN DER

UNTERNEHMUNGSFÜHRUNG

1. Planung als Instrument zum Abbau der Principal Agent-

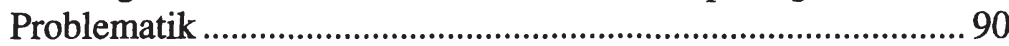

2. Planung als Prämisse einer ordnungsgemäßen Überwachung.... 93 
III. ENTWURF EINES AUFSICHTSRATSPLANUNGSSYSTEMS 98

A. ÜBERBLICK ZU DEN ELEMENTEN DES PLANUNGSSYSTEMS ...................98

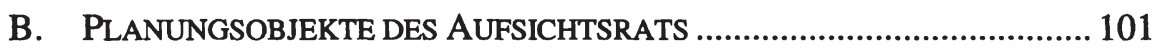

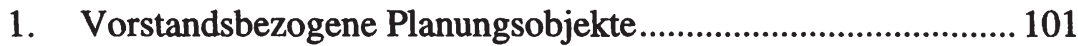

a) Organisatorische Planungsobjekte im Rahmen der Geschäftsordnungskompetenz............................................ 101

(1) Bedeutung der Geschäftsordnungskompetenz .......... 101

(2) Vorstandsorganisation ................................................ 103

(3) Zusammenarbeit von Vorstand und Aufsichtsrat...... 107

b) Personelle Planungsobjekte............................................... 108

(1) Besetzung des Vorstands.............................................. 108

(2) Gestaltung des Anreizsystems .....................................111

c) Die Berücksichtigung von Grundsätzen ordnungsmäßiger

Unternehmungsleitung durch den Aufsichtsrat.................. 113

2. Strategische Unternehmungsführung......................................... 117

a) Generelle Unternehmungsziele ........................................117

b) Strategische Kontrolle ...........................................................119

c) Planungs-, Organisations- und Kontrollsystem ................. 122

3. Jahresabschluß und Zusammenarbeit mit dem

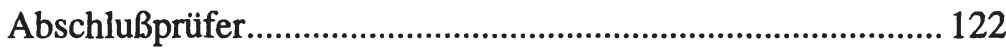

4. Organisatorische Angelegenheiten des Aufsichtsrats................ 124

C. DIE PlanUNGSORganisation DES AUfSICHTSRATS ...........................126

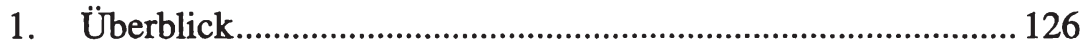

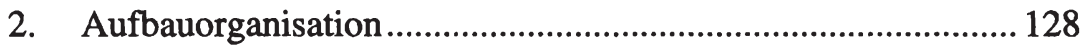

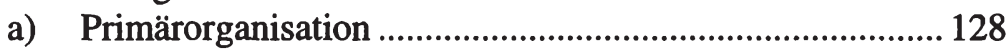

(1) Ressortierung des Aufsichtsrats ................................ 128

(2) Übertragung von Planungsaufgaben auf Ressorts..... 130

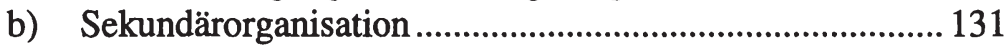

(1) Begriff und Gestaltungsprinzipien ............................ 131

(2) Stabsprinzip ............................................................ 132

(3) Arbeitsgruppenprinzip ..............................................133

(a) Überblick ....................................................... 133

(b) Empirische Analyse von Aufsichtsratsausschüssen ........................................................ 135

(c) Einrichtung von Planungsausschüssen .............. 138

(d) Entscheidungsdelegation an Ausschüsse............ 140

c) Kommunikationswegegestaltung ....................................... 143

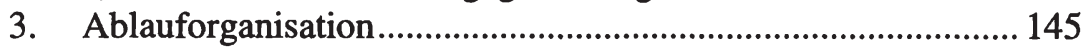

4. Personelle Angelegenheiten des Aufsichtsrats .......................... 146

D. Der AufsichtSRat als PlanUngSSUBJekT .................................... 148

1. Rollenverteilung im Planungsprozeß ...................................... 148 
2. Entwicklung eines Anforderungsprofils für

Aufsichtsratsmitglieder

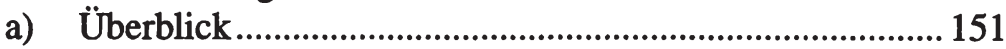

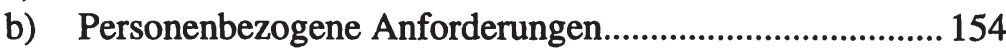

c) Managementbezogene Anforderungen ..............................156

(1) Allgemeine Charakterisierung .................................... 156

(2) Technische Kompetenz .............................................. 156

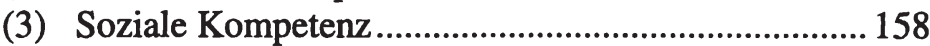

(4) Konzeptionelle Kompetenz ....................................... 159

E. GRUNDLAGEN EINES COMPUTERGESTÜTZTEN AUFSICHTSRATS-

UNTERSTÜTZUNGSSYSTEMS ALS PLANUNGSINSTRUMENT .................... 160

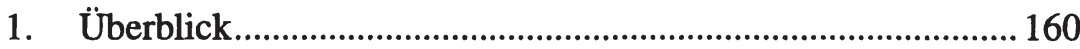

2. Das Informationsproblem des Aufsichtsrats.............................161

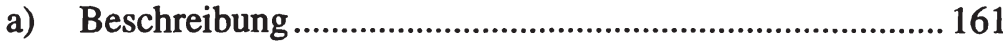

b) Informationsschrägverteilungen...........................................163

3. Das computergestützte Aufsichtsratsunterstützungssystem ..... 168

a) Systemtechnische Einordnung ……..................................... 168

(1) Managementunterstuitzungssysteme ........................... 168

(2) Weiterentwicklungen von Managementunterstützungssystemen ........................ 171

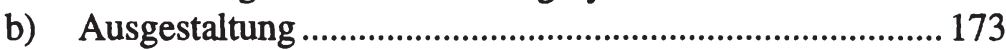

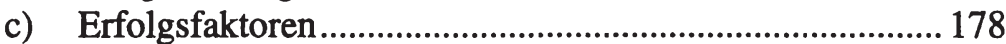

F. EXEMPLARISCHE GESTALTUNG EINES AUFSICHTSRATS-

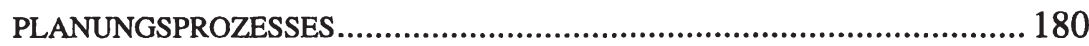

1. Einführung und Integrationsnotwendigkeit ............................. 180

2. Simultane Integration von Vorstandsorganisations- und

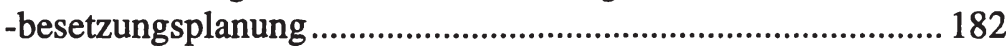

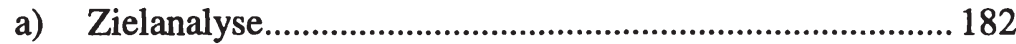

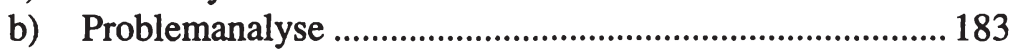

c) Alternativenanalyse ...........................................................185

d) Prognose, Bewertung und Entscheidung............................. 189

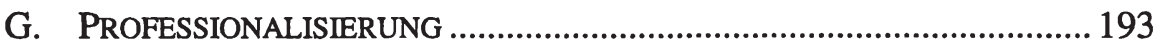

IV. VERHALTENSBEEINFLUSSUNG VON AUFSICHTSRATSMITGLIEDERN

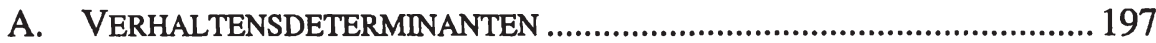

B. GeSTALTUNG DES MATERIELLEN ANREIZSYSTEMS ..............................200

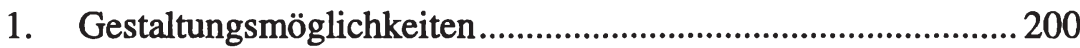

2. Analyse empirischer Befunde ....................................................203

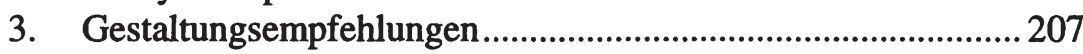

a) Wertorientierte Vergütungssysteme....................................2207

b) Anforderungen und Konsequenzen.................................... 209 
C. UNTERSTÜTZENDE SANKTIONSMECHANISMEN ................................214

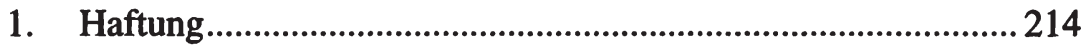

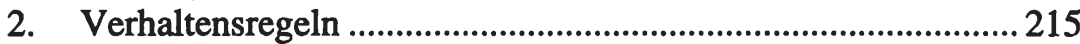

a) Grundsätze ordnungsmäßiger Überwachung .....................2215

b) Corporate Governance-Grundsätze ....................................2216

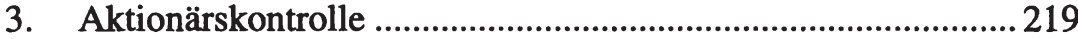

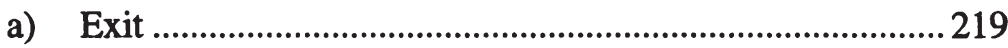

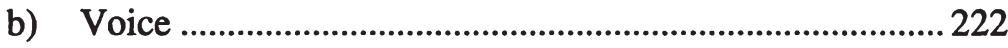

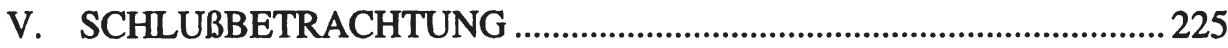

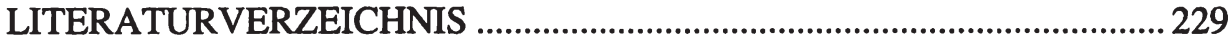

VERZEICHNIS DER RECHTSQUELLEN.................................................. 272 
Peter Ruhwedel - 978-3-631-75491-7 Downloaded from PubFactory at 01/11/2019 04:17:06AM 


\section{Abbildungsverzeichnis}

Abb. 1: Untersuchungsaufbau..................................................................

Abb. 2: Zusammenhang von Führungsaufgaben und -funktionen ...................9

Abb. 3: Mechanismen zur Zielkonfliktlösung .............................................. 21

Abb. 4: Die Unternehmung im Stakeholdernetzwerk ................................... 23

Abb. 5: Beiträge und Forderungen der verschiedenen Anspruchsgruppen .... 25

Abb. 6: Die Zusammensetzung des Aufsichtsrats nach dem MitbestG.......... 47

Abb. 7: Klassifikation der Überwachungsaufgabe ..........................................5 50

Abb. 8: Entscheidungsaufgaben des Aufsichtsrats ........................................ 55

Abb. 9: Dimensionen der Verdünnung von Property Rights............................69

Abb. 10: Probleme und Lösungsmöglichkeiten aus Sicht der Principal Agent-Theorie .................................................................................. 78

Abb. 11: Vertikale Arbeitsteilung im Leitungsorgan ..................................... 105

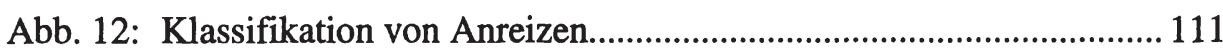

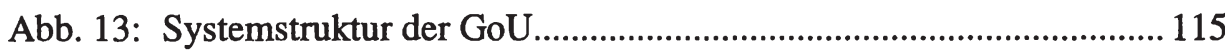

Abb. 14: Hauptstufen der Argumentationsrationalität....................................... 116

Abb. 15: Auszug aus der Satzung der ThyssenKrupp AG .............................. 121

Abb. 16: Aufsichtsratsausschüsse in DAX-Unternehmungen 1999 bzw. 1998/99.................................................................................. 136

Abb. 17: Kriterien zur Einrichtung von Planungs- oder

Entscheidungsausschüssen

Abb. 18: Planungs- und Entscheidungsinformationsbedarf, -angebot und -nachfrage ...................................................................................... 162

Abb. 19: Managementunterstützungssysteme …........................................ 170

Abb. 20: Referenzarchitektur für Analytische Informationssysteme ............. 172

Abb. 21: Anforderungen an MUS und AUS................................................... 174 
Abb. 22: Zusammenhang von Vorstandsorganisations- und -besetzungsplanung.

Abb. 23: Führungseigenschaften und Beurteilungskriterien in Abhängigkeit von Strategien 184

Abb. 24: Vorstandsorganisation in der Ausgangssituation. 186

Abb. 25: Vorstandsorganisation nach Neuzuschnitt des Ressorts. 186

Abb. 26: Entscheidungsmatrix zur integrierten Vorstandsorganisationsund -besetzungsplanung.....

Abb. 27: Verhältnis von durchschnittlicher Aufsichtsratsvergütung pro Kopf zur durchschnittlichen Vorstandsvergütung pro Kopf in \%.... 204 Abb. 28: Wertorientierte Vergütungssysteme. 208 


\section{Abkürzungsverzeichnis}

Abb.

Abs.

AG

AHGB

AktG

Anm.

Art.

AUS

BB

Bd.

BetrVG

BFuP

BGB

BGBl.

BGH

bzw.

CEO

CIS

CFROI

CSCW

DAV

DAX

DB

DBW

DCF

d.h.

DSS

DStR

DSW

DWS

EIS
Abbildung

Absatz

Aktiengesellschaft / Die Aktiengesellschaft (Zeitschrift)

Allgemeines Deutsches Handelsgesetzbuch

Aktiengesetz

Anmerkung

Artikel

(computergestütztes) Aufsichtsratsunterstützungssystem Betriebs-Berater (Zeitschrift)

Band

Betriebsverfassungsgesetz

Betriebswirtschaftliche Forschung und Praxis (Zeitschrift)

Bürgerliches Gesetzbuch

Bundesgesetzblatt

Bundesgerichtshof

beziehungsweise

Chief Executive Officer

Chefinformationssystem

Cash-Flow Return on Investment

Computer Supported Cooperative Work

Deutscher Anwaltverein

Deutscher Aktienindex

Der Betrieb (Zeitschrift)

Die Betriebswirtschaft (Zeitschrift)

Discounted Cash-Flow

das heißt

Decision Support System

Deutsches Steuerrecht (Zeitschrift)

Deutsche Schutzvereinigung für Wertpapierbesitz e.V.

Deutsche Gesellschaft für Wertpapiersparen $\mathrm{mbH}$

Executive Information System 
etc. et cetera

e.V.

eingetragener Verein

EVA

Economic Value Added

f. (ff.)

(fort)folgende Seite(n) / Spalte(n)

FAZ

Frankfurter Allgemeine Zeitung

FN

Fußnote

FuE

Forschung und Entwicklung

GG

Grundgesetz

GoF

Grundsätze ordnungsmäßiger Unternehmungsführung

GoU

Grundsätze ordnungsmäßiger Unternehmungsleitung

GoÜ

Grundsätze ordnungsmäßiger Überwachung

HGB

Handelsgesetzbuch

Hrsg.

Herausgeber

i.V.m.

in Verbindung mit

IuK-System

(computergestuitztes) Informations- und

Kommunikationssystem

Jh.

Jahrhundert

Jg.

Jahrgang

$\mathrm{kA}$

keine Angabe

KonTraG

Gesetz zur Kontrolle und Transparenz im Unternehmensbereich

KStG Körperschaftsteuergesetz

LG

Landgericht

m.w.N.

mit weiteren Nachweisen

Mio.

Millionen

MIS

Management Information System

MitbestG

Mitbestimmungsgesetz

Mrd.

Milliarden

MUS

Managementunterstützungssystem

NJW

Neue Juristische Wochenschrift (Zeitschrift)

$\mathrm{Nr}$.

Nummer

OECD

Organization for Economic Co-Operation and Development

o.J.

ohne Jahresangabe 
OLG

o.O.

o.S.

o.V.

OLAP

PIM

RGBl.

S.

s.

Sp.

syn.

$\mathrm{SzU}$

u.a.

US

USA

usw.

vgl.

VIS

vs.

WiSt

WISU

WM

WpG

z.B.

ZfB

$\mathrm{ZfbF}$

ZfhF

zfo

ZfP

ZGR

ZHR

ZIP
Oberlandesgericht

ohne Ortsangabe

ohne Seitenangabe

ohne Verfasser

On-Line Analytical Processing

Personal Information System

Reichsgesetzblatt

Seite / Satz

siehe

Spalte

synonym

Schriften zur Unternehmensführung

unter anderem / und andere

United States (Vereinigte Staaten)

United States of America (Vereinigte Staaten von Amerika)

und so weiter

vergleiche

Vorstandsinformationssystem

versus

Wirtschaftswissenschaftliches Studium (Zeitschrift)

Das Wirtschaftsstudium (Zeitschrift)

Wertpapier-Mitteilungen (Zeitschrift)

Die Wirtschaftsprüfung (Zeitschrift)

zum Beispiel

Zeitschrift für Betriebswirtschaft

Zeitschrift für betriebswirtschaftliche Forschung

Zeitschrift für handelswissenschaftliche Forschung

Zeitschrift Führung + Organisation

Zeitschrift für Planung

Zeitschrift für Unternehmens- und Gesellschaftsrecht

Zeitschrift für das gesamte Handels- und Wirtschaftsrecht

Zeitschrift für Wirtschaftsrecht und Insolvenzpraxis 
Peter Ruhwedel - 978-3-631-75491-7 Downloaded from PubFactory at 01/11/2019 04:17:06AM 


\section{Einführung}

\section{A. Problemstellung und Zielsetzung}

Aufsichtsräte von Aktiengesellschaften standen in der Vergangenheit aufgrund spektakulärer Unternehmungskrisen bei Balsam, coop, Metallgesellschaft oder VW und zuletzt bei Holzmann sowie der HypoVereinsbank massiv in der Kritik. ${ }^{1}$ In der öffentlichen Diskussion wurde insbesondere in Publikumsaktiengesellschaften ${ }^{2}$ eine Überwachungslücke des Aufsichtsrats diagnostiziert, die u.a. auf einen zu großen Einfluß der Banken, das „Oldboys“-Network in der „Deutschland $\mathrm{AG}^{\text {‘3 }}$, überlastete oder sogar inkompetente Mandatsträger und mangelhafte institutionelle Regelungen zur Verhinderung dieses persönlichen Fehlverhaltens zurückgeführt wurde. ${ }^{4}$

„Die gesetzlichen Funktionen werden nicht so wahrgenommen, wie der betriebswirtschaftlich versierte Betrachter der Normen dies erwarten darf, sondern es besteht für die gesamte Aufgabenstellung des Aufsichtsrats sozusagen eine informelle, aber sowohl nach der entsprechenden Unternehmenspraxis als auch nach der herrschenden $\mathrm{Ge}$ setzesinterpretation durch die Rechtswissenschaften konsensuale Verfahrens- und Vorgehensweise, die deutliche Unterschiede zu den gesetzlichen und statutarischen Vorgaben aufweisen. “5

Diese fehlende normative Kraft gesetzlicher Regelungen zeigt sich in verschiedenen Bereichen: Es kommt beispielsweise zu einer Aushöhlung der Willensbildung im Vorstand und einer unzureichenden Wahrnehmung seiner Personalkompetenz durch den Aufsichtsrat, die zu einer faktischen Kooptation der Aufsichtsratsmitglieder durch den Vorstand selbst führt. ${ }^{6}$ So beklagt MUTTER, daß

1 Einen Überblick zu spektakulären Fällen geben Hofmann, Aufsichtsrat, 1996, S. 40ff., und Wenger, Organisation, 1996, S. 175-180; aktuell Scheffler, Rollenverständnis, 2000, S. 433, sowie Schneider, Unternehmensüberwachung, 2000, S. 5-14; zu ähnlichen Problemen bereits Schmalenbach, Überwachungspflicht, 1910/1911, S. 271-283; kritisch zur Situation bei der Volkswagen AG Rother/Wildhagen/Fischer, Versagen, 1998, S. 44f.

2 Vgl. zu dieser und weiteren Formen von Publikumsgesellschaften (KGaA, GmbH \& Co. KG, eingetragene Genossenschaft) Jansch, Publikumsgesellschaften, 1999, S. 21-64.

3 Vgl. hierzu IWD, Deutschland AG, 2000, S. 6f.; Hillebrandt, Deutschland AG, 2001.

4 Vgl. zu einem Überblick Bernhardt, Aufsichtsrat, 1995, sowie Schmidt u.a., Corporate Governance, 1997, S. 109-121, u.a. mit einer Analyse der Verflechtungsstrukturen; auch Wagner, Aufsichtsgremien, 1998, S. 69; mit der Beschreibung sehr ähnlicher Probleme in amerikanischen Publikumsgesellschaften Hess, Corporate Governance, 1996, S. 14ff., sowie Werther Jr./Kerr, corporate governance, 1995, S. 63f.; mit einer kritischen Beurteilung des Aufsichtsratssystems im internationalen Vergleich Reischauer, Eitelkeit, 1999, S. 76-78.

5 Theisen, Aufsichtsrats-Informationssysteme, 1998, S. 85; zustimmend Scheffler, Rollenverständnis, 2000, S. 433.

6 Vgl. m.w.N. von Werder, Grundsätze, 1999, S. 2223; Dufey/Hommel/Riemer-Hommel, Corporate Governance, 1998, S. 54. 
die realiter zu beobachtende Auswahl zukünftiger Mitglieder des Vorstands durch aktive Vorstandsmitglieder bereits bei einigen Juristen zur Annahme entsprechender Pflichten des Vorstands führt, obwohl dies dem geltenden Aktienrecht widerspricht. ${ }^{7}$

Eine Mitte der 90er Jahre einsetzende Reformdiskussion zur „angemessenen Unternehmensorganisation“ (Corporate Governance ${ }^{8}$ ) deutscher Unternehmungen fand mit dem Gesetz zur Kontrolle und Transparenz im Unternehmensbereich (KonTraG) im Jahre 1998 ihren vorläufigen Höhepunkt. ${ }^{9}$ Nach umfangreichen und zum Teil konträren Beratungen wurden Teile der in der Literatur diskutierten Reformvorschläge aufgegriffen und entsprechende Änderungen des Aktiengesetzes (AktG) und des Handelsgesetzbuches (HGB) durchgeführt. ${ }^{10}$ Ziel des KonTraG war es nicht nur, den Fortbestand einer Unternehmung gefährdende Krisen zu verhindern, es sollte aufgrund der zunehmenden Bedeutung internationaler Kapitalmärkte für die Unternehmungsfinanzierung auch dazu beitragen, deutsche Aktiengesellschaften für ausländische Kapitalgeber attraktiver zu machen. ${ }^{11}$ Erst seit der Vorlage verschiedener Corporate GovernanceGrundsätze, beispielsweise des Vorschlags der Grundsatzkommission Corporate Governance $^{12}$, gewinnt die Rolle des Aufsichtsrats im Prozeß der Unternehmungsführung wieder verstärkt an Bedeutung. So fordert KOPPER die Übernahme einer aktiveren Rolle von Aufsichtsräten in den unternehmungsintern und

7 Vgl. m.w.N. Mutter, Aktiengesellschaft, 1994, S. 71.

8 Vgl. Feddersen/Hommelhoff/Schneider, Corporate Governance, 1996, S. 1; eine prozeßbezogene Sichtweise der Corporate Governance beispielsweise bei Hess, Corporate Governance, 1996, S. 10; zur hier vorgenommenen begrifflichen Abgrenzung Abschnitt II.B.2.a)(1).

9 Potthoff, Aufsichtsrat, 1998, S. I, ging sogar von dem Ende der Diskussion aus.

10 Vgl. umfassend AG, Sonderheft August 1997: Die Aktienrechtsreform 1997, sowie Zimmer, Gesetz, 1998, S. 3521-3534; Kienbaum, Aufsichtsratsstudie, 1997, kommt auf Grundlage einer Befragung von 116 amtierenden Aufsichtsratsmitgliedern zu dem Ergebnis, daß die Gesetzesänderungen des KonTraG überwiegend auf Zustimmung stoßen. Dies muß insoweit überraschen, als es bereits vorher zumindest teilweise im Ermessen der Aufsichtsratsmitglieder lag, solche Änderungen auf freiwilliger Basis durchzuführen. Ein ähnliches Ergebnis ergibt eine Befragung von Führungskräften börsennotierter Unternehmungen durch Förschle/Glaum/Mandler, Unternehmungen, 1998, S. 895.

11 Vgl. Allgemeiner Teil der Begründung des Regierungsentwurfs zum KonTraG in Ernst/Seibert/Stuckert, KonTraG, 1998, S. 29ff.; zu den Auswirkungen der Internationalisierung auf die Corporate Governance deutscher Unternehmungen umfassend Nassauer, Internationalisierung, 2000.

12 Vgl. Grundsatzkommission Corporate Governance, Corporate Governance-Grundsätze, 2000, S. 238-241; aktualisierte Version ist unter http://www.corgov.de/download/code0700d.pdf verfügbar; Mitglieder der Kommission, die einen Vorschlag zu Corporate Governance-Grundsätzen erarbeitet haben, sind Theodor Baums, Dieter Feddersen, Ulrich Hartmann, Ulrich Hocker, Robert Koehler, Rolf Nonnenmacher, Rüdiger von Rosen, Kim Schindelhauer, Uwe H. Schneider und Christian Strenger. 
unternehmensextern induzierten Veränderungsprozessen der Unternehmungen, indem er gemeinsam mit dem Vorstand die „Positionierung“ findet, die der Unternehmung den notwendigen Erfolg am Markt nachhaltig sichert. ${ }^{13}$ Mit der Vorlage des Berichts der Regierungskommission "Corporate Governance“ liegen neue Vorschläge vor, die zu einer Modernisierung des deutschen Aktienrechts beitragen wollen, um die internationale Kapitalmarktfähigkeit deutscher Unternehmungen zu verbessern. ${ }^{14}$

$\mathrm{Zu}$ einer erhöhten Wettbewerbsfähigkeit auf den Kapitalmärkten gehört neben weiteren Aspekten, beispielsweise einer Verbesserung der Rechnungslegungspublizität, ${ }^{15}$ insbesondere die Qualität der Arbeit von Aufsichtsräten. ${ }^{16}$ Sie dokumentiert sich jedoch nicht nur in der Vermeidung von krisenhaften Unternehmungssituationen (einige Autoren sprechen dem Aufsichtsrat sogar das Potential zur Vermeidung deliktisch begründeter Unternehmungskrisen wie bei Balsam oder Metallgesellschaft vollkommen $\mathrm{ab}^{17}$ ); die Bewertung der Arbeit von Aufsichtsräten muß sich vielmehr an ihrem Beitrag zur Erreichung der Unternehmungsziele orientieren. ${ }^{18}$ Eine Studie aus dem Jahr 2000 zeigt, daß Maßnahmen $\mathrm{zu}$ einer Verbesserung der Corporate Governance vom Kapitalmarkt tatsächlich honoriert werden könnten. ${ }^{19}$ Auf Grundlage einer Befragung kommen die Autoren der Studie zu dem Ergebnis, daß unzureichende GovernanceStandards zu höheren Renditeforderungen der Kapitalgeber führten:

„High governance standards will prove essential to attracting and retaining investors in globalized capital markets, while failure to reform is likely to hinder those companies with global ambitions. “20

So würden $79 \%$ aller befragten Investoren in Deutschland eine Prämie von durchschnittlich $20,2 \%$ für eine Unternehmung mit vorbildlicher Governance-

13 Vgl. Kopper, Vorwort, 1999, S. VIII; kritisch Peltzer/von Werder, Corporate Governance, 2001, S. 2 .

14 Vgl. Regierungskommission „Corporate Governance“, Unternehmensführung, 2001.

15 Vgl. hierzu Gassen, Rechnungslegungspublizität, 2000.

16 So auch Feddersen, Aufsichtsrat, 2000, S. 396.

17 Vgl. Lutter, Unternehmensverwaltung, 1995, S. 22; die Verantwortung des Vorstands betonend auch Wagner, Aufsichtsgremien, 1998, S. 68.

18 Vgl. zustimmend Bernhardt, Qualitätsmessung, 2000, S. 27; eine verstärkte Fokussierung der Corporate Governance-Diskussion auf den Erfolgsbeitrag des Aufsichtsrats fordert auch Sünner, Corporate Governance, 2000, S. 493.

19 Vgl. McKinsey, Investor, 2000, S. 3.

20 McKinsey, Investor, 2000, S. 3. 
Struktur bezahlen. Mit anderen Worten: Der Unternehmungswert könnte hierdurch signifikant gesteigert werden. ${ }^{21}$

Für den Aufsichtsrat ist daher die Forderung aufzustellen, daß er sein Handeln zukunftsbezogen auf die Verfolgung der Unternehmungsziele ausrichtet. Er soll im Rahmen seiner Überwachungstätigkeit die Geschäftsführung des Vorstands nicht nur ex-post zustimmend oder mißbilligend zur Kenntnis nehmen, als Sounding Board muß er Ziele und Strategien des Vorstands beurteilen, um Fehlentwicklungen möglichst frühzeitig entgegen zu wirken. ${ }^{22} \mathrm{Zu}$ den Aufgaben des Aufsichtsrats zählt jedoch nicht nur die Kontrolle des Vorstands, ihm kommen darüber hinaus originäre Entscheidungskompetenzen $\mathrm{zu}$, beispielsweise im Rahmen der Berufung von Vorstandsmitgliedern ( $\$ 84$ Abs. 1 S. 1 AktG) oder der Festlegung der Vorstandsbezüge ( $\$ 87$ Abs. 1 AktG). Zur Wahrnehmung seiner Aufgaben ist der Aufsichtsrat auf vielfältige zukunftsgerichtete Informationen angewiesen.

Zukunftsgerichtete Informationen können systematisch nur mit Hilfe der Unternehmungsplanung gewonnen werden. Daher wird hier als erste These vertreten, daß für den Aufsichtsrat eine entsprechende organisatorische, personelle und instrumentelle Unterstützung einer eigenen Planung notwendig ist. Mit Bezug auf die Unternehmungsplanung bedeutet dies, daß der Aufsichtsrat in ein Planungssystem eingebunden werden sollte, das für ihn näher zu konkretisieren ist. Hierbei läßt es sich aufgrund der Aufgabenkomplexität nicht vermeiden, daß Elemente oder Beziehungen des Aufsichtsratsplanungssystems redundant zu Elementen oder Beziehungen weiterer Subsysteme des Aufsichtsrats (z.B. des Kontrollsystems) sind. Die Ausgestaltung des Planungssystems hat die oben erwähnten Probleme zu berücksichtigen und sollte dazu beitragen, daß sie zukünftig vermieden werden können.

Nach LUTTER liegt eine weitere Ursache der Krise des Aufsichtsrats in dem oben bemängelten Fehlverhalten von Aufsichtsratsmitgliedern begründet. ${ }^{23}$ Verhaltensänderungen, die zu einer pflichtgemäßen Wahrnehmung des Aufsichtsratsmandats führen, werden jedoch nur dann erfolgen, wenn die Aufsichtsratsmitglieder durch entsprechende Sanktions- bzw. Anreizmechanismen hierzu motiviert werden. Neben einer erleichterten Haftung von Aufsichtsratsmitgliedern für schuldhaftes oder pflichtwidriges Verhalten ist eine Verhaltensänderung insbesondere durch eine verstärkte Teilhabe der Aufsichtsratsmitglieder am Unter-

\footnotetext{
21 Vgl. McKinsey, Investor, 2000, S. 16.

22 Vgl. Bleicher, Management, 1999, S. 211 .

23 Vgl. Lutter, Aufsichtsratstätigkeit, 1995, S. 295.
} 
nehmungserfolg zu erwarten. Während im Vorstandsbereich entsprechende Anreizsysteme inzwischen zum Standard gehören, kommt die Vergütung von Aufsichtsräten in der Regel noch immer eher einer Anwesenheitsprämie gleich. ${ }^{24}$ Nicht ohne Grund ist eine Form des Entgelts das sogenannte Sitzungsgeld. Daher lautet die zweite These, daß neben dem Aufsichtsratsplanungssystem ein Anreizsystem erforderlich ist, das eine verstärkte Ausrichtung der Aufsichtsratstätigkeit auf die Unternehmungsziele sicherstellt. Pflichtwidriges Verhalten muß durch geeignete Haftungsregelungen verhindert werden.

Ausgehend von der Forderung nach einer zukunftsorientierten Aufgabenerfüllung des Aufsichtsrats ist es Ziel dieser Arbeit, über die bisher im Vordergrund stehenden kontrollzentrierten Maßnahmen hinauszugehen und dem Aufsichtsrat neue Funktionsfelder in der Unternehmungsführung der Publikumsaktiengesellschaft zu eröffnen. Als Bestandteil eines umfassenden aufsichtsratsbezogenen Planungssystems soll er auf dieser Grundlage bei der Wahrnehmung seiner gesetzlich vorgesehenen Aufgaben unterstützt sowie durch ein entsprechendes Anreizsystem motiviert werden. Dieses Hauptziel der Untersuchung wird über zwei Subziele verfolgt: Das erste kognitive Ziel ist die Beschreibung und Erklärung der Notwendigkeit einer eigenständigen Planung durch den Aufsichtsrat zur Bewältigung seiner Aufgaben. Darauf aufbauend soll als zweites praktisches Ziel sowohl der Aufbau eines solchen Planungssystems als auch eines Anreizsystems verfolgt werden. Durch die Orientierung am rechtlichen Handlungsrahmen ist gewährleistet, daß die Gestaltungsvorschläge in der unternehmerischen Praxis grundsätzlich umsetzbar sind. Dort, wo einzelne Anregungen de lege ferenda erfolgen, müßten entsprechende gesetzliche Änderungen nachfolgen. $^{25}$

\section{B. Gang der Untersuchung}

Zur Erreichung des Ziels dieser Untersuchung ist im folgenden zweiten Kapitel zunächst das Gestaltungsfeld zu analysieren. Daher wird in Abschnitt II.A.1 der notwendige betriebswirtschaftliche „Begriffshaushalt“ vorgestellt. Dies beinhaltet insbesondere eine Beschreibung der Unternehmungsführung. Da Unternehmungsführung die Existenz von Unternehmungen voraussetzt, ist anschließend danach zu fragen, welche Anspruchsgruppen ein Interesse an Unternehmungen besitzen und welche Ziele sie mit der Teilnahme an der Unternehmung verfolgen (Abschnitt II.A.2). In Abschnitt II.B werden die für diese Arbeit erforderli-

\footnotetext{
24 Vgl. Kienbaum, Vergütungsstudie, 2000, S. 36, 56.

Vgl. Theisen, Reform, 1999, S. 220, der die lange Dauer einer Übernahme betriebswirtschaftlicher Erkenntnisse in Rechtsnormen bemängelt.
} 
chen juristischen Grundlagen erarbeitet, die dokumentieren, welche Vorstellungen der Gesetzgeber von der arbeitsteiligen Aufgabenerfüllung insbesondere zwischen Vorstand und Aufsichtsrat in Aktiengesellschaften hat. Er hat den Prozeß der Zielerreichung in Aktiengesellschaften über eine Delegation in Auftraggeber-/Auftragnehmerbeziehungen gestaltet. Eine institutionenökonomische Analyse der Spitzenverfassung (Abschnitt II.C.1) zeigt Probleme und erste Lösungsmöglichkeiten auf. Wie zu begründen sein wird, finden sich Lösungsmöglichkeiten u.a. in einer konsequenten Verfolgung des Shareholder ValueAnsatzes (II.C.2). Auf Grundlage der bis dahin vorgenommenen Ausführungen erfolgt in Abschnitt II.D die Begründung einer eigenständigen Planungsnotwendigkeit durch den Aufsichtsrat als Funktionserweiterung der Aufsichtsratstätigkeit.

Nachdem die notwendige Funktionserweiterung des Aufsichtsrats begründet wurde, wird in Kapitel III ein Aufsichtsratsplanungssystem entworfen. Auf Grundlage des institutionalen Planungsbegriffs werden hierzu seine Planungsobjekte ermittelt (Abschnitt III.B) und seine Planungsorganisation (Abschnitt III.C) gestaltet. Die anschließende Diskussion der Aufsichtsratsmitglieder als Planungssubjekte (Abschnitt III.D) bietet die Grundlage für die Formulierung ihrer Anforderungsprofile. Im Rahmen der in Abschnitt III.E analysierten instrumentellen Unterstützung werden Möglichkeiten zu einer informationstechnologischen Unterstützung der Aufsichtsratsmitglieder aufgezeigt. Die Diskussion der Elemente des Planungssystems schließt mit einer exemplarischen Gestaltung des Planungsprozesses (Abschnitt III.F). Hieran schließt sich eine kurze Betrachtung von Professionalisierungsmöglichkeiten (Abschnitt III.G) an.

Die Einführung von Teilen oder des gesamten Aufsichtsratsplanungssystems ist nur zu erwarten, wenn eine entsprechende Verhaltensbeeinflussung der Aufsichtsratsmitglieder (Kapitel IV) erfolgt. Ausgehend von der Analyse ihrer Verhaltensdeterminanten (Abschnitt IV.A) werden Gestaltungsvorschläge für das materielle Anreizsystem des Aufsichtsrats entwickelt (Abschnitt IV.B) und unterstützende Sanktionsmechanismen aufgezeigt (Abschnitt IV.C). Die Arbeit endet mit einer zusammenfassenden Schlußbetrachtung (Kapitel V). Den Untersuchungsaufbau verdeutlicht Abb. 1. 


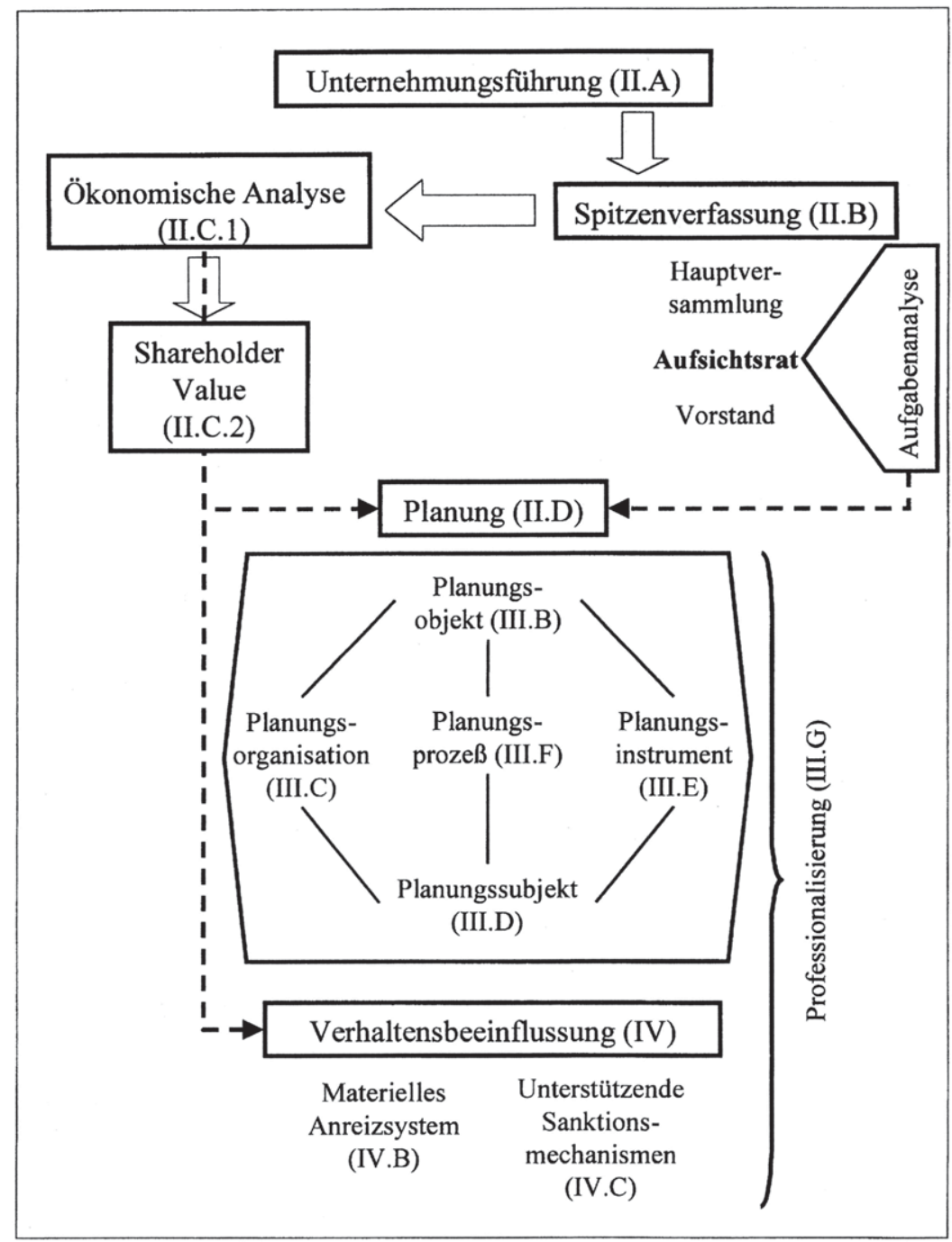

Abb. 1: Untersuchungsaufbau 


\section{Unternehmungsführung in der Aktiengesellschaft}

\section{A. Wesen der Unternehmungsfiuhrung}

\section{Begriffliche Grundlagen}

a) Begriff der Unternehmungsführung

Der Begriff Unternehmungsführung besteht aus den Teilbegriffen Unternehmung und Führung. Betrachtet man zunächst den Unternehmungsbegriff, ist in einer betriebswirtschaftlichen Untersuchung mit dem Erfahrungsobjekt der Aktiengesellschaft die Begriffsklärung sowohl aus einer juristischen als auch einer betriebswirtschaftlichen Perspektive möglich. Bei dem Versuch einer Aufklärung aus juristischer Sicht findet sich zwar das Unternehmen in einschlägigen Paragraphen des Aktiengesetzes, ${ }^{26}$ ohne aber eine eindeutige Umschreibung zu erfahren. ${ }^{27}$ Aktiengesetzlich handelt es sich bei Unternehmen um einen polysemantischen Begriff, der in Abhängigkeit von der Rechtsnorm unterschiedliche Begriffsinhalte haben kann, ${ }^{28}$ so daß die Abgrenzung aus betriebswirtschaftlicher Perspektive erfolgen soll.

Auch in der Betriebswirtschaftslehre existieren unterschiedliche Definitionen von Unternehmungen (syn. Unternehmen ${ }^{29}$ ). ${ }^{30}$ Sie seien die „versachlichte Institution der Tätigkeit des Unternehmers“, „Wirtschaftseinheiten [..], die ihren Erwerbsüberschuß an private Unternehmer abführen“ oder „für den anonymen Markt produzierende Betriebe “. ${ }^{31}$ Diese Begriffsbeschreibungen beinhalten für den hier zu verwendenden Unternehmungsbegriff relevante Charakteristika, ohne jedoch einzeln für eine abschließende Begriffsdefinition auszureichen. Daher soll auf eine systemtheoretische Definition zurückgegriffen werden. Danach wird unter einer Unternehmung ein offenes (Vielzahl von Umweltbeziehungen), dynamisches (intertemporale Variabilität der Elemente und Beziehungen), stochastisches (unsicheres Systemverhalten), komplexes (Vielzahl von Elementen und Beziehungen), sozio-technisches (Zusammenwirken menschlicher und maschineller Aufgabenträger) System verstanden. Das System verfolgt dauerhaft

\footnotetext{
26 Vgl. beispielsweise $\S 3,4,15 \mathrm{ff}$. AktG.

27 Vgl. Kropff, Aktiengesetz, 1965, S. $27 \mathrm{ff}$.

28 Vgl. Koppensteiner, Aktiengesellschaft, 1988, S. 157; demgegenüber ist der Unternehmerbegriff seit 2000 in § 14 BGB definiert.

29 So auch Wöhe, Einführung, 1990, S. 6, FN 4.

30 Vgl. Grochla, Unternehmung, 1993, Sp. 374ff.; für Kosiol, Organisation, 1976, S. 19, handelt es sich bei einer Unternehmung mit Bezug auf die Organisation um ein von Menschen geschaffenes Gebilde eigener Art.

31 Vgl. zu den Definitionen Grochla, Unternehmung, 1993, Sp. 380.
} 
(nicht nur einmalige Aufgabenerfüllung) durch die Erfüllung ökonomisch produktiver Aufgaben (Sach- bzw. Dienstleistungsproduktion) ein ökonomisches Ziel (beispielsweise Gewinn- oder Vermögensmaximierung). ${ }^{32}$

Zur Gewährleistung einer zielorientierten Gestaltung und Steuerung ist in Unternehmungen Führung notwendig, die die Erfüllung verschiedener Aufgaben erfordert: die Willensbildung, Willensdurchsetzung und Willenssicherung (vgl. Abb. 2): ${ }^{33}$

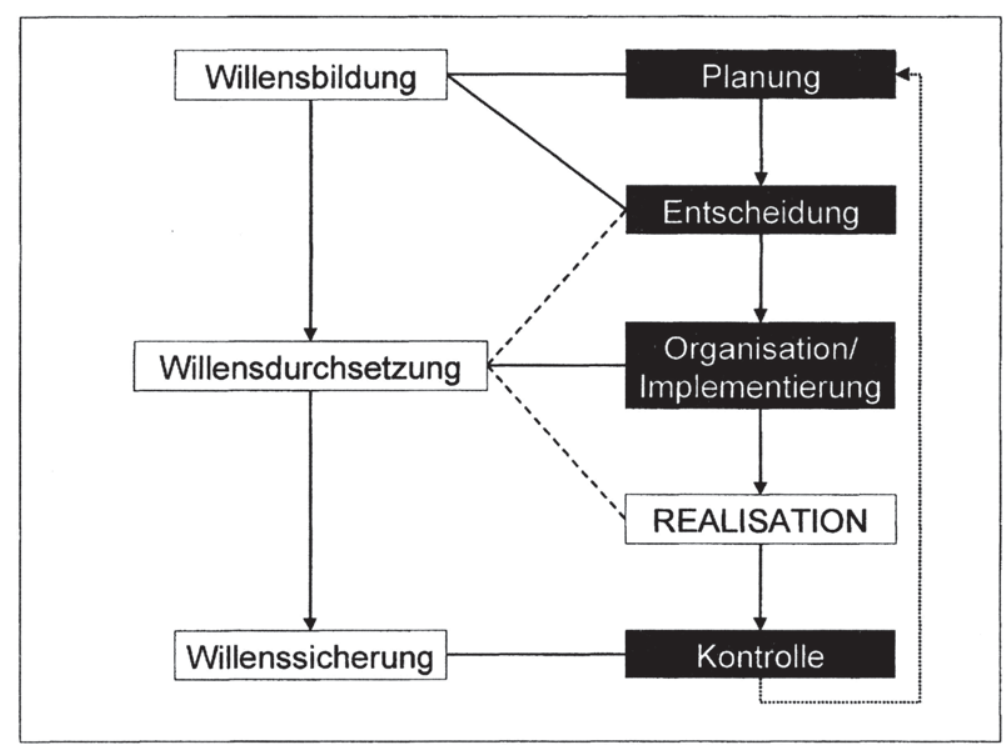

Abb. 2: Zusammenhang von Führungsaufgaben und-funktionen

Die Bewältigung dieser Aufgaben wird durch den Einsatz spezifischer Gestaltungsfunktionen bewerkstelligt, die hier synonym als Aufgaben bezeichnet werden sollen. ${ }^{34}$ Sie beschreiben aus funktionaler Perspektive Unternehmungsführung - da hier ausschließlich Unternehmungen betrachtet werden, soll zukünftig synonym von Führung gesprochen werden - als Führungsproze $B:{ }^{35}$ Planung als prospektives Denkhandeln ${ }^{36}$ zur Vorbereitung der Entscheidung, anschließend

32 Vgl. Mag, Unternehmung, 1978, S. 259ff.

33 Vgl. Steinmann/Schreyögg, Management, 2000, S. 6f.; Wild, Unternehmungsplanung, 1982, S. 13; zu den Aufgaben Mag, Unternehmensführung, 1992, S. 64; Mag, Unternehmungsplanung, 1995, S. 178; ähnlich Hahn, Planung, 1994, S. 44, jedoch ohne Willenssicherung; die Kontrolle zur Willensdurchsetzung zählend auch Heinen, Betriebswirtschaftslehre, 1985, S. 23; die besondere Bedeutung der Willensbildung betont Gutenberg, Einführung, 1985, S. 39f.

34 Vgl. Steinmann/Schreyögg, Management, 2000, S. 6f., die Aufgaben und Funktionen ebenfalls synonym verwenden; so auch schon Kosiol, Organisation, 1962, S. 45.

35 Vgl. Mag, Unternehmensführung, 1992, S. 60ff.

36 Vgl. Wild, Unternehmungsplanung, 1982, S. 13. 
die technische und materielle Vorbereitung der Realisation durch die Organisation bzw. Implementierung zum frühzeitigen Abbau von Veränderungswiderständen Betroffener und schließlich Kontrolle zur Ermittlung und Analyse von Abweichungen zwischen Planung und Realisation. STEINMANN/SCHREYÖGG sprechen bei der Planung kritisch von einer Primärfunktion in dem Sinne, daß alle anderen Funktionen ihre Bestimmung aus der Planung erfahren und so dem Regiment der Planung unterworfen seien. ${ }^{37}$ Alle Phasen des Führungsprozesses nicht jedoch die Realisierung, bei der es sich um keine Führungsfunktion handelt - sind reine Informationsprozesse und untereinander wiederum informatorisch verbunden. ${ }^{38}$ Über die bereits genannten Funktionen hinausgehende personelle Führungsaspekte (Führungsverhalten) bleiben hier unberücksichtigt. ${ }^{39}$

Neben dieser funktionalen Sichtweise als Führungsprozeß kann Unternehmungsführung als Institution bezeichnet werden. ${ }^{40}$ Aus dieser Perspektive werden die in Unternehmungen führenden Personen näher betrachtet, d.h. alle Personen, die Führungsaufgaben wahrnehmen. Die Führungskräfte beinhalten, vereinfachend klassifiziert, eine obere, mittlere und untere Führungsebene. Sie bilden insgesamt die Gruppe der Führungskräfte (syn. Manager ${ }^{41}$ bzw. Management) der Unternehmung. ${ }^{42}$ Zur Vermeidung begrifflicher Unklarheiten wird nur noch der funktionale Führungsbegriff Verwendung finden. Statt von Unternehmungsführung im institutionalen Sinne wird dagegen von den jeweiligen Personenmehrheiten gesprochen, die in Unternehmungen zur Führung legitimiert sein können.

Die vorliegende Problemstellung legt es nahe, die Betrachtung der Führungskräfte auf den Vorstand sowie den Aufsichtsrat zu fokussieren. Der Vorstand bildet in der Aktiengesellschaft den Kern der oberen Führung. Daneben zählt HAHN zur sogenannten externen Führung auch den Aufsichtsrat der Aktienge-

37 Vgl. Steinmann/Schreyögg, Management, 2000, S. 9, $123 \mathrm{ff}$.

38 Vgl. Wild, Unternehmungsplanung, 1982, S. 38.

39 So auch Mag, Unternehmensführung, 1992, S. 60; mit Berücksichtigung des Führungsverhaltens beispielsweise Bleicher, Management, 1999, S. 83.

40 Vgl. Hahn, Planung, 1994, S. 30; Mag, Unternehmensführung, 1992, S. 60; Steinmann/Schreyögg, Management, 2000, S. 6; mit einer engen Abgrenzung von Unternehmensführung beispielsweise Korndörfer, Unternehmensführungslehre, 1999, S. 23; zu weiteren möglichen Abgrenzungen Reiß/Corsten, Unternehmungsführung, 1995, S. 7.

41 Vgl. zur Mehrdeutigkeit von Manager unter Berücksichtigung einer industrieökonomischen Perspektive Steinmann/Schreyögg, Management, 2000, S. 6, mit Verweis auf Berle/Means, corporation, 1968, sowie Schreyögg/Steinmann, Verfügungsgewalt, 1981, S. 533-558.

42 Vgl. Hahn, Planung, 1994, S. 30f. 
sellschaft. ${ }^{43}$ Wie zu zeigen sein wird, nimmt der Aufsichtsrat zwar wichtige Führungsaufgaben wahr, da jedoch keine Weisungsbeziehung zwischen dem Aufsichtsrat und dem Vorstand besteht, ${ }^{44}$ sollen Mitglieder des Aufsichtsrats nicht zu den Führungskräften bzw. zum Management gerechnet werden. ${ }^{45}$

Aufgrund der im weiteren Verlauf der Arbeit besonderen Bedeutung des Planungs-, des Entscheidungs- sowie des Kontrollbegriffs werden diese nachfolgend genauer betrachtet. Der hier entwickelte Begriffsapparat ist die notwendige Voraussetzung für die spätere Einordnung der juristisch vorgesehenen Aufgaben des Aufsichtsrats.

\section{b) Relevante Führungsfunktionen}

\section{(1) Planung}

Für GUTENBERG ist Planung eine wesentliche Voraussetzung, um eine möglichst hohe Qualität von Entscheidungen sicherzustellen. ${ }^{46}$ Dabei wird mit dem funktionalen Planungsbegriff ein systematisch-methodischer Prozeß der Erkenntnis und Lösung von Zukunftsproblemen bezeichnet, der gewährleistet, daß Entscheidungen nicht auf unsystematischer Informationsbeschaffung und -auswertung fußen und damit eher an Improvisation erinnern, sondern auf Grundlage eines systematischen Informationsprozesses gefällt werden. ${ }^{47}$ Dabei können Informationen als entscheidungsorientiertes oder allgemeiner als zweckorientiertes Wissen definiert werden, das von einem oder von mehreren Entscheidungsträgern in einer konkreten Entscheidungssituation benötigt wird. ${ }^{48}$

Daneben bezeichnet der institutionale Planungsbegriff ein Entscheidungsvorbereitungssystem, das neben dem Planungsprozeß als weitere Elemente Planungsobjekte, Planungsorganisation, Planungssubjekte sowie Planungsinstru-

43 Die ebenfalls erwähnte Hauptversammlung bleibt hier unberücksichtigt.

44 Vgl. zur fehlenden Weisungsbeziehung zwischen Aufsichtsrat und Vorstand Lutter, Unternehmensplanung, 1991, S. 348, sowie Wagner, Aufsichtsgremien, 1998, S. 72.

45 Abweichend Hahn, Planung, 1994, S. 31, sowie Hutzschenreuter, Unternehmensverfassung, 1998, S. 13.

46 Vgl. Gutenberg, Grundlagen, 1983, S. 132.

47 Vgl. Wild, Unternehmungsplanung, 1982, S. 13; mit dem Versuch einer ethymologischen Herleitung des Planungsbegriffs beispielsweise Berens/Delfmann, Planung, 1995, S. 9.

48 Vgl. Mag, Unternehmungsplanung, 1995, S. 9; eine andere Abgrenzung mit Bezug auf den Aufsichtsrat beispielsweise bei Theisen, Information, 1996, S. 8, der Informationen als Maßnahmen bezeichnet, die ergriffen werden müssen, um den Aufsichtsrat mit Kenntnissen zu versorgen; die Definition von Information als entscheidungsorientiertes Wissen geht auf Wittmann, Unternehmung, 1959, S. 14f., zurück. 
mente beinhaltet. ${ }^{49}$ In diesem Kapitel wird der funktionale Planungsbegriff verwendet, der im nachfolgenden dritten Kapitel zum institutionalen erweitert wird.

Als gedankliche Vorstrukturierung späterer Entscheidungen zeichnet sich Planung durch bestimmte Merkmale aus: ${ }^{.0}$

1. Zukunftsbezogenheit: Planung findet im Zustand der unvollkommenen Informiertheit statt, da zukünftige Realisierungen vorgedacht werden. Hierzu müssen innerhalb der Planung Prognosen und Erwartungen gebildet werden.

2. Rationalität: Im Gegensatz zu intuitivem Handeln ist Planung ein methodisch-systematisches Vorgehen.

3. Gestaltungscharakter: Planung ist nicht nur auf das Erkennen von Problemen beschränkt, es sollen vielmehr Lösungsalternativen entwickelt und hinsichtlich ihrer Wirksamkeit beurteilt werden.

4. Informationsorientierung: Als Input benötigt Planung Informationen; in ihr werden aber auch Informationen generiert, gespeichert, verarbeitet und übertragen. $^{51}$

Das Ergebnis des Planungsprozesses ist eine Anzahl bewerteter Handlungsmöglichkeiten. Einer dieser Problemlösungsvorschläge wird mit der Entscheidung endgültig ausgewählt. ${ }^{52}$

\section{(2) Entscheidung}

Das Fällen von Entscheidungen macht das Wesen der Unternehmungsführung und damit eine der bedeutsamsten Tätigkeiten der Führungskräfte aus. ${ }^{53}$ Folgerichtig stehen Entscheidungen für UlRICH im Mittelpunkt des Führungsprozes-

49 Vgl. Mag, Unternehmungsplanung, 1995, S. 3; eine andere Aufzählung wählt Hammer, Unternehmensplanung, 1998, S. 36ff.

50 Vgl. Mag, Kontrolle, 1999, S. 4; Mag/Henselek/Ruhwedel, Personalplanung, 2000, S. 8; Wild, Unternehmungsplanung, 1982, S. 13f.; mit einer ähnlichen Aufzählung Kuhn, Unternehmensführung, 1990, S. 9.

51 Vgl. zur Bedeutung von Informationen für die Planung Wild, Unternehmungsplanung, 1982, S. $118 \mathrm{ff}$.

52 Vgl. Wild, Unternehmungsplanung, 1982, S. 41.

53 Vgl. Frese, Unternehmungsführung, 1987, S. 93; Gutenberg, Grundlagen, 1983, S. 131ff.; Heinen, Betriebswirtschaftslehre, 1985, S. 22ff.; Mag, Unternehmensführung, 1992, S. 61; Schildbach, Entscheidung, 1999, S. 70f.; zur entscheidungstheoretischen Sichtweise beispielsweise Homburg, Betriebswirtschaftslehre, 1998, S. 447-601; Mag, Entscheidungstheorie, 1990; Schildbach, Entscheidung, 1999, S. 65-105. 
ses. ${ }^{54}$ Entscheidungen sind zukunftsbezogene Willensakte, die dem Handeln vorausgehen und in der Regel nicht beobachtbar sind. ${ }^{55}$

Entscheidungen machen aus Handlungsmöglichkeiten Handlungen und stehen daher an der Schnittstelle zwischen gedanklicher und realisierender Phase des Führungsprozesses. ${ }^{56}$ Sie schließen die gedankliche Phase durch die Auswahl einer innerhalb der Planung entwickelten Handlungsalternative ab, über die Erklärung der Vollzugsverbindlichkeit der ausgewählten Alternative wird gleichzeitig die realisierende Phase eingeleitet. ${ }^{57}$ Ergebnis der Entscheidung ist der zur Realisierung vorgesehene Plan bzw. ein entsprechendes Programm (Komplex von Teilplänen). ${ }^{58}$ Planung kann insoweit als Planaufstellung bezeichnet werden, die Entscheidung dagegen als Planverabschiedung. ${ }^{59}$

Die Entscheidungsfunktion wird in der Literatur unterschiedlich eingegrenzt bzw. zugeordnet. Aufgrund ihrer herausgehobenen Bedeutung ist es gerechtfertigt, sie trotz einer ebenfalls möglichen Zuordnung zur Planung als eigenständige Führungsfunktion aufzuführen. ${ }^{60}$ Darüber hinaus ist es so möglich, eine unterschiedliche Aufgabenverteilung zwischen den einzelnen Phasen vorzunehmen. Dies ist für das Verhältnis von Planung und Entscheidung immer dann von besonderer Bedeutung, wenn Planer und Entscheider auseinanderfallen. ${ }^{61}$

\section{(3) Kontrolle}

Der zielorientierte Planvollzug soll mit Hilfe der Kontrollfunktion sichergestellt werden. ${ }^{62}$ Sie ist für WILD als Abschluß des Führungsprozesses die sinnstiftende Voraussetzung der Planung, da Planung ohne Kontrolle sinnlos sei und Kontrolle ohne Planung unmöglich. ${ }^{63}$

\footnotetext{
54 Vgl. Ulrich, Unternehmung, 1970, S. 204.

5s Vgl. Schneider, Entscheidungstheorie, 1995, S. 1.

56 Vgl. Mag, Unternehmensführung, 1992, S. 61.

57 Vgl. Mag, Unternehmungsplanung, 1995, S. 8.

58 Vgl. Wild, Unternehmungsplanung, 1982, S. 41.

59 Vgl. Hahn, Planung, 1994, S. 42, der hierbei von einer Planung im engeren Sinne und im weiteren Sinne spricht.
}

60 So auch Wild, Unternehmungsplanung, 1982, S. 116; sehr weit dagegen Heinen, Betriebswirtschaftslehre, 1985, S. 22, der auch alle Tätigkeiten der Planungs-, Vollzugs- und Kontrollphase, die im Zusammenhang mit der Entscheidung stehen, zum Entscheidungsprozeß rechnet; von einem die Entscheidung einschließenden Planungsverständnis ausgehend wohl auch Hahn, Planung, 1994, S. 41f.

61 Mit gleicher Begründung auch Wild, Unternehmungsplanung, 1982, S. 39.

62 Vgl. Gutenberg, Einführung, 1958, S. 51; Wild, Unternehmungsplanung, 1982, S. 44.

Vgl. Wild, Unternehmungsplanung, 1982, S. 44; so auch Staehle, Management, 1999, S. 539. 
Kontrolle kann allgemein als Vergleich von mindestens zwei Daten (Zeichen, Zeichenfolgen oder Symbole) definiert werden. ${ }^{64}$ Als Abschlußfunktion des Führungsprozesses leitet sie gegebenenfalls neue Planungen ein. So verstanden beinhaltet sie die Ermittlung von Ist-Werten, einen Vergleich von in der Planung bestimmten Soll-Werten mit diesen Ist-Werten sowie eine Abweichungsanaly$\mathrm{se}^{65}$

Diese Begrenzung der Kontrollfunktion auf eine führungsprozeßabschließende Soll-Ist-Kontrolle ist in der Literatur kritisiert worden. ${ }^{66}$ Es wurde insbesondere bemängelt, daß bei in ihren Konsequenzen weit in die Zukunft reichenden Entscheidungssituationen Probleme nicht frühzeitig genug festgestellt werden können, um geeignete Gegenmaßnahmen zu ergreifen. Daher wurden verschiedene Erweiterungen des Kontrollbegriffs vorgenommen, die auf eine führungsprozeßbegleitende Kontrolle hinauslaufen: die Planfortschrittskontrolle sowie die Prämissenkontrolle. ${ }^{67}$ Während bei der Planfortschrittskontrolle Teilziele bzw. Meilensteine definiert werden, die eine realisierungsbegleitende Kontrolle ermöglichen, soll die Prämissenkontrolle parallel zur Planrealisierung die Gültigkeit der prognostizierten Ausgangsannahmen der Planung (Planungsprämissen ${ }^{68}$ kontrollieren, da ein Wegfall der Planungsprämissen auch den Plan unbrauchbar werden läßt.

SCHREYÖGG hält auch dieses erweiterte Kontrollverständnis für unzulänglich. ${ }^{69}$ Er begründet dies mit der jeder Planung inhärenten Selektionsnotwendigkeit, die es aufgrund der Umweltkomplexität erforderlich mache, Teile der Umwelt bei der Formulierung des Planungsproblems unberücksichtigt zu lassen. Hierdurch entstehe in jedem Planungsprozeß ein gewisses Maß an Willkür. Durch die Unsicherheit von Prognosen werde dieses Problem zusätzlich verschärft, so daß zwei Modifikationen notwendig seien. Als erstes habe die Prämissenkontrolle nicht erst mit der Realisierung einzusetzen; sie sollte bereits parallel zur Planungserstellung beginnen. Hierdurch sollen die Selektionsentscheidungen im Planungsproze $ß$ von Beginn an fortlaufend auf ihre weitere Tragfähigkeit über-

\footnotetext{
64 Vgl. Mag, Kontrolle, 1999, S. 54; Wild, Unternehmungsplanung, 1982, S. 36.

65 Vgl. Frese, Unternehmungsführung, 1987, S. 184; zu weiteren oder engeren Begriffsabgrenzungen beispielsweise Kuhn, Unternehmensführung, 1990, S. 55, sowie Mag, Kontrolle, 1999, S. 55.

66 Vgl. m.w.N. Schreyögg, Kontrolle, 1994, S. 345ff.

67 Vgl. Kuhn, Unternehmensführung, 1990, S. 59; Mag, Kontrolle, 1999, S. 57; Wild, Unternehmungsplanung, 1982, S. 44.

68 Vgl. Mag, Kontrolle, 1999, S. 57.

69 Vgl. Schreyögg, Kontrolle, 1994, S. 348ff.
} 
prüft werden, um das strukturelle Risiko der Planung zu vermindern. ${ }^{70}$ Des weiteren müsse neben der Prämissenkontrolle eine allgemeine Überwachungskontrolle durchgeführt werden, um alle relevanten Vorgänge erfassen zu können, die nicht schon in den Planungsprämissen berücksichtigt worden sind. Es gelte, diejenigen Tatbestände nach Beginn der Planung zu erfassen, die in der Planungsphase fälschlicherweise ausgeblendet oder erst nach Abschluß der Prämissensetzung relevant wurden.

Im Ergebnis entsteht eine aus drei Kontrolltypen bestehende Kontrolle, wobei hier zur Vermeidung terminologischer Unklarheiten der Begriff der ,allgemeinen Überwachungskontrolle“ durch „ungerichtete Kontrolle“ ersetzt werden soll: ${ }^{71}$

1. die während bzw. am Ende der Realisierung durchzuführende zwischenund endergebnisorientierte Soll-Ist-Kontrolle, die als Realisierungskontrolle bezeichnet werden soll,

2. eine parallel zum Führungsprozeß erfolgende Prämissenkontrolle,

3. die den gesamten Führungsprozeß umspannende ungerichtete Kontrolle.

Bei der Kontrolle handelt es sich somit nicht nur um eine den Führungsprozeß abschließende Funktion, sie umfaßt mit ihren einzelnen Kontrolltypen den gesamten Führungsprozeß $B$ und gewinnt damit in dieser Ausprägung den Charakter einer zweiten, zum Führungsprozeß komplementären Funktion. ${ }^{72}$ Gerade in Situationen mit einem weit in der Zukunft liegenden Planungshorizont ist nur eine so verstandene Kontrolle in der Lage, die planungsimmanente Selektivität zu kompensieren. ${ }^{73}$ In solchen strategischen Entscheidungssituationen wird daher auch von einer strategischen Kontrolle gesprochen. ${ }^{74}$

Im Zusammenhang mit dem Begriff der Kontrolle wird oft auch von Überwachung gesprochen, wobei die Begriffe nicht immer einheitlich verwendet werden. ${ }^{75}$ Es findet sich teilweise eine synonyme Verwendung von Überwachung

70 Vgl. Schreyögg, Kontrolle, 1994, S. 350f.

71 Vgl. Steinmann/Schreyögg, Management, 2000, S. $245 \mathrm{ff}$.

72 So auch Heinen, Betriebswirtschaftslehre, 1985, S. 23.

73 Vgl. Steinmann/Schreyögg, Management, 2000, S. 245 f.

74 Vgl. zum Konzept der strategischen Kontrolle Schreyögg/Steinmann, Strategic Control, 1987, S. 91-103; Steinmann/Schreyögg, Management, 2000, S. 243-251.

75 Vgl. Theisen, Überwachung, 1987, S. 5ff., mit dem dort vorgenommenen umfassenden Begriffsüberblick; exemplarisch für die juristische Sichtweise beispielsweise Lutter, Prüfungsbefugnisse, 1992, Sp. 96ff.; mit einem unterschiedlichen Begriffsverständnis beispielsweise auch Er- 
und Kontrolle ${ }^{76}$ teilweise wird eine Hierarchisierung vorgenommen. ${ }^{77}$ Hier wird mit Überwachung die aktienrechtliche Gesamtaufgabe des Aufsichtsrats bezeichnet, die, wie zu zeigen sein wird, inhaltlich weiter ist als die betriebswirtschaftliche Kontrolle. ${ }^{78}$ Diese sichtenspezifische Trennung ist notwendig, da unter Überwachung aus juristischer Perspektive unterschiedliche Teilaufgaben gefaßt werden. ${ }^{79}$

Statt von Kontrolle wird teilweise auch von Prüfung (oder Revision ${ }^{80}$ ) gesprochen. ${ }^{81}$ Unter Prüfung soll hier jedoch eine retrospektive Untersuchung mehr oder weniger regelmäßig erhobener Sachverhalte verstanden werden, wie beispielsweise die Prüfung des Jahresabschlusses ( $\$ 316$ HGB) ${ }^{82}$ Daher wird dieser Begriff nur dann Verwendung finden, wenn der Prüfungscharakter einer Kontrollhandlung hervorgehoben werden soll.

\section{c) Hierarchische Vermaschung von Führungsprozessen}

Führungsprozesse treten prinzipiell auf jeder der angesprochenen Führungsebenen auf. ${ }^{83}$ WILD interpretiert eine Unternehmung daher als mehrstufige Hierarchie miteinander vermaschter Regelkreise. Für ihn vollzieht sich Führung nach diesem Regelungsmodell, so daß der Führungsprozeß der Gesamtunternehmung aus ineinandergeschachtelten, untergeordneten Führungsprozessen für die hierarchisierten Organisationseinheiten, d.h. für die verschiedenen Führungsebenen, besteht. ${ }^{84}$

Zur Unterscheidung unterschiedlicher Führungsaufgaben innerhalb dieser Hierarchie kann eine sachlich, zeitlich und institutionell begründete Einteilung in

lei/Leschke/Sauerland, Institutionenökonomik, 1999, S. 75, sowie Heinen, Betriebswirtschaftslehre, 1985, S. 23.

Vgl. Henn, Handbuch, 1998, S. 296ff.; Mertens, Kölner Kommentar, 1996, Anm. 28 zu § 111 AktG.

77 Vgl. Raiser, Kapitalgesellschaften, 1992, S. 117; in diesem Sinne auch Lutter, Prüfungsbefugnisse, 1992, Sp. $97 f$.

78 Anders dagegen Semler, Überwachung, 1996, S. 59, FN 155, der einen weiteren Kontrollbegriff verwendet und auf dieser Grundlage juristische Überwachung und betriebswirtschaftliche Kontrolle gleichsetzt.

79 Anders dagegen Theisen, Überwachung, 1993, Sp. 4218-4229.

80 Vgl. Zünd, Revisionslehre, 1982, S. 21.

81 Vgl. Lutter, Prüfungsbefugnisse, 1992, Sp. 97f.

82 Vgl. Thom, Kontrolle, 1993, Sp. 1141; zur Abgrenzung von Kontrolle und Prüfung beispielsweise auch Schewe/Littkemann/Beckermeier, Kontrollsysteme, 1999, S. 1484.

83 Vgl. Steinmann/Schreyögg, Management, 2000, S. 7; Wild, Unternehmungsplanung, 1982, S. 36.

84 Vgl. auch Hahn, Entwicklungstendenzen, 1999, S. 15; Hahn, Planung, 1994, S. 3ff.; Hahn, Unternehmungsführung, 1999, S. 29ff. 
die strategische, die taktische sowie die operative Führung erfolgen. ${ }^{85}$ Die strategische Führung steckt mit ihren grundlegenden, die gesamte Unternehmung betreffenden Entscheidungen den Handlungsrahmen der Unternehmung ab. Sie ist langfristig ausgerichtet, komplex und zeichnet sich durch ein großes $\mathrm{Maß}$ an Unsicherheit aus. Institutionell wird sie der oberen Führungsebene zugeordnet. Die taktische Führung der mittleren Führungsebene ist dagegen auf bestimmte unternehmerische Teilbereiche begrenzt und eher mittelfristig ausgerichtet. Die kurzfristige operative Führung der unteren Führungsebene schließlich bildet die Nahtstelle zur Realisierung. Sie konzentriert sich auf Anweisungen an Mitarbeiter der Realisationsebene.

Da mit dem Vorstand und dem Aufsichtsrat die oberste Führungsebene der Unternehmung angesprochen ist, erfolgt im vorliegenden Problemzusammenhang eine Beschränkung auf die strategische Ebene. HAHN spezifiziert verschiedene Aufgabenkomplexe der strategischen Führung: ${ }^{86}$

1. Festlegung der unternehmungspolitischen generellen Ziele,

2. Geschäftsfeldstrategieplanung sowie grundlegende Planung der Funktionsbereichs- und Regionalstrategien,

3. Planung der Organisations- und Rechtsstruktur sowie -form,

4. Planung des Führungskräftesystems einschließlich des Führungskräfteplanungssystems, des Anreizsystems und des Informationssystems,

5. Gestaltung und Durchführung der Steuerungs- und Kontrollprozesse.

HAHN zählt auch die Gestaltung der Unternehmungskultur sowie die Festlegung der Unternehmungsphilosophie zu den Führungsaufgaben. Sie werden hier jedoch lediglich als nicht näher betrachtete Rahmenbedingungen erfaßt, da sowohl die Unternehmungskultur als auch die Unternehmungsphilosophie nur in Ansätzen bewußt gestaltbar sind und somit dem rationalen Gestaltungsanspruch der Unternehmungsführung nur begrenzt zugänglich scheinen. ${ }^{87}$

Im Mittelpunkt der strategischen Führung steht das integrierte Planungssystem der Unternehmung. Es besteht neben der generellen Zielplanung zur Festle-

85 Vgl. Henselek, Management, 1996, S. $11 \mathrm{ff}$.

86 Vgl. Hahn, Unternehmungsführung, 1999, S. 35; mit einer Übersicht zu verschiedenen Konzepten der strategischen Führung Hahn, Konzepte, 1998, S. 563-579, sowie Henselek, Management, 1996, S. 16-23.

87 So auch Bleicher, Management, 1999, S. 73ff.; die Unternehmungskultur ebenfalls als Rahmenbedingung der Planung einordnend Mag, Unternehmungsplanung, 1995, S. 171; zur Unternehmungskultur beispielsweise Steinmann/Schreyögg, Management, 2000, S. 603ff. 
gung der Unternehmungspolitik, der strategischen Planung (Programm-, Potential- und Potentialstrukturplanung) und der operativen Planung (Programm- und Aktionsplanung bei gegebenen Potentialen) zusätzlich aus der gesamtunternehmungsbezogenen Ergebnis- und Finanzplanung sowie aus spezifischen Projektplanungen, die im Gegensatz zu den übrigen Planungen aperiodisch stattfinden. Die generelle Zielplanung sowie die strategische und die operative Planung werden mittels der monetären und nichtmonetären Planungs- und Kontrollrechnung quantifiziert. In Anknüpfung an das hier zugrunde gelegte Kontrollverständnis ist es nicht ausreichend, Kontrolle nur als Abschluß des strategischen Führungsprozesses zu begreifen; sie muß im Sinne einer strategischen Kontrolle den gesamten Führungsproze $ß$ beginnend mit der Planung begleiten.

Innerhalb eines Führungsprozesses kommt den Zielen eine zentrale Funktion $\mathrm{zu}^{88} \mathrm{Da}$ Ziele entwickelt und koordiniert, geprüft sowie in Maßnahmen und Entscheidungen umgesetzt werden müssen und darüber hinaus bei Abweichungen geeignete Anpassungsmaßnahmen und gegebenenfalls Zieländerungen vorzubereiten sind, kommt auch der Planung eine wichtige Rolle innerhalb dieser Prozesse zu.

\section{Ziele als Handlungsmaßstäbe einer Unternehmung}

\section{a) Ziele}

Unternehmungsführung wurde als zielorientierte Gestaltung und Steuerung von Unternehmungen definiert. Es ist daher zu klären, um welche Ziele es sich für die hier zu untersuchenden Unternehmungen handeln könnte und welche Probleme bei deren Formulierung und Durchsetzung möglich sind. Bevor dies geschehen kann, muß jedoch zunächst der Ziel- bzw. Unternehmungszielbegriff ${ }^{89}$ näher abgegrenzt und erläutert werden.

Ein Vergleich unterschiedlicher Zieldefinitionen zeigt, daß Ziele übereinstimmend als zukünftig angestrebte Zustände der Realität beschrieben und als Beurteilungsmaßstäbe verwendet werden. ${ }^{90}$ Sie können als Aussagen mit normativem Charakter definiert werden, die einen von einem Entscheidungsträger gewünschten, von ihm oder anderen anzustrebenden, zukünftigen Zustand der Realität beschreiben. ${ }^{91}$ Diese Unternehmungsziele dienen den Führungskräften als Beurteilungsmaßstab zukünftiger Handlungen, d.h., sie werden sowohl als Entschei-

88 Vgl. Wild, Unternehmungsplanung, 1982, S. 36.

89 Da nur Ziele für Unternehmungen untersucht werden, werden beide Begriffe synonym verwendet.

90 Vgl. zu einem Überblick unterschiedlicher Zielbegriffe Macharzina, Unternehmensführung, 1999, S. 155.

91 Vgl. Hauschildt, Entscheidungsziele, 1977, S. 9. 
dungskriterium für die Auswahl einer möglichst zielentsprechenden Alternative als auch als Vergleichsmaßstab für das realisierte Ist verwendet. Optimale Entscheidungen können daher auch als zielentsprechende Entscheidungen bezeichnet werden. ${ }^{92}$ Ziele sind aber nur dann in der Lage, diese handlungsleitende Funktion für Unternehmungen zu erfüllen, wenn sie eindeutig beschrieben und operationalisiert sind.

Zur eindeutigen Beschreibung sind Ziele in drei Dimensionen festzulegen: ${ }^{93}$

1. Der Zielinhalt nennt anzustrebende ökonomische oder nichtökonomische Zielgrößen, von denen hier aufgrund des Untersuchungsobjekts nur ökonomische Ziele relevant sind. ${ }^{94}$ Ökonomische Ziele können weiter in das Formalziel (beispielsweise Gewinnerzielung) oder leistungserstellungsbezogene Sachziele unterschieden werden. ${ }^{95}$ Beide Zielgruppen sind jedoch nicht als gleichgewichtig anzusehen. Das leistungserstellungsbezogene Sachziel ist dem ökonomischen Formalziel untergeordnet:

„Gewinnerzielung stellt den Primäreffekt betrieblicher Betätigung dar, die Leistungserstellung dagegen den Sekundäreffekt, insofern Leistungserstellung Mittel zum Zweck maximaler Gewinnerzielung ist. “"96

2. Die Zielvorschrift legt fest, ob die Zielgröße minimal bzw. maximal (Extremierung) werden soll oder ob sie genau (Fixierung), mindestens (Satisfizierung mit Untergrenze) oder höchstens (Satisfizierung mit Höchstgrenze) zu erreichen ist.

3. Die Zieldauer bezeichnet den Gültigkeitszeitraum des Ziels; in Abhängigkeit von der zeitlichen Erstreckung können kurzfristige (Nah-)Ziele und langfristige (Fern-)Ziele unterschieden werden.

Die Beschreibung der Zielinhalte zeigt, daß in Unternehmungen mehrere Zielgrößen gleichzeitig verfolgt werden können. Es ist daher nach der Art der Beziehungen zwischen diesen Zielen zu fragen.

\section{b) Zielbeziehungen}

In Abhängigkeit von der Art der Beziehung einzelner Ziele kann Zielidentität (Ziele stimmen überein), Zielkomplementarität (positive Korrelation zwischen

\footnotetext{
Vgl. Grochla, Einführung, 1978, S. 12.

93 Vgl. Heinen, Betriebswirtschaftslehre, 1985, S. 98ff.; Macharzina, Unternehmensführung, 1999, S. 157f.; Mag, Entscheidungstheorie, 1990, S. 29ff.; Mag, Unternehmungsplanung, 1995, S. 47f.

94 Vgl. die anfangs gefundene Definition von Unternehmung in Abschnitt II.A.1.a).

95 Vgl. Kuhn, Unternehmensführung, 1990, S. 30; Staehle, Management, 1999, S. 440.

96 Gutenberg, Grundlagen, 1983, S. 465.
} 
Zielen), Zielindifferenz (keine Beziehung zwischen Zielen), Zielkonkurrenz (negative Korrelation zwischen Zielen) oder Zielantinomie (Ziele schließen sich gegenseitig aus) vorliegen, ${ }^{97}$ wobei jedoch nur die Fälle der Zielkomplementarität sowie der Zielkonkurrenz praktische Relevanz besitzen. ${ }^{98}$ Im Fall komplementärer Zielgrößen kann eine Hierarchisierung der Ziele in Ober-, Zwischen- und Unterziele eine Lösung bringen.

Liegen dagegen konfliktäre Beziehungen zwischen einzelnen Zielgrößen vor, sind leistungsfähige Lösungsmechanismen zu finden, die die negative Korrelation der Zielgrößen berücksichtigen. ${ }^{99}$ Hier ist neben der Zielgewichtung bzw. mischung mit den Unterfällen der Zieldominanz und des Zielkompromisses vor allem die einfache und mehrfache Zielrangordnung zu nennen. Bei der Zieldominanz wird der Zielkonflikt gelöst, indem nur noch das Hauptziel berücksichtigt wird, die weiteren Ziele bleiben vollkommen unberücksichtigt (monistische Zielvorstellung). Der Zielkompromiß versucht dagegen, eine echte Mischung herbeizuführen. Hierbei liegt das Problem in der Ermittlung der Gewichtungsfaktoren. Zudem müssen verschiedendimensionale Einzelziele in Nutzengrößen transformiert und anschließend zu einem Gesamtziel synthetisiert werden. Im Fall der praktisch bedeutsamen einfachen Rangordnung wird ein Hauptziel unter der Berücksichtigung von Nebenbedingungen (Nebenzielen) verfolgt, wobei diese als Restriktionen bei der Erreichung des Hauptziels wirken. Bei der Zielrangordnung können verschiedendimensionierte Ziele ohne eine Transformation in Nutzenziffern gleichzeitig berücksichtigt werden. So ist es beispielsweise möglich, einem Geschäftsbereich eine Zielumsatzrendite von $12 \%$ bei gleichzeitiger Beachtung der Nebenbedingung „Keine betriebsbedingten Kündigungen! " vorzugeben. Wird die Nebenbedingung nicht eingehalten, ist die zugehörige Alternative nicht zulässig. Bei der Festlegung der Restriktionen ist darauf zu achten, daß nicht alle Handlungsmöglichkeiten ausgeschlossen werden, d.h., die Nebenbedingungen sind in einem angemessenen Verhältnis zu berücksichtigen. Die mehrfache Rangordnung löst den Zielkonflikt, indem nicht nur eine Rangordnung für das Vorrangziel gebildet wird, sondern für alle konkurrierenden Ziele. Einen zusammenfassenden Überblick der Lösungsmöglichkeiten für Zielkonflikte gibt Abb. 3.

97 Vgl. Mag, Entscheidungstheorie, 1990, S. 32f.

98 Vgl. Heinen, Betriebswirtschaftslehre, 1985, S. 101ff.; Macharzina, Unternehmensführung, 1999, S. 158; Mag, Unternehmungsplanung, 1995, S. 49ff.; zu Mehrfachzielen auch Mag, Mehrfachziele, 1976, S. 49-55.

99 Vgl. Mag, Entscheidungstheorie, 1990, S. 38ff.; Mag, Mehrfachziele, 1976, S. 54f. 


\begin{tabular}{|c|c|c|c|c|}
\hline \multicolumn{5}{|c|}{ Mechanismen zur Zielkonfliktlösung } \\
\hline & \multicolumn{2}{|c|}{ Zielgewichtung } & \multicolumn{2}{|c|}{ Zielrangordnung } \\
\hline & Zielkompromi $\beta$ & Zieldominanz & $\begin{array}{c}\text { Einfache } \\
\text { Rangordnung }\end{array}$ & $\begin{array}{c}\text { Mehrfache } \\
\text { Rangordnung }\end{array}$ \\
\hline $\begin{array}{l}\text { Charakteri- } \\
\text { sierung }\end{array}$ & \begin{tabular}{|l|} 
Gewichtung \\
der einzelnen \\
Zielgrößen mit \\
Faktoren \\
ungleich Null \\
(echte \\
Mischung)
\end{tabular} & $\begin{array}{l}\text { Gewichtung } \\
\text { einer Zielgröße } \\
\text { mit Eins, aller } \\
\text { übrigen mit } \\
\text { Null (unechte } \\
\text { Mischung) }\end{array}$ & $\begin{array}{l}\text { ein Ziel wird } \\
\text { zum Hauptziel, } \\
\text { die übrigen } \\
\text { Ziele sind } \\
\text { Nebenbedin- } \\
\text { gungen } \\
\text { (Nebenziele), } \\
\text { die satisfiziert } \\
\text { werden } \\
\text { müssen }\end{array}$ & $\begin{array}{l}\text { lexikographi- } \\
\text { sche Anord- } \\
\text { nung der } \\
\text { einzelnen Ziele }\end{array}$ \\
\hline $\begin{array}{l}\text { Zielverhältnis } \\
\text { nach Verfah- } \\
\text { rensdurchfüh- } \\
\text { rung }\end{array}$ & $\begin{array}{l}\text { jedem Ziel } \\
\text { kommt eine } \\
\text { eigenständige } \\
\text { Bedeutung bei } \\
\text { der Verfolgung } \\
\text { des Gesamt- } \\
\text { ziels zu; eine } \\
\text { identische } \\
\text { Gewichtung } \\
\text { zeigt eine } \\
\text { gleich hohe } \\
\text { Bedeutung der } \\
\text { Ziele für den } \\
\text { Entscheider; } \\
\text { unterschied- } \\
\text { liche Gewich- } \\
\text { tungsfaktoren } \\
\text { drücken seine } \\
\text { Zielpräferenz } \\
\text { aus }\end{array}$ & $\begin{array}{l}\text { aus Sicht des } \\
\text { Entscheiders } \\
\text { ist nur eine } \\
\text { Zielgröße von } \\
\text { Bedeutung }\end{array}$ & $\begin{array}{l}\text { sowohl Haupt- } \\
\text { als auch } \\
\text { Nebenziele } \\
\text { sind für den } \\
\text { Entscheider } \\
\text { relevante } \\
\text { Zielgrößen }\end{array}$ & $\begin{array}{l}\text { sowohl Haupt- } \\
\text { als auch } \\
\text { Nebenziel sind } \\
\text { für den } \\
\text { Entscheider } \\
\text { grundsätzlich } \\
\text { relevante } \\
\text { Zielgrößen, } \\
\text { wobei jedoch } \\
\text { unter Umstän- } \\
\text { den nicht alle } \\
\text { Nebenziele } \\
\text { berücksichtigt } \\
\text { werden }\end{array}$ \\
\hline
\end{tabular}

Abb. 3: Mechanismen zur Zielkonfliktlösung

\section{c) Zielsystem}

Die systematische Ordnung der unterschiedlichen Zielgrößen in einer Unternehmung wird als Zielsystem bezeichnet. ${ }^{100}$ Ausgehend von einem oder mehreren Oberzielen erfolgt unter Berücksichtigung von Ursache-WirkungsZusammenhängen eine Ableitung derivativer Ziele. Die Dekomposition operationaler Subziele entwickelt Handlungsmaßstäbe für die in einer Unternehmung agierenden Personen. MACHARZINA gibt einen Überblick unterschiedlicher Mo-

100 Vgl. Heinen, Betriebswirtschaftslehre, 1985, S. 106ff.; Macharzina, Unternehmensführung, 1999, S. $157 \mathrm{ff}$. 
delle von Unternehmungszielsystemen, kommt jedoch zu dem Schluß, daß ein generelles Modell bisher noch nicht vorgelegt wurde. ${ }^{101}$ Während in den älteren Zielsystemen ${ }^{102}$ eine Dominanz finanzieller Zielgrößen festzustellen ist, greifen jüngere Entwicklungen wie die Balanced Scorecard ${ }^{103}$ explizit weitere, nichtfinanzielle Größen auf, wodurch eine simultane Betrachtung von leistungswirtschaftlichen und finanziellen Zielgrößen erfolgt.

Die detaillierte Ausgestaltung eines Zielsystems hängt von den obersten Unternehmungszielen $a b$. Dies sind mit der Unternehmungspolitik festzulegende generelle Unternehmungsziele, die als Leitlinien, Normen und Prinzipien die Unternehmung als Ganzes betreffen. ${ }^{104}$ Generelle Ziele werden für einen längeren Zeitraum oder sogar unbefristet auf der Grundlage der Unternehmungsverfassung festgelegt; sie können jedoch, beispielsweise ausgelöst durch Veränderungen in den Unternehmungsumfeldern, variiert werden. Ihre primäre Funktion besteht in der grundsätzlichen Ausrichtung der Unternehmung. Dies geschieht über die Gestaltung eines die Unternehmung und ihre Mitglieder verbindlich zusammenfügenden Rahmens fundamentaler Leitsätze.

Bisher ist unbeantwortet geblieben, an welchen oder wessen Zielen Handlungen in Unternehmungen auszurichten sind. Unter Rückgriff auf die Definition von Unternehmungen offene Systeme liegt es nahe, potentiell zielbeeinflussende Gruppen im Unternehmungskontext zu identifizieren. ${ }^{105}$

\section{d) Anspruchsgruppen der Unternehmung}

Unternehmungen erfüllen keinen Selbstzweck, sie entstehen durch individualrationale Überlegungen als Instrument zur Verwirklichung eigener Interessen von an der Unternehmung bzw. ihren Aktivitäten beteiligten Personen oder Personengruppen. ${ }^{106}$ Somit kann eine Unternehmung weder eigene Bedürfnisse noch eigene Ziele haben; diese werden durch näher zu bestimmende Stakeholder ${ }^{107}$ der Unternehmung empfunden bzw. gesetzt. Ausgangspunkt einer Zielermitt-

101 Vgl. Macharzina, Unternehmensführung, 1999, S. 160ff.

102 Vgl. beispielsweise das DuPont-Kennzahlensystem bei Davis, performance, 1950 (1967), sowie das ZVEI-Kennzahlensystem in ZVEI, ZVEI-Kennzahlensystem, 1989.

103 Vgl. Kaplan/Norton, Balanced scorecard, 1997; Horváth, Balanced Scorecard, 1998.

104 Vgl. Bleicher, Organisation, 1991, S. 4; Bleicher, Management, 1999, S. 74, 264; Hahn, Unternehmungsziele, 1999, S. 305; Mag, Unternehmungsplanung, 1995, S. 1566.

Vgl. Bleicher u.a., Spitzenorganisation, 1984, S. 21; Schmidt, Unternehmung, 1977, S. 48ff.; Staehle, Management, 1999, S. 437; zum methodologischen Individualismus Richter/Furubotn, Institutionenökonomik, 1996, S. 3.

Englisch „stake“: (Wett-, Spiel-)Einsatz, Interesse oder Risiko übernehmen. 
lung sind daher nicht Ziele der Unternehmung, sondern die Bedürfnisse und Interessen bzw. Ziele von Individuen. ${ }^{108}$

$\mathrm{Zu}$ den Stakeholdern einer Unternehmung können alle Personen(-gruppen) gezählt werden, die in der Unternehmung etwas einsetzen, an der Unternehmung bzw. an ihrem Wirken interessiert sind oder in dieser ein Risiko tragen. ${ }^{109}$ Mit Bezug auf die Ziele der Unternehmung konkretisiert FREEMAN:

„A stakeholder in an organization is [...] any group or individual who can affect or is affected by the achievement of the organization's objectives. “110

Dies sind u.a. Anteilseigner, aber auch Arbeitnehmer, Top-Manager, Kunden, Lieferanten oder ganz allgemein die Öffentlichkeit (vgl. Abb. 4). ${ }^{11}$

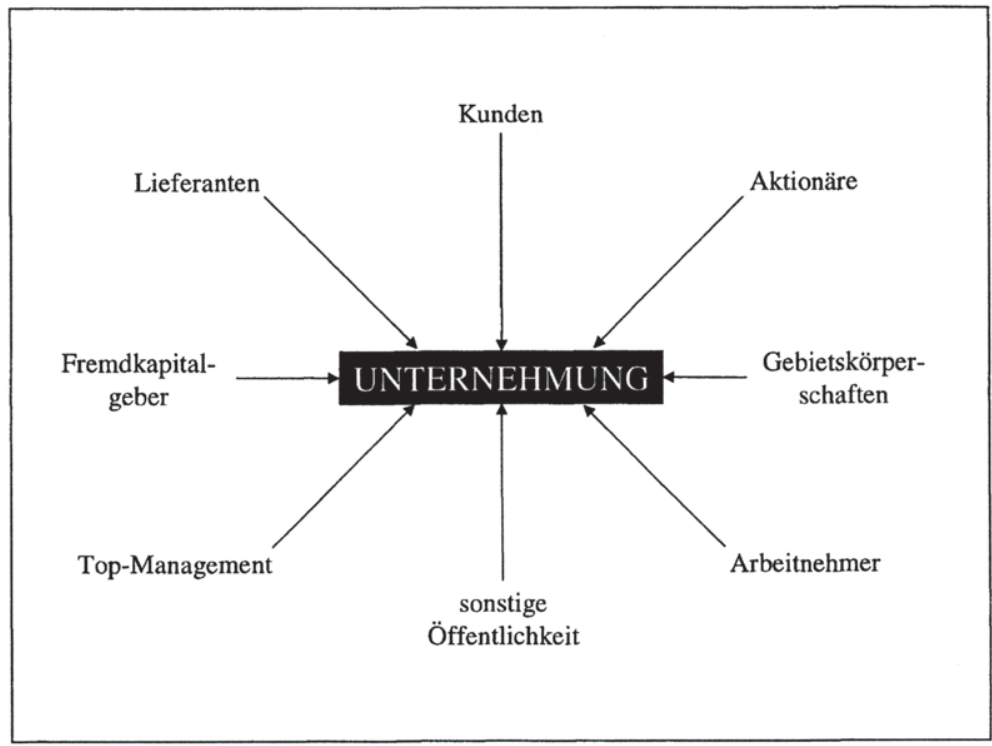

Abb. 4: Die Unternehmung im Stakeholdernetzwerk

Quelle: In Anlehnung an Baetge, Gesellschafterorientierung, 1997, S. 106.

108 Vgl. Drukarczyk, Finanzierung, 1993, S. 19.

109 Vgl. zum Stakeholder-Ansatz grundlegend Cyert/March, Theory, 1963; Dill, Environment, 1958, S. 409-433; zur differenzierten Unterscheidung einzelner Stakeholder-Kategorien s. Achleitner, Unternehmungen, 1985, S. 76.

110 Freeman, Strategic management, 1984, S. 46; ähnlich mit Bezug auf Entscheidungen in Unternehmungen auch Steinmann/Schreyögg, Management, 2000, S. 75f.

111 Vgl. Freeman, Strategic management, 1984 S. 55; zur Gestaltung einer interessenpluralistischen Unternehmungsverfassung auf der Grundlage einer koalitionstheoretischen Sicht der Unternehmung Steinmann/Gerum, Unternehmung, 1978; kritisch hierzu Wagner, Shareholder Value, 1997, S. $487 f f$. 
Zur Gruppe des Managements, genauer des Top-Managements, zählen alle Personen, die Rechte der Eigentümer unmittelbar wahrnehmen, beispielsweise in der Aktiengesellschaft der Vorstand.

Um generelle Unternehmungsziele als Ausgangspunkt des Zielsystems ableiten zu können, muß zunächst Klarheit über die Interessen und Bedürfnisse der einzelnen internen und externen Stakeholdergruppen bestehen. Die in Abb. 5 aufgezeigten Gegenleistungsansprüche der Anspruchsgruppen, d.h. ihre mit Hilfe der Unternehmung verfolgten Ziele, sind unterschiedlich und teilweise sogar gegensätzlich. $^{112}$

\begin{tabular}{|c|c|c|}
\hline Anspruchsgruppe & $\begin{array}{l}\text { Erbrachte Leistung fïr } \\
\text { die Unternehmung }\end{array}$ & $\begin{array}{c}\text { Geforderte } \\
\text { Gegenleistung von der } \\
\text { Unternehmung }\end{array}$ \\
\hline \multicolumn{3}{|c|}{ Interne Anspruchsgruppen: } \\
\hline Aktionäre & - Eigenkapital & $\begin{array}{ll}\text { - } & \text { Einkommen } \\
\text { - Wertsteigerung des } \\
\text { Kapitals } \\
\text { - Einfluß auf die } \\
\text { Unternehmungspolitik }\end{array}$ \\
\hline Top-Management & $\begin{array}{ll}\text { - } & \text { Kompetenz } \\
\text { - } & \text { Engagement } \\
\text { - } & \text { Leistung } \\
\end{array}$ & $\begin{array}{ll}\text { - } & \text { Einkommen } \\
\text { - } & \text { Macht und Prestige } \\
\text { - } & \text { Selbstverwirklichung }\end{array}$ \\
\hline Arbeitnehmer & $\begin{array}{l}\text { - Einordnung in die } \\
\text { Unternehmung } \\
\text { - Übernahme } \\
\text { fremdbestimmter } \\
\text { Tätigkeiten } \\
\text { - Leistung }\end{array}$ & $\begin{array}{ll}\text { - } & \text { Einkommen } \\
\text { - } & \text { Arbeitsplatzsicherheit } \\
\text { - } & \text { Arbeitszufriedenheit } \\
\text { - } & \text { Aus- und Fortbildung } \\
\text { - } & \text { Selbstverwirklichung }\end{array}$ \\
\hline \multicolumn{3}{|c|}{ Externe Anspruchsgruppen: } \\
\hline Fremdkapitalgeber & - Fremdkapital & $\begin{array}{ll}\text { - } & \text { fristgemäße } \\
\text { Zinszahlung } \\
\text { - } \\
\text { vereinbarungsgemäße } \\
\text { Tilgung } \\
\end{array}$ \\
\hline Lieferanten & $\begin{array}{ll}\text { - } & \text { termingerechte } \\
& \text { Leistung } \\
\text { - } & \text { hochwertige Güter }\end{array}$ & $\begin{array}{l}\text { langfristige und } \\
\text { sichere Geschäfts- } \\
\text { beziehung } \\
\text { - Zahlungsfähigkeit des } \\
\text { Abnehmers }\end{array}$ \\
\hline
\end{tabular}

112 Vgl. Bühner/Tuschke, Shareholder Value, 1997, S. 503; als Kernkonflikt wird oftmals das Verhältnis von Anteilseignern und Arbeitnehmern gesehen; vgl. Richter, Konzeption, 1996, S. 3; von Werder, Shareholder Value, 1998, S. 74; der Nachweis einer langfristigen Interessenkonvergenz dagegen bei Bughin/Copeland, Shareholder Value, 1997. 


\begin{tabular}{|c|c|c|}
\hline Kunden & $\begin{array}{l}\text { - Kauf der Produkte } \\
\text { - Markentreue }\end{array}$ & $\begin{array}{l}\text { - qualitativ und } \\
\text { quantitativ } \\
\text { angemessenes Preis- } \\
\text { Leistungsverhältnis } \\
\text { - Service }\end{array}$ \\
\hline $\begin{array}{l}\text { Sonstige Öffentlichkeit } \\
\text { und } \\
\text { Gebietskörperschaften }\end{array}$ & $\begin{array}{l}\text { - öffentliche Sicherheit } \\
\text { und Ordnung } \\
\text { - Infrastruktur } \\
\text { - international } \\
\text { wettbewerbsfähiges } \\
\text { Rechtssystem }\end{array}$ & $\begin{array}{ll} & \text { Steuerzahlung } \\
\text { - } & \text { Sicherung der } \\
\text { Arbeitsplätze } \\
\text { - Einhaltung von } \\
\text { Rechtsvorschriften }\end{array}$ \\
\hline
\end{tabular}

Abb. 5: Beiträge und Forderungen der verschiedenen Anspruchsgruppen

Quelle: In Anlehnung an Schmid, Anspruchsgruppen-Konzept, 1997, S. 633.

Aus Sicht der sogenannten Stakeholder-Theorie ist es Aufgabe der Unternehmungsleitung, einen Interessenausgleich zwischen allen Einzelinteressen der Stakeholder herbeizuführen. Hierbei soll eine Wertschaffung oder -steigerung alle Interessengruppen berücksichtigen, so daß die Summe der Einzelnutzen maximiert wird (Stakeholder Value) ${ }^{113}$ Dies bedeutet als Zielkonfliktlösungsmechanismus eine Zielgewichtung. BÜHNER/TUSCHKE bemängeln jedoch, daß es dem Stakeholder-Ansatz weder gelänge, die Gruppe relevanter Stakeholder abschließend abzugrenzen, noch eine angemessene Balance zwischen den einzelnen Interessen herbeizuführen, so daß sie insgesamt konstatieren, daß der Stakeholder Value-Ansatz keine klare Zielfunktion aufweist. ${ }^{114}$ Daneben ist der durch die Trennung von Eigentum und Verfügungsmacht entstehende Konflikt zwischen Aktionären und Managern evident, da die Manager durch die Ausnutzung diskretionärer Handlungsspielräume eigene Ziele zu Lasten der Anteilseigner verfolgen können. ${ }^{115}$ Diese Gefahr würde durch eine Stakeholderorientierung noch vergrößert. Im Gegensatz dazu liegt mit dem Shareholder Value-Ansatz eine eindeutige Konzeption vor, die es erlaubt, operationale Unternehmungsziele zu ermitteln und gleichzeitig diskretionäre Handlungsspielräume des Managements zu verringern. ${ }^{116}$ Bevor die Konkretisierung der Unternehmungsziele auf

113 Vgl. grundlegend Freeman, Strategic management, 1984, mit einem Literaturüberblick auf S. 3147; Busse von Colbe, Shareholder Value, 1997, S. 272; Schmid, Anspruchsgruppen, 1997, S. 634f.; kritisch zur Ermittlung eines Stakeholder Value Gomez, Shareholder Value, 1995, Sp. 1728.

114 Vgl. m.w.N. Bühner/Tuschke, Shareholder Value, 1997, S. 502f.; ähnlich Wagner, Shareholder Value, 1997, S. 492.

115 Vgl. Bühner/Tuschke, Shareholder Value, 1997, S. 514; Steinmann/Schreyögg, Management, 2000, S. 94.

116 Vgl. Bühner/Tuschke, Shareholder Value, 1997, S. 500, 514; zum Shareholder ValueManagement grundlegend Rappaport, Shareholder-Value, 1986. 
der Grundlage des Shareholder Value-Ansatzes erfolgt, sollen aufgrund ihrer Bedeutung zunächst die rechtlichen Rahmenbedingungen näher berücksichtigt werden, die einen wesentlichen Einfluß auf die Zielerreichung in Aktiengesellschaften ausüben. ${ }^{117}$

\section{B. Die Spitzenverfassung der Aktiengesellschaft}

\section{Grundlagen der Aktiengesellschaft}

\section{a) Geschichtliche Entwicklung des Rechts der Aktiengesellschaft}

Zum Verständnis der Ausgangssituation des Aufsichtsrats ist es hilfreich, die historische Entwicklung der Aktiengesellschaft unter besonderer Berücksichtigung des Aufsichtsrats zu betrachten. Der Ursprung der Aktiengesellschaft findet sich in den im 17. Jh. in Europa entstandenen Handelskompagnien, die der Kapitalaufbringung für überseeische Unternehmungen dienten. ${ }^{118}$ Mit ihrer Gründung wurden drei Ziele verfolgt: Zunächst konnte durch die Kapitalaufnahme bei einer Vielzahl von Anlegern der immense Kapitalbedarf der Unternehmungen befriedigt werden, daneben konnten die Anteilseigner mit einer vergleichbar geringen Einlage verbriefte Kapital- und Gewinnanteile (Anteil[schein], niederländisch „Aktie“) erwerben und schließlich gleichzeitig das Risiko auf die Höhe ihrer Kapitaleinlage beschränken. Solche Personenvereinigungen gewannen durch staatliche Verleihung - Octroi - körperschaftlichen Charakter, wodurch die Haftung der Kapitalgeber nach außen ausgeschlossen wurde. Die Gesellschaften wurden von den Hauptanteilseignern geleitet, die hierzu ein Direktorium zur Führung und zur Überwachung bildeten. ${ }^{119}$ Wesentlichen Einfluß auf die Gesellschaften übte der Staat aus, dem umfassende Überwachungs- und Mitspracherechte zukamen. Einen Aufsichtsrat oder ein ähnliches Gremium kannten die Handelskompagnien noch nicht.

Die im 18. Jh. in Deutschland stark zunehmende Bedeutung der Aktiengesellschaft als Rechtsform von Unternehmungen fand erst mit dem Preußischen Gesetz über die Aktiengesellschaften von 1843 eine erste generelle aktienrechtliche Regelung. ${ }^{120}$ Der Staat fungierte als Überwachungsorgan der Gesellschaften und überließ die Gestaltung und Funktionszuweisung der Organe weitgehend den individuellen Gesellschaftssatzungen. Wie im Octroisystem unterlag die Grün-

\footnotetext{
117 Vgl. Kräkel, Management, 1999, S. 259ff.; Steinmann/Schreyögg, Management, 2000, S. 101ff.

118 Vgl. Gadow u.a., Aktiengesetz, 1961, S. 1; Lehmann, Aktiengesellschaften, 1898, S. 4ff.; Raiser, Kapitalgesellschaften, 1992, S. 3f.

119 Vgl. Lehmann, Code de Commerce, 1895.

120 Vgl. Bleicher/Leberl/Paul, Unternehmungsverfassung, 1989, S. 52; Raiser, Kapitalgesellschaften, 1992, S. 3f.
} 
dung von Aktiengesellschaften auch in diesem Konzessionssystem dem staatlichen Genehmigungsvorbehalt. ${ }^{121}$

Mit dem Allgemeinen Deutschen Handelsgesetzbuch (AHGB) 1861 erfolgte erstmals eine vollständige Kodifizierung des Rechts der Aktiengesellschaft, zu diesem Zeitpunkt jedoch noch nicht als eigenständiges Gesetz. ${ }^{122}$ Weiterhin wurde die Grundlegung der inneren Ordnung von Aktiengesellschaften mit dem Vorstand zur Führung der Geschäfte der Gesellschaft, der Zuweisung gewisser Mindestkompetenzen an die Generalversammlung als Vertretung der Anteilseigner sowie einem fakultativen Aufsichtsrat als Überwachungsorgan vorgenommen. ${ }^{123}$ Aufgrund der unzureichenden Beschreibung seiner Funktion und der gesellschaftlichen Satzungsfreiheit beschränkte sich die Aufsichtsratstätigkeit zumeist jedoch nicht auf die Überwachung des Vorstands; er nahm auch Führungsaufgaben wahr und ähnelte hierdurch mehr dem bis dahin üblichen Verwaltungsrat.

Mit der Aktienrechtsnovelle 1870 wurde die Einrichtung eines Aufsichtsrats aufgrund der vollständigen Abschaffung staatlicher Überwachung und Genehmigungsvorbehalte Pflicht (Artikel 209 Nr. 6 AHGB 1870). ${ }^{124}$ Die Aktionäre mußten von nun an selbst für die Wahrnehmung ihrer Interessen Sorge tragen. Das „Überwachungssubstitut" Aufsichtsrat ist somit von seiner Grundkonzeption als ein auf die Interessen der Anteilseigner ausgerichtetes Organ zu bezeichnen. ${ }^{125}$ PASSOW zeigt jedoch, daß die Einführung des überwachenden Aufsichtsrats ein Zufallsprodukt war. ${ }^{126}$ Eine rein sprachliche Umbenennung des Verwaltungsrats der Aktienkommanditgesellschaft in einen Aufsichtsrat habe bei der Abschaffung der Staatsaufsicht für Aktiengesellschaften und der hierdurch ausgelösten Suche nach einem anderen Aufsichtsinstrument zu einer inhaltlichen Änderung der Aufgaben dieses Organs geführt. Da jedoch keine Beschränkung der aufsichtsratlichen Tätigkeit auf die Überwachung des Vorstands erfolgte, nahmen Vorstand und Aufsichtsrat weiterhin zum Teil in Personalunion gemeinsam die Leitung der Gesellschaft wahr. ${ }^{127}$ Dies führte in Verbindung mit einer unzureichenden Wahrnehmung der Überwachungsfunktion zu einer

\footnotetext{
121 Vgl. Gadow u.a., Aktiengesetz, 1961, S. 1.

122 Vgl. Assmann, Aktiengesetz, 1992, Anm. 71ff.

123 Vgl. Baltzer, Aufsichtsrat, 1983, S. 118.

124 Vgl. Hahn, Handelsgesetzbuch, 1871, S. 640; Lehmann, Aktiengesellschaften, 1898, S. 79.

125 Vgl. Hahn, Handelsgesetzbuch, 1871, S. 592f., 599; auch Monopolkommission, Leitlinien, 1998, S. 82.

126 Vgl. Passow, Aktiengesellschaft, 1909, S. 28, 50f.

127 Vgl. Baltzer, Aufsichtsrat, 1983, S. 119 f.
} 
ersten Krise der Aktiengesellschaft als Rechtsform. Der Gesetzgeber reagierte auf diese Krise des Aktienrechts mit einer zweiten Aktienrechtsnovelle von 1884, mit der die Generalversammlung als Vertretung der Anteilseigner gestärkt sowie eine gleichzeitige Zugehörigkeit zu Aufsichtsrat und Vorstand einer Aktiengesellschaft verboten wurde. ${ }^{128}$ Zudem war die Einrichtung eines Verwaltungsrats neben Vorstand und Aufsichtsrat nicht mehr möglich. Es bestand für den Aufsichtsrat jedoch weiterhin die Möglichkeit, seine gesetzliche Überwachungsfunktion durch Satzung zu erweitern, so daß er auch als Leitungsorgan agieren konnte.

Erst seit dem AktG 1937 liegt eine eigenständige Kodifizierung des Aktienrechts vor. ${ }^{129}$ Eine wesentliche Änderung durch die Reform bestand in dem Ausschluß der Vertragsfreiheit hinsichtlich der gesetzlich vorgesehenen Zuständigkeiten und Verantwortlichkeiten der Verwaltungsträger. Die Verteilung der Kompetenzen zwischen den Organen der Aktiengesellschaft wurde zwingend vom Gesetz festgelegt. ${ }^{130}$ Hierdurch kam es zu einer strikten Funktionstrennung von Vorstand und Aufsichtsrat: Der Vorstand hatte die Gesellschaft unter eigener Verantwortung zu leiten, der Aufsichtsrat die Geschäftsführung zu überwachen. ${ }^{131}$ Dabei hat der Aufsichtsrat die Interessen aller Aktionäre zu schützen. ${ }^{132}$

Das AktG 1965 paßte das Aktienrecht an die veränderten Rahmenbedingungen an. ${ }^{133}$ Ziel war dabei weniger die Beseitigung von Mißständen, als vielmehr die Anpassung an wirtschafts- und gesellschaftspolitische Veränderungen. ${ }^{134} \mathrm{We}$ sentliche Änderungen fanden sich u.a. in der völligen Neugestaltung des Konzernrechts, der Stärkung der Hauptversammlung gegenüber der Verwaltung, der Ablösung des „Führerprinzips“ im Vorstand durch das Kollegialprinzip sowie Veränderungen im Bereich der Rechnungslegung. ${ }^{135}$ Hinsichtlich des Aufsichtsrats wurden die Informationspflichten des Vorstands erweitert, indem der Vorstand verpflichtet wurde, den Aufsichtsrat einmal im Jahr über die beabsichtigte

\footnotetext{
128 Vgl. Lehmann, Aktiengesellschaften, 1898, S. 80; Lutter, Unternehmensverwaltung, 1995, S. 8 .

129 Vgl. Assmann, Aktiengesetz, 1992, Anm. 152ff.

130 Vgl. Schmidt/Meyer-Landrut, Aktiengesetz, 1961, S. 428.

131 Vgl. Lutter, Unternehmensverwaltung, 1995, S. 10.

132 Vgl. Hax, Aktiengesellschaft, 1967, S. 205.

133 Vgl. Lutter, Unternehmensverwaltung, 1995, S. 10.

134 Vgl. Bleicher/Leberl/Paul, Unternehmungsverfassung, 1989, S. $55 f$.

135 Vgl. Henn, Handbuch, 1998, S. 3, sowie Bleicher/Leberl/Paul, Unternehmungsverfassung, 1989, S. 55.
} 
Geschäftspolitik zu unterrichten. ${ }^{136}$ Darüber hinaus wurde die Höchstzahl von Aufsichtsratsmandaten auf zehn begrenzt.

Seit Inkrafttreten hat das AktG 1965 zahlreiche Änderungen erfahren. ${ }^{137}$ So wurde beispielsweise durch das Gesetz über kleine Aktiengesellschaften vom 10.8.1994 für Aktiengesellschaften mit weniger als 500 Beschäftigten die Mitbestimmung im Aufsichtsrat abgeschafft. ${ }^{138}$ Die letzte gravierende Änderung des Aktienrechts erfolgte mit der Aktienrechtsreform 1997/98. Im Mittelpunkt standen das Bankenstimmrecht und die Macht der Banken, das Rechnungslegungsund das Prüfungsrecht sowie das Aufsichtsratsrecht. ${ }^{139}$ Sie fanden ihre Berücksichtigung vor allem im KonTraG vom 1.5.1998, das sowohl das AktG als auch das HGB aktienrechtlich modifizierte. ${ }^{140}$ In bezug auf den Aufsichtsrat zielt das KonTraG auf eine Revitalisierung seiner Überwachungstätigkeit und dessen verstärkte Einbeziehung in den gesamten Führungsprozeß. Diese Entwicklung ist jedoch noch nicht beendet. In der laufenden Legislaturperiode wurde eine Expertenkommission „Corporate Governance: Unternehmensführung - Unternehmenskontrolle - Modernisierung des Aktienrechts" eingesetzt, die inzwischen Vorschläge zu einer Fortentwicklung des Aktienrechts vorgelegt hat, mit der das System der Corporate Governance in Deutschland international wettbewerbsfähig gehalten werden soll. ${ }^{141}$

\section{b) Konkretisierung des Untersuchungsobjekts}

\section{(1) Bedeutung und Charakteristika der Publikumsaktiengesellschaft}

Die Aktiengesellschaft gilt als die reinste Form der Kapitalgesellschaft. ${ }^{142}$ Als Rechtsform, die es einer Vielzahl kleiner Anleger ermöglicht, Vorhaben mit einem großen Finanzierungsbedarf zu realisieren, ist sie vor allem für größere Unternehmungen von Bedeutung. Ende 2000 existierten in Deutschland insgesamt 10.582 Aktiengesellschaften mit einem nominalen Grundkapital von zusammen

136 Vgl. Potthoff, Aufsichtsratstätigkeit, 1998, S. 320.

137 Vgl. Henn, Handbuch, 1998, S. 3.

138 Vgl. Lutter, Unternehmensverwaltung, 1995, S. 10; zur kleinen AG beispielsweise umfassend Rust/Ulbert, Aktiengesellschaft, 1995, sowie Lutter, Aktiengesellschaften, 1994, S. 429-447.

139 Vgl. zusammenfassend Claussen, Aktiengesetz, 1998, S. 177ff.

140 Vgl. im Überblick Ernst/Seibert/Stuckert, KonTraG, 1998.

141 Vgl. Pressemitteilungen Nr. 301/00 und 321/00 des Bundespresseamtes, sowie den Bericht der Regierungskommission „Corporate Governance“, Unternehmensführung, 2001; zur jüngeren Rechtsprechung des Bundesgerichtshofs zur Aktiengesellschaft Kurzwelly, Aktiengesellschaft, 2000, S. 337-342; mit Bezug auf den Aufsichtsrat jüngst Feddersen, Aufsichtsrat, 2000, S. 385396.

142 Vgl. Potthoff/Trescher, Aufsichtsratsmitglied, 1999, S. 1. 
288,7 Mrd. DM. ${ }^{143}$ Dies ist der höchste Stand seit Bestehen der Bundesrepublik Deutschland, wodurch die zunehmende Attraktivität dieser Rechtsform dokumentiert wird.

Trotz der Einheitlichkeit der Rechtsform lassen sich wesentliche Charakteristika von Aktiengesellschaften unterscheiden. ${ }^{144}$ Hierzu zählen u.a. die Frage der Börsennotierung, die Anteilseignerstruktur und -zahl, die Größe gemessen in Gewinn bzw. Umsatz oder die Anzahl der beschäftigten Arbeitnehmer. Auch wenn die in dieser Arbeit zu analysierende Problemstellung in jeder Aktiengesellschaft vorliegen kann, so sind es doch bestimmte Unternehmungen, die für das hier vorliegende Problemfeld besonders anfällig sind. Hierzu gehören vor allem Publikumsaktiengesellschaften mit heterogener und breit gestreuter Anteilseignerstruktur und überwiegend Führungskräften anderer Unternehmungen als Anteilseignervertreter im Aufsichtsrat. ${ }^{145}$ In diesen Gesellschaften werden Manager durch andere Manager statt durch Anteilseigner überwacht, die sich zudem nur noch eingeschränkt als Treuhänder der Anteilseigner fühlen. ${ }^{146}$

Zu den Publikumsaktiengesellschaften können typischerweise die im Deutschen Aktienindex (DAX) enthaltenen Gesellschaften gezählt werden, eine ebenso eindeutige Zuordnung der MDAX $^{\circledR}$-Gesellschaften ist aufgrund der teilweise dominierenden Großaktionäre dagegen problematischer. Unabhängig davon, ob nur die DAX- oder auch die MDAX $^{\circledR}$-Gesellschaften berücksichtigt werden, haben Publikumsaktiengesellschaften einen zahlenmäßig geringen Anteil an der Gesamtzahl der (Aktien-)Gesellschaften. ${ }^{147}$ Hieraus darf jedoch nicht auf eine geringe Bedeutung geschlossen werden, da sie zum „Hochadel der Industrie“"148 in der Bundesrepublik Deutschland gehören und Schlüsselstellungen in der Wirtschaft besetzen. Zudem werden Aktiengesellschaften zukünftig eine immer größere Bedeutung in der privaten Altersversorgung spielen, sei es direkt über privaten Aktienbesitz oder indirekt durch Investmentfonds. ${ }^{149}$ Es ist somit zu erwarten, daß die Bedeutung der Publikumsaktiengesellschaften in der Zukunft weiter zunehmen wird.

\footnotetext{
143 Vgl. Hansen, AG Report, 2001, R 67.

144 Vgl. Potthoff/Trescher, Aufsichtsratsmitglied, 1999, S. 2.

145 Vgl. Chmielewicz, Unternehmensverfassung, 1993, Sp. 4409f.; Busse von Colbe/Pellens, Rechnungswesen, 1998, S. 587; mit weiteren Kriterien Jansch, Publikumsgesellschaften, 1999, S. $21 \mathrm{ff}$.

146 Vgl. Chmielewicz, Unternehmensverfassung, 1993, Sp. 4410.

147 Mit dieser Feststellung bereits Schneider, Pensionskassenkorporatismus, 1990, S. 318.

148 Schneider, Pensionskassenkorporatismus, 1990, S. 318.

149 Vgl. Leven, Aktienbesitz, 1998, S. 2
} 


\section{(2) Mitbestimmung}

Die Eingrenzung des Untersuchungsobjekts wäre unvollständig, wenn nicht auch zur Mitbestimmung Stellung bezogen würde. Mitbestimmung wird in einem weiten Sinne als Beteiligung von Betroffenen an Entscheidungen in verschiedenen Lebensbereichen verstanden. ${ }^{150}$ Dies konkretisiert sich in Unternehmungen zu einem engeren Begriffsverständnis, das Mitbestimmung als eine Beteiligung von Arbeitnehmern oder ihrer Vertreter an Entscheidungen im Rahmen einer Rechtsbeziehung (z.B. Gesetz oder Vertrag) oder einer Machtbeziehung definiert. ${ }^{151}$ In Unternehmungen tritt sie als Mitbestimmung am Arbeitsplatz, im Betrieb und auf Unternehmungsebene auf. ${ }^{152}$

Während Regelungen sowohl zur Mitbestimmung am Arbeitsplatz als auch zur betrieblichen Mitbestimmung hier irrelevant sind, sind Regelungen zur unternehmerischen Mitbestimmung bei der vorliegenden Problemstellung näher zu erläutern. Es ist daher zunächst zu klären, welche gesetzlichen Regelungen gegebenenfalls zu beachten sind. Da sich diese Untersuchung an dem Leitbild einer Publikumsaktiengesellschaft, die regelmäßig mehr als 2000 Arbeitnehmer beschäftigt, orientiert, kommt eine Mitbestimmung auf Unternehmungsebene lediglich nach dem Montan-Mitbestimmungsgesetz 1951, dem Mitbestimmungsergänzungsgesetz 1956 oder dem Mitbestimmungsgesetz 1976 (MitbestG) in Frage. Aufgrund der abnehmenden Bedeutung der Montanindustrie können Besonderheiten für montanmitbestimmte Unternehmungen aus dieser Untersuchung ausgeschlossen werden, ${ }^{153}$ so daß lediglich das MitbestG im vorliegenden Kontext zu beachten wäre.

Doch auch dessen Regelungen können in ihren Konsequenzen für den Unternehmungsführungsprozeß überwiegend unberücksichtigt bleiben. Dies ist aus zwei Gründen gerechtfertigt: Erstens ist die normative Entscheidungsdominanz der Eigentümer innerhalb der paritätischen Mitbestimmung des MitbestG durch die Abstimmungsregelungen im Aufsichtsrat gesichert, da im Ergebnis die Anteilseignervertreter den Aufsichtsratsvorsitzenden auch gegen den Willen der Arbeitnehmervertreter bestimmen können, der über sein Zweitstimmrecht Entscheidungen des Aufsichtsrats jederzeit zu Gunsten der Aktionäre beeinflussen

150 Vgl. Chmielewicz u.a., Mitbestimmung, 1977, S. 105.

151 Vgl. Mag, Mitbestimmung, 1996, S. 16.

152 Vgl. Chmielewicz u.a., Mitbestimmung, 1977, S. 105ff.; Frese, Unternehmungsführung, 1987, S. 305; umfassend Heymann/Seiwert/Theisen, Mitbestimmungsmanagement, 1983, sowie Mag, Mitbestimmung, 1996, S. 16.

153 Die Zahl aller Unternehmungen, die der Montanmitbestimmung unterliegen, lag 1998 bei 45 ; vgl. hierzu Kommission Mitbestimmung, Mitbestimmung, 1998, S. 43. 
kann. ${ }^{154}$ Daher spricht auch das Bundesverfassungsgericht nicht von paritätischer Mitbestimmung, da ein faktisches Übergewicht der Anteilseignervertreter besteht. ${ }^{155}$ Zweitens erfolgt die Bestellung eines Arbeitsdirektors als ,normales“ Vorstandsmitglied ohne Beeinflussung der Arbeitnehmervertreter, denn dieser kann im Gegensatz zur Montanmitbestimmung auch gegen die Stimmen der Arbeitnehmervertreter im Aufsichtsrat gewählt werden. ${ }^{156}$ Daher werden Mitbestimmungsregelungen lediglich im jeweiligen Problemkontext berücksichtigt, wenn die Analyse dies erfordert. ${ }^{157}$

\section{Ausgestaltung der Spitzenverfassung}

a) Die Verfassung der Aktiengesellschaft

\section{(1) Begriffliche Eingrenzung}

Zur Beschreibung der Funktionsweise von Leitung und Überwachung in der Aktiengesellschaft ist es erforderlich, deren normative und faktische Regelungsbasis zu analysieren. Dies ist die Unternehmungsverfassung (syn. Organisationsverfassung ${ }^{158}$ als Gesamtheit der grundlegenden und langfristig gültigen normativen und faktischen Strukturregelungen der Unternehmung. ${ }^{159}$ Sie dient als konstitutioneller Rahmen der Unternehmungsführung, d.h. ihre primär rechtlich determinierte Konkretisierung regelt die arbeitsteilige Aufgabenerfüllung im Entscheidungsprozeß der Unternehmung.

Zur Analyse der Organisationsverfassung muß der Organisationsbegriff abgegrenzt werden. Organisation kann als Funktion, als Institution oder als Regelsystem verstanden werden. ${ }^{160}$ Während der funktionale Organisationsbegriff die Tätigkeit des Organisierens bezeichnet, werden Organisationen als Institutionen bzw. als Regelsystem unter dem institutionalen Organisationsbegriff subsumiert.

154 Vgl. Henn, Handbuch, 1998, S. 251; Niedenhoff, Mitbestimmung, 2000, S. 432; Bernhardt, Anforderungen, 2000, S. 59.

155 Vgl. Bundesverfassungsgericht, Mitbestimmung, 1979, S. 593.

156 Vgl. Chmielewicz, Gremien, 1980, Sp. 2279ff.; Hahn, Mitbestimmung, 1999, S. 796.

157 Vgl. zu Fragen der Mitbestimmung umfassend Niedenhoff, Mitbestimmung, 2000, sowie mit Bezug auf die Tätigkeit von Arbeitnehmervertretern im Aufsichtsrat Kittner/Köstler/Zachert, Aufsichtsratspraxis, 1995; zum Zusammenhang von Mitbestimmung und Corporate Governance Gerum, Corporate Governance, 1998.

Die Verfassungsbestandteile von Unternehmungen werden unterschiedlich weit gefaßt; Chmielewicz, Unternehmensverfassung, 1993, Sp. 4400ff., subsumiert hierunter auch die Marktverfassung sowie die Finanzverfassung, die jedoch nachfolgend unberücksichtigt bleiben; die Gleichsetzung von Unternehmungsverfassung und Organisationsverfassung beispielsweise auch bei Bleicher, Organisation, 1991, S. $15 \mathrm{ff}$.

159 Vgl. Chmielewicz, Unternehmensverfassung, 1993, Sp. 4400ff.

Vgl. Kesten, Organisation, 1998, S. $28 \mathrm{ff}$. 
Der abstrakt-institutionale Begriff (Organisation als Regelsystem) bezeichnet alle generellen expliziten Regelungen zur Gestaltung von Aufbau- und Ablaufstrukturen in Unternehmungen. ${ }^{161}$ Er ist in dem weiteren, konkret-institutionalen Begriff (Organisation als Institution) enthalten, der über das System von Regelungen hinaus Organisationen als soziale Gebilde versteht. Es werden zusätzlich die handelnden Personen berücksichtigt, so daß eine Organisation dann vorliegt, wenn mehrere Personen zur gemeinsamen Aufgabenerfüllung Technologien (Computer, Modelle, Methoden und Verfahren) einsetzen und hierzu eine Kommunikationsstruktur aufbauen. ${ }^{162}$

Für die weitere Analyse muß einer der drei Begriffe zweckentsprechend ausgewählt werden. Im vorliegenden Problemzusammenhang soll der konkretinstitutionale Begriff Anwendung finden, da insbesondere im Zusammenhang mit dem später zu betrachtenden Planungssystem neben den organisatorischen Regelungen, die bei der Betrachtung der Organisationsverfassung im Vordergrund stehen, auch die handelnden Personen betrachtet werden.

Unter Berücksichtigung der jeweiligen Rechtsform sowie der Eigentumsverhältnisse gehören zur Organisationsverfassung folgende Regelungsbereiche: ${ }^{163}$

1. Grundstruktur der Ziele der Unternehmung,

2. Grundstruktur, Zusammensetzung und Arbeitsweise der Gremien,

3. ihre Aufgaben, Kompetenzen und Verantwortung,

4. Gestaltung und Vollzug des Führungsprozesses,

5. Grundrechte und -pflichten der Unternehmungsbeteiligten,

6. Gestaltung des Außenverhältnisses der Unternehmung.

Im Mittelpunkt aller Regelungen steht die Generierung, Implementierung sowie die Kontrolle aller für die Realisierung des jeweiligen Unternehmungsziels bedeutsamen Entscheidungen. ${ }^{164}$

Steht wie hier die Spitzenorganisation von Unternehmungen im Mittelpunkt, so ist eine Eingrenzung des Begriffs Organisationsverfassung sinnvoll. Die Spitzenverfassung bezeichnet die Teilmenge der Organisationsverfassung, die sich

\footnotetext{
161 Vgl. Bleicher, Organisation, 1991, S. 35.

162 Vgl. Mag, Unternehmungsplanung, 1995, S. 120; Mag, Unternehmensorganisation, 1970, S. 26; grundlegend Leavitt, Change, 1965, S. 1144-1170.

163 Vgl. Chmielewicz, Unternehmensverfassung, 1993, Sp. 4400.

164 Vgl. Frese, Unternehmensverfassung, 1993, S. 993.
} 
ausschließlich mit langfristigen und konstitutiven Fragen der Spitzenorgane von Unternehmungen befaßt. ${ }^{165}$ Für die deutsche Aktiengesellschaft zählen hierzu die Regelung der Verteilungs- und Arbeitsbeziehungen von Hauptversammlung, Vorstand und Aufsichtsrat. Synonym mit Spitzenverfassung soll hier der Begriff Corporate Governance verwendet werden. ${ }^{166}$ Im internationalen Vergleich der Spitzenverfassungen bestehen trotzt der unterschiedlichen formalen Ausgestaltung grundlegende Gemeinsamkeiten, auf die hier jedoch nicht näher eingegangen werden muß. ${ }^{167}$ Nachfolgend werden ausschließlich die Organe der deutschen Aktiengesellschaft betrachtet.

(2) Organe der Aktiengesellschaft

Organ ist die gesellschaftsrechtliche Bezeichnung eines Gremiums der Aktiengesellschaft. ${ }^{168}$ Der Gremienbegriff selbst wird uneinheitlich abgegrenzt. ${ }^{169}$ In seiner weitesten Fassung werden alle statusmäßig undifferenzierten Personenmehrheiten mit beliebiger Funktion als Gremium bezeichnet. ${ }^{170}$ Diese sehr allgemeine Definition bietet jedoch keine hinreichende Beschreibung aller relevanten Charakteristika. Andererseits grenzt die engste Definition von Gremium durch die Zuordnung zur betrieblichen Sekundärorganisation die hier zu betrachtenden Organe aus, so daß die weiteste Fassung durch die Hinzufügung weiterer Charakteristika näher spezifiziert wird. In Anlehnung an LUDWIG soll als Gremium eine Personenmehrheit bezeichnet werden,

1. die eine spezifische (Dauer-)Funktion hat,

2. die abgesehen von der Sonderstellung eines eventuell vorhandenen Vorsitzenden keine formal hierarchische Struktur besitzt und

165 Vgl. Bleicher/Leberl/Paul, Unternehmungsverfassung, 1989, S. 23.

166 Vgl. Bleicher, Organisation, 1991, S. 16; die hier verwendete Abgrenzung von Corporate Governance ist enger als die üblicherweise verwendeten Definitionen, da sie Fragen der Rechnungslegung, Publizität und der Abschlußprüfung ausgrenzt; im internationalen Kontext sehr weit beispielsweise auch Shleifer/Vishny, Corporate Governance, 1997, S. 737; zur Abgrenzung aus amerikanischer Perspektive, Hess, Corporate Governance, 1996, S. 10; anders Hakelmacher, Corporate Governance, 2001, S. 177.

Vgl. zu einem Vergleich der Spitzenverfassung der deutschen Aktiengesellschaft mit dem amerikanischen Boardsystem beispielsweise Potthoff, Unternehmensverwaltung, 1996, S. 253ff.; für einen internationalen Vergleich Gerum, Unternehmensführung, 1998, S. 135-153, Hopt/Wymeersch, corporate governance, 1997, sowie Schneider, Unternehmensüberwachung, 2000, S. 2366; umfassend zur Corporate Governance in amerikanischen Unternehmungen Monks/Minow, Corporate Governance, 1996.

Vgl. Frese, Unternehmungsführung, 1987, S. 305.

Vgl. Seidel, Gremienorganisation, 1992, Sp. 714; Ludwig, Leitungsgremien, 1997, S. 16; Chmielewicz, Gremien, 1980, Sp. 2272.

Vgl. Seidel, Gremienorganisation, 1992, Sp. 714. 
3. deren Zusammenarbeit sich weitgehend auf Sitzungstermine beschränkt. ${ }^{171}$

In Deutschland ist die Ausgestaltung der Spitzenverfassung der Entscheidungsautonomie der Anteilseigner von Aktiengesellschaften entzogen, da die Entscheidungskompetenzen zwingend drei Organen zugeordnet sind: der Hauptversammlung, dem Aufsichtsrat und dem Vorstand. ${ }^{172}$

Hier erfolgt eine Konkretisierung der betrachteten Organe hinsichtlich Umfang und Detaillierungsgrad. Hinsichtlich des Umfangs betrifft dies die weitgehende Vernachlässigung der Hauptversammlung. ${ }^{173}$ Obwohl es sich bei dieser formal um das oberste Organ der Aktiengesellschaft handelt, ${ }^{174}$ hat sie in Publikumsaktiengesellschaften materiell nur sehr begrenzte Einflußmöglichkeiten. ${ }^{175}$ Es ist daher gerechtfertigt, in dieser Untersuchung auf eine nähere Betrachtung der Hauptversammlung zu verzichten und sich auf die eigentliche „Verwaltung der Aktiengesellschaft ${ }^{\star 176}$, den Aufsichtsrat und den Vorstand, zu konzentrieren, um nur dort näher auf Einflußmöglichkeiten der Hauptversammlung einzugehen, wo dies für die Arbeits- und Funktionsweise der Verwaltung von Bedeutung ist. Die Begrenzung des Detaillierungsgrades bezieht sich auf die Darstellung der Rechtsregelungen zum Vorstand und zum Aufsichtsrat. Es wird nachfolgend ein juristisches Grundgerüst für diese beiden Organe erarbeitet, das dem eigentlichen Ziel einer betriebswirtschaftlichen Analyse als Basis dient. ${ }^{177}$ Eine vertiefende Analyse erfolgt im jeweiligen Problemzusammenhang.

\section{b) Vorstand}

\section{(1) Bildung und Zusammensetzung}

Im vierten Teil des Aktiengesetzes wird die „Verfassung der Aktiengesellschaft“ normiert. Dies betrifft den Vorstand (§§ 76-94 AktG), den Aufsichtsrat ${ }^{178}$ (§§

171 Vgl. Ludwig, Leitungsgremien, 1997, S. 16.

172 Vgl. Bleicher/Leberl/Paul, Unternehmungsverfassung, 1989, S. 24; Chmielewicz, Unternehmensverfassung, 1993, Sp. 4407ff.

173 Zur Hauptversammlung beispielsweise Wellkamp, Aufsichtsrat, 2000, S. 49ff.

174 Vgl. Henn, Handbuch, 1998, S. 243.

175 Vgl. Frese, Unternehmungsführung, 1987, S. 306ff; mit dem gleichen Ansatz auch Grundsatzkommission Corporate Governance, Corporate Governance-Grundsätze, 2000, S. 9.

176 Mertens, Aktiengesellschaft, 1996, S. 2.

$177 \mathrm{Zu}$ einer umfassenden juristischen Analyse der Leitung und Überwachung der Aktiengesellschaft Semler, Überwachung, 1996.

178 Mit Vorstand wird nachfolgend das Gesamtorgan bezeichnet, während Vorstandsmitglied(er) einzelne Personen des Gesamtorgans meint. Gleiches gilt für den Aufsichtsrat als Organ und das einzelne Aufsichtsratsmitglied. 
95-116 AktG), die rechtswidrige Einflußnahme auf Verwaltungsmitglieder bzw. Leitende Angestellte (Prokuristen und Handlungsbevollmächtigte) zum Schaden der Gesellschaft ( $§ 117$ AktG) sowie die Hauptversammlung ( $\S$ 118-147 AktG).

Zentrale Vorschrift für die Berufung und Abberufung von Vorstandsmitgliedern ist $\S 84$ AktG. Er bestimmt, daß sie für höchstens fünf Jahre durch den Aufsichtsrat kraft Beschluß bestellt bzw. wiederbestellt werden ( $\S 84$ Abs. 1 S. 1, 2, 108 AktG). Beides bedarf der ausdrücklichen Zustimmung der bestellten Person. ${ }^{179}$ Bei der Abberufung eines Vorstandsmitglieds, die aus wichtigem Grund, beispielsweise wegen grober Pflichtverletzung oder Unfähigkeit zur ordnungsgemäßen Geschäftsführung ( $\S 84$ Abs. 3 AktG), durch den Aufsichtsrat erfolgen kann, ist diese Zustimmung nicht erforderlich. ${ }^{180}$ Einen solchen Grund können auch Divergenzen über die Unternehmungsplanung darstellen. ${ }^{181}$

Der Aufsichtsrat wählt alle Mitglieder des Vorstands, auch den Arbeitsdirektor, mit qualifizierter Mehrheit seiner Mitglieder ( $\$ 31$ Abs. 2 MitbestG). Kommt diese Mehrheit nicht zustande, wird ein gemäß $\S 27$ Abs. 3 MitbestG bestehender Vermittlungsausschuß des Aufsichtsrats mit der Erarbeitung eines Personalvorschlags beauftragt ( $\$ 31$ Abs. 3 S. 1 MitbestG). Über diesen Vorschlag entscheiden die Mitglieder des gesamten Aufsichtsrats mit einfacher Mehrheit ( $\$ 31$ Abs. 3 S. 2 MitbestG). Sollte auch hierbei keine Einigung erzielt werden, hat der Aufsichtsratsvorsitzende in einer dritten Abstimmung zwei Stimmen ( $\$ 31$ Abs. 4 S. 1 MitbestG).

Vom organschaftlichen Akt der Bestellung bzw. Abberufung des Vorstandsmitglieds ist gemäß der der herrschenden Meinung entsprechenden Trennungstheorie der Abschluß bzw. die Beendigung des Anstellungsvertrages abzugrenzen. ${ }^{182}$ Bei dem Anstellungsvertrag handelt es sich um einen Dienstvertrag ( $\$ 611$ BGB), so daß die Mitglieder des Vorstands aus juristischer Sicht nicht zu den abhängig Beschäftigten und somit nicht zum Personal einer Unternehmung gehören. ${ }^{183}$ Während die Bestellung, die Wiederbestellung sowie die Abberufung von Vorstandsmitgliedern nur durch den gesamten Aufsichtsrat erfolgen kann,

\footnotetext{
179 Vgl. Henn, Handbuch, 1998, S. 256; Mertens, Kölner Kommentar, 1996, Anm. 3 zu § 84 AktG.

180 Vgl. Henn, Handbuch, 1998, S. 254.

181 Vgl. m.w.N. Mutter, Aktiengesellschaft, 1994, S. 46, 80.

182 Vgl. Henn, Handbuch, 1998, S. 255, und die dort angegebenen Quellen; Mertens, Kölner Kommentar, 1996, Anm. 2 zu § 84 AktG.

183 Vgl. Mag, Personalplanung, 1998, S. $5 f$.
} 
sind der Abschluß und die Aufhebung des Anstellungsvertrages vom Plenum an einen Aufsichtsratsausschuß gemäß § 107 Abs. 3 AktG delegierbar. ${ }^{184}$

Der Vorstand einer Aktiengesellschaft kann aus einer oder aus mehreren Personen bestehen ( $\$ 76$ Abs. 2 S. 1 AktG). Ab einem Grundkapital von drei Mio. Euro ist eine Mindestanzahl von zwei Personen vorgeschrieben ( $\$ 76$ Abs. 2 S. 2 AktG). Bei Unternehmungen, die dem MitbestG unterliegen, ist immer ein Arbeitsdirektor zu bestimmen, so daß bei paritätisch mitbestimmten Unternehmungen ein mindestens zweiköpfiger Vorstand entsteht (§ 76 Abs. 2 S. 3 AktG i.V.m. $§ 33$ Abs. 1 MitbestG). ${ }^{185}$

Die Zahl der Mitglieder des Vorstands oder die Regel, nach der diese Zahl ermittelt wird, müssen in der Satzung der Gesellschaft festgelegt werden ( $\$ 23$ Abs. 3 Nr. 6 AktG). Eine absolute Fixierung der Zahl der Vorstandsmitglieder ist im Hinblick auf eine eventuelle spätere Umstrukturierung nicht sinnvoll und wird in der Praxis zumeist vermieden. ${ }^{186}$ Statt dessen wird die Regel aufgestellt, daß der Aufsichtsrat die Anzahl der Vorstandsmitglieder bestimmt.

\section{(2) Aufgaben}

Dem Vorstand werden an verschiedenen Stellen des Aktiengesetzes Aufgaben zugewiesen, wobei der Gesetzgeber jedoch unterschiedliche Begriffe verwen$\operatorname{det}^{187}$

1. Der Vorstand leitet die Gesellschaft ( 76 Abs. 1 AktG).

2. Der Vorstand ist zur Geschäftsführung befugt ( $\$ 77$ Abs. 1 AktG).

3. Der Vorstand vertritt die Gesellschaft nach außen ( 78 Abs. 1 AktG).

Der Leitung, der Geschäftsführung und der Vertretung der Aktiengesellschaft liegt ein funktionales Begriffsverständnis zugrunde, da so bezeichnete Institutionen in der Aktiengesellschaft nicht existieren. ${ }^{188}$ Während unter Vertretung nach übereinstimmender Auffassung das nach außen wirkende rechtsgeschäftliche Handeln des Vorstands im Namen der Gesellschaft verstanden wird, ${ }^{189}$ fällt eine einheitliche Begriffsbestimmung der Leitung und der Geschäftsführung

\footnotetext{
184 Vgl. Henn, Handbuch, 1998, S. 259; Mertens, Kölner Kommentar, 1996, Anm. 10 zu § 84 AktG.

185 Vgl. Niedenhoff, Mitbestimmung, 2000, S. 432.

186 Vgl. Münchner Handbuch, Anm. 23 zu § 19 AktG; bestätigend der Beitrag „Dresdner Bank mit anderen Vorstandszuständigkeiten" in der FAZ vom 21.11.1998.

187 Vgl. Henn, Handbuch, 1998, S. 245.

188 Vgl. Henn, Handbuch, 1998, S. 296; Scheffler, Aufsichtsrat, 1993, S. $65 f$.

189 Vgl. Mertens, Kölner Kommentar, 1996, Anm. 3 zu § 78 AktG.
} 
schwieriger. ${ }^{190}$ Dies ist jedoch wichtig, da dem Aufsichtsrat gemäß § 111 AktG die Überwachung der Geschäftsführung übertragen wird. Grundsätzlich sind vier unterschiedliche Begriffsbeziehungen denkbar: Einmal könnten die Begriffe synonym verwendet werden, zum anderen könnte es sich bei der Geschäftsführung um eine Teilmenge der Leitung handeln oder als dritte Möglichkeit bei der Leitung um einen Ausschnitt der Geschäftsführung. Schließlich könnten die beiden Begriffe etwas vollkommen unterschiedliches bezeichnen. Betrachtet man die einschlägige juristische Literatur, so können die letzten beiden Alternativen ausgeschlossen werden. ${ }^{191}$ Bei den verbleibenden Möglichkeiten ist dies problematischer, so daß eine detailliertere Analyse erforderlich wird.

$\S 76$ Abs. 1 AktG beinhaltet nicht nur das Recht, sondern auch die Pflicht zur Leitung der Gesellschaft durch den Vorstand. ${ }^{192}$ Dieser Leitungsbegriff wurde durch den Gesetzgeber über eine Generalklausel geregelt. ${ }^{193}$ HEFERMEHL versteht unter Leitung einen entscheidungsorientierten Prozeß, zu dem einmal die Führung der Unternehmung einschließlich der Entscheidungen über die zu übernehmenden geschäftlichen und finanziellen Risiken, zum anderen die routinemäßige Verwaltung zählen. ${ }^{194}$ Ein für die Leitung konstitutiver Führungsbestandteil wird auch von MERTENS betont, nach dessen Ansicht der Vorstand durch die Führung eigenverantwortlich die Unternehmerfunktion in der Aktiengesellschaft wahrnimmt. ${ }^{195}$

Gerade durch die Wahrnehmung der Unternehmerfunktion geht für MEYERLANDRUT der Leitungsbegriff über die Geschäftsführung hinaus. ${ }^{196}$ Ähnlich argumentiert auch DOSE, der daher von einem gegenüber der Geschäftsführung erweiterten Leitungsbegriff ausgeht. ${ }^{197}$ Problematisch ist an dieser Sichtweise jedoch, daß entsprechend der Wortwahl des $§ 111$ AktG bei Erweiterung der Leitungsaufgabe im Verhältnis zur Geschäftsführung der Eindruck entstehen könnte, daß diejenigen Bestandteile der Vorstandstätigkeit, die über die Ge-

\footnotetext{
190 Vgl. Semler, Überwachung, 1996, S. 6 (hier besonders FN 8); zur Abgrenzung beispielsweise Henze, Leitungsverantwortung, 2000, S. 209-210.

191 Vgl. exemplarisch Mertens, Kölner Kommentar, 1996, Anm. 4 zu § 76 AktG; Hefermehl, Aktiengesetz, 1973, Anm. 10 zu § 76 AktG; Hüffer, Aktiengesetz, 1999, Anm. 3 zu § 77 AktG.

192 Vgl. Hefermehl, Aktiengesetz, 1973, Anm. 9 zu § 76 AktG.

193 Vgl. Henn, Handbuch, 1998, S. 271; Mertens, Kölner Kommentar, 1996, Anm. 10ff. zu § 76 AktG.

194 Vgl. Hefermehl, Aktiengesetz, 1973, Anm. $10 \mathrm{zu} \S 76$ AktG.

195 Vgl. Mertens, Kölner Kommentar, 1996, Anm. 4 zu § 76 AktG.

196 Vgl. Meyer-Landrut, Aktiengesetz, 1970, Anm. 2 zu § 76 AktG.

197 Vgl. Dose, Aktiengesellschaft, 1975, S. 40.
} 
schäftsführung hinausgehen, nicht der Überwachung durch den Aufsichtsrat unterliegen. ${ }^{198}$ In dem von MEYER-LANDRUT und DOSE gewählten Begriffsverständnis würde sogar die wesentliche Führungsaufgabe des Vorstands nicht zum Überwachungsfeld des Aufsichtsrats zählen. Eine solche Aushöhlung der Überwachungsaufgabe scheint nicht hinnehmbar und somit ist es zweckmäßig, von einem übereinstimmenden Begriffsinhalt der Leitung und der Geschäftsführung auszugehen. ${ }^{199}$

\section{(3) Vorstandsorganisation}

\section{(a) Aufbauorganisation}

Die Organisation des Vorstands ist auf eine effiziente Entscheidungsvorbereitung und -findung auszurichten. ${ }^{200}$ Hierzu finden sich im AktG jedoch nur vereinzelt Regelungen. Um diese einer Systematisierung zu erschließen, werden sie in aufbau- und ablauforganisatorische Regelungen untergliedert. Zur Aufbauorganisation als dem statischen Gefüge der Aufgabenverteilung und -zuordnung sowie der Gestaltung der Kommunikations- und Weisungsbeziehungen gehören alle Regelungen zur internen und externen Strukturierung des Vorstands. ${ }^{201}$ Während die externe Strukturierung (das ist die Einbindung des Organs in die Gesamtunternehmung) eindeutig durch die aktienrechtlich determinierte Einordnung des Vorstandsorgans in die Spitzenverfassung der Aktiengesellschaft geregelt ist, existieren für die interne Strukturierung unterschiedliche Gestaltungsalternativen. $^{202}$

Der Vorstand ist vom Aktienrecht als Kollegialorgan konzipiert, so daß Weisungen zwischen den einzelnen Organmitgliedern nicht zulässig sind. ${ }^{203}$ Das Gesetz sieht sowohl eine Gesamtgeschäftsführung ( 77 Abs. 1 AktG) als auch eine Gesamtvertretung der Gesellschaft durch den Vorstand ( $\$ 78$ Abs. 1 AktG) vor, d.h. alle Vorstandsmitglieder müssen einzelnen Maßnahmen zustimmen. Abweichungen sind jedoch von beiden Prinzipien möglich. Während die Gesamtvertretung nur durch die Satzung aufgehoben werden kann bzw. die Möglichkeit besteht, daß diese dem Aufsichtsrat explizit das Recht zur Aufhe-

\footnotetext{
198 Vgl. Semler, Überwachung, 1996, S. 6.

199 So auch Hefermehl, Aktiengesetz, 1973, Anm. 10 zu § 76 AktG; Potthoff/Trescher, Aufsichtsratsmitglied, 1999, S. $61(\mathrm{FN} *)$.

200 Vgl. Schmidt, Gesellschaftsrecht, 1997, S. 820.

201 Vgl. grundlegend zur Aufbauorganisation beispielsweise Hoffmann, Aufbauorganisation, 1992, Sp. $208 \mathrm{ff}$.

202 Vgl. zur internen und externen Strukturierung von Leitungsgremien Ludwig, Aufsichtsräte, 1997, S. 59ff., $69 \mathrm{ff}$.

203 Vgl. von Werder, Unternehmungsleitung, 1987, S. 2266.
} 
bung zuweist, existieren für die Gestaltung der Geschäftsführung unterschiedliche Alternativen. ${ }^{204}$ Zuallererst können die Aktionäre über die Satzung ihren Einfluß auf die Geschäftsverteilung geltend machen (§ 77 Abs. 1 S. 2 AktG) ${ }^{205}$ Daneben können abweichende Regelungen auch in einer Geschäftsordnung für den Vorstand normiert werden, wobei die Geschäftsordnungskompetenz unterschiedlich verteilt sein kann ( $\$ 77$ Abs. 2 AktG):

1. Regelung von Einzelfragen der Geschäftsordnung durch die Satzung ( $\$ 77$ Abs. 2 S. 2 AktG),

2. Übertragung der Geschäftsordnungskompetenz durch die Satzung an den Aufsichtsrat ( 77 Abs. 2 S. 1 AktG),

3. Erlaß der Geschäftsordnung durch den Aufsichtsrat ohne besondere Ermächtigung durch die Satzung ( $\$ 77$ Abs. 2 S. 1 AktG),

4. bei Fehlen anderer Regelungen kann sich der Vorstand eine eigene Geschäftsordnung geben (§ 77 Abs. 2 S. 1 AktG).

$\mathrm{Zu}$ den bedeutsamsten Regelungsobjekten einer Geschäftsordnung gehört zur horizontalen Strukturierung die Festlegung der Geschäftsverteilung zwischen den einzelnen Mitgliedern des Vorstands. ${ }^{206}$ Abweichungen vom Prinzip der Gesamtgeschäftsführung können zu verschiedenen Formen der Geschäftsverteilung führen. ${ }^{207}$ Eng mit der horizontalen Strukturierung des Vorstands verbunden sind Fragen der vertikalen Strukturierung, wozu sowohl die Gestaltung der Weisungsbeziehungen innerhalb des Gremiums als auch der Weisungsbeziehungen zu nachgelagerten Hierarchieebenen gehören. ${ }^{208}$

Die Grenzen einer Ressortierung im Vorstand sowie der Delegation von Aufgaben und Kompetenzen an nachgelagerte Führungsebenen liegen dort, wo die „organschaftlichen Mindestkompetenzen“ betroffen sind. ${ }^{209}$ Die in diesen Be-

204 Vgl. Semler, Überwachung, 1996, S. 17; Schmidt, Gesellschaftsrecht, 1997, S. 821.

$205 \mathrm{Vgl}$. Lutter/Krieger, Rechte, 1993, S. 142.

206 Vgl. Hoffmann-Becking, Vorstand, 1998, S. 499.

207 Vgl. Mertens, Kölner Kommentar, 1996, Anm. 15ff. zu § 77 AktG, sowie aus organisationstheoretischer Perspektive Krüger, Organisation, 1994, S. 258f., der auch Mischformen vorstellt. Explizit vorgesehen ist in paritätisch mitbestimmten Unternehmungen ein für das Personalund Sozialwesen zuständiger Arbeitsdirektor ( $\$ 33$ MitbestG).

Vgl. Chmielewicz, Unternehmungsleitung, 1992, Sp. 2477ff.; Krüger, Organisation, 1994, S. 253f., 260.

209 Vgl. Semler, Überwachung, 1996, S. 17; Mertens, Kölner Kommentar, 1996, Anm. 43ff. zu § 76 Abs. 1 AktG; Raiser, Kapitalgesellschaften, 1992, S. 93. 
reich fallenden Aufgaben müssen zwingend vom Vorstand als Gesamtorgan wahrgenommen werden. Hierzu gehören

1. Aufgaben, die das Verhältnis zu einem anderen Organ betreffen,

2. Aufgaben, die im öffentlichen Interesse liegen,

3. die Wahrnehmung der unternehmerischen Führungsfunktionen,

4. die Beschlußfassung über Führungsentscheidungen.

Schließlich kann eine weitere Strukturierung des Vorstands durch die Hervorhebung einzelner Mitglieder erfolgen. Obwohl der Vorstand grundsätzlich als hierarchiefreies Kollegialorgan konzipiert ist, kann ein einzelnes Vorstandsmitglied als ,primus inter pares“ hervorgehoben werden. ${ }^{210}$ Hierzu bestehen zwei Möglichkeiten: Nach $\S 84$ Abs. 2 AktG kann der Aufsichtsrat bei mehreren Vorstandsmitgliedern ein Mitglied zum Vorstandsvorsitzenden ernennen. Hierüber entscheidet der Aufsichtsrat mit Mehrheitsbeschluß. ${ }^{211}$ Dem Vorstandsvorsitzenden kommen kraft Gesetzes verschiedene Rechte zu: Er kann Vorstandssitzungen einberufen und leiten sowie hierfür eine vorläufige Tagesordnung festlegen. Zusätzlich kann ihm durch Satzung oder Geschäftsordnung die entscheidende Stimme bei der Entscheidungsfindung im Vorstandsgremium zugewiesen werden.

Sofern die Satzung nichts anderes bestimmt, ist es dem Vorstand alternativ möglich, im Rahmen seiner Geschäftsordnungskompetenz aus seinen Reihen einen Vorstandssprecher zu bestimmen. ${ }^{212}$ Dies hat große ablauforganisatorische Bedeutung, da diesem primär koordinierende Tätigkeiten im Hinblick auf die Zusammenarbeit der einzelnen Vorstandsmitglieder zukommen. ${ }^{213}$ Insoweit deckt sich der Aufgabenbereich des Vorstandssprechers mit dem des Vorstandsvorsitzenden. ${ }^{214}$ Beide repräsentieren darüber hinaus die Gesellschaft nach auBen. ${ }^{215}$

\footnotetext{
210 Vgl. Schmidt, Gesellschaftsrecht, 1997, S. 820; Lutter/Krieger, Rechte, 1993, S. 147.

211 Vgl. Mertens, Kölner Kommentar, 1996, Anm. 87 zu § 84 AktG und Anm. 90 zu § 84 AktG, auBerdem Hoffmann-Becking, Vorstand, 1998, S. 517.

212 Vgl. Mertens, Kölner Kommentar, 1996, Anm. 89 zu § 84 AktG.

213 Vgl. Henn, Handbuch, 1998, S. 252; Raiser, Kapitalgesellschaften, 1992, S. 92.

214 Vgl. Raiser, Kapitalgesellschaften, 1992, S. 92.

215 Vgl. Hoffmann-Becking, Vorstand, 1998, S. 517.
} 


\section{(b) Ablauforganisation}

Im Gegensatz zur statischen Aufbauorganisation beinhaltet die Ablauforganisation die raum-zeitliche Koordination der arbeitsteiligen Aufgabenerfüllung. ${ }^{216}$ Hierunter fallen innerhalb der Vorstandstätigkeit zum einen die Koordination der auf einzelne Vorstandsmitglieder bzw. Vorstandsausschüsse delegierten Aufgaben und andererseits die Zusammenarbeit des Gesamtorgans. ${ }^{217}$ In den Fällen, in denen eine gemeinsame Entscheidungsfindung durch alle Vorstandsmitglieder vorgesehen ist, erfolgen die Entscheidungen des Gesamtvorstands in hierzu notwendigen Sitzungen. Der Vorstand diskutiert und entscheidet auf diesen Sitzungen über Entscheidungsvorlagen, in denen Probleme analysiert und Lösungsalternativen vorbereitet worden sind.

Hinsichtlich der Arbeitsweise und der Beschlußfassung des Vorstands haben BLEICHER/LEBERL/PAUL in einer empirischen Untersuchung einige Charakteristika aufgedeckt: ${ }^{218}$

1. Vorstandssitzungen werden (gegebenenfalls auf Verlangen eines Vorstandsmitglieds) durch den Vorsitzenden/Sprecher einberufen,

2. die Sitzungen werden durch den Vorsitzenden/Sprecher geleitet, wobei bei wichtigen Entscheidungen auch die Meinung abwesender Vorstandsmitglieder eingeholt wird,

3. nach erfolgter Aussprache entscheidet der Vorstand gemeinschaftlich,

4. die Stimmenmehrheit gibt bei unterschiedlichen Auffassungen den Ausschlag,

5. sofern die Geschäftsordnung keine Regelungen enthält, wird die Art der Abstimmung durch den Sitzungsleiter bestimmt,

6. über die Beschlüsse des Vorstands wird eine Niederschrift angefertigt, die von allen Sitzungsteilnehmern unterzeichnet und jedem Vorstandsmitglied zugeleitet wird.

In der Praxis kann bei Vorstandsentscheidungen ein Trend zur Einstimmigkeit beobachtet werden. ${ }^{219}$ Kommt es dennoch zu einem Mehrheitsbeschluß durch Abstimmung, so kann dies entweder mit einfacher oder mit qualifizierter Mehr-

216 Vgl. Frese, Organisation, 2000, S. 7.

217 Vgl. Bleicher/Leberl/Paul, Spitzenverfassung, 1987, S. 104f.

218 Vgl. Bleicher/Leberl/Paul, Spitzenverfassung, 1987, S. 104f.; zum Sprecher Raiser, Kapitalgesellschaften, 1992, S. 90.

219 Vgl. Trenkle, Vorstandsentscheidung, 1983, S. 107. 
heit erfolgen. ${ }^{20}$ Der ARBEITSKREIS KRÄHE DER SCHMALENBACH-GESELLSCHAFT hat hierfür den Begriff der Abstimmungskollegialität geprägt. ${ }^{221}$ Lediglich Entscheidungen des Vorstands über seine eigene Geschäftsordnung müssen zwingend einstimmig erfolgen ( $\$ 77$ Abs. 2 S. 3 AktG).

\section{(4) Verantwortlichkeit und Haftung der Vorstandsmitglieder}

Bei der Aufgabenerfüllung unterliegen die Vorstandsmitglieder einer besonderen Verantwortung und Haftung. Hierfür ist $\S 93$ AktG die zentrale Norm. Ähnlich dem handelsrechtlichen Sorgfaltsmaß des „ordentlichen Kaufmanns“ ( $\$ 347$ Abs. 1 HGB) müssen die Vorstandsmitglieder bei der Geschäftsführung die Sorgfalt eines ordentlichen und gewissenhaften Geschäftsleiters anwenden (§ 93 Abs. 1 S. 1 AktG). ${ }^{222}$ Die an das Sorgfaltsmaß geknüpfte gesamtschuldnerische Haftung der Vorstandsmitglieder ( $\$ 93$ Abs. 2 S. 1 AktG) ergibt sich aus ihrer herausgehobenen Stellung als Organ der Aktiengesellschaft, weniger aus ihrem Anstellungsvertrag. ${ }^{223}$ Bei dem Vorwurf des pflichtwidrigen Verhaltens kehrt sich die Beweislast um (§93 Abs. 2 S. 2 AktG); es wird in Schadensfällen vermutet, daß der gesamte Vorstand die Verantwortung für den Schaden trägt. ${ }^{224}$ Es ist Aufgabe des einzelnen Vorstandsmitglieds, den Beweis für sein pflichtgemäßes Verhalten anzutreten. Die Ersatzpflicht der Vorstandsmitglieder tritt abgesehen von der Exkulpation auch dann nicht ein, wenn die Handlung auf einem gesetzmäßigen Beschluß der Hauptversammlung beruht ( $\$ 93$ Abs. 4 S. 1 AktG). Die Zustimmung des Aufsichtsrats allein ist hierfür nicht ausreichend (§93 Abs. 4 S. 2 AktG).

Dabei sind die umfangreichen Pflichten und die Ermessensspielräume des Vorstands nur teilweise einer Detailregelung zugänglich. ${ }^{225}$ Eine mögliche Klassifikation und Enumeration hat RAISER entwickelt: ${ }^{26}$

1. Spezifische Pflichten kraft Gesetzes, zu denen beispielsweise die unter $\S$ 93 Abs. 3 AktG aufgelisteten Pflichten sowie vor allem die mit dem KonTraG erweiterten Berichterstattungspflichten an den Aufsichtsrat (§

\footnotetext{
$220 \mathrm{Vgl}$. Chmielewicz, Unternehmungsleitung, 1992, Sp. 2476.

221 Vgl. Arbeitskreis Krähe, Geschäftsführung, 1971, S. 62.

222 Vgl. Henn, Handbuch, 1998, S. 288.

223 Vgl. Schmidt, Gesellschaftsrecht, 1997, S. 823.

Vgl. Henn, Handbuch, 1998, S. 290; Hoffmann-Becking, Vorstand, 1998, S. 506, und die dort angegebene Literatur.

225 Vgl. Schmidt, Gesellschaftsrecht, 1997, S. 823.

226

Vgl. Raiser, Kapitalgesellschaften, 1992, S. 104ff.; eine alternative Klassifikation findet sich beispielsweise bei Hübner, Managerhaftung, 1992, S. $2 \mathrm{ff}$.
} 
90 AktG, hier insbesondere Abs. 1) sowie die Pflicht zur Einrichtung eines Überwachungssystems ( $\$ 91$ Abs. 2 AktG) zählen,

2. Beachtung der für die innere Organisation des Vorstands relevanten Vorschriften, zu denen insbesondere die Satzung sowie gegebenenfalls die Geschäftsordnung gehören,

3. Berücksichtigung der im Außenverhältnis relevanten Rechtsvorschriften (beispielsweise Wettbewerbs- oder Steuerrecht),

4. Pflicht zur kollegialen Zusammenarbeit mit den übrigen Vorstandsmitgliedern,

5. Pflicht zur sorgfältigen Geschäftsführung,

6. Treuepflicht, die erfordert, daß das Vorstandsmitglied seine eigenen Interessen hinter denen der Gesellschaft zurückstellt,

7. Einhaltung der Grenzen der unternehmerischen Ermessensfreiheit.

Bei pflichtwidrigem Verhalten sind die Vorstandsmitglieder zum Ersatz des hierdurch entstehenden Schadens verpflichtet. ${ }^{227}$ Schadensersatzansprüche können entweder durch den Aufsichtsrat ( $\$ 112 \mathrm{AktG}$ ) oder durch einen Teil der in der Hauptversammlung vertretenen Aktionäre ( $\$ 147$ AktG) geltend gemacht werden. In der Praxis kam beiden Möglichkeiten lange Zeit nur eine untergeordnete Bedeutung $\mathrm{zu}^{228}$ erst mit dem KonTraG wurde durch eine Verringerung des für eine Aktionärsklage notwendigen Quorums die Möglichkeit solcher Klagen verbessert. ${ }^{229}$ Es sind nach neuem Recht bereits $5 \%$ des Grundkapitals oder der anteilige Betrag von 500.000 Euro für die Erzwingung einer solchen Klage ausreichend ( $\$ 147$ Abs. 3 AktG). Zur Vermeidung einer Flut von Schadensersatzklagen sieht das Gesetz jedoch die Einrichtung eines besonderen Vertreters vor, der die Erfolgsaussichten der Klage zu beurteilen hat und diese gegebenenfalls geltend macht.

Neben der Einführung des KonTraG weisen weitere Indizien auf eine zunehmende haftungsrechtliche Inanspruchnahme von Vorstandsmitgliedern hin. ${ }^{230}$ Zum einen existiert eine wachsende Bereitschaft zur gerichtlichen Durchsetzung von Ansprüchen, zum anderen werden durch die Globalisierung seit langem im Ausland bekannte Anspruchsmentalitäten auf Deutschland übertragen, was

\footnotetext{
$227 \mathrm{Vgl}$. Hommelhoff/Mattheus, Corporate Governance, 1998, S. 258.

228 Vgl. Raiser, Kapitalgesellschaften, 1992, S. 113ff.

229 Vgl. Hommelhoff/Mattheus, Corporate Governance, 1998, S. 259.

230

Vgl. Krüger, D\&O, 1999, S. 54f.
} 
durch die Internationalisierung der Anteilseignerstruktur noch verstärkt wird. Bei pflichtwidrigem Verhalten des Vorstands bzw. einzelner Vorstandsmitglieder ist es schließlich möglich, daß deren Bestellung durch den Aufsichtsrat widerrufen wird ( $\$ 84 \mathrm{Abs} .3 \mathrm{AktG}$ ).

c) Aufsichtsrat

\section{(1) Persönliche Anforderungen an Aufsichtsratsmitglieder}

Der Aufsichtsrat ist das gesetzlich vorgeschriebene Überwachungsorgan der Aktiengesellschaft. ${ }^{231}$ Um eine ordnungsgemäße Aufgabenwahrnehmung durch die Aufsichtsratsmitglieder zu gewährleisten, werden an sie durch den Gesetzgeber verschiedene persönliche Anforderungen gestellt ( $\S 100,105 \mathrm{AktG}):{ }^{232}$

1. Aufsichtsratsmitglied können nur natürliche und unbeschränkt geschäftsfähige Personen werden,

2. sie dürfen maximal neun weitere Aufsichtsratsmandate innehaben, wobei fünf Konzernmandate nicht angerechnet werden,

3. es darf sich nicht um einen gesetzlichen Vertreter einer von der Gesellschaft abhängigen Unternehmung handeln,

4. es darf keine Überkreuzverflechtung mit anderen Kapitalgesellschaften bestehen,

5. es darf sich nicht um ein Vorstandsmitglied, einen Prokuristen oder Generalbevollmächtigten der Gesellschaft handeln (Inkompatibilitätsregel).

Dabei ist insbesondere die Höchstzahl weiterer Aufsichtsratsmandate immer wieder Gegenstand der Kritik. ${ }^{233}$ Mit dem KonTraG konnte sich der Gesetzgeber jedoch lediglich zu einer doppelten Anrechnung von Vorsitzmandaten auf die Höchstzahl der Mandate durchringen, nicht aber auf eine Verminderung der absoluten Zahl. Begründet wurde dies mit der stark unterschiedlichen Ausgangssituation in den verschiedenen Aktiengesellschaften und den abweichenden per-

231 Vgl. Mertens, Kölner Kommentar, 1996, Anm. 1 der Vorbemerkungen zu § 95 AktG; Schmidt, Gesellschaftsrecht, 1997, S. 825.

232 Vgl. Semler, Kompetenzen, 1999, S. 9ff., sowie Semler, Wahlverfahren, 1999, S. 79f.; in diesem Zusammenhang sei darauf hingewiesen, daß für den Bundeskanzler und die Bundesminister vorbehaltlich einer Zustimmung des Bundestages gemäß Art. 66 GG das Verbot der Zugehörigkeit zu Aufsichtsräten in Unternehmungen besteht. 
sönlichen Voraussetzungen der einzelnen Mitglieder, denen eine pauschale Absenkung der Höchstzahl an Mandaten nicht gerecht würde. ${ }^{234}$

Die Hauptversammlung hat auf freiwilliger Basis die Möglichkeit, an die Vertreter der Anteilseigner höhere Anforderungen zu stellen. ${ }^{235}$ De lege lata existieren weitere persönliche Voraussetzungen aber nur für die Vertreter der Arbeitnehmer: ${ }^{236}$ Als unternehmungsinterne Vertreter sind nur Beschäftigte wählbar, die wenigstens ein Jahr der Unternehmung angehören und die weiteren Voraussetzungen für die Wahl zum Betriebsrat ( $\$ 8$ BetrVG) erfüllen.

Neben den bereits genannten formalen Anforderungen an Aufsichtsratsmitglieder müssen diese auch über bestimmte inhaltliche Mindestkenntnisse verfügen: ${ }^{237}$ Jedes einzelne Mitglied muß nicht nur Bilanzkenntnisse haben, sondern zusätzlich in der Lage sein, die Vorstandsberichte gemäß § 90 AktG sowie den Prüfungsbericht der Abschlußprüfer zu verstehen. Richtungsweisend ist hier das Hertie-Urteil des BGH, in dem verlangt wird, daß Aufsichtsratsmitglieder diejenigen Mindestkenntnisse besitzen bzw. sich aneignen müssen, die sie brauchen, um alle regulären Geschäftsvorfälle ohne fremde Hilfe verstehen und sachgerecht beurteilen zu können. ${ }^{238}$ Diese Anforderungen sind mit dem KonTraG insbesondere für Ausschußmitglieder verschärft worden, so daß diese nicht nur über allgemeine betriebswirtschaftliche und juristische Kenntnisse verfügen müssen, sie müssen zudem in dem jeweiligen Arbeitsgebiet des Ausschusses Spezialkenntnisse besitzen. ${ }^{239}$

\section{(2) Bildung und Zusammensetzung}

In den hier zu betrachtenden Gesellschaften richten sich die Anzahl, die Wahl und die Zusammensetzung des Aufsichtsrats nach den einschlägigen Vorschriften des AktG und des MitbestG. ${ }^{240}$ Die Anzahl der Aufsichtsratsmitglieder hängt von der Zahl der Mitarbeiter der Unternehmung ab (§95 Abs. 1 AktG i.V.m. § 7 Abs. 1 MitbestG). Für Gesellschaften mit in der Regel bis zu 10.000 Mitarbeitern sind zwölf Aufsichtsratsmitglieder vorgesehen, mit in der Regel bis

\footnotetext{
234 Vgl. Begründung aus dem Regierungsentwurf zum KonTraG in Ernst/Seibert/Stuckert, KonTraG, 1998, S. 54.

235 Vgl. Wellkamp, Aufsichtsrat, 2000, S. 43.

236 Vgl. Raiser, Kapitalgesellschaften, 1992, S. $125 \mathrm{f}$

237 Vgl. Raiser, Kapitalgesellschaften, 1992, S.151; Mertens, Kölner Kommentar, 1996, Anm. 7 zu § 116 AktG.

238 Vgl. BGH, Beratung, 1983, S. 55, 56.

239 Vgl. Hommelhoff/Mattheus, Corporate Governance, 1998, S. 255.

240 Vgl. Raiser, Kapitalgesellschaften, 1992, S. 121ff.
} 
zu 20.000 Mitarbeitern 16 und mit in der Regel mehr als 20.000 Mitarbeitern 20 Mitglieder. Per Satzung kann die Zahl jeweils auf 16 oder 20 Mitglieder erhöht werden ( $\$ 7$ Abs. 1 MitbestG).

Die Sitze im Aufsichtsrat sind je zur Hälfte mit Anteilseigner- sowie Arbeitnehmervertretern zu besetzen, wobei sich die Wahlvorschriften für die Anteilseignervertreter von denen der Arbeitnehmervertreter unterscheiden. ${ }^{241}$ Während die frei bestimmbaren Vertreter der Anteilseigner in der Regel durch die Hauptversammlung auf Vorschlag des Aufsichtsrats mit einfacher oder relativer Mehrheit in einer Listenwahl gewählt ( $\S 101$ Abs. 1, 119 Abs. 1 Nr. 1, 124 Abs. 3, 133 Abs. 1, 2 AktG), ${ }^{242}$ sie auf Grundlage der Satzung durch bestimmte Aktionäre entsendet oder unvollständig besetzte Aufsichtsräte durch das Gericht ergänzt werden, gilt für die Wahl der Arbeitnehmervertreter ein separates, differenziertes Wahlverfahren durch die Arbeitnehmer der Unternehmung. ${ }^{243} \mathrm{Im}$ Ergebnis wählen die Arbeitnehmer einer Unternehmung unmittelbar oder über Wahlmänner unternehmungsinterne Vertreter (Arbeiter, Angestellte und Leitende Angestellte) sowie Repräsentanten der in der Unternehmung vertretenen Gewerkschaften. Deren Anzahl richtet sich nach der Zahl der Mitarbeiter bzw. der Größe des Aufsichtsrats ( $\$ 7$ Abs. 2 MitbestG, vgl. Abb. 6):

\begin{tabular}{|ccccc|}
\hline $\begin{array}{c}\text { Zahl der } \\
\text { Mitarbeiter }\end{array}$ & $\begin{array}{c}\text { Aufsichts- } \\
\text { ratsmitglie- } \\
\text { der } \\
\text { insgesamt }\end{array}$ & $\begin{array}{c}\text { Unterneh- } \\
\text { mungsinterne } \\
\text { Vertreter }\end{array}$ & $\begin{array}{c}\text { Gewerk- } \\
\text { schafts- } \\
\text { vertreter }\end{array}$ & $\begin{array}{c}\text { Anteilseigner- } \\
\text { vertreter }\end{array}$ \\
\hline $2000-10000$ & 12 & 4 & 2 & 6 \\
$10001-20000$ & 16 & 6 & 2 & 8 \\
$>20000$ & 20 & 7 & 3 & 10 \\
\hline
\end{tabular}

Abb. 6: Die Zusammensetzung des Aufsichtsrats nach dem Mitbest $G$

Quelle: Niedenhoff, Mitbestimmung, 2000, S. 405.

Mit der Wahl werden die Mitglieder des Aufsichtsrats bestellt. ${ }^{244}$ Die Annahme der Bestellung erfolgt konkludent oder durch ausdrückliche Willenserklärung, ohne daß für das hierdurch entstehende Schuldverhältnis eine explizite vertragliche Regelung erforderlich ist. Neben dem durch die Wahl und ihrer Annahme begründeten organschaftlichen Verhältnis entsteht implizit auch ein Anstel-

\footnotetext{
$241 \mathrm{Zu}$ den Wahlverfahren beispielsweise Semler, Wahlverfahren, 1999, S. 67ff.

242 Vgl. Henn, Handbuch, 1998, S. 307f.; m.w.N. Wellkamp, Aufsichtsrat, 2000, S. 36, der darauf hinweist, daß die Listenwahl, bei der nur die gesamte Liste angenommen oder abgelehnt werden kann, rechtlich nicht unumstritten ist.

243 Vgl. zu Einzelheiten Niedenhoff, Mitbestimmung, 2000, S. 405ff.

244 Vgl. Schmidt, Gesellschaftsrecht, 1997, S. 839f.
} 
lungsverhältnis des Aufsichtsratsmitglieds. ${ }^{245}$ Für den geleisteten Zeiteinsatz erhalten Aufsichtsratsmitglieder einen Anspruch auf Aufwandsentschädigung, zu der durch die Satzung oder die Hauptversammlung eine Vergütung gewährt werden kann ( $\$ 113 \mathrm{AktG}){ }^{246}$ Die $\S \S 114$ und 115 AktG verhindern außerdem, daß die Vergütung einzelner Mitglieder durch die Gewährung von Beraterverträgen oder von Krediten willkürlich erhöht wird, indem solche Verträge einem Zustimmungsvorbehalt des gesamten Aufsichtsrats unterliegen. Zudem ist durch die Klärung der sehr umfassenden, auch beratenden Aufgaben des Aufsichtsrats die Gewährung zusätzlicher Beraterverträge stark eingeschränkt worden. ${ }^{247}$

Die Amtsdauer der Aufsichtsratsmitglieder beträgt pro Amtszeit maximal fünf Jahre ( $\$ 102$ Abs. 1 AktG), wobei eine Wiederbestellung unbegrenzt oft möglich ist. ${ }^{248}$ Für die Aufsichtsratsmitglieder werden keine Stellvertreter, sondern lediglich Ersatzmitglieder gewählt ( $\$ 101$ Abs. 3 AktG), die beispielsweise bei Tod oder Niederlegung des Mandats in den Aufsichtsrat nachrücken. ${ }^{249}$ Eine Abberufung von Mitgliedern des Aufsichtsrat ist im Vergleich zum Widerruf der Bestellung von Vorstandsmitgliedern problemlos: ${ }^{250}$ Aufsichtsratsmitglieder, die von der Hauptversammlung ohne Bindung an einen Wahlvorschlag gewählt wurden, können jederzeit mit einer Mehrheit von $3 / 4$ der abgegebenen Stimmen abberufen werden. Die Satzung kann das notwendige Quorum erhöhen oder vermindern. Daneben kann die Abberufung von Aufsichtsratsmitgliedern aus wichtigem Grund durch ein Gericht auf Antrag der Mehrheit der Aufsichtsratsmitglieder erfolgen ( $\$ 103$ Abs. $3 \mathrm{AktG}$ ). ${ }^{251}$ Hierdurch besteht für die Mehrheit des Aufsichtsorgans die Möglichkeit, bestimmte Mitglieder aus dem Gremium zu entfernen.

\footnotetext{
245 Vgl. Raiser, Kapitalgesellschaften, 1992, S. 141.

246 Vgl. Schmidt, Gesellschaftsrecht, 1997, S. 840.

247 Vgl. Boujong, Mindestanforderungen, 1995, S. 203ff.; Deckert, Beratungspflichten, 1997, S. 114.

248 Vgl. Hüffer, Aktiengesetz, 1999, Anm. 6 zu § 102 AktG; Meyer-Landrut, Aktiengesetz, 1970, Anm. 3 zu § 102 AktG.

249 Vgl. Raiser, Kapitalgesellschaften, 1992, S. 128f.

250 Vgl. Mertens, Kölner Kommentar, 1996, Anm. 11, 13ff. zu § 103 AktG; Schmidt, Gesellschaftsrecht, 1997, S. 841; Semler, Wahlverfahren, 1999, S. $76 f$.

251 Vgl. Raiser, Kapitalgesellschaften, 1992, 131f.; Semler, Wahlverfahren, 1999, S. 76.
} 


\section{(3) Die Überwachungsaufgabe des Aufsichtsrats}

\section{(a) Begriffliche Einordnung}

Sowohl den Anteilseigner- als auch den Arbeitnehmervertretern im Aufsichtsrat wird gemeinsam durch das AktG die Überwachungsaufgabe in Form einer Generalklausel zugewiesen: ${ }^{252}$

„Der Aufsichtsrat hat die Geschäftsführung zu überwachen“ (§ 111 Abs. 1 AktG).

Zur Konkretisierung der Überwachungsaufgabe leitet SEMLER aus den aktienrechtlichen Kompetenzen des Aufsichtsrats seine Teilaufgaben und aus diesen den Inhalt der Überwachungsaufgabe ab. ${ }^{253}$ Der Inhalt besteht für ihn in der Tatsachenfeststellung, der Urteilsbildung sowie der Einwirkung. Auch POTTHOFF/TRESCHER bezeichnen komplementär zur Leitungsaufgabe des Vorstands die Überwachung als Gesamtaufgabe des Aufsichtsrats. ${ }^{254}$ Sie unterteilen die Überwachung in Prüfungsaufgaben, in eine begleitende Überwachung sowie in eine gestaltende Überwachung. Die gestaltende Überwachung zeichnet sich analog der Einwirkung bei SEMLER durch das Eingreifen des Aufsichtsrats in das Unternehmungsgeschehen aus. Sie beinhaltet u.a. die Auswahl und Bestellung der Vorstandsmitglieder einschließlich des Abschlusses und der Auflösung der Vorstandsverträge, ${ }^{255}$ was für LUTTER gerade nicht zur Überwachung zählt. ${ }^{256}$

Es zeigt sich, daß die Überwachungsaufgabe des Aufsichtsrats ein Bündel verschiedenartiger Tätigkeiten bzw. Teilaufgaben darstellt. Dieses Bündel ist unter Berücksichtigung der in Abschnitt II.A.1.b) vorgenommenen begrifflichen Abgrenzung aus der hier einzunehmenden betriebswirtschaftlichen Perspektive näher zu klassifizieren (vgl. Abb. 7). ${ }^{257}$

Die Prüfungsaufgaben und die Aufgaben innerhalb der begleitenden Überwachung, die die eigentlichen Kontrolltätigkeiten im Rahmen der Überwachungsaufgabe beinhalten, sollen hier zur Kontrolle zusammengefaßt werden. Die Einwirkungskompetenzen als Gegenstand der gestaltenden Überwachung wer-

\footnotetext{
252 Vgl. Potthoff/Trescher, Aufsichtsratsmitglied, 1999, S. 61; Semler, Überwachung, 1996, S. 55.

253 Vgl. Semler, Überwachung, 1996, S. 56ff.

Vgl. Potthoff/Trescher, Aufsichtsratsmitglied, 1999, S. 3f.; ganz ähnlich auch Wagner, Aufsichtsgremien, 1998, S. 73f. Vgl. Wagner, Aufsichtsgremien, 1998, S. 76.

Vgl. Lutter, Aufsichtsratstätigkeit, 1995, S. 285, im Gegensatz zu Potthoff/Trescher, Aufsichtsratsmitglied, 1999, S. 4, sowie Wagner, Aufsichtsgremien, 1998.

257 Vgl. auch Gawrisch, Ermessensentscheidungen, 2000, S. 20.
} 
den dagegen als eigenständiger Aufgabenbereich betrachtet. Sie zeichnen sich durch Mit- sowie Alleinentscheidungsaufgaben des Aufsichtsrats aus.

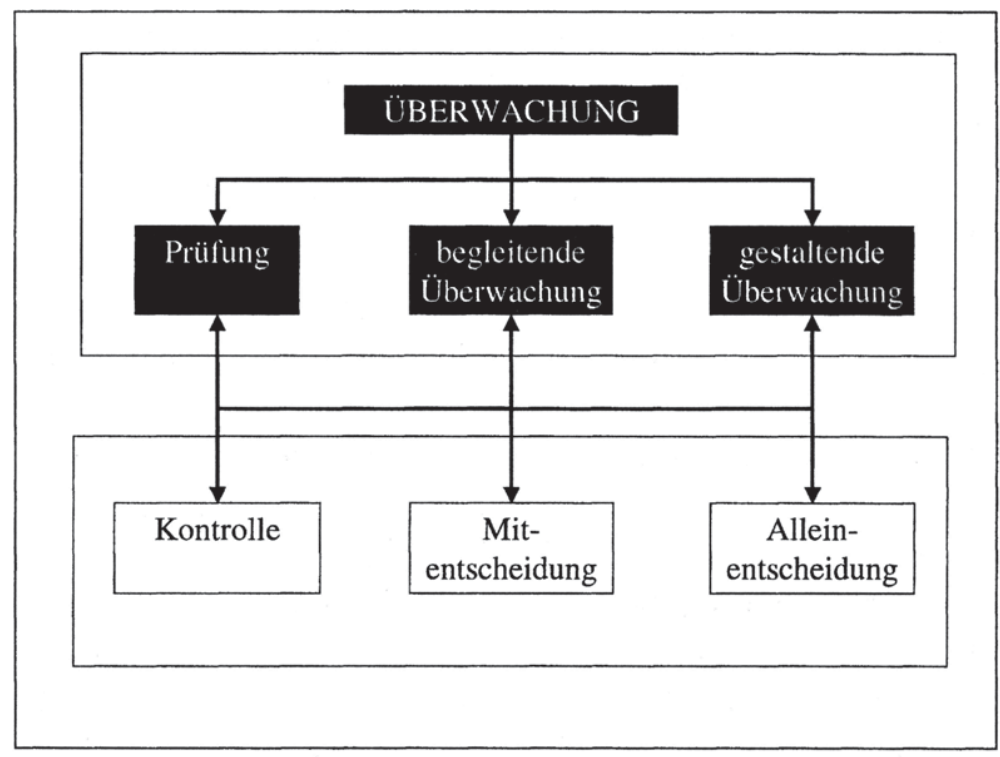

Abb. 7: Klassifikation der Überwachungsaufgabe

\section{(b) Kontrolle}

Als erstes sind die Kontrollaufgaben des Aufsichtsrats näher zu betrachten. In der juristischen Literatur findet sich inzwischen ganz überwiegend ein weites, in die Zukunft gerichtetes Verständnis von Kontrolle: ${ }^{258}$

„Die Kontrolle des Aufsichtsrats darf nicht nur retrospektiv sein, sondern muß sich gerade auch in die Zukunft richten (Ex-anteKontrolle). ${ }^{\text {c259 }}$

Demnach umfaßt die Kontrolle sowohl eine ergebnisorientierte retrospektive Kontrolle als auch eine in die Zukunft orientierte präventive Kontrolle einschließlich einer Beratung mit dem Vorstand über geplante Maßnahmen. ${ }^{260}$ POTTHOFF/TRESCHER sprechen daher auch von einer begleitenden Überwachung, innerhalb derer sich der Aufsichtsrat alle relevanten Informationen notfalls selbst beschafft und auf dieser Grundlage gegebenenfalls als Ausdruck ei-

258 Vgl. m.w.N. Mertens, Kölner Kommentar, 1996, Anm. 11 zu § 111 AktG; Semler, Überwachung, 1996, S. 53; in diesem Sinne bereits Raiser, Kapitalgesellschaften, 1992, S. 117; für Thümmel, Aufsichtsräte, 1999, S. 1892, ist gerade die präventive Kontrolle Ausdruck einer unternehmerischen Tätigkeit des Aufsichtsrats, im Rahmen der er an laufenden oder geplanten Maßnahmen mitwirkt.

259 Begtündung aus dem Regierungsentwurf, Art. $1 \S 90$ KonTraG, 1998, S. 52.

260 Vgl. m.w.N. Mutter, Aktiengesellschaft, 1994, S. 40f. 
ner gestaltenden Überwachung auf den Vorstand einwirkt. ${ }^{261}$ Die Kontroll- und Beratungstätigkeit des Aufsichtsrats muß parallel zu der des Vorstands erfolgen, ${ }^{262}$ so daß eine weitgehende Übereinstimmung mit dem in dieser Arbeit als Bestandteil der Führungsfunktionen verwendeten Kontrollbegriff konstatiert werden kann. ${ }^{263}$

Mit Geschäftsführung wurde jedes rechtliche und tatsächliche Handeln des Vorstands für die Gesellschaft bezeichnet. Auf Grundlage des eben gefundenen Kontrollverständnisses bedeutet dies, daß nicht nur die Konsequenzen vergangenen Vorstandshandelns der Kontrolle durch den Aufsichtsrat unterliegen, sondern ebenfalls die zukünftigen Auswirkungen getroffener bzw. noch zu treffender Entscheidungen. ${ }^{264}$ Im Mittelpunkt der Kontrolle durch den Aufsichtsrat steht dabei nicht die Durchführung der Vorstandsentscheidungen, sondern die Entscheidungsvorbereitung (Planung) bzw. die Entscheidung selbst. ${ }^{265}$ Folgerichtig betont LUTTER die besondere Bedeutung der Beteiligung des Aufsichtsrats an der Planung und der Entscheidung neben der Erfolgskontrolle des unternehmerischen Handelns des Vorstands. ${ }^{266}$ Bezüglich der vom Vorstand delegierten Entscheidungsdurchführung ist lediglich eine Systemkontrolle möglich, die die Einrichtung und Gestaltung eines effizienten Planungs-, Organisations- und Kontrollsystems durch den Vorstand beinhaltet. ${ }^{267}$

Die wesentlichen Kontrollobjekte lassen sich anhand des AktG konkretisieren:

1. Gegenüber dem Aufsichtsrat sind Regelberichte zu erstatten ( $\$ 90$ Abs. 1 AktG), zu deren Inhalt neben der Unternehmungsplanung die aktuelle wirtschaftliche Situation und die Entwicklung der Unternehmung, gravierende Einzelgeschäfte sowie sonstige wichtige Anlässe gehören; die Be-

\footnotetext{
261 Vgl. Potthoff/Trescher, Aufsichtsratsmitglied, 1999, S. 3f.

262 Vgl. Lutter, Aufsichtsratstätigkeit, 1995, S. 291.

263 Vgl. zur Abgrenzung des Kontrollbegriffs Abschnitt II.A.1.b)(3).

264 Vgl. m.w.N. nur Lutter, Aufsichtsratstätigkeit, 1995, S. $290 \mathrm{ff}$.

265 Vgl. Bleicher/Leberl/Paul, Unternehmungsverfassung, 1989, S. 59; Raiser, Kapitalgesellschaften, 1992, S. 117.

266 Vgl. Lutter, Aufsichtsratstätigkeit, 1995, S. 291; Lutter/Krieger, Rechte, 1993, S. 35f.; auch Mertens, Kölner Kommentar, 1996, Anm. 26 zu § 111 AktG.

267 Vgl. Mertens, Kölner Kommentar, 1996, Anm. 3 der Vorbemerkungen zu $§ 95$ AktG; Potthoff/Trescher, Aufsichtsratsmitglied, 1999, S. 63, unter Verweis auf Frese, Unternehmungsführung, 1987, S. 111, 113.
} 
richtsinhalte unterliegen gleichzeitig der Kontrolle durch den Aufsichts$\mathrm{rat}^{268}$

2. Internes Überwachungssystem ( 991 Abs. 2 AktG);

3. Jahresabschluß, Lagebericht und Gewinnverwendungsvorschlag (§ 171 Abs. 1 AktG); ${ }^{269}$

4. etwaiger Abhängigkeitsbericht ( $\$ 314$ Abs. 2 AktG).

Bei seiner Kontrolltätigkeit kann der Aufsichtsrat ebenso wie im Rahmen seiner weiteren Aufgaben auf umfassende interne und externe Informationsquellen zugreifen, die es ihm erlauben, detaillierte und umfassende Informationen über alle Angelegenheiten der Unternehmung zu erhalten. Hierbei ist er nicht nur auf die Berichterstattung des Vorstands beschränkt ( $\$ 90 \mathrm{AktG}$ ), er kann zusätzliche Informationsquellen, beispielsweise den Abschlußprüfer, nutzen. ${ }^{270}$ Gerade dessen Funktion als Informant des Aufsichtsrats ist durch das KonTraG erheblich gestärkt worden. ${ }^{271}$ In der Begründung zum KonTraG wird darüber hinaus explizit auf die Möglichkeit zum Erlaß einer Informationsordnung hingewiesen, die eine umfassende und kontinuierliche Information des Aufsichtsrats sicherstellt. ${ }^{272}$ Es kann daher davon ausgegangen werden, da $\beta$ den Mitgliedern des Aufsichtsrats die für die Wahrnehmung ihrer Kontrollaufgabe erforderlichen Informationen verfügbar sind. Dies ist gerade im Hinblick auf die Kontrolle der Planung von besonderer Bedeutung, da es hier nicht darauf ankommt, Ergebnisse vergangener Entscheidungen zu prüfen, sondern die Vorbereitung zukünftiger Entscheidungen (Planung) zu kontrollieren.

\section{(c) Kontrollmaßstäbe}

Die Kontrolle der Geschäftsführung des Vorstands orientiert sich an vier Kontrollmaßstäben: ${ }^{273}$ Rechtmäßigkeit, Ordnungsmäßigkeit, Zweckmäßigkeit und Wirtschaftlichkeit. Der Aufsichtsrat hat zu gewährleisten, daß der Vorstand sein Handeln an den einschlägigen Gesetzen, der Satzung und gegebenenfalls seiner Geschäftsordnung ausrichtet (Rechtmäßigkeit), ordnungsgemäße Planungs-,

\footnotetext{
268 Vgl. Feddersen, Aufsichtsrat, 2000, S. 388; Lutter/Krieger, Rechte, 1993, S. 36; Wagner, Aufsichtsgremien, 1998, S. 74f.

Bei Mutterunternehmungen im Sinne des $\S 290$ HGB auch den Konzernabschluß und Konzernlagebericht (§ 171 Abs. 1 S. 1 AktG).

Vgl. Henn, Handbuch, 1998, S. 297ff.; Potthoff/Trescher, Aufsichtsratsmitglied, 1999, S. 166.

Vgl. Hommelhoff/Mattheus, Corporate Governance, 1998, S. 256.

Vgl. Begründung aus dem Regierungsentwurf zum KonTraG in Ernst/Seibert/Stuckert, KonTraG, 1998, S. 52. 
Organisations- und Kontrollsysteme eingerichtet hat (Ordnungsmäßigkeit) sowie die Effektivität (Zweckmäßigkeit) und Effizienz (Wirtschaftlichkeit) seiner Maßnahmen sicherstellt. Eine Konkretisierung kann darüber hinaus anhand der beschriebenen Vorstandspflichten erfolgen. ${ }^{274}$

POTTHOFF/TRESCHER heben als fünften Kontrollmaßstab die Ausrichtung der Vorstandstätigkeit auf die Verfolgung des Unternehmensinteresses und der Unternehmungsziele hervor, zwischen denen eine Zweck-Mittel-Beziehung bestehe. ${ }^{275}$ Sie betonen jedoch, daß der Inhalt des Unternehmensinteresses, insbesondere als Kontrollmaßstab der Vorstandstätigkeit, abschließend noch nicht konsensual ermittelt werden konnte. ${ }^{276}$ Hierauf kann jedoch verzichtet werden, da eine Ausrichtung der Vorstandstätigkeit auf die Verfolgung der Unternehmungsziele nur unter Beachtung des Prinzips der Wirtschaftlichkeit sowie der Zweckmäßigkeit sinnvoll ist. Der fünfte Kontrollmaßstab ist daher in den übrigen bereits enthalten.

Insbesondere die Kontrollmaßstäbe der Zweckmäßigkeit und der Wirtschaftlichkeit machen es erforderlich, daß der Aufsichtsrat die Zielkonzeption des Vorstands mit seiner eigenen abgleicht und

„erforderlichenfalls auch in die seiner Ansicht nach beanstandungsfreie Richtung lenken muß.“277

Hierzu stehen dem Aufsichtsrat über die Wahrnehmung seiner weiteren Aufgaben umfassende Sanktions- und Gestaltungspotentiale zur Verfügung.

\section{(d) Einwirkungsmöglichkeiten}

Über die Wahrnehmung ihrer weiteren Aufgaben verfügen die Aufsichtsratsmitglieder de jure über Sanktionspotentiale gegenüber einzelnen Vorstandsmitgliedern oder dem Gesamtvorstand. Sie sollen es ihnen ermöglichen, Verhaltensänderungen beim Vorstand $\mathrm{zu}$ bewirken. LUTTER/KRIEGER subsumieren diese Aufgaben daher unter die Einwirkungsmöglichkeiten des Aufsichtsrats, ${ }^{278}$ die eigene Entscheidungsfelder beinhalten. ${ }^{279}$ Bei diesen Entscheidungsaufgaben handelt es sich erstens um Mitentscheidungsrechte gemeinsam mit dem Vor-

\footnotetext{
274 Vgl. Henn, Handbuch, 1998, S. 297.

275 Vgl. Potthoff/Trescher, Aufsichtsratsmitglied, 1999, S. 79f.; Jaschke, Überwachungsfunktion, 1989, S. 34.

276 Vgl. Potthoff/Trescher, Aufsichtsratsmitglied, 1999, S. 79, unter Verweis auf Semler, Überwachung, 1996, S. 34f., FN 94 mit weiteren Hinweisen.

277 Henn, Handbuch, 1998, S. 298.

278 Vgl. Lutter/Krieger, Rechte, 1993, S. 47.

279 Vgl. Wagner, Aufsichtsgremien, 1998, S. 72ff.
} 
stand oder zweitens um Alleinentscheidungsrechte des Aufsichtsrats (vgl. Abb. 8). ${ }^{280}$ In den Fällen der Mitentscheidung liegt die Initiativverantwortung beim Vorstand. ${ }^{281}$ Dies enthebt die Aufsichtsratsmitglieder jedoch nicht von der Verantwortung zur Gewinnung und Verarbeitung aller für ihre persönliche Entscheidung erforderlichen Informationen. Im Rahmen der Alleinentscheidung dokumentiert sich dagegen in besonderem Maße die unternehmerische Verantwortung des Aufsichtsrats: Er hat in den Fällen, in denen er nicht auf Verlangen Dritter tätig wird, das Initiativrecht bzw. die Initiativverantwortung für die Maßnahme, d.h., er muß die personellen und organisatorischen Rahmenbedingungen sicherstellen, die erforderlichen Informationen beschaffen, diese Informationen bewerten und hieraus die richtigen Ergebnisse ableiten. ${ }^{282}$

\begin{tabular}{|c|c|c|}
\hline \multicolumn{3}{|c|}{ Entscheidung $^{283}$} \\
\hline Mitentscheidung & Alleinentscheidung & $\begin{array}{l}\text { Initiative: } \\
\text { Aufsichtsrat }\end{array}$ \\
\hline $\begin{array}{l}\text { Bildung von Gewinnrücklagen } \\
(\S 58 \mathrm{Abs} .2 \mathrm{AktG})\end{array}$ & $\begin{array}{l}\text { Gestaltung und Erlaß der } \\
\text { Geschäftsordnung des } \\
\text { Vorstands } \\
(\S 77 \text { Abs. 2 S. 1 AktG) }\end{array}$ & $\begin{array}{l}\text { in den } \\
\text { Satzungs- } \\
\text { grenzen }\end{array}$ \\
\hline $\begin{array}{l}\text { Zustimmung zu Abschlags- } \\
\text { zahlungen auf den } \\
\text { Bilanzgewinn } \\
(\S 59 \text { Abs. } 3 \text { AktG) }\end{array}$ & $\begin{array}{l}\text { Bestellung, Wiederbestellung } \\
\text { und Abberufung der } \\
\text { Vorstandsmitglieder } \\
\text { einschließlich der Gestaltung, } \\
\text { des Abschlusses und der } \\
\text { Beendigung der } \\
\text { Anstellungsverträge } \\
\text { ( } \$ 84 \text { Abs. 1, } 3 \text { AktG) } \\
\end{array}$ & $X$ \\
\hline $\begin{array}{l}\text { Zustimmung zu Krediten an } \\
\text { Leitungspersonen } \\
\text { ( } 889 \text { Abs. } 2 \text { AktG) }\end{array}$ & $\begin{array}{l}\text { Bestellung und Abberufung } \\
\text { eines Vorstandsvorsitzenden } \\
\text { ( } \S 84 \text { Abs. } 2 \text { AktG) }\end{array}$ & $\mathrm{X}$ \\
\hline $\begin{array}{l}\text { Entscheidung über } \\
\text { zustimmungspflichtige } \\
\text { Geschäfte } \\
\text { (§111 Abs. 4 S. 2 AktG) }\end{array}$ & $\begin{array}{l}\text { Genehmigung der } \\
\text { Nebentätigkeiten von } \\
\text { Vorstandsmitgliedern } \\
\text { ( } 888 \mathrm{Abs} .1 \mathrm{AktG}) \\
\end{array}$ & \\
\hline
\end{tabular}

280 Vgl. Potthoff/Trescher, Aufsichtsratsmitglied, 1999, S. 165ff., 205ff., 245ff., 289ff.; eine abweichende Unterscheidung bei Hahn, Mitbestimmung, 1999, S. 794.

281 Vgl. Potthoff/Trescher, Aufsichtsratsmitglied, 1999, S. $289 \mathrm{f}$.

282 Vgl. Potthoff/Trescher, Aufsichtsratsmitglied, 1999, S. 245.

283 Vgl. Henn, Handbuch, 1998, S. 297; Lutter/Krieger, Rechte, 1993, S. 47ff.; Schmidt, Gesellschaftsrecht, 1997, S. 829; Wagner, Aufsichtsgremien, 1998, S. 72ff.; mit einer umfassenden Auflistung Semler, Überwachung, 1996, S. 56ff.

284 Vgl. Potthoff/Trescher, Aufsichtsratsmitglied, 1999, S. 4. 


\begin{tabular}{|c|c|c|}
\hline $\begin{array}{l}\text { Zustimmung zu Beraterver- } \\
\text { trägen mit Mitgliedern des } \\
\text { Aufsichtsrats } \\
(\S 114 \text { AktG) }\end{array}$ & $\begin{array}{l}\text { Kreditvergabe an } \\
\text { Vorstandsmitglieder } \\
\text { ( } 889 \text { Abs. 1 AktG) }\end{array}$ & \\
\hline $\begin{array}{l}\text { Zustimmung zu Krediten an } \\
\text { Aufsichtsratsmitglieder } \\
\text { (§115 AktG) }\end{array}$ & $\begin{array}{l}\text { Erteilung des Prüfungsauftrags } \\
\text { an den Abschlußprüfer ( } \S 111 \\
\text { Abs. } 2 \text { S. } 3 \text { AktG und } \S 318 \\
\text { Abs. } 1 \text { S. } 4 \text { HGB) sowie } \\
\text { Entgegennahme des } \\
\text { Prüfungsberichts ( } \S 321 \text { Abs. } \\
5 \text { S. } 2 \text { HGB) }\end{array}$ & $X$ \\
\hline $\begin{array}{l}\text { Feststellung des } \\
\text { Jahresabschlusses } \\
(\S 172 \text { AktG) }\end{array}$ & $\begin{array}{l}\text { Einberufung einer außer- } \\
\text { ordentlichen Hauptversamm- } \\
\text { lung, wenn das Wohl der } \\
\text { Gesellschaft dies erfordert } \\
(\S 111 \mathrm{Abs} .3 \mathrm{AktG})\end{array}$ & X \\
\hline \multirow[t]{5}{*}{$\begin{array}{l}\text { Zustimmung zur Ausgabe } \\
\text { neuer Aktien aus } \\
\text { genehmigtem Kapital } \\
(\S 204 \text { Abs. } 1 \text { AktG i.V.m. } \\
\S 202 \text { AktG) } \\
\S\end{array}$} & $\begin{array}{l}\text { Festlegung zustimmungs- } \\
\text { pflichtiger Geschäfte } \\
(\$ 111 \text { Abs. } 4 \text { AktG); } \\
\text { können vom Aufsichtsrat auch } \\
\text { ad hoc beschlossen werden }{ }^{286} \\
\end{array}$ & $X$ \\
\hline & $\begin{array}{l}\text { Vertretung der Gesellschaft } \\
\text { gegenüber Vorstands- } \\
\text { mitgliedern } \\
(\S 112 \mathrm{AktG}) \\
\end{array}$ & \\
\hline & $\begin{array}{l}\text { Vorschlag zur Wahl von } \\
\text { Aufsichtsratsmitgliedern } \\
\text { ( }(124 \text { Abs. } 3 \text { AktG) } \\
\end{array}$ & $X$ \\
\hline & $\begin{array}{l}\text { Vorschläge für die } \\
\text { Beschlußfassung zu Punkten } \\
\text { der Tagesordnung der } \\
\text { Hauptversammlung } \\
(\S 124 \text { Abs. } 3 \text { AktG) } \\
\end{array}$ & X \\
\hline & $\begin{array}{l}\text { Selbstorganisationspflicht des } \\
\text { Aufsichtsrats }{ }^{287}\end{array}$ & $\mathrm{X}$ \\
\hline
\end{tabular}

Abb. 8: Entscheidungsaufgaben des Aufsichtsrats

Von den vielfältigen (Mit-)Entscheidungsaufgaben eröffnen dem Aufsichtsrat insbesondere die Besetzung der Vorstandsposten, die Organisation der Vorstandstätigkeit, die Gestaltung des monetären Anreizsystems der Vorstandsmit-

285 Vgl. Kropff, Geschäftsführung, 1999, S. 388; Lutter/Krieger, Rechte, 1993, S. $159 f$.

286 Vgl. Lutter/Krieger, Rechte, 1993, S. 49; Raiser, Kapitalgesellschaften, 1992, S. 119; Theisen, Unternehmungsführung, 1987, S. 352.

287 Vgl. Lutter/Krieger, Rechte, 1993, S. 166f.; eine Geschäftsordnung des Aufsichtsrats fordernd auch Berliner Initiativkreis German Code of Corporate Governance, Code, 2000, S. 67, IV.3.1., sowie Feddersen, Aufsichtsrat, 2000, S. 436f. 
glieder und die unternehmerische Mitentscheidung des Aufsichtsrats in bestimmten Geschäftsführungsfragen ein beträchtliches Potential zur Verhaltensbeeinflussung des Vorstands. Diese Einwirkungsmöglichkeiten des Aufsichtsrats zeichnen sich durch das Fällen unternehmerischer Entscheidungen unter Risiko aus. ${ }^{288}$

Um die Bedeutung der durch den Aufsichtsrat zu fällenden Entscheidungen für die Unternehmung zu erfassen, können sie in Klassifikationen besonders relevanter Entscheidungen eingeordnet werden. Hierbei handelt es sich um Versuche, solche Entscheidungen zu identifizieren, die vom obersten Leitungsorgan mit dem Ziel der Ermittlung des optimalen (De-)Zentralisationsgrades auf nachgelagerte hierarchische Ebenen delegiert werden können. ${ }^{289}$

GUTENBERG bezeichnet alle nicht delegierbaren Entscheidungen als sogenannte echte Führungsentscheidungen: ${ }^{290}$

1. Festlegung der Unternehmungspolitik auf weite Sicht,

2. Koordinierung der großen betrieblichen Teilbereiche,

3. Beseitigung von Störungen im laufenden Betriebsprozeß,

4. geschäftliche Maßnahmen von außergewöhnlicher betrieblicher Bedeutsamkeit,

5. Besetzung der Führungsstellen in der Unternehmung.

Eine teilweise nur sprachlich, zum Teil aber auch inhaltlich abweichende Beschreibung von Kernaufgaben der Unternehmungsführung wählt dagegen FRESE, der hierzu

1. alle strategischen Grundsatzentscheidungen mit Auswirkungen auf die Erfolgspotentiale der Unternehmung,

2. Entscheidungen zum Planungs- und Kontrollsystem,

3. Entscheidungen zum Organisationssystem und

4. Entscheidungen zur Organisation der Unternehmungsführung zählt. ${ }^{291}$

288 Mit einer umfassenden Analyse unternehmerischer Entscheidungen des Aufsichtsrats Mutter, Aktiengesellschaft, 1994.

Vgl. Frese, Unternehmungsführung, 1987, S. 111.

Vgl. Gutenberg, Unternehmensführung, 1962, S. 59ff.; sehr ähnlich Semler, Überwachung, 1996, S. $10 \mathrm{f}$. 
Die Einordnung der mit einem Initiativrecht verbundenen Alleinentscheidungsaufgaben des Aufsichtsrats in diese Entscheidungsklassifikationen kommt zu folgendem Ergebnis: Aus der Sicht GUTENBERGs handelt es sich bei der Besetzung von Führungsstellen in der Unternehmung, zu der auch die Vorstandsbesetzung zählt, um eine echte Führungsentscheidung. In der Klassifikation von FRESE kann darüber hinaus die Geschäftsordnungskompetenz des Aufsichtsrats eindeutig als Kernaufgabe der Unternehmungsführung identifiziert werden.

Daneben kommen dem Aufsichtsrat im Rahmen der Zustimmungsvorbehalte Mitentscheidungsrechte bei der „Festlegung der Unternehmungspolitik auf weite Sicht", den „geschäftlichen Maßnahmen von außergewöhnlicher betrieblicher Bedeutsamkeit" sowie allen ,strategischen Grundsatzentscheidungen mit Auswirkungen auf die Erfolgspotentiale der Unternehmung " $\mathrm{zu}^{292}$ Es zeigt sich somit, daß der Aufsichtsrat umfassende und für die Unternehmung wesentliche unternehmerische Entscheidungskompetenzen besitzt. ${ }^{293}$

\section{(4) Aufsichtsratsorganisation}

\section{(a) Überblick}

Die externe Strukturierung der Organisation des Aufsichtsrats ist analog der des Vorstands durch das AktG zwingend vorgegeben. Dagegen finden sich zur internen Strukturierung auch beim Überwachungsorgan nur wenige Vorschriften. ${ }^{294} \S 107$ Abs. 1 S. 1, 2, Abs. 3 AktG normieren die Aufbauorganisation des Aufsichtsrats, $\S \S 107$ Abs. 2, 108-110 AktG die Ablauforganisation. Es handelt sich hierbei aber lediglich um einen Rahmen, den der Aufsichtsrat innerhalb seiner Selbstorganisationspflicht als Konkretisierung der allgemeinen Überwachungspflicht weiter auszugestalten hat.

Die Unternehmungen können durch die organisatorische Gestaltung über Satzungsregelungen oder subsidiär durch den Aufsichtsrat selbst im Rahmen des geltenden Organisationsrechts ihren Spielraum zu einer unternehmungsindividuellen und funktionsfähigen Strukturierung nutzen. ${ }^{295}$ HOMMELHOFF/MATTHEUS weisen zu Recht bei Fehlen entsprechender Satzungsstatuten auf die besondere Bedeutung des Erlasses einer Geschäftsordnung durch den Aufsichtsrat hin. ${ }^{296}$

\footnotetext{
292 So konstatiert bereits Gutenberg, Einführung, 1958, S. 40, eine aktive Mitarbeit des Aufsichtsrats bei den entscheidenden Fragen der Unternehmenspolitik; ebenso Gutenberg, Funktionswandel, 1970, S. 1-10.

293 Vgl. Frese, Organisation, 2000, S. 545.

294 Vgl. Schmidt, Gesellschaftsrecht, 1997, S. 841.

295 Vgl. Dreher, Organisation, 1996, S. 35f.

Vgl. Hommelhoff/Mattheus, Corporate Governance, 1998, S. $254 \mathrm{f}$.
} 
Auch wenn sich der Aufsichtsrat in Einzelfällen über seine eigene Geschäftsordnung hinwegsetzen kann, ${ }^{297}$ wird mit der organisatorischen Strukturierung eine zielorientierte Aufgabenerfüllung unterstützt. Dies zu ermöglichen, gehört zu den Grundpflichten des Aufsichtsrats. ${ }^{298}$

Wollen die Aufsichtsratsmitglieder ihre Aufgaben jedoch pflichtgemäß erfüllen, müssen sie aufgrund des nebenamtlichen Charakters ihrer Tätigkeit sowie des Aufgabenumfangs eine Aufgabendelegation vornehmen. ${ }^{299}$ Dies geschieht innerhalb der Aufbauorganisation erstens über die Zuweisung von über seinen gesetzlichen Auftrag hinausgehenden Aufgaben an den Aufsichtsratsvorsitzenden sowie zweitens durch die Installierung von Aufsichtsratsausschüssen. Mit der Ablauforganisation wird die arbeitsteilige Aufgabenerfüllung koordiniert.

\section{(b) Aufbauorganisation}

\section{(i) Aufsichtsratsvorsitzender}

Der Aufsichtsratsvorsitzende und sein Stellvertreter werden mit 2/3-Mehrheit durch die Aufsichtsratsmitglieder gewählt (§27 Abs. 1 MitbestG) ${ }^{300}$ Kommt im ersten Wahlgang keine Entscheidung zustande, wählen in einem zweiten Wahlgang die Vertreter der Anteilseigner den Vorsitzenden des Aufsichtsrats, die Vertreter der Arbeitnehmer wählen seinen Stellvertreter (§ 27 Abs. 2 MitbestG). Dieses Wahlverfahren gewährleistet die Besetzung des Aufsichtsratsvorsitzes mit einem Anteilseignervertreter. ${ }^{301}$

Durch die normative Zuweisung bestimmter Aufgaben wird der Aufsichtsratsvorsitzende aus dem Gesamtgremium hervorgehoben. ${ }^{302} \mathrm{Zu}$ diesen Aufgaben gehören: ${ }^{303}$

1. Koordination der arbeitsteiligen Aufgabenerfüllung des Gesamtgremiums und seiner Ausschüsse,

2. Repräsentation des Aufsichtsrats,

3. Vertretung der Aktiengesellschaft in bestimmten Angelegenheiten und

4. Leitung der Hauptversammlung.

\footnotetext{
297 Vgl. Lutter/Krieger, Rechte, 1993, S. 166.

298 Vgl. Semler, Kompetenzen, 1999, S. 11.

Vgl. Lutter, Aufsichtsrat, 1992, Sp. 104.

Vgl. zum Aufsichtsratsvorsitzenden beispielsweise Sarrazin, Aufgaben, 1995, S. 125-146.

Vgl. Lutter/Krieger, Rechte, 1993, S. 174.

Vgl. Lutter/Krieger, Rechte, 1993, S. 171.

Vgl. Peus, Aktiengesetz, 1983, S. 16ff.; Starrsinn, Aufsichtsratsvorsitzender, 1995, S. 128ff.
} 
Innerhalb der Aufbauorganisation kommt der Repräsentationspflicht eine besondere Bedeutung zu, da sie die Rolle des Aufsichtsratsvorsitzenden als Informationsmittler zwischen den weiteren Organen der Aktiengesellschaft und dem Aufsichtsrat beinhaltet. ${ }^{304}$ Diese Informationsversorgung ist eine conditio sine qua non für die Wahrnehmung der Aufgaben des Aufsichtsrats. ${ }^{305}$ Sie bezieht sich nicht nur auf die Regelberichterstattung ( $\$ 90$ Abs. 1 AktG), sondern auch auf eventuell notwendige Anforderungsberichte ( $\$ 90$ Abs. 2 AktG). Daneben muß der Aufsichtsratsvorsitzende den Bericht des Aufsichtsrats über die Prüfung des Jahresabschlusses, des Lageberichts und des Gewinnverwendungsvorschlags der Hauptversammlung erläutern ( $\S 171$ Abs. 1, 2 i.V.m. § 176 Abs. 1 AktG). Hierdurch besteht für den Aufsichtsrat die Möglichkeit, den Aktionären problemorientiert die Lage der Gesellschaft darzulegen, um so ihren wachsenden Informationsansprüchen Rechnung zu tragen. ${ }^{306}$

Die Aufgaben des Aufsichtsratsvorsitzenden können alternativ einem Präsidium des Aufsichtsrats zugewiesen werden. ${ }^{307}$ Es besteht zumeist aus dem Aufsichtsratsvorsitzenden, seinem Stellvertreter und mindestens einem weiteren Mitglied. ${ }^{308}$ Dem Präsidium obliegt ebenso wie dem Vorsitzenden der ständige Kontakt zum Vorstand, wodurch sich unabhängig von einer Aufgabenwahrnehmung durch den Aufsichtsratsvorsitzenden oder durch ein Präsidium die besondere Bedeutung eines stetigen Informationsflusses zwischen den beiden Gremien manifestiert. Werden dem Präsidium weitere Aufgaben zugewiesen, die über den eigentlichen Aufgabenbereich des Aufsichtsratsvorsitzenden hinausgehen, wird es als regulärer Ausschuß des Aufsichtsrats tätig.

\section{(ii) Ausschüsse}

Der Aufsichtsrat hat de jure lediglich einen Pflichtausschuß zu bilden: Dies ist der Vermittlungsausschuß zur Ermittlung von Wahlvorschlägen für Vorstandsmitglieder, der unmittelbar nach der Wahl des Aufsichtsratsvorsitzenden und dessen Stellvertreters einzurichten ist ( $\$ 27$ Abs. 3 S. 1 MitbestG). Er wird immer dann tätig, wenn im ersten Wahlgang eines Vorstandskandidaten keine 2/3-Mehrheit erzielt wird ( 331 Abs. 2, 3 MitbestG). Zusätzlich zum Vermittlungsausschuß kann der Aufsichtsrat weitere Ausschüsse zur Wahrnehmung besonderer Aufgaben einsetzen ( $\$ 107$ Abs. 3 AktG). Der Gesetzgeber konnte sich

\footnotetext{
304 Vgl. Lutter/Krieger, Rechte, 1993, S. 175; Peus, Aktiengesetz, 1983, S. 138ff.

305 Vgl. Dreher, Organisation, 1996, S. 51.

306 Vgl. Hommelhoff/Mattheus, Corporate Governance, 1998, S. 257.

307 Vgl. Lutter/Krieger, Rechte, 1993, S. $214 f$.

308 Vgl. Vogel, Aktienrecht, 1980, S. 86.
} 
auch im KonTraG lediglich zur Setzung von Anreizen für eine vermehrte Ausschußbildung durchringen, nicht jedoch zu einer gesetzlichen Pflicht. ${ }^{309}$ Somit verbleibt die Einrichtung von Ausschüssen weiterhin im Autonomiebereich der Gesellschaften.

Es können zwei Arten von Ausschüssen unterschieden werden: Beschließende Ausschüsse sowie Beschlüsse des Gesamtplenums vorbereitende Ausschüsse (§ 107 Abs. 3 S. 2 AktG). ${ }^{310}$ Folgende Aufgaben dürfen einem Ausschuß nicht zur Beschlußfassung übertragen werden ( $\$ 107$ Abs. 3 S. 2 AktG):

1. die Wahl und die Abberufung des Aufsichtsratsvorsitzenden oder seiner Stellvertreter (§ 107 Abs. 1 S. 1 AktG),

2. die Zahlung eines Abschlags auf den Bilanzgewinn ( $\$ 59$ Abs. 3 AktG),

3. der Erlaß einer Geschäftsordnung für den Vorstand (§ 77 Abs. 2 S. 1 AktG),

4. die Bestellung und Abbestellung von Vorstandsmitgliedern ( 84 Abs. 1 S. 1, 3, Abs. 3 S. 1 AktG),

5. die Ernennung und Abberufung eines Vorstandsvorsitzenden ( $\$ 84$ Abs. 2, Abs. 3 S. 1 AktG),

6. die Einberufung einer Hauptversammlung, wenn das Wohl der Gesellschaft dies erfordert ( $\$ 111$ Abs. $3 \mathrm{AktG}$ ),

7. die Prüfung von Jahresabschluß, Geschäftsbericht und Gewinnverwendungsvorschlag sowie die Beschlußfassung über den Aufsichtsratsbericht und dessen Vorlage an den Vorstand ( $\S \S 171,314$ Abs. 2, 3 AktG),

8. die Beschlußfassung über den Kreis zustimmungspflichtiger Geschäfte (§ 111 Abs. 4 S. 2 AktG).

POTTHOFF/TRESCHER betrachten daneben bestimmte Fragen der Selbstorganisation des Aufsichtsgremiums als nicht delegierbar: ${ }^{311}$

1. die Wahrnehmung der gesamten Überwachungsaufgabe, von der nur einzelne Überwachungsfelder übertragbar seien,

2. den Einsatz und die Besetzung von Ausschüssen sowie

\footnotetext{
309 Vgl. Siebel, Ausschüsse, 1999, S. 290ff.

310 Vgl. Henn, Handbuch, 1998, S. 333, sowie umfassend Rellermeyer, Aufsichtsratsausschüsse, 1986.

311 Vgl. Potthoff/Trescher, Aufsichtsratsmitglied, 1999, S. 154.
} 
3. den Erlaß oder die Änderung einer Geschäftsordnung des Aufsichtsrats.

Ansonsten ist der Aufsichtsrat in seiner Möglichkeit, die Entscheidungsvorbereitung an Ausschüsse zu delegieren, nicht beschränkt. ${ }^{312}$ Sowohl bei entscheidenden als auch bei vorbereitenden Ausschüssen verändern sich die Auskunftsrechte von nicht zum Ausschuß gehörenden Aufsichtsratsmitgliedern. ${ }^{313}$ Dies ist bei beschließenden Ausschüssen von besonderer Bedeutung, da Nichtmitglieder nur noch sehr eingeschränkte Informationsrechte besitzen, wodurch verhindert werden kann, daß sensible Detailinformationen allen Aufsichtsratsmitgliedern bekannt werden. Während bei vorbereitenden Ausschüssen die Personenzahl de jure nicht fixiert ist, müssen entscheidungsbefugte Ausschüsse mindestens aus drei Mitgliedern bestehen. ${ }^{314}$ Ihre Besetzung ist in mitbestimmten Gesellschaften strittig. ${ }^{315}$ Zwischen den beiden Extrempositionen des vollkommenen Ausschlusses von Arbeitnehmervertretern und der paritätischen Besetzung von Ausschüssen setzt sich wohl eine vermittelnde Auffassung durch, nach der nur der totale Ausschluß einer Repräsentationsgruppe unzulässig ist. ${ }^{316}$ Dies ist auf die Gesamtheit der Ausschüsse bezogen, nicht auf jeden einzelnen, d.h., wenn insgesamt eine ausgewogene Beteiligung der Arbeitnehmervertreter gewährleistet ist, können einzelne Ausschüsse ohne sie gebildet werden. ${ }^{317}$

Im Einzelfall können sachliche Gründe vorliegen, die den Ausschluß von Arbeitnehmervertretern aus einem Ausschuß rechtfertigen. ${ }^{318}$ Da seit dem KonTraG bei der Auswahl geeigneter Ausschußmitglieder zudem deren Qualifikation als entscheidungsrelevantes Selektionskriterium heranzuziehen ist und die Übernahme von Aufgaben ohne entsprechende Qualifikation eine Haftung des Aufsichtsratsmitglieds begründen kann, werden diese abzuwägen haben, ob sie die erforderlichen Qualifikationen für die Wahrnehmung der Aufgaben des

\footnotetext{
312 Vgl. hierzu Siebel, Ausschüsse, 1999, S. 300f.

313 Vgl. Potthoff/Trescher, Aufsichtsratsmitglied, 1999, S. 163.

314 Vgl. Henn, Handbuch, 1998, S. 335; m.w.N. Lutter/Krieger, Rechte, 1993, S. 203.

315 Vgl. Henn, Handbuch, 1998, S. 334f.; Lutter/Krieger, Rechte, 1993, S. 204f.; Schmidt, Gesellschaftsrecht, 1997, S. 843f., und die dort jeweils angegebene Literatur.

316 Vgl. Schmidt, Gesellschaftsrecht, 1997, S. 844.

317 Vgl. Jaeger, Aufsichtsratsausschüsse, 1995, S. 1739.

318 Vgl. Siebel, Ausschüsse, 1999, S. $290 \mathrm{ff}$.
} 
Ausschusses besitzen. ${ }^{319}$ LUTTER/KRIEGER haben dies schon frühzeitig als Eignungsprinzip bezeichnet. ${ }^{320}$

\section{(c) Ablauforganisation}

Die Gestaltung der Ablauforganisation beinhaltet neben den Regelungen für Aufsichtsratssitzungen insbesondere die Koordination der arbeitsteilig wahrgenommenen Teilaufgaben. Hierbei steht die Einbindung der Tätigkeit von Ausschüssen in die Arbeit des Gesamtgremiums im Vordergrund. Zur Diskussion und zur Beschlußfassung trifft sich der Aufsichtsrat in hierfür vorgesehenen Sitzungen. ${ }^{321}$ Diese werden durch den Aufsichtsratsvorsitzenden vorbereitet, einberufen und geleitet. Mit dem KonTraG ist die Mindestzahl der Aufsichtsratssitzungen angehoben worden ( $\$ 110$ Abs. $3 \mathrm{AktG}$ ): Der Aufsichtsrat muß bei börsennotierten Kapitalgesellschaften zweimal im Kalenderhalbjahr zusammentreten, bei nicht börsennotierten Gesellschaften nur einmal. In seiner allgemeinen Begründung zum Regierungsentwurf betont der Gesetzgeber, daß es sich bei jährlich vier Sitzungen des Gremiums um eine Untergrenze handelt. ${ }^{322}$

Jedes Aufsichtsratsmitglied ist zur Teilnahme an den Sitzungen verpflichtet. ${ }^{323}$ Dies gilt auf Verlangen auch für den Vorstand - er kann aber auch von den Aufsichtsratssitzungen ausgeschlossen werden. Zudem ist die fallweise Teilnahme von Beratern möglich, die die Aufsichtsratsmitglieder bei ihrer Entscheidungsfindung unterstützen ( $§ 109$ Abs. 1 S. 2 AktG).

Bei der Wahrnehmung seiner Aufgaben wird der Aufsichtsrat grundsätzlich als Kollegialorgan tätig und entscheidet durch Beschluß (§ $108 \mathrm{Abs.} 1 \mathrm{AktG}$ ). ${ }^{324}$ Zur Beschlußfassung des Aufsichtsrats ist eine Abstimmung erforderlich. Ein Beschluß gilt als gefaßt, wenn er die Mehrheit der abgegebenen Stimmen erhält ( 29 Abs. 1 MitbestG). Durch die Zweitstimme des Vorsitzenden ( 29 Abs. 2 MitbestG) in Verbindung mit seiner Wahl durch die Anteilseignervertreter ist sichergestellt, daß diese Bank des Aufsichtsrats ihre Interessen durchsetzen kann. Sollte ein Mitglied des Aufsichtsrats verhindert sein, kann es seine Stim-

\footnotetext{
319 Vgl. Hommelhoff/Mattheus, Corporate Governance, 1998, S. 256; Siebel, Ausschüsse, 1999, S. $290 \mathrm{ff}$.

320 Vgl. Lutter/Krieger, Rechte, 1993, S. 204.

321 Vgl. Henn, Handbuch, 1998, S. 328; Raiser, Kapitalgesellschaften, 1992, S. 132.

322 Vgl. Begründung aus dem Regierungsentwurf zum KonTraG in Ernst/Seibert/Stuckert, KonTraG, 1998, S. 56f.

323 Vgl. Lutter/Krieger, Rechte, 1993, S. 182.

324 Vgl. Lutter/Krieger, Rechte, 1993, S. 165; Schmidt, Gesellschaftsrecht, 1997, S. 841.
} 
me auch schriftlich abgeben (sogenannte Stimmbotenschaft gemäß § 108 Abs. 3 AktG).

\section{(5) Verantwortlichkeit und Haftung der Aufsichtsratsmitglieder}

Bei der Erfüllung ihrer Aufgaben haben die Aufsichtsratsmitglieder verschiedene Pflichten zu berücksichtigen, deren Nichtbeachtung haftungsbegründend sein kann. Der Gesetzgeber hat diese Verantwortlichkeit und die Haftung der Aufsichtsratsmitglieder mit einem Verweis auf die entsprechende Norm für den Vorstand geregelt ( $\$ 116 \mathrm{AktG}) .{ }^{325}$ Somit gilt für den Aufsichtsrat ebenfalls das Sorgfaltsmaß des „ordentlichen und gewissenhaften Geschäftsleiters“ ( $\$ 116$ i.V.m. § 93 AktG), das analog die Anwendung der Sorgfalt eines ordentlichen und gewissenhaften Aufsichtsratsmitglieds erfordert. ${ }^{326} \mathrm{Da}$ es sich bei der Aufsichtsratstätigkeit um die Überwachung der Geschäftsführung des Vorstands handelt, konkretisiert sich dieses Sorgfaltsmaß zur Sorgfalt eines ordentlichen und gewissenhaften Überwachers. ${ }^{327}$ HENN betont darüber hinaus die Sorgfalt des Beraters. Diese Erweiterung auf die Beratung ist jedoch tautologisch, da es sich bei der Beratung lediglich um eine Teilaufgabe der Überwachung handelt und somit in der Sorgfalt des Überwachers enthalten ist. ${ }^{328}$

In der Literatur erfolgt die Systematisierung der Pflichten von Aufsichtsratsmitgliedern uneinheitlich. ${ }^{329}$ Werden die unterschiedlichen Einteilungen zusammengefaßt, so lassen sich drei verschiedene Gruppen von aufsichtsratlichen Sorgfaltspflichten unterscheiden: Die Überwachungspflicht, die Treuepflicht sowie die Verschwiegenheitspflicht.

Die Überwachungspflicht wird durch $\S 111 \mathrm{AktG}$ begründet und durch die sich aus § 116 AktG ergebenden Pflichten zur sorgfältigen Wahrnehmung der Organfunktion vertieft bzw. ergänzt. ${ }^{330}$ Hierzu zählen: ${ }^{331}$

1. Pflicht zur Mitarbeit: Dies betrifft die Vorbereitung auf sowie die Teilnahme an Sitzungen des Gesamtplenums und gegebenenfalls von Aus-

325 Vgl. Dreher, Ermessen, 1994, S. 614

326

327

328

329

330

331

Vgl. Raiser, Kapitalgesellschaften, 1992, S. 144; Schmidt, Gesellschaftsrecht, 1996, S. 833.

Vgl. Henn, Handbuch, 1998, S. 344.

Vgl. Mertens, Kölner Kommentar, 1996, Anm. 34 zu § 111 AktG.

Vgl. die abweichenden Zusammenstellungen bei Henn, Handbuch, 1998, S. 343ff.; Raiser, Kapitalgesellschaften, 1992, S. 144ff.; Mertens, Kölner Kommentar, 1996, Anm. 4ff. zu § 116 AktG.

Vgl. Mertens, Kölner Kommentar, 1996, Anm. 5ff. zu § 116 AktG; zur Überwachungspflicht auch Henze, Leitungsverantwortung, 2000, S. 213-216.

Vgl. Mertens, Kölner Kommentar, 1996, Anm. 10ff. zu § 116 AktG; Raiser, Kapitalgesellschaften, S. $147 \mathrm{ff}$. 
schüssen, d.h. die Einbringung seiner Arbeitsleistung durch das einzelne Mitglied.

2. Organisationspflicht: Schaffung einer funktionsfähigen Aufbau- und Ablauforganisation des Aufsichtsrats.

3. Informationspflicht: Wahrnehmung der aktiven und passiven Informationsrechte.

4. Prüfungspflicht: Prüfung des Jahresabschlusses, des Lageberichts und des Vorschlags zur Verwendung des Bilanzgewinns.

5. Pflicht einer persönlichen Urteilsbildung über die Eignung von Vorstandsmitgliedern.

6. Interventionspflicht bei Hinweisen auf eine Pflichtverletzung durch den Vorstand oder durch einzelne Mitglieder.

7. Methodenpflicht: Pflicht zur Anwendung anerkannter technischer, finanzund betriebswirtschaftlicher Methoden bei der Überprüfung zustimmungspflichtiger Geschäfte.

Durch ihre Treuepflicht werden alle Aufsichtsratsmitglieder auf die Verfolgung der Ziele der Unternehmung verpflichtet. ${ }^{332} \mathrm{Da}$ der Aufsichtsrat in börsennotierten Gesellschaften als Repräsentant der Kapitalanleger agiert, müssen die Mitglieder sowohl ihre privaten als auch die Interessen der von ihnen vertretenen Gruppen zurückstellen. ${ }^{333}$ MERTENS spricht in diesem Zusammenhang vom „Prinzip der Homogenität des Aufsichtsrats“, das eine Differenzierung des Gremiums nach einzelnen Interessengruppen nicht zuläßt. ${ }^{334}$ Durch die Pflicht zur persönlichen und weisungsfreien Amtswahrnehmung hat die Mitbestimmung in Aufsichtsräten formal keine Auswirkung, d.h., eine Einflußnahme der Entsendergruppe ist rechtswidrig. ${ }^{335}$ Für POTTHOFF/TRESCHER sind die Bezeichnungen „Arbeitnehmervertreter" bzw. „Anteilseignervertreter“ daher auch nur Ausdruck des unterschiedlichen Bestellungsvorgangs, nicht aber eine Klassifizierung für unterschiedlich Verpflichtete oder Bevorrechtigte. ${ }^{336}$ Kommt es bei einem Auf-

332 Vgl. Henn, Handbuch, 1998, S. 345; Hüffer, Aktiengesetz, 1999, Anm. 5 zu § 116 AktG; Mertens, Kölner Kommentar, 1996, Anm. 27 zu § 116 AktG; Raiser, Kapitalgesellschaften, 1992, S. 144.

So ausdrücklich auch Hommelhoff/Mattheus, Corporate Governance, 1998, S. 252.

Vgl. Mertens, Kölner Kommentar, 1996, Anm. 15 der Vorbemerkungen zu § 95 AktG.

Vgl. Raiser, Kapitalgesellschaften, 1992, S. 144f.; Mertens, Kölner Kommentar, 1996, Anm. 15 der Vorbemerkungen zu § 95 AktG.

Vgl. Potthoff/Trescher, Aufsichtsratsmitglied, 1999, S. 106. 
sichtsratsmitglied zu einer Interessenkollision, so muß entweder das Aufsichtsratsmandat oder die kollidierende Tätigkeit aufgegeben werden. ${ }^{337}$ Auf keinen Fall dürfen weitere Haupt- oder Nebenämter Einfluß auf die Entscheidung des Aufsichtsratsmitglieds haben. ${ }^{338}$ Dieses hat seine Entscheidungen ausschließlich an der Verfolgung der Unternehmungsziele auszurichten.

Für die Aufsichtsratsmitglieder gilt eine absolute Verschwiegenheitspflicht (§ 116 i.V.m. § 93 Abs. 1 S. 2 AktG), die auch Vorrang vor den Informationsbedürfnissen Dritter besitzt. ${ }^{339}$ Sie ist in dieser Strenge erforderlich, da der Aufsichtsrat aufgrund seiner umfassenden Informationsrechte einerseits und seiner heterogenen Zusammensetzung andererseits der Gefahr unterliegt, da $ß$ entweder von Seiten des Vorstands eine restriktive Informationsversorgung erfolgt oder wesentliche Unternehmungsinterna beispielsweise durch Gewerkschafts- oder auch Bankenvertreter Unbefugten weitergegeben werden. ${ }^{340}$

Pflichtverletzungen durch Aufsichtsratsmitglieder begründen durch die sinngemäße Anwendung des § 93 Abs. 2 - 4 AktG einen Schadensersatzanspruch der Gesellschaft. ${ }^{341}$ Hierbei gelten sowohl für Anteilseignervertreter als auch für Arbeitnehmervertreter die gleichen Regeln. ${ }^{342}$ Das strenge Haftungsrecht wurde mit dem KonTraG implizit weiter verschärft, indem der Pflichtenkatalog des Vorstands konkretisiert worden ist und so gleichzeitig die Überwachungspflicht des Aufsichtsrats weiter spezifiziert wurde. ${ }^{343}$ Ansprïche gegen den Aufsichtsrat bzw. einzelne Mitglieder können entweder durch den Vorstand oder aufgrund des bereits erwähnten verminderten Aktionärsquorums geltend gemacht werden ( $\$ 147$ Abs. 3 AktG). Diese Vereinfachung einer Aktionärsklage zielt auf eine verbesserte Überwachung des Vorstands durch den Aufsichtsrat.

Zusammenfassend kann festgestellt werden, daß de jure umfangreiche Regelungstatbestände zum Aufsichtsrat sowie zum Vorstand vorliegenden. Dies betrifft sowohl die Organe selbst als auch ihr Zusammenwirken im Unterneh-

337 Vgl. m.w.N. Henn, Handbuch, 1998, S. 345.

338 Vgl. Potthoff/Trescher, Aufsichtsratsmitglied, 1999, S. 115.

Vgl. Henn, Handbuch, 1998, S. 346; Hüffer, Aktiengesetz, 1999, Anm. 7 zu § 116 AktG; Raiser, Kapitalgesellschaften, 1992, S. 146f; Schmidt, Gesellschaftsrecht, 1997, S. 830.

340 Vgl. Schmidt, Gesellschaftsrecht, 1997, S. 832.

341 Vgl. Raiser, Kapitalgesellschaften, 1992, S. 144; Hüffer, Aktiengesetz, 1999, Anm. 8 zu § 116 AktG.

342 Vgl. Edenfeld/Neufang, Aufsichtsrat, 1999, S. 57.

343 Vgl. Vogler/Gundert, Einführung, 1998, S. 2377; Hommelhoff/Mattheus, Corporate Governance, 1998, S. 252; im Überblick zur Vorstands- und Aufsichtsratshaftung bei Pflichtverletzungen nach dem AktG Kau/Kukat, Haftung, 2000, S. 1045-1050. 
mungsführungsprozeß. Insbesondere die Betrachtung der Aufgaben des Aufsichtsrats konnte zeigen, daß ihm wichtige Entscheidungsaufgaben rechtlich übertragen sind. Es ist daher aus betriebswirtschaftlicher Sicht zu fragen, welche Voraussetzungen für eine ordnungsgemäße Erfüllung dieser Aufgaben vorhanden sein sollten. Bevor dies geschieht, soll jedoch nachfolgend zunächst analysiert werden, welche ökonomischen Konsequenzen aus der juristisch determinierten Situation resultieren.

\section{Zielerreichung in Publikumsaktiengesellschaften}

\section{1. Ökonomische Analyse der Spitzenverfassung}

\section{a) Überblick}

Mit der Spitzenverfassung ist ein wesentlicher Teil der unternehmungsbezogenen Handlungsrechte und -pflichten von an der Unternehmung beteiligten Personengruppen festgelegt. ${ }^{344}$ Diese Rechte und Pflichten können entweder qua Gesetz zwingenden Charakter haben (beispielsweise die Arbeitnehmermitbestimmung im Aufsichtsrat) oder den Unternehmungen werden im Rahmen der gesetzlichen Vorschriften gewisse Handlungsoptionen überlassen. ${ }^{345}$ Dies gilt beispielsweise für die im KonTraG enthaltenen Anregungsnormen, die zwar eine vermehrte Ausschußbildung im Aufsichtsrat bewirken wollen, sie aber nicht verbindlich vorschreiben. ${ }^{346}$ Hierzu zählt ebenso die Möglichkeit des Aufsichtsrats, für den Vorstand eine Geschäftsordnung erlassen zu können, dies aber ohne entsprechende Satzungsbestimmung nicht zu müssen ( $\$ 77$ Abs. 2 AktG). Für Publikumsaktiengesellschaften ist diese Ausgangssituation mit Bezug auf die Spitzenverfassung im vorangegangenen Abschnitt dargestellt worden. Sie soll nachfolgend hinsichtlich der hiermit verbundenen ökonomischen Konsequenzen analysiert werden. ${ }^{347}$

Den Ausgangspunkt der Analyse bildet die in Publikumsaktiengesellschaften bestehende personelle Trennung von Eigentümern und Managern. ${ }^{348}$ Dieses bereits 1932 von BERLE und MEANS beschriebene Phänomen moderner Kapitalgesellschaften kennzeichnet das Wesen der Spitzenverfassung dieses Unterneh-

\footnotetext{
344 Vgl. Picot/Dietl/Franck, Organisation, 1997, S. 186.

345 Vgl. Hartmann-Wendels, Kontrollrechte, 1995, Sp. 1217.

346 Vgl. Feddersen, Aufsichtsrat, 2000, S. 386; für Möllers, Kapitalmarkttauglichkeit, 1999, S. 439, gehen diese Anregungsnormen über den Charakter eines Symbolgesetzes hinaus und er geht davon aus, daß sie von den Adressaten befolgt und angewendet werden.

347 Zur Ökonomischen Analyse des Rechts grundlegend Posner, Analysis, 1998, sowie Ott/Schäfer, Analyse, 1993.

348 Vgl. Bleicher u.a., Spitzenorganisation, 1984, S. 22; Chmielewicz, Führung, 1988, S. 569.
} 
mungstyps. ${ }^{349}$ Zur Erklärung hieraus resultierender Konsequenzen für die Formulierung genereller Unternehmungsziele wird diese Situation zunächst mit Hilfe der Verfügungsrechtstheorie unter zusätzlicher Verwendung der Transaktionskostentheorie analysiert, um hierauf aufbauend Probleme und geeignete Lösungsansätze auf Grundlage der Principal Agent-Theorie diskutieren zu können. ${ }^{350}$

Im Mittelpunkt der institutionenökonomischen Ansätze (dies sind die Property Rights-Theorie, die Transaktionskostentheorie sowie die Principal AgentTheorie) stehen Institutionen und deren Auswirkungen auf menschliches Handeln. ${ }^{351}$ RICHTER/FURUBOTN weisen darauf hin, daß der Institutionenbegriff noch nicht hinreichend exakt abgegrenzt werden konnte. ${ }^{352}$ Sie definieren Institution als ein System formgebundener (formaler) und formungebundener (informaler) Regeln einschließlich der Vorkehrungen zu deren Umsetzung. ${ }^{353}$ Institutionen beabsichtigen die zielgerichtete Verhaltensbeeinflussung von Individuen, um hierdurch Ordnung zu schaffen und Unsicherheit zu vermindern. ${ }^{354}$ Als Beispiele für Institutionen können Gesetze, Gerichtsurteile, Verträge oder organisatorische Regelungen genannt werden. ${ }^{355}$ Wie die aufgezählten Beispiele zeigen, zählen hierzu auch die Determinanten der Spitzenverfassung der Aktiengesellschaft. ${ }^{356}$ Ziel der institutionenökonomischen Ansätze ist einerseits eine positive Analyse und andererseits die normative Ableitung von Gestaltungsempfehlungen für ein möglichst effizientes Design solcher Institutionen. ${ }^{357}$ Diese Analyse aus dem Blickwinkel des methodologischen Individualismus, in der soziale Gebilde wie eine Unternehmung aus der Perspektive des Individuums und seiner Entscheidungen untersucht werden, geht von bestimmten menschlichen Ver-

$349 \mathrm{Vgl}$. grundlegend Berle/Means, corporation, 1932.

350 Zur situativen Eignung einer solch komplementären Vorgehensweise Picot/Diet//Franck, Organisation, 1997, S. 94; eine alleinige Verwendung der Principal Agent-Theorie in der Praxis m.w.N. ablehnend Elschen, Agency-Theorie, 1991, S. 1003.

351 Vgl. Picot/Dietl/Franck, Organisation, 1997, S. 55, 91.

352 Vgl. Richter/Furubotn, Institutionenökonomik, 1996, S. 7, unter Verweis auf Arrow, Theory, 1970, S. 224.

353 Ähnlich Picot/Diet1/Franck, Organisation, 1997, S. 11ff., die Institutionen als sanktionierbare Erwartungen definieren; für North, Institutions, 1990, S. 3, sind sie ganz allgemein „the rules of the game in a society".

354 Vgl. North, Institutions, 1990, S. 3.

355 Vgl. mit weiteren Beispielen Picot/Dietl/Franck, Organisation, 1997, S. 10ff.

356 Mit Bezugnahme auf das Gesellschaftsrecht und seine Auswirkungen auf die Transaktionskosten beispielsweise Posner, Analysis, 1998, S. 452.

357 Vgl. Picot/Diet1/Franck, Organisation, 1997, S. 91. 
haltensannahmen aus: Dies sind begrenzte Rationalität und individuelle Nutzenmaximierung. ${ }^{358}$

Als erste dieser Theorien soll die Property Rights-Theorie herangezogen werden, um die in Aktiengesellschaften vorzufindende Verfügungsrechtsstruktur zu analysieren.

\section{b) Verfügungsrechtsdelegation in der Publikumsaktiengesellschaft}

\section{(1) Verfügungsrechte}

Die Property Rights-Theorie (Verfügungsrechtstheorie) untersucht die Gestaltung und Verteilung von Verfügungsrechten (englisch Property Rights). ${ }^{359}$ Verfügungsrechte sind alle denkbaren mit dem Eigentum an einem wirtschaftlichen $\mathrm{Gut}^{360}$ verbundenen Individualrechte an allen möglichen Ressourcen (im weitesten Sinne), die in irgendeiner Weise individuellen Nutzen spenden. ${ }^{361}$ Hierzu zählen das Recht

1. zur Nutzung eines Gutes,

2. zu seiner Veränderung,

3. zur Aneignung von Erträgen aus der Nutzung des Gutes und

4. zur Veräußerung des Gutes einschließlich der Aneignung eines Liquidationserlöses.

Der Wert eines Gutes hängt daher nicht nur von seinen Eigenschaften ab, er wird darüber hinaus wesentlich durch die mit ihm verbundenen Verfügungsrechte bestimmt. ${ }^{362}$ Zwischen Wirtschaftssubjekten werden die mit den Gütern verbundenen Verfügungsrechte durch Verträge übertragen. Unternehmungsintern erfolgt dies durch organisatorische Regelungen auf Grundlage der Unternehmungsverfassung.

Können Wirtschaftssubjekte Verfügungsrechte nur eingeschränkt ausüben, liegt eine Verdünnung dieser Rechte vor. ${ }^{363}$ Sie können sowohl auf eine unterschied-

358 Der Grad der Nutzenindividualität ist abhängig vom jeweiligen Modellansatz; Williamson, Markets, 1975, S. 26-30, geht beispielsweise von Opportunismus aus.

359 Vgl. Picot/Diet//Franck, Organisation, 1997, S. 54ff., 92; grundlegend die Arbeiten von Coase, Nature, 1937, S. 386-405, sowie Alchian, Economics, 1977, S. 127-149.

360 Vgl. zu wirtschaftlichen Gütern beispielsweise Busse von Colbe/Laßmann, Betriebswirtschaftstheorie, 1991, S. 72.

361 Vgl. Alchian/Demsetz, Production, 1972, S. 777-795; Neus, Betriebswirtschaftslehre, 1998, S. 99; Picot/Dietl/Franck, Organisation, 1997, S. 54f.; Ridder-Aab, Aktiengesellschaft, 1980, S. 38.

362 Vgl. Neus, Betriebswirtschaftslehre, 1998, S. 99; Picot/Diet//Franck, Organisation, 1997, S. 55.

363 Vgl. Neus, Betriebswirtschaftslehre, 1998, S. 100; Picot/Diet//Franck, Organisation, 1997, S. 55. 
liche Zahl von Personen verteilt werden als auch unvollständig zugeordnet sein. Insgesamt sind vier mögliche Verdünnungszustände zu unterscheiden (vgl. Abb. 9).

\begin{tabular}{|c|c|c|c|}
\hline \multirow{5}{*}{ 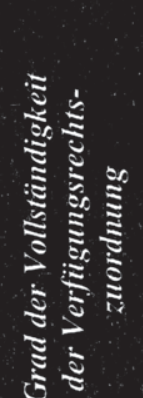 } & & Niedrig & Hoch \\
\hline & \multirow{2}{*}{ 造 } & $\begin{array}{c}\text { konzentrierte } \\
\text { Verfügungsrechtsstruktur }\end{array}$ & $\begin{array}{c}\text { verdünnte } \\
\text { Verfügungsrechtsstruktur }\end{array}$ \\
\hline & & Bsp.: Einzelunternehmung & $\begin{array}{c}\text { Bsp.: OHG mit großer } \\
\text { Gesellschafterzahl }\end{array}$ \\
\hline & \multirow{2}{*}{ 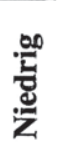 } & $\begin{array}{c}\text { verdünnte } \\
\text { Verfügungsrechtsstruktur }\end{array}$ & $\begin{array}{c}\text { stark verdünnte } \\
\text { Verfügungsrechtsstruktur }\end{array}$ \\
\hline & & Bsp.: Stiftung & $\begin{aligned} \text { Bsp.: } & \text { Publikumsaktien- } \\
& \text { gesellschaft }\end{aligned}$ \\
\hline
\end{tabular}

Abb. 9: Dimensionen der Verdünnung von Property Rights

Quelle: In Anlehnung an Picot/Diet/Franck, Organisation, 1997, S. 55.

Verfügungsrechte an Gütern im allgemeinen Sinne sind für Unternehmungen zu konkretisieren. $\mathrm{Zu}$ den unternehmungsbezogenen Rechten gehören beispielsweise

1. die Festlegung der Unternehmungspläne,

2. ein Weisungs- und Sanktionsrecht gegenüber den Mitarbeitern,

3. ein unsicherer Residualeinkommensanspruch sowie

4. eine Veräußerungsmöglichkeit. ${ }^{364}$

NEUS faßt diese Rechte zu zwei Gruppen von Verfügungsrechten zusammen: Zum einen den Residualeinkommensanspruch, zum anderen das Recht zur Unternehmungsleitung, das alle übrigen Verfügungsrechte umfaßt und in dessen Mittelpunkt das Treffen unternehmerischer Entscheidungen steht. ${ }^{365}$ Dies korrespondiert mit der angloamerikanischen Terminologie einer Trennung von „ownership“ und „control“, die zu externen Effekten führt. ${ }^{366}$

\footnotetext{
364 Vgl. Neus, Betriebswirtschaftslehre, 1998, S. 113.

365 Vgl. Neus, Betriebswirtschaftslehre, 1998, S. 128f.

366 Vgl. Posner, Analysis, 1998, S. 451f., für den diese Trennung in der Publikumsgesellschaft ein effizientes Arrangement darstellt; Shleifer/Vishny, Corporate Governance, 1997, S. 740, sprechen hierbei auch von einer Trennung von "finance“ und „management“.
} 


\section{(2) Externe Effekte}

Durch eine unvollständige Zuordnung von Verfügungsrechten entstehen sogenannte externe Effekte, die insbesondere in Publikumsaktiengesellschaften Ineffizienzen begründen können. ${ }^{367}$ Externe Effekte sind durch wirtschaftliches Handeln ausgelöste unkompensierte Nutzenminderungen (negativ) oder Nutzenmehrungen (positiv) Dritter. ${ }^{368} \mathrm{Im}$ Falle negativer externer Effekte muß das handelnde Wirtschaftssubjekt nicht alle negativen Folgen seines Handelns tragen. Es kann vielmehr einen Teil der anfallenden Nutzenminderungen auf Dritte überwälzen. Positive externe Effekte führen dagegen zu Nutzenmehrungen bei Dritten, ohne daß diesen für die Nutzensteigerung entsprechende Kosten entstehen. Das Ausmaß solcher externen Effekte ist um so geringer, je eindeutiger jedem einzelnen Wirtschaftssubjekt die Folgen seines Handelns zugeordnet werden können, d.h. desto vollständiger Verfügungsrechte zugewiesen sind. ${ }^{369}$ Hieraus ist zu folgern, daß der effiziente Einsatz ökonomischer Ressourcen positiv mit dem Umfang der zugewiesenen Verfügungsrechte korreliert ist.

Die Verbindung des Residualeinkommensanspruchs mit dem Recht auf Nutzung und Veränderung des Gutes stellt sicher, daß den Handelnden alle Folgen seiner Handlungen selbst treffen. Er hat einen sehr großen Anreiz, die ihm zur Verfügung stehenden Ressourcen effizient einzusetzen. ${ }^{370}$ Als allgemeine Organisationsempfehlung fordern PICOT/DIETL/FrANCK daher eine möglichst vollständige Zuordnung von Rechtebündeln. ${ }^{371}$ Mit Blick auf die Unternehmungsverfassung bedeutet dies die Etablierung einer eigentümergeleiteten Unternehmung, in der die Eigenkapitalgeber direkt die Unternehmungsleitung ausüben. ${ }^{372}$

Die vollständige und exklusive Zuweisung von Verfügungsrechten scheitert in der Realität jedoch an der Existenz von Transaktionskosten. Dies sind alle Mühen und Nachteile, die von den beteiligten Akteuren bei der Zuordnung und Durchsetzung von Property Rights zu tragen sind. ${ }^{373}$ In der Publikumsaktiengesellschaft verhindert die Vielzahl der Anteilseigner aufgrund prohibitiv hoher

367 Vgl. Kräkel, Management, 1999, S. 260.

368 Vgl. grundlegend Pigout, Welfare, 1932/1962.

369 Vgl. Coase, Social Cost, 1960, S. 8; Demsetz, Property Rights, 1967, S. 348.

370 Vgl. Picot, Verfügungsrechtstheorie, 1995, Sp. 2107; Picot/Diet1/Franck, Organisation, 1997, S. 57.

371 Vgl. Picot/Dietl/Franck, Organisation, 1997, S. 62.

372 Vgl. Neus, Betriebswirtschaftslehre, 1998, S. $147 f$.

373 Vgl. Picot/Dietl/Franck, Organisation, 1997, S. 57; umfassend zu Transaktionskosten Williamson, Transaction-cost economics, 1979, S. 3-61, sowie Richter/Furubotn, Institutionenökonomik, 1996, S. 49ff. 
Transaktionskosten die Teilhabe aller Aktionäre an der Unternehmungsleitung, so daß hier eine Verdünnung der Verfügungsrechte vorliegt. ${ }^{374}$ Dem Nachteil der Entstehung von externen Effekten im Vergleich zur eigentümergeleiteten Unternehmung steht der Vorteil der Spezialisierung der Eigentümerfunktionen entgegen. ${ }^{375}$ Verfügungsrechte sollen dorthin geleitet werden, wo für deren Ausübung spezifische Vorteile vorhanden sind. $\mathrm{Zu}$ diesen zählt NEUS die Möglichkeit der Kapitalbereitstellung, die Fähigkeit und die Bereitschaft zur Risikoübernahme sowie die Fähigkeit, eine Unternehmung zu leiten. ${ }^{376}$ Während die Eigenkapitalgeber die Unternehmung mit Kapital ausstatten und als Residualeinkommensempfänger das mit ihr verbundene Risiko tragen, können sie für die Leitung der Unternehmung geeignete Agenten auswählen. Hierdurch entsteht eine Principal Agent-Beziehung zwischen Anteilseignern und Leitungsträgern. ${ }^{377}$

\begin{abstract}
„The separation of ownership and control is a false issue. Separation is efficient, and indeed inescapable, given that for most shareholders the opportunity costs of active participation in the management of the firm would be prohibitively high. What is necessary in the interests of the shareholders is not participatory shareholder democracy but machinery for discouraging management from deflecting too much of the firm's net income from the shareholders to itself." ${ }^{\text {"378 }}$
\end{abstract}

Nachfolgend soll daher das durch den Gesetzgeber institutionalisierte Verfügungsrechtsarrangement der Aktiengesellschaft mit Hilfe der Principal AgentTheorie analysiert werden. Ziel muß es dabei sein, für die hieraus resultierenden Probleme geeignete Lösungsmöglichkeiten aufzuzeigen.

\title{
c) Principal Agent-Beziehungen als Folge der Verfügungsrechtsdelegation
}

In Aktiengesellschaften ist die Hauptversammlung als Vertretungsorgan der Aktionäre de jure nicht nur von der Unternehmungsleitung ausgeschlossen (§ 119 Abs. 2 AktG), darüber hinaus ist auch das Überwachungsrecht delegiert. ${ }^{379}$ Durch diese Übertragung von Verfügungsrechten entstehen in Publikumsaktiengesellschaften mehrstufige Auftraggeber/Auftragnehmer-Beziehungen zwischen

\footnotetext{
374 Vgl. Kräkel, Management, 1999, S. 260; Neus, Betriebswirtschaftslehre, 1998, S. 149f.

375 So auch Shleifer/Vishny, Corporate Governance, 1997, S. 740. Vgl. Neus, Betriebswirtschaftslehre, 1998, S. 150; Jaschke, Überwachungsfunktion, 1989, S. 42.

377 Vgl. Chmielewicz, Führung, 1988, S. 568; Chmielewicz, Unternehmensverfassung, 1993, Sp. 4408ff.

378 Posner, Analysis, 1992, S. 411; Shleifer/Vishny, Corporate Governance, 1997, S. 741, sprechen von einer Enteignung der Kapitalgeber durch die Manager.

379 Vgl. Jaschke, Überwachungsfunktion, 1989, S. 48.
} 
der Hauptversammlung, dem Aufsichtsrat und dem Vorstand. ${ }^{380}$ Principal Agent-Beziehungen zeichnen sich dadurch aus, daß Handlungen des Agent nicht nur sein eigenes Nutzenniveau beeinflussen, sondern auch das des Principal. ${ }^{381}$

Ungeachtet der gesetzlichen Notwendigkeit könnten die Aktionäre aufgrund ihrer Gruppengröße, dem mangelhaften Organisationsgrad, ihrer Heterogenität sowie zur Ausnutzung von Spezialisierungsvorteilen als erster Principal der Unternehmung den Aufsichtsrat mit der Überwachung des Vorstands beauftragen. ${ }^{382}$ Dies beinhaltet die Auswahl der zur Leitung der Unternehmung befugten Vorstandsmitglieder, so da $\beta$ die notwendigen Bedingungen sowohl des Verbleibs der Personalkompetenz als auch der Überwachungskompetenz bei den Delegierenden in der Aktiengesellschaft nicht erfüllt sind. ${ }^{383}$ Auf dieser ersten Stufe wird der Aufsichtsrat zum Agenten der Aktionäre, er ist jedoch gleichzeitig auf der zweiten Stufe Principal des Vorstands, dem Agenten des Aufsichtsrats. $^{384}$

Der Vollständigkeit halber sei erwähnt, daß der Vorstand auf einer dritten Stufe als Principal der von ihm als Agents beauftragten Führungskräfte der Unternehmung agiert. ${ }^{385}$ Durch diese dritte Stufe ergeben sich Rückwirkungen auf die ersten beiden Agency-Beziehungen, da auf der Arbeitnehmerbank im Aufsichtsrat auch Leitende Angestellte der Unternehmung zu finden sind. Daneben können weitere Principal Agent-Beziehungen in sehr unterschiedlicher Form zwischen den genannten Personengruppen vorliegen: So könnte etwa der Vorstand als Agent der Mitarbeiter bei der Durchsetzung ihrer Interessen gegenüber den Aktionären der Gesellschaft gesehen werden. Nachfolgend soll jedoch ausschließlich das mehrstufige Principal Agent-Verhältnis einerseits zwischen Ak-

380 Vgl. Bernhardt/Witt, Unternehmensleitung, 1999, S. 838f.; Chwolka, Aufsichtsrat, 1999, S. 636ff.; Jaschke, Überwachungsfunktion, 1989, S. 48f.; Picot/Dietl/Franck, Organisation, 1997, S. 82; Semler, Unternehmensüberwachung, 1995, S. 49f.

Vgl. zur Principal Agent-Theorie beispielsweise Pratt/Zeckhauser, Agents, 1985; Levinthal, Agency, 1988, S. 153-188; Eisenhardt, Agency, 1989, S. 57-74.

Vgl. Bernhardt/Witt, Unternehmensleitung, 1999, S. 838f.; Jaschke, Überwachungsfunktion, 1989, S. 48f; es zeigt sich, daß sich die Aktionäre bei einer Wahlmöglichkeit, wie sie in Frankreich zwischen dem monistischen und dem dualistischen System besteht, überwiegend für eine monistische Struktur entscheiden, vgl. hierzu Dufey/Hommel/Riemer-Hommel, Corporate Governance, 1998, S. 55.

Vgl. hierzu Bleicher u.a., Spitzenorganisation, 1984, S. 22.

Vgl. Jaschke, Überwachungsfunktion, 1989, S. 50; m.w.N. Erlei/Leschke/Sauerland, Institutionenökonomik, 1999, S. 162. mik, 1999, S. 74f.; Picot/Diet//Franck, Organisation, 1997, S. 82. 
tionären und Aufsichtsrat sowie andererseits zwischen Aufsichtsrat und Vorstand betrachtet werden.

Zudem werden hier zwei Annahmen für die weitere ökonomische Analyse eingeführt: $^{386}$

1. Aktionäre, Aufsichtsrat und Vorstand werden jeweils in ihrer Gesamtheit betrachtet, so daß Individualinteressen unberücksichtigt bleiben.

2. Im Aufsichtsrat wird ausschließlich die Bank der Anteilseignervertreter betrachtet, d.h., es wird Interessenmonismus unterstellt.

\section{d) Probleme in Principal Agent-Beziehungen}

Zum Verständnis der in der Principal Agent-Theorie beschriebenen Auftragsprobleme und hieraus abzuleitender Lösungsvorschläge muß zunächst geklärt werden, was Gegenstand dieser Theorie ist. ${ }^{387}$ Die sehr große Anzahl von Beiträgen wird von JENSEN in sogenannte positive und normative Modelle unterschieden, die einer positiven bzw. einer normativen Principal Agent-Theorie zugeordnet werden ${ }^{388}$ Alle Beiträge machen in ihrer Gesamtheit die Principal Agent-Theorie aus. Sie analysiert und gestaltet Auftragsbeziehungen zwischen Auftraggeber und Auftragnehmer, in denen

1. aufgrund von Eigeninteresse und individuell nutzenmaximierendem Verhalten Zielkonflikte zwischen den Beteiligten bestehen,

2. exogene Einflußgrößen dazu führen, daß das Handlungsergebnis nicht nur vom Anstrengungsniveau des Agenten abhängt,

386 So auch Chwolka, Aufsichtsrat, 1999, S. 631, sowie Jaschke, Überwachungsfunktion, 1989, S. 54.

387 Vgl. hierzu grundlegend in historischer Reihenfolge zur positiven Principal Agent-Theorie Jensen/Meckling, Theory, 1976, S. 305-360; Fama, Agency, 1980, S. 288-307; Fama/Jensen, Separation, 1983, S. 301-325, sowie zur normativen Principal Agent-Theorie Wilson, Incentives, 1969, S. 287-307; Berhold, theory, 1971, S. 460-482; Ross, Agency, 1973, S. 134-139; Harris/Raviv, Contracts, 1978, S. 20-30; Holmström, Moral hazard, 1979, S. 74-91; Shavell, incentives, 1979, S. 55-73. 
3. der Beauftragte die Höhe seines Anstrengungsniveaus zumindest teilweise verbergen kann, so daß der Auftraggeber aufgrund dieses Informationsdefizits das Verhalten des Beauftragten nur unvollständig beobachten kann. ${ }^{389}$

Solche Informationsvorsprünge bzw. allgemein Informationsasymmetrien entstehen dadurch, daß realiter aufgrund prohibitiver Kosten der Informationsbeschaffung das Wissen der ökonomischen Akteure unvollständig und ungleich verteilt ist. ${ }^{390}$ Informationsvorsprünge sind jedoch nicht nur die Ursache für die Entstehung von Principal Agent-Problemen, sie sind - neben den oben beschriebenen Transaktionskostenvorteilen - gleichzeitig die Voraussetzung für die Realisierung von Spezialisierungsvorteilen innerhalb der Auftragsbeziehung. ${ }^{391}$ Der Auftraggeber darf also nicht durch explizite Verhaltensnormen versuchen, die Entscheidungen des Auftragnehmers vorzubestimmen, da dann der Wert der Entscheidungsdelegation durch die Vorwegnahme der zu delegierenden Tätigkeiten den Delegationswert vermindern oder sogar aufzehren würde. ${ }^{392}$

Die vorliegenden Informationsasymmetrien lassen sich in drei Typen unterscheiden: ${ }^{393}$

1. Kann der Auftraggeber die Eigenschaften des Beauftragten nicht vor der Entscheidungsdelegation, sondern erst ex post vollständig feststellen, liegen Hidden Characteristics vor. Diese Informationsasymmetrien treten in der Vorkontraktphase auf, so daß sie üblicherweise als eigenständiger Problemkomplex behandelt werden. ${ }^{394}$ Aufgrund ihrer Bedeutung sollen sie mit unter die Principal Agent-Problematik gefaßt werden. ${ }^{395}$

\footnotetext{
389 Vgl. Elschen, Agency-Theorie, 1991, S. 1004; Richter/Furubotn, Institutionenökonomik, 1996, S. $163 \mathrm{f}$.

390 Vgl. Picot/Diet1/Franck, Organisation, 1997, S. 83; mit dem Hinweis auf die in Publikumsaktiengesellschaften existierenden hohen Informationskosten für den einzelnen Aktionär Pellens/Hillebrandt, Vorzugsaktien, 2001, S. 59.

391 Vgl. Elschen, Agency-Theorie, 1991, S. 1004; Shleifer/Vishny, Corporate Governance, 1997, S. 741.

392 Vgl. zum Delegationswertkonzept Laux/Liermann, Organisation, 1997, S. $218 \mathrm{ff}$.

393 Vgl. Picot/Diet//Franck, Organisation, 1997, S. 85f.; Richter/Furubotn, Institutionenökonomik, 1996, S. 163ff.; Spremann, Information, 1990, S. 561-586.

394 Vgl. Günther, Controlling, 1997, S. 43f., m.w.N. zu Abgrenzungsproblemen in der Nachvertragsphase.

395 So auch Picot/Diet//Franck, Organisation, 1997, S. 85ff.
} 
2. Kann der Auftraggeber nicht alle Handlungen des Auftragnehmers beobachten (Hidden Action) bzw. gelingt es dem Auftragnehmer selbst im Falle beobachtbarer Handlungsergebnisse, dem Auftraggeber Informationen über die Entscheidungssituation und mögliche Risiken vorzuenthalten (Hidden Information), entstehen auch im Verlauf der Principal AgentBeziehung Informationsasymmetrien. Es ist für den Auftraggeber daher nicht möglich, von den Handlungsergebnissen auf das Anstrengungsniveau des Agenten zu schließen.

3. Hidden Intention liegt dann vor, wenn der Principal das den individuellen Nutzen maximierende Verhalten des Agenten zwar erkennen, aber nicht verhindern kann. Durch Sunk Costs (dies sind beispielsweise irreversible Investitionen für die Rekrutierung und Einstellung von Mitarbeitern) gerät der Auftraggeber in ein Abhängigkeitsverhältnis gegenüber dem Auftragnehmer. ${ }^{396}$ Dies ermöglicht ihm, zu Lasten des Auftraggebers zu handeln, ohne daß der dieses Verhalten verhindern könnte.

Aus den unterschiedlichen Informationsasymmetrien resultieren spezifische Verhaltensprobleme: ${ }^{397}$

1. Hidden Characteristics können zu Adverse Selection führen. ${ }^{398}$ Hierbei wird aufgrund der unzureichenden Kenntnis des Auftraggebers über relevante Eigenschaften des Auftragnehmers der falsche Vertragspartner ausgewählt.

2. $\mathrm{Zu}$ Moral Hazard kann es im Fall von Hidden Action bzw. Hidden Information kommen. Aufgrund seiner Informationsvorsprünge ist es dem Agenten möglich, eigene Zielvorstellungen zu Lasten des Auftraggebers zu realisieren. Hierzu gehören Drückebergerei (Shirking) und Konsum am Arbeitsplatz (Consumption on the job). ${ }^{399}$

3. Im Falle von Hidden Intention besteht die Gefahr des Hold Up. Hierbei führen die verborgenen Absichten des Agenten in Verbindung mit den spezifischen Investitionen des Auftraggebers zu einer möglichen Erpressung des Principal durch den Agenten.

\footnotetext{
396 Vgl. Picot/Neuburger, Agency Theorie, 1995, Sp. 20.

397 Vgl. Picot/Dietl/Franck, Organisation, 1997, S. 87ff.; Spremann, Information, 1990, S. 567ff.

$398 \mathrm{Vgl}$. hierzu exemplarisch Richter/Furubotn, Institutionenökonomik, 1996, S. 236-240.

Vgl. Elschen, Agency-Theorie, 1991, S. 1005.
} 
Übertragen auf die hier vorliegenden Principal Agent-Beziehungen bedeutet dies, daß sowohl Aufsichtsrat als auch Vorstand in ihrer jeweiligen Auftragnehmerrolle gegenüber Aktionären bzw. Aufsichtsrat Informationsvorsprünge besitzen, die sie zu Lasten des Auftraggebers ausnutzen können. ${ }^{400}$ Gleichzeitig sind beide Agency-Beziehungen dadurch gekennzeichnet, daß der Agent durch das Auftragshandeln nicht nur sein eigenes Nutzenniveau beeinflußt, sondern auch das des Principal. In der Vorvertragsphase müssen daher die Aktionäre bzw. der Aufsichtsrat sicherstellen, daß sie als Principal die richtigen Vertragspartner auswählen. ${ }^{401}$

Im Verlauf der Agency-Beziehung geht es hauptsächlich nicht um die Verminderung des üblicherweise vermuteten Moral Hazard-Risikos, das bei Führungskräften aufgrund ihres „Arbeitsleids“ einen Hang zur Minimierung des Arbeitseinsatzes unterstellt, es müssen vielmehr alle Entscheidungen des Agenten verhindert werden, die seinen Entlohnungsanteil über den vertraglich geregelten Betrag hinaus zu Lasten des Principal verbessern. ${ }^{402}$ Vorstände neigen zur Verfolgung eigener Interessen, beispielsweise zu einer Kapitalakkumulation durch Gewinnthesaurierung, um hierdurch ihren Einflußbereich auszudehnen und zusätzliche Freiheitsgrade zu gewinnen. ${ }^{403}$ Dieses Verhalten geht immer dann zu Lasten der Aktionäre, wenn die unternehmungsinterne Kapitalverzinsung geringer als die einer unternehmungsexternen Alternative ist. Daneben können sie versuchen, durch überhöhte, gewinnmindernde Aufwendungen für repräsentative Zwecke ihren individuell kostenlosen Konsum am Arbeitsplatz zu erhöhen. Schließlich können Aufwendungen zur Förderung des persönlichen Ansehens von Vorstandsmitgliedern (z.B. Mäzenatentum oder die Verfolgung gemeinwirtschaftlicher Interessen) zu Lasten der Aktionäre gehen.

Die Verfolgung solcher Zielsetzungen ermöglicht den Agenten durch den Aufbau von Organizational Slack die Erhöhung ihres individuellen Nutzens zu Lasten der Aktionäre, da sie einen Teil des Gewinns der Unternehmung, der über den Mindestanspruch der Aktionäre hinausgeht, gemäß ihren individuellen Zie-

\footnotetext{
400 Vgl. Jaschke, Überwachungsfunktion, 1989, S. 50.

401 Vgl. Günther, Controlling, 1997, S. 44.

402 Vgl. Bernhardt/Witt, Unternehmensleitung, 1999, S. 839; kritisch zur Annahme der Arbeitsaversion von Mitgliedern der Unternehmungsleitung m.w.N. Erlei/Leschke/Sauerland, Institutionenökonomik, 1999, S. 162f.; Gedenk, Agency-Theorie, 1998, S. 34; zu aus Management Discretion resultierenden Problemen im Überblick m.w.N. Shleifer/Vishny, Corporate Governance, 1997, S. $742 \mathrm{ff}$.

${ }^{403}$ Vgl. Neus, Betriebswirtschaftslehre, 1998, S. 150 f.
} 
len verwenden können. ${ }^{404}$ Sie werden daher im Rahmen der an sie delegierten Entscheidungsbefugnisse nicht immer diejenige Handlungsalternative auswählen, die auch der Auftraggeber in seinem eigenen Interesse gewählt hätte. ${ }^{405}$ Hierdurch ist eine Ausrichtung der in der Unternehmung verfolgten Ziele an den Interessen der Anteilseigner nicht mehr gewährleistet. Zur Verringerung bzw. Vermeidung solcher Probleme stehen dem Auftraggeber verschiedene Möglichkeiten zur Verfügung, deren Nutzung jedoch mit Kosten verbunden ist.

\section{e) Lösungsansätze zum Abbau der Principal Agent-Problematik}

Durch die abweichenden Nutzenfunktionen von Auftraggeber und Auftragnehmer bestehen für die Auftragnehmer Anreize, von den für die Auftraggeber nutzenmaximierenden Entscheidungen abzuweichen. ${ }^{406}$ Alle Versuche zur Verringerung der Informationsasymmetrien verursachen entweder beim Auftraggeber oder beim Auftragnehmer Kosten. ${ }^{407}$ Agency-Kosten entstehen zur Durchführung von Kontrollen (Monitoring Costs des Principals), für Rechenschafts- und Garantieaufwendungen aufgrund von Fehlverhalten (Bonding Costs des Beauftragten) sowie als Residual Loss, der den Nutzenverlust des Principals bezeichnet. Er entsteht dadurch, daß der Agent nicht diejenige Alternative auswählt, für die sich auch der Principal entschieden hätte. Normatives Ziel der Principal Agent-Theorie ist es, das kostenminimale Arrangement zu finden, bei dem die Summe der durch die Entscheidungsdelegation entstehenden Nutzenminderungen (gemessen als Opportunitätskosten) und die Kosten zur Verringerung der Agency-Problematik minimal werden. ${ }^{408}$ JENSEN/MECKLING verstehen diese als Differenz zwischen der theoretisch möglichen First-best-Lösung, die in einer Welt ohne Informationsasymmetrien erreicht werden könnte, und der realiter möglichen Second-best-Lösung. ${ }^{409}$

In Abhängigkeit von der betrachteten Informationsasymmetrie werden unterschiedliche Lösungsmöglichkeiten vorgeschlagen (vgl. Abb. 10): ${ }^{410}$

\footnotetext{
404 Vgl. zum Begriff des Organizational Slack Scharfenkamp, Gestaltung, 1987, S. 22ff.; zu einer Definition insbesondere S. 38 unter zusätzlichem Verweis auf Williamson, Behavior, 1964, S. $35 \mathrm{f}$.

Vgl. Elschen, Agency-Theorie, 1991, S. 1004f.; Günther, Controlling, 1997, S. 47f.; Staehle, Management, 1999, S. 423f.

406 Vgl. Gerke, Agency-Theorie, 1995, Sp. 19; Meinhövel, Principal-Agent-Theorie, 1999, S. 42.

407 Vgl. Erlei/Leschke/Sauerland, Institutionenökonomik, 1999, S. 75; Jensen/Meckling, Theory, 1976, S. 308; Picot/Diet1/Franck, Organisation, 1997, S. 87; Richter/Furubotn, Institutionenökonomik, 1996, S. 166f.

408 Vgl. Picot/Dietl/Franck, Organisation, 1997, S. 83.

409 Vgl. Jensen/Meckling, Theory, 1976, S. 327.

$410 \mathrm{Vgl}$. Picot/Diett/Franck, Organisation, 1997, S. 87ff
} 


\begin{tabular}{|c|c|c|c|c|c|c|}
\hline $\begin{array}{l}\text { Informations- } \\
\text { asymmetrie }\end{array}$ & \multicolumn{3}{|c|}{ (1) } & \multicolumn{2}{|c|}{ (2) } & (3) \\
\hline $\begin{array}{l}\text { Unter- } \\
\text { scheidlungs- } \\
\text { kriterium }\end{array}$ & \multicolumn{3}{|c|}{$\begin{array}{c}\text { Hidden } \\
\text { Characteristics }\end{array}$} & \multicolumn{2}{|c|}{$\begin{array}{l}\text { Hidden } \\
\text { Information / Action }\end{array}$} & $\begin{array}{l}\text { Hidden } \\
\text { Intention }\end{array}$ \\
\hline $\begin{array}{l}\text { Informations- } \\
\text { problem des } \\
\text { Principal }\end{array}$ & \multicolumn{3}{|c|}{$\begin{array}{l}\text { Qualitätseigenschaften } \\
\text { der Leistung des } \\
\text { Vertragspartners } \\
\text { unbekannt }\end{array}$} & \multicolumn{2}{|c|}{$\begin{array}{l}\text { Anstrengungen des } \\
\text { Vertragspartners nicht } \\
\text { beobachtbar bzw. } \\
\text { nicht beurteilbar }\end{array}$} & $\begin{array}{l}\text { Absichten des } \\
\text { Vertragspartners } \\
\text { unbekannt }\end{array}$ \\
\hline $\begin{array}{l}\text { Problemursache } \\
\text { oder } \\
\text { wesentliche } \\
\text { Einflußgröße }\end{array}$ & \multicolumn{3}{|c|}{$\begin{array}{l}\text { Verbergbarkeit von } \\
\text { Eigenschaften }\end{array}$} & \multicolumn{2}{|c|}{$\begin{array}{l}\text { Ressourcenplastizi- } \\
\text { tät; Überwachungs- } \\
\text { möglichkeiten und } \\
\text {-kosten }\end{array}$} & $\begin{array}{l}\text { Ressourcenabhän- } \\
\text { gigkeit, -einmalig- } \\
\text { keit und -entzieh- } \\
\text { barkeit }\end{array}$ \\
\hline $\begin{array}{l}\text { Verhaltens- } \\
\text { spielraum des } \\
\text { Agenten }\end{array}$ & \multicolumn{3}{|c|}{ vor Vertragsabschluß } & \multicolumn{2}{|c|}{$\begin{array}{l}\text { nach } \\
\text { Vertragsabschluß }\end{array}$} & $\begin{array}{l}\text { nach } \\
\text { Vertragsabschluß }\end{array}$ \\
\hline Beispiel & \multicolumn{3}{|c|}{$\begin{array}{l}\text { Einstellung von } \\
\text { Personal }\end{array}$} & \multicolumn{2}{|c|}{$\begin{array}{l}\text { Leistungsverhalten } \\
\text { von Personal }\end{array}$} & $\begin{array}{l}\text { Verweilabsicht von } \\
\text { Personal }\end{array}$ \\
\hline Problem & \multicolumn{3}{|c|}{ Adverse Selection } & \multicolumn{2}{|c|}{ Moral Hazard } & Hold Up \\
\hline \multirow[t]{2}{*}{$\begin{array}{l}\text { Art der Pro- } \\
\text { blembewälti- } \\
\text { gung }\end{array}$} & \multicolumn{2}{|c|}{$\begin{array}{l}\text { Beseitigung } \\
\text { der } \\
\text { Informatons- } \\
\text { asymmetrie } \\
\text { durch }\end{array}$} & \multirow[t]{2}{*}{$\begin{array}{l}\text { Inter- } \\
\text { essen- } \\
\text { anglei- } \\
\text { chung }\end{array}$} & \multirow{2}{*}{$\begin{array}{l}\text { Reduzie- } \\
\text { rung der } \\
\text { Informa- } \\
\text { tionsa- } \\
\text { sym- } \\
\text { metrie } \\
\text { (Moni- } \\
\text { toring) }\end{array}$} & \multirow[t]{2}{*}{$\begin{array}{l}\text { Interes- } \\
\text { senan- } \\
\text { gleichung }\end{array}$} & \multirow[t]{2}{*}{$\begin{array}{l}\text { Interessenanglei- } \\
\text { chung }\end{array}$} \\
\hline & $\begin{array}{l}\text { Sig- } \\
\text { nal- } \\
\text { ling/ } \\
\text { Scree- } \\
\text { ning }\end{array}$ & $\begin{array}{l}\text { Self- } \\
\text { selec- } \\
\text { tion }\end{array}$ & & & & \\
\hline $\begin{array}{l}\text { Möglichkeiten } \\
\text { zur Problembe- } \\
\text { grenzung } \\
\text { (beispielhaft) }\end{array}$ & $\begin{array}{l}\text { Bilan- } \\
\text { zen, } \\
\text { Zeug- } \\
\text { nisse, } \\
\text { Güte- } \\
\text { siegel }\end{array}$ & $\begin{array}{l}\text { diffe- } \\
\text { ren- } \\
\text { zierte } \\
\text { Ko- } \\
\text { opera- } \\
\text { tions- } \\
\text { ver- } \\
\text { träge }\end{array}$ & $\begin{array}{l}\text { Repu- } \\
\text { tation } \\
\text { des } \\
\text { Ver- } \\
\text { trags- } \\
\text { part- } \\
\text { ners }\end{array}$ & $\begin{array}{l}\text { z.B. } \\
\text { durch } \\
\text { Planungs- } \\
\text { und } \\
\text { Kontroll- } \\
\text { systeme, } \\
\text { Berichts- } \\
\text { wesen } \\
\text { etc. }\end{array}$ & $\begin{array}{l}\text { Ergebnis- } \\
\text { beteili- } \\
\text { gung des } \\
\text { Vertrags- } \\
\text { partners } \\
\text { (z.B. } \\
\text { Prämien- } \\
\text { system } \\
\text { oder } \\
\text { Kapital- } \\
\text { beteili- } \\
\text { gung) }\end{array}$ & $\begin{array}{l}\text { Sicherheiten (z.B. } \\
\text { Leistungsgarantien, } \\
\text { Bürgschaften, } \\
\text { Gegengeschäfte), } \\
\text { vertikale Integration } \\
\text { (langfristige Ver- } \\
\text { träge, Sicherung von } \\
\text { Verfügungsrechten, } \\
\text { soziale Wert- und } \\
\text { Strafsysteme) }\end{array}$ \\
\hline
\end{tabular}

Abb. 10: Probleme und Lösungsmöglichkeiten aus Sicht der Principal Agent-Theorie Quelle: In Anlehnung an Picot/Dietl/Franck, Organisation, 1997, S. 88. 
1. Die Auswahl falscher Vertragspartner kann durch den Abbau von Informationsasymmetrien vor der Vertragsbeziehung verhindert werden. ${ }^{411}$ Signalling (bspw. durch Zeugnisse, Gütesiegel, Mitgliedschaft in Berufsverbänden usw.) erlaubt es Agenten mit einem hohen Leistungsvermögen, sich von solchen mit einem geringeren abzuheben, indem sie den Principal davon überzeugen, daß sie über die geforderten Leistungsmerkmale verfügen. Der Auftraggeber kann seinerseits durch Screening (hierzu zählen bei der Bewerberauswahl u.a. Einstellungstests oder auch die Einschaltung eines Headhunters) versuchen, seinen Informationsstand $\mathrm{zu}$ verbessern, um Informationen über die für ihn relevanten Qualitätseigenschaften des Agenten zu erhalten. Daneben kann der Auftraggeber dem Agenten unterschiedliche Verträge mit abweichenden Vertragsklauseln anbieten, von denen der Auftragnehmer denjenigen auswählen wird, der seinem Leistungsvermögen am ehesten entspricht (Selfselection). Hierdurch erhält der Auftraggeber implizit weitere Informationen über den Agenten. Neben dem Abbau von Informationsasymmetrien kann versucht werden, einen Interessenausgleich zwischen Auftraggeber und Auftragnehmer herbeizuführen. Hierzu zählt zum Beispiel die Gefahr des Reputationverlustes des Beauftragten durch Nachrede des Principal.

2. Insbesondere im Falle hoher Kontrollkosten entstehen für den Agenten umfangreiche Verhaltensspielräume. Dies gilt um so mehr, je weniger das Verhalten des Agenten durch den Principal beobachtbar ist bzw. Beurteilungsschwierigkeiten bezüglich des Ergebnisses bestehen. Um die Gefahr des Moral Hazard möglichst gering zu halten, können die Principals Anreizsysteme für den Agenten implementieren, die über die Orientierung der Belohnung des Agenten am Handlungsergebnis einen Interessenausgleich zwischen Principal und Agent herbeiführen. ${ }^{412}$ Zusätzlich kann die Kontrolle des Agenten durch die Einrichtung von Planungs- und Kontrollsystemen (Monitoring) zur systematischen Informationsgewinnung dessen Verhaltensspielräume einengen.

3. Wenn der Principal vom Agenten ein Gegenpfand fordert, vermindert er die Erpressungsgefahr durch Hidden Intention. Hierzu zählen die Reputation des Agenten, aber beispielsweise auch eine Kapitalbeteiligung an einer Unternehmung. Mit Bezug auf Mitarbeiter der Unternehmung könnte

411 Vgl. Günther, Controlling, 1997, S. 44ff.; zu einer modelltheoretischen Beschreibung Erlei/Leschke/Sauerland, Institutionenökonomik, 1999, S. 144ff.

412 Vgl. Elschen, Shareholder Value, 1991, S. 209-219; m.w.N. Shleifer/Vishny, Corporate Governance, 1997, S. 744 f. 
der Principal versuchen, durch die vertragliche Gewährung von Pensionszusagen oder Abfindungen, deren Inanspruchnahme von bestimmten zu erbringenden Leistungen des Beschäftigten abhängt, eine bilaterale Hold Up-Situation herzustellen. ${ }^{413}$

Trotz des unterschiedlichen Problembezugs sowie der konkreten Ausgestaltung der Problemlösung können zwei Lösungsansätze unterschieden werden: Zum einen sind dies Maßnahmen zum Abbau von Informationsasymmetrien, die es dem Principal ermöglichen sollen, Eigenschaften, Handlungen und Absichten des Agenten besser beurteilen zu können; zum anderen fallen hierunter Handlungen, die eine Interessenkonvergenz von Principal und Agent herbeiführen. ${ }^{414}$ Beide Maßnahmengruppen sollen eine Ausrichtung des Agenten auf die Ziele des Principals gewährleisten.

SCHNEIDER bemängelt an der Principal Agent-Theorie die fehlende Meßbarkeit der Agency-Kosten insgesamt und insbesondere des Residual Loss. ${ }^{415}$ Für ihn ist daher der gesamte Agency-Costs-Ansatz eine „Seifenblase bei der Theorienbildung “. ${ }^{416}$ SCHMIDT hält dem neben einer grundsätzlichen Kritik an der Argumentation SCHNEIDERS entgegen, daß es sich bei dem Agency-Ansatz lediglich um ein heuristisches Konzept handele. ${ }^{417}$ Agency-Kosten seien in der Realität nur sehr schwer bzw. gar nicht zu messen. Sie können daher lediglich als heuristisches Beurteilungskriterium herangezogen werden, um im Rahmen einer komparativ statischen Analyse über die Ableitung von Tendenzaussagen unterschiedliche Formen der Arbeitsteilung zwischen Auftraggeber und Auftragnehmer ableiten zu können. Dabei seien die Trade-off-Beziehungen zwischen den einzelnen Kostenarten zu berücksichtigen, um bei der Beurteilung arbeitsteiliger Aufgabenerfüllungen zu einem Minimum der Gesamtkosten zu kommen.

Trotz der grundsätzlichen Anwendbarkeit der Principal Agent-Theorie auf die hier zu untersuchende Problemstellung findet nachfolgend keine agencytheoretische Analyse des betrachteten Problemfeldes statt. ${ }^{418}$ Die Ursache hierfür liegt

413 Vgl. Picot/Neuburger, Agency Theorie, 1995, Sp. 20.

414 Vgl. Elschen, Shareholder Value, 1991, S. $210 \mathrm{f}$.

415 Vgl. Schneider, Agency Costs, 1987, S. 481ff.; Schneider, Grundlagen, 1995, S. 276ff.; zur Kritik an den Grundannahmen der Principal Agent-Theorie aus der Sicht verhaltenswissenschaftlicher Untersuchungsansätze m.w.N. Staehle, Management, 1999, S. 424f.; die Principal Agent-Theorie als „Grundlage für eine ökonomische Theorie des Auftragshandelns“ ablehnend Meinhövel, Principal-Agent-Theorie, 1999, S. 215.

416 Vgl. Schneider, Grundlagen, 1995, S. 279.

417 Vgl. Schmidt, Agency Costs, 1987, S. 498f.; m.w.N. Günther, Controlling, 1997, S. 49.

$418 \mathrm{Vgl}$. hierzu beispielsweise Jaschke, Überwachungsfunktion, 1989. 
in dem gewählten gestaltungsorientierten Ansatz, der auf die Ableitung von konkret umsetzbaren Handlungsempfehlungen abzielt. Der Erkenntnisgewinn, den eine agencytheoretische Analyse hierfür bieten könnte, scheint aufgrund der geäußerten Kritik nur sehr begrenzt zu sein, so daß nachfolgend lediglich Lösungsvorschläge der Agency-Theorie aufgegriffen werden sollen. Dies ist trotz der an ihr geäußerten Kritik berechtigt. ${ }^{419}$

Potentielle Eigenkapitalgeber werden nur dann bereit sein, die Aktien einer Unternehmung zu erwerben, wenn die aus der Verdünnung der Verfügungsrechte resultierenden Agency-Probleme wirksam eingegrenzt werden können. ${ }^{420}$ Neben der Marktkontrolle, beispielsweise durch den Gütermarkt ${ }^{421}$, den Kapitalmarkt ${ }^{422}$ oder den Arbeitsmarkt für Manager, ist für Aktiengesellschaften insbesondere der Aufsichtsrat ${ }^{423}$ zu nennen. Bevor dessen Rolle in der Unternehmungsführung konkretisiert wird, soll jedoch zunächst mit dem Shareholder Value-Konzept eine inhaltliche Bestimmung der Unternehmungsziele erfolgen.

\section{Der Shareholder Value-Ansatz als unternehmungspolitisches Para- digma}

a) Überprïfung der Zweckmäßigkeit als unternehmerische Zielgröße

Die vorstehenden Ausführungen haben gezeigt, daß die durch den Gesetzgeber bewirkte Ausgestaltung der Spitzenverfassung von Publikumsaktiengesellschaften und die hiermit verbundene Determinierung des Unternehmungsführungsprozesses aus agencytheoretischer Perspektive umfangreiche Probleme für die Zielerreichung hervorruft. Mit dem Shareholder Value-Ansatz liegt eine unternehmerische Zielkonzeption vor, die dazu beitragen will, solche Probleme zu vermindern. $^{424}$

419 Vgl. Staehle, Management, 1999, S. 425.

420 Vgl. Gerum, Eigentümerführung, 1995, Sp. 1464f.; Neus, Betriebswirtschaftslehre, 1998, S. 151f.; Picot/Dietl/Franck, Organisation, 1997, S. 196.

421 Skeptisch zur Wirksamkeit des Gütermarktes Shleifer/Vishny, Corporate Governance, 1997, S. 738.

${ }^{422} \mathrm{Zu}$ dessen Rolle als Markt für Unternehmungskontrolle Wenger, Unternehmenskontrolle, 1995, Sp. 1409-1419.

423 Für Neus, Betriebswirtschaftslehre, 1998, S. 152, kann der Aufsichtsrat diese Rolle trotz der unternehmerischen Mitbestimmung erfüllen; Gerum, Eigentümerführung, 1995, Sp. 1464, geht sogar davon aus, daß dies die einzig verbleibende strukturell gesicherte interne Fremdkontrolle darstellt.

424 Vgl. Neus, Betriebswirtschaftslehre, 1998, S. 127ff., sowie Zimmermann/Wortmann, Shareholder-Value-Ansatz, 2001, S. 289-294, die zu den konstituierenden Komponenten des Shareholder Value-Konzepts u.a. Vergütungssysteme, Investor Relations und Corporate Governance zählen (S. 293). 
Bereits für HEINEN war das Gewinnstreben von Unternehmungen Ausdruck des erwerbswirtschaftlichen Grundsatzes, demzufolge es Ziel der Unternehmung sei, Einkommen für jene Haushalte zu erwirtschaften, die das erforderliche Eigenkapital zur Verfügung stellen. ${ }^{425}$ Diese Orientierung an den Interessen der Anteilseigner steht auch im Vordergrund des Shareholder Value-Ansatzes. ${ }^{426}$ Potentiellen Eigenkapitalgebern stehen am Kapitalmarkt vielfältige Anlagemöglichkeiten mit verschiedenen Rendite-Risiko-Positionen zur Verfügung. ${ }^{427}$ Sie werden diejenige Alternative auswählen, die unter Berücksichtigung ihrer individuellen Risikoneigung den eigenen Nutzen maximiert. $\mathrm{Da}$ insbesondere global agierende Publikumsaktiengesellschaften auf dem Markt für Eigenkapital im Wettbewerb mit anderen Unternehmungen und Anlageformen stehen, ist das Management dieser Unternehmungen gezwungen, die Wettbewerbsfähigkeit der Unternehmung auf diesem Markt zu erhöhen, d.h., es muß die für die Eigenkapitalgeber relevanten Entscheidungskriterien berücksichtigen. ${ }^{428}$

Ausgehend von stark vereinfachenden modelltheoretischen Annahmen kann unter sukzessiver Erhöhung des Realitätsbezugs gezeigt werden, daß das Ziel der Marktwertmaximierung des Eigenkapitals realiter ein widerspruchsfreies Unternehmungsziel im Sinne aller Eigenkapitalgeber darstellt. ${ }^{429}$ Konkret ergibt sich die Argumentation wie folgt: Die Anteilseigner der Unternehmung streben nach Optimierung ihres für Konsumzwecke zur Verfügung stehenden Einkommensstroms unter Berücksichtigung ihrer individuellen Zeit- und Risikopräferenzen. ${ }^{430}$ Eine Optimierung der Einkommensströme aller Anteilseigner durch die

425 Vgl. Heinen, Betriebswirtschaftslehre, 1985, S. 106.

426 Vgl. Rappaport, Shareholder-Value, 1986; aufgrund der zwischenzeitlich auch im deutschen Schrifttum erschienenen zahlreiche Beiträge sei hier nur auf Bischoff, Shareholder-ValueKonzept, 1994; Bühner, Shareholder-Value-Report, 1994, sowie Richter, Konzeption, 1996, S. 1, und die dort angegebene Literatur verwiesen.

427 Vgl. Baetge, Gesellschafterorientierung, 1997, S. 107f.; Bühner/Tuschke, Shareholder Value, 1997, S. 501.

428 Vgl. Bühner/Tuschke, Shareholder Value, 1997, S. 501, sowie Hommelhoff, Shareholder ValuePrinzip, 1997, S. 18; anders von Werder, Shareholder Value, 1998, S. 81f., der sich bei seiner Argumentation unter anderem auf die unbeeinträchtigten Finanzierungsmöglichkeiten der Siemens AG bezieht, die expressis verbis keine strikte Ausrichtung am Shareholder Value-Ansatz verfolge; doch auch die Siemens AG war inzwischen gezwungen, ihre Unternehmungspolitik verstärkt am Shareholder Value auszurichten (vgl. die Rede von Heinrich von Pierer zur Siemens Hauptversammlung am 24. Februar 2000).

Vgl. zur finanztheoretischen Begründung Kürsten, Shareholder Value, 2000, S. 359-381; Wilhelm, Marktwertmaximierung, 1983, S. 516-534; auch Ballwieser/Schmidt, Finanztheorie, 1981, S. 662; Bühner/Tuschke, Shareholder Value, 1997, S. 501; m.w.N. Günther, Controlling, 1997, S. 3.

430 Vgl. Fisher, Theory, 1930, S. 61ff.; Schmidt/Terberger, Finanzierungspolitik, 1997, S. 46-48, $100 f$. 
Unternehmung würde aber die Kenntnis sämtlicher individueller Zeit- und Risikopräferenzen voraussetzen und eine Anpassung der Investitions- und Ausschüttungspolitik bedingen. Die Maximierung des Marktwertes des Eigenkapitals kann hier als konfliktfreie Zielsetzung aus Sicht aller Anteilseigner auf vollkommenen und vollständigen Kapitalmärkten Abhilfe schaffen. ${ }^{431}$ Durch die Realisierung der optimalen Investitionspolitik wird der Marktwert des Eigenkapitals maximiert, die jeweiligen Anteilseigner stellen sich daraus am Kapitalmarkt ihren individuellen Konsumstrom durch Geldanlage bzw. Kreditaufnahme selbst zusammen. Durch die Maximierung des Marktwertes und gleichzeitige Anpassung an die individuellen Konsumwünsche durch Kapitalmarkttransaktionen wird der Nutzen aus Sicht aller Eigenkapitalgeber maximal.

Auch auf unvollständigen und unvollkommenen Kapitalmärkten stellt die Marktwertmaximierung eine sinnvolle Zielsetzung dar, wenn die schwächeren Voraussetzungen der Spanning Property und Competitivity erfüllt sind. ${ }^{432}$ Spanning Property ist gewährleistet, wenn der Kapitalmarkt die Nachbildbarkeit der aus Sicht der Anteilseigner wünschenswerten Zahlungsströme gewährleistet; Competitivity liegt vor, wenn einzelne Kapitalmarktteilnehmer keinen Einfluß auf die Kapitalmarktpreise haben. ${ }^{433}$ Aufgrund des Zusammenwachsens der internationalen Kapitalmärkte und des verstärkten Handels mit derivativen Finanzinstrumenten können diese Bedingungen in der Realität zunehmend als erfüllt angesehen werden, so daß die Marktwertsteigerung als konfliktfreie Zielsetzung aus Sicht aller Aktionäre angesehen werden kann.

Unterstützend wird durch die Befürworter des Shareholder Value-Ansatzes ausgeführt, daß die Interessen anderer Stakeholder als Nebenbedingungen mit zu berücksichtigen seien, da von einer guten Zusammenarbeit mit ihnen der Erfolg der Unternehmung abhänge. ${ }^{434}$ Zudem werden im Einzelfall existierende Zielkonflikte bei einer langfristigen Betrachtung aufgelöst. Außerdem sind alle Ansprüche der Stakeholder durch Marktprozesse, das Rechtssystem sowie Verträge abgesichert, so daß das Ziel der Marktwertmaximierung des Eigenkapitals als alleiniges Oberziel der Unternehmung angesehen werden sollte. ${ }^{435}$

431 Vgl. Schmidt/Terberger, Finanzierungspolitik, 1997, S. 111-114.

432 Vgl. Arrow, Risk-bearing, 1964, S. 91ff.; Debreu, Analysis, 1959, S. 98ff.

433 Vgl. Breuer, Marktwertmaximierung, 1997, S. 224.

434 Vgl. Baetge, Gesellschafterorientierung, 1997, S. 114; Bischoff, Shareholder-Value-Konzept, 1994, S. 180f.; Busse von Colbe, Shareholder Value, 1997, S. 289f.; Rappaport, shareholder value, 1986, S. 12.

435 Vgl. Neus, Betriebswirtschaftslehre, 1998, S. 162ff.; Bühner/Tuschke, Shareholder Value, 1997, S. 502. 
„Meines Erachtens geht [..] der Shareholder Value vor dem Stakeholder-Value, denn das Unternehmen gehört den Gesellschaftern und nicht den Kunden und nicht den Managern und nicht den Arbeitnehmern. In der Unternehmens-Zielhierarchie ist demzufolge der Shareholder Value als oberstes Ziel zu installieren. Die Wünsche und Bedürfnisse der übrigen Stakeholder bilden in einem solchen Zielsystem gegebenenfalls einzuhaltende Nebenbedingungen. ${ }^{\text {4436 }}$

Diese Auffassung ist in der Literatur nicht unumstritten:

1. VON WERDER bemängelt die fehlende exogene Vorgabe von Befriedigungsniveaus der Nebenbedingungen. ${ }^{437} \mathrm{Da}$ deren Festlegung in den Händen der Unternehmungsleitung liegt, könnten die Nebenbedingungen aufgrund der Variabilität ihre restringierende Funktion nicht erfüllen. Als Alternative schlägt er eine mehreren Anspruchsgruppen verpflichtete Unternehmungspolitik vor, ${ }^{438}$ was gleichbedeutend mit einer gewichteten $\mathrm{Be}$ rücksichtigung konfliktärer Ziele ist.

2. Darüber hinaus bezweifelt er zwar nicht das langfristige gemeinsame Interesse von Anteilseignern und Arbeitnehmern an einer wirtschaftlich erfolgreichen Unternehmung, er hält dies jedoch für nicht tragfähig, da im Einzelfall sehr wohl massive Zielkonflikte zwischen einem einzelnen Arbeitnehmer und den Anteilseignern, beispielsweise bei Betriebsstillegungen, bestehen könnten. ${ }^{439}$

3. Ein mit den Punkten 1 und 2 eng zusammenhängender dritter Kritikpunkt resultiert aus Marktineffizienzen und der fehlenden Möglichkeit des Abschlusses perfekter Verträge zwischen den übrigen Stakeholdern mit der Unternehmung. ${ }^{440}$ Da zum Zeitpunkt des Vertragsabschlusses nicht alle möglichen zukünftigen Ereignisse berücksichtigt werden können, entstehen neben den in den Verträgen enthaltenen expliziten Ansprüchen zusätzliche implizite Ansprüche. Bei diesen bestehe die Gefahr, daß das

436 Baetge, Gesellschafterorientierung, 1997, S. 115; Speckbacher, Shareholder Value, 1998, S. 97, irrt, wenn er den Stakeholder-Ansatz als deutsche Version einer eigentümerorientierten Unternehmungsführung betrachtet, da in diesem nicht eine Zweck-Mittel-Beziehung zwischen Anteilseignerinteressen und den Interessen der übrigen Stakeholder besteht, sondern deren Interessen als Selbstzweck verfolgt werden; vgl. auch Bühner/Tuschke, Shareholder Value, 1997, S. 501.

Vgl. von Werder, Shareholder Value, 1998, S. 74f.

Vgl. von Werder, Shareholder Value, 1998, S. 90f., der hier von einer "multiplen Exzellenz spricht"; dies heißt für von Werder, Corporate Governance, 2000, S. 14, ein „austarieren“ der Ziele unterschiedlicher Anspruchsgruppen durch die Unternehmungsleitung.

Vgl. von Werder, Shareholder Value, 1998, S. 75.

Vgl. Bühner/Tuschke, Shareholder Value, 1997, S. 502; Schmidt/Spindler, Shareholder-Value, 1997, S. 526. 
Management die impliziten Verträge bricht und so die Stakeholder ausbeutet. Als Beispiel können durch Bildungsmaßnahmen vorgenommene firmenspezifische Investitionen der Mitarbeiter in ihr Humankapital genannt werden, die bei vorzeitiger Entlassung ihren Wert verlieren würden. $^{441}$

Gegen diese Vorbehalte ist erstens einzuwenden, daß eine einfache Rangordnung konfliktärer Ziele, wie sie mit dem Shareholder Value-Ansatz vorgenommen wird, eine exogene Festlegung von Anspruchsniveaus der Restriktionen nicht voraussetzt. ${ }^{442}$ Deren Festlegung liegt im unternehmerischen Ermessen und sollte einfacher möglich sein, als die bei VON WERDER vorgeschlagene Festlegung von Zielgewichten. ${ }^{443}$ Außerdem liegen aufgrund gesetzlicher und vertraglicher Bestimmungen (beispielsweise Wettbewerbsrecht, Arbeitsrecht, Insolvenzrecht, Produkthaftungsrecht, Umweltrecht, Handels- und Gesellschaftsrecht, Steuerrecht) operationale Mindestniveaus vor. ${ }^{44}$

Zum zweiten Kritikpunkt ist festzustellen, daß zwar zwischen Anteilseignern und Arbeitnehmern ein Zielkonflikt auf individueller Ebene grundsätzlich möglich ist, hier ist jedoch den überindividuellen Interessen der Gesamtheit der Beschäftigten einer Unternehmung der Vorrang zu geben, die nur in einer wirtschaftlich erfolgreichen Unternehmung langfristige Beschäftigungsperspektiven besitzt. $^{445}$ Auch wenn manche Entscheidungen für das Individuum nachteilig sind, können sie aus Systemsicht dennoch rational sein. ${ }^{446}$ Für HOMMELHOFF besteht sogar eine Verpflichtung der Unternehmungsleitung zum Abbau unrentabler Arbeitsplätze, solange dies nicht kurzfristig erfolgt. ${ }^{447}$

Schließlich können SCHMIDT/SPINDLER zeigen, daß ein Bruch von impliziten Verträgen durch das Management nicht im Interesse der gegenwärtigen und zu-

\footnotetext{
$441 \mathrm{Zu}$ impliziten Ansprüchen beispielsweise Cornell/Shapiro, Finance, 1987, S. 6.

Vgl. Mag, Mehrfachziele, 1976, S. 54f.

Vgl. von Werder, Shareholder Value, 1998, S. 78f.; hier sei nochmals auf die am StakeholderAnsatz geäußerte Kritik in Abschnitt II.A.2.d) verwiesen.

Vgl. Neus, Betriebswirtschaftslehre, 1998, S. 164ff.; die besondere Schutzwürdigkeit der Anteilseignerinteressen betonen auch Shleifer/Vishny, Corporate Governance, 1997, S. 751 f.

Vgl. im Ergebnis zustimmend Koch, Unternehmensinteresse, 1983, S. 206f., der mit den dominanten Mehrheitsinteressen der weiter beschäftigten Arbeitnehmer im Verhältnis zu den Minderheitsinteressen der im Falle einer Betriebsschließung zu entlassenden Arbeitnehmer argumentiert.

Vgl. Hommelhoff, Shareholder Value-Prinzip, 1997, S. 18f.
} 
künftigen Eigentümer ist. ${ }^{48}$ Langfristig kommen Vertragsbeziehungen zwischen der Unternehmung und Mitgliedern der Anspruchsgruppen nur dann zustande, wenn die genannten Risiken abgegolten werden. Im Falle der Mitarbeiter werden diese daher nur solange in unternehmungsspezifisches Humankapital investieren, wie sie zusätzlich zu ihrer Entlohnung einen angemessenen Ausgleich für eventuelle Wertverluste erhalten. ${ }^{449}$ Zudem ist die Gefahr des Vertragsbruchs für die übrigen Stakeholder um so geringer, je umfassender gesetzliche Regelungen den Bruch impliziter Verträge verhindern und die Stakeholder ihrerseits mächtig genug sind, entsprechende Verhaltensweisen zu Lasten der Unternehmung zu sanktionieren. ${ }^{450}$

Vollständig kann die Shareholder Value-Konzeption als generelles Unternehmungsziel von Publikumsaktiengesellschaften wie folgt definiert werden:

Die Unternehmungsleitung maximiert langfristig (Zielvorschrift) im Interesse aller Aktionäre der Gesellschaft den Marktwert des Eigenkapitals (Zielinhalt). Dieses Ziel ist unbefristet gültig (Zieldauer). Hierbei muß sie im Interesse der Aktionäre die Ziele der übrigen Stakeholder berücksichtigen, da sich diese anderenfalls von der Unternehmung abwenden werden (Nebenbedingung). ${ }^{451}$

Diese Restriktionen werden sowohl hinsichtlich ihres Umfangs als auch des Ausmaßes periodisch überprüft und gegebenenfalls angepaßt.

BÜHNER/TUSCHKE sprechen in diesem Zusammenhang von einem Paradigmawechsel innerhalb der deutschen Unternehmungsverfassung, da ein Übergang von einer "Stakeholder-Wirtschaft" zu einer "Shareholder-Wirtschaft" stattfände. ${ }^{452}$ Die grundlegende Ausrichtung der Unternehmungsaktivitäten auf die Interessen der Anteilseigner betrifft die unternehmungspolitische Dimension des Shareholder Value-Ansatzes und seine paradigmatische Wirkungsweise. ${ }^{453}$ Das

448 Vgl. Schmidt/Spindler, Shareholder-Value, 1997, S. 529ff.; Schmidt, Corporate Governance, 1997, S. $7 \mathrm{ff}$.

449 Vgl. Bühner/Tuschke, Shareholder Value, 1997, S. 502; Fama, Agency, 1980, S. 292.

450 Vgl. Neus, Betriebswirtschaftslehre, 1998, S. 165ff.; Schmidt/Spindler, Shareholder-Value, 1997, S. 555.

451 In diesem Sinne auch Schmidt/Spindler, Shareholder-Value, 1997, S. 533, die von der moderaten Form des Shareholder Value-Ansatzes sprechen.

452 Vgl. Bühner/Tuschke, Shareholder Value, 1997, S. 499; zustimmend Groh, Aktienrecht, 2000, S. 2153, 2158; weniger optimistisch Matthes, Corporate-Governance-System, 2000, S. 57.

453 Zu den Dimensionen des Shareholder Value-Ansatzes beispielsweise von Werder, Shareholder Value, 1998, S. 69ff., sowie Schmidt/Spindler, Shareholder-Value, 1997, S. 518f.; Busse von Colbe, Shareholder Value, 1997, S. 290; Baetge, Gesellschafterorientierung, 1997, S. 105, oder Potthoff, Shareholder Value, 1998, S. I; zur rechentechnischen Dimension des Shareholder Value-Ansatzes Gomez, Shareholder Value, 1995; Günther, Controlling, 1997; Lorson, Shareholder 
Shareholder Value-Paradigma konkretisiert sich durch ein Wertziel, das als Beurteilungsinstrument der Sachziele der Unternehmung dient. Sachziele und die im Rahmen des Shareholder Value-Ansatzes zu berücksichtigenden Restriktionen stehen bei langfristiger Betrachtung in einer Zweck-Mittel-Beziehung zum Formalziel „Shareholder Value“. Die Restriktionen finden ihren Ausdruck in den Sozialzielen der Unternehmung, die Mindestanforderungen an die Berücksichtigung der Stakeholderinteressen darstellen. Alle drei Zielkategorien bilden den Ausgangspunkt des unternehmerischen Zielsystems.

\section{b) Verringerung der Principal Agent-Problematik}

Mit der Ausrichtung der Unternehmung auf den Shareholder Value ist es auch unter Berücksichtigung von Interessen der verschiedenen Stakeholdergruppen nicht nur möglich, ein konsistentes Unternehmungszielsystem abzuleiten, darüber hinaus wird ein Beitrag zur Lösung der Principal Agent-Problematik geleistet. ${ }^{454} \mathrm{Je}$ weniger Einkommensanreize die Unternehmungsleitung hat, die Interessen der Anteilseigner zu berücksichtigen, und je größer die Informationsasymmetrien zwischen den Aktionären und der Unternehmungsleitung sind, desto größer sind die Anreize der Unternehmungsleitung, Anteilseignerinteressen zu Gunsten der eigenen Position und der weiterer Stakeholder zu mißachten. ${ }^{455}$ Die Umsetzung des Shareholder Value-Ansatzes vermindert diese Ausbeutungspotentiale und trägt so zum Abbau von Agency-Problemen bei. Für SCHMIDT/SPINDLER richtet sich das Shareholder Value-Konzept daher gegen das Management, um zu verhindern, daß es Handlungsspielräume zu Lasten der Aktionäre, aber auch der übrigen Stakeholder ausnutzt. ${ }^{456}$ Der Problemlösungsansatz des Shareholder Value-Konzepts liegt neben der Vorgabe operationaler Zielgrößen insbesondere darin, daß es in Verbindung mit einer aktienwertorientierten Entlohnung des Managements eine am Marktwert des Eigenkapitals orientierte Unternehmungsführung sicherstellt. So können über die Bindung der

\footnotetext{
Value-Ansätze, 1999; Pape, Unternehmensführung, 2000; Siegert, Shareholder-Value, 1995; zum Konzept einer wertorientierten Planungs- und Kontrollrechnung Dirrigl, Unternehmensrechnung, 1998; kritisch zur Umsetzung in der Praxis Pellens/Rockholtz/Stienemann, Konzerncontrolling, 1997; mit Bezugnahme auf das Value Reporting Pellens/Hillebrandt/Tomaszewski, Value Reporting, 2000, S. 177-207.

$454 \mathrm{Vgl}$. Elschen, Shareholder Value, 1991, S. 218.

455 Vgl. Wagner, Shareholder Value, 1997, S. 482.

$456 \mathrm{Vgl}$. Schmidt/Spindler, Shareholder-Value, 1997, S. 533
} 
Managemententlohnung an das Ziel der Anteilseigner aus der Auftragsbeziehung resultierende Zielkonflikte vermindert werden. ${ }^{457}$

Die hier vorliegende zweistufige Agency-Beziehung bietet zwei unterschiedliche Anknüpfungspunkte: Zum einen muß der Aufsichtsrat als Principal des Vorstands sicherstellen, daß das Anreizsystem des Vorstands durch eine Ausrichtung am Aktienkurs an der Erhöhung des Shareholder Value ausgerichtet ist. ${ }^{458}$ Darüber hinaus ist zu prüfen, inwieweit auch für den Aufsichtsrat entsprechende Vergütungsformen zu einem Abbau der Principal Agent-Problematik zwischen ihm und der Hauptversammlung beitragen können. Hierzu erfolgt im Anschluß an die Vorstellung des Aufsichtsratsplanungssystems eine Analyse der gegenwärtigen Aufsichtsratsvergütung. Darauf aufbauend wird gezeigt, wie eine aktienkursorientierte Vergütung von Aufsichtsratsmitgliedern ausgestaltet werden kann.

Um zu klären, ob die hier verfolgte Shareholder Value-Konzeption vor dem Hintergrund der Verfassung deutscher Publikumsaktiengesellschaften umsetzbar ist, soll nachfolgend ihre rechtliche Zulässigkeit überprüft werden.

\section{c) Überprüfung der Rechtmäßigkeit als unternehmerische Zielgröße}

Im Rahmen ihrer Treuepflicht sind die Aufsichtsratsmitglieder zur Verfolgung der Unternehmungsziele verpflichtet. Die aus juristischer Perspektive geführte Diskussion nach dem richtigen Inhalt der Unternehmungsziele hat jedoch ebenso wie ihr betriebswirtschaftliches Pendant noch zu keinem abschließenden Ergebnis geführt. ${ }^{459}$ GROBMANN stellt fest, daß es nicht möglich sei, aktienrechtlich abgeleitet Ziele einer Publikumsaktiengesellschaft zu ermitteln. ${ }^{460}$ Es ist daher danach zu fragen, ob das oben abgeleitete generelle Unternehmungsziel der Marktwertmaximierung des Eigenkapitals rechtlich unzulässig ist. ${ }^{461}$ Ist dies

457 Vgl. Baetge, Gesellschafterorientierung, 1997, S. 110f.; Bughin/Copeland, shareholder value, 1997, S. 164; Busse von Colbe, Shareholder Value, 1997, S. 288f.; Richter, Konzeption, 1996, S. $16 \mathrm{ff}$.

458 Vgl. Berliner Initiativkreis German Code of Corporate Governance, Code, 2000, S. 61f., III.6.; zu aktienkursorientierten Vergütungsformen m.w.N. Kräkel, Management, 1999, S. 288, sowie mit einer modelltheoretischen Überlegung zu risikofreien Anreizen für Top-Manager S. 322ff.; Schmidt, Shareholder Value-Konzept, 1993, S. 288.

459 Vgl. m.w.N. von Werder, Shareholder Value, 1998, S. 77; ausführlich setzt sich Wagner, Shareholder Value, 1997, S. 473-498 mit den rechts- und wirtschaftshistorischen Wurzeln der Ablehnung einer eigentümerorientierten Ausrichtung von Unternehmungen in Deutschland auseinander.

Vgl. Großmann, Unternehmensziele, 1980, S. 258.

461 Ähnlich von Werder, Shareholder Value, 1998, S. 76ff.; mit einer Fokussierung auf die kapitalmarkttheoretischen Grundlagen Mülbert, Shareholder Value, 1997, S. 129-172. 
nicht der Fall, so ist die Unternehmungsleitung an einer expliziten Verfolgung der Shareholder-Interessen nicht gehindert.

SCHMIDT/SPINDLER zeigen auf Grundlage der auch hier vorgenommenen Interpretation des Shareholder Value-Ansatzes, daß diese Zielkonzeption sowohl mit dem Gesellschafts- als auch mit dem Unternehmensinteresse vereinbar ist. ${ }^{462}$ Beide Konstrukte werden von verschiedenen Seiten als handlungsbestimmend für die Unternehmungsleitung in der Aktiengesellschaft angesehen. ${ }^{463}$

Das Gesellschaftsinteresse ist eine überindividuelle und von den konkreten Interessen der Verbandsmitglieder losgelöste, für alle Gesellschaftsorgane gleichermaßen verbindliche Leitmaxime. ${ }^{464}$ Hiervon ausgehend muß für jeden Einzelfall das konkrete Gesellschaftsinteresse im Sinne der überindividuell aggregierten Anteilseignerinteressen entwickelt werden. Für eine Aktiengesellschaft bedeutet dies eine Orientierung an der Zielsetzung der langfristigen Gewinnmaximierung. Aus juristischer Perspektive besteht insoweit kein Konflikt zu einer Ausrichtung der Unternehmung am Marktwert des Eigenkapitals. ${ }^{465}$ Für SCHMIDT/SPINDLER handelt es sich hierbei sogar um die einzig mögliche Konkretisierung dessen, was mit Gesellschaftsinteresse gemeint sein könnte. Sie schlagen daher vor, die Verfolgung des Shareholder Value in der Satzung verbindlich vorzuschreiben. ${ }^{466}$

Auch das Unternehmensinteresse als Bezugspunkt der Interessen aller an der Unternehmung beteiligten Stakeholder spricht nicht gegen die Verfolgung des Shareholder Value-Ansatzes, da das Unternehmensinteresse als Rechtsfigur juristisch zweifelhaft sowie nur begrenzt operationalisierbar ist und daher nicht

462 Vgl. Schmidt/Spindler, Shareholder-Value, 1997, S. 515-555, insbesondere S. 548; im Ergebnis anderes dagegen von Werder, Shareholder Value, 1998, S. 69-91, insbesondere S. 89. Hüffer, Aktiengesetz, 1999, Anm. 9 zu $\S 82$ AktG. Grundlegend zur Idee des Unternehmensinteresses Rathenau, Aktienwesen, 1918; zu den unterschiedlichen Sichtweisen zum Unternehmensinteresse Schmidt/Spindler, Shareholder-Value, 1997, S. 542f.; die Figur des Unternehmensinteresses mangels dogmatischer Reflektierung und inhaltlicher Bestimmtheit ablehnend schon Großmann, Unternehmensziele, 1980, S. 256; umfassend mit Bezug auf den Aufsichtsrat Koch, Unternehmensinteresse, 1983.

Vgl. Mülbert, Shareholder Value, 1997, S. 141.; m.w.N. Schmidt/Spindler, Shareholder-Value, 1997, S. 535.

Vgl. Schmidt/Spindler, Shareholder-Value, 1997, S. 539f.; kritisch dagegen Mülbert, Shareholder Value, 1997, S. 161, der eine Ausrichtung am Marktwert des Eigenkapitals jedoch insoweit für rechtlich zulässig erachtet, als dies die zukünftigen Finanzierungsmöglichkeiten am Kapitalmarkt verbessert.

Vgl. Schmidt/Spindler, Shareholder-Value, 1997, S. 541. 
handlungsleitend sein kann. ${ }^{467}$ Selbst wenn das Unternehmensinteresse operational wäre, sollte es für die Unternehmungsleitung nicht handlungsleitend sein, ${ }^{468}$ denn der Vorstand könnte dann unter Berufung auf das juristische Unternehmensinteresse sowie das hiermit verbundene Stakeholder-Konzept Handlungsspielräume zu Lasten aller übrigen Stakeholder einschließlich der Aktionäre ausnutzen, da es in sein Ermessen gestellt wäre, wessen Interessen in welchem Ausmaß berücksichtigt würden. ${ }^{469}$

Die hier vorgeschlagene Zielkonzeption des Shareholder Value kann dagegen juristisch abgesichert eine Harmonisierung aller Interessen soweit wie möglich und nötig sicherstellen. ${ }^{470}$ Dort, wo die Verfolgung der Interessen der Aktionäre zu Lasten der übrigen Stakeholder gehen sollte, sind diese durch marktliche oder rechtliche Mechanismen hinreichend abgesichert. ${ }^{471}$ So sieht HOMMELHOFF auch unter Verweis auf die Sozialpflichtigkeit des Eigentums und den notwendigen Interessenausgleich keine Bedenken dagegen, Geschäftsbereiche zu schließen, die die vom Kapitalmarkt geforderte Mindestrendite nicht erzielen. ${ }^{472}$

\section{Funktionsfelder des Aufsichtsrats in der Unternehmungsfuih- rung}

1. Planung als Instrument zum Abbau der Principal Agent-Problematik Nachdem in den vorangegangenen Abschnitten die Unternehmungsführung in Aktiengesellschaften näher betrachtet und institutionelle Hemmnisse bei der arbeitsteiligen Zielerreichung zwischen Hauptversammlung, Aufsichtsrat und Vorstand analysiert wurden, soll es jetzt darum gehen, die hier vorzuschlagende Funktionserweiterung des Aufsichtsrats im Führungsprozeß zu begründen. Hierzu werden zwei komplementäre Begründungsstränge herangezogen: in diesem

467 Vgl. Schmidt/Spindler, Shareholder-Value, 1997, S. 542ff.; zustimmend Großmann, Unternehmensziele, 1980, S. 256. Anders dagegen beispielsweise Potthoff/Trescher, Aufsichtsratsmitglied, 1999, S. 79, die Unternehmens- und Gesellschaftsinteresse als weitgehend synonym ansehen, jedoch ebenfalls die fehlende Operationalisierbarkeit beklagen. Kritisch zu den Konsequenzen einer falschen, am Unternehmensinteresse ausgerichteten Zielsetzung Wagner, Shareholder Value, 1997, S. 487ff. der Value, 1997, S. 503; kritisch zu den Folgen bei der Berufung auf „diffuse“ Stakeholderinte-
ressen auch Wenger, Unternehmenskontrolle, 1995, Sp. 1411, sowie Groh, Aktienrecht, 2000, S. 2158, der hier von Vorstandsherrlichkeit spricht.

470 den Gesetzgeber feststellt.

471 Vgl. Neus, Betriebswirtschaftslehre, 1998, S. 164ff., sowie Schmidt/Spindler, Shareholder-Value, 1997, S. 555.

472 Vgl. Hommelhoff, Shareholder Value-Prinzip, 1997, S. 18. 
Abschnitt die institutionenökonomische Analyse der Delegationsbeziehungen in der Publikumsaktiengesellschaft, im nachfolgenden Abschnitt die Analyse der Aufgaben des Aufsichtsrats.

Auf Grundlage der ökonomischen Analyse der Spitzenverfassung kann der Aufsichtsrat aus agencytheoretischer Perspektive als eine der Institutionen zum Abbau der Agency-Problematik charakterisiert werden. ${ }^{473}$ Der Aufsichtsrat nimmt für die Anteilseigner einer Aktiengesellschaft die Überwachungsfunktion einschließlich der Personalkompetenz gegenüber dem Vorstand wahr, so daß von einer einstufigen Principal Agent-Beziehung zwischen dem Aufsichtsrat und dem Vorstand ausgegangen werden soll. ${ }^{474}$ Wie gezeigt wurde, schlägt die Principal Agent-Theorie zur Vermeidung hieraus resultierender Probleme u.a. die Durchführung von Monitoringaktivitäten zur Verminderung der Informationsasymmetrie zwischen Auftraggeber und Auftragnehmer vor, ${ }^{475}$ da eine vollständige Lösung des Agency-Problems allein mit Hilfe des Anreizsystems nicht zu erwarten ist. ${ }^{476}$ Für JASCHKE ist gerade der Aufsichtsrat für diese Aktivitäten prädestiniert, da er im Gegensatz zur Öffentlichkeit über deutlich bessere Informationsmöglichkeiten verfügt. ${ }^{477}$ Die Monitoring-Aktivitäten setzen neben Kontroll- und Berichtssystemen auch die Einführung geeigneter Planungssysteme voraus. ${ }^{478}$ Es scheint daher aus agencytheoretischer Perspektive notwendig zu sein, zum Abbau von Informationsasymmetrien zwischen Aufsichtsrat und Vorstand ein Planungssystem für den Aufsichtsrat einzurichten, um die Monitoringaktivitäten des Aufsichtsrats zu unterstützen.

Zusätzlich wird der Aufsichtsrat selbst als Agent der Aktionäre tätig, so daß auch innerhalb dieser Agency-Beziehung Maßnahmen zum Abbau der Principal Agent-Problematik ergriffen werden sollten. Daher wird hier eine Interessenan-

473 Vgl. Bernhardt/Witt, Unternehmensleitung, 1999, S. 838; Semler, Unternehmensüberwachung, 1995, S. 50.

474 So beispielsweise Chwolka, Aufsichtsrat, 1999, S. 631, die später hierarchische Beziehungen betrachtet; zu dynamischen und mehrstufigen Principal Agent-Beziehungen Petersen, Anreizsysteme, 1989; Elschen, Agency-Theorie, 1988, S. 250, weist auf aus der Partialbetrachtung resultierende Probleme hin, die hier jedoch nicht betrachtet werden sollen; neuere agencytheoretische Untersuchungen zum Aufsichtsrat beispielsweise von Auge-Dickhut, Aufsichtsrat, 1999, Martens, Corporate Governance, 2000, und Portisch, Überwachung, 1997, der jedoch vor dem Hintergrund, daB der Aufsichtsrat nicht nur den Anteilseignerinteressen, sondern auch weiteren Stakeholdern verpflichtet sei, die Agency-Theorie mit dem Stakeholder-Ansatz zusammenführt.

475 Vgl. Picot/Diet1/Franck, Organisation, 1997, S. 90; Günther, Controlling, 1997, S. 49.

476 Vgl. Shleifer/Vishny, Corporate Governance, 1997, S. 745.

477 Vgl. Jaschke, Überwachungsfunktion, 1989, S. 50; so wohl auch Semler, Unternehmensüberwachung, 1995, S. 52.

478 Vgl. Picot/Diet//Franck, Organisation, 1997, S. 90. 
gleichung durch Anreize und Sanktionen vorgeschlagen, um Handlungen von Aufsichtsräten zu Lasten der Aktionäre zu verhindern. ${ }^{479}$ Im Mittelpunkt der vorliegenden Untersuchung werden die in jüngster Zeit auch in der Praxis an Bedeutung gewinnenden wertorientierten Anreizsysteme für Aufsichtsratsmitglieder stehen, ${ }^{480}$ da durch sie gewährleistet werden kann, daß die Aufsichtsratsmitglieder die ihnen de jure übertragenen Aufgaben im Interesse der Aktionäre und nicht irgendeiner anderen Interessengruppe wahrnehmen. ${ }^{481}$ BERNHARDT/WITT unterstellen zu Recht, daß Aufsichtsratsmitglieder aufgrund des gar nicht oder nur in geringem Maße vorhandenen Aktienbesitzes

„nicht primär Aktionärsinteressen, sondern andere Ziele, z.B. den Schutz eines Kreditengagements (Bankenvertreter), die Sicherung der eigenen Arbeitsplätze (Arbeitnehmervertreter), die Verfestigung von Firmenbeziehungen (Industrievertreter) usw. “482

verfolgen werden.

Neben der Notwendigkeit einer Interessenangleichung zwischen Aktionären und Aufsichtsratsmitgliedern durch eine entsprechende Ergebnisbeteiligung liefert die Principal Agent-Theorie weitere relevante Gestaltungshinweise. Hierzu sind beispielsweise geeignete Signalling- bzw. Screeningaktivitäten zum Abbau von vorvertraglichen Informationsasymmetrien bei der Besetzung der Aufsichtsratsmandate zu rechnen, die es bei der Gestaltung des Planungs- sowie des Anreizsystems zu berücksichtigen gilt.

Neben den Gestaltungshinweisen der Principal Agent-Theorie kann auf Grundlage der vorgenommenen Aufgabenanalyse des Aufsichtsrats gezeigt werden, $\mathrm{da} ß$ für ihn eine Neupositionierung im Unternehmungsführungsprozeß zu fordern ist.

479 So auch Knoll/Knoesel/Probst, Aufsichtsratsvergütungen, 1997, S. 237, sowie unter EinschluB der Mitbestimmung Neus, Betriebswirtschaftslehre, 1998, S. 152.

480 Vgl. Einladung zur Hauptversammlung der Siemens AG am 18. Februar 1999; aus juristischer Perspektive Friedrichsen, Aktienoptionsprogramme, 2000, S. 195ff.; allgemein Bernhardt/Witt, Stock Options, 1997, S. 85-101; Pellens, Entlohnungssysteme, 1998; Rosen, Vergütungssysteme, o. J.; Schmidt, Stock Options, 1998, S. 76-79; Wenger/Knoll/Kaserer, Stock options, 1999, S. 3538, sowie empirisch für Deutschland Schwalbach/Graßhoff, Managervergütung, 1997, S. 203217.

481 Vgl. Bernhardt/Witt, Unternehmensleitung, 1999, S. 840; Portisch, Überwachung, 1997, S. 164, sieht den Aufsichtsrat dagegen gerade als Forum des Interessenausgleichs unterschiedlicher Stakeholder. Eine ausschließlich ergebnisorientierte Entlohnung von Aufsichtsräten auf Grundlage einer formalen agencytheoretischen Analyse ablehnend Chwolka, Aufsichtsrat, 1999, S. 639; zum praktischen Aussagegehalt normativer Agencymodelle kritisch Meinhövel, Principal-AgentTheorie, 1999, S. 213.

482 Bernhardt/Witt, Unternehmensleitung, 1999, S. 842, FN 42. 


\section{Planung als Prämisse einer ordnungsgemäßen Überwachung}

Im Zentrum unternehmerischer Aktivitäten steht das Fällen von Entscheidungen, mit denen Handlungsalternativen ausgewählt und für vollzugsverbindlich erklärt werden. Alleiniger Qualitätsmaßstab einer Entscheidung ist ihr Beitrag zur Erreichung der Unternehmungsziele, d.h., die mit einer Entscheidung ausgewählte Handlungsmöglichkeit muß den größtmöglichen Zielbeitrag zur langfristigen Maximierung des Shareholder Value aufweisen. Damit Entscheidungen diesen Anspruch erfüllen können, sind sie informatorisch vorzubereiten. Dies geschieht mit Hilfe der Planung, die zur systematischen Suche nach neuen Zielen, Mitteln und Maßnahmen beiträgt und über die Schaffung eines Anpassungsvorrats zu einer Erhöhung der unternehmerischen Flexibilität führt. Durch die Systematisierung der Suche nach optimalen Handlungsalternativen führt sie außerdem zur Versachlichung von Entscheidungen.

Wie die Analyse der arbeitsteiligen Aufgabenerfüllung von Vorstand und Aufsichtsrat gezeigt hat, entspricht die oftmals vorzufindende Reduktion des Aufsichtsrats auf reine Kontrolltätigkeiten nicht der aktienrechtlichen Sollvorstellung. Dem Aufsichtsrat kommen innerhalb seiner Überwachungsaufgabe neben der Kontrolle des Vorstands weitere Mitentscheidungsaufgaben gemeinsam mit dem Vorstand sowie eigenständige Entscheidungsaufgaben zu. Sie beinhalten primär die Personalhoheit des Aufsichtsrats über den Vorstand einschließlich der Konzipierung seines Anreizsystems sowie die Gestaltung der inneren Organisation des Vorstandsorgans. Diese Entscheidungen konnten im Rahmen der Aufgabenanalyse des Aufsichtsrats als originäre Führungsentscheidungen bzw. Kernaufgaben der Unternehmungsführung identifiziert werden, wobei gerade die Wahrnehmung der Personalhoheit über den Vorstand Grundvoraussetzung für die Funktionsfähigkeit des deutschen Aufsichtsratsmodells ist. ${ }^{483}$ Verzichtet der Aufsichtsrat auf die Wahrnehmung seiner Personalhoheit, wird nicht nur gegen geltendes Recht verstoßen, eine effektive Überwachung des Vorstands ist ohne Nutzung dieser Gestaltungs- und Sanktionspotentiale unmöglich.

„Leitung und Überwachung sind nach dem Aktiengesetz unternehmerische Tätigkeiten im Sinne des Festlegens von Zielen, des Planens von Vorhaben und des Abwickelns von geplanten Maßnahmen. “484

Während die Pflicht des Vorstands zur Planung inzwischen auch aus juristischer Perspektive akzeptiert ist, muß dies für den Aufsichtsrat begründet werden. ${ }^{485}$

483 Vgl. Bleicher, Organisation, 1991, S. 22; den unternehmerischen Charakter der Entscheidungen und einen hieraus resultierenden Ermessensspielraum konstatiert auch Gawrisch, Ermessensentscheidungen, 2000, S. 20.

484 Semler, Überwachung, 1996, S. 53. 
Hierzu ist an seinen Aufgaben anzuknüpfen: Der Aufsichtsrat kann das Vorstandshandeln nur zweckentsprechend kontrollieren, wenn er selbst plant. Dies läßt sich damit begründen, daß er innerhalb seiner Kontrollfunktion nicht nur Handlungen, sondern auch Unterlassungen des Vorstands zu beurteilen hat. Um solche Unterlassungen feststellen zu können, benötigt der Aufsichtsrat eigene Vorstellungen über die zukünftige Entwicklung der Unternehmung, d.h., er muß - beispielsweise ein Produkt-Markt-Konzept - planen. ${ }^{486}$ Somit wird seine Planung zu einer Komplementärfunktion seiner Kontrolle. Dies gilt um so mehr, als zukunftsorientierte Entscheidungen des Vorstands mit einem hohen Maß an Unsicherheit verbunden sind. Die Bestimmung des Soll-Zustands der Objekte bzw. Kontrollmaßstäbe des Aufsichtsrats unterliegt daher einem sehr hohen $\mathrm{Ma} B$ an subjektiver Einschätzung. ${ }^{487}$ Will sich der Aufsichtsrat bei der Entwicklung der Kontrollmaßstäbe nicht nur auf die von seinem Überwachungsobjekt „Vorstand“ angefertigten Unterlagen und sein eigenes Fingerspitzengefühl - um nicht zu sagen Willkür - verlassen, muß er im Rahmen der ihm zur Verfügung stehenden Möglichkeiten eigene Überlegungen über den Sollzustand des Kontrollobjekts anstellen und weitere Informationsquellen nutzen. ${ }^{488}$ Sinnvoll kann dies nur mit Hilfe der Planung erfolgen.

Noch mehr als im Rahmen der Vorstandskontrolle kommt dem Aufsichtsrat bei einer Mitentscheidung zusammen mit dem Vorstand, beispielsweise im Rahmen zustimmungspflichtiger Geschäfte, eine originäre unternehmerische Verantwortung zu, die es erforderlich macht, daß sich Aufsichtsratsmitglieder eine eigene Meinung über die Entscheidungen des Vorstands bilden und dessen Planung kritisch reflektieren. ${ }^{489}$ Ein bloßes Nachvollziehen der Vorstandsargumen-

485 Vgl. Feddersen, Unternehmensplanung, 1993, S. 114-117, sowie Lutter, Aufsichtsratstätigkeit, 1995, S. 291f., und den dort skizzierten Disput zur rechtlichen Verpflichtung des Vorstands hinsichtlich der Erstellung einer Unternehmungsplanung; so sah sich der Gesetzgeber noch mit dem KonTraG genötigt, diese Pflicht klarzustellen, obwohl dies aus betriebswirtschaftlicher Perspektive seit langem unstrittig ist; vgl. hierzu Theisen, Reform, 1999, S. 220; ablehnend Kallmeyer, Unternehmensplanung, 1993, S. 104-113.

So bereits Chmielewicz, Führung, 1988, S. 569; auch Mutter, Aktiengesellschaft, 1994, S. 132f., fordert ,in gewissem Umfang“ die Entwicklung einer eigenen planerischen Konzeption durch den Aufsichtsrat zur Wahrnehmung seiner Kontrollfunktion insbesondere im Hinblick auf die Unternehmungsplanung des Vorstands; dem folgend Wellenkamp, Aufsichtsrat, 2000, S. 182; im Ergebnis wohl auch Scheffler, Überwachungsaufgabe, 1994, S. 794, wenn er fordert, daß der Aufsichtsrat danach zu fragen habe, ob ein alternatives Vorgehen zweckmäßiger wäre oder nicht.

Vgl. Theisen, Geschäftsführung, 1993, Sp. 4226f.

So auch Mutter, Aktiengesellschaft, 1994, S. 134f.

Vgl. Lutter, Aufsichtsratstätigkeit, 1995, S. 291f.; Mertens, Kölner Kommentar, 1996, Anm. 26 zu § 111 AktG.; Henze, Entscheidungen, 2001, S. 58, betont die selbständige Entscheidungsbefugnis bei der Mitentscheidung; wie ausgeführt wurde, ist die Pflicht zur Anwendung betriebs- 
tation kann als nicht ausreichend angesehen werden. Der Aufsichtsrat hat ebenso wie bei der Kontrolle seine Sicht der Dinge mit der des Vorstands abzugleichen und diesem als kritischer Diskussionspartner beratend zur Verfügung zu stehen. ${ }^{490}$ Dabei muß er auf den Meinungsbildungsproze $ß$ des Vorstands einwirken, ohne diesen in seiner unternehmerischen Ermessensfreiheit zu beschränken. Will er sich hierbei nicht auf die Informationen des Vorstands beschränken, ist hierzu ebenfalls eine eigenständige Planung erforderlich.

Die Planungsnotwendigkeit des Aufsichtsrats verdeutlicht sich schließlich insbesondere im Kontext seiner eigenen unternehmerischen Entscheidungen. Wie gezeigt wurde, erfordern die hier zu fällenden langfristig wirksamen Entscheidungen aufgrund des mit ihnen verbundenen hohen Maßes an Unsicherheit umfangreiche planerische Vorbereitungen. Da sich die Entscheidungen zudem oftmals unmittelbar auf den Vorstand beziehen, ist die Willensbildung des Aufsichtsrats als unzureichend zu bezeichnen, wenn sie auf Planungsüberlegungen des Vorstands selbst beruht. Besonders gravierend wird dies bei der Auswahl, der Bestellung und der Abberufung von Vorstandsmitgliedern deutlich, die ein funktionsfähiges „Metamanagement" erfordern, das den Vorstand als Objekt erfaßt und beeinflußt. ${ }^{491}$ Daher sollte der Aufsichtsrat insbesondere in allen eigenen unternehmerischen Entscheidungsangelegenheiten, für die er zudem in der Regel das Initiativrecht besitzt, eigene Planungsüberlegungen anstellen.

Es zeigt sich, daß auf der Ebene der strategischen Führung eine Vernetzung von Aufsichtsrat und Vorstand vorliegt. ${ }^{492}$ Zur eindeutigen Trennung der Tätigkeitsbereiche beider Organe werden daher alle Aufgaben des Aufsichtsrats einem eigenen Führungsprozeß als Element des unternehmerischen Systems vermaschter Führungsprozesse zugeordnet. ${ }^{493}$ Dieser Prozeß umfaßt sowohl Kontroll- als auch (Mit-)Entscheidungsaufgaben. Als neues Funktionsfeld in der Unternehmungsführung tritt die Notwendigkeit einer eigenständigen Planung durch

wirtschaftlicher Methoden im Rahmen zustimmungspflichtiger Geschäfte Ausdruck einer Methodenpflicht des Aufsichtsrats; vgl. hierzu Abschnitt II.B.2.c)(5).

Vgl. Henn, Handbuch, 1998, S. 297.

Vgl. Oesterle, Führungswechsel, 1999, S. 305.

Dieses Verhältnis bezeichnet Frese, Führung, 1993, Sp. 1291, zutreffend als eines der organisatorischen Kernprobleme der Unternehmungsverfassung. Professionalisierung, 1995, S. 1133, diesen als Überwachungsprozeß, was mit der hier vorliegenden terminologischen Trennung übereinstimmt. 
den Aufsichtsrat hinzu, die über die bloße Beurteilung der Planung des Vorstands hinausgeht. ${ }^{494}$

Zur Wahrnehmung seiner Planungsfunktion wird für den Aufsichtsrat in Kapitel III ein Planungssystem entworfen, das alle erforderlichen Systemelemente beinhaltet. Gesetzliche Vorgaben zur Gestaltung der Entscheidungsvorbereitung finden sich nur vereinzelt, so zum Beispiel für die Bildung von Ausschüssen oder die Gewinnung relevanter Informationen. ${ }^{495}$ Dabei wird der Aufsichtsrat in seiner Maßnahmenwahl nur gering eingeschränkt:

„Das Aktiengesetz bietet alles, was der Aufsichtsrat zur Erfüllung seiner Aufgaben benötigt. “496

An das Aufsichtsratsplanungssystem sind verschiedene Anforderungen zu stellen. Als Metaziel sollte es den Aufsichtsrat in die Lage versetzen, seine Aufgaben unabhängig vom Vorstand zu erfüllen. ${ }^{497}$ Um dieses Ziel zu erreichen, sind verschiedene Voraussetzungen zu schaffen:

1. die Aufsichtsratsarbeit hat kontinuierlich stattzufinden,

2. der Aufsichtsrat muß regelmäßig, zeitnah und in gleichbleibender Form über alle notwendigen Informationen verfügen können,

3. er muß außerdem Zugriff auf Ressourcen der Gesellschaft erhalten.

Für LUTTER kann nur so die notwendige Professionalität des Aufsichtsrats realisiert werden, die erforderlich ist, damit er den „Vorstands-Professionals“ gewachsen ist. ${ }^{498}$

Diese Veränderungen dürfen jedoch nicht nur die Tätigkeit des Aufsichtsrats betreffen, seine Mitgliedern müssen darüber hinaus eine entsprechende Vergütung erhalten. Daher ist das Aufsichtsratsplanungssystem durch ein entspre-

494 Zustimmend Link, Unternehmungsplanung, 1999, S. 817, sowie Martin, Aufsichtsrat, 1990, S. 22, der auf der Grundlage einer handelsrechtlichen Begründung (gemeint ist das Gesellschaftsrecht) den Aufsichtsrat als Träger des strategischen Controlling sieht; kritisch zu seinen Ausführungen Römer, Aufsichtsrat, 1990, S. 126, der Martin zwar im Ergebnis zustimmt, seine Begründung jedoch auf der Aufsichtsratspraxis aufbauen will.

Vgl. Frese, Führung, 1993, Sp. 1292.

Servatius, Vorstandskontrolle, 1995, S. 223; zustimmend Schmidt u.a., Corporate Governance, 1997, S. 110.

497 Mit einer entsprechenden Forderung im Hinblick auf die Aufsichtsratstätigkeit Dufey/Hommel, Shareholder-Value-Ansatz, 1997, S. 197f.

Vgl. Lutter, Professionalisierung, 1995, S. 1133; zustimmend auch Dufey/Hommel, ShareholderValue-Ansatz, 1997, S. 197f., sowie bereits Bleicher/Leberl/Paul, Unternehmungsverfassung, 1989, S. 264. 
chendes Anreizsystem zu ergänzen. Dies erfolgt unter Berücksichtigung weiterer Anreizbestandteile in Kapitel IV.

„Strukturell bedingte Informationsdefizite, mangelnde Sachkompetenz und suboptimale Anreizstrukturen haben dieses Organ nicht selten in völlige Abhängigkeit des Vorstands geraten lassen.““999

Dies gilt es, mit dem Aufsichtsratsplanungssystem in Verbindung mit einem zweckentsprechenden Anreizsystem zu verhindern. Hierdurch soll eine dialogische Unternehmungsführung bewirkt werden, in der sowohl der Vorstand als auch der Aufsichtsrat in ihren jeweiligen Rollen gemeinsam auf die Erreichung der Unternehmungsziele hinarbeiten. ${ }^{500}$

499 Hommel/Riemer-Hommel, Unternehmensüberwachungssysteme, 1999, S. 169.

500 Zur Idee einer dialogischen Führung Servatius, Vorstandskontrolle, 1995, S. 224. 


\section{Entwurf eines Aufsichtsratsplanungssystems}

\section{A. Überblick zu den Elementen des Planungssystems}

Wie die bisherigen Ausführungen gezeigt haben, steht im Mittelpunkt jeder unternehmerischen Tätigkeit das Fällen zielentsprechender Entscheidungen. Planung wurde im zweiten Kapitel aus funktionaler Perspektive als Entscheidungsvorbereitungsprozeß definiert, an den insbesondere bei bedeutsamen und komplexen unternehmerischen Entscheidungen, wie sie im Entscheidungsfeld des Aufsichtsrats vorliegen, hohe Anforderungen zu stellen sind. ${ }^{501}$ Es reicht nicht aus, Entscheidungen intuitiv auf der Grundlage unternehmerischer Erfahrung zu fällen, sie sollten unter Einsatz geeigneter Instrumente detailliert vorbereitet werden.

Ausgehend von seinen unternehmerischen Entscheidungsaufgaben konnte für den Aufsichtsrat Planung als neues Funktionsfeld im Prozeß der Unternehmungsführung identifiziert werden, das ihm die ordnungsgemäße Wahrnehmung seiner übrigen Aufgaben erst ermöglicht und zum Abbau der Principal AgentProblematik zwischen ihm und dem Vorstand beiträgt. Es ist daher im vorliegenden dritten Kapitel danach zu fragen, wie eine Planung durch den Aufsichtsrat aussehen sollte. Dies betrifft nicht nur die Planungsfunktion, da es für eine umfassende Beschreibung erforderlich ist, alle Elemente des Planungssystems zu betrachten: Hierzu zählen das Planungsobjekt, die Planungsorganisation, das Planungssubjekt, die Planungsinstrumente sowie der Planungsproze $B{ }^{502}$

Am Anfang der Analyse stehen Planungsobjekte oder Gegenstände bzw. Inhalte der Planung. ${ }^{503}$ Sie beschreiben die Aufgabenfelder, mit denen sich der Aufsichtsrat im Rahmen seiner Tätigkeit zu befassen hat. Ihre Analyse bildet den Ausgangspunkt der anschließenden organisatorischen Gestaltung. ${ }^{504}$ Über die Planungsorganisation ist eine zweckentsprechende Aufgabenerfüllung zu ge-

501 Vgl. zur Bedeutung einer systematischen Entscheidungsvorbereitung Schoemaker/Russo, Entscheidung, 1994, S. 105.

502 Vgl. Mag, Unternehmungsplanung, 1995, S. 3f.; Mag, Kontrolle, 1999, S. 4f.; für abweichende Elementaufzählungen beispielsweise Wild, Unternehmungsplanung, 1982, S. 153, oder Hammer, Unternehmensplanung, 1998, S. 37ff.

Vgl. Mag, Unternehmungsplanung, 1995, S. 38ff., sowie Hammer, Unternehmensplanung, 1998, S. 22f., der von Objektbereichen der Unternehmungsplanung spricht, und Szyperski/Winand, Unternehmungsplanung, 1980, S. 43f.

504 Von einem Primat der Aufgabe für die Organisation geht beispielsweise auch Kosiol, Organisation, 1962, S. 41, aus. 
währleisten. ${ }^{505}$ In Abhängigkeit von der gewählten organisatorischen Ausgestaltung bestehen unterschiedliche Anforderungen an die Planungssubjekte, d.h. alle Personen, die aktiv oder passiv mit der Planung in Berührung kommen, ${ }^{506}$ da mit der Organisation deren arbeitsteilige Aufgabenerfüllung strukturiert und koordiniert wird. Die Planungssubjekte sollen daher im Anschluß an die Planungsorganisation dargestellt werden. Zur Erfüllung ihrer Planungsaufgaben setzen die Planungssubjekte Planungsinstrumente als mentale und reale Hilfsmittel ein. ${ }^{507}$ In der abschließenden Analyse des Planungsprozesses erfolgt eine Integration der übrigen Systemelemente. Der Planungsprozeß ist identisch mit dem funktionalen Planungsbegriff. ${ }^{508}$ Er beinhaltet eine Folge verschiedener mentaler Arbeitsschritte, die idealtypisch linear dargestellt werden, in der Praxis aber mit Rückkoppelungen oder unter Auslassung einzelner Teilschritte durchlaufen werden: ${ }^{509}$

1. Zielanalyse zur Planung von Maßstäben bzw. Sollvorstellungen für zukünftiges Handeln.

2. Problemanalyse mit der Problemerkenntnis und Problemformulierung als Grundlage der Entwicklung von Lösungsvorschlägen.

3. Alternativenanalyse zur Generierung von Handlungsmöglichkeiten.

4. Prognose zur Gewinnung von Vorwissen über Daten oder Umweltzustände (Lage- und Entwicklungsprognose), über die Auswirkungen der Realisation möglicher Handlungsalternativen (Wirkungsprognose) sowie über die Unsicherheitsstruktur (Wahrscheinlichkeitsprognose).

5. Bewertung der Handlungsalternativen im Hinblick auf ihren Zielerreichungsgrad bzw. ihren Zielbeitrag.

Die konkrete Ausgestaltung dieser Arbeitsschritte variiert in Abhängigkeit von dem betrachteten Planungsobjekt, so daß aufgrund der Vielzahl möglicher Pla-

505 Vgl. Mag, Unternehmungsplanung, 1995, S. 120ff.; Hammer, Unternehmensplanung, 1998, S. $91 \mathrm{ff}$.

506 Vgl. Mag, Kontrolle, 1999, S. 32.

507 Vgl. Mag, Kontrolle, 1999, S. 38f.; Mag, Unternehmungsplanung, 1995, S. 19ff.; zum Teil wird auch der Begriff Methoden verwendet, vgl. Hammer, Unternehmensplanung, 1998, S. $71 \mathrm{ff}$.

508 Vgl. Mag, Unternehmungsplanung, 1995, S. 46.

509 Vgl. m.w.N. Mag, Unternehmungsplanung, 1995, S. 8; zum Planungsprozeß ausführlich Mag, Unternehmungsplanung, 1995, S. 46ff., sowie Wild, Unternehmungsplanung, 1982, S. 46ff.; mit einer abweichenden Einteilung Hahn, Planung, 1994, S. 42; zur Kritik am Phasenschema Mag, Unternehmungsplanung, 1995, S. 98ff.; ähnlich auch Schneider, Betriebswirtschaftslehre, 1997, S. 84. 
nungsobjekte des Aufsichtsrats nur ein Teilbereich der diskutierten Objekte exemplarisch betrachtet wird. Wegen ihrer Bedeutung handelt es sich dabei um die personellen Angelegenheiten des Vorstands sowie die hiermit eng verbundenen organisatorischen Regelungen. ${ }^{510}$

Im vorliegenden Problemzusammenhang dient diese Klassifizierung einer umfassenden und strukturierten Analyse aller notwendigen Voraussetzungen für eine systematische Entscheidungsvorbereitung im bzw. durch den Aufsichtsrat. Sie soll erstens dazu beitragen, die in der Regel problembezogenen Gestaltungsempfehlungen zum Aufsichtsrat zu systematisieren und Einzelvorschläge in einen Gesamtzusammenhang einzubinden. ${ }^{511}$ Aufgrund des zweiten wesentlichen Unterschieds zu den bisherigen aufsichtsratsbezogenen Gestaltungsempfehlungen, der hier vorgenommenen inhaltlichen Erweiterung und Betonung der Planungsaufgaben, wird es drittens notwendig sein, über die bisherigen Gestaltungsvorschläge hinaus zu gehen und weitere Vorschläge zu entwickeln. Hierbei ist zu betonen, daß auch die bisher diskutierten Gestaltungsempfehlungen ganz überwiegend (noch) nicht in der Aufsichtsratspraxis umgesetzt sind. ${ }^{512}$

Die verschiedenen Planungssystemelemente zeichnen sich durch vielfältige Interdependenzen aus, wodurch eine unterschiedliche Vorgehensweise bei ihrer Beschreibung möglich ist. ${ }^{513}$ Als erste Alternative scheint eine integrierende Gesamtbetrachtung grundsätzlich gangbar zu sein. Hierdurch könnte jedoch das Ziel, eine Klassifikation der in der Literatur diskutierten Gestaltungsvorschläge vorzunehmen und diese systematisch weiterzuentwickeln, nicht erreicht werden. Ein Systematisierungsgewinn wäre so nicht realisierbar. Daher erfolgt nachfolgend eine sequentielle Beschreibung der verschiedenen Elemente, wobei nicht unberücksichtigt bleiben darf, daß aufgrund der vorhandenen Interdependenzen eine vollständige Isolation der einzelnen Elemente nicht möglich ist. Die Reihenfolge der Behandlung aller Elemente stellt hierbei keine Priorisierung dar. Insbesondere deutet die abschließende Behandlung des Planungsprozesses nicht

510 So bezeichnet der Berliner Initiativkreis German Code of Corporate Governance, Code, 2000, S. 43-47, II.1., u.a. die personelle Besetzung des Vorstands als Kernprozeß der Corporate Governance.

511 Vgl. beispielsweise Claussen, Aktienrechtsreform, 1996, S. 481-494; Claussen, Aktiengesetz, 1998, S. 177-186; Götz, Überwachung, 1995, S. 337-353.

$512 \mathrm{Zu}$ einer empirischen Untersuchung der Umsetzung neuer gesetzlicher Anforderungen Feddersen, Aufsichtsrat, 2000, S. 392f.; Theisen, Berichterstattung, 1997, S. 114; Theisen, Reform, 1999, S. 243, bemängelt jedoch zu Recht, daB über die tatsächliche Überwachungstätigkeit des Aufsichtsrats nur unzureichende Informationen verfügbar sind.

513 Vgl. zu einer von der hier gewählten Reihenfolge abweichenden Beschreibung Mag, Unternehmungsplanung, 1995, S. 38. 
auf seine eventuelle Nachrangigkeit hin; dies erfolgt vielmehr aus Zweckmäßigkeitsüberlegungen, da die Analyse des Planungsprozesses die Beschreibung der übrigen Systembestandteile am nachhaltigsten voraussetzt.

\section{B. Planungsobjekte des Aufsichtsrats}

\section{Vorstandsbezogene Planungsobjekte}

a) Organisatorische Planungsobjekte im Rahmen der Geschäftsordnungskompetenz

\section{(1) Bedeutung der Geschäftsordnungskompetenz}

Ziel einer Ermittlung von Planungsobjekten des Aufsichtsrats muß es sein, nicht alle möglichen, sondern lediglich die wesentlichen Planungsgegenstände zu ermitteln und zu systematisieren. ${ }^{514}$ Daher setzt die Systematisierung der für den Aufsichtsrat relevanten Planungsobjekte an seinen unterschiedlichen Teilaufgaben an, bei der die Bildung von Aufgabenbündeln nach inhaltlichen Gesichtspunkten erfolgt ist. Sie berücksichtigt außerdem Rechte und Pflichten des Aufsichtsrats zu einer ordnungsgemäßen Aufgabenwahrnehmung. Dies umfaßt vor allem die sich aus der Überwachungspflicht ergebenden Anforderungen.

Wie die Aufgabenanalyse des Aufsichtsrats gezeigt hat, zählt zu seinen wichtigsten Tätigkeiten der Erlaß einer Geschäftsordnung für den Vorstand, die er ohne Beteiligung des Vorstands wahrnehmen sollte. ${ }^{515}$ Immer dann, wenn der Vorstand sich selbst eine Geschäftsordnung gibt, besteht die Gefahr, daß er über eine entsprechende Geschäftsverteilung die Personalkompetenz des Aufsichtsrats untergräbt. ${ }^{516}$ Es ist daher nachdrücklich zu fordern, daß sowohl die Organisations- als auch die Personalkompetenz für den Vorstand als zwei untrennbare Aufgaben in den Händen des Aufsichtsrats liegen ${ }^{517}$ Die große Bedeutung die-

$514 \mathrm{Zu}$ einer priorisierenden Auflistung von Aufsichtsratsaufgaben beispielsweise Berliner Initiativkreis German Code of Corporate Governance, Code, 2000, S. 65f., VI.2.

sis Vgl. Scheffler, Rollenverständnis, 2000, S. 435; im Ansatz auch Berliner Initiativkreis German Code of Corporate Governance, Code, 2000, S. 65, IV.2.2.; Hoffmann-Becking, Vorstand, 1998, S. 501ff, spricht von der primären Regelungskompetenz des Aufsichtsrats und vermutet mangels empirischer Belege, daß sehr häufig Vorstand und Aufsichtsrat gemeinsam die Geschäftsordnung erlassen; das hierin liegende Gestaltungspotential hebt auch Mutter, Aktiengesellschaft, 1994, S. $66,153 f$., hervor.

516 Vgl. Hoffmann-Becking, Vorstand, 1998, S. 502; die enge Verbindung von personeller und organisatorischer Kompetenz betont auch Meyer-Lohmann, Aufsichtsrat, 1997, S. 47; die Deutsche Bank verpflichtet dagegen in ihren Corporate Governance-Grundsätzen den Vorstand, sich selbst eine Geschäftsordnung zu geben; vgl. Deutsche Bank, Corporate Governance-Grundsätze, 2001, S. 5.

$517 \mathrm{Da}$ dies in der Praxis nicht so gesehen wird, zeigt das Beispiel der Deutschen Telekom AG, bei der der Vorstandsvorsitzende Sommer den Telekom-Vorstand neu strukturiert; vgl. hierzu Riedel, Telekom-Vorstand, 2001, S. 15. 
ser Entscheidungen des Aufsichtsrats für die Unternehmung betont auch WELLENKAMP ${ }^{518}$ so daß von einer umfassenden Planungsnotwendigkeit auszugehen ist.

Die Geschäftsordnung enthält verbindliche Regelungen, die sich primär auf die arbeitsteilige Aufgabenerfüllung innerhalb des Vorstandsorgans sowie die $\mathrm{Zu}$ sammenarbeit mit dem Aufsichtsrat beziehen. Insbesondere bietet seine Geschäftsordnungskompetenz dem Aufsichtsrat die Möglichkeit, für den Vorstand die Aufbau- und Ablauforganisation über die gesetzlichen Mindestanforderungen hinaus verbindlich zu regeln, um hierdurch die Arbeit der „Steuerungszentrale" einer Unternehmung zu gestalten. ${ }^{519}$ Solche organisatorischen Regelungen als Teilmenge präsituativer Regelungen erfordern vorausschauendes Denken, d.h. sie sind das Ergebnis von Planung. ${ }^{520}$ Zur inhaltlichen Ausgestaltung der Geschäftsordnung ist daher eine revolvierende Organisationsplanung durch den Aufsichtsrat erforderlich, die gegebenenfalls an veränderte Bedingungen angepaßt werden sollte. ${ }^{521}$ Hiermit nimmt der Aufsichtsrat die zukünftige Aufgabenerledigung des Vorstands vorweg und strukturiert sie, wobei unterschiedliche Teilplanungsobjekte zu berücksichtigen sind. Die Frage nach dem Umfang der in der Geschäftsordnung erfaßten Teilplanungsobjekte kann unterschiedlich beantwortet werden. So fordert beispielsweise der BERLINER INITIATIVKREIS GERMAN CODE OF CORPORATE GOVERNANCE eine auf die Grundzüge beschränkte Festlegung der Geschäftsordnung. ${ }^{522}$ Diese Forderung ist jedoch unzureichend, da zum einen nicht eindeutig bestimmt ist, worum es sich bei diesen Grundzügen handeln sollte. Zum anderen werden hierdurch wiederum unerwünschte Ausweichhandlungen des Aufsichtsrats ermöglicht, der unnötigerweise aus seiner Organisationspflicht entlassen würde, so daß der Aufsichtsrat innerhalb des vom Satzungsgeber gesteckten Rahmens sämtliche erforderlichen organisatorischen Tatbestände für das Vorstandsorgan regeln sollte.

\footnotetext{
518 Vgl. Wellenkamp, Aufsichtsrat, 2000, S. 184f.

519 Vgl. Mutter, Aktiengesellschaft, 1994, S. 153.

520 Vgl. Krüger, Organisation, 1994, S. 18f.

521 Vgl. Servatius, Vorstandskontrolle, 1995, S. 225.

522 Vgl. Berliner Initiativkreis German Code of Corporate Governance, Code, 2000, S. 65, IV.2.2.; Peemöller, Aufsichtsrat, 1995, S. 184, spricht hierbei allgemeiner von Organisationsgrundsätzen.
} 


\section{(2) Vorstandsorganisation}

Der sachgerechten Organisation des Vorstands kommt vor dem Hintergrund sich stark wandelnder wirtschaftlicher Rahmenbedingungen eine herausragende Bedeutung $\mathrm{zu}^{523}$ Vor allem die Globalisierung der Wirtschaft stellt hohe Anforderungen an eine zweckentsprechende organisatorische Gestaltung, die in der Unternehmungspraxis zu sehr unterschiedlichen Ausprägungen geführt hat. ${ }^{524} \mathrm{Ne}$ ben der Notwendigkeit einer unternehmungsindividuellen Ausgestaltung sollte der Aufsichtsrat bei der organisatorischen Gestaltung des Vorstands berücksichtigen, da $ß$ er aufgrund der gravierenden Auswirkungen der aufbauorganisatorischen Strukturierung des Vorstands auf die Gesamtstruktur der Unternehmung über einen umfangreichen Gestaltungseinfluß verfügt. ${ }^{525}$

Wie bei jeder organisatorischen Gestaltung hat sich auch die des Vorstands an seinen Aufgaben zu orientieren. ${ }^{526}$ Die analytisch separierbaren Aufgabenbündel werden zu verschiedenen Stellen zusammengefügt, die die Grundlage für die einzelnen Stellenbeschreibungen bilden. ${ }^{527}$ Die Stellenbildung als abstrakte Aufgabenbeschreibung unterscheidet sich von der Stellenbesetzung als Zuweisung konkreter Personen auf einzelne Ressorts. ${ }^{528}$ Dabei hat der Aufsichtsrat zu berücksichtigen, daß Stellenbildung und Stellenbesetzung in den oberen Rängen der unternehmerischen Hierarchie nicht unabhängig voneinander erfolgen können. ${ }^{529}$ Die Aufgabenbündel werden insbesondere für den Vorstand zumindest ansatzweise auf die vorhandenen bzw. potentiellen Aufgabenträger zugeschnitten, so daß eine Stellenbildung ad personam stattfindet und hierdurch das Prinzip der Personenunabhängigkeit der Stellenbildung aufgehoben wird. ${ }^{530}$ Trotz dieser grundsätzlichen Verbindung werden hier zunächst die Organisationspla-

523 Vgl. Endres, Organisation, 1999, S. 442f.; mit der Beschreibung eines Disputs über die „richtige“ Vorstandsorganisation bei ThyssenKrupp s. Bialdiga, Kompromiss, 2000, o.S.

524 Vgl. zu entsprechenden Beispielen Endres, Organisation, 1999, S. 445-448.

$525 \mathrm{Vgl}$. Bleicher, Organisationspolitik, 1980, S. 104ff.

526 Vgl. Frese, Organisation, 2000, S. 556.

527 Mit dem Beispiel einer Stellenbeschreibung für die Stelle eines Finanzvorstands s. Chini, Aufsichtsrats-Informationssysteme, 1988, S. 95-99; zum Analyse-Synthese-Konzept beispielsweise Schulte-Zurhausen, Organisation, 1999, S. 39-47, sowie grundlegend Kosiol, Organisation, 1976, S. 32f., $42 \mathrm{ff}$.

528 Vgl. Hoffmann-Becking, Vorstand, 1998, S. 499f., der sogar die Stellenbesetzung in der Geschäftsordnung regeln möchte.

529 Vgl. Krüger, Organisation, 1994, S. 47.

530 Vgl. hierzu Schreyögg, Organisation, 1999, S. 124; zu weiteren Stellenbildungsprinzipien Schulte-Zurhausen, Organisation, 1999, S. 144ff. 
nung und die personelle Planung getrennt vorgestellt. Ihre Integration erfolgt bei der späteren Betrachtung des Planungsprozesses.

Die mit der Vorstandsressortierung erfolgende horizontale Arbeitsteilung kann zu einer Aufgabenspezialisierung nach Funktionen, Objekten oder nach beiden Prinzipien gleichzeitig führen. ${ }^{531}$ Hiermit wird die Unternehmungsorganisation vorbestimmt, ${ }^{532}$ wobei jedoch nicht unberücksichtigt bleiben darf, daß eine Veränderung der Gesamtstruktur der Unternehmung nicht friktionsfrei möglich scheint, so daß Rückkopplungen einer bestehenden Unternehmungsstruktur auf die Vorstandsorganisation bestehen. ${ }^{533}$ Der Aufsichtsrat kann diese strategische Organisationsplanung daher nur in enger Abstimmung mit der Festlegung der Unternehmungsaufgabe sowie der Strategischen Planung durchführen.

Neben der horizontalen Strukturierung der arbeitsteiligen Aufgabenerfüllung des Vorstands muß gerade der Aufsichtsrat von Publikumsaktiengesellschaften eine vertikale Strukturierung des Vorstands vornehmen, da hierdurch Spezialisierungseffekte genutzt werden können. Dies beinhaltet die Gestaltung der Entscheidungsbefugnisse innerhalb des Gremiums und hiermit verbunden der Weisungsbeziehung zur unmittelbar nachgelagerten zweiten Hierarchieebene. ${ }^{534}$

In einem unressortierten Vorstand werden alle Entscheidungen gemeinsam gefällt, so daß Anweisungen an nachgelagerte Hierarchieebenen dem gesamten Gremium vorbehalten sind (Gesamtgeschäftsführung). ${ }^{535}$ KRÜGER spricht hier vom Teamprinzip. Eine Entscheidungsvorbereitung durch einzelne Vorstandsmitglieder in der Rolle ähnlich der eines Stabes ist nicht ausgeschlossen. ${ }^{536}$ Sie fungieren als Ansprechpartner der nachgelagerten Bereiche, nicht jedoch als Vorgesetzte. Diese Rolle hat das gesamte Gremium, weshalb im Verhältnis zu den nachgelagerten Bereichen eine Gremienanbindung besteht.

Kommt es dagegen zu einer Ressortierung des Vorstands, werden Aufgaben, die nicht unter die organschaftlichen Mindestkompetenzen des Vorstands fallen, zumeist durch das für ein Ressort zuständige Vorstandsmitglied (Einzelge-

\footnotetext{
531 Vgl. Krüger, Organisation, 1994, S. 258.

532 Vgl. Siebel, Selbstorganisation, 1999, S. 112.

533 Vgl. zur Verbindung der Führungsorganisation mit der Organisationsstruktur der Unternehmung Hahn, Führungskräfteplanung, 1999, S. 621-624.

534 Vgl. Chmielewicz, Unternehmungsleitung, 1992, Sp. 2477ff.; Krüger, Organisation, 1994, S. 253f., 260.

535 Vgl. Ludwig, Aufsichtsräte, 1997, S. 66

536 Vgl. Krüger, Organisation, 1994, S. 253; Frese, Unternehmungsführung, 1987, S. 337f., bezeichnet dies als Sprechermodell.
} 
schäftsführung) oder einen mit besonders qualifizierten Vorstandsmitgliedern besetzten Ausschuß (unechte Gesamtgeschäftsführung) entschieden. ${ }^{537}$ Hier findet das Hierarchieprinzip Anwendung, bei dem die Ressortleiter im Vorstand Vorgesetztenfunktion gegenüber den nachgelagerten Bereichen übernehmen (Individualanbindung). ${ }^{538}$ Sie sind in Personalunion Teilbereichsleiter und Vorstandsmitglied. ${ }^{539}$ In diesem Zusammenhang hat der Aufsichtsrat außerdem darüber zu entscheiden, ob die Anbindung als Einlinien- oder als Mehrliniensystem erfolgt. Im nachfolgenden Beispiel der Abb. 11 liegt ein Einliniensystem vor.

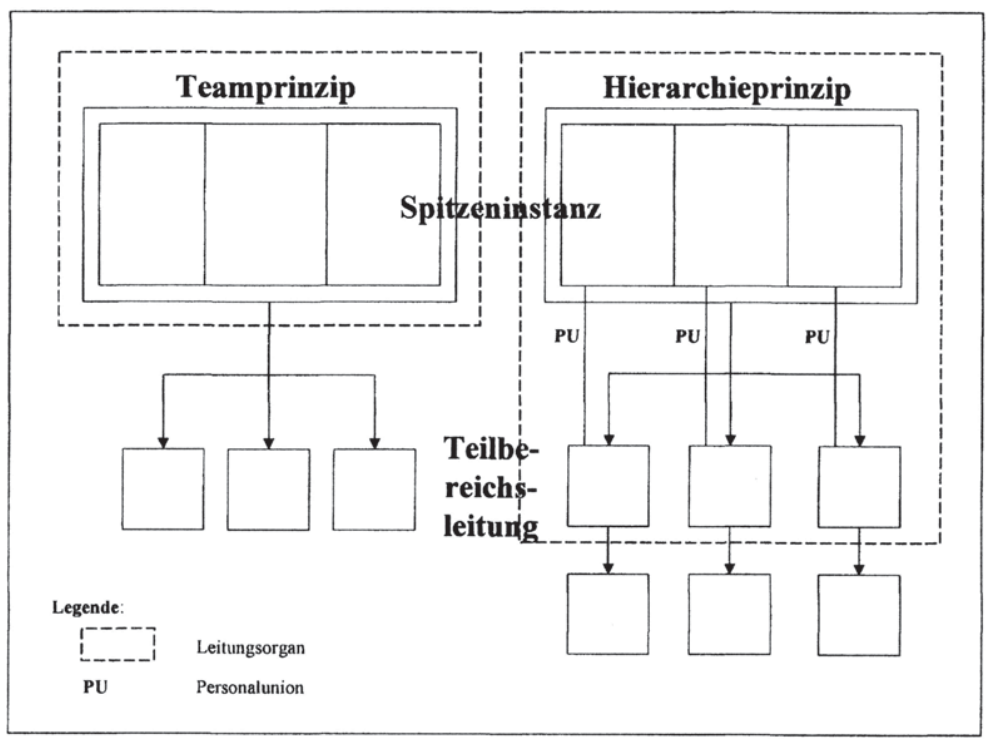

Abb. 11: Vertikale Arbeitsteilung im Leitungsorgan

Quelle: In Anlehnung an Krüger, Organisation, 1994, S. 254.

Die Bewertung der Zweckmäßigkeit einer Ressortierung des Vorstands kann nur vor dem Hintergrund der spezifischen Unternehmungssituation erfolgen. ${ }^{540}$ Hierbei hat der Aufsichtsrat zu berücksichtigen, daß dem Vorteil der eine unternehmerische Perspektive fördernden Gesamtverantwortung einer unressortierten

537 Vgl. Raiser, Kapitalgesellschaften, 1992, S. 92; Hoffmann-Becking, Vorstand, 1998, S. 508f.

538 Vgl. Krüger, Organisation, 1994, S. 254; Frese, Unternehmungsführung, 1987, S. 337f., bezeichnet dies als Modell der Personalunion.

539 Vgl. Bleicher/Leberl/Paul, Unternehmungsverfassung, 1989, S. 99; Krüger, Organisation, 1994, S. 259.

540 Vgl. zum Versuch der Ermittlung einer relativen Vorteilhaftigkeit Frese, Organisation, 2000, S. 565ff.; der Berliner Initiativkreis German Code of Corporate Governance, Code, 2000, S. 57f., III.3.5., spricht sich grundsätzlich für eine Ressortierung aus. 
Gesamtgeschäftsführung verschiedene Nachteile gegenüberstehen. ${ }^{541} \mathrm{Zu}$ diesen zählen unklare personale Zuständigkeiten gegenüber den Teilbereichsleitungen und fehlende Verantwortungsübernahme für Teilprobleme durch einzelne Vorstandsmitglieder. Für eine Ressortierung sprechen dagegen die größere Transparenz, die eindeutige Strukturierung und hieraus folgend klare Verantwortung der einzelnen Vorstandsmitglieder sowie die Möglichkeit einer professionalisierten Ressortführung aufgrund der Aufgabenspezialisierung. Dem steht jedoch das Problem widerstreitender Ressortinteressen gegenüber.

Zur Strukturierung des Vorstands gehört auch die Ernennung eines Vorstandsvorsitzenden. Der BERLINER INITIATIVKREIS GERMAN CODE OF CORPORATE GOVERNANCE weist zu Recht darauf hin, daß es sich hierbei um ein gleichberechtigtes Vorstandsmitglied ohne Weisungsbefugnis handelt. Daher hat der Aufsichtsrat bei der Ausgestaltung der Vorstandsgeschäftsordnung darauf hinzuwirken, daß die heute moderne Umbenennung des Vorsitzenden bzw. Sprechers in den Chief Executive Officer (CEO) des amerikanischen Direktorialprinzips nicht im Ergebnis zu einer entsprechenden Aufgabenübernahme führt. $^{542}$

Abschließend hat der Aufsichtsrat im Rahmen seiner aufbauorganisatorischen Gestaltung des Vorstands die Anzahl der Vorstandsmitglieder festzulegen. ${ }^{543}$ Diese ist abhängig von der Komplexität der Gesamtaufgabe. ${ }^{544}$ Bei seiner Planung sollte der Aufsichtsrat die Gesamtgröße der Unternehmung, die unternehmungsinterne- und -externe Dynamik, aber auch die Kosten des Vorstands berücksichtigen. FRESE geht unabhängig von den aktienrechtlichen Vorschriften aufgrund der in großen Unternehmungen herrschenden Aufgabenkomplexität immer von einer multipersonalen Aufgabenerfüllung aus. ${ }^{545}$

Neben der Aufbauorganisation sollte der Aufsichtsrat im Rahmen der ablauforganisatorischen Regelung insbesondere die Entscheidungs- und Beschlußver-

541 Vgl. Krüger, Organisation, 1994, S. $258 \mathrm{f}$.

542 Vgl. Berliner Initiativkreis German Code of Corporate Governance, Code, 2000, S. 57, III.3.3.; Bernhardt, Anforderungen, 2000, S. 59; ein Sprecher ist in der hier vorgeschlagenen Aufgabenverteilung nicht möglich, da der Aufsichtsrat die Strukturierung vornimmt, der einen Vorsitzenden des Vorstands ernennt.

Vgl. Krüger, Organisation, 1994, S. 258; Ludwig, Aufsichtsräte, 1997, S. 60.

Der Berliner Initiativkreis German Code of Corporate Governance, Code, 2000, S. 57, III.3.1., schlägt im Normalfall drei bis neun Mitglieder vor.

Vgl. Frese, Organisation, 2000, S. 556. 
fahren des Vorstands festlegen. ${ }^{546}$ Dabei muß die Art der Beschlußfassung des Vorstandsorgans seine Entscheidungsfähigkeit gewährleisten. ${ }^{547}$ Hierzu können abweichend vom Einstimmigkeitsprinzip problemadäquate Mehrheitsregeln vorgegeben werden (Abstimmungskollegialität). ${ }^{548}$ Daneben sind insbesondere in international organisierten Vorständen organisatorische Hilfsmittel wie beispielsweise die Beschlußfassung in Videokonferenzen vorzusehen. ${ }^{549}$

\section{(3) Zusammenarbeit von Vorstand und Aufsichtsrat}

Im Rahmen seiner Geschäftsordnungskompetenz für den Vorstand kann der Aufsichtsrat nicht nur dessen organinterne Organisation regeln, darüber hinaus sollte die Geschäftsordnung auch Regelungen zur Zusammenarbeit von Vorstand und Aufsichtsrat enthalten. Dies beinhaltet vor allem die Ausgestaltung der Berichtspflichten. Sie beziehen sich primär auf die Regelberichterstattung des Vorstands, die über die Berichtserfordernisse des $§ 90$ Abs. 2 AktG hinaus eine kontinuierliche und an die jeweilige Unternehmungssituation angepaßte stetige Informationsversorgung des Aufsichtsrats sicherstellen sollte. FEDDERSEN hält eine eigene Informationsordnung für zu bürokratisch, so daß er diese Regelungen zum Bestandteil der Geschäftsordnung des Vorstands machen möchte. ${ }^{550}$ Da der Erlaß einer eigenständigen Informationsordnung bzw. alternativ die Aufnahme entsprechender Regelungen in die Geschäftsordnung für die Planungstätigkeit des Aufsichtsrats keinen materiellen Unterschied ausmachen, kann die Wahl der Ausgestaltung dem jeweiligen Gremium überlassen bleiben. Sowohl die Geschäftsordnung als auch eine eventuelle Informationsordnung dürfen sich jedoch nicht auf die Auflistung der Regelberichte beschränken, sie müssen darüber hinaus auch die aktiven Informationsbeschaffungsmöglichkeiten und -notwendigkeiten des Aufsichtsrats situationsadäquat beschreiben.

Neben den aus der Geschäftsordnungskompetenz abgeleiteten organisatorischen Planungsobjekten des Aufsichtsrats kommen als weiterer Bereich die personellen Planungsobjekte hinzu. Sie beinhalten sowohl die Besetzung des Vorstands als auch die Gestaltung seines Anreizsystems.

\footnotetext{
546 Vgl. zu entsprechenden Vorschlägen Berliner Initiativkreis German Code of Corporate Governance, Code, 2000, S. 59, III.4; deren besondere Bedeutung betont auch Mutter, Aktiengesellschaft, 1994, S. 66.

547 Vgl. Krüger, Organisation, 1994, S. 255.

$548 \mathrm{Vgl}$. zu verschiedenen Abstimmungsregeln Laux/Liermann, Organisation, 1997, S. 88ff.

$549 \mathrm{Vgl}$. Endres, Organisation, 1999, S. 447.

550 Vgl. Feddersen, Aufsichtsrat, 2000, S. 388.
} 


\section{b) Personelle Planungsobjekte}

\section{(1) Besetzung des Vorstands}

In enger Verbindung zur Organisationsplanung mit der Festlegung der Führungsorganisation der Aktiengesellschaft ist der Aufsichtsrat ebenfalls für die Besetzung des Vorstands zuständig. Da er das Überwachungsobjekt „Unternehmung“ immer weniger selbst überblicken kann, ergänzt die personelle Dimension der Überwachung zunehmend die sachliche. ${ }^{551}$ Dies bedeutet, daß der Aufsichtsrat über die Auswahl der Vorstandsmitglieder und die mit diesen Personen verbundenen strategischen Grundrichtungen die Erreichung der Unternehmungsziele fördern muß. ${ }^{552}$

Es können zwei unterschiedliche Bestellungsanlässe unterschieden werden: Die erstmalige Bestellung eines Vorstandsmitglieds und die Wiederbestellung im Rahmen der Verlängerung der Amtszeit. OESTERLE spricht in diesem Zusammenhang von einem ,strategischen Management von Führungswechseln“ ${ }^{.553}$ Dieses beinhaltet als zusätzliche dritte Komponente eine situationsinduzierte Abberufung von Vorstandsmitgliedern, so daß insgesamt von der Besetzung des Vorstands gesprochen werden kann.

Mit Blick auf die Praxis stellt SCHEFFLER jedoch fest, daß der Aufsichtsrat sein „Bestellobligo“ hinsichtlich Berufung, Wiederberufung und Abberufung nur unzureichend erfülle. ${ }^{554}$ Während die Wiederbestellung eines Vorstandsmitglieds als Selbstverständnis angesehen werde, suche bei Neubesetzungen der amtierende Vorstandsvorsitzende einen Kandidaten aus und bespricht dies mit dem Aufsichtsratsvorsitzenden, so daß der Aufsichtsrat abschließend nur noch über diese Vorauswahl beschließt. Hierdurch besteht die Gefahr, daß sich ein starker Vorstand bzw. ein starker Vorstandsvorsitzender weitere Vorstandsmitglieder qua Kooptation selbst auswählt. ${ }^{555}$ Durch die Vernachlässigung seiner Personalkompetenz wird der Aufsichtsrat weitgehend entmachtet und ist nur noch eingeschränkt in der Lage, seine Überwachungspflichten ordnungsgemäß zu erfüllen.

551 Vgl. Bleicher/Leberl/Paul, Unternehmungsverfassung, 1989, S. 61f.; zustimmend m.w.N. Gollnick, Beurteilung, 1997, S. 66.

552 Die Bedeutung einer zweckentsprechenden Besetzung des Vorstandsorgans verdeutlicht die Krise der Philipp Holzmann AG, die durch falsche personale Auswahlentscheidungen des Aufsichtsrats ausgelöst worden sei; vgl. Gaertringen/Wildhagen, überfordert, 1999, S. $76 f$.

Vgl. Oesterle, Führungswechsel, 1999, S. $297 \mathrm{ff}$.

554

Vgl. Scheffler, Rollenverständnis, 2000, S. 434; o.V., Ära, 1998, S. 14, mit der Feststellung, daß diese Aufgabe bei der Deutschen Bank AG endlich vom Aufsichtsrat angegangen werden solle.

Vgl. Krüger, Organisation, 1994, S. 257; Potthoff/Trescher, Aufsichtsratsmitglied, 1999, S. 252; von Werder, Grundsätze, 1999, S. 2223. 
Dies gilt insbesondere in den Fällen, in denen die im Rahmen der Besetzung des Vorstands durch die Aufsichtsratsmitglieder zu treffenden Entscheidungen zu einem formalen Akt denaturieren.

Aufgrund der Bedeutung der Personalentscheidungen ist daher zu fordern, daß der Aufsichtsrat nicht nur formell die Entscheidung fällt, sondern ebenso wie im Rahmen der organisatorischen Gestaltung diese personellen Entscheidungen als Ausdruck seiner Verpflichtung zur Urteilsbildung über die Eignung von Vorstandsmitgliedern planerisch vorbereitet. ${ }^{556}$ Als Teilmenge der Führungskräfte wird der Vorstand damit zum Objekt einer Führungskräfteplanung des Aufsichtsrats. Allgemein beinhaltet die Führungskräfteplanung die Beschaffung, den Einsatz, die Entwicklung und gegebenenfalls die Freisetzung von Führungskräften. ${ }^{557}$ Die Entwicklungsplanung kann weiter in die Bildungs- und die Förderungsplanung unterteilt werden, die selbst wiederum aus der unternehmungsbezogenen Nachfolgeplanung sowie der mitarbeiterbezogenen Karriereplanung besteht. ${ }^{558}$ Diesen Maßnahmenplanungen sind die Zielplanung und die Bedarfsplanung vorgeschaltet. ${ }^{559}$

Da es hier um die Abgrenzung relevanter Planungsobjekte des Aufsichtsrats mit Bezug auf die Besetzung des Vorstands geht, sind zwei Fragen zu beantworten: Es ist erstens danach zu fragen, welche Teile der Führungskräfteplanung durch den Aufsichtsrat erfolgen müssen; zweitens muß geklärt werden, welche Führungsebenen als Nachwuchsreservoir von dieser Planung erfaßt werden.

Zur Beantwortung der ersten Frage kann zunächst auf einen Vorschlag des BERLINER INITIATIVKREISES GERMAN CODE OF CORPORATE GOVERNANCE verwiesen werden, der dem Aufsichtsrat lediglich die Besetzungsentscheidung zuweist. ${ }^{560}$ Für eine Nachfolgeplanung soll allein der Vorstand zuständig sein, der in dem zur Regel erklärten Fall einer Besetzung aus den eigenen Reihen dem Aufsichtsrat geeignete Kandidaten vorzuschlagen habe. Doch selbst dann, wenn der Aufsichtsrat eine eigenständige Beurteilung der potentiellen und gegenwärtigen

556 Den eher situativen und nicht planerisch vorbereiteten Charakter der Besetzungsentscheidung stellten bereits Bleicher/Leberl, Aufsichtsrat, 1987, S. 61, auf der Grundlage einer empirischen Untersuchung fest. Zur Begründung der Notwendigkeit einer Planung im personalen Bereich Mag, Personalplanung, 1998, S. 1f.

557 Vgl. Hahn, Planung, 1994, S. 341f.; Hahn, Führungskräfteplanung, 1999, S. 617; weiter dagegen Mag, Personalplanung, 1998, S. 80ff., wobei hier jedoch die Anreizsystemplanung separat diskutiert wird.

Vgl. Mag, Personalplanung, 1998, S. 146; mit Beschränkung auf die Nachfolge- und die Karriereplanung auch Hahn, Planung, 1994, S. 341.

559 Vgl. Mag, Personalplanung, 1998, S. 57ff., 63ff.

560 Vgl. Berliner Initiativkreis German Code of Corporate Governance, Code, 2000, S. 43, II.1.4. 
Vorstandsmitglieder durchführen sollte, besteht weiterhin die oben beschriebene Kooptationsgefahr, d.h. eine Auswahl der Mitglieder des Leitungsorgans durch das Leitungsorgan selbst, da der Aufsichtsrat aufgrund der Vorselektion durch den Vorstand und der hiermit verbundenen Reduktion der Handlungsalternativen in seiner Entscheidungsautonomie zu sehr eingegrenzt wird. ${ }^{561}$

Dagegen faßt die GRUNDSATZKOMMISSION CORPORATE GOVERNANCE den Kreis der Aufsichtsratszuständigkeiten weiter, indem sie dem Aufsichtsrat zusätzlich zu der Besetzungsentscheidung gemeinsam mit dem Vorstand die Nachfolgeplanung zuordnet. ${ }^{562}$ Noch weiter geht SCHEFFLER, der für diese Aufgabe allein den Aufsichtsrat zuständig sieht. ${ }^{563}$ Doch auch dies ist noch nicht hinreichend, so daß hier vorgeschlagen wird, dem Aufsichtsrat zur Vorbereitung seiner Besetzungsentscheidung nicht nur die Nachfolgeplanung zu übertragen, sondern darüber hinaus auch die Karriereplanung sowie die Bildungsplanung, d.h. die gesamte Entwicklungsplanung. ${ }^{564}$ Dies kann damit begründet werden, daß zwischen Förderung und Bildung ein enger Zusammenhang besteht, da für die Übernahme höherrangiger Aufgabenbereiche eine entsprechende Qualifikation vorhanden sein muß bzw. nach einer Beförderung noch nicht vorhandene Qualifikationen erworben werden müssen. ${ }^{565}$ Diese Entwicklungsplanung ist um die Beschaffungsplanung sowie die Freisetzungsplanung zu ergänzen, wobei diesen Teilplanungen insgesamt eine Ziel- und Bedarfsplanung vorangehen sollte.

Zur Beantwortung der zweiten Frage schlägt SCHEFFLER vor, daß der Aufsichtsrat bei seiner Nachfolgeplanung alle Führungskräfte der zweiten Ebene berücksichtigen soll. ${ }^{566}$ Dem ist mit Bezug auf die vorgeschlagene Entwicklungsplanung zuzustimmen, da eine darüber hinausgehende Erweiterung in den hier betrachteten Publikumsaktiengesellschaften für den Aufsichtsrat nicht mehr praktikabel scheint. In allen weiteren relevanten Führungskräfteteilplanungen wird als Objekt nur der Vorstand einbezogen.

561 Vgl. Hahn, Entwicklungstendenzen, 1999, S. 20, unter Verweis auf Cassier, Großunternehmer, 1962, S. 130f.

562 Vgl. Grundsatzkommission Corporate Governance, Corporate Governance-Grundsätze, 2000, S. 240; ebenso Römer, Aufsichtsrat, 1990, S. 130; so auch die Corporate Governance-Grundsätze der Deutschen Bank; vgl. Deutsche Bank AG, Corporate Governance-Grundsätze, 2001, S. 6.

563 Vgl. Scheffler, Rollenverständnis, 2000, S. 434.

564 Ähnlich auch Servatius, Vorstandskontrolle, 1995, S. 225.

565 Zum Zusammenhang von Förderungsplanung und Bildungsplanung als Bestandteile der Entwicklungsplanung allgemein Mag, Personalplanung, 1998, S. 155f.

566

Vgl. Scheffler, Rollenverständnis, 2000, S. 435. 


\section{(2) Gestaltung des Anreizsystems}

Unter Anreizen werden situative Gegebenheiten verstanden, die als Stimuli das Verhalten von Individuen in bestimmter Weise beeinflussen ${ }^{567}$ Die Gesamtheit aller bewußt in Form von materiellen und immateriellen Stimuli gestalteten Arbeitsbedingungen kann als Anreizsystem bezeichnet werden. ${ }^{568}$ Neben den unternehmungsexternen Anreizen, wie beispielsweise geographische oder soziokulturelle Faktoren, zählen zu den unternehmungsinternen Anreizen sowohl materielle als auch immaterielle. ${ }^{569}$ Die materiellen Anreize können weiter in finanzielle und nicht-finanzielle unterschieden werden (vgl. mit einer exemplarischen Auflistung Abb. 12).

\begin{tabular}{|c|c|c|}
\hline materie & e Anreize & immateriolle A nroizz \\
\hline finanzielle & nicht-finanzielle & 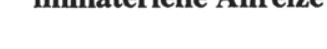 \\
\hline $\begin{array}{ll}\text { - } & \text { fixe Grundvergütung } \\
\text { - } & \text { kurzfristige variable } \\
\text { Vergütung } \\
\text { - } & \text { langfristige } \\
\text { wertorientierte } \\
\text { Vergütung }\end{array}$ & 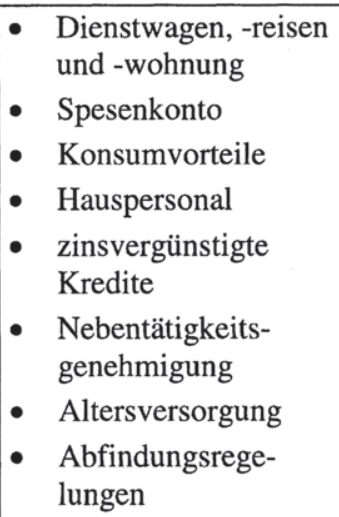 & $\begin{array}{ll}\text { - } & \text { Arbeitsinhalt } \\
\text { - } & \text { Aufgabenfeld und } \\
\text { Entwicklungsmög- } \\
\text { lichkeiten } \\
\text { - unternehmerische } \\
\text { Entfaltungsmöglich- } \\
\text { keiten } \\
\text { - Verantwortung } \\
\text { - Image der } \\
\text { - Unternehmung } \\
\text { Beziehungen zu } \\
\text { Mitarbeitern }\end{array}$ \\
\hline
\end{tabular}

Abb. 12: Klassifikation von Anreizen

Quelle: In Anlehnung an Welge/Hüttemann/Al-Laham, Anreizsystemgestaltung, 1996, S. 82 .

Es ist danach zu fragen, über welche Gestaltungsparameter der Aufsichtsrat Einfluß auf das Anreizsystem des Vorstands nimmt bzw. nehmen sollte.

\footnotetext{
567 Vgl. Becker, Anreizsysteme, 1995, Sp. 36; Hahn, Planung, 1994, S. 343; Hungenberg, Anreizsysteme, 1999, S. 720ff.; mit einer entsprechenden Unterscheidung in monetäre und nichtmonetäre Anreize Mag, Personalplanung, 1998, S. $122 \mathrm{f}$.

568 Vgl. Wild, Organisation, 1973, S. 47.

569 Vgl. Becker, Anreizsysteme, 1995, Sp. 40f.; Hahn, Planung, 1994, S. 343.
} 
Der Aufsichtsrat ist nach $\S \S 84$ Abs. 1 S. 5, 112 AktG für den Abschluß der Anstellungsverträge der Vorstandsmitglieder verantwortlich. ${ }^{570}$ Sie beinhalten u.a. eine Festsetzung der Gesamtbezüge der einzelnen Vorstandsmitglieder, zu denen $\S 87$ Abs. 1 AktG sein Gehalt, Gewinnbeteiligungen, Aufwandsentschädigungen, Versicherungsentgelte, Provisionen und Nebenleistungen jeder Art zählt. Diese Auflistung ist nicht abschließend zu verstehen, so daß hierzu alle finanziellen und nicht-finanziellen materiellen Anreize gezählt werden können. ${ }^{571} \mathrm{Zu}$ den nicht-finanziellen Anreizen gehören auch die Genehmigung der Nebentätigkeiten von Vorstandsmitgliedern sowie die Kreditvergabe an Vorstandsmitglieder ( $\S 88,89$ AktG). Doch der Aufsichtsrat ist nicht nur in der Lage, das materielle Anreizsystem des Vorstands zu gestalten, über die Ausgestaltung der Führungsorganisation kann der Aufsichtsrat zusätzlich positive immaterielle Anreizwirkungen erzielen, indem er beispielsweise den einzelnen Vorstandsmitgliedern unternehmerischen Handlungsspielraum und Entscheidungsmöglichkeiten einräumt. Dies beinhaltet eine offene Diskussionskultur, die es allen Beteiligten ermöglicht, ihren Leistungsbeitrag zu erbringen. ${ }^{572}$

Besonderes Augenmerk sollte der Aufsichtsrat auf die Gestaltung der finanziellen Anreize der Vorstandsmitglieder richten, da durch die in Publikumsaktiengesellschaften erfolgte Trennung von Eigentum und Leitung eine besondere Notwendigkeit besteht, das Handeln des Vorstands an den Interessen der Aktionäre auszurichten.$^{573}$ In Abschnitt II.C.2.a) wurde gezeigt, daß der Shareholder Value-Ansatz mit dem Marktwert des Eigenkapitals eine sowohl an den Interessen der Eigentümer als auch der übrigen Stakeholder ausgerichtete Zielgröße liefert. Das Anreizsystem des Vorstands sollte daher ebenfalls am Shareholder Value orientiert werden. ${ }^{574}$ Dies ist über langfristige aktienwertbasierte Vergütungssysteme möglich, die über die unternehmungsindividuelle Ausgestaltung die Vergütung des Vorstands an der Wertentwicklung der Unternehmung aus-

570 Vgl. Nagel, Gesellschaftsrecht, 2000, S. 121; der Berliner Initiativkreis German Code of Corporate Governance, Code, 2000, S. 43, II.1.2., rechnet dies mit zum Kernprozeß der personellen Besetzung des Vorstands.

571 Vgl. Mertens, Kölner Kommentar, 1996, Anm. 8 zu § 87; Knoll, Entlohnung, 1997, S. 22.

572 Vgl. Berliner Initiativkreis German Code of Corporate Governance, Code, 2000, S. 53, I.4.

573 Vgl. Becker, Anreizsysteme, 1997, S. 112; zur Funktion ergebnisabhängiger Vorstandsbezüge als Eigentumssurrogat aus Sicht der Principal Agent-Theorie Picot/Dietl/Franck, Organisation, 1997, S. 196; kritisch zum Stand der theoretischen und empirischen Forschung Backes-Gellner/Geil, Managervergütung, 1997, S. 468-475.

574 Vgl. Grundsatzkommission Corporate Governance, Corporate Governance-Grundsätze, 2000, S. 239; Schätzle, Unternehmenswert, 1998, S. 50. 
richten. ${ }^{575}$ Hierdurch erfolgt nicht nur ein Abbau der Principal AgentProblematik, darüber hinaus wird dies vom Kapitalmarkt als positives Signal aufgefaßt, daß die Unternehmungspolitik an der Steigerung des Shareholder Value ausgerichtet wird. ${ }^{576}$

Ausgelöst durch Forderungen des Kapitalmarkts hat auch der Gesetzgeber die Notwendigkeit einer wertorientierten Vergütung von Vorständen erkannt und mit dem KonTraG die Einführung von Stock Option-Programmen für Aktiengesellschaften vereinfacht ( $\$ 192$ Abs. 2 Nr. 3 AktG) ${ }^{577}$ An die Ausgestaltung der Optionsprogramme sind jedoch differenzierte Anforderungen zu stellen, da ansonsten ihr grundsätzlicher Wert zur Ausrichtung des Vorstandshandelns an den Aktionärsinteressen in Frage zu stellen ist. ${ }^{578}$ Als wichtigste Forderung zählt hierzu die konkrete Ausgestaltung des Optionsprogramms durch den Aufsichtsrat bzw. einen hierzu ermächtigten Ausschuß und nicht durch den Vorstand. Daneben sollte der Aufsichtsrat in Publikumsaktiengesellschaften den Teilnehmerkreis am Optionsprogramm auf die obersten Führungskräfte beschränken sowie eine Sperrfrist und außerdem Ausübungshürden vorsehen. ${ }^{579}$

Die mit der Einführung von Stock Options verbundene Variabilisierung und Erhöhung der Vergütung von Vorstandsmitgliedern bedeutet eine Abkehr von den bisherigen Vergütungsusancen. ${ }^{580}$ Insbesondere die potentielle Höhe der Vorstandsvergütung, aber auch die schwierige Ausgestaltung der Optionsprogramme machen daher eine detaillierte Planung erforderlich.

\section{c) Die Berücksichtigung von Grundsätzen ordnungsmäßiger Unterneh- mungsleitung durch den Aufsichtsrat}

Die innerhalb der vorstandsbezogenen Planungsobjekte angesprochenen Problembereiche sind neben weiteren Gegenstand von in der Literatur entwickelten

$575 \mathrm{Zu}$ wertorientierten Anreizsystemen beispielsweise Becker, Anreizsysteme, 1997, S. 112-119; Evers, Wertorientierung, 1998, S. 53-67; Pellens/Crasselt/Rockholtz, Entlohnungssysteme, 1998, S. 1-28.

576 Vgl. Langner/Bursee, Aktienoptionsprogramme, 2000, S. 531.

57 Vgl. Seibert, Stock Options, 1998, S. 29-52; kritisch Nassauer, Internationalisierung, 2000, S. 252.

Vgl. Knoll, Entlohnung, 1997, S. 24; zur Ausgestaltung von Optionsprogrammen beispielsweise Bernhardt/Witt, Stock Options, 1997, S. 85-101.

579 Dies wird durch eine Befragung institutioneller Kapitalmarktteilnehmer durch Langner/Bursee, Aktienoptionsprogramme, 2000, S. 531ff., gestützt, wobei die Forderungen zum Teil durch den Gesetzgeber bereits umgesetzt wurden. nehmer Langner/Bursee, Aktienoptionsprogramme, 2000, S. 530-534; mit einem Überblick über aktuelle Vergütungstrends Abel/Becker, Vergütung, 2000, S. 388-392. 
Grundsätzen ordnungsmäßiger Unternehmungsleitung (GoU), die zu den Grundsätzen ordnungsmäßiger Unternehmungsführung zählen. ${ }^{581}$ Es ist daher zu überlegen, inwieweit GoU im Rahmen der Aufsichtsratstätigkeit Berücksichtigung finden sollten. ${ }^{582}$

Bei den GoU handelt es sich um allgemein anerkannte Leitlinien für die Aktivitäten des Vorstands, mit denen eine systematische und umfassende Ausfüllung des generalklauselhaft formulierten aktienrechtlichen Auftrags zur eigenverantwortlichen Leitung der Gesellschaft ( $\$ 93$ Abs. 1 S. 1 AktG) bezweckt wird. ${ }^{583}$ Ihnen werden zwei Funktionen zugeschrieben: Zum einen sollen sie den Leitungsauftrag des Vorstands konkretisieren (Leitlinienfunktion), zum anderen erfüllen sie eine Schutzfunktion des Vorstands, da sie ihn vor überzogenen Anforderungen schützen. ${ }^{584}$ Die GoU können in Allgemeine und Besondere Grundsätze unterschieden werden (vgl. Abb. 13). ${ }^{585}$

Die für alle Vorstandsmaßnahmen gültigen Allgemeinen Grundsätze schreiben die rechtliche Zulässigkeit, die ökonomische Zweckmäßigkeit sowie die soziale und ethische Zuträglichkeit der Vorstandsmaßnahmen vor. Sie werden durch die Besonderen Grundsätze konkretisiert. Innerhalb der Systemgrundsätze beinhalten die Aufgabengrundsätze eine Aufgabenbeschreibung des Vorstands. Sie bilden die Grundlage seiner Stellenbeschreibung und nennen im wesentlichen die Kernaufgaben der Unternehmungsführung. ${ }^{586}$ Diese sind in Abhängigkeit von der organisatorischen Strukturierung des Vorstands für jede Vorstandsstelle zu konkretisieren. Sie stehen in enger Beziehung zu den Organisationsgrundsätzen, die Prinzipien für die Strukturierung des Vorstands enthalten. Mit den Kooperationsgrundsätzen wird die Zusammenarbeit des Vorstands mit dem Abschlußprüfer und dem Aufsichtsrat geregelt. $\mathrm{Zu}$ den Personalgrundsätzen

581 Vgl. grundlegend zu Grundsätzen ordnungsmäßiger Unternehmungsleitung von Werder, Management, 1995, S. 2177-2183; von Werder, Leitlinien, 1996, S. 27-73, sowie von Werder, Praxis, 1998, S. 1193-1198; die GoU zählen neben den Grundsätzen ordnungsmäßiger Überwachung und denen zur Abschlußprüfung zu den Grundsätzen ordnungsmäßiger Unternehmungsführung, vgl. hierzu von Werder, Grundsätze, 1996; kritisch zu den Grundsätzen ordnungsmäBiger Unternehmungsleitung Scherer, Managementpraxis, 2000, S. 84-98.

Mit einer entsprechenden Forderung von Werder, Grundsätze, 1999, S. 2221; ebenso Berliner Initiativkreis German Code of Corporate Governance, Code, 2000, S. 65, IV.2.3.

Vgl. von Werder, Grundsätze, 1999, S. 2221; von Werder, Management, 1995, S. 2178.

Vgl. von Werder, Leitlinien, 1996, S. 31f.; zum Spannungsfeld zwischen juristischen Sorgfaltspflichten und betriebswirtschaftlichem Sorgfaltspotential von Werder, Vorstandsentscheidungen, 1997, S. 901-922.

Vgl. zum nachfolgenden von Werder, Grundsätze, 1999, S. 2221f., sowie von Werder, Leitlinien, 1996, S. 34ff. 
zählt VON WERDER Fragen der Qualifikation und Auswahl der Vorstandsmitglieder. Sie haben zum einen klarstellenden Charakter bezüglich der erforderlichen Qualifikationen der Vorstandsmitglieder (Qualifikationsgrundsatz), zum anderen verdeutlichen sie die klare Zuweisung der Auswahl- und Bestellungsaufgabe für Mitglieder des Vorstands an den Aufsichtsrat (Auswahlgrundsatz). ${ }^{587}$

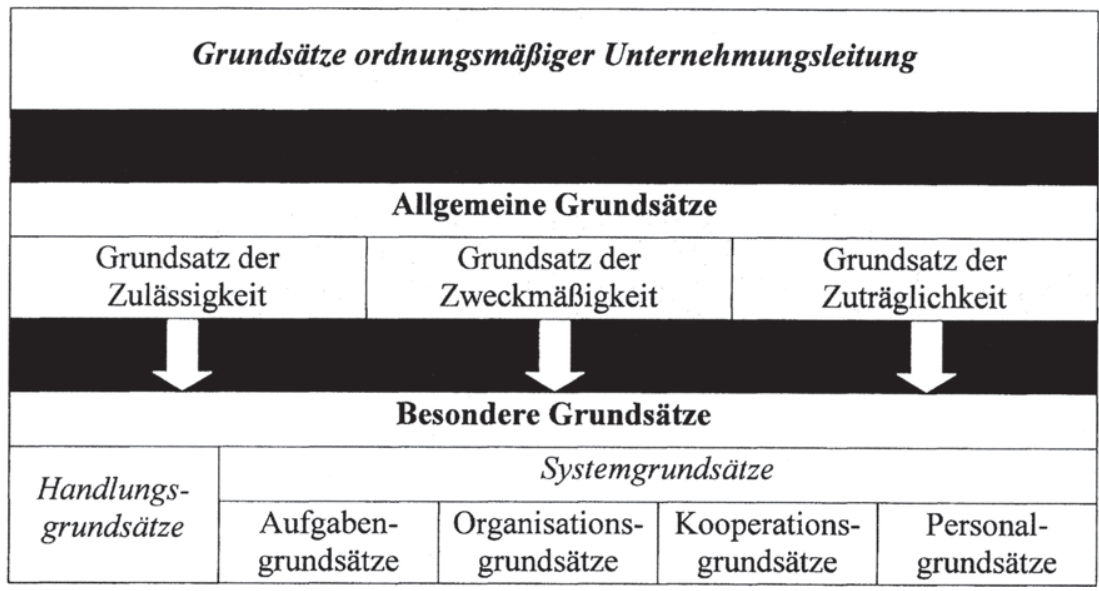

Abb. 13: Systemstruktur der GoU

Quelle: In Anlehnung an von Werder, Grundsätze, 1999, S. 2222.

In den Handlungsgrundsätzen werden Vorgaben für die Modalitäten der Aufgabenerfüllung durch den Vorstand gesetzt. Entscheidungen sollen nicht intuitiv, sondern auf der Grundlage systematischer Planungen erfolgen. ${ }^{588}$ Hierzu fordert VON WERDER, daß Vorstandsentscheidungen, die durch ein hohes Maß an Unsicherheit gekennzeichnet sind und darüber hinaus durch den Aufsichtsrat auch nur schwer beobachtet werden können, argumentationsrational sein sollen. Dies stellt sicher, daß Entscheidungen nicht intuitiv gefällt werden, sondern unter systematischer Ausnutzung möglichst umfangreicher Informationen. Außerdem werden Vorstandsentscheidungen als Kontrollobjekt des Aufsichtsrats für ihn so transparenter und nachvollziehbarer. Die verbindliche Vorgabe von Handlungsgrundsätzen erlaubt es dem Aufsichtsrat, die Arbeitsweise und Problemlösungsansätze des Vorstands so weit wie möglich vorzustrukturieren, ohne ihm die unternehmerische Handlungsfreiheit zu nehmen. Gleichzeitig zwingt er den Vorstand, seine Entscheidungsfindung dem Aufsichtsrat zu begründen. In Abhängigkeit von dem anstehenden Entscheidungsproblem kann der Aufsichtsrat dem

587 Vgl. Bernhardt/Witt, Unternehmensleitung, 1999, S. 827.

588 Vgl. von Werder, Leitlinien, 1996, S. $51 \mathrm{ff}$. 
Vorstand verschiedene Rationalitätsstufen vorschreiben (vgl. Abb. 14). ${ }^{589}$ Unrationale Problemlösungen versuchen nicht einmal, eine Begründung zu suchen. Sie sind daher grundsätzlich abzulehnen. Schwach-rationale Problemlösungen zeichnen sich dagegen dadurch aus, daß zumindest die positiven Konsequenzen einer Maßnahme angeführt werden. Eine wirkliche Begründung erfolgt jedoch erst durch mäßig- bis hoch-rationale Problemlösungen, die versuchen, für die globalen Konsequenzaussagen Begründungen zu finden und die verfügbaren Informationen zu nutzen. Voll-rationale Problemlösungen, deren Argumente das verfügbare Wissen vollständig ausschöpfen, sind dagegen in der Praxis nicht denkbar.

\begin{tabular}{|c|c|c|}
\hline Rationalitätsstufen & Rationalgehalt & Beispiel $^{590}$ \\
\hline $\begin{array}{l}\text { Unbegründete } \\
\text { Problemlösung }\end{array}$ & null (unrational) & $\begin{array}{l}\text { „Der Kauf des } \\
\text { amerikanischen } \\
\text { Automobilbauers ist } \\
\text { vorteilhaft.“ }\end{array}$ \\
\hline $\begin{array}{l}\text { Globalbegründete } \\
\text { Problemlösung }\end{array}$ & niedrig (schwach-rational) & $\begin{array}{l}\text { „Der Kauf des } \\
\text { amerikanischen } \\
\text { Automobilbauers ist } \\
\text { vorteilhaft, da hierdurch } \\
\text { Synergien realisiert } \\
\text { werden können.“ }\end{array}$ \\
\hline $\begin{array}{l}\text { Detailbegründete } \\
\text { Problemlösung }\end{array}$ & $\begin{array}{l}\text { mittel (mäßig-rational) bis } \\
\text { hoch (hoch-rational) }\end{array}$ & $\begin{array}{l}\text { „Wir können durch den } \\
\text { Kauf des amerikanischen } \\
\text { Automobilherstellers } \\
\text { Synergien realisieren, } \\
\text { weil..." } \\
\end{array}$ \\
\hline $\begin{array}{l}\text { Qualifizierte } \\
\text { Problemlösung }\end{array}$ & maximal (voll-rational) & unmöglich \\
\hline
\end{tabular}

Abb. 14: Hauptstufen der Argumentationsrationalität

Quelle: In Anlehnung an von Werder, Unternehmensleitung, 1996, S. 56.

Durch die Vorgabe von Argumentationsstufen zu Entscheidungen des Vorstands könnte der Aufsichtsrat die Qualität der Vorstandsentscheidungen nachhaltig erhöhen. Die Zuordnung erfordert jedoch eine umfassende planerische Vorbereitung, da eine Systematisierung und Priorisierung grundsätzlich denkbarer Entscheidungen ex ante erfolgen müßte. Aus pragmatischen Gründen erscheint es daher zweckmäßig, bei allen Geschäftsführungsmaßnahmen, die einem Zustim-

\footnotetext{
589 Vgl. zur Argumentationsrationalität umfassend von Werder, Argumentationsrationalität, 1994.

590 Vgl. zu einem weiteren Beispiel von Werder, Leitlinien, 1996, S. 55, FN 119-121.
} 
mungsvorbehalt des Aufsichtsrats unterliegen, eine detailbegründete Problemlösung einzufordern.

Wegen ihrer inhaltlichen Nähe zu den Regelungstatbeständen der Geschäftsordnung sollten GoU als expliziter Bestandteil in die Geschäftsordnung des Vorstands aufgenommen werden. Hierdurch entfalten sie unmittelbar rechtliche Wirksamkeit für den Vorstand, so daß ihre Verletzung gleichzeitig haftungsbegründend sein kann. Zudem wird durch den Rückgriff auf die allgemein anerkannten GoU die Planung der Regelungstatbestände der Geschäftsordnung, d.h. insbesondere die Teilplanungsobjekte der Vorstandsorganisation, systematisiert. Bei Anwendung der GoU durch den Aufsichtsrat können sie eine Qualitätssicherungsfunktion erfüllen, die die Leistungsfähigkeit sowohl des Vorstands als auch des Aufsichtsrats erhöht. ${ }^{591}$

Neben den vorstandsbezogenen Planungsobjekten, die der Aufsichtsrat mit Blick auf die organisatorischen und personellen Angelegenheiten des Vorstands zu berücksichtigen hat, existieren weitere Aufgabenfelder, die grundsätzliche Planungsobjekte des Aufsichtsrats beinhalten können. Diese sind nachfolgend $\mathrm{zu}$ analysieren.

\section{Strategische Unternehmungsführung}

\section{a) Generelle Unternehmungsziele}

Wie die Aufgabenanalyse des Aufsichtsrats gezeigt hat, nimmt nicht nur der Vorstand, sondern auch der Aufsichtsrat im Prozeß der strategischen Führung der Unternehmung wichtige Führungsaufgaben entweder alleine oder gemeinsam mit dem Vorstand wahr.

„Gute Unternehmensführung im Sinne moderner Corporate Governance resultiert aus dem durchdachten Zusammenwirken beider Orga$n e^{\text {“592. }}$.

Dies bedingt eine arbeitsteilige Aufgabenerfüllung durch den Vorstand und den Aufsichtsrat im strategischen Führungsprozeß ${ }^{593}$ Es ist daher nachfolgend zu ermitteln, welche Planungsobjekte des Aufsichtsrats hier existieren, wobei die organisatorischen und personellen Angelegenheiten des Vorstands aufgrund ihrer ausführlichen Behandlung im vorherigen Abschnitt ausgeklammert werden.

\footnotetext{
591 Vgl. von Werder, Grundsätze, 1999, S. 221, 224.

592 Malik, Kritik, 1998, S. 27.

593 Vgl. Malik, Kritik, 1998, S. 27, sowie Servatius, Vorstandskontrolle, 1995, S. 224; s. hierzu nochmals die Auflistung in Abschnitt II.A.1.c).
} 
$\mathrm{Zu}$ den Planungsobjekten des Aufsichtsrats sind zunächst die generellen Unternehmungsziele zu zählen. ${ }^{594} \mathrm{Ihr}$ Inhalt ist durch die paradigmatische Ausrichtung auf das Shareholder Value-Konzept beeinflußt. Aufgabe des Aufsichtsrats ist es, den notwendigen Interessenausgleich zwischen den hauptsächlich zu verfolgenden Interessen der Aktionäre und den ebenfalls zu berücksichtigenden Stakeholderinteressen herbeizuführen. Hierzu müssen als Rahmenbedingungen für den Vorstand Leitlinien bzw. generelle Ziele geplant und formuliert werden. ALBACH bezeichnet solche Leitlinien als Unternehmungsgrundsätze, die als Bezugsrahmen und Basis der strategischen Führung durch den Vorstand dienen sollen. ${ }^{595}$ Sie beschreiben das Selbstverständnis der Unternehmung und enthalten über die Satzung hinausgehend die Ziele, denen die Tätigkeit der Unternehmung dient.

ALBACH verdeutlicht die Notwendigkeit einer Verabschiedung von generellen Zielen der Unternehmung durch den Aufsichtsrat an einem Beispiel, in dem der Vorstand durch die Unternehmungsgrundsätze auf einen angemessenen Interessenausgleich zwischen den Aktionären, den Mitarbeitern, den Lieferanten, den Kunden und den Nachbarn verpflichtet wird, da es nicht ersichtlich sei, warum die Ziele der Aktionäre zu Gunsten der Interessen weiterer Gruppen vernachlässigt werden sollten. ${ }^{596}$ Die sich hinter einer solchen Formulierung verbergende Stakeholderorientierung wird aktuell durch zwei unterschiedliche Corporate Governance-Grundsatzkataloge unterstützt, die den Vorstand bei seiner Leitung auf das „Unternehmensinteresse“597 bzw. auf ein „Austarieren unterschiedlicher Interessengruppen ${ }^{\text {‘598 }}$ verpflichten wollen. ${ }^{599}$ Hierdurch entstehen unerwünschte diskretionäre Handlungsspielräume des Vorstands, der in diesem Fall eigene Ziele zu Lasten der übrigen Stakeholder durchsetzen kann. Sie können nur verhindert werden, wenn nicht der Vorstand, sondern der Aufsichtsrat nicht nur für

594 Vgl. Bleicher, Organisation, 1991, S. 31; Bleicher, Management, 1999, S. 74ff.; Kuhn, Unternehmensführung, 1990, S. 32; Lukarsch, Aufsichtsrat, 1998, S. 46; anderer Ansicht Hahn, Planungs- und Kontrollsysteme, 1999, S. 668; auch Römer, Aufsichtsrat, 1990, S. 129, sowie Potthoff/Trescher, Aufsichtsratsmitglied, 1999, S. 64.

Vgl. Albach, Aufsichtsrat, 1997, S. 37.

Vgl. Albach, Aufsichtsrat, 1997, S. 36f., sowie zur Lösung der Principal Agent-Problematik mit Hilfe des Shareholder Value-Konzepts Abschnitt II.C.2.b); ein Beispiel der hier angesprochenen Leitlinien findet sich bei der Deutschen Bank AG, Unternehmensprofil, o.S., die für die Mitarbeiter, die Aktionäre, die Kunden sowie die Gesellschaft Wert schaffen und einen Interessenausgleich herbeiführen will.

Vgl. Grundsatzkommission Corporate Governance, Corporate Governance-Grundsätze, 2000, S. 239.

598 Vgl. Berliner Initiativkreis German Code of Corporate Governance, Code, 2000, S. 37, I.3.

Kritisch zu diesen Tendenzen auch Groh, Aktienrecht, 2000, S. 2158. 
die Verabschiedung, sondern darüber hinaus auch für die Formulierung der generellen Unternehmungsziele verantwortlich ist, um so die Verfolgung der Interessen der Anteilseigner zu gewährleisten.

\section{b) Strategische Kontrolle}

Im Rahmen der vom Aufsichtsrat verabschiedeten Unternehmungsziele ist der Vorstand für die strategische Führung der Unternehmung verantwortlich. Parallel zu dem Führungsprozeß des Vorstands, der selbst eine strategische Kontrolle umfaßt, liegt der Führungsprozeß des Aufsichtsrats. ${ }^{600}$ Er beinhaltet eine eigenständige strategische Kontrolle. Zu den Kontrollobjekten des Aufsichtsrats zählen alle strategischen Entscheidungen des Vorstands mit Auswirkungen auf die Erfolgspotentiale der Unternehmung. ${ }^{601}$ Zum Aufbau und Erhalt der langfristigen Erfolgspotentiale sind Strategien der Unternehmung festzulegen und geeignete Dispositionen über die Kapitalbeschaffung und den zweckentsprechenden Einsatz unternehmerischer Ressourcen vorzunehmen. Dies beinhaltet sowohl Investitionen in Sachkapital als auch in das Humankapital der Unternehmung. ${ }^{602}$

Der geplante Aufbau von strategischen Erfolgspotentialen und ihre Ausbeutung manifestiert sich in den strategischen Unternehmungsplänen. Es ist daher nicht ausreichend, dem Aufsichtsrat lediglich vergangenheitsorientierte Rechnungswesendaten bzw. den Jahresabschluß als Informationsgrundlage zur Verfügung zu stellen. Für Aussagen über die zukünftige Unternehmungsentwicklung muß der Aufsichtsrat zusätzlich auf die strategische Planung des Vorstands zugreifen und diese auf der Grundlage eigener Planungsüberlegungen und gegebenenfalls weiterer Informationen nicht nur kritisch nachvollziehen - dies ist Bestandteil der strategischen Kontrolle des Aufsichtsrats -, sondern eigene Zukunftsprojektionen entwickeln. $\mathrm{Zu}$ seinen Planungsobjekten können somit alle Inhalte zählen, mit denen sich der Vorstand planerisch auseinandersetzt. ${ }^{603} \mathrm{Um}$ zu gewährleisten, daß dem Aufsichtsrat die strategischen Pläne vollständig zur Verfügung

600 Vgl. Hahn, Planung, 1994, S. 32, der die Notwendigkeit einer unabhängigen Kontrolle betont, sowie Claussen/Bröcker, Corporate Governance-Grundsätze, S. 490, die aus diesem Grund eine Annäherung des Board-Systems an das deutsche Aufsichtsratssystems als notwendig erachten; Lukarsch, Aufsichtsrat, 1998, S. 213, betont ebenfalls die Notwendigkeit der Parallelität, um so das bereits angesprochene Selektionsrisiko der strategischen Planung kompensieren zu können.

Vgl. Frese, Unternehmungsführung, 1987, S. 113; Scheffler, Rollenverständnis, 2000, S. 435, sowie die in $\S 90$ Abs. 1 AktG enthaltenen Informationsobjekte; ablehnend wohl nur Bea/Scheurer, Kontrollfunktion, 1994, S. 2147. 
gestellt werden, sollten sie einem Zustimmungsvorbehalt des Aufsichtsrats unterworfen werden. ${ }^{604}$

Der Aufsichtsrat sollte nicht nur bei der Unternehmungsplanung, sondern auch bei bestimmten Einzelmaßnahmen von seiner Möglichkeit Gebrauch machen, diese einem Zustimmungsvorbehalt zu unterwerfen. ${ }^{605}$ Dafür ist die Einführung bzw. die Veränderung eines Katalogs zustimmungspflichtiger Geschäfte erforderlich. Durch die Aufstellung eines Katalogs zustimmungspflichtiger Geschäfte gewährleistet der Aufsichtsrat, daß er über die Ausübung der $\mathrm{Zu}$ stimmungsvorbehalte unternehmerische Entwicklungen verhindern kann, die nicht den obersten Zielen der Unternehmung entsprechen. Auf der anderen Seite darf weder der Vorstand seiner unternehmerischen Freiheit beraubt noch der Aufsichtsrat gleichzeitig durch die Beschlußfassung über weniger bedeutende Geschäfte von seiner eigentlichen Überwachungstätigkeit abgehalten werden. ${ }^{606}$

Auf Grundlage der Satzung, der Geschäftsordnung oder eines Aufsichtsratsbeschlusses zählen zu möglichen zustimmungspflichtigen Maßnahmen Änderungen der Fertigungs- und Absatzprogrammstruktur, größere Investitionen oder Desinvestitionen, Finanzierungsmaßnahmen beispielsweise im Rahmen von Kapitalerhöhungen, Organisationsänderungen, Rechtsform- und Rechtsstrukturänderungen sowie die Einstellung von Führungskräften unterhalb der Vorstandsebene. ${ }^{607}$ Exemplarisch sei hierzu auf die entsprechende Satzungsbestimmung der ThyssenKrupp AG verwiesen (vgl. Abb. 15).

Neben der konkreten Nennung einzelner Maßnahmen enthält die Satzungsbestimmung eine Ermächtigungsklausel für den Aufsichtsrat zum Erlaß weiterer zustimmungspflichtiger Geschäfte (Abs. 2).

604 Vgl. Albach, Aufsichtsrat, 1997, S. 32; Lutter/Krieger, Rechte, 1993, S. 50f.; Kropff, Geschäftsführung, 1999, S. 361; im Ergebnis wohl zustimmend Rechenberg, Unternehmensplanung, 1990, S. 1363; grundlegend Götz, Zustimmungsvorbehalte, 1990, S. 633-656.

So unterscheidet Gerum, Führungsorganisation, 1995, S. 359-361, das Ausmaß der unternehmungspolitischen Kompetenz des Aufsichtsrats anhand des Umfangs zustimmungspflichtiger Geschäfte, wodurch die Bedeutung dieses Instruments deutlich wird.

Ein Beispiel für einen Katalog zustimmungspflichtiger Geschäfte einschlieBlich der Unternehmungsplanung als Bestandteil der Geschäftsordnung des Vorstands findet sich bei Kropff, Anlage H 1, 1999, S. 785ff.

607 Vgl. Hahn, Mitbestimmung, 1999, S. 795. 
„§ 7 Zustimmungspflichtige Geschäftsvorfälle

(1) Der Vorstand bedarf der Zustimmung des Aufsichtsrates für die nachstehend aufgeführten Geschäfte der Gesellschaft und der mit ihr verbundenen Unternehmen:

a) grundsätzliche Änderungen der Konzernorganisation;

b) die jährliche Investitionsplanung des Konzerns und deren Finanzierung;

c) grundsätzliche Änderungen der Grundstückspolitik des Konzerns;

d) Erwerb, Veräußerung oder Belastung von Grundstücken, grundstücksgleichen Rechten oder Rechten an Grundstücken, soweit der Wert der Maßnahme im Einzelfall den Betrag von 10.000.000,- EURO übersteigt;

e) Beteiligungen an anderen Unternehmen oder Aufgabe solcher Beteiligungen, sofern der Wert der Maßnahme im Einzelfall den Betrag von 25.000.000,- EURO übersteigt;

f) Erschließung neuer Geschäftsfelder oder Einschränkung oder Aufgabe bestehender Geschäftsfelder, soweit die Maßnahme für den Konzern von wesentlicher Bedeutung ist;

g) Übernahme von Bürgschaften, Garantien oder ähnlichen Haftungen außerhalb des üblichen Geschäftsbetriebes, soweit die Maßnahme für den Konzern von wesentlicher Bedeutung ist;

h) Gewährung von Darlehen oder sonstigen Krediten außerhalb des üblichen Geschäftsbetriebes, soweit die Maßnahme für den Konzern von wesentlicher Bedeutung ist.

(2) Der Aufsichtsrat kann bestimmen, dass auch andere Geschäfte nur mit seiner Zustimmung vorgenommen werden dürfen.

(3) Die nach Absatz 1 erforderliche Zustimmung des Aufsichtsrates kann auch in Form einer allgemeinen Ermächtigung für einen Kreis der vorbezeichneten Geschäfte erfolgen."

Abb. 15: Auszug aus der Satzung der ThyssenKrupp AG

Quelle: ThyssenKrupp AG, Satzung, 2000, S. 4.

Da bei jeder zustimmungspflichtigen Maßnahme eine Entscheidung sowie eine vorbereitende Planung des Aufsichtsrats erforderlich sind, handelt es sich hierbei gleichzeitig um mögliche Planungsobjekte des Aufsichtsrats. Auch die Aufstellung des Katalogs zustimmungspflichtiger Geschäfte selbst muß entsprechend vorbereitet werden, um eine wirkungsvolle Einflußnahme des Aufsichtsrats zu ermöglichen. ${ }^{608}$ Eine allgemeine Ermächtigung, wie sie Abs. 3 des obigen Satzungsparagraphen der ThyssenKrupp AG vorsieht, sollte jedoch nur sparsam eingesetzt werden, da ansonsten die Gefahr besteht, daß die Einflußmöglichkeit des Aufsichtsrats über den Zustimmungsvorbehalt ausgehöhlt wird. Die Zustimmungspflicht des Aufsichtsrats und die hierdurch ausgelöste Diskussion mit dem Vorstand sind eine wichtige Voraussetzung für die anzustrebende dialogische Unternehmungsführung. ${ }^{609}$ Bei gravierenden Differenzen über die

608 Vgl. Kropff, Geschäftsführung, 1999, S. 360ff.

609 Vgl. Servatius, Vorstandskontrolle, 1995, S. 224. 
Unternehmungsplanung ist es als Ultima ratio möglich, daß der Aufsichtsrat den Vorstand auf Grundlage von $\S 84$ Abs. 3 AktG abberuft. ${ }^{610}$

\section{c) Planungs-, Organisations- und Kontrollsystem}

Im Rahmen seiner Führungsaufgabe hat der Vorstand geeignete Planungs-, Organisations- und Kontrollsysteme einzurichten. Seit der Einführung des KonTraG zählt dazu insbesondere ein Überwachungssystem (syn. Frühwarnsystem, Risikomanagementsystem) der Unternehmung (§ 91 Abs. 2 AktG) ${ }^{611}$ Die Gewährleistung eines funktionsfähigen Überwachungssystems soll bestandsgefährdende Risiken für die Unternehmung vermeiden helfen. Der Aufsichtsratskontrolle unterliegt sowohl die Einrichtung dieses Systems als auch dessen Funktionsfähigkeit. ${ }^{612}$ Dabei kann jedoch ebensowenig von einer Planungsnotwendigkeit ausgegangen werden wie bei den übrigen Systemen. Hiervon auszunehmen sind lediglich grundlegende organisatorische Änderungen. So sind gegenwärtig umfangreiche Reorganisationsprozesse $\mathrm{zu}$ beobachten, die entweder durch Veränderungen der Unternehmungsstrategien oder durch die Einführung neuer Softwaresysteme ausgelöst werden. Während im ersten Fall eine Befassung des Aufsichtsrats im Rahmen der strategischen Führung stattfindet, können im zweiten Fall hiervon losgelöst Planungsüberlegungen notwendig sein. ${ }^{613}$

\section{Jahresabschluß und Zusammenarbeit mit dem Abschlußprüfer}

Als weiteres Aufgabenfeld, in dem Planungsobjekte des Aufsichtsrats grundsätzlich möglich scheinen, sind Tätigkeiten im Zusammenhang mit der Feststellung bzw. der Prüfung des Jahresabschlusses zu nennen. ${ }^{614}$ Der Aufsichtsrat muß den Jahresabschluß der Unternehmung einschließlich des ihm direkt zugeleiteten Prüfungsberichtes des Abschlußprüfers prüfen; bei Mutterunternehmungen im Sinne des $\S 290$ HGB zusätzlich den Konzernabschluß und den Konzernlagebericht. Dabei geht die Prüfungspflicht des Aufsichtsrats weiter als die des Abschlußprüfers, da er nicht nur die Gesetzmäßigkeit des Jahresabschlusses festzustellen hat, er muß darüber hinaus einen Vergleich mit der operativen Planung des betreffenden Jahres durchführen sowie analysieren, welche Maßnah-

\footnotetext{
610 Vgl. Mutter, Aktiengesellschaft, 1994, S. 46, 80.

611 Vgl. Salzberger, Aufsichtsrat, 2000, S. 757ff.

612 Vgl. Vogler/Gundert, Einführung, 1998, S. 2378; auch Kromschröder/Lück, Unternehmensüberwachung, 1998, S. 1573-1576; Kuhl/Nickel, Risikomanagement, 1999, S. 133-135; Lück, Elemente, 1998, S. 8-14; Perlitz, Frühwarnsysteme, 1993, Sp. 679-688.

613 Vgl. Potthoff, Aufsichtsrat, 1998, S. 135.

614 Vgl. hierzu umfassend Kropff, Geschäftsführung, 1999, S. 397ff., sowie Potthoff/Trescher, Aufsichtsratsmitglied, 1999, S. $205 \mathrm{ff}$.
} 
men der Vorstand bei eventuellen Abweichungen ergriffen hat und wie Abweichungen zukünftig vermieden werden können. ${ }^{615}$ Ergeben sich aus dem Prüfungsbericht des Abschlußprüfers jedoch keine Hinweise auf Unstimmigkeiten oder Probleme, kann sich der Aufsichtsrat hinsichtlich der Ordnungsmäßigkeit des Abschlusses auf das Urteil des Prüfers verlassen. ${ }^{616}$ Insgesamt können die hier anstehenden Tätigkeiten zur Kontrolle gerechnet werden.

Neben diesen Kontrollaufgaben entscheidet der Aufsichtsrat mit über die Billigung des Jahresabschlusses ( $§ 171$ Abs. $2 \mathrm{~S} .4$ AktG), wodurch dieser gleichzeitig festgestellt wird, und stimmt gegebenenfalls Einstellungen eines Teils des Gewinns in andere Gewinnrücklagen ( $\S 58 \mathrm{Abs} .2 \mathrm{AktG}$ ) sowie Abschlagszahlungen auf den Bilanzgewinn ( $\$ 59 \mathrm{Abs} .3 \mathrm{AktG}) \mathrm{zu} .{ }^{617}$ Auch bei diesen Teilaufgaben bleibt die zukünftige Entwicklung der Unternehmung weitgehend unberücksichtigt. Eigenständige Planungsüberlegungen des Aufsichtsrats werden lediglich erstens im Rahmen seiner Zweckmäßigkeitskontrolle bilanzpolitischer Entscheidungen des Vorstands sowie zweitens bei der Auswahl und Vergütung des Abschlußprüfers einschließlich der Festlegung von Prüfungsschwerpunkten erforderlich.

Mit der Bilanzpolitik beabsichtigt der Vorstand eine zweckorientierte Steuerung der handels- und steuerrechtlich relevanten Jahresergebnisse und Vermögensdarstellungen in Handelsbilanzen und Steuerbilanzen. ${ }^{618}$ Durch die Ausnutzung handelsrechtlicher Bilanzierungs- und Bewertungswahlrechte soll das Bild der Vermögens-, Finanz- und Ertragslage gestaltet werden. Dies zielt auf die Beeinflussung der Steuerlasten der Unternehmung, den Ergebnisausweis und insbesondere auf die Kapitalmarktinformation. Da hierdurch beispielsweise geplante Finanzierungsmöglichkeiten der Unternehmung beeinflußt werden können, sollte der Aufsichtsrat auch die zukünftigen Auswirkungen dieser Maßnahmen hinsichtlich ihrer Zweckmäßigkeit hinterfragen. ${ }^{619}$

Schließlich ist mit dem KonTraG die Verpflichtung zum Vertragsabschluß mit dem Abschlußprüfer vom Vorstand auf den Aufsichtsrat übergegangen (§ 111

615 Vgl. Albach, Aufsichtsrat, 1997, S. 32f.; Potthoff, Abschlußprüfung, 1997, S. I; Scheffler, Rollenverständnis, 2000, S. 435.

616 Vgl. Lutter/Krieger, Rechte, 1993, S. 158.

617 Vgl. Lutter/Krieger, Rechte, 1993, S. 158; Potthoff/Trescher, Aufsichtsratsmitglied, 1999, S. 206.

$618 \mathrm{Vgl}$. Busse von Colbe/Pellens, Rechnungswesen, 1998, S. $117 \mathrm{ff}$.

619 Vgl. Potthoff/Trescher, Aufsichtsratsmitglied, 1999, S. 207f.; m.w.N. auch Mutter, Aktiengesellschaft, 1994, S. 148f.; auf die besondere Bedeutung der Steuerbilanz hinweisend Theisen, Aufsichtsrats-Informationssysteme, 1998, S. 81. 
Abs. 2 AktG) ${ }^{620}$ Hierdurch wird beabsichtigt, daß der Abschlußprüfer seine Hilfsfunktion für den Aufsichtsrat erfüllen kann. ${ }^{621}$ Zudem sollen so Qualitätsprobleme der Abschlußprüfung vermieden werden. ${ }^{622}$ Hierzu ist neben der Auswahl des Abschlußprüfers insbesondere zu planen, in welchen Unternehmungsbereichen der Abschlußprüfer in Zukunft verstärkt zu prüfen hat.

Insgesamt kann jedoch festgestellt werden, daß trotz dieser Planungsnotwendigkeiten mit Bezug auf den Jahresabschluß der Kontrollaspekt weiter dominiert, so $\mathrm{da}$ die hier betrachteten Planungsobjekte im weiteren Verlauf der Untersuchung vernachlässigt werden können.

\section{Organisatorische Angelegenheiten des Aufsichtsrats}

Abschließend ist auf die organisatorischen Angelegenheiten des Aufsichtsrats einzugehen. ${ }^{623}$ Wie die Analyse der rechtlichen Regelungen zur Organisation des Aufsichtsrats zeigen konnte, besteht für ihn aufgrund des Mangels an normativen Vorgaben eine Verpflichtung, seine Aufgabenwahrnehmung eigenständig zu organisieren ${ }^{624}$ In der bisherigen Praxis ist jedoch kritisch festzustellen, $\mathrm{da}$ die Aufsichtsorgane nur unzureichend dieser Organisationspflicht nachkommen. ${ }^{625}$ So zählen für MALIK neben der zu großen Zahl von Aufsichtsratsmitgliedern insbesondere die schlechte Organisation des Aufsichtsorgans, eine zu geringe Zahl von zu kurzen Sitzungen und das unprofessionelle Sitzungsmanagement zu den ausschlaggebenden Mängeln für die unzureichende Funktionsfähigkeit des Aufsichtsrats. ${ }^{626}$ Daher hängt eine Verbesserung der Überwachungstätigkeit von Aufsichtsräten wesentlich von einer Veränderung der Organisation $a b .{ }^{627}$ LUTTER weist jedoch zu Recht darauf hin, daß, solange die Zahl

620 Vgl. Coenenberg/Reinhart/Schmitz, Audit Committees, 1997, S. $994 f$.

621 Vgl. Theisen, Reform, 1999, S. 224f.

622 Vgl. Streim, Haftung, 2000, S. 618, sowie umfassend zu Qualitätsproblemen Quick, Abschlußprüfer, 2000, S. 525-548; auch Ewert/Feess/Nell, Prüfungsqualität, 2000, S. 572-593; mit einer empirischen Analyse des Prüferwechsels Marten, Analyse, 1995, S. 703-727.

${ }^{623}$ Zur Organisation der Entscheidungsprozesse im Aufsichtsrat Kruse, Aufsichtsrat, 1972.

624 Vgl. Lutter/Krieger, Rechte, 1993, S. 166; m.w.N. Hommelhoff, Aufsichtsrats-Überwachung, 1995, S. 3; die Notwendigkeit einer persönlichen Beteiligung aller Aufsichtsratsmitglieder an der Entscheidung zur Organisationsgestaltung explizit betonend Semler, Kompetenzen, 1999, S. 11.

Vgl. Hommelhoff/Mattheus, Corporate Governance, 1998, S. 254; zu Defiziten der Arbeitsweise des Gesamtgremiums und der hieraus abgeleiteten Forderung nach einer vermehrten AusschuBbildung die Auswertung eines Interviews mit Semler bei Schneider, Unternehmensüberwachung, 2000, S. 227f.

Vgl. Malik, Kritik, 1998, S. 27; äußert kritisch zur aktuellen Funktionsfähigkeit der Aufsichtsratsorganisation auch Sünner, Corporate Governance, 2000, S. 496.

Vgl. Lutter, Aufsichtsratstätigkeit, 1995, S. 298f. 
der Aufsichtsratsmitglieder nicht nachhaltig verringert wird, ${ }^{628}$ eine Erhöhung der Sitzungszahl nur zu einer noch größeren Anzahl ineffektiver Sitzungen führt. Da eine mit dem KonTraG geplante Verringerung der Aufsichtsratsgröße u.a. an dem Widerstand der Gewerkschaften gescheitert ist, ${ }^{629}$ haben sich alle organisatorischen Gestaltungsvorschläge darauf zu konzentrieren, die Organisation des Aufsichtsrats im Rahmen dieser Restriktion zu verbessern. Für HOMMELHOFF/MATTHEUS ist von einer funktionsfähigen Organisation sogar eine größere Qualitätssteigerung der Aufsichtsratsarbeit zu erwarten als von einer Verkleinerung des Gremiums. ${ }^{630}$

Ebenso wie für die organisatorische Gestaltung des Vorstands sollte der Aufsichtsrat im Rahmen seiner Selbstorganisation eine Organisationsplanung durchführen. Sie beinhaltet nicht nur die Gestaltung der Planungsorganisation, sondern darüber hinausgehend die der Organisation des gesamten Aufsichtsgremiums. ${ }^{631}$ Die konkrete Ausgestaltung erfolgt in Abhängigkeit von unterschiedlichen Determinanten, zu denen die Unternehmungsgröße, die Unternehmungsaufgabe, der Diversifikationsgrad oder die geographische Reichweite zu rechnen sind. ${ }^{632}$

Aufgrund der Besonderheiten der Aufgabenerfüllung des Aufsichtsrats ist ein ausgeprägter Formalisierungsgrad der organisatorischen Regelungen erforderlich. ${ }^{633}$ FEDDERSEN weist daher zu Recht mit Nachdruck darauf hin, daß die umfangreichen organisatorischen Gestaltungsregelungen des Aufsichtsrats der Fixierung innerhalb einer Geschäftsordnung bedürfen. ${ }^{634}$ Neben den Regelungen $\mathrm{zu}$ organisatorischen und personellen Angelegenheiten des Aufsichtsrats kann die Geschäftsordnung außerdem solche zur Behandlung von Insiderwissen enthalten. Solange der Katalog zustimmungspflichtiger Geschäfte nicht Bestandteil

628 Entsprechende Forderungen werden immer wieder erhoben; vgl. beispielsweise Sünner, Corporate Governance, 2000, S. 496; die Anzahl der Aufsichtsratsmitglieder erreicht in den hier betrachteten Gesellschaften regelmäBig die maximale Zahl von 20 Mitgliedern.

Vgl. m.w.N. Claussen, Aktiengesetz, 1998, S. 182f.

Vgl. Hommelhoff/Mattheus, Corporate Governance, 1998, S. 255; anderer Auffassung Sünner, Corporate Governance, 2000, S. 496.

Vgl. mit Überwachungsbezug Theisen, Unternehmungsführung, 1987, S. 265; zur Organisation des Aufsichtsrats aus betriebswirtschaftlicher Perspektive Chini, Aufsichtsrats-Informationssystem, 1988, S. 81-92, sowie Ludwig, Aufsichtsräte, 1997, S. 148-178.

Vgl. unter Bezugnahme auf Ausschüsse Berliner Initiativkreis German Code of Corporate Governance, Code, 2000, S. 67, IV.3.4.

Vgl. Hommelhoff, Aufsichtsrats-Überwachung, 1995, S. 3.

Vgl. Feddersen, Aufsichtsrat, 2000, S. 394; auch Potthoff, Aufsichtsrat, 1998, S. 140, sowie unter Bezugnahme auf Ausschüsse Siebel, Arbeit, 1999, S. 311 f. 
der Geschäftsordnung des Vorstands ist, sollte er ebenfalls in der des Aufsichtsrats enthalten sein.

$\mathrm{Da}$ in der vorliegenden Untersuchung die Entwicklung eines Planungssystems im Vordergrund steht, läge es nahe, bei der Gestaltung der Planungsorganisation ebenso wie im Rahmen der anderen Planungssystemelemente die übrigen Funktionsfelder des Aufsichtsrats unberücksichtigt zu lassen. Da jedoch im organisatorischen Bereich umfangreiche Interdependenzen bestehen, sollen bei der Betrachtung der Aufsichtsratsorganisation auch seine weiteren Aufgabenfelder berücksichtigt werden. Diese üben zum Teil eine restringierende Wirkung für die Durchführung der Planungsaufgaben aus. Schließlich sei darauf hingewiesen, daß aufgrund der engen Verbindung zwischen den organisatorischen und den personellen Angelegenheiten des Aufsichtsrats diese im Zusammenhang mit der Planungsorganisation diskutiert werden.

\section{Die Planungsorganisation des Aufsichtsrats}

\section{1. Überblick}

Im Mittelpunkt der nachfolgenden Überlegungen steht die Planungsorganisation des Aufsichtsrats und die Analyse der grundsätzlich möglichen Strukturierungsalternativen. Die organisatorische Gestaltung des Gremiums erfolgt mit dem Ziel, die arbeitsteilige Aufgabenerfüllung der Aufsichtsratsmitglieder zu strukturieren. ${ }^{635}$ Ausgehend von den Aufgaben des Aufsichtsrats sind im Rahmen einer Organisationsplanung zweckentsprechende Regelungen zu gestalten, die den Aufsichtsrat insgesamt in die Lage versetzen, die an ihn gestellten Anforderungen zu erfüllen. Aufgrund der bisher zu beobachtenden Defizite in der Arbeitsweise des Gesamtgremiums sowie die mit der Einführung des Planungssystems gestiegenen Anforderungen an die Tätigkeit des Aufsichtsrats ist zu überprüfen, inwieweit Planungsaufgaben entweder an einzelne Aufsichtsratsstellen oder an Ausschüsse delegiert werden sollten. ${ }^{636}$ Im Rahmen dieser Delegationsentscheidung ist darüber hinaus festzulegen, ob ein Ausschuß zusätzlich die mit dem Planungsobjekt verbundenen Entscheidungskompetenzen übertragen bekommen sollte. Dies kann nur unter Beachtung der beschriebenen rechtlichen Delegationsverbote erfolgen. Eine Entscheidungsdelegation auf einzelne Mitglieder ist grundsätzlich nicht möglich. ${ }^{637}$

\footnotetext{
635 Vgl. zum Zusammenhang von Struktur und Organisation Kosiol, Organisation, 1976, S. 19f.

636 Vgl. mit der Forderung nach einer verstärkten Einsetzung von Ausschüssen nur Deckert, Ausschüsse, 1996, S. 986, sowie Hommelhoff, Aufsichtsrats-Überwachung, 1995, S. 3, die ihre Ausführungen jedoch auf die Kontrollaufgaben des Aufsichtsrats fokussieren.

637 Vgl. m.w.N. Ludwig, Aufsichtsräte, 1997, S. 166.
} 
Die Diskussion der Planungsobjekte des Aufsichtsrats hat gezeigt, daß in unterschiedlichen Bereichen Planungsaufgaben identifiziert werden können. Um zu überprüfen, ob eine Zuordnung zu den verschiedenen organisatorischen Gestaltungsalternativen möglich ist, ist es sinnvoll, sie nach inhaltlichen Gesichtspunkten zu gruppieren:

1. Vorstandsbezogene Planungsobjekte, die alle organisatorischen und personellen Angelegenheiten des Vorstands einschließlich der Detaillierung von Grundsätzen ordnungsmäßiger Unternehmungsleitung umfassen;

2. alle im Zusammenhang mit der strategischen Unternehmungsführung stehenden Planungsobjekte des Aufsichtsrats, zu denen die generellen Unternehmungsziele, die strategische Führung des Vorstands, die Festlegung des Katalogs zustimmungspflichtiger Geschäfte sowie die Entscheidung über einzelne Geschäfte zählen;

3. fallweise Aktivitäten, wie beispielsweise einmalige Reorganisationen der gesamten Unternehmung oder wesentlicher Bereiche außerhalb einer Veränderung der Unternehmungsstrategien;

4. organisatorische und personelle Angelegenheiten des Aufsichtsrats einschließlich der Verabschiedung seiner Geschäftsordnung.

Für den hier verfolgten gestaltungsorientierten Ansatz wird zur Entwicklung der Planungsorganisation des Aufsichtsrats zunächst eine Unterscheidung in die Aufbau- und die Ablauforganisation vorgenommen. ${ }^{638}$ Mit der Aufbauorganisation erfolgt seine externe und interne Strukturierung. Da die externe Strukturierung der Aufbauorganisation des Aufsichtsrats in der Einheitsunternehmung juristisch determiniert ist und somit unberücksichtigt bleiben kann, finden sich Gestaltungspotentiale nur bei der internen Strukturierung. ${ }^{639}$ Sie beinhaltet sowohl die Primär- als auch die Sekundärorganisation. Innerhalb der nachfolgenden Analyse werden daher zunächst aufbauorganisatorische Gestaltungsoptionen zur Primär- sowie zur Sekundärorganisation entwickelt. Dabei ist zusätzlich die Gestaltung der organinternen und der organexternen Kommunikationswege zu

638 Vgl. grundlegend Bleicher, Organisation, 1991, S. 35ff.; Schulte-Zurhausen, Organisation, 1999, S. 39ff., sowie mit Bezug auf die Planungsorganisation Hammer, Unternehmensplanung, 1998, S. 91ff.; Mag, Unternehmungsplanung, 1995, S. 120ff.; Wild, Unternehmungsplanung, 1983, S. 183ff.; Link, Organisation, 1999, S. 804-829; Vancil/Lorange, Strategic Planning, 1999, S. 830843.

639 Vgl. zur externen Strukturierung von Aufsichtsräten im Konzern Ludwig, Aufsichtsräte, 1997, S. $170 \mathrm{ff}$. 
berücksichtigen. ${ }^{640}$ In der anschließenden Betrachtung der Ablauforganisation ist die organinterne Zusammenarbeit der Aufgabenträger zu gestalten ${ }^{641}$ Den Abschluß bildet die Berücksichtigung personeller Angelegenheiten der Aufsichtsratsmitglieder durch den Aufsichtsrat.

\section{Aufbauorganisation}

a) Primärorganisation

\section{(1) Ressortierung des Aufsichtsrats}

Die Primärorganisation umfaßt allgemein alle dauerhaften hierarchisch verbundenen Organisationseinheiten. ${ }^{642}$ Für den Aufsichtsrat ist diese Definition zu modifizieren, da er als Kollegialorgan grundsätzlich hierarchiefrei ist. ${ }^{643} \mathrm{Hier}$ ist CHINI nachhaltig zu widersprechen, der zur Aufbauorganisation des Aufsichtsrats auch Weisungsbeziehungen zählt. ${ }^{644}$ Daher wird unter Primärorganisation des Aufsichtsrats seine dauerhafte grundlegende (hierarchiefreie) Organisationsstruktur verstanden.

Bevor eine Verteilung von Planungsaufgaben innerhalb der Primärorganisation erfolgen kann, ist zunächst danach zu fragen, in welcher Form die Primärorganisation des Aufsichtsgremiums grundsätzlich gestaltet werden sollte. Wie die Aufgabenanalyse des Aufsichtsrats gezeigt hat, beinhaltet die Überwachungsaufgabe vielfältige Teilaufgaben. Diese werden üblicherweise durch den gesamten Aufsichtsrat in hierfür vorgesehenen und teilweise ritualisierten Sitzungen bearbeitet, wobei oftmals eine vorherige Absprache innerhalb der beiden Aufsichtsratsbänke stattfindet. Daneben kommt dem Aufsichtsratsvorsitzenden eine besondere Bedeutung $\mathrm{zu}$, da er den Kontakt zum Vorstand hält und die Beschlüsse des Aufsichtsrats vorbereitet. Eine weitergehende Arbeitsteilung, d.h. die Übertragung eines Tätigkeitskomplexes auf mehrere Personen, findet außer durch die Einsetzung von Ausschüssen üblicherweise nicht statt, ${ }^{645}$ so daß in der bisherigen Praxis auf die Vorteile einer arbeitsteiligen Aufgabenerfüllung weitgehend verzichtet wird. $\mathrm{Zu}$ diesen Vorzügen zählen eine höhere quantitative

640 Vgl. zur Kommunikation als Bestandteil der Aufbauorganisation Kosiol, Organisation, 1976, S. $178 f$.

641 Vgl. zur Ablauforganisation umfassend Kosiol, Organisation, 1976, S. 185-241.

642 Vgl. Schulte-Zurhausen, Organisation, 1999, S. 237.

$643 \mathrm{Vgl}$. Lutter/Krieger, Rechte, 1993, S. 34.

644 Vgl. Chini, Aufsichtsrats-Informationssysteme, 1988, S. 83; Schulte-Zurhausen, Organisation, 1999, S. 279, zählt zur Primärorganisation auch die Matrixorganisation, die in diesem Fall jedoch mit Über- und Unterstellungsverhältnissen verbunden ist und somit ausgeschlossen werden kann.

645 Vgl. mit einer entsprechenden Forderung beispielsweise Schneider, Unternehmensüberwachung, 2000, S. 226. 
Kapazität sowie eine verbesserte Bewältigung von Aufgabenvielfalt und -komplexität. ${ }^{646}$ Daher wird eine Ressortierung des Aufsichtsrats vorgeschlagen, ${ }^{647}$ die eine Aufgabenspezialisierung erfordert, mit der unterschiedlichen Aufsichtsratsstellen - und somit den Aufsichtsratsmitgliedern als Stelleninhaber - einzelne Teilaufgaben dauerhaft zur Erledigung zugewiesen werden. Wie bereits festgestellt wurde, dürfen einzelnen Aufsichtsratsmitgliedern grundsätzlich keine Entscheidungskompetenzen übertragen werden, so daß nur eine dem Teamprinzip ähnliche horizontale Arbeitsteilung möglich ist, bei der abweichend vom Prinzip der Gesamtkollegialität eine Ressortkollegialität entsteht. ${ }^{648}$ Sie hat den Vorteil größerer Transparenz, einer eindeutigeren Struktur sowie klarer Verantwortung und ermöglicht eine professionelle Ressortführung.

Die Ressortierung des Aufsichtsrats kann nach Funktionen oder nach Objekten erfolgen, wodurch einzelnen Aufsichtsratsmitgliedern beispielsweise die Überwachung der Kreditvergabe oder wesentlicher Produkte bzw. Produktgruppen übertragen würde ${ }^{649}$ LUDWIG schlägt vor, diese nicht an der Ressortierung des Vorstands zu orientieren, da hierdurch ein zu großes Abhängigkeitsverhältnis zwischen den beteiligten Personen im Aufsichtsrat und im Vorstand entstünde. ${ }^{650}$ In Abhängigkeit davon, ob alle Aufsichtsratsmitglieder ein Ressort übertragen bekommen oder einzelne Mitglieder eine mehrfache Ressortverantwortung tragen, sind unterschiedliche Mischformen der Ressortkollegialität möglich. ${ }^{651}$ Neben diesen Ressortstellen beinhaltet die Primärorganisation zusätzlich den Aufsichtsratsvorsitzenden sowie seine(n) Stellvertreter. Obwohl insbesondere dem Aufsichtsratsvorsitzenden primär koordinierende Tätigkeiten zukommen, ist nicht ausgeschlossen, daß er ebenso wie sein(e) Stellvertreter eine bestimmte Ressortverantwortung übernimmt.

646 Vgl. Schulte-Zurhausen, Organisation, 1999, S. 130f.

647 Vgl. Chini, Aufsichtsrats-Informationssysteme, 1988, S. 83f.; Ludwig, Aufsichtsräte, 1997, S. 165f., die feststellt, daß dem bisher in der Literatur kaum Beachtung geschenkt wurde; Lutter/Krieger, Rechte, 1993, S. 166f.; mit entsprechenden Forderungen auch Bernhardt, Qualitätsmessung, 2000, S. 2, Lutter, Qualitätsmessung, 2000, sowie Meyer-Lohmann, Aufsichtsrat, 1997, S. 269f.; in Ansätzen auch Arbeitskreis „Externe und interne Überwachung der Unternehmung“", Grundsätze, 1995, S. 2; Hutzschenreuter, Unternehmensverfassung, 1997, S. 188ff., beschränkt dagegen die Aufbauorganisation auf die Ausschußbildung.

Vgl. Krüger, Organisation, 1994, S. 258f.; Hommelhoff, Aufsichtsrats-Überwachung, 1995, S. 3, spricht in diesem Zusammenhang von Berichterstattern.

Vgl. Hommelhoff, Organstreit, 1979, S. 298; zu den sich auch hieraus ergebenden Anforderungen an Aufsichtsratsmitglieder s. Abschnitt III.D.2.

Vgl. Ludwig, Aufsichtsräte, 1997, S. 165.

Vgl. zu den Mischformen Krüger, Organisation, 1994, S. 259. 


\section{(2) Übertragung von Planungsaufgaben auf Ressorts}

Der bisherige Gestaltungsvorschlag bezog sich auf eine funktions- bzw. objektorientierte Arbeitsteilung im Aufsichtsrat, ohne eine Trennung in einzelne Überwachungsteilaufgaben vorzunehmen. Nachfolgend ist daher zu überlegen, inwieweit Aufsichtsratsmitgliedern die hier betrachteten Planungsaufgaben zur Vorbereitung der Entscheidungen des Gesamtgremiums übertragen werden sollten.

Die Delegationsmöglichkeit von Aufgaben an einzelne Aufsichtsratsstellen ist abhängig von der Kapazität potentieller Stelleninhaber. ${ }^{652}$ Da hier zunächst nur einzelne Stellen betrachtet werden, wird die Kapazität lokal, zeitlich und qualitativ determiniert. Unter Vernachlässigung der lokalen Dimension wird das auf eine Aufsichtsratsstelle delegierbare Aufgabenbündel durch die potentiell vorhandenen zeitlichen und qualitativen Kapazitäten des Stelleninhabers begrenzt. Bei einzelnen Aufsichtsratsmitgliedern ist davon auszugehen, daß die zeitliche Kapazität stark eingeschränkt ist. Aufsichtsratsmitgliedern, vor allem solchen mit Ressortverantwortung, sollten daher nur sehr begrenzt über die Ressortätigkeit hinaus zusätzliche Planungsaufgaben übertragen werden.

Neben der unzureichenden zeitlichen Kapazität spricht auch die zu geringe qualitative Kapazität gegen eine Übertragung der oben genannten Planungsaufgaben auf einzelne Aufsichtsratsmitglieder, da nicht davon auszugehen ist, daß sie über alle benötigten Qualifikationen verfügen. Dies gilt insbesondere im Vergleich zur Einrichtung von Ausschüssen, da diese aufgrund ihrer Multipersonalität komplementäre Qualifikationen zusammenführen können. ${ }^{653}$ Trotz des häufig größeren zeitlichen Einsatzes trifft dies grundsätzlich auch für den Aufsichtsratsvorsitzenden $\mathrm{zu}$, da seine Arbeitsbelastung bereits heute deutlich höher als die eines einfachen Aufsichtsratsmitglieds ist. Der Gesetzgeber berücksichtigt dieses über die Doppeltzählung eines Aufsichtsratsvorsitzes bei der Ermittlung der Höchstzahl von Aufsichtsratsmandaten entsprechend ( $\S 100$ Abs. 2 $\mathrm{AktG)}{ }^{654}$

Insgesamt kann festgestellt werden, daß innerhalb der Primärorganisation des Aufsichtsrats über eine Ressortierung und die hiermit verbundene Arbeitsteilung eine verbesserte Bearbeitung der Überwachungsaufgaben erreicht werden kann. Die Übernahme der hier betrachteten Planungsaufgaben durch einzelne Auf-

652 Vgl. hierzu Mag, Personalplanung, 1998, S. 63ff., 83ff.

653 Vgl. Redel, Führungsgremien, 1995, Sp. 709.

654 Vgl. Ludwig, Aufsichtsräte, 1997, S. 166; Arbeitskreis „Externe und interne Überwachung der Unternehmung“, Grundsätze, 1995, S. 1. 
sichtsratsmitglieder ist jedoch aufgrund der begrenzten zeitlichen und qualitativen Kapazitäten einzelner Stelleninhaber kritisch zu beurteilen, so daß nachfolgend zu analysieren ist, welche Lösungsmöglichkeiten im Rahmen der Sekundärorganisation bestehen.

\section{b) Sekundärorganisation}

\section{(1) Begriff und Gestaltungsprinzipien}

Mit Sekundärorganisation werden üblicherweise alle hierarchieergänzenden bzw. -übergreifenden Organisationsstrukturen bezeichnet. ${ }^{655}$ Mangels Hierarchisierung des Aufsichtsrats zählen hierzu im vorliegenden Problemzusammenhang alle die Primärorganisation ergänzenden Strukturformen. Als Gliederungsprinzipien der Sekundärorganisation kommen das Ausgliederungsprinzip, das Matrixprinzip, das Stabsprinzip und das Arbeitsgruppenprinzip in Betracht. ${ }^{656}$

Das Ausgliederungsprinzip führt zu einer Ausgliederung bestimmter abgrenzbarer Aufgaben bzw. Aufgabenteile aus der Primärorganisation in neue, autonome Organisationseinheiten. ${ }^{657}$ Diese Organisationseinheiten werden mit allen zur Problemlösung notwendigen Ressourcen und Kompetenzen ausgestattet, so daß die Aufgabenbearbeitung vollkommen losgelöst von der Primärorganisation erfolgt. Da Aufgaben des Aufsichtsrats jedoch nicht dauerhaft auf andere Personen übertragen werden dürfen ( $\S 111 \mathrm{Abs} .5 \mathrm{AktG})$ und somit eine permanente Hinzuziehung sachverständiger Dritter verboten ist, könnte die Anwendung des Ausgliederungsprinzips lediglich zu einer Reorganisation der Primärorganisation führen, nicht aber eine Ausprägung der Sekundärorganisation begründen. Somit kann die Anwendung dieses Prinzips für den Aufsichtsrat ausgeschlossen werden.

Bei Anwendung des Matrixprinzips werden zwei organisatorische Gliederungsprinzipien gleichzeitig angewendet. ${ }^{658}$ Die Matrixschnittstelle bildet ein gemeinsames Problemfeld, über das sich die betroffenen Matrixstellen abstimmen müssen. Dabei bestehen bei Anwendung im Rahmen der Sekundärorganisation enge Verbindungen zum Stabsprinzip sowie zum Arbeitsgruppenprinzip, die auch als Sonderfälle des Matrixprinzips aufgefaßt werden können. ${ }^{659}$ Das

655 Vgl. Redel, Führungsgremien, 1995, Sp. 707; Schulte-Zurhausen, Organisation, 1999, S. 273; anders dagegen Krüger, Organisation, 1994, S. 41, der zur Sekundärorganisation nur Einheiten zur Erfüllung von Spezialaufgaben zählt, so daß bei ihm Ausschüsse Bestandteil der Primärorganisation sind.

Vgl. Frese, Organisation, 2000, S. 363ff.; Schulte-Zurhausen, Organisation, 1999, S. $275 \mathrm{ff}$.

Vgl. Schulte-Zurhausen, Organisation, 1999, S. 280. 
Matrixprinzip soll daher nachfolgend vernachlässigt werden, um nur die Eignung des Stabs- und des Arbeitsgruppenprinzip als grundsätzliche Strukturierungsmuster zu untersuchen.

\section{(2) Stabsprinzip}

Mit dem Stabsprinzip werden entscheidungsvorbereitende Aufgaben aus der Primärorganisation ausgelagert und ihr angegliederten Stabsstellen zugeordnet. ${ }^{660}$ Diesen Stabsstellen kommen primär Koordinationsaufgaben zu, die eine intensive Informationssammlung und -verarbeitung erfordern. Dem Stabsprinzip entspricht ein 1998 im Gesetzgebungsverfahren zum KonTraG gemachter Vorschlag des Bundesrats zur Einrichtung eines Aufsichtsratsassistenten, der ähnlich einer Stabsstelle agieren sollte, ohne selbst Mitglied des Aufsichtsrats zu sein. Der Vorschlag enthielt detaillierte Regelungen zur Einrichtung, Organisation und Unabhängigkeit des Assistenten und sah vor, daß er primär der vom Vorstand unabhängigen und zeitnahen Information des Aufsichtsrats dienen und ihn darüber hinaus koordinierend unterstützen sollte. ${ }^{661}$ Dieser Vorschlag fand jedoch wie eine Vielzahl weiterer keinen Eingang in das verabschiedete Gesetz.

Vor dem Hintergrund der hier diskutierten Planungsaufgaben ist der Frage nachzugehen, welche Bedeutung der Einrichtung eines Aufsichtsratsassistenten zukommen könnte. Dabei ist festzustellen, daß insbesondere im Hinblick auf die mit der vorgeschlagenen Ressortierung stark gestiegenen Kommunikations- und Koordinationserfordernisse die Einrichtung eines Aufsichtsratsassistenten als unternehmungsinterne Ressource deutliche Potentiale zur Unterstützung des Aufsichtsrats bietet. Darüber hinaus wäre es durch die Einrichtung eigener unternehmungsinterner Ressourcen möglich, Geheimhaltungspflichten sorgfältiger einzuhalten als bei der Unterstützung des Aufsichtsratsmitglieds durch Mitarbeiter einer anderen Unternehmung. Dazu kann beispielsweise die Nutzung personeller Ressourcen eines Aufsichtsratsmitglieds aus der Unternehmung, in der er als hauptamtlicher Vorstand tätig ist, gezählt werden. ${ }^{662}$

FEDDERSEN kritisiert an diesem Vorschlag jedoch, daß der Aufsichtsratsassistent in eine zu große Abhängigkeit vom Vorstand geraten könne und außerdem die

\footnotetext{
660 Vgl. beispielsweise Frese, Organisation, 2000, S. 346ff.; Grochla, Einführung, 1972, S. 181ff.; Kosiol, Organisation, 1976, S. 131ff.; Ulrich, Organisationslehre, 1949, S. 149ff.

${ }^{661}$ Vgl. Deutscher Bundestag: Drucksache 13/9716 vom 29.01.1998; auch schon Lutter, Professionalisierung, 1995, S. 1133; kritisch Feddersen, Aufsichtsrat, 2000, S. 395; eigene personelle Ressourcen des Aufsichtsrats fordert auch Wenger, Organisation, 1996, S. 179.
}

662 Vgl. hierzu Lutter/Krieger, Hilfspersonen, 1995, S. 257-260; Mülbert, Aufsichtsratsmitglieder, 1996, S. 104ff. 
Gefahr einer zu starken Einbindung in das Tagesgeschäft bestehe. ${ }^{663}$ Dem ist jedoch zweierlei entgegen zu halten: Erstens ist gegenüber dem Aufsichtsratsassistenten nur der Aufsichtsrat weisungsbefugt, zweitens ist die Einstellung einer solchen Hilfsperson durch den Aufsichtsrat selbst möglich, so daß der Vorschlag wieder aufgegriffen werden sollte. ${ }^{664}$ Es ist jedoch nicht davon auszugehen, daß trotz Unterstützung durch einen Aufsichtsratsassistenten die oben angesprochenen komplexen Planungsaufgaben durch ein einzelnes Aufsichtsratsmitglied bearbeitbar sind, so daß weitere Alternativen ermittelt werden müssen.

\section{(3) Arbeitsgruppenprinzip \\ (a) Überblick}

Aufgrund der fehlenden Eignung der bisher diskutierten Strukturierungsalternativen zur Bewältigung der Planungsaufgaben wird abschließend die Anwendung des Arbeitsgruppenprinzips analysiert. Hiernach werden Aufgaben oder/und Entscheidungsbefugnisse auf einzelne Arbeitsgruppen übertragen, in denen sachkundige Personen aus der Primärorganisation in Form der Gruppenarbeit sämtliche problembezogenen Aspekte sammeln und auswerten. ${ }^{665}$ Auch wenn die Arbeitsgruppen aus Mitgliedern der Primärorganisation bestehen, können sie fallweise durch externe Sachverständige ergänzt werden. Mit Bezug auf den Aufsichtsrat wird als Ausdruck des Arbeitsgruppenprinzips in der Literatur seit langem die verstärkte Einrichtung von Ausschüssen zur Bewältigung der anstehenden Überwachungsaufgaben diskutiert. ${ }^{666}$

Zur Charakterisierung von Ausschüssen können verschiedene Merkmale herangezogen werden: ${ }^{667}$

1. Beteiligung von zwei oder mehr Aufgabenträgern,

663 Vgl. Feddersen, Aufsichtsrat, 2000, S. 395

664 Vgl. Lutter/Krieger, Rechte, 1993, S. 164; Feddersen, Aufsichtsrat, 2000, S. 395, hat ermittelt, $\mathrm{da} B$ in sechs der $30 \mathrm{DAX}$-Gesellschaften bereits ein mehr oder weniger deutlich so bezeichneter Aufsichtsratsassistent existiert.

Vgl. Schulte-Zurhausen, Organisation, 1999, S. 166, 280; zu Gruppen beispielsweise umfassend Staehle, Management, 1999, S. 265-413.

666 Vgl. beispielsweise Lutter/Krieger, Rechte, 1993, S. 199, oder Deckert, Ausschüsse, 1996, S. 985-994; ablehnend Sünner, Corporate Governance, 2000, S. 496; aus organisationstheoretischer Perspektive Mag, Ausschüsse, 1992, Sp. 252ff., sowie Krüger, Organisation, 1994, S. 55ff.; Schulte-Zurhausen, Organisation, 1999, S. 165, weist m.w.N. auf die häufige Verwendung ähnlicher Begriffe wie Gremium, Komitee, Kommission, Sitzung, Besprechung, Kollegium, Konferenz, Task Force, Team, Projektgruppe usw. hin, die hier nachfolgend unberücksichtigt bleiben; zu einem partiellen Abgrenzungsversuch Schulte-Zurhausen, Organisation, 1999, S. 167.

Vgl. Mag, Ausschüsse, 1992, Sp. 252; Schulte-Zurhausen, Organisation, 1999, S. 168. 
2. Mitglieder kommen aus unterschiedlichen Bereichen der Primärorganisation,

3. keine interne formal-hierarchische Strukturierung,

4. Erfüllung bestimmter oder gelegentlich auftretender Aufgaben,

5. ein Ausschuß tritt nur diskontinuierlich zusammen oder er ist nur zeitlich befristet tätig.

Von den in der Literatur genannten Gründen für die Einrichtung von Ausschüssen sind im vorliegenden Problemzusammenhang Vorteile bei der Problembewältigung sowie die rasche Reaktionszeit bei veränderten Umweltbedingungen zu nennen. Ausschüsse führen aufgrund ihrer im Vergleich zum Gesamtgremium geringeren Größe zu einer effektiveren Arbeitsweise und erlauben außerdem eine leichtere Vereinbarkeit von Zusammenkünften. ${ }^{668}$ Darüber hinaus führt die größere Nähe der Ausschußmitglieder zu verbesserten zwischenmenschlichen Beziehungen, die eine größere Kreativität und Motivation bewirken können. ${ }^{669}$ Nachteilig können sich dagegen beispielsweise Informationsasymmetrien zwischen dem Ausschuß und den übrigen Mitgliedern der Primärorganisation oder Risikoverschiebungen (Risky-Shift) auswirken.

Für den Aufsichtsrat ermöglichen Ausschüsse neben den oben genannten allgemeinen Vorteilen vor allem eine anforderungsgerechte Arbeitsteilung, so daß nicht jedes einzelne Aufsichtsratsmitglied über alle erforderlichen Qualifikationen verfügen muß. ${ }^{670}$ Eine qualitativ hochwertige Ausschußbesetzung ist gleichzeitig Voraussetzung für eine konstruktive Zusammenarbeit und Diskussion mit dem Vorstand. ${ }^{671}$ Die Untergrenze für die Anzahl der Mitglieder in Aufsichtsratsausschüssen beträgt zwei bzw. drei im Falle entscheidender Ausschüsse, für die Obergrenze schlägt LUDWIG maximal sieben Mitglieder vor. Als optimale Gruppengröße werden drei bis fünf Personen angesehen. ${ }^{672}$

Die Vorteilhaftigkeit von Aufsichtsratsausschüssen hat auch der Gesetzgeber erkannt und mit dem KonTraG Anregungen zur vermehrten Einrichtung von Aus-

668 Vgl. Deckert, Ausschüsse, 1996, S. 987; Frese, Organisation, 2000, S. 338ff.; Ludwig, Aufsichtsräte, 1997, S. 169; Mag, Ausschüsse, 1992, Sp. 252.

669 Vgl. zur Beurteilung von Gruppenarbeit Staehle, Management, 1999, S. 284-299.

670 Vgl. Deckert, Ausschüsse, 1996, S. 987; Lutter/Krieger, Rechte, 1993, S. 239; Schneider, Unternehmensüberwachung, 2000, S. 228.

671 Vgl. Deckert, Ausschüsse, 1996, S. 989.

672 Vgl. Bales, Konferenz-Führung, 1957, S. 232; Berliner Initiativkreis German Code of Corporate Governance, Code, 2000, S. 67, IV.3.3.; Hoff, Gestaltung, 1986, S. 59ff.; Ludwig, Aufsichtsräte, 1997, S. 169; Köhler, Effizienz, 1975, S. 68f.; Scheffler, Überwachung, 1995, S. 210. 
schüssen gegeben. ${ }^{673}$ Aufgrund der Aufgabenvielfalt und ihrer Heterogenität in den jeweiligen Unternehmungen wurde die Ausgestaltung des Ausschußwesens jedoch den einzelnen Gesellschaften überlassen. Auch wenn sich der Gesetzgeber im KonTraG nur zu einer Anregungsnorm durchringen konnte, so muß doch davon ausgegangen werden, daß gerade in großen Aufsichtsräten eine ordnungsgemäße Wahrnehmung der Überwachungsaufgabe nur über die Einrichtung von Ausschüssen möglich ist. ${ }^{674}$ Dies gilt aufgrund der Vorteile der Ausschußarbeit, für die insbesondere die Multipersonalität bei gleichzeitiger Begrenzung der Anzahl der Mitglieder auf eine arbeitsfähige Größe kausal ist, auch für die hier betrachteten Planungsaufgaben. Daher sollten in Aufsichtsräten Ausschüsse vorhanden sein, die Planungsaufgaben bewältigen können, so daß die Aufgabenduplizität der Aufsichtsratsmitglieder als Ressortvertreter in Verbindung mit einer Ausschußtätigkeit insgesamt zu einer eingeschränkten Matrixorganisation des Aufsichtsrats ohne Leitungsbeziehung führt. ${ }^{675}$

Nachfolgend soll analysiert werden, inwieweit in der unternehmerischen Praxis das Ausschußwesen bereits etabliert ist. Hierbei ist insbesondere der Frage nachzugehen, ob die hier diskutierten Planungsaufgaben bereits erfüllt werden könnten.

\section{(b) Empirische Analyse von Aufsichtsratsausschüssen}

Grundlage der empirischen Analyse der Aufsichtsratsausschüsse bildet eine Auswertung der Geschäftsberichte der DAX-Unternehmungen aus dem Jahre 1999 bzw. 1998/99 (vgl. Abb. 16). ${ }^{676}$ In den Geschäftsberichten müssen seit Einführung des KonTraG Angaben zu den vom Aufsichtsrat gebildeten Ausschüssen sowie ihrer Sitzungshäufigkeit gemacht werden ( $\$ 171$ Abs. 2 S. 2 AktG). ${ }^{677}$ Trotz der teilweise noch geltenden Übergangsvorschriften haben alle Unternehmungen die geforderten Angaben publiziert. Ergänzend wird eine Auswertung von 26 Aufsichtsratsgeschäftsordnungen der ,bedeutendsten deutschen Aktiengesellschaften" von FEDDERSEN aus dem Jahre 1999 herangezogen. ${ }^{678}$

\footnotetext{
673 Vgl. Feddersen, Aufsichtsrat, 2000, S. 386.

674 Zustimmend Feddersen, Aufsichtsrat, 2000, S. 386, 390, 394.

675 Vgl. Bleicher, Organisation, 1991, S. 566ff.; Chini, Aufsichtsrats-Informationssysteme, 1988, S. 85.

676 Der gesetzlich zu bildende Vermittlungsausschuß bleibt hierbei unberücksichtigt; vgl. mit entsprechenden Zahlen beispielsweise Schneider, Unternehmensüberwachung, 2000, S. 213f.

677 Vgl. hierzu kritisch Theisen, Reform, 1999, S. 243.

678 Vgl. Feddersen, Aufsichtsrat, 2000, S. 392; hierbei handelt es sich nahezu ausschließlich um DAX-Gesellschaften.
} 
Anzahl DAX-Unternehmen mit Ausschuß

(Werte in Klammern in \%)

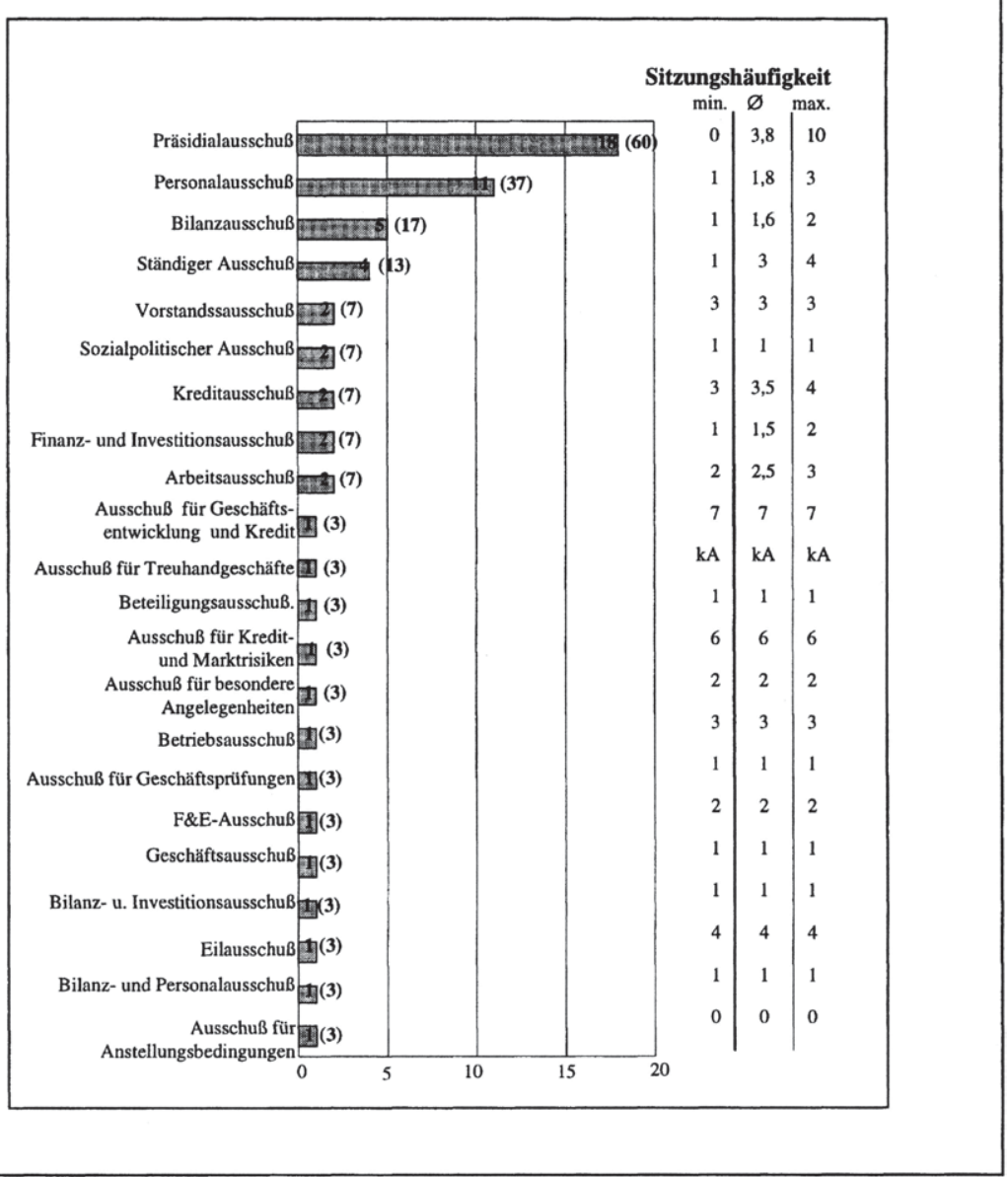

Abb. 16: Aufsichtsratsausschüsse in DAX-Unternehmungen 1999 bzw. 1998/99

Quelle: Eigene Auswertung.

In allen untersuchten Geschäftsberichten waren Angaben zu Ausschüssen enthalten. Die Auswertung zeigt jedoch ein sehr heterogenes Bild: ${ }^{679}$ Während 60\% der Unternehmungen einen Präsidialausschuß eingerichtet hatten, der im Durchschnitt 3,8 mal im Berichtsjahr tagte, und immerhin noch 37\% einen Personalausschuß (durchschnittliche Sitzungszahl im Berichtsjahr 1,8) besaßen, fallen

679 Vgl. ähnlich Feddersen, Aufsichtsrat, 2000, S. 393, dessen Aussagen sich inhaltlich nahezu mit der hier gemachten Auswertung decken. 
die übrigen Ausschüsse von ihrer Bedeutung her stark ab. Auch vor dem Hintergrund, daß sich teilweise verschieden benannte Ausschüsse mit ähnlichen Themenstellungen befassen, kann ihre Bedeutung bis auf den Bilanzausschuß als untergeordnet angesehen werden, da von den insgesamt vorhandenen 22 Ausschußarten fünf jeweils nur zweimal eingerichtet waren und 13 sogar nur einmal. Zudem lassen der Gesamtdurchschnitt aller Ausschüsse pro Gesellschaft von 2,03 auf eine sehr geringe Bedeutung des Ausschußwesens in der Praxis schließen. Diese Aussage bestätigt sich im internationalen Vergleich, bei dem entsprechende Zahlen für US-amerikanische Corporations auf einen zweieinhalb mal so hohen Wert von 5,1 kommen. ${ }^{60}$

Um eine abschließende Bewertung zu ermöglichen, ist zusätzlich die inhaltliche Ausrichtung der Ausschüsse zu berücksichtigen. Zur Klassifikation können verschiedene Kriterien herangezogen werden. ${ }^{681}$ Ausschüsse können nach der Bildungsrichtung, der Dauer des Einsatzes, der Art der Mitgliedschaft, dem Gegenstand und nach der Stellung im Führungsprozeß unterschieden werden. Da nur horizontale und auf Dauer eingerichtete Ausschüsse mit freiwilligen Mitgliedern betrachtet werden, ist für die Aufsichtsratsausschüsse lediglich der Gegenstand und ihre Stellung im Führungsprozeß variabel. Wie die obige Auflistung zeigt, orientieren sich die Gegenstände an den Überwachungsobjekten des Aufsichtsrats. Sie richten sich nach den spezifischen Gegebenheiten der jeweiligen Unternehmung und variieren stark, wobei der Arbeitsinhalt im Ausschußnamen ausgedrückt wird. ${ }^{682}$

Ihre Stellung im Führungsprozeß, d.h. die vom Aufsichtsratsausschuß wahrgenommenen Führungsfunktionen, sind weniger eindeutig. Grundsätzlich sind Planungs-, Entscheidungs- und Kontrollausschüsse von Bedeutung. ${ }^{683}$ Planungsausschüsse dienen ausschließlich der Entscheidungsvorbereitung, Entscheidungsausschüsse fassen zusätzlich Beschlüsse und Kontrollausschüsse nehmen Kontroll- und Prüfungsaufgaben ${ }^{684}$ wahr. Beide Auswertungen kommen jedoch

680 Vgl. Schneider, Unternehmensüberwachung, 2000, S. 213, und eine dort zitierte Untersuchung von Spencer Stuart aus dem Jahre 1996; so bereits auf Grundlage einer empirischen Untersuchung Bleicher/Leberl, Aufsichtsrat, 1987, S. 63.

681 Vgl. Mag, Ausschüsse, 1992, Sp. 254ff.; anders dagegen Kosiol, Organisation, 1962, S. 161ff.; dem folgend auch Schulte-Zurhausen, Organisation, 1999, S. 169.

682 Vgl. Feddersen, Aufsichtsrat, 2000, S. 393.

683 Vgl. Mag, Ausschüsse, 1992, Sp. 254ff.; Schneider, Unternehmensüberwachung, 2000, S. 211, spricht von vorbereitenden, beschließenden und überwachenden Ausschüssen; aus juristischer Perspektive auch Siebel, Ausschüsse, 1999, S. 314ff.

684 Mit der Forderung nach einer verstärkten Einrichtung von Audit Committees in Aktiengesellschaften Arbeitskreis „Externe und interne Überwachung der Unternehmung“, Prüfungsausschüsse, 2000, S. 2285; vgl. auch Coenenberg/Reinhart/Schmitz, Audit Committees, 1997, S. 989-997; 
zum Ergebnis, daß sowohl Entscheidungs- und vor allem Kontrollaufgaben dominieren. Eine Beschlußvorbereitung wird zwar bei einigen Ausschüssen explizit genannt, die geringe Häufigkeit solcher Ausschüsse in Verbindung mit der sehr begrenzten Sitzungsfrequenz läßt jedoch vermuten, daß bei den hier betrachteten DAX-Gesellschaften den entscheidungsvorbereitenden Ausschüssen insgesamt nur eine untergeordnete bis gar keine Bedeutung beikommt. Daher werden nachfolgend für die Bearbeitung der Planungsaufgaben geeignete Ausschüsse vorgeschlagen. ${ }^{685}$

\section{(c) Einrichtung von Planungsausschüssen}

Wie die Analyse der Ausschußpraxis im Aufsichtsrat zeigt, ist zu bezweifeln, daß selbst die bisher im Vordergrund stehenden Entscheidungs- und vor allem Kontrollaufgaben zweckentsprechend erfüllt werden können. Darüber hinausgehende Potentiale zur Durchführung der notwendigen Planungsaufgaben scheinen dagegen nicht vorhanden zu sein. Dies gilt sowohl inhaltlich als auch bezüglich der bisherigen Arbeitsweise, da Sitzungen üblicherweise nur sporadisch oder sogar gar nicht stattfinden, d. h. der Ausschuß existiert in diesen Fällen nur formal, ohne umfassend tätig zu werden. Aufgrund der angesprochenen Aufgabenkomplexität, des Aufgabeninhalts und des Aufgabenumfangs sollten daher alle oben genannten Planungsaufgaben speziellen Planungsausschüssen ${ }^{686}$ übertragen werden, die nicht oder nur partiell mit den bisher bekannten Ausschüssen identisch sind. Hierzu wird die zusätzliche Einrichtung folgender Ausschüsse vorgeschlagen, denen die oben gruppierten Planungsaufgaben zu Durchführung zu übertragen sind:

1. Vorstandsausschuß zur Behandlung aller personellen Angelegenheiten und zur Gestaltung der Organisation des Vorstands. ${ }^{687}$ Beide Aufgaben zeichnen sich durch ausgeprägte Interdependenzen aus, so daß eine ausschließliche Behandlung der personellen Angelegenheiten unzureichend wäre.

Girnghuber, Audit Committee, 1998; Langenbucher/Blaum, Audit Committees, 1994, S. 2197 2206; Lück, Audit Committees, 1999, S. 507-523; Lück, Prüfungsausschüsse, 1999, S. 441-443; Niehus, Audit Committees, 1999, S. 1765-1769.

Mit der Empfehlung zu einer vermehrten Ausschußbildung auch Feddersen, Aufsichtsrat, 2000, S. $393 f$.

Vgl. Krüger, Organisation, 1994, S. 56, der die besondere Eignung von Ausschüssen zur Entscheidungsvorbereitung betont.

687 Feddersen, Aufsichtsrat, 2000, S. 394, schlägt die Übernahme personeller Aufgaben durch den Vermittlungsausschuß nach $\S \S 27$ Abs. 3, 31 Abs. 3, 5 MitbestG als Zeichen einer zustimmenden Position zur Mitbestimmung vor; mit der Forderung zur Einrichtung eines Personalausschusses beispielsweise Grundsatzkommission Corporate Governance, Corporate Governance-Grundsätze, 2000, S. 241. 
2. Strategieausschuß zur Behandlung aller Fragestellungen im Kontext des strategischen Unternehmungsführungsprozesses. ${ }^{688}$ Dies umfaßt neben der strategischen Kontrolle insbesondere auch die strategische Planung. ${ }^{689}$

3. Organisationsausschuß zur ordnungsgemäßen Aufsichtsratsorganisation einschließlich des Entwurfs einer Geschäftsordnung und der Beschlußvorbereitung im Rahmen der personellen Angelegenheiten der Aufsichtsratsmitglieder. ${ }^{690}$

Für neuartige und nicht vorhersehbare Aufgaben ist es darüber hinaus jederzeit möglich, kurzfristig entsprechende Ausschüsse zu bilden. Zu solchen Aufgaben zählen beispielsweise die ebenfalls angesprochenen Reorganisationen. Dort, wo Ausschüsse in verwandten Themenfeldern bereits existieren, sollten deren Aufgaben in die entsprechenden Planungsausschüsse integriert werden.

Neben den Planungsausschüssen kommt einigen der bereits heute bekannten Ausschüsse eine besondere Bedeutung in bezug auf die Planungsaufgaben sowie die hier insgesamt vorgeschlagene Aufsichtsratsorganisation zu:

1. Das Präsidium sollte aufgrund der steigenden Organisationskomplexität des Aufsichtsrats primär zur Koordination der Aufsichtsratsarbeit dienen. Die hierzu notwendigen Freiräume entstehen durch die Auslagerung von Fachaufgaben auf Spezialausschüsse. Hierzu zählen beispielsweise die bisher häufig vom Präsidium wahrgenommenen Vorstandspersonalia.

2. Darüber hinaus sollte immer auch ein Arbeitsausschuß zur Erledigung eilbedürftiger Aufgaben vorhanden sein. Er kann mit dem Strategieausschuß identisch sein, da in dessen Aufgabenbereich die zustimmungspflichtigen Geschäfte fallen.

3. Ein Technischer Ausschuß ist zur sachgerechten Vorbereitung technologieorientierter Entscheidungen erforderlich. Die Einrichtung eines solchen Ausschusses ist jedoch abhängig von den technologischen Rahmenbedin-

688 Vgl. Deckert, Ausschüsse, 1996, S. 988; DWS, Corporate Governance, o.J., S. 7; Möllers, Professionalisierung, 1995, S. $1730 \mathrm{f}$.

689 Vgl. Claussen, Corporate Governance-Grundsätze, 2000, S. 491, der „die Vorbereitung von strategischen Entscheidungen" dem Präsidial- und Strategieausschuß zuweist; ebenso Lutter, Aufsichtsratstätigkeit, 1995, S. 289, der hierzu die Einrichtung eines Planungsausschusses fordert.

690 Vgl. Grundsatzkommission Corporate Governance, Corporate Governance-Grundsätze, 2000, S. 241, die einen Auswahl- und Ernennungsausschuß des Aufsichtsrats vorschlagen, der sich primär mit personellen aber auch mit organisatorischen Angelegenheiten des Aufsichtsrats zu befassen hat. 
gungen der Unternehmung und der Branche, so daß er nicht grundsätzlich vorhanden sein muß. ${ }^{691}$

Abschließend muß auch für den Aufsichtsratsausschuß dessen externe und interne Strukturierung festgelegt werden. Ebenso wie der Aufsichtsrat verfügt jeder Ausschuß über einen Vorsitzenden, der primär für die Koordination der Ausschußarbeit sowie für die Informationsversorgung und -weitergabe zuständig ist.

\section{(d) Entscheidungsdelegation an Ausschüsse}

Grundsätzlich können im Rahmen der rechtlichen Delegationsmöglichkeiten neben Planungsaufgaben auch die korrespondierenden Entscheidungsaufgaben an Ausschüsse delegiert werden. Daher ist jetzt zu untersuchen, ob und wenn ja, wann dies geschehen sollte. Dabei sollte sich der Aufsichtsrat an dem Grundsatz orientieren, nur die sachlich unbedingt notwendigen Entscheidungskompetenzen zu delegieren, da nur hierdurch gewährleistet werden kann, daß jedes Aufsichtsratsmitglied seine unternehmerische Verantwortung über die Plenumsentscheidung tatsächlich trägt. ${ }^{692}$ Dies schließt eine Entscheidungsvorbereitung durch einen Ausschuß oder bei weniger komplexen Problemen durch ein einzelnes Aufsichtsratsmitglied nicht aus. Zur Festlegung, ob ein Ausschuß neben den Planungs- auch die korrespondierenden Entscheidungsaufgaben übertragen bekommt, werden nachfolgend verschiedene Kriterien betrachtet.

$\mathrm{Zu}$ den Determinanten können die Möglichkeit einer Zusammenarbeit in der Gruppe, die Dauer der Entscheidungsfindung, die Notwendigkeit der Diskretion und die Problemlösungsfähigkeit gezählt werden. Während die in Ausschüssen im Vergleich zum Plenum einfachere Zusammenarbeit, die Schnelligkeit und die größere Diskretion für die möglichst weitgehende Bündelung sowohl von Planungs- als auch von Entscheidungsaufgaben in Entscheidungsausschüssen sprechen, kann die bessere Problemlösungsfähigkeit auch genutzt werden, wenn lediglich die Entscheidungen des Plenums vorbereitende Ausschüsse vorhanden sind. Die Problemlösungsfähigkeit ist daher als Entscheidungskriterium zu vernachlässigen. Statt dessen sollte als weiteres Kriterium die Bedeutung des Planungsobjekts herangezogen werden. Im Falle nachrangiger Angelegenheiten ist eine zusätzliche Delegation von Entscheidungsaufgaben zweckmäßig, da in solchen Fällen ein möglichst breiter Konsens im Aufsichtsrat nicht unbedingt er-

691 So hat der Aufsichtsrat der Schering AG, Geschäftsbericht 1999, S. 89, beispielsweise einen Ausschuß für Forschung und Entwicklung eingerichtet. 
forderlich ist. ${ }^{693}$ Dieser sollte jedoch immer bei bedeutsamen Fragen angestrebt werden.

Die Wirkungsweise der Kriterien kann an zwei Beispielen verdeutlicht werden. In Abb. 17 wurden hierzu zwei unterschiedliche Situationen eingezeichnet:

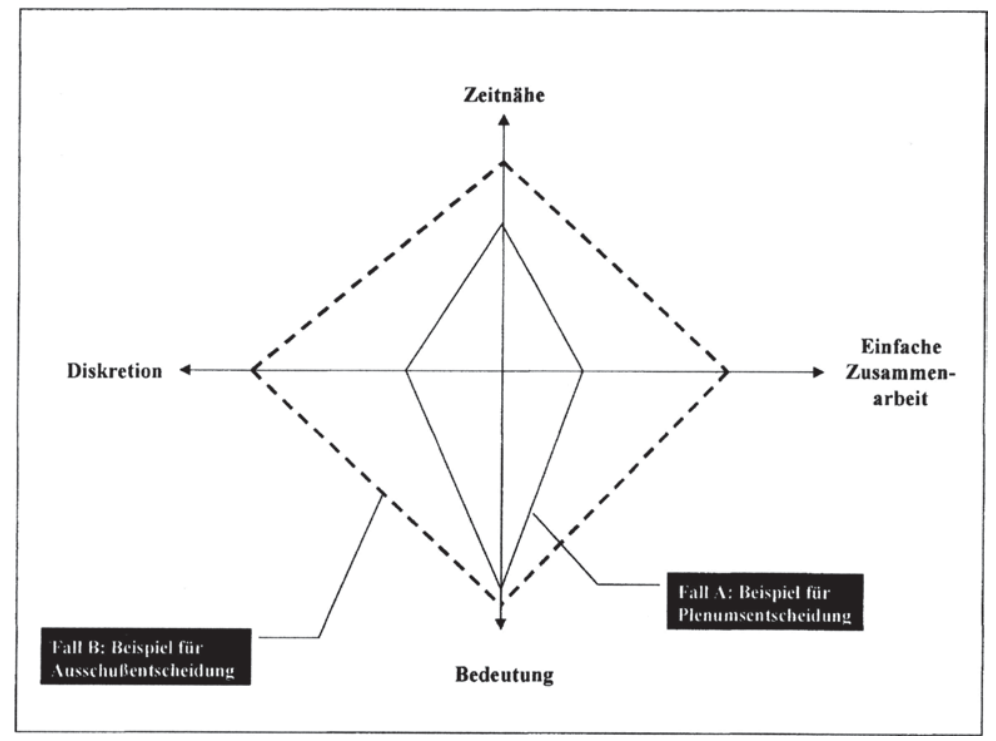

Abb. 17: Kriterien zur Einrichtung von Planungs- oder Entscheidungsausschüssen

Bei Fall A, einem Beispiel für eine Plenumsentscheidung, sind keine besonderen Ansprüche an die Diskretion zu stellen, es besteht kein Zeitdruck und somit kein großes Erfordernis einer einfachen Zusammenarbeit. Daher ist aufgrund der überaus großen Bedeutung der Entscheidung (Vorrangigkeit) eine Plenumsentscheidung anzustreben. In Fall B liegt zwar ebenfalls eine große Bedeutung der Entscheidung für die Unternehmung vor, aufgrund der hohen Erfordernisse hinsichtlich Diskretion, Zeitnähe und Zusammenarbeit ist jedoch eine Entscheidung bereits im Ausschuß herbeizuführen.

Die jeweilige Entscheidung, ob nur Planungs- oder subsidiär auch Entscheidungsaufgaben delegiert werden, kann nur im konkreten Einzelfall gefällt werden. ${ }^{694}$ Dazu werden verschiedene Planungsobjekte betrachtet.

Der erste Fall ist die Vorstandsbestellung. Dabei handelt es sich insoweit um eine besondere Situation, als die letztendliche Bestellungsentscheidung zwar nicht delegierbar ist, aufgrund der großen Bedeutung der Diskretion die Vorbe-

\footnotetext{
693 Mit einer entsprechenden Forderung Ludwig, Aufsichtsräte, 1997, S. 169.

694 Vgl. Mag, Ausschüsse, 1992, Sp. 256.
} 
reitung der Vorstandsbestellung jedoch möglichst umfassend an den Vorstandsausschuß delegiert werden sollte. ${ }^{695}$ Damit dem Plenum im Ergebnis nicht nur eine einzige Handlungsalternative präsentiert wird, können Zwischenberichte vereinbart werden, die das Plenum über den Ablauf des Bestellungsverfahrens informieren. So kann verhindert werden, daß die Entscheidungskompetenz des Plenums zu einem Vetorecht denaturiert. Es sollte aber darauf geachtet werden, $\mathrm{da} ß$ durch die Preisgabe zu umfangreicher Details die Bindung eines potentiellen Vorstandsmitglieds nicht gefährdet wird. Die sich anschließende Vertragsgestaltung mit dem Vorstandsmitglied sollte dagegen ganz dem Vorstandsausschuß zufallen. ${ }^{696}$ Um das Plenum seiner Kompetenz nicht vollständig zu entheben, ist die Verwendung von Musterverträgen für Vorstandsmitglieder anzuregen. ${ }^{697}$ Diese werden vom Aufsichtsrat verabschiedet und in den Vertragsverhandlungen zwischen Vorstandsausschuß und Vorstandskandidaten beispielsweise hinsichtlich der Vergütung konkretisiert.

Ein weiteres wichtiges Planungsobjekt sind die zustimmungspflichtigen Geschäfte. Aus Gründen der Eilbedürftigkeit und der Diskretion sollte die Entscheidung hierüber dem auch für die Planung verantwortlichen Arbeitsausschuß zugewiesen werden. ${ }^{698}$ Dieser sollte mit den Vorsitzenden der übrigen Ausschüsse besetzt sein, da hierdurch eine weitgehende Informationsfundierung der Entscheidungen realisierbar ist. LUDWIG argumentiert zwar, daß strategische Planungen und Maßnahmen für die zukünftige Entwicklung der Unternehmung von zu großer Bedeutung seien, um sie durch einen Ausschuß entscheiden zu lassen. ${ }^{699}$ Dem ist jedoch entgegen zu halten, daß gerade im Falle zustimmungspflichtiger Geschäfte sowohl die Zeitnähe als auch die Diskretion zwei wesentliche Erfolgsfaktoren darstellen. Daher sollte zwischen der Zustimmung zur strategischen Planung und den Einzelmaßnahmen zur Erreichung der strategischen Ziele unterschieden werden. ${ }^{700}$ Während die strategische Planung der Entscheidung des Plenums vorbehalten bleibt, sollte über die Einzelmaßnahmen durch einen Ausschuß entschieden werden. Soll die Entscheidungsautonomie des Aus-

\footnotetext{
695 Vgl. Feddersen, Aufsichtsrat, 2000, S. 394; Lutter/Krieger, Rechte, 1993, S. 104.

696 Vgl. Deckert, Ausschüsse, 1996, S. 990, die sogar nicht-mitbestimmte Ausschüsse vorschlägt; zustimmend Jaeger, Aufsichtsratsausschüsse, 1995, S. 1739; ablehnend dagegen Mertens, Kölner Kommentar, 1996, Anm. 111 zu § 107 AktG.

697 Vgl. hierzu beispielsweise Bredow, Management, 1998, S. 380-382, mit einer Mustervereinbarung zu Aktienoptionsplänen sowie Fonk, Personalentscheidungen, 1999, S. 788-802, mit einem Musterdienstvertrag für das Vorstandsmitglied einer AG.

698 Vgl. Deckert, Ausschüsse, 1996, S. 987.

699 Vgl. Ludwig, Aufsichtsräte, 1997, S. 169.

700 Vgl. Deckert, Ausschüsse, 1996, S. 988.
} 
schusses weiter eingeschränkt werden, kann das Plenum Vorratsbeschlüsse einsetzen. Deren Wirkungsweise kann an dem Beispiel eines geplanten Unternehmungskaufs oder einer Investition verdeutlicht werden: In einem solchen Fall gibt das Gesamtplenum seine grundsätzliche Zustimmung zu einer Maßnahme, der Arbeitsausschuß entscheidet anschließend nur noch über den konkreten Kauf. Im Ergebnis sind sowohl der Vorstands- als auch der Arbeitsausschuß in Abhängigkeit von der Aufgabe sowohl Planungs- als auch Entscheidungsausschuß.

\section{c) Kommunikationswegegestaltung}

Zur Aufbauorganisation gehört als letzter Bestandteil die Gestaltung der externen und internen Kommunikation, d.h. der Kommunikation innerhalb des Aufsichtsrats sowie die unternehmungsinterne und -externe Kommunikation des Aufsichtsrats bzw. einzelner Mitglieder. Dabei bezeichnet Kommunikation jede Verbindung zwischen zwei oder mehr Kommunikationssubjekten, bei der informationelle Kommunikationsobjekte raum-zeitlichen Veränderungen unterliegen. ${ }^{701}$ Damit der Aufsichtsrat bzw. die einzelnen Mitglieder kommunizieren können, ist die Gestaltung zweckentsprechender Kommunikationswege erforderlich. ${ }^{702}$ Von besonderer Bedeutung ist dabei die Informationsversorgung des Aufsichtsrats. ${ }^{703}$ Hier existiert ein grundlegendes Problem, da der Vorstand als diejenige Instanz, die der Aufsichtsrat überwachen soll, gleichzeitig das Monopol an überwachungsrelevanten Informationen besitzt. ${ }^{704}$ Üblicherweise werden dem Aufsichtsratsvorsitzenden durch den Vorstand die überwachungsrelevanten Informationen zur Verfügung gestellt. ${ }^{705}$ Hierdurch werden die Informationen zweifach gefiltert: Durch den Vorstand bei der Weitergabe an den Aufsichtsratsvorsitzenden und durch den Aufsichtsratsvorsitzenden bei der Weitergabe an die Aufsichtsratsmitglieder. ${ }^{706}$ Es sind daher zwei unterschiedliche Problemfelder anzusprechen: Die Informationsversorgung des Aufsichtsrats mit unterneh-

\footnotetext{
701 Vgl. Mag, Kommunikation, 1980, Sp. 1032.

702 Vgl. Mag, Unternehmungsplanung, 1995, S. $117 \mathrm{f}$.

703 Die von Mutter, Aktiengesellschaft, 1994, S. 50, angesprochenen informellen Informationsmöglichkeiten des Aufsichtsrats bleiben hier unberücksichtigt.

704 Vgl. beispielsweise Becker, Überwachungskonzepte, 1993, S. 1, 45; Wenger, Organisation, 1996 , S. 179; Werther Jr./Kerr, corporate governance, 1995, S. 64, konstatieren im Board-System ganz ähnliche Problem für das Verhältnis von Chief Executive Officer zum Board of Directors .

$705 \mathrm{Vgl}$. Chini, Aufsichtsrats-Informationssysteme, 1988, S. 86.

706 Kritisch zur Informationsfilterung durch den Aufsichtsratsvorsitzenden auch Deckert, Ausschüsse, 1996, S. 991.
} 
mungsexternen und -internen Informationen sowie die Informationsweitergabe innerhalb des Gremiums. ${ }^{707}$

Zur besseren Informationsversorgung des Aufsichtsrats wird eine aufgabenbezogene Erweiterung seiner Informationswege vorgeschlagen. ${ }^{708}$ Der Aufsichtsrat oder einzelne seiner Mitglieder sollten unmittelbar und aktiv auf Informationen der Unternehmung zugreifen können. ${ }^{709}$ Daneben ist eine passive Informationsversorgung erforderlich, indem der Aufsichtsrat regelmäßiger Berichtsempfänger innerhalb des unternehmerischen Berichtssystems wird. Dabei sollten entsprechende Fachberichte direkt an einen hierfür im Aufsichtsrat zuständigen Ausschuß oder an ein für dieses Ressort verantwortliches Aufsichtsratsmitglied gerichtet sein. HOFMANN fordert beispielsweise die unmittelbare Information des Aufsichtsrats bzw. eines Audit Committees durch das Controlling und die Interne Revision. ${ }^{710}$ Obwohl dies der herrschenden juristischen Auffassung widerspricht, verfügt der Aufsichtsrat über seine faktischen Sanktionspotentiale gegenüber dem Vorstand jederzeit über die Möglichkeit, den Vorstand zur Aufgabe gegebenenfalls vorhandener Widerstände zu bewegen. ${ }^{711}$

Daneben müssen die organinternen Informationswege gestaltet werden. Wenn das Aufsichtsratsmitglied oder der Ausschuß die notwendigen Informationen erhalten haben, verarbeiten sie diese Informationen im Falle beschlußvorbereitender Tätigkeiten zu einer Entscheidungsvorlage und leiten sie an das Plenum weiter. $^{712}$ Die Entscheidungsvorlage darf nicht nur die unterschiedlichen Alternativen mit ihrer jeweiligen Bewertung enthalten. Damit der Aufsichtsrat den Planungsproze $B$ nachvollziehen kann, sollten ebenfalls Annahmen, Restriktionen oder die eingesetzten Methoden bzw. Verfahren angegeben werden. Im Falle der Ausschußarbeit sind darüber hinaus Ausschußberichte anzufertigen, in denen das Plenum komprimiert über die Tätigkeit des Ausschusses informiert wird. ${ }^{713}$

$707 \mathrm{Zu}$ weiteren, hier nicht relevanten Informationsbeziehungen vgl. Deckert, Ausschüsse, 1996, S. 990.

708 Im Kontext der Unternehmungsplanung eine Reduktion auf die Information durch den Vorstand ablehnend auch Mutter, Aktiengesellschaft, 1994, S. 134f.

Vl. Servatius, Vorstandskontrolle, 1995, S. 225.

Vgl. Hofmann, Aufsichtsrat, 1996, S. 53; für das Verhältnis von Audit Committee und Interner Revision Langenbucher/Blaum, Audit Committees, 1994, S. 2205; auch Dreher, Organisation, 1996, S. 55; Wenger, Organisation, 1996, S. 179, verlangt sogar Weisungsrechte des Aufsichtsrats gegenüber der Innenrevision oder vergleichbaren Kontrollabteilungen.

711 Vgl. m.w.N. Möllers, Professionalisierung, 1995, S. 1728.

712 Unter ausschlieBlicher Betrachtung eines einzelnen Aufsichtsratsmitglieds Chini, AufsichtsratsInformationssysteme, 1988, S. 89.

713 Vgl. Langenbucher/Blaum, Audit Committees, 1994, S. 2205. 
Bei entscheidenden Ausschüssen wird diese Information gegebenenfalls auf die Bekanntgabe des Ergebnisses beschränkt.

Für die informationsbezogene Koordination des Gesamtorgans ist der Aufsichtsratsvorsitzende bzw. das Präsidium zuständig, ausschußintern dagegen der jeweilige Ausschußvorsitzende. Zur Systematisierung und zur Vereinfachung der Informationsversorgung, -speicherung, -verarbeitung und -weitergabe kann ein computergestütztes Aufsichtsrats-Informationssystem eingesetzt werden. ${ }^{714}$

\section{Ablauforganisation}

Neben der Aufbauorganisation muß der Aufsichtsrat bei seiner organisatorischen Gestaltung auch seine Ablauforganisation berücksichtigen, mit der die Durchführung der Planungs- und Entscheidungsprozesse strukturiert wird. ${ }^{715}$ Sie wird durch vier Ablaufordnungskomponenten beschrieben: den Arbeitsinhalt, die Arbeitszeit, den Arbeitsort sowie die Arbeitszuordnung.

Der Arbeitsinhalt beschreibt die verschiedenen durch den Aufsichtsrat zu bearbeitenden Objekte. Daneben umfaßt er die Aufgliederung des Planungs- und Entscheidungsprozesses in seine einzelnen Schritte. Die Arbeitszeit regelt die späteste Fertigstellung der einzelnen Teilaktivitäten, wohingegen der Arbeitsort eine lokale Zuordnung vornimmt. Wenn die einzelnen Arbeitsschritte, die Arbeitszeit und der Arbeitsort feststehen, wird mit der Arbeitszuordnung die Rollenverteilung innerhalb des Planungsprozesses vorgenommen. Zum Beispiel kann die Durchführung einer Organisationsplanung für den Aufsichtsrat (Arbeitsinhalt) bis zum Jahresultimo (Arbeitszeit) dem regelmäßig in der Unternehmungszentrale (Arbeitsort) zusammentretenden Vorstandsausschuß (Arbeitszuordnung) zugewiesen werden.

Ebenso wie für den Aufsichtsrat ist auch die Ablauforganisation seiner Ausschüsse zu regeln. ${ }^{716}$ Hierzu zählt insbesondere die Festlegung der vier Ablaufordnungskomponenten für ihre Sitzungen. Es ist darauf zu achten, daß der Ausschußvorsitzende den Ausschuß nicht dominiert, sondern neben seinen inhaltlichen Aufgaben nur koordinierende und moderierende Tätigkeiten übernimmt. Wie die Mängelliste der Aufsichtsratsorganisation gezeigt hat, sollte innerhalb der Ablauforganisation sowohl bei den Plenumssitzungen als auch bei denen der Ausschüsse insbesondere auf eine effektive und effiziente Sitzungsplanung und

\footnotetext{
714 Vgl. in Ansätzen Chini, Aufsichtsrats-Informationssysteme, 1988, S. 87ff.

715 Vgl. Mag, Unternehmungsplanung, 1995, S. 125ff.; abweichend zur Ablauforganisation die Gestaltung der Informationswege zählend dagegen Chini, Aufsichtsrats-Informationssysteme, 1988, S. 86ff.

716 Vgl. Mag, Ausschüsse, 1992, Sp. 260f.
} 
-durchführung geachtet werden. ${ }^{717}$ Zudem ist es für eine ordnungsgemäße Erfüllung der Ausschußaufgaben unbedingt erforderlich, daß sowohl die Zahl der Ausschußsitzungen deutlich erhöht als auch die Arbeit zwischen den Sitzungen intensiviert wird.

\section{Personelle Angelegenheiten des Aufsichtsrats}

In enger Verbindung mit seiner Organisation hat der Aufsichtsrat in verschiedenen personellen Angelegenheiten seiner Mitglieder zu entscheiden. Neben der Entscheidung über Beraterverträge mit Aufsichtsratsmitgliedern sowie über Kredite ( $\S 114,115$ AktG), bei denen eine detaillierte Entscheidungsvorbereitung nicht erforderlich ist, zählt hierzu die Aufgabe, für die verschiedenen Aufsichtsratsstellen geeignete Personen zu finden, ${ }^{718}$ da für die Wahl von Aufsichtsratsmitgliedern der Anteilseignervertreter der Aufsichtsrat alleine ein Vorschlagsrecht hat ( $\$ 124$ Abs. 3 S. 1 AktG). Diese Aufgabe wird jedoch in der bisherigen Praxis nur unzureichend wahrgenommen. ${ }^{719}$

Damit der Aufsichtsrat über die bestgeeigneten Mitglieder verfügt, ist zu fordern, daß er die Wahlvorschläge planerisch vorbereitet. ${ }^{720} \mathrm{Da}$ die Aufsichtsratsmitglieder ebenso wenig wie die Vorstandsmitglieder zum Personal der Unternehmung zählen, kann hier von einer Aufsichtsratsbesetzungsplanung gesprochen werden. Damit sie zweckentsprechend erfolgen kann, ist es erforderlich, daß bei der Wahl der Aufsichtsratsmitglieder von der häufig zu beobachtenden Listenwahl abgewichen wird und statt dessen der Wahlvorschlag stellenbezogen erfolgt. $^{721}$ Dies würde dazu führen, daß Aufsichtsratsmitglieder zu wählen sind, die als Vorsitzender, als Mitglied in einem bestimmten Ausschuß oder für ein vorher festgelegtes Ressort tätig werden sollen. Hierdurch wäre die Hauptversammlung in die Lage versetzt, für die von ihr zu besetzenden Aufsichtsratsstellen geeignete Kandidaten auszuwählen und gleichzeitig die Kooptationsgefahr durch den Vorstand vermindert. ${ }^{722}$ Wie die juristische Analyse gezeigt hat, könnte sie darüber hinaus bereits de lege lata über das gesetzliche Mindestmaß

717 Vgl. Malik, Kritik, 1998, S. 27, der hier von Sitzungsmanagement spricht.

718 Die zunehmende Bedeutung spezifischer Kenntnisse zeigt die Berufung Karel van Mierts für den ehemaligen Vorstandsvorsitzenden Gieske in den Aufsichtsrat der RWE AG; er soll bei der Lösung kartellrechtlicher Probleme helfen; vgl. o.V., Märkte, 2001, o.S.

719 Für Frühauf, Unternehmenspraxis, 1998, S. 417, erscheint die Auswahl neuer Mitglieder unter maßgeblichen Einfluß des Vorstandsvorsitzenden aus Sicht der Unternehmenspraxis zwar nicht ganz systemkonform, sie sei im Falle herausragender Persönlichkeiten jedoch effizient.

Aufgrund der Wahlvorschriften ist dies nur für die Anteilseignervertreter sinnvoll. 
hinaus höhere Anforderungen an die Vertreter der Anteilseigner formulieren. Erfüllt ein Aufsichtsratsmitglied die Erwartungen nicht, wäre auch eine Nichtwiederwahl einfacher möglich. ${ }^{723}$ Zudem könnte die Zusammensetzung des Aufsichtsrats flexibler gehandhabt werden, wenn nicht nur von der Listenwahl abgewichen würde, sondern darüber hinaus die Wahl der einzelnen Aufsichtsratsmitglieder an unterschiedlichen Hauptversammlungsterminen stattfände. ${ }^{724}$

SCHNEIDER bemängelt ebenfalls, daß der Aufsichtsrat die Aufgabe einer systematischen Gewinnung von Aufsichtsratsmitgliedern nur unzureichend verfolgt. ${ }^{725} \mathrm{Er}$ schlägt daher vor, daß der für die Vorstandspersonalia zuständige Ausschuß auch die Besetzung des Aufsichtsgremiums vorbereiten solle. So notwendig die Wahrnehmung dieser Aufgabe durch den Aufsichtsrat zwar ist, ist dennoch zu fordern, daß beide personellen Bereiche strikt getrennt bleiben. Durch die Separation können Junktims verhindert werden, wodurch die Unabhängigkeit beider Entscheidungen unterstützt wird. Daher wird hier angeregt, daß der für die Organisationsfragen zuständige Organisationsausschuß des Aufsichtsrats auch über die personellen Angelegenheiten der Aufsichtsratsmitglieder entscheidet.

Zur Gewinnung qualifizierter Aufsichtsratsmitglieder sollte der Aufsichtsrat bzw. der beauftragte Organisationsausschuß ebenso wie für den Vorstand Personalberatungsunternehmungen einschalten. ${ }^{726}$ Diese Aktivitäten können zu einer Verbesserung des Informationsstandes der Aktionäre über die Qualifikation potentieller Aufsichtsratsmitglieder führen (Screening) und über den Abbau von

723 Mit entsprechenden Vorschlägen zu einer durch den Einfluß institutioneller Investoren ausgelösten Leistungsbeurteilung des amerikanischen Board of Directors s. Conger/Finegold/Lawler, Performance, 1998, S. 136-148; die auch in Deutschland zunehmende Relevanz einer Leistungsbeurteilung von Aufsichtsräten verdeutlicht eine Studie der DWS aus dem Jahre 2000; vgl. hierzu Nölting, Druck, 2000, S. 132-137; der Berliner Initiativkreis German Code of Corporate Governance, Code, 2000, S. 67, IV.2.6., schlägt außerdem die Durchführung von Evaluationen des Aufsichtsrats vor; mit einem umfassenden Beurteilungs- und Analyseinstrument Bassen u.a., Scorecard, 2000, S. 693-698, sowie Deutsche Vereinigung für Finanzanalyse und Asset Management, Scorecard, 2000; auch Déminor, Corporate Governance, 2000.

724 Vgl. mit entsprechenden Regelungen in ihren Corporate Governance-Grundsätzen Deutsche Bank AG, Corporate Governance-Grundsätze, 2001, S. 7, die zudem die Anzahl ehemaliger Vorstandsmitglieder im Aufsichtsrat auf zwei begrenzt.

Vgl. Schneider, Unternehmensüberwachung, 2000, S. 215.

Vgl. Lutter, Unternehmensverwaltung, 1995, S. 21, sowie Lutter, Aufsichtsratstätigkeit, 1995, S. 301, mit dem Vorschlag von Stellenanzeigen zur Gewinnung von Aufsichtsratsmitgliedern; zuletzt nochmals eindringlich fordernd Lutter, Qualitätsmessung, 2000; vor dem Hintergrund eines prognostizierten „dramatischen“ Nachfrageanstiegs nach qualifizierten Aufsichtsratsmitgliedern gewinnt dies zusätzlich an Bedeutung; so Henzler in o.V., Unternehmensverfassung, 2001, S. 23. 
Informationsasymmetrien eine Verringerung der Agency-Problematik zwischen Aufsichtsrat und Hauptversammlung bewirken. ${ }^{727}$

Mit der Gestaltung der Aufsichtsratsorganisation wurde das Regelungssystem beschrieben, in dem die Aufsichtsratsmitglieder als Stelleninhaber tätig werden. Dabei ist die Auswahl geeigneter Stelleninhaber mit Bezug auf das hier betrachtete Planungssystem von den Anforderungen abhängig, die sowohl organisatorisch und vor allem aufgabenabhängig variieren. Daher sollen nachfolgend zunächst die verschiedenen Planungssubjektrollen der Aufsichtsratsmitglieder unterschieden werden, um anschließend ein Anforderungsprofil für Aufsichtsratsmitglieder zu entwickeln.

\section{Der Aufsichtsrat als Planungssubjekt}

\section{Rollenverteilung im Planungsprozeß}

Den Ausgangspunkt der Betrachtung bildet die im Rahmen der Planungsorganisation aufgezeigte institutionelle Trennung von Planungs- und Entscheidungsaufgaben. Es ist danach zu fragen, welche Rollen der Aufsichtsrat bzw. einzelne seiner Mitglieder innerhalb des Planungsprozesses wahrnehmen. Zusätzlich ist zu analysieren, welche aufsichtsratsexternen Personen den Aufsichtsrat unterstützen.

Die Planungsverantwortlichen lassen Entscheidungen durch Planung systematisch vorbereiten und sind als Empfänger der Planungsempfehlung darüber hinaus für die Institutionalisierung des Planungssystems verantwortlich. ${ }^{728}$ Beide Anforderungen sind für den gesamten Aufsichtsrat in all den Fällen erfüllt, in denen er seine Entscheidungen nur vorbereiten läßt, so daß er als Planungsverantwortlicher identifiziert werden kann. In den Fällen, in denen der Aufsichtsrat dagegen nicht nur Planungs-, sondern auch Entscheidungsaufgaben auf einen Ausschuß delegiert, soll von einer subsidiären Planungsverantwortung des Ausschusses gesprochen werden. Sie muß als subsidiär bezeichnet werden, da das Gesamtplenum auch bei der Einsetzung von Ausschüssen „Herr des Verfahrens“ bleibt. ${ }^{729}$ Dies bedeutet, daß der entscheidungsbefugte Ausschuß nur so lange Planungsverantwortlicher ist, wie ihm diese Kompetenz nicht wieder vom Plenum entzogen wird.

\footnotetext{
727 Vgl. zum Screening beispielsweise Stiglitz, Information, 1975, S. 27-52, sowie im Rahmen der Personalbeurteilung und -auswahl Schulte-Florian, Karriere, 1999, S. 143f.

728 Vgl. Mag, Unternehmungsplanung, 1995, S. 114f.

729

Vgl. Lutter/Krieger, Rechte, 1993, S. 201.
} 
Planungsträger (Planer) führen die eigentlichen Planungsaktivitäten im Auftrag der Planungsverantwortlichen durch ${ }^{730}$ Hierfür kommen neben den Aufsichtsratsausschüssen in begrenztem Maße einzelne Aufsichtsratsmitglieder und auch aufsichtsratsexterne Personen in Betracht. Die aufsichtsratsexternen Personen können weiter in unternehmungsinterne oder unternehmungsexterne unterschieden werden. $\mathrm{Zu}$ den potentiellen unternehmungsinternen Planungsträgern zählen alle Personen, die in der Unternehmung grundsätzlich mit Planungsaufgaben befaßt sind. Dies sind beispielsweise Mitarbeiter einer zentralen Planungsabteilung, die Planungsaufgaben für den Aufsichtsrat übernehmen könnten. Daneben sollten unternehmungsexterne Planungsträger verstärkt Berücksichtigung finden, $\mathrm{zu}$ denen vor allem Mitarbeiter von Unternehmungsberatungen zu rechnen sind, die als Sachverständige den Aufsichtsrat bei Einzelfragen unterstützen ( $\$ 111$ Abs. 2 S. 2 AktG). ${ }^{731}$ Als Beispiel ist hier die Einschaltung eines Unternehmungsberaters im Rahmen der strategischen Planung zu nennen. ${ }^{732}$

Sowohl die unternehmungsinternen als auch die unternehmungsexternen Personen können darüber hinaus als bloße Planungsinformanten (Planungsinformatoren) eingesetzt werden, zu denen alle Personen zählen, von denen der Planungsträger Informationen erhält. ${ }^{733} \mathrm{Zu}$ den unternehmungsinternen Informanten zählen an erster Stelle der Vorstand sowie Mitarbeiter des Rechnungswesens, des Controlling oder der Internen Revision. Wie bereits bei der Vorstellung der notwendigen Kommunikationswege gefordert wurde, sollten insbesondere zu den Unternehmungsbereichen außerhalb des Vorstands sowohl verstetigte Berichtswege eingerichtet werden als auch ein unmittelbarer Zugriff durch den Aufsichtsrat bzw. den jeweiligen Planungsträger möglich sein.

Daneben kommt dem Aufsichtsratsassistenten eine besondere Bedeutung als Planungsinformant zu. Indem dem Aufsichtsrat dauerhaft eine unternehmungsinterne Person zur Verfügung gestellt wird, kann eine Verstetigung und Intensivierung sowohl der Überwachungsarbeit im allgemeinen als auch der Planungstätigkeit im besonderen erfolgen, da der Assistent seine gesamte oder zumindest einen großen Teil seiner Arbeitszeit in der Unternehmung verbringt und dabei

730 Vgl. Mag, Unternehmungsplanung, 1995, S. 115.

731 Vgl. Mag, Unternehmungsplanung, 1995, S. 115; Unternehmungsberatungen werden bei einem inhaltsorientierten Ansatz zum Planungsträger, weniger jedoch bei einem prozeßorientierten; zu dieser Unterscheidung Kirsch/Eckert, Strategieberatung, 2000, S. 265-310.

732 Vgl. m.w.N. Möllers, Professionalisierung, 1995, S. 1730, der eine uneingeschränkte Möglichkeit zur Hinzuziehung Sachverständiger im Rahmen der Überwachungsaufgabe des Aufsichtsrats sieht.

733 Vgl. Mag, Unternehmungsplanung, 1995, S. 117. 
primär mit der Informationsversorgung des Aufsichtsrats befaßt ist. ${ }^{734}$ Neben seiner Funktion als Planungsinformant kann der Aufsichtsratsassistent in begrenztem Umfang auch als Planungsträger eingesetzt werden. Zur Gewährleistung einer umfassenden Unterstützung der Aufsichtsratsmitglieder fordert Dreyer u.a. die Installierung eines Aufsichtsrats-Controllers, der ausschließlich dem Aufsichtsrat unterstellt werden solle. ${ }^{735}$

Aufgrund der besonderen Bedeutung des Abschlußprüfers für die Tätigkeit des Aufsichtsrats ist abschließend zu klären, welche Rolle ihm im Rahmen der Planungsaufgaben des Aufsichtsrats zukommen kann. Die Funktion des Abschlußprüfers als Hilfsperson des Aufsichtsrats ist zuletzt vom Gesetzgeber mit dem KonTraG gestärkt worden. ${ }^{736}$ FEDDERSEN sieht gerade in der Reorientierung und Fokussierung des Abschlußprüfers auf die Unterstützung des Aufsichtsrats den Kern der auf den Abschlußprüfer bezogenen Reformbestandteile. ${ }^{737}$ Diese wurde notwendig, da die dem Abschlußprüfer seit seiner Einführung mit der Notverordnung von 1931 ursprünglich zugewiesene Funktion rechtstatsächlich immer mehr in den Hintergrund getreten war. Durch die Erteilung des Prüfungsauftrags an den Abschlußprüfer durch den Aufsichtsrat (§ 111 Abs. 2 AktG) und die hiermit verbundene Festlegung der Prüfungsschwerpunkte, die unmittelbare Zuleitung des Prüfungsberichts an den Aufsichtsrat (§321 Abs. 5 HGB) sowie die zwingende Teilnahme des Abschlußprüfers an der Bilanzsitzung des Aufsichtsrats bzw. eines hiermit beauftragten Aufsichtsratsausschusses $(\S 171 \text { Abs. } 1 \text { S. } 2 \text { AktG) })^{738}$ hat der Gesetzgeber die ursprünglich beabsichtigte Nähe wieder hergestellt.

Es kann jedoch festgestellt werden, daß sich aus der Reorientierung des Abschlußprüfers keine aktive Beteiligung am Planungsprozeß ergibt, da er primär zur Unterstützung des Aufsichtsrats in Kontroll- bzw. Prüfungsfragen dient. ${ }^{739}$ Auch die Veränderung der Prüfungsschwerpunkte des Abschlußprüfers - er hat das vom Vorstand einzurichtende zukunftsgerichtete Risikomanagement zu beurteilen ( $\S 321$ Abs. 4, 317 Abs. 4 HGB) - ändert hieran nichts, da sich

734 Vgl. Feddersen, Aufsichtsrat, 2000, S. 395.

735 Vgl. Dreyer, Informationskonzeptionen, 1980, S. 220f.

736 Vgl. Begründung aus dem Regierungsentwurf zum KonTraG in Ernst/Seibert/Stuckert, KonTraG, 1998, S. 57.

737 Vgl. Feddersen, Aufsichtsrat, 2000, S. 387, mit Verweis auf Hommelhoff/Mattheus, Corporate Governance, 1998, S. 249; auch Theisen, Vergabe, 1999, S. 341; kritisch auch schon vorher zum Spannungsfeld Vorstand-Abschlußprüfer-Aufsichtsrat Theisen, Abschlußprüfer, 1995, S. 186.

738 Vgl. hierzu Bischof/Oser, Teilnahmepflicht, 1998, S. 539-543.

739 Vgl. hierzu beispielsweise die Beschreibung aus Sicht des Wirtschaftsprüfers bei Rürup, Kontrolle, 1995, S. 220. 
diese Prüfung lediglich auf die Funktionsfähigkeit des Systems bezieht. ${ }^{740}$ Andererseits erhält der Aufsichtsrat durch den unmittelbaren Zugriff auf den auf ihn zugeschnittenen Prüfungsbericht in Verbindung mit den Erläuterungen durch den Abschlußprüfer detaillierte Informationen über die Unternehmung, die auch im Rahmen der Planungsaufgaben Verwendung finden sollten. Somit kann festgehalten werden, daß im Hinblick auf die Kontrollaufgaben des Aufsichtsrats eine verstärkte Zusammenarbeit mit dem Abschlußprüfer möglich ist, dem Abschlußprüfer hinsichtlich der Planungstätigkeit des Aufsichtsrats aber lediglich die Rolle eines Planungsinformanten zukommt. ${ }^{741}$

\section{Entwicklung eines Anforderungsprofils für Aufsichtsratsmitglieder a) Überblick}

In der Diskussion über die Verbesserung der Arbeit von Aufsichtsräten ist immer wieder die Forderung nach der Entwicklung eines Anforderungsprofils für Aufsichtsratsmitglieder erhoben worden. ${ }^{742}$ Diesem kommt im Zusammenhang mit dem hier diskutierten Planungssystem eine besondere Bedeutung zu, da die Qualität des Planungsergebnisses und damit die Entscheidungsqualität neben der organisatorischen Gestaltung sowie der Qualität der verarbeiteten Informationen ganz wesentlich von der Qualifikation des Planers abhängt. ${ }^{743}$ Die Ermittlung geeigneter Personen setzt mit Blick auf die Organisation die Feststellung von Anforderungen der zu besetzenden Stelle voraus, die wiederum von den einzelnen Aufgaben abhängen. ${ }^{744}$ Hierbei kann mit Blick auf die Praxis festgehalten

740 Vgl. Feddersen, Aufsichtsrat, 2000, S. 387f., sowie Salzberger, Überwachung, 2000, S. $760 f$.

741 Die Rolle des Aufsichtsrats als Überwachungsorgan des Vorstands ebenfalls betonend Potthoff, AbschluBprüfung, 1997, S. I.

742 Mit einem Beispiel Meyer-Lohmann, Aufsichtsrat, 1997, S. 156; ein Anforderungsprofil vermissend beispielsweise Lutter, Aufsichtsratstätigkeit, 1995, S. 301, sowie Möllers, Professionalisierung, 1995, S. 1733; auch Peemöller, Aufsichtsrat, 1995, S. 195f., und Semler, Wahlverfahren, 1999, S. 79, der die Notwendigkeit einer Ermittlung relevanter Fähigkeiten potentieller Aufsichtsratsmitglieder betont; der Berliner Initiativkreis German Code of Corporate Governance, Code, 2000, S. 69, IV.4.1., spricht von transparenten Kriterien als Beurteilungsgrundlage des Wahlvorschlags für die Aufsichtsratsmitglieder; Bleicher/Leberl, Aufsichtsrat, 1987, S. 64, ermittelten empirisch, daß kein Konsens über das notwendige Anforderungsprofil bestehe.

743 Vgl. Mag, Unternehmungsplanung, 1995, S. 115; mit Bezug auf die Besetzungsentscheidung des Vorstands auch Berliner Initiativkreis German Code of Corporate Governance, Code, 2000, S. 43, II.1.1.

744 Vgl. Mag, Personalplanung, 1998, S. 101f., sowie Mag/Henselek/Ruhwedel, Personalplanung, 2000, S. 27ff.; die für einen Profilvergleich ebenfalls notwendige Ermittlung der Anforderungshöhen soll hier unberücksichtigt bleiben. 
werden, daß heute die erforderlichen Fähigkeiten bei den Aufsichtsratsmitgliedern überwiegend nicht vorhanden sind. ${ }^{745}$

In einem Anforderungsprofil erfolgt die differenzierte Feststellung von Art und Höhe der benötigten Fähigkeiten. Für potentielle Stelleninhaber ist ein entsprechendes Fähigkeitsprofil zu erstellen, um durch einen Profilvergleich von Anforderungs- und Fähigkeitsprofil den am besten geeigneten Stelleninhaber ermitteln zu können. ${ }^{746}$ Bei Eignung handelt es sich um einen relativen Begriff, da nicht diejenige Person mit den maximalen Fähigkeiten eine Aufgabe übertragen bekommt, sondern diejenige, die den Anforderungen am besten entspricht. Diese Trennung in stellenbezogene Anforderungen und personenbezogene Fähigkeiten ist erforderlich, da insbesondere in der juristischen Literatur beide Dimensionen teilweise verwechselt werden, wodurch die Ermittlung von Anforderungsprofilen von Aufsichtsratsstellen zusätzlich erschwert wird. ${ }^{747}$

Grundlegend für das Anforderungsprofil sind die beschriebenen rechtlichen Mindestanforderungen. ${ }^{748}$ Vor dem Hintergrund einer seit längerem stattfindenden Diskussion wünschenswerter Fähigkeiten von Aufsichtsratsmitgliedern ist es jedoch nicht ausreichend, nur für alle Aufsichtsratsmitglieder gültige Basisanforderungen zu beschreiben, es sollten darüber hinaus spezifische Anforderungen bzw. relevante Einflußgrößen ermittelt werden. ${ }^{749}$ Da Anforderungs- und Fähigkeitsprofil hinsichtlich der enthaltenen Anforderungs- bzw. Fähigkeitsarten übereinstimmen müssen, werden mit der Nennung der Anforderungen auch die bei den potentiellen Aufsichtsratsmitgliedern notwendigen Fähigkeiten beschrieben. Während die Ermittlung der Anforderungen auf Grundlage einer Stellenbeschreibung erfolgt, können für die Fähigkeiten verschiedene fähigkeitsdiagnostische Verfahren eingesetzt werden. ${ }^{750}$

Bevor mit der Ermittlung konkreter Anforderungen begonnen werden kann, ist zunächst die Frage zu beantworten, ob dies für den Aufsichtsrat überhaupt möglich ist. Sowohl THEISEN als auch FEDDERSEN bezweifeln, daß die vom Aufsichtsrat $\mathrm{zu}$ bewältigende Überwachungsaufgabe eine allgemeingültige Be-

745 Vgl. insbesondere mit Blick auf die Arbeitnehmervertreter Möllers, Professionalisierung, 1995, S. 1733.

746 Vgl. für den Aufsichtsrat ähnlich Semler, Wahlverfahren, 1999, S. 81.

747 Vgl. beispielsweise Feddersen, Aufsichtsrat, 2000, S. 389, der von Anforderungen, Qualifikationen, Kriterien, Qualifikationsmerkmal oder Persönlichkeitsprofil spricht.

748 Vgl. hierzu Abschnitt II.B.2.c)(1).

749 Vgl. Lutter, Qualitätsmessung, 2000; Möllers, Professionalisierung, 1995, S. 1733.

750 Vgl. hierzu allgemein Mag, Personalplanung, 1998, S. $103 \mathrm{f}$. 
schreibung notwendiger Fähigkeiten zuläßt. ${ }^{751}$ Sie gehen bei ihren Ausführungen von den Fähigkeiten „des Aufsichtsratsmitglieds“ aus. Wie die obigen Ausführungen zur Planungsorganisation sowie zum Planungssubjekt jedoch gezeigt haben, gibt es nicht eine typische Aufsichtsratsstelle und hieraus folgend eine allgemein verbindliche Stellenbeschreibung. Aufgrund der Aufgabenspezialisierung innerhalb des Aufsichtsrats können vielmehr verschiedene Stellen unterschieden werden. Sie bieten einen ersten Anknüpfungspunkt für die Ermittlung eines über bloße Grundfähigkeiten hinausgehenden Anforderungsprofils. ${ }^{752}$ Als Randbedingung ist hierbei zu berücksichtigen, daß die im Aufsichtsrat insgesamt vorhandenen Fähigkeiten ihm ermöglichen sollten, seinen Überwachungsauftrag ordnungsgemäß zu erfüllen, so daß von einer Komplementarität spezifischer Fähigkeiten ausgegangen werden kann. ${ }^{753}$

Die bisher in der Literatur vorgefundenen Versuche zur Ableitung von Anforderungen an Aufsichtsratsmitglieder haben eher grundsätzlichen Charakter. ${ }^{754}$ Für eine weitergehende Systematisierung ist es erforderlich, zunächst unterschiedliche Anforderungsgruppen zu unterscheiden. ${ }^{755}$ Hierbei sind sowohl die besondere Ausgangssituation der Aufsichtsratsmitglieder zu berücksichtigen als auch ihre Aufgabenfelder. Es wird daher vorgeschlagen, die Anforderungen zunächst in zwei Hauptgruppen zu unterscheiden: die personenbezogenen Anforderungen sowie die managementbezogenen Anforderungen.

Die erste Gruppe der personenbezogenen Anforderungen beinhaltet alle Anforderungen, die in der Person und der Beziehung zum sonstigen Umfeld be-

751 Vgl. Feddersen, Aufsichtsrat, 2000, S. 389; Theisen, Unternehmungsführung, 1987, S. 258.

752 Vgl. Hommelhoff/Mattheus, Corporate Governance, 1998, S. 255; die besondere Bedeutung einer qualifikationsorientierten Besetzung von Ausschüssen betonend Deckert, Ausschüsse, 1996, S. 987.

753 Vgl. Lutter/Krieger, Rechte, 1993, S. 30; Semler, Wahlverfahren, 1999, S. 81.

754 Vgl. Bernhardt, Qualitätsmessung, 2000, S. 2; Feddersen, Aufsichtsrat, 2000, S. 389f.; Hutzschenreuter, Unternehmensverfassung, 1997, S. 187, der zudem den Aufsichtsratsvorsitzenden in den Vordergrund stellt; Hommelhoff/Mattheus, Corporate Governance, 1998, S. 255f.; Lutter/Krieger, Rechte, 1993, S. 30; Lutter, Professionalisierung, 1995, S. 1133f.; Scheffler, Aufsichtsrat, 1993, S. 69f.; Scheffler, Überwachung, 1995, S. 209f.; Scheffler, Rollenverständnis, 2000, S. 433f.; Schneider, Unternehmensüberwachung, 2000, S. 251ff.; Theisen, Unternehmungsführung, 1987, S. $251 \mathrm{ff}$.

755 Vgl. beispielsweise Mag, Personalplanung, 1998, S. 101f.; ohne inhaltliche Abgrenzung der Begriffe ähnlich Semler, Wahlverfahren, 1999, S. 79. 
gründet sind. Mit ihnen soll die persönliche Ausgangssituation des (potentiellen) Aufsichtsratsmitglieds erfaßt werden. ${ }^{756}$

Mit den Anforderungen der zweiten Gruppe werden solche berücksichtigt, die aus den unterschiedlichen Aufgaben der Aufsichtsratsmitglieder resultieren. Wie festgestellt werden konnte, unterscheiden sich diese nicht grundsätzlich von denen von Managern. Daher soll hier auf eine Einteilung notwendiger Kompetenzen bzw. Fähigkeiten zurückgegriffen werden, die als Schlüsselkompetenzen die Grundlage für eine erfolgreiche Bewältigung der Managementfunktionen bilden. ${ }^{757}$ Diese werden als managementbezogene Anforderungen bezeichnet. Beide Anforderungsgruppen werden nachfolgend für den Aufsichtsrat detailliert.

\section{b) Personenbezogene Anforderungen}

$\mathrm{Zu}$ den personenbezogenen Anforderungen gehören neben den rechtlichen Mindestanforderungen die Verschwiegenheit, die Eigenverantwortlichkeit, die Unabhängigkeit und die Gewissenhaftigkeit: ${ }^{758}$

Die Verschwiegenheit erfordert striktes Stillschweigen über alle Angelegenheiten der Unternehmung, von denen die Aufsichtsratsmitglieder bei der Ausübung ihrer Tätigkeit Kenntnis erlangen (§§ 116, 93 Abs. 1 S. 2 AktG) ${ }^{759}$ Diese Anforderung ist für mit Planungsaufgaben befaßte Aufsichtsratsmitglieder von groBer Bedeutung, da sie sich in dieser Rolle sehr detailliert mit der zukünftigen Entwicklung der Unternehmung und ihren Erfolgspotentialen befassen.

Eigenverantwortlichkeit bedeutet, daß das Aufsichtsratsmitglied nicht weisungsgebunden als Vertreter unternehmungsexterner Interessen handeln darf. ${ }^{760}$ So weist SÜNNER auf die notwendige Ausrichtung der Tätigkeit aller Aufsichtsratsmitglieder auf den Gesamtunternehmungserfolg hin und bemängelt, daß in

756 Auf die Diskussion von Altersgrenzen oder Amtsdauern sei hier jedoch verzichtet; vgl. hierzu beispielsweise Malik, Unternehmensaufsicht, 1997, S. 178-180, sowie Schneider, Unternehmensüberwachung, 2000, S. 265-268.

757 Vgl. zu der Kompetenzeinteilung Katz, Skills, 1974, S. 90ff.; Steinmann/Schreyögg, Management, 2000, S. 20; Whitley, Nature, 1989, S. 215ff.; anderer Auffassung zu den notwendigen Fähigkeiten von Aufsichtsratsmitgliedern Gollnick, Beurteilung, 1997, S. 217, der nicht „Managementqualitäten" verlangt, sondern nur die Fähigkeit, das Management zu bewerten.

758 Vgl. Scheffler, Überwachung, 1995, S. 209; Arbeitskreis „Externe und interne Überwachung der Unternehmung" der Schmalenbach-Gesellschaft/Deutsche Gesellschaft für Betriebswirtschaft e.V., Grundsätze, 1995, S. 1; ähnlich Peemöller, Aufsichtsrat, 1995, S. 197.

759 Vgl. m.w.N. Möllers, Professionalisierung, 1995, S. 1728, unter besonderer Betonung der Verschwiegenheitspflicht der Arbeitnehmerseite, sowie Theisen, Unternehmungsführung, 1987, S. 261.

760 Für Peemöller, Aufsichtsrat, 1995, S. 191, ist diese Interessenvertretung einer der wesentlichen Kritikpunkte am Aufsichtsratssystem. 
der Praxis beispielsweise bei notwendigen Betriebsschließungen oder Arbeitsplatzabbaumaßnahmen häufig eine Benachteiligung ausländischer Tochterunternehmungen zu beobachten sei. ${ }^{761}$ Er führt dies auf die Dominanz deutscher Arbeitnehmervertreter im Aufsichtsrat zurück, die die Interessen ihrer eigenen Klientel vor den Erfolg der Gesamtunternehmung stellten. Daneben beinhaltet die Eigenverantwortlichkeit die höchstpersönliche Aufgabenwahrnehmung durch das Aufsichtsratsmitglied. Dem steht die fallweise Hinzuziehung von Sachverständigen jedoch nicht entgegen.

Durch die Unabhängigkeit soll gewährleistet werden, daß das Aufsichtsratsmitglied unabhängig von seinem Überwachungsobjekt ist. ${ }^{762}$ Sowohl die Eigenverantwortlichkeit als auch die Unabhängigkeit stellen sicher, daß sich die Aufsichtsratsmitglieder in ihrem Handeln nur von den Zielen der Unternehmung leiten lassen.

Der letzte Punkt der personenbezogenen Voraussetzungen, die Gewissenhaftigkeit, erfordert einen ausreichenden zeitlichen Einsatz der Aufsichtsratsmitglieder. Vor dem Hintergrund einer verstärkten zeitlichen Beanspruchung durch die Planungsaufgaben darf das Aufsichtsratsmitglied nicht durch andere berufliche Verpflichtungen von der ordnungsgemäßen Erfüllung seiner Aufgaben abgehalten werden. ${ }^{763}$ In diesem Zusammenhang ist auf die Forderung hinzuweisen, daß zumindest der Aufsichtsratsvorsitzende in großen Publikumsaktiengesellschaften sein Amt hauptberuflich ausschließlich für eine Gesellschaft ausübt. ${ }^{764}$ Dem ist insbesondere im Fall großer Publikumsaktiengesellschaften zuzustimmen, da die hier anfallenden Aufgaben im Rahmen einer nebenamtlichen Tätigkeit nicht zu bewältigen sind. Aber auch für die übrigen Aufsichtsratsmitglieder gilt, daß sie ihrem Mandat ausreichend Zeit zur Verfügung stellen können müssen.

761 Vgl. Sünner, Corporate Governance, 2000, S. 497f.; kritisch zu Interessenkonflikten bei Bankenvertretern Monopolkommission, Leitlinien, 1998, S. 83, sowie Heermann, Interessenkonflikte, 1997, S. 1689-1728, mit Bezugnahme auf Unternehmensübernahmen.

762 Vgl. Regierungskommission „Corporate Governance“, Unternehmensführung, 2001, Rdz. 54, die fordert, daß Aufsichtsratsmitglieder keine Mandate bei Wettbewerbern einnehmen sollten.

763 Dies korrespondiert mit der Forderung der Regierungskommission „Corporate Governance“, Unternehmensführung, 2001, Rdz. 52, die Höchstzahl der konzernexternen Mandate auf fünf zu begrenzen.

$764 \mathrm{Vgl}$. Begründung aus dem Regierungsentwurf zu § $100 \mathrm{AktG}$ in Ernst/Seibert/Stuckert, KonTraG, 1998, S. 55; ablehnend u.a. Möllers, Professionalisierung, 1995, S. 1732. 


\section{c) Managementbezogene Anforderungen}

\section{(1) Allgemeine Charakterisierung}

Zur Bewältigung der Managementfunktionen sollten die (potentiellen) Aufsichtsratsmitglieder Anforderungen in drei Kompetenzfeldern erfüllen: Der technischen Kompetenz, der sozialen Kompetenz sowie der konzeptionellen Kompetenz. ${ }^{765}$

1. Mit technischer Kompetenz wird das notwendige Managementwissen bezeichnet sowie die Fähigkeit, theoretische Kenntnisse, Techniken und Methoden auf neue Problemzusammenhänge zu adaptieren. Diese Fähigkeit wird auch als Können bezeichnet.

2. Daneben gewinnt die soziale Kompetenz immer mehr an Bedeutung. Sie beinhaltet die Fähigkeit, mit anderen Personen effektiv zusammenzuarbeiten und darüber hinaus in der Lage zu sein, durch andere Menschen zu wirken.

3. Schließlich ist eine konzeptionelle Kompetenz erforderlich. Das ist die Fähigkeit, unübersichtliche komplexe Problemfelder zu strukturieren und in ein operationales Handlungskonzept zu überführen.

Es ist nachfolgend zu überlegen, wie diese einzelnen Kompetenzfelder für den Aufsichtsrat konkretisiert werden können.

\section{(2) Technische Kompetenz}

Bei der technischen Kompetenz handelt es sich um die am einfachsten zu vermittelnde und daher lange Zeit im Vordergrund stehende Kompetenz innerhalb der Managementlehre. ${ }^{766}$ Mit Blick auf den Aufsichtsrat zählen hierzu grundlegende betriebswirtschaftliche und juristische Kenntnisse. ${ }^{767}$ Sie werden ergänzt durch personelle Kenntnisse zur Beurteilung der Vorstandskandidaten, Kenntnisse zur sachgerechten Organisation eines geschäftsleitenden und eines überwachenden Gremiums, des Rechnungswesens und der Bilanzierung sowie aus dem Bereich der strategischen Führung. ${ }^{768}$

\footnotetext{
765 Vgl. zu dieser Abgrenzung Steinmann/Schreyögg, Management, 2000, S. $20 \mathrm{f}$.

Vgl. Steinmann/Schreyögg, Management, 2000, S. 20.

Vgl. Arbeitskreis „Externe und interne Überwachung der Unternehmung“ der SchmalenbachGesellschaft/Deutsche Gesellschaft für Betriebswirtschaft e.V., Grundsätze, 1995, S. 1; Feddersen, Aufsichtsrat, 2000, S. 389; Scheffler, Aufsichtsrat, 1993, S. 70; Scheffler, Überwachung, 1995, S. 209. 
Aufgrund der hier vorgeschlagenen Organisation des Aufsichtsrats, d.h. der Delegation von Aufgaben auf einzelne spezialisierte Aufsichtsratsmitglieder bzw. auf Ausschüsse, handelt es sich bei diesen Anforderungen jedoch nur um notwendige Grundkenntnisse. ${ }^{769}$ Sie werden stellenbezogen durch aufgabenspezifische Spezialkenntnisse erweitert. ${ }^{770}$ Mit Bezug auf Einzelpersonen ist hierbei zunächst an Spezialkenntnisse von Ressortvertretern sowie des Aufsichtsratsvorsitzenden zu denken. Dieser sollte außerdem Anforderungen zur Organisation und Koordination des Gesamtgremiums erfüllen sowie in der Lage sein, die Aufsichtsratssitzungen zu leiten.

Für die einzelnen Ausschußmitglieder sind darüber hinausgehende Spezialkenntnisse in dem Arbeitsgebiet des jeweiligen Ausschusses zu verlangen. ${ }^{771}$ Für Mitglieder des Organisationsausschusses ist hierbei an spezielle Organisationskenntnisse zu denken, Mitglieder des Vorstandsausschusses müssen zusätzlich Kenntnisse aus dem Bereich der Beurteilung und Auswahl von Führungskräften besitzen sowie insgesamt zur Gestaltung von Anreizsystemen unter besonderer Berücksichtigung von Stock Option-Programmen. ${ }^{772}$

Die Aufsichtsratsmitglieder sollten jedoch nicht nur über entsprechende Kenntnisse verfügen, sie müssen in der Lage sein, diese auf neue Problemstellungen zu übertragen. Dies beinhaltet die Adaption von Erfahrungen aus einer Beratungstätigkeit oder einer eigenen unternehmerischen Tätigkeit. ${ }^{773}$ FEDDERSEN unterscheidet die unternehmerische Erfahrung von der Erfahrung, Unternehmer überwachen zu können. ${ }^{774}$ Wenn potentielle Stelleninhaber lediglich über unternehmerische Erfahrung verfügen, kommt der Fähigkeit, diese auf die Tätigkeit als Aufsichtsrat zu transformieren, eine herausgehobene Bedeutung bei.

769 Mit der Forderung nach entsprechenden Grundkenntnissen aller Aufsichtsratsmitglieder beispielsweise Semler, Wahlverfahren, 1999, S. 83f.; ohne Aufgabenbezug die Kenntnisse ebenfalls in Mindest- und Fachkenntnisse unterscheidend Potthoff/Trescher, Aufsichtsratsmitglied, 1999, S. 110.

770 Vgl. Hommelhoff/Mattheus, Corporate Governance, 1998, S. 255; Semler, Wahlverfahren, 1999, S. 85.

771 Vgl. Semler, Wahlverfahren, 1999, S. 85.

772 Vgl. Baums, Aktienoptionen, 1996, S. 24, der die Notwendigkeit betont, „professionellen Sachverstand" hinzuziehen.

773 Vgl. Feddersen, Aufsichtsrat, 2000, S. 389; o.V., Aufsichtsräte, 1997, S. 18, sowie zusätzlich zur erforderlichen Beratungserfahrung Scheffler, Rollenverständnis, 2000, S. 434.

774 Vgl. Feddersen, Aufsichtsrat, 2000, S. 389. 


\section{(3) Soziale Kompetenz}

Die soziale Kompetenz zielt auf eine Interaktionsfähigkeit der Aufsichtsratsmitglieder mit weiteren Personen in ihrem Aufgabenumfeld. ${ }^{775} \mathrm{Da}$ in der vorliegenden Untersuchung der Aufsichtsrat nicht mehr als ein Gremium gesehen wird, das zu sporadischen Sitzungen zusammentritt und dessen soziale Interaktion sich auf die Aufsichtsratsmitglieder sowie von Unternehmungsseite primär auf den Vorstand beschränkt, ist die soziale Kompetenz von herausragender Bedeutung. Dies gilt sowohl im Verhältnis zwischen den Aufsichtsratsmitgliedern, die in sehr viel engerem Kontakt als bisher üblich stehen sollten, als auch in ihrer Zusammenarbeit mit weiteren unternehmungsexternen und -internen Personen. Hierzu ist eine grundsätzliche Kooperationsbereitschaft erforderlich sowie die Fähigkeit, andere Menschen zu verstehen und sich in sie hineinzuversetzen.

Eine ausgeprägte soziale Kompetenz der Aufsichtsratsmitglieder ist auch die Voraussetzung zur Umsetzung einer Forderung des BERLINER INITIATIVKREISES German Code Of CORPORATE GovernanCE, der als einen der Kernprozesse der Corporate Governance die Pflege der Diskussionskultur ansieht. ${ }^{776}$ Hierunter fallen eine offene und gelebte, engagierte Diskussionskultur in den Unternehmungsorganen. Dies wird jedoch nur dann zu erwarten sein, wenn die Aufsichtsratsmitglieder aufgrund ihrer sozialen Kompetenzen dazu in der Lage sind.

Das interkulturelle Verstehen gehört ebenfalls zur sozialen Kompetenz. ${ }^{777}$ Hiermit wird die Fähigkeit bezeichnet, über kulturelle Grenzen hinweg zu kommunizieren und gemeinschaftlich zu handeln. ${ }^{778}$ Das Vorhandensein der Fähigkeit zum interkulturellen Verstehen gewinnt vor dem Hintergrund der weiter zunehmenden Globalisierung als Voraussetzung für eine interkulturelle Zusammenarbeit zunehmend an Bedeutung. Dies geht einher mit der Forderung einer verstärkten Gewinnung unternehmungsexterner Fachleute aus dem Ausland als Aufsichtsratsmitglieder. ${ }^{779}$

\footnotetext{
775 Vgl. Steinmann/Schreyögg, Management, 2000, S. 20.

776 Vgl. Berliner Initiativkreis German Code of Corporate Governance, Code, 2000, S. 51, II.4.

777 Vgl. Steinmann/Schreyögg, Management, 2000, S. 20f.

778 Vgl. grundlegend Hansen, Kultur, 2000, S. 317ff.

779 Vgl. Lückmann, Kontrolle, 2000, S. 16, zu einer Überlegung der auf Regierungsebene installierten Grundsatzkommission Corporate Governance; Panel Qualitätsmessung von Aufsichtsräten, Thesenpapier, 2000
} 


\section{(4) Konzeptionelle Kompetenz}

Abschließend ist zu ermitteln, über welche konzeptionellen Kompetenzen Aufsichtsratsmitglieder in welchem $A u s m a ß$ verfügen sollten. Vor dem Hintergrund des hier entwickelten Aufsichtsratsplanungssystems ist zu fordern, daß zumindest die mit Planungsaufgaben beauftragten Aufsichtsratsmitglieder eine umfassende konzeptionelle Kompetenz besitzen. Hierzu zählen folgende Fähigkeiten: ${ }^{780}$

1. holistisches, kreatives sowie abstraktes Denkvermögen zur Entwicklung alternativer unternehmerischer Vorstellungen,

2. hohes Informationsverarbeitungspotential,

3. hohes Kommunikations- und Kooperationspotential, das ebenfalls Bestandteil der sozialen Kompetenz sein kann, sowie

4. ausgeprägte Eigen- und Fremdmotivation.

Diese Fähigkeiten sollen sie in die Lage versetzen, auch wenig standardisierbare Problemfelder analytisch zu durchdringen, um zweckentsprechende Gestaltungsalternativen zu entwickeln. Dies setzt eine grundsätzliche Strukturierungsund Urteilsfähigkeit voraus. ${ }^{781}$

Voraussetzung zur Entwicklung einer konzeptionellen Kompetenz ist ein grundsätzliches Verständnis für die Zusammenhänge und die Determinanten des Leistungsprozesses, da nur so für Einzelprobleme Anschlüsse an andere Entscheidungen gefunden werden können. ${ }^{782}$ Dies knüpft an den in der Literatur geforderten unternehmungs- und branchenspezifischen Kenntnissen von Aufsichtsratsmitgliedern an. ${ }^{783}$ Als bedeutsamste konzeptionelle Kompetenz ist schließlich eine grundsätzliche Lernfähigkeit zu nennen, die die Aufsichtsratsmitglie-

780 Vor dem Hintergrund der VW-Krise forderte bereits Fischer, Lehren, 1975, S. 293, die „Fähigkeit zum Denken in langfristigen Entwicklungsräumen und -zeiten"; wo diese nicht vorhanden seien, müßten sie erlernt werden; grundlegend m.w.N. Mag, Unternehmungsplanung, 1995, S. 116, sowie mit Bezug auf den Aufsichtsrat Semler, Wahlverfahren, 1999, S. 85.

781 Vgl. Steinmann/Schreyögg, Management, 2000, S. 21; zur Bedeutung der Urteilsfähigkeit Scheffler, Rollenverständnis, 2000, S. 434.

782 Vgl. Steinmann/Schreyögg, Management, 2000, S. 21.

783 Vgl. Arbeitskreis „Externe und interne Überwachung der Unternehmung“ der SchmalenbachGesellschaft/Deutsche Gesellschaft für Betriebswirtschaft e.V., Grundsätze, 1995, S. 1; Feddersen, Aufsichtsrat, 2000, S. 389; Scheffler, Aufsichtsrat, 1993, S. 70; Scheffler, Überwachung, 1995, S. 209. 
der in die Lage versetzen soll, sich permanent an veränderte Problemstellungen anzupassen. ${ }^{784}$

\section{E. Grundlagen eines computergestiitzten Aufsichtsratsunter- stiitzungssystems als Planungsinstrument}

\section{1. Überblick}

Die bisherige Diskussion des Aufsichtsratsplanungssystems hat gezeigt, daß die Aufsichtsratsmitglieder als Planungssubjekte innerhalb der Planungsorganisation vielfältige Planungs- und Entscheidungsaufgaben zu bearbeiten haben. Diese Aufgaben sind komplementär zu ihren weiteren Überwachungsaufgaben. Um ihre Tätigkeit wirkungsvoll ausüben zu können, ist es erforderlich, daß die Aufgabenträger verschiedene Instrumente einsetzen. ${ }^{785}$ Von diesen sind im vorliegenden Problemzusammenhang nur die Planungsinstrumente von Belang.

$\mathrm{Zu}$ den Planungsinstrumenten zählen als generelle phasenübergreifende Instrumente Computer und Modelle sowie als spezielle phasenbezogene Instrumente qualitative Methoden und quantitative Verfahren. Da für den Aufsichtsrat keine der Unternehmungspraxis fremden Planungsfelder erschlossen werden sollen, sondern ihm lediglich bekannte Planungsfelder neu zuzuweisen sind, kann an dieser Stelle auf eine umfassende Diskussion aller möglichen (strategischen) Planungsinstrumente verzichtet werden. ${ }^{786}$ Ihr Einsatz durch den Aufsichtsrat unterscheidet sich nicht von dem durch andere Planungsträger. Wesentliche Neuerungen können dagegen aus einer verstärkten Computerunterstützung des Aufsichtsrats erwachsen. Sie wird daher nachfolgend im Mittelpunkt stehen.

Sowohl Planung als auch die anschließende Entscheidung konnten als informationsabsorbierende, -verarbeitende und -generierende Prozesse identifiziert werden. Die Rolle computergestützter Systeme kann entweder allein darin bestehen, die Versorgung des Aufsichtsrats mit relevanten Informationen sicherzustellen, oder es können zusätzlich Möglichkeiten zur Unterstützung der Planungs- und Entscheidungsprozesse Berücksichtigung finden. ${ }^{787}$ Aufgrund der Aufgabenkomplexität des Aufsichtsrats und seiner organisatorischen Besonderheiten soll hier der zweiten, weiteren Abgrenzung gefolgt werden. Um die Potentiale einer

\footnotetext{
784 Vgl. Steinmann/Schreyögg, Management, 2000, S. 21.; mit einer entsprechenden Forderung Scheffler, Rollenverständnis, 2000, S. 434.

785 Diese Notwendigkeit betont auch Martin, Aufsichtsrat, 1990, S. 24.

786 Vgl. zu strategischen Planungsinstrumenten statt vieler anderer beispielsweise Hammer, Unternehmensplanung, 1998, S. 175ff.; Mag, Unternehmungsplanung, 1995, S. 158ff.; umfassend auch Homburg, Betriebswirtschaftslehre, 1998, sowie zu wertorientierten Instrumenten Günther, Controlling, 1997.

787 Vgl. allgemein Vetschera, Informationssysteme, 1995, S. 3.
} 
Computerunterstützung des Aufsichtsrats umfassend darzustellen, wird daher zunächst das Informationsproblem des Aufsichtsrats analysiert, um darauf aufbauend Lösungsmöglichkeiten durch ein computergestütztes Aufsichtsratsunterstützungssystem aufzuzeigen. Dabei steht nicht die detaillierte Konzipierung eines computergestuitzten Informationssystems im Vordergrund; die hier erfolgenden Ausführungen sind vielmehr als Grundlage für die Entwicklung eines solchen Systems zu verstehen.

Da sich die nachfolgenden Ausführungen sowohl mit betriebswirtschaftlichen Sachverhalten befassen als auch mit Fragestellungen aus dem Bereich der Wirtschaftsinformatik, ist eine begriffliche Abgrenzung von Informationen und Daten erforderlich. Während Informationen als zweckorientiertes Wissen bezeichnet wurden, handelt es sich bei Daten um Zeichen bzw. Zeichenfolgen (z.B. Wörter, Sätze, ganze Texte) oder Symbole (z.B. Grafiken), mit deren Hilfe ein bestimmter Sachverhalt abgebildet oder maschinell verarbeitet werden kann. Im Gegensatz zu Informationen, die nicht maschinell verarbeitbar sein müssen, steht bei Daten gerade dieser Aspekt im Vordergrund. Sie sind immer an bestimmte Träger oder Medien (z.B. Papier, Diskette, Festplatte, CD-ROM) gebunden, müssen aber nicht zwangsläufig einen Zweckbezug aufweisen ${ }^{788}$ Sind beide Kriterien erfüllt, liegen sowohl Daten als auch Informationen vor. Nachfolgend wird nur dann von Daten gesprochen, wenn der Datenverarbeitungsaspekt im Vordergrund stehen soll; ansonsten soll allgemein von Informationen gesprochen werden. ${ }^{789}$

\section{Das Informationsproblem des Aufsichtsrats}

\section{a) Beschreibung}

Das Informationsproblem des Aufsichtsrats besteht darin, daß er zum einen nicht ex ante die Menge aller benötigten Informationen bestimmen kann; zum anderen ist es dem Vorstand möglich, sich bei seiner Informationsweitergabe strategisch zu verhalten, indem er verfälschte, unvollständige oder auch zu viele Informationen (informational overload) an den Aufsichtsrat gibt, um diesen bei der Aufgabenbewältigung zu behindern. ${ }^{790}$ Daher ist zunächst eine Abgrenzung des Informationsbedarfs des Aufsichtsrats notwendig, der in Abhängigkeit von

788 Vgl. hierzu unter zusätzlicher Berücksichtigung von Wissen m.w.N. Müller, Daten, 2000, S. 5-8; auch Krcmar, Informationsmanagement, 2000, S. 10-18, sowie Voß/Gutenschwager, Informationsmanagement, 2001, S. 8-16.

789 Becker, Überwachungskonzepte, 1993, S. 37, geht sogar von einem Daten vollständig umfassenden Informationsbegriff aus.

790 Vgl. m.w.N. Becker, Überwachungskonzepte, 1993, S. 38f.; zur Informationsversorgung des Aufsichtsrats umfassend beispielsweise Dreyer, Informationskonzeptionen, 1980, oder Theisen, Information, 1996. 
dem verfolgten Zweck bzw. der zu erfüllenden Aufgabe ermittelt werden sollte. ${ }^{791}$ Dieser liegt in der Planung sowie der Entscheidung, so daß der Planungsund Entscheidungsinformationsbedarf als Menge aller erforderlichen Informationen zur Erfüllung der Planungs- und Entscheidungsaufgaben definiert werden kann. ${ }^{792}$ Hierbei soll vom objektiven Informationsbedarf gesprochen werden ${ }^{793}$ wobei jedoch nicht angenommen werden darf, daß er abschließend ermittelbar sei, da er neben der organisatorisch determinierten Aufgabe zusätzlich von subjektiven Einflüssen der informationsverarbeitenden Personen sowie situativen Faktoren abhängig ist. ${ }^{794}$ Diesem objektiven Informationsbedarf können das tatsächliche Informationsangebot der Informationssender und die tatsächliche Informationsnachfrage der Informationsempfänger gegenübergestellt werden (vgl. Abb. 18).

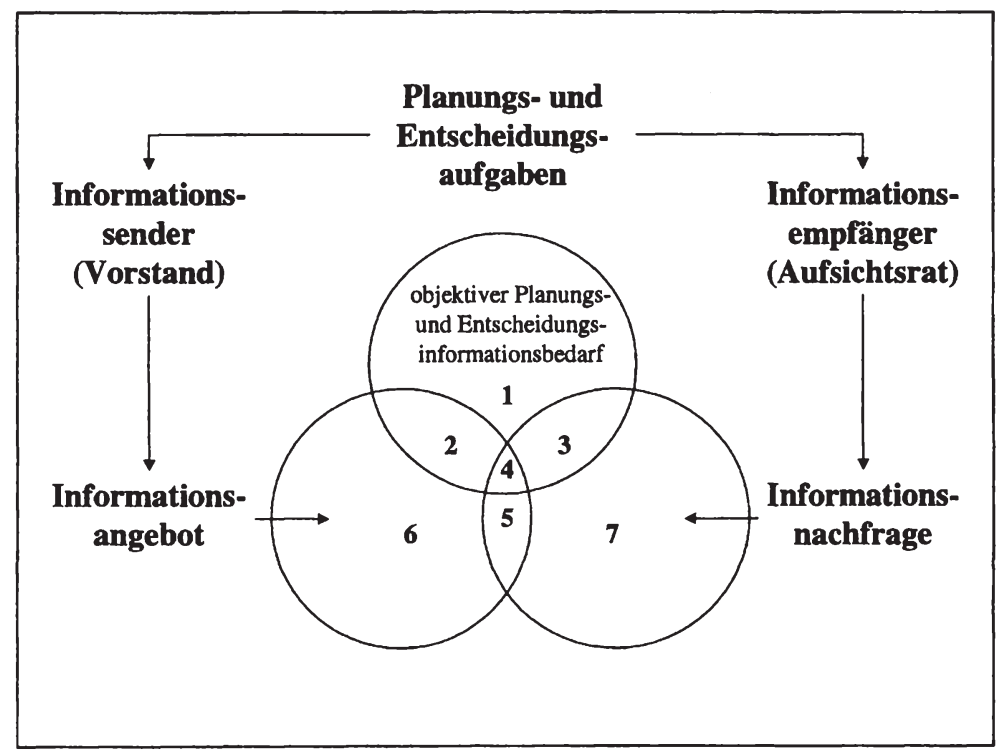

Abb. 18: Planungs- und Entscheidungsinformationsbedarf, -angebot und-nachfrage Quelle: In Anlehnung an Theisen, Information, 1996, S. 7.

791 Vgl. Krcmar, Informationsmanagement, 2000, S. 38; Becker, Überwachungskonzepte, 1993, S. 22, 41 ff.; Theisen, Information, 1996, S. 5; dies spricht die pragmatische Ebene des Übertragungsmodells der Informationstheorie an; vgl. hierzu Mag, Kommunikation, 1980, Sp. 1033; Vetschera, Informationssysteme, 1995, S. 4 f.

792 Vgl. mit Bezug auf Überwachung Theisen, Information, 1996, S. 6; auch Becker, Überwachungskonzepte, 1993, S. 42.

$793 \mathrm{Vgl}$. Picot/Franck, Information, 1988, S. 548.

794 Vgl. zur Unterscheidung von objektivem und subjektivem Informationsbedarf Picot/Franck, Information, 1988, S. 609; auch Krcmar, Informationsmanagement, 2000, S. 38; kritisch zur Ermittlung eines objektiven Informationsbedarfs Streubel, Informationsmanagement, 2000, S. $76 f$. 
In Abb. 18 sind insgesamt sieben unterschiedliche Situationen dargestellt, deren Flächengröße von der willkürlich gewählten Anordnung und Größe der Kreise abhängig ist. Sie soll verdeutlichen, daß Informationsbedarf, Informationsangebot und Informationsnachfrage in der Regel nicht übereinstimmen. ${ }^{795}$ Setzt man Informationssender mit dem Vorstand und Informationsempfänger mit dem Aufsichtsrat gleich, fragt der Aufsichtsrat alleine im Feld 4 die zur Bewältigung seiner Planungs- und Entscheidungsaufgaben benötigten Informationen nach und erhält diese auch vom Vorstand angeboten. Die Felder 5, 6 und 7 zeichnen sich dagegen dadurch aus, daß irrelevante Informationen entweder vom Vorstand angeboten, aber nicht nachgefragt werden (Feld 6), der Aufsichtsrat nicht angebotene Informationen nachfragt (Feld 7) oder sowohl nachfragt als auch angeboten bekommt (Feld 5). In Feld 2 werden dem Aufsichtsrat zwar relevante Informationen angeboten, er fragt sie jedoch nicht nach; Feld 3 beschreibt dagegen eine Situation, in der der Aufsichtsrat relevante Informationen nachfragt, aber nicht angeboten bekommt. Feld 1 beinhaltet dagegen objektiv benötigte Informationen, die jedoch weder vom Aufsichtsrat nachgefragt noch vom Vorstand angeboten werden, wodurch sogenannte Informationsschrägverteilungen entstehen.

\section{b) Informationsschrägverteilungen}

Mit Informationsschrägverteilung wird eine Situation bezeichnet, in der Informationsbedarf, Informationsangebot und Informationsnachfrage auseinanderfallen. ${ }^{796}$ THEISEN sieht hierfür verschiedene Gründe:

1. zeitliche, inhaltliche oder qualitative Informationsblockade durch die Informationssender,

2. die Informationsempfänger haben nur unzureichende Kenntnisse über die Informationsrechte sowie die Informationswege oder/und -erfordernisse,

3. subjektiv sind sowohl der Informationssender als auch der Informationsempfänger zufrieden über die bereitgestellte bzw. gebotene Informationsversorgung, obwohl diese unzureichend ist,

4. die Informationsversorgung ist objektiv hinreichend, die vorhandenen Informationen werden jedoch nur unzureichend eingesetzt.

795 Vgl. Becker, Überwachungskonzepte, 1993, S. 43ff.; Theisen, Unternehmungsführung, 1987, S. 279ff.; Theisen, Information, 1996, S. 6f.; zur grundlegenden Systematik Berthel, Informationssysteme, 1975, S. $27 \mathrm{ff}$.

796 Vgl. Theisen, Information, 1996, S. 6. 
Die Gründe der Informationsschrägverteilungen sprechen verschiedene Problemfelder an. Zur Vermeidung von Informationsblockaden sollte der Aufsichtsrat darauf drängen, daß ihm der Vorstand sämtliche benötigten Informationen zur Verfügung stellt. Dies kann durch den Vorstand selbst erfolgen oder durch andere unternehmungsinterne und -externe Informationsgeber. ${ }^{797}$

Zur Durchsetzung seiner Informationsansprüche verfügt der Aufsichtsrat über umfassende Informationsrechte, die er aufgrund seiner Informationspflicht auf jeden Fall wahrnehmen muß. ${ }^{798}$ Im Gegensatz zur juristischen Sichtweise, die teilweise nur von eingeschränkten Informationsrechten des Aufsichtsrats ausgeht, weist THEISEN aus betriebswirtschaftlicher Perspektive darauf hin, daß für die Erfüllung der Überwachungsaufgaben uneingeschränkte und uneinschränkbare Informationsrechte des Aufsichtsrats erforderlich seien. ${ }^{799}$ SEMLER sieht ebenfalls einen umfassenden Informationsanspruch in allen Mitentscheidungsangelegenheiten des Aufsichtsrats. ${ }^{800}$ Dies ist bei allen zustimmungspflichtigen Angelegenheiten der Fall, also beispielsweise im Rahmen der Zustimmung zur Unternehmungsplanung. Sind aber bereits bei Mitentscheidungsrechten umfassende Informationsansprüche begründet, können sie für die originären Entscheidungsaufgaben einschließlich der notwendigen Planungsüberlegungen nicht geringer sein.

Damit der Aufsichtsrat seine Informationsrechte wahrnehmen kann, sind zwei Voraussetzungen zu erfüllen: Er sollte zum einen die möglichen Informationswege kennen, zum anderen sollte er in der Lage sein, die notwendigen Informationen umfassend abzugrenzen. Für das erste Problem der Ermittlung von Informationswegen ist es hilfreich, die verschiedenen Informationen zunächst $\mathrm{zu}$ systematisieren. Dies kann anhand verschiedener substitutionaler oder komplementärer Kriterien erfolgen, wobei unterschiedliche Zustände der Informationen vorliegen: ${ }^{801}$ sektorale, regionale oder zeitraumbezogene Informationen können quantitativ oder qualitativ sein und sich auf unternehmungsbezogene oder unternehmungsexterne Sachverhalte beziehen. Daneben können die Informationen permanent bzw. regelmäßig oder nur einmalig vorliegen. $\mathrm{Zu}$ den unterneh-

797 Vgl. zu einer Beschreibung relevanter Informationsgeber Theisen, Information, 1996, S. 8ff.

798 Vgl. Theisen, Aufsichtsrats-Informationssysteme, 1998, S. 78; auch Becker, Überwachungskonzepte, 1993, S. 40.

799 Vgl. Theisen, Aufsichtsrats-Informationssysteme, 1998, S. 78, und der dort skizzierte Disput; die umfassenden Informationsansprüche aus juristischer Perspektive unterstützend Lutter/Krieger, Rechte, 1993, S. 219; nachhaltig ablehnend dagegen Mertens, Grundsätze, 1992, S. 208.

800 Vgl. Semler, Kompetenzen, 1999, S. 20.

${ }^{801}$ Vgl. zur Informationsklassifizierung umfassend Theisen, Information, S. 67ff.; unterschiedliche Informationszustände beschreibt Vetschera, Informationssysteme, 1995, S. 5ff. 
mungsbezogenen permanenten Informationen zählen die „Bücher und Schriften“ der Gesellschaft, Berichte des Vorstands an den Aufsichtsrat gemäß $§ 90$ AktG, Berichte der Internen Revision und des Controlling, der Jahresabschluß einschließlich Lagebericht und Abschlußprüferbericht und die Unternehmungspläne. ${ }^{802}$ Schließlich liegen die Informationen einmalig in Form von Sonderberichten der Unternehmungsführung, internen Expertisen und Stellungnahmen, externen Gutachten und Berichten sowie Sonderprüfungsberichten vor. ${ }^{803}$ Während sich die unternehmungsbezogenen Informationen ausschließlich auf unternehmungsinterne Sachverhalte beziehen, sollen die unternehmungsexternen Informationen Entwicklungen im Umfeld der Unternehmung erfassen und systematisieren. $^{804}$

Große Teile dieser Informationen sind bereits aggregiert, d.h. sie wurden über mehrere Erhebungsobjekte hinweg verdichtet, so daß sie dem Aufsichtsrat problemlos zur Verfügung gestellt werden können. ${ }^{805}$ Anders verhält es sich dagegen bei den „Büchern und Schriften“ der Gesellschaft. Dies sind alle zur Dokumentation geeigneten Datenträger, d.h. alle Dokumente, Daten-, Ton- und Bildträger sowie alle Formen der elektronischen und optischen Speicherung. ${ }^{806}$ Aufgrund seines Zugriffsrechts auf die „Bücher und Schriften“ der Gesellschaft ist der Aufsichtsrat in der Lage, auch auf disaggregierte, d.h. unverdichtete Informationen bzw. Daten innerhalb der betrieblichen Informationssysteme zugreifen. Er sollte diese Möglichkeit verstärkt nutzen, um insbesondere mögliche Informationsblockaden des Vorstands zu umgehen. Eine wichtige Voraussetzung bildet hierfür eine Computerunterstützung.

Das zweite Problemfeld einer Abgrenzung der erforderlichen Informationen des Aufsichtsrats beinhaltet die Ermittlung derjenigen betrieblichen Informationssysteme, auf die der Aufsichtsrat bei seinen Planungs- und Entscheidungsaufgaben zugreifen können sollte. Die vom Aufsichtsrat verwendeten Informationssysteme sollten in der Lage sein, für seine Planungs- und Entscheidungsaufgaben vorhandene oder beschaffbare Informationen zu sammeln, zu dokumentieren, zu verarbeiten und weiterzugeben. ${ }^{807}$ Sie umfassen sowohl Informationssysteme, die die Umwelt der Unternehmung zum Gegenstand haben, als

\footnotetext{
802 Vgl. Theisen, Information, 1996, S. 85ff.; mit einer Beschreibung notwendiger Informationen auch Becker, Überwachungskonzepte, 1993, S. 45-54.

${ }^{803}$ Vgl. Theisen, Information, 1996, S. 112ff.

$804 \mathrm{Vgl}$. Theisen, Information, 1996, S. 115ff.

Vgl. zur Verdichtung von Informationen Vetschera, Informationssysteme, 1995, S. 6.

Vgl. Theisen, Information, 1996, S. 85.

Vgl. allgemein Grünewald, Informationssysteme, 1989, Sp. 696.
} 
auch solche mit unternehmungsinternen Informationen. Informationssysteme werden an dieser Stelle allgemein als ein aufeinander abgestimmtes Arrangement verschiedener Elemente verstanden, die dazu dienen, Handlungsträger mit zweckorientiertem Wissen für die Aufgabenerfüllung zu versorgen. ${ }^{808}$

In Anlehnung an GRÜNEWALD kann die Vielzahl der für die Planung eingesetzten betrieblichen Informationssysteme nach der Abhängigkeit des Informationsinputs der Systeme vom Unternehmungsplanungssystem abgegrenzt werden. ${ }^{809}$ Durch die Verwendung dieses Kriteriums und unter Hinzufügung weiterer Informationssysteme sind folgende Informationssystemgruppen zu unterscheiden:

1. Informationssysteme, die Informationen unabhängig vom Unternehmungsplanungssystem aufnehmen. Hierzu zählen die sozioökonomische Umfeldanalyse, die technologische Trendanalyse und die Konkurrenzanalyse.

2. Daneben existieren Informationssysteme, die sowohl unternehmungsplanungsunabhängige als auch unternehmungsplanungsabhängige Informationen aufnehmen. Dies sind die Marktforschung, das Frühwarnsystem, das Risiko-Controlling ${ }^{810}$, das Personalinformationssystem ${ }^{811}$ sowie das Finanz- und Kapitalmarktinformationssystem.

3. Diese ersten beiden Gruppen werden durch unternehmungsinterne Informationssysteme ergänzt. Hierzu können die Informationssysteme Strategie, Marktpositionierung, Stärken und Schwächen der Unternehmung, Rechnungswesen, technisch-forscherische Entwicklung der Unternehmung sowie das Interne Überwachungssystem gezählt werden.

Zwischen den einzelnen Informationssystemen existieren vielfältige Informationsbeziehungen.

Zusammenfassend kann festgestellt werden, daß der Aufsichtsrat über umfassende Rechte zur Informationsbeschaffung verfügt, wozu insbesondere der Zugriff auf die „Bücher und Schriften“ der Unternehmung zählt. Über die hier erfolgte Nennung von für die Planung und Entscheidung relevanten betrieblichen Informationssystemen ist es darüber hinaus möglich, seinen Informations-

${ }^{808}$ Vgl. Picot/Maier, Informationssysteme, 1992, Sp. 923.

${ }^{809}$ Vgl. Grünewald, Informationssysteme, 1989, Sp. 696ff.

810 Zur Abgrenzung von Risiko-Controlling, Frühwarnsystem und Internem Überwachungssystem vgl. Lück, Internes Überwachungssystem, 1998, S. 183.

811 Vgl. Henselek/Mag/Ruhwedel, Personalplanung, 2001, S. 122-127. 
bedarf abzugrenzen. ${ }^{812}$ Die konkrete Informationsnachfrage ist jedoch von dem zu lösenden Planungsproblem abhängig. Daher ist zu fordern, daß für den Aufsichtsrat nicht detailliert alle möglicherweise relevanten Informationen beschrieben werden, sondern er durch den Zugriff auf sein Informationssystem über umfassende Informationsbeschaffungspotentiale verfügt, über deren Nutzung das einzelne Aufsichtsratsmitglied im jeweiligen Problemkontext individuell entscheiden sollte. ${ }^{813}$ Die Informationen stammen aus verschiedenen unternehmerischen Subsystemen, die entweder vollständig oder teilweise das Informationssystem des Aufsichtsrats bilden bzw. dieses mit Informationen versorgen. ${ }^{814}$ Für den Vorstand kann in diesem Zusammenhang von einer Bringschuld gesprochen werden, der Aufsichtsrat hat dagegen eine Holschuld. ${ }^{815}$

Der planungs- und entscheidungsbezogene Informationsbedarf des Aufsichtsrats kann nur begrenzt durch ein standardisiertes Berichtswesen gedeckt werden, wie es beispielsweise für reine Kontrolltätigkeiten möglich ist. ${ }^{816}$ Zur Lösung seines Informationsproblems und zur Deckung des aufgezeigten Planungs- und Entscheidungsinformationsbedarfs ist daher in der Literatur die Einrichtung eines computergestützten Aufsichtsratsinformationssystems vorgeschlagen worden, bei dem jedoch bisher die reine Informationsversorgung im Vordergrund steht. ${ }^{817}$ Dieses muß in der Lage sein, den Aufsichtsrat „online“ mit allen relevanten Informationen zu versorgen. ${ }^{818}$ Hier sollen dagegen über die reine Informationsbereitstellung hinaus weitere Gestaltungsmöglichkeiten aufgezeigt werden, die den Aufsichtsrat zusätzlich bei seinen Planungs- und Entscheidungsprozessen instrumentell und organisatorisch unterstützen.

${ }_{812} \mathrm{Zu}$ einer systemtechnischen Abgrenzung betrieblicher Informationssysteme vgl. Vetschera, Informationssysteme, 1995, S. 7f.

813 Mit Bezug auf die Entscheidungsunterstützung des Top-Managements eine entspechende Flexibilität fordernd auch Voß/Gutenschwager, Informationsmanagement, 2001, S. 328.

814 Vgl. Chini, Aufsichtsrats-Informationssysteme, 1988, S. 86f.; Potthoff/Trescher, Aufsichtsratsmitglied, 1999, S. 201; so geht beispielsweise Schröder, Unternehmens-Controlling, 2000, S. 130 , davon aus, daß der Aufsichtsrat einer der Informationsempfänger des Controlling ist; weitere Subsysteme nennt Theisen, Aufsichtsrats-Informationssysteme, 1998, S. 79.

$815 \mathrm{Vgl}$. Theisen, Information, 1996, S. 89; Liesen in Kaden/Wilhelm, überwachen, 1998, S. 96.

816 Selbst dies wird von Becker, Überwachungskonzepte, 1993, S. 42, bei den hier zu kontrollierenden Entscheidungssituationen bezweifelt.

817 Vgl. Chini, Aufsichtsrats-Informationssysteme, 1988, S. 87.

818 Vgl. Holzer/Makowski, Corporate Governance, 1997, S. 691. 


\section{Das computergestützte Aufsichtsratsunterstützungssystem}

a) Systemtechnische Einordnung

\section{(1) Managementunterstützungssysteme}

Aufgrund der mit Führungsaufgaben verbundenen Komplexität und der Notwendigkeit, eine Vielzahl von Informationen gewinnen, übertragen, verarbeiten und speichern zu müssen, werden für das Management von Unternehmungen seit langem computergestützte Informations- und Kommunikationssysteme eingesetzt. ${ }^{819}$ Dieses Erfordernis hat in den letzten Jahren zunehmend an Bedeutung gewonnen, so daß eine zeitnahe Informationsversorgung von Entscheidungsträgern mit qualitativ hochwertigen Informationen inzwischen als wesentlicher Wettbewerbsfaktor erkannt wurde. ${ }^{820} \mathrm{Da}$ ebenso wie für das Top-Management der Unternehmung auch für den Aufsichtsrat eine Planungs- und Entscheidungsunterstützung im Vordergrund steht, soll nachfolgend analysiert werden, inwieweit bestehende systemtechnische Möglichkeiten für den Aufsichtsrat eingesetzt werden können und welche spezifischen Anforderungen hierbei zu berücksichtigen sind. Hierzu erfolgt zunächst eine systemtechnische Einordnung von Managementunterstützungssystemen, die als Ausgangspunkt der zu entwickelnden Aufsichtsratsunterstützungssysteme herangezogen werden sollten.

Computergestützte Planungssysteme setzen sich aus verschiedenen Komponenten zusammen: Anwender oder Benutzer, das Anwendungsprogrammsystem, das Betriebssystem und das Maschinensystem (Hardware) ${ }^{821}$ Bei der Hardware dominieren inzwischen Client-Server-Strukturen, die unterschiedliche Rechnersysteme miteinander verbinden. ${ }^{822}$ Für die hier vorliegende Problemstellung ist primär die Gestaltung des Anwendungsprogrammsystems von Interesse, da seine Ausgestaltung wesentlich für die Nutzung durch das Planungssubjekt ist. Mit dem Anwendungsprogrammsystem erfolgt die Transformation von Input- zu Outputdaten, die die Basis für die Gewinnung von Informationen für das Planungsproblem bilden. ${ }^{823}$ Idealtypisch können Datenbanken und Methoden- bzw. Modellbanken als Bestandteile des Anwendungsprogrammsystems unterschieden werden, wobei insbesondere für den Nutzer die programmtechnische Tren-

\footnotetext{
819 Vgl. Gabriel, Informationssysteme, 1999, S. 419.

820 Vgl. Behme/Mucksch, Informationsversorgung, 1998, S. 4; Chamoni/Gluchowski, Informationssysteme, 1999, S. 4; zur Bedeutung von Information und Kommunikation für das Management Gluchowski/Gabriel/Chamoni, Systeme, 1997, S. 19ff.

Vgl. Mag, Unternehmungsplanung, 1995, S. 30. 
nung nicht transparent wird. ${ }^{824}$ Die Inhalte aller oben aufgeführten planungsrelevanten Informationssysteme werden im Falle einer computergestützten Planung in den Datenbanken vorgehalten, die Methoden- oder Modellbanken enthalten dagegen Algorithmen zur Durchführung von Datentransformationen. Zusätzlich werden alle notwendigen Kommunikationsprozesse unterstïtzt. Daher kann umfassender von computergestützten Informations- und Kommunikationssystemen (IuK-Systeme) gesprochen werden. ${ }^{825}$

Zur Planungs- und Entscheidungsunterstützung dienen in der betrieblichen Praxis verschiedene Klassen von IuK-Systemen. ${ }^{826}$ Im Gegensatz zu den transaktionsorientierten operativen Systemen, bei denen es sich überwiegend um betriebswirtschaftliche Standardsoftware handelt, zeichnen sich diese Management Support Systeme bzw. Managementunterstützungssysteme (MUS) durch eine explizite Unterstïtzung dispositiver Tätigkeiten aus. Allgemein bezeichnen sie alle Datenverarbeitungs-, Informations- und Kommunikationstechnologien zur Unterstuitzung unternehmerischer Aufgaben. ${ }^{827}$

Hierzu greifen die MUS auf die operativen Daten der untersten Ebene zu und verdichten sie für den Einsatz in Entscheidungsprozessen. Damit dies friktionsfrei möglich ist, müssen beide Systemklassen sowohl vertikal als auch horizontal integriert sein. ${ }^{828}$ MUS können in allen betrieblichen Bereichen und auf allen Managementebenen eingesetzt werden. ${ }^{829}$ Sie umfassen verschiedene Gruppen managementunterstützender Systeme, die in Abhängigkeit von ihrer Funktionalität abgegrenzt werden können (vgl. Abb. 19).

824 Vgl. Gluchowski/Gabriel/Chamoni, Systeme, 1997, S. 39ff.; Mag, Unternehmungsplanung, 1995, S. 31 .

Zur Abgrenzung eines computergestützten Informationssystems beispielsweise Picot/Maier, Informationssysteme, 1992, Sp. 923. 1997, S. 309; Voß/Gutenschwager, Informationsmanagement, 2001, S. 323ff. Vgl. Krallmann/Rieger, ESS, 1987, S. 29.

Vgl. Gabriel/Gluchowski, Systeme, 1997, S. 310; ebenso Behme/Mucksch, Informationsversorgung, 1998, S. 22f.

Vgl. Gabriel/Gluchowski, Systeme, 1997, S. 312. 


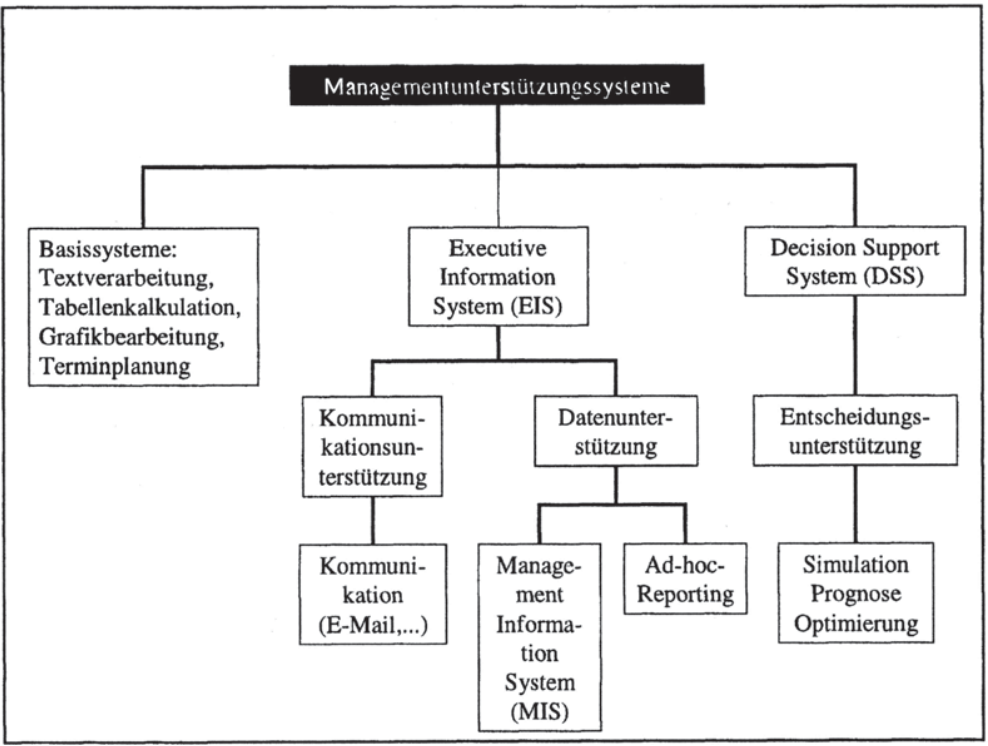

Abb. 19: Managementunterstützungssysteme

Quelle: In Anlehnung an Gluchowski/GabrieV/Chamoni, Systeme, 1997, S. 244.

Management Information Systems (MIS) dienen primär der Automatisierung von Standardberichten. ${ }^{830}$ Dies geschieht über die periodische Verdichtung und Auswertung operativer Datenbestände. Ergänzt werden diese Standardinformationen durch die Möglichkeit von Ad-hoc-Auswertungen. Da der Schwerpunkt nicht auf dem Einsatz von Methoden oder Modellen, sondern auf einer Datenunterstützung liegt, werden MIS primär für Kontrolltätigkeiten eingesetzt.

Executive Information Systems (EIS, syn. Führungsinformationssystem [FIS], Chefinformationssystem [CIS] oder Vorstandsinformationssystem [VIS] $)^{831}$ stellen in mehrfacher Hinsicht eine Erweiterung und Ergänzung der MIS dar. ${ }^{832}$ Über intuitiv nutzbare und anwenderfreundliche Benutzeroberflächen ermöglichen sie Entscheidungsträgern die Selektion und Analyse aktueller interner und externer Informationen. Hierdurch bietet sich der Einsatz von EIS bereits in frühen Phasen des Planungsprozesses, aber auch im Rahmen von Kontrolltätigkei-

830 Vgl. Chamoni/Gluchowski, Informationssysteme, 1999, S. 6f.; Gabriel/Gluchowski, Systeme, 1997, S. 422f.; ebenso Hess, Führungsinformationssysteme, 1999, S. 1503f.; zur Entwicklung und zur Kritik an der frühen Generation der MIS beispielsweise Voß/Gutenschwager, Informationsmanagement, 2001, S. 325-328.

831 Vgl. Möllmann, Unternehmensführung, 1992, S. 366.

832 Vgl. Chamoni/Gluchowski, Informationssysteme, 1999, S. 8f.; Gabriel/Gluchowski, Systeme, 1997, S. 424f.; für ein Visualisierungsbeispiel Behme/Mucksch, Informationsversorgung, 1998, S. 21; Voß/Gutenschwager, Informationsmanagement, 2001, S. 330-335. 
ten an. Daneben verfügen sie über ausgeprägte Kommunikationskomponenten, so daß nicht nur die Tätigkeit einzelner Entscheidungsträger, sondern auch Gruppenentscheidungen unterstützt werden.

Im Gegensatz zu MIS und EIS zeichnen sich Decision Support Systems (DSS) durch eine ausgeprägte Modell- und Methodenorientierung aus. ${ }^{833}$ Sie sollen Entscheidungsträger insbesondere in eher schlecht-strukturierten Entscheidungssituationen instrumentell unterstützen. ${ }^{834}$

Neben diesen verschiedenen Ausprägungen von MUS zählen zu diesen auch Basissysteme des Personal Information Management (PIM) ${ }^{835}$ Dies beinhaltet unter anderem Textverarbeitungs-, Grafik- und Terminplanungsprogramme. Die Zusammenfügung der einzelnen Bestandteile soll Entscheidungsträger in allen Phasen ihrer Aufgabenerfüllung unterstützen.

Aufgrund fehlender Interaktivität, einer übermäßigen Starrheit der Systeme, einer Informationsüberflutung bei gleichzeitig mangelnder Datenversorgung sowie einer unzureichenden Integrierbarkeit in bestehende Datenverarbeitungsund Organisationsstrukturen sind für die MUS unterschiedliche Ergänzungen entwickelt worden. Dies umfaßt unter anderem die Analytischen Informationssysteme. ${ }^{836}$

\section{(2) Weiterentwicklungen von Managementunterstuitzungssystemen}

$\mathrm{Zu}$ den Analytischen Informationssystemen können Data WarehouseKonzepte, das Konzept des On-Line Analytical Processing (OLAP) sowie das Data-Mining gezählt werden, das aufgrund der geringen praktischen Relevanz jedoch vernachlässigt werden kann. ${ }^{837}$ Ein Data Warehouse ist ein unternehmungsweites Konzept, das über den Aufbau einer logisch zentralen, einheitlichen und konsistenten Datenbasis eine informationsorientierte Entscheidungsunterstützung von Führungskräften anstrebt. Die aufzubauende Datenbasis wird

833 Vgl. umfassend Gluchowski/Gabriel/Chamoni, Systeme, 1997, S. 165ff.; Voß/Gutenschwager, Informationsmanagement, 2001, S. 335-341.

834 Vgl. Gabriel/Gluchowski, Systeme, 1997, S. 423f.

835 Vgl. Chamoni/Gluchowski, Informationssysteme, 1999, S. 9.

$836 \mathrm{Vgl}$. Gluchowski/Gabriel/Chamoni, Systeme, 1997, S. 266ff., sowie zu Analytischen Informationssystemen Chamoni/Gluchowski, Informationssysteme, 1999; zum Data Warehouse-Konzept außerdem Mucksch/Behme, Data Warehouse-Konzept, 1998; die Analytischen Informationssysteme als eigenständige Gruppe der MUS einordnend auch Voß/Gutenschwager, Informationsmanagement, 2001, S. 323.

837 Vgl. Chamoni/Gluchowski, Informationssysteme, 1999, S. 11ff., sowie Gabriel/Chamoni/Gluchowski, Management, 2000, S. 74-93; zum Data Warehouse-Konzept beispielsweise Behme/Mucksch, Data Warehouse-Konzept, 1998, S. 44ff.; Voß/Gutenschwager, Informationsmanagement, 2001, S. 249-277. 
losgelöst von den operativen Datenbanken betrieben ${ }^{838}$ Mit OLAP wird es Entscheidungsträgern ermöglicht, intuitiv dynamische, multidimensionale Analysen auf konsolidierten Unternehmungsdatenbeständen durchzuführen. ${ }^{839}$ Besondere Bedeutung wird der Multidimensionalität beigemessen, da durch sie betriebswirtschaftlich relevante Größen anhand verschiedener Dimensionen analysiert werden können.

GABRIEL/CHAMONI/GLUCHOWSKI beschreiben eine Referenzarchitektur für analytische Informationssysteme (vgl. Abb. 20): ${ }^{840}$

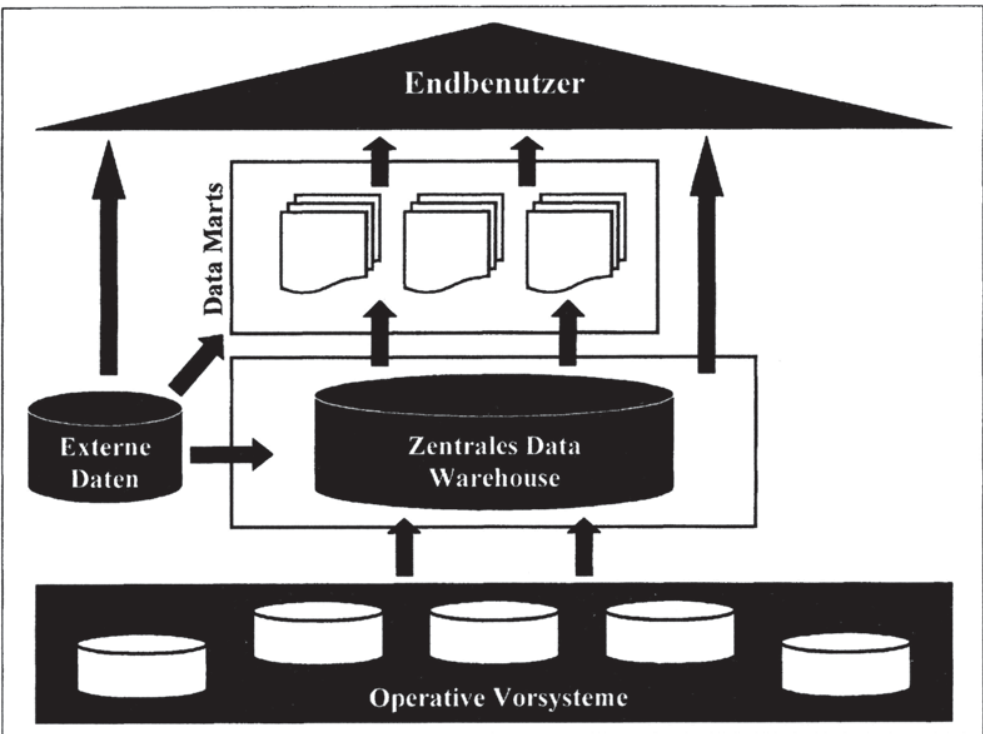

Abb. 20: Referenzarchitektur für Analytische Informationssysteme

Quelle: Gabriel/Chamoni/Gluchowski, Management, 2000, S. 83.

Im Mittelpunkt steht das Zentrale Data Warehouse, das periodisch mit Daten aus den operativen Vorsystemen sowie aus externen Informationssystemen versorgt wird. ${ }^{841} \mathrm{Um} \mathrm{zu}$ verhindern, daß durch den interaktiven Zugriff auf ein unternehmungsweites, zentrales Data Warehouse dieses zu unflexibel und schwerfällig wird, ist es möglich, funktionsbereichs- oder personengruppenzentrierte Data Marts zu bilden. Hierbei handelt es sich um themenzentrierte, auf

\footnotetext{
838 Vgl. zur Datenextraktion für Data Warehouses Müller, Data Warehouse, 1999, S. 95-117.

839 Vgl. Gabriel/Chamoni/Gluchowski, Management, 2000, S. 78.

$840 \mathrm{Vgl}$. Gabriel/Chamoni/Gluchowski, Management, 2000, S. $82 \mathrm{ff}$.

${ }^{841}$ Vgl. Chamoni/Gluchowski, Informationssysteme, 1999, S. 13; Gabriel/Chamoni/Gluchowski, Management, 2000, S. 82ff.; da es sich hierbei um quantitative Daten handelt, wird zur Integration qualitativer, unstrukturierter Daten die Erweiterung von Data Warehouse-Konzepten zu Knowledge Warehouse-Konzepten gefordert, vgl. hierzu Dittmar, Wissen, 2000, S. 14-17.
} 
die Informationsbedürfnisse der späteren Nutzer abgestimmte Datenextrakte. Sie führen zu Verbesserungen der Leistungsfähigkeit und können kostengünstiger als unternehmungsweite Data Warehouses realisiert werden. Für die Datenabfragen sowohl aus den Data Marts als auch aus dem Zentralen Data Warehouse kommen häufig OLAP-Technologien zum Einsatz. Der Systemzugriff durch den Endbenutzer erfolgt über Front-End-Werkzeuge auf den Desktop- oder LaptopRechnern. Durch den Einsatz entsprechender Oberflächengeneratoren können die Benutzeroberflächen vergleichsweise unproblematisch an die Bedürfnisse der Endbenutzer angepaßt werden. Als Weiterentwicklung der bekannten EIS unterstützen Analyseorientierte Informationssysteme die Unternehmungsleitung im Rahmen ihrer Planungs- und Entscheidungs-, aber auch der Kontrollaufgaben. ${ }^{842}$

Die übrigen Weiterentwicklungen der MUS betreffen verbesserte Visualisierungs- und Präsentationsmöglichkeiten (Multimedia-Systeme), leistungsfähigere Navigations- und Suchwerkzeuge (Hypertext- und Hypermediasysteme, Internet und Intranet), Verbesserungen im Decision Support (Wissensbasierte und Neuronale Systeme), vereinfachte Kommunikationsmöglichkeiten (Telekommunikationssysteme, Multimedia-Systeme) sowie die Unterstützung von Gruppenarbeit durch Computer Supported Cooperative Work-Systeme (CSCW-Systeme). ${ }^{843}$ Ihre Nutzungspotentiale gilt es bei der Ausgestaltung des IuK-Systems für den Aufsichtsrat ebenfalls zu berücksichtigen.

\section{b) Ausgestaltung}

Nach der systemtechnischen Einordnung von MUS ist jetzt zu analysieren, inwieweit dieses Konzept auf den Aufsichtsrat übertragbar ist. Bei dem Einsatz von MUS für den Aufsichtsrat sollen sie als Aufsichtsratsunterstützungssysteme (AUS) bezeichnet werden. Der Begriff ist weiter als der des Aufsichtsratsinformationssystems, da nicht nur die informatorische Versorgung des Aufsichtsrats betrachtet wird, sondern auch Möglichkeiten zur Kommunikation.

Die Ausrichtung von MUS auf die Unterstützung unternehmerischer Aufgaben gibt einen ersten Hinweis auf eine grundsätzliche Übertragbarkeit auch auf den Aufsichtsrat, da dieser ebenfalls unternehmerische Aufgaben wahrnimmt. Zusätzlich kann geprüft werden, ob die Anforderungen an die einzusetzenden Technologien identisch sind. Sollte dies der Fall sein, kann davon ausgegangen werden, daß anforderungsgerechte MUS grundsätzlich auch als AUS einsetzbar

\footnotetext{
842 Vgl. Gabriel/Chamoni/Gluchowski, Management, 2000, S. 90.

${ }^{843}$ Vgl. Gluchowski/Gabriel/Chamoni, Systeme, 1997, S. 247ff.
} 
sind. Daher müssen die Anforderungen an MUS (vgl. Abb. 21) auf ihre Notwendigkeit im Kontext des AUS beurteilt werden. ${ }^{844}$

\begin{tabular}{|ll|}
\hline \multicolumn{1}{c|}{ Anforderung } \\
\hline - & Einfacher und schneller Zugang zu internen Informationen \\
\hline - & Einfacher und schneller Zugang zu externen Informationen \\
\hline & Methodische Unterstützung allgemeiner und spezifischer Planungs- und \\
- & Untscheidungsaufgaben \\
\hline - & Umfassende Kommunikationsmöglichkeiten \\
\hline - & Unterstützung von Gruppenarbeit \\
\hline - & Benutzerfreundlichkeit \\
\hline
\end{tabular}

Abb. 21: Anforderungen an MUS und AUS

Wie die Analyse des Informationsproblems des Aufsichtsrats gezeigt hat, ist für die Aufsichtsratsmitglieder zur Erfüllung ihrer Planungs- und Entscheidungsaufgaben sowohl die Versorgung mit internen als auch mit externen Informationen eine wesentliche Voraussetzung. Darüber hinaus ist es erforderlich, daß sie beispielsweise im Rahmen des strategischen Planungsprozesses oder bei der Evaluierung von Organisationsalternativen methodisch unterstützt werden. Aufgrund der Besonderheiten der Arbeitsweise des Gremiums (seltene, diskontinuierliche und kurze Sitzungen im Plenum, Delegation vielfältiger Aufgaben an einzelne Mitglieder oder Ausschüsse, räumlich dezentralisierte Arbeitsplätze der Aufsichtsratsmitglieder außerhalb der Unternehmungszentrale, hohes Erfordernis zur Gruppenarbeit), die sich durch ausgeprägte Virtualität auszeichnet, ${ }^{845}$ kommt der administrativen und organisatorischen Unterstützung ein hoher Stellenwert $\mathrm{zu}$, so daß besondere Anforderungen an die Unterstützung allgemeiner Bürotätigkeiten, an die Kommunikation und an die Unterstützung von Gruppenarbeit bestehen. Und schließlich ist auch bei Aufsichtsräten auf die Benutzerfreundlichkeit als allgemeiner Anforderung zu achten. Diese Analyse zeigt, da $\beta$ die Anforderungen, die an MUS zur Unterstützung von Entscheidungsträgern gestellt werden, auch an AUS zu stellen sind. Daher soll nachfolgend die Ausgestaltung eines AUS skizziert werden, indem hierzu auf geeignete MUSFunktionalitäten zurückgegriffen wird und diese auf die AUS übertragen werden.

844 Vgl. zu den Anforderungen an MUS Gluchowski/Gabriel/Chamoni, Systeme, 1997, S. 54ff.; mit weiteren notwendigen Eigenschaften Voß/Gutenschwager, Informationsmanagement, 2001, S. 329.

845 Vgl. zur Virtualisierung umfassend Picot/Reichwald/Winand, Unternehmung, 2001, S. 387-449. 
Das AUS muß die Aufsichtsratsmitglieder in die Lage versetzen, einfach und schnell alle gewünschten internen Informationen erhalten und geeignet darstellen zu können. ${ }^{846}$ Hierzu bieten die vorliegenden Analyseorientierten Informationssysteme durch eine intuitive, multidimensionale Informationsversorgung umfangreiche Potentiale für die Aufsichtsratsmitglieder ${ }^{847}$ Dabei scheint aufgrund der abgrenzbaren Informationsbedürfnisse des Aufsichtsrats die Einrichtung von Data Marts sinnvoll zu sein, um hierdurch zum einen eine Informationsüberfrachtung durch den Zugriff auf das gesamte Data Warehouse zu vermeiden und zum anderen eine akzeptable Systemperformance zu gewährleisten. Von der Systemstruktur und der Organisationsstruktur hängt ab, ob ein gemeinsamer Data Mart für den gesamten Aufsichtsrat oder ausschußspezifische Marts eingerichtet werden. So wäre es beispielsweise denkbar, zur Unterstützung des Vorstandsausschusses einen Data Mart mit unternehmungsinternen und externen Führungskräftearbeitsmarktdaten zusammenzustellen. Im Hinblick auf den gesamten Aufsichtsrat ist außerdem zu überlegen, ob zur Vermeidung einer Informationsüberfrachtung ein Ausnahmeberichtswesen (Exception-Reporting) installiert wird, das jedoch primär im Rahmen der Kontrollfunktion von Bedeutung ist.

Neben den intuitiven Recherchemöglichkeiten sollte das AUS außerdem den Zugriff auf Texte und Grafiken ermöglichen. ${ }^{848}$ Dies sind alle für den Aufsichtsrat relevanten Berichte oder beispielsweise in Form eines Monatsabschlusses aufbereitete Rechnungswesendaten. Mit der Ablage solcher Dokumente in papierloser Form im AUS werden die Zugriffs- und Auswertungsmöglichkeiten für die einzelnen Aufsichtsratsmitglieder deutlich verbessert.

Über die Versorgung mit internen Informationen hinaus spielt insbesondere für die Planung der Zugriff auf externe Informationen eine große Rolle. Das AUS muß den Zugang zu externen Datenbanken gewährleisten, wie beispielsweise Börsendaten oder Branchenkennzahlen. ${ }^{849}$ Dies kann durch die Nutzung des Internet wesentlich vereinfacht werden. Es kann sowohl als originäre Informationsquelle genutzt werden als auch als Zugangsmedium auf Informationsdienste, elektronische Zeitungs- und Zeitschriftenarchive sowie auf Wirtschaftsdatenbanken. ${ }^{850}$ Deren Nutzung sollte schwerpunktmäßig durch den Aufsichtsratsas-

\footnotetext{
846 Vgl. Gluchowski/Gabriel/Chamoni, Systeme, 1997, S. 55.

847 Vgl. Gabriel/Chamoni/Gluchowski, Management, 2000, S. 90.

848 Vgl. Gluchowski/Gabriel/Chamoni, Systeme, 1997, S. 55; Dittmar, Wissen, 2000, S. 15f., mit Bezug auf Knowledge Management.

849 Vgl. Gluchowski/Gabriel/Chamoni, Systeme, 1997, S. 55.

850 Vgl. Meier/Mertens, Führungsinformationssysteme, 2000, S. 355.
} 
sistenten als Informationsintermediär des Aufsichtsrats erfolgen. Über die Nutzung eines Redaktionssystems wäre es darüber hinaus möglich, aus dem Internet gewonnene Daten in das unternehmungsinterne Informationssystem zu integrieren. Zur Gewährleistung einer integrierten Sicht auf interne und externe sowie quantitative und qualitative Daten schlagen daher MEIER/MERTENS die Entwicklung eines Management-Portals vor. ${ }^{851}$ In ähnlicher Weise könnte für das Überwachungsorgan die Einrichtung eines Aufsichtsrats-Portals in Betracht gezogen werden.

Ebenso wie das Management einer Unternehmung benötigen auch mit Planungsaufgaben betraute einzelne Aufsichtsratsmitglieder oder Aufsichtsratsausschüsse instrumentelle Unterstützung durch Entscheidungsunterstützungssysteme. ${ }^{852}$ Neben einer allgemeinen instrumentellen Unterstützung zählt hierzu der Einsatz spezifischer Methoden. Dies könnte beispielsweise im Rahmen des strategischen Planungsprozesses der informationstechnologisch unterstützte Einsatz einer Balanced Scorecard sein, wie sie im SAP ${ }^{\circledR}$ Strategic Enterprise Management ${ }^{\mathrm{TM}}$ enthalten ist. ${ }^{853}$

Neben der informatorischen und der instrumentellen Unterstützung sollte das AUS die einzelnen Aufsichtsratsmitglieder und den Aufsichtsratsassistenten bei allgemeinen Bürotätigkeiten unterstützen. Hierzu zählen Textverarbeitung, Tabellenkalkulation oder Terminplanung.

Darüber hinaus sind umfassende Kommunikationsmöglichkeiten vorzusehen. Dies beinhaltet sowohl die Sprachkommunikation als auch den digitalisierten Versand bzw. Empfang von Texten, Daten oder Grafiken. ${ }^{854}$ Hier verspricht insbesondere die Nutzung von E-Mail die Realisierung großer Nutzenpotentiale. ${ }^{855}$ Auch der Einsatz multimedialer Kommunikationstechnologien wie beispielsweise Videokonferenzen bedeutet eine wesentliche Unterstützung für den Aufsichtsrat. ${ }^{856}$ Hierdurch können gemeinsame Konferenzen abgehalten werden,

851 Vgl. Meier/Mertens, Führungsinformationssysteme, 2000, S 358f.; zur Verbindung von Data Warehouses mit Internet/Intranet-Technologien vgl. Mucksch/Behme, Data Warehouse-Konzept, 1998, S. 74ff.

852 Vgl. Gluchowski/Gabriel/Chamoni, Systeme, 1997, S. 55f.

853 Vgl. hierzu und zu weiteren Funktionalitäten im Rahmen des strategischen Planungsprozesses SAP AG, SAP ${ }^{\circledast}$ Strategic Enterprise Management ${ }^{\mathrm{TM}}$, 2000, sowie Müller/Schöne, BalancedScorecard-Anwendung, 2001, S. 90, die mit Verweis auf das Hyperion Wired for OLAP eine grundsätzliche Einsetzbarkeit auf Top-Management-Ebene sehen.

Vgl. Gluchowski/Gabriel/Chamoni, Systeme, 1997, S. 54f.

855 Vgl. Gabriel/Gluchowski, Systeme, 1997, S. 538. nutzt; vgl. hierzu Fresenius Medical Care AG, Geschäftsbericht 1999, S. 85. 
ohne daß das gesamte Gremium oder ein Ausschuß an einem Ort versammelt sein muß. Diese Kommunikationsmöglichkeiten führen zu einer nachhaltigen Vereinfachung von Gruppenarbeit im Aufsichtsrat.

E-Mail und Videokonferenzen können zu den sogenannten CSCW-Konzepten gezählt werden, die auf die Unterstützung von Gruppenarbeitsprozessen ausgerichtet sind. ${ }^{857}$ Im Vordergrund steht dabei die Möglichkeit zur zeitgleichen oder zeitversetzten Tätigkeit der Gruppenmitglieder an verschiedenen Orten. CSCWKonzepte beinhalten verschiedene Technologien, die sowohl die Kommunikation als auch die Informationsverarbeitung und den Informationszugang vereinfachen und somit eine Gruppenentscheidungsfindung in unterschiedlichen Szenarien ermöglichen. ${ }^{858}$ Für den Aufsichtsrat bzw. einzelne Ausschüsse bieten sie den Vorteil, nicht nur zeit- oder/und ortsversetzt kommunizieren, sondern darüber hinaus auch Entscheidungen fällen zu können, da weniger bzw. effektivere Treffen der Entscheidungsträger notwendig sind, eine Integration von geographisch getrennten Gruppen erfolgen kann und eine verbesserte globale Koordination möglich ist. Diese Potentiale bilden eine wesentliche Voraussetzung für die Umsetzung der Forderung nach einer internationalen Besetzung der Aufsichtsgremien in Verbindung mit der hier geforderten Intensivierung der Aufsichtsratstätigkeit. Als Beispiele solcher Softwarepakete können erstens Sitzungsunterstützungssysteme (Electronic Meeting Room-Systeme) genannt werden, die eine synchrone Tätigkeit einer Gruppe an einem Ort unterstützen. ${ }^{859}$ Zweitens können Whiteboarding- und Videokonferenzsysteme eingesetzt werden, mit deren Hilfe räumlich verteilte, synchrone Gruppenarbeit möglich ist. Drittens ist beispielsweise an den Einsatz von Computerkonferenzsystemen zu denken, die eine asynchrone Gruppenarbeit unterstützen.

Eine breite Unterstützung erfahren diese Planungs- und Entscheidungsprozesse durch die Dokumentation der Planungsunterlagen in Dokumentenmanagementsystemen. ${ }^{860}$ Dieses dient gleichzeitig der Dokumentation der Tätigkeit des Aufsichtsrats, wenn nicht nur die Planungsunterlagen enthalten sind, sondern auch

857 Vgl. Gabriel/Gluchowski, Systeme, 1997, S. 539; Voß/Gutenschwager, Informationsmanagement, 2001, S. 361-375.

Vgl. Gluchowski/Gabriel/Chamoni, Systeme, 1997, S. 294ff.; VoB/Gutenschwager, Informationsmanagement, 2001, S. 362. CSCW-Technologien Reif-Mosel, Kooperation, 2000, S. 79-138. 
die Entscheidungsvorlagen des Aufsichtsrats sowie seine Protokolle und auch weitere Dokumente. ${ }^{861}$

Die vorangehenden Ausführungen haben gezeigt, daß durch den Einsatz von AUS umfangreiche Unterstützungsmöglichkeiten für die Aufsichtsratsmitglieder im Rahmen ihrer Planungs- und Entscheidungsaufgaben bestehen. ${ }^{862}$ Die erfolgreiche Einführung und der Einsatz eines AUS ist jedoch ebenso wie im Falle eines MUS von verschiedenen Erfolgsfaktoren abhängig.

\section{c) Erfolgsfaktoren}

Voraussetzung für den effizienten Einsatz eines MUS und somit auch für den des hier vorgestellten AUS sind die Akzeptanz sowie die Nutzung des Systems durch den Endanwender. ${ }^{863}$ VOB/GUTENSCHWAGER leiten daher verschiedene Kriterien ab, deren Vorliegen die Voraussetzung für den erfolgreichen Einsatz eines solchen Systems in der Unternehmung bilden. Sie können weiter in Erfolgsfaktoren innerhalb der Einführungsphase und Faktoren im Rahmen des Einsatzes unterschieden werden. Um die erfolgreiche Einführung eines AUS in der Unternehmung zu gewährleisten, sollte das Projekt einen ranghohen Sponsor haben. Hierbei ist an den Aufsichtsratsvorsitzenden oder den Vorsitzenden des für die Einführung verantwortlichen Organisationsausschusses zu denken. Dies gewährleistet zum einen die Berücksichtigung der Anforderungen der Aufsichtsratsmitglieder, zum anderen ist es so möglich, gegebenenfalls vorhandenen Einführungswiderständen auf Seiten des Vorstands gezielt entgegen wirken zu können. Daneben sollte in dem verantwortlichen Projektteam nicht nur entsprechendes fachliches sowie unternehmungsspezifisches Know-how vorhanden sein, zusätzlich ist sowohl eine Beteiligung der Informationslieferanten als auch der Endanwender erforderlich. Hierbei ist auf Seiten des Aufsichtsrats insbesondere an die Mitglieder des Organisationsausschusses zu denken, die möglichst in die Entwicklung eingebunden werden sollten.

861 Die Dokumentationsfunktion eines Aufsichtsratsinformationssystems betont auch Chini, Aufsichtsrats-Informationssystem, 1988, S. 89; zum Dokumentenmanagement beispielsweise Voß/Gutenschwager, Informationsmanagement, 2001, S. 277-284.

${ }^{862}$ Ein Beispiel mit möglichen Themenbereichen eines solchen Informationssystems für das TopManagement der Bayer AG gibt Krcmar, Informationsmanagement, 2000, S. 61f., das folgende Themenbereiche enthält: Strategie (Portfolios, Investitionen, Forschung), Markt/Börse (Wettbewerber, Preise für Rohstoffe, Börseninformationen, Marktinformationssysteme), Geschäft (Controllingdaten: Umsatz, Ergebnis, CFROI), Organisation (u.a. Organisationspläne), Personal (u.a. Qualifikationsprofile, Altersstrukturen, Personalaufwand) und Beschlüsse (u.a. Protokolle, Sitzungsunterlagen).

${ }^{863} \mathrm{Vgl}$. Voß/Gutenschwager, Informationsmanagement, 2001, S. 342; auch Kaiser, Unternehmensinformation, 1999, S. 61. 
Im Rahmen des Einsatzes des AUS sind verschiedene Faktoren als erfolgsrelevant zu bezeichnen. Zunächst ist für den erfolgreichen Einsatz des AUS eine breite Unterstützung durch den gesamten Aufsichtsrat, daneben eine kompetente systemtechnische Betreuung des AUS sowie die realistische Einschätzung der eingesetzten Technologien zu nennen ${ }^{864}$ Um eine breite Unterstützung und Akzeptanz im gesamten Aufsichtsrat zu erreichen, sind möglichst frühzeitige Informationen und Schulungen der Aufsichtsratsmitglieder sowie weiterer Nutzer, beispielsweise des Aufsichtsratsassistenten, erforderlich. Da nicht zwangsläufig alle Aufsichtsratsmitglieder mit Planungsaufgaben betraut werden und ebenso eine fachspezifische Systemunterstützung nur für hierfür zuständige Einzelpersonen oder Ausschüsse erforderlich ist, können gestufte Schulungen erfolgen. Während alle Aufsichtsratsmitglieder mit den Grundfunktionalitäten des Systems vertraut sein müssen, sind sonderfunktionalitätsspezifische Schulungen nur für einen Teil des Aufsichtsrats durchzuführen. Daneben ist für die Akzeptanz und Nutzung des Systems eine ausgeprägte Benutzerfreundlichkeit von großer Bedeutung. ${ }^{865}$ Dies gilt insbesondere im vorliegenden Fall, da überwiegend davon auszugehen sein wird, daß die potentiellen Nutzer bisher keine ausgeprägte Affinität zu modernen Informations- und Kommunikationstechnologien besaßen.

Als letzter Erfolgsfaktor ist das Berechtigungskonzept zu nennen. ${ }^{866}$ Um durch ein für jedes Aufsichtsratsmitglied offenes AUS nicht die durch die Bildung von Ausschüssen gewünschte Aufgabendelegation und die hiermit verbundene Einschränkung der Informationsrechte von nicht zum Ausschuß gehörenden Aufsichtsratsmitgliedern zu unterlaufen, muß über ein Berechtigungskonzept sichergestellt werden, daß auf die Daten des AUS nur hierzu berechtigte Personen zugreifen können. Dies gilt sowohl innerhalb des Aufsichtsgremiums, beispielsweise eine alleinige Zugriffsmöglichkeit auf die Personaldaten des Vorstands nur durch Mitglieder des Vorstandsausschusses, als auch für unbefugte Zugriffe auf das AUS durch Dritte. Hierfür sind geeignete Schutzmaßnahmen auch gegenüber Externen einzurichten. ${ }^{867}$

Doch trotz aller Möglichkeiten, die Arbeitsweise des Aufsichtsrats durch den Einsatz eines AUS zu verbessern, ist abschließend festzustellen, daß die Computerunterstützung nur dann zu einer höheren Effektivität und Effizienz des Organs

\footnotetext{
${ }^{864}$ Vgl. zu Erfolgsfaktoren vom MUS Gabriel/Gluchowski, Systeme, 1997, S. 310f.; auch Voß/Gutenschwager, Informationsmanagement, 2001, S. 342.

Vgl. Gluchowski/Gabriel/Chamoni, Systeme, 1997, S. 342.
} 
führen kann, wenn die Aufsichtsratsmitglieder die ihnen zugedachte Rolle realiter wahrnehmen.

\begin{abstract}
„Computergestützte Management-Informationssysteme können zu einem Informationsniveau führen, das mit den traditionellen Verfahren des Rechnungswesens und der statistischen Analyse nicht zu erreichen ist. Wie groß aber immer in Zukunft die Leistungen derartiger Systeme sein werden, sie vermögen entschlußfähige Persönlichkeiten nicht zu ersetzen." ${ }^{\text {“868 }}$
\end{abstract}

Die prozessurale Ausgestaltung des Planungs- und Entscheidungsprozesses ist Gegenstand des folgenden Abschnitts. Er beinhaltet als letztes Element des Aufsichtsratsplanungssystems den Planungsproze $\beta$, innerhalb dessen die übrigen Systemelemente integriert werden können.

\title{
F. Exemplarische Gestaltung eines Aufsichtsratsplanungsprozes- ses
}

\section{Einführung und Integrationsnotwendigkeit}

Die Diskussion des Planungsprozesses des Aufsichtsrats scheint auf unterschiedlichem Wege möglich zu sein. Zum einen könnte der Versuch unternommen werden, Sachprobleme des Aufsichtsratsplanungsprozesses allgemeingültig zu beschreiben. ${ }^{869}$ Andererseits könnte versucht werden, alle möglichen (Teil-)Planungsprozesse mit den zugehörigen Planungsobjekten des Aufsichtsrats bzw. seiner Ausschüsse oder einzelner Mitglieder enumerativ zu beschreiben, wodurch jedoch umfangreiche Redundanzen entstünden. Beide Vorgehensweisen ließen einen Beitrag für den hier verfolgten gestaltungsorientierten Ansatz vermissen, so daß nachfolgend ein bestimmtes Planungsobjekt exemplarisch im Systemzusammenhang betrachtet wird.

Bei dem hier betrachteten Objekt handelt es sich um die Planung der Besetzung des Vorstands, der eine herausgehobene Bedeutung für eine funktionsfähige Unternehmungsführung zukommt. ${ }^{870}$ Zur Vermeidung sprachlicher Überschneidungen mit der Führungskräfteplanung durch den Vorstand wird nachfolgend von der Vorstandsbesetzungsplanung des Aufsichtsrats gesprochen. Dies bedingt keine grundsätzlichen Unterscheidungsnotwendigkeiten, da die Vor-

868 Gutenberg, Grundlagen, 1983, S. 133.

${ }^{869}$ Vgl. hierzu aus planungstheoretischer Perspektive Mag, Unternehmungsplanung, 1995, S. 46ff.; von Zeitproblemen wird in der vorliegenden Arbeit abstrahiert; vgl. hierzu Mag, Zeitprobleme, 1990, S. 694-699.

870 Vgl. hierzu auch die Ausführungen bei Chini, Aufsichtsrats-Informationssysteme, 1988, S. 93106; aus US-amerikanischer Perspektive zum lange Zeit intransparenten Auswahlprozeß des Chief Executive Officer, die teilweise an die Papstwahl erinnere, Lorsch/Khurana, Leaders, 1999, S. 96-105. 
standsmitglieder eine Teilmenge der obersten Führungskräfte einer Unternehmung darstellen. Im Gegensatz zur Führungskräfteplanung ist der Vorstand hier jedoch nicht Planungssubjekt, sondern Planungsobjekt.

Wie die Ausführungen zu den vorstandsbezogenen Planungsobjekten gezeigt haben, bestehen verschiedene Interdependenzen zwischen der Vorstandsorganisationsplanung und der Vorstandsbesetzungsplanung, die es notwendig erscheinen lassen, beide Teilplanungen aufeinander abzustimmen. ${ }^{871}$ Während die Organisationsplanung den gedanklichen Entwurf der internen Struktur des Vorstands vornimmt, befaßt sich die personenbezogene Planung mit dem gedanklichen Entwurf von personellen Bedarfsumfängen und Bedarfsstrukturen sowie geeigneten Maßnahmen zu ihrer Deckung. Ihr gemeinsamer, integrationsbegründender Kern liegt in der vorzunehmenden sachlich-personellen Zuordnung (vgl. Abb. 22). ${ }^{872}$

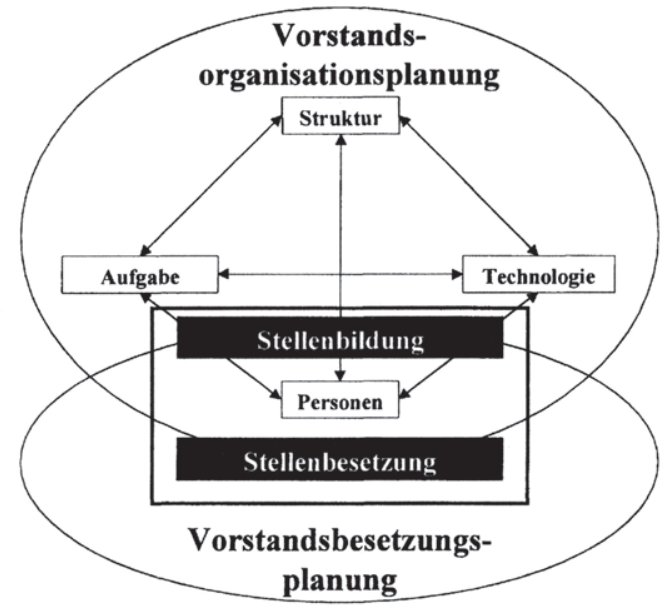

Abb. 22: Zusammenhang von Vorstandsorganisations- und -besetzungsplanung Quelle: In Anlehnung an Mag, Organisations- und Personalplanung, 1999, S. 380.

Die hieraus entstehende Integrationsnotwendigkeit kann unterschiedlich gelöst werden: Entweder erfolgt zunächst eine Organisationsplanung, aus der im Anschluß die personelle Planung abgeleitet wird, oder es wird erst eine Vor-

871 Die ausgeprägten Interdependenzen der strategischen Organisations- und Führungskräfteplanung ebenfalls betonend Hahn, Führungskräfteplanung, 1999, S. 618; zum grundsätzlichen Verhältnis von Organisations- und Personalplanung Mag, Organisations- und Personalplanung, 1999, S. 376f.

872 Vgl. Mag, Organisations- und Personalplanung, 1999, S. $379 f$. 
standsbesetzungsplanung durchgeführt, die als Grundlage für die spätere Organisationsplanung dient. Schließlich kann versucht werden, beide Teilplanungen simultan durchzuführen. ${ }^{873}$ Die ersten beiden Alternativen stellen nur eine partielle Integration beider Planungen dar, da die nachfolgende Planung sukzessiv aus der vorgelagerten abgeleitet wird. Weil die Berücksichtigung von Interdependenzen eine gegenseitige Beeinflussungsmöglichkeit innerhalb eines Planungsprozesses erfordert, sind beide partiellen Integrationsformen unbefriedigend. ${ }^{874}$ Daher ist eine Simultanintegration von Organisations- und Vorstandsbesetzungsplanung anzustreben. Die Notwendigkeit einer Simultanintegration ergibt sich darüber hinaus aus den Besonderheiten der Planungsobjekte. Hochqualifizierte Führungskräfte stellen einen bedeutenden Erfolgs-, aber auch einen Engpaßfaktor dar. ${ }^{875}$ Dies wird bei einer derivativen Vorstandsbesetzungsplanung vernachlässigt. Andererseits stößt eine derivative Organisationsplanung ebenfalls sehr schnell an ihre Grenzen, da die organisatorischen Anpassungspotentiale, beispielsweise durch die bereits bestehende Vorstandsressortierung, begrenzt sind.

HAHN nennt als Voraussetzung für eine erfolgreiche Simultanintegration beider Planungen die Identität des Planungsträgers. ${ }^{876}$ Dies ist hier gewährleistet, da der Vorstandsausschuß sowohl für die Organisationsplanung des Vorstands als auch für die Besetzungsplanung zuständig ist. Als geeignete Anwendungsfelder kommen für ihn grundlegende Veränderungen der Strategischen Planung, der Organisationsstruktur oder die Besetzung der obersten Führungsstellen der Unternehmung in Frage. Darüber hinaus ist die Anzahl der Planungsobjekte im vorliegenden Problemzusammenhang begrenzt, so daß insgesamt günstige Ausgangsbedingungen für eine simultane Organisations- und Vorstandsbesetzungsplanung vorliegen.

\section{Simultane Integration von Vorstandsorganisations- und -besetzungs- planung}

\section{a) Zielanalyse}

Die simultane Integration von Vorstandsorganisations- und besetzungsplanung erfolgt in einem mehrstufigen Planungsprozeß, der mit der Entscheidung für eine Handlungsalternative seinen Abschluß findet. Am Anfang steht die Zielana-

\footnotetext{
873 Vgl. Mag, Organisations- und Personalplanung, 1999, S. 380ff.

874 Vgl. Hahn, Führungskräfteplanung, 1999, S. 635, für den die simultane Integration der Idealfall ist; zustimmend auch Mag, Organisations- und Personalplanung, 1999, S. 382.

875 Vgl. Hahn, Führungskräfteplanung, 1999, S. 631; Mag, Organisations- und Personalplanung, 1999, S. 382.

876 Vgl. Hahn, Führungskräfteplanung, 1999, S. 635f.
} 
lyse, die der Ermittlung von Maßstäben bzw. Sollvorstellungen für zukünftiges Handeln dient. Mit ihrer Hilfe werden ausgehend von der Unternehmungszielsetzung sowohl für die organisatorische als auch für die personelle Dimension des Vorstands spezifische Subziele abgeleitet. Organisatorische Subziele sind als nach außen gerichtete Ziele die Markt- und Wettbewerbsorientierung, Flexibilität und Innovationsfähigkeit sowie als nach innen gerichtete Ziele die Führungsprozeßeffizienz, Humanressourceneffizienz, Finanz- und Sachressourceneffizienz sowie Geschäftsprozeßeffizienz. ${ }^{877} \mathrm{Zu}$ den personellen Subzielen für den Vorstand zählt primär die jederzeitige quantitative und qualitative Verfügbarkeit der für die Erreichung der Unternehmungsziele am besten geeigneten Personen. ${ }^{878}$ Neben den Zielen der Unternehmung sind in der Vorstandsbesetzungsplanung als personenbezogener Planung die Ziele der betroffenen Personen zu berücksichtigen und in das unternehmerische Zielsystem einzubinden.

\section{b) Problemanalyse}

Nachdem geeignete Ziele abgeleitet wurden, erfolgt in der Problemanalyse eine Beurteilung der Ausgangssituation. Hierzu wird überprüft, ob die gegenwärtige Organisation mit den verfügbaren Vorstandsmitgliedern zur bestmöglichen Zielerreichung führt. Diese Evaluierung sollte durch den Vorstandsausschuß jährlich durchgeführt und mit dem Strategieausschuß abgestimmt werden, ${ }^{879}$ da für die Evaluierung zukunftsgerichtete Informationen der Strategischen Planung zu berücksichtigen sind. Zur frühzeitigen Identifikation von Krisen umfaßt dies insbesondere die strategische Frühaufklärung. ${ }^{880} \mathrm{Da}$ nicht davon auszugehen ist, daß eine optimale Übereinstimmung von Strategie, Organisation und verfügbaren Vorstandsmitgliedern vorliegt, ${ }^{881}$ schließt sich eine Bedarfsplanung an, bei der die geplanten Stellenbeschreibungen des Vorstands bzw. die hieraus abgeleiteten zukünftigen Anforderungsprofile den zukünftigen Fähigkeitsprofilen der Vorstandsmitglieder gegenübergestellt werden. ${ }^{882}$ Ziel muß es sein, die für die geplanten Strategien der Unternehmung bestgeeigneten Führungskräfte einzusetzen.

877 Vgl. Krüger, Organisation, 1994, S. 14, der jedoch von Human-Ressourcen-Orientierung spricht, sowie Hahn, Führungskräfteplanung, 1999, S. 637.

878 Vgl. Hinterhuber, Führungskräfteentwicklung, 1999, S. 642

879 Vgl. mit Bezug auf eine Evaluierung des Vorstands Berliner Initiativkreis German Code of Corporate Governance, Code, 2000, S. 45, II.1.10.

880 Vgl. Oesterle, Führungswechsel, 1999, S. 306, sowie zur Frühaufklärung Baetge/Jerschensky, Frühwarnsysteme, 1999, S. 171-176, und Mikus, Führungsprozeß, 1999, S. 85-110.

881 Vgl. Hinterhuber, Führungskräfteentwicklung, 1999, S. 641.

${ }_{882}$ Vgl. Hahn, Führungskräfteplanung, 1999, S. 625, sowie grundlegend Mag, Personalplanung, 1998 , S. 63ff. 
HINTERHUBER zählt relevante Fähigkeiten von Führungskräften auf, die für vier verschiedene Strategietypen erforderlich seien. ${ }^{883}$ Die Beurteilung der Führungskräfte sollte auf der Grundlage laufender oder zukünftiger Ergebnisse erfolgen (vgl. Abb. 23):

\begin{tabular}{|c|c|c|c|}
\hline \multirow{2}{*}{ Strategien } & \multirow{2}{*}{$\begin{array}{l}\text { wichtigste } \\
\text { Führungs- } \\
\text { eigenschaften }\end{array}$} & \multicolumn{2}{|c|}{$\begin{array}{c}\text { Beurteilung der Fïhrungskräfte } \\
\text { aufgrund }\end{array}$} \\
\hline & & $\begin{array}{l}\text { laufender } \\
\text { Ergebnisse }\end{array}$ & $\begin{array}{l}\text { zuküinftiger } \\
\text { Ergebnisse }\end{array}$ \\
\hline Offensivstrategien & $\begin{array}{l}\text { unternehmerische } \\
\text { Fähigkeiten, } \\
\text { Innovations- } \\
\text { verhalten }\end{array}$ & nicht sinnvoll & sinnvoll \\
\hline $\begin{array}{l}\text { Investitions- und } \\
\text { Wachstumsstrategien }\end{array}$ & $\begin{array}{l}\text { unternehmerische } \\
\text { Fähigkeiten }\end{array}$ & nicht sinnvoll & sinnvoll \\
\hline Defensivstrategien & Urteilsfähigkeit & sinnvoll & teilweise sinnvoll \\
\hline $\begin{array}{l}\text { Umstrukturierungs-I } \\
\text { Defensivstrategien }\end{array}$ & $\begin{array}{l}\text { administrative } \\
\text { Fähigkeiten }\end{array}$ & sinnvoll & nicht sinnvoll \\
\hline
\end{tabular}

Abb. 23: Führungseigenschaften und Beurteilungskriterien in Abhängigkeit von Strategien Quelle: In Anlehnung an Hinterhuber, Führungskräfteentwicklung, 1999, S. 647.

Zur Ermittlung des zukünftigen Bedarfs wird der Soll-Bestand dem voraussichtlichen Ist-Bestand gegenübergestellt. ${ }^{884}$ Unter Berücksichtigung von langfristig geplanten $\mathrm{Zu}$ - und Abgängen, d.h. in vergangenen Perioden geplanten und verabschiedeten personellen Maßnahmen, die noch nicht wirksam geworden sind, entsteht gegebenenfalls ein Ersatzbedarf, ein Neubedarf oder ein Freistellungsbedarf. Besonderes Augenmerk muß auf die qualitative Bedarfsdimension gerichtet werden, da eine Fehlbesetzung auf der Vorstandsebene erhebliche negative Konsequenzen für die Unternehmung mit sich bringt und die Erreichung der unternehmerischen Ziele verhindern kann. ${ }^{885}$

Daneben müssen ebenso wie für die Personen auch organisatorische Probleme des Vorstandsorgans aufgezeigt werden, die in enger Verbindung zu den Organisationsproblemen der Gesamtunternehmung stehen. ${ }^{886}$ Für international tätige

${ }^{883}$ Vgl. Hinterhuber, Führungskräfteentwicklung, 1999, S. 646f.; zustimmend Hahn, Führungskräfteplanung, 1999, S. 625; mit lebenszyklusspezifischen und weiteren Anforderungen Bleicher/Hahn, Management-Potential, 1995, S. 426ff., 431; verschiedene Manager-Typen in Abhängigkeit von Alter, Marktposition, Branche, finanzieller Lage und strategischer Position der Unternehmung unterscheidet Hengst, Management Audit, 1997, S. 200.

Vgl. Hahn, Führungskräfteplanung, 1999, S. 625.

Vgl. Hinterhuber, Führungskräfteentwicklung, 1999, S. 641f.; zur qualitativen Bedarfsermittlung Bleicher/Hahn, Management-Potential, 1995, S. 425. 
Publikumsaktiengesellschaften zählen hierzu unter anderem das durch die Globalisierung in Verbindung mit einem in den letzten Jahrzehnten zu beobachtenden Größenwachstum induzierte Erfordernis zur Verringerung ihres Komplexitätsgrades sowie die Notwendigkeit einer verstärkten Kundenorientierung. ${ }^{887}$ Mit Bezug auf die Gesamtunternehmung ist es in der Folge zur Entwicklung verschiedener Holdingkonzepte gekommen, die in Abhängigkeit von der konkreten Ausgestaltung für den Vorstand unterschiedliche Strukturierungsalternativen bedingen. ${ }^{888}$

\section{c) Alternativenanalyse}

Innerhalb der Alternativenanalyse entwickelt der Vorstandsausschuß organisatorische und personelle Handlungsalternativen. In dieser Planungsphase ist die Unterstützung durch weitere Planungssubjekte am größten. Aufgrund der Interdependenz der Teilplanungen ist es jedoch nicht ausreichend, lediglich Rückkopplungen zwischen im Ergebnis wiederum sukzessiv vorgenommenen Planungen vorzunehmen, da diese Vorgehensweise nur dann akzeptabel wäre, wenn einer der beiden Problembereiche so sehr dominiert, daß Maßnahmen in dem anderen Bereich reaktiv erfolgen könnten. Hier wird dagegen davon ausgegangen, daß der Vorstandsausschuß Handlungsalternativen entwickelt, die organisatorische und personelle Maßnahmen simultan vornehmen.

Um die Vorgehensweise bei einer integrierenden Alternativenanalyse zu beschreiben, soll nachfolgend ein abstraktes Beispiel vorgestellt werden. Die Grundlage hierfür bildet eine Modellbetrachtung von MAG zur simultanen Organisations- und Personalplanung, die auf den vorliegenden Problemzusammenhang adaptiert wird. ${ }^{889}$ Dabei wird eine Einschränkung der Betrachtung auf den Zusammenhang von Aufgaben und Personen vorgenommen. ${ }^{890}$

Die Gesamtaufgabe „Unternehmungsführung“ kann idealtypisch beispielsweise in folgende Teilaufgaben unterschieden werden (vgl. Abb. 24):

887 Vgl. Endres, Organisation, 1999, S. 442, 444f.

${ }^{888}$ Vgl. statt vieler anderer Theisen, Konzern, 2000, S. 153ff.; aufsichtsratsbezogene Aspekte im Konzern bleiben hier jedoch unberücksichtigt, vgl. hierzu umfassend Ludwig, Aufsichtsräte, 1997, sowie Hoffmann-Becking, Aufsichtsrat, 1995, S. 325-345, Martens, Aufsichtsrat, 1995, S. 567-592, und Theisen, Aufsichtsratsprobleme, 1998, S. 441-460.

889 Vgl. zur grundsätzlichen Vorgehensweise Mag, Organisations- und Personalplanung, 1999, S. $383 \mathrm{ff}$.

890 Vgl. zum Struktur-Personen-Zusammenhang Mag, Organisations- und Personalplanung, 1999, S. 389ff., sowie zum Aufgabe-Struktur-Personen-Zusammenhang S. 393ff. 


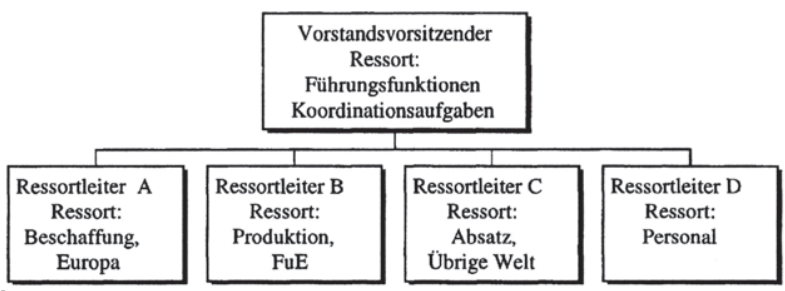

Abb. 24: Vorstandsorganisation in der Ausgangssituation

Im vorliegenden Beispiel gibt es neben dem Vorstandsvorsitzenden vier weitere Vorstandsmitglieder als Ressortleiter. Stellt der Vorstandsausschuß im Rahmen seiner Problemanalyse fest, daß die Fähigkeitsprofile der Vorstandsmitglieder den aus den Teilaufgaben abgeleiteten Anforderungsprofilen nicht entsprechen, muß er geeignete personelle und organisatorische Anpassungsmaßnahmen planen. Wird angenommenen, daß Ressortleiter C Qualifikationsdefizite aufweist, kann entweder versucht werden, geeignete Bildungsmaßnahmen zu ergreifen, oder es erfolgt ein Neuzuschnitt des Ressorts. Dies bedeutet jedoch noch keine simultane Integration, die erst dann vorliegt, wenn sowohl Ressortleiter C Qualifikationsdefizite abbaut als auch gleichzeitig einen Teil seiner Ressortkompetenzen verliert, die in diesem Fall einem neuen Vorstandsmitglied übertragen werden sollen (vgl. Abb. 25).

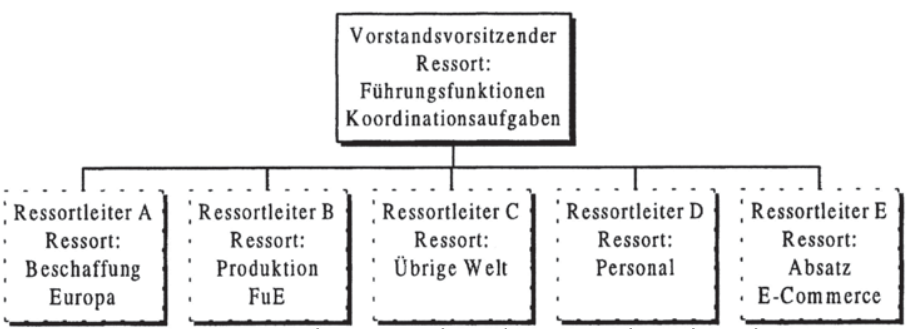

Abb. 25: Vorstandsorganisation nach Neuzuschnitt des Ressorts

Hierbei wird angenommen, daß der neue Ressortleiter E als bisheriges Mitglied der dem Vorstand unmittelbar nachfolgenden zweiten Führungsebene der Unternehmung ursprünglich nur für die auf Vorstandsebene hinzukommenden ECommerce-Aktivitäten vorgesehen war. Er bekommt jedoch zusätzlich das gesamte Absatzressort übertragen. Für den zukünftigen Ressortleiter E können frühzeitig geeignete Bildungsmaßnahmen einsetzen. ${ }^{891}$ Die Durchführung von Bildungsmaßnahmen sowohl für $\mathrm{C}$ als auch für $\mathrm{E}$ dient der Deckung des qualitativen Personalbedarfs. Dies kann nur in enger Abstimmung mit den Betroffenen erfolgen. Die Aufgabe des Aufsichtsratsausschusses beschränkt sich aufgrund

891 Vgl. hierzu Hahn, Führungskräfteplanung, 1999, S. 628ff. 
des operativen Charakters dieser Tätigkeit jedoch auf das Aufzeigen eines entsprechenden Bedarfs.

Die bisherige Lösung bewegt sich innerhalb der bereits verabschiedeten Nachfolgeplanung, da Ressortleiter E bereits vor dem Neuzuschnitt des Ressorts als Vorstandsmitglied vorgesehen war. Es wurden jedoch interne Beschaffungsmaßnahmen in Form von zusätzlichen Bildungsmaßnahmen erforderlich. Sollte zusätzlich eine Erhöhung der quantitativen Kapazität des Vorstands notwendig werden, löst dies entsprechende Beschaffungsaktivitäten des Vorstandsausschusses aus. ${ }^{892}$ Dies umfaßt sowohl eine Beschaffung über den internen als auch über den externen Führungskräftearbeitsmarkt. ${ }^{893}$

Die Relevanz des internen Arbeitsmarktes für die Vorstandsbesetzung und damit die Auswahlmöglichkeiten des Vorstandsausschusses hängen von einer funktionsfähigen Führungskräfteentwicklungsplanung des Vorstands ab. ${ }^{894} \mathrm{Nur}$ wenn durch diesen möglichst frühzeitig und in enger Abstimmung mit der Unternehmungsstrategie unternehmungsintern sogenannte Highpotentials als mögliche Kandidaten für Vorstandspositionen identifiziert wurden, verfügt der Aufsichtsrat über geeignete Wahlmöglichkeiten. Außerdem ist es erforderlich, daß zumindest der Vorstandsausschuß, am besten jedoch der gesamte Aufsichtsrat die Mitglieder der zweiten Führungsebene persönlich kennt. ${ }^{895}$ Möglichkeiten des Kennenlernens von Führungskräften nachgelagerter Hierarchieebenen bieten beispielsweise Präsentationen vor dem Aufsichtsrat. Daneben können Informationen über potentielle interne Vorstandskandidaten einer Führungskräftedatenbank entnommen werden, deren Aufbau entsprechend an den Informationsbedürfnissen des Aufsichtsrats auszurichten ist und Bestandteil des AUS werden sollte.

Bei der externen Beschaffung geht es um die Bestellung von Personen zu Vorstandsmitgliedern, die bisher nicht der Unternehmung angehört haben. Hierzu muß der Vorstandsausschuß versuchen, Führungskräfte außerhalb der Unternehmung in seiner Nachfolgeplanung zu erfassen. Dazu führt der Vorstandsausschuß unter Einschaltung der übrigen Mitglieder des Aufsichtsrats eine permanente Analyse des externen Arbeitsmarktes durch. Hierbei unterstützen ihn seine vielfältigen externen Kontakte. SERVATIUS weist diese Aufgabe alleine

\footnotetext{
892 Vgl. für diesen Fall der simultanen Organisations- und Personalplanung Mag, Organisations- und Personalplanung, 1999, S. 387.

893 Vgl. zu entsprechenden Maßnahmen Hahn, Führungskräfteplanung, 1999, S. $625 f$.

894 Vgl. Chini, Aufsichtsrats-Informationssysteme, 1988, S. 101.

$895 \mathrm{Vgl}$. Potthoff/Trescher, Aufsichtsratsmitglied, 1999, S. 152.
} 
dem Aufsichtsratsvorsitzenden zu. ${ }^{896}$ Dies verkennt jedoch die Verantwortung des Vorstandsausschusses für die Vorstandsbesetzungsplanung.

Zur Ansprache potentieller Vorstandsmitglieder stehen verschiedene Wege zur Verfügung. Für interne Kandidaten könnte neben der direkten Ansprache von dem Aufsichtsrat persönlich bekannten Mitarbeitern der Unternehmung eine interne Stellenausschreibung erfolgen. ${ }^{897}$ Sowohl die schriftlichen Bewerbungen an den Vorstandsausschuß als auch Gespräche mit Kandidaten sind dabei mit äußerster Vertraulichkeit zu behandeln. ${ }^{898}$ Bei der Ansprache externer Kandidaten ebenso wie im gesamten Beschaffungsprozeß einschließlich der Formulierung der Anforderungsprofile sollte die Hinzuziehung externer Personalberater (Headhunter) in Erwägung gezogen werden. Aufgrund ihrer Erfahrung in Verbindung mit der relativen Seltenheit von Besetzungsvorgängen in Unternehmungen können sie wertvolle Hilfestellungen leisten. ${ }^{899}$

Die internen und externen personellen Alternativen sind mit den Organisationsalternativen abzustimmen. Dabei kann es notwendig sein, eine Stellenbildung ad personam durchzuführen, bei der unter Berücksichtigung der Bedürfnisse der zukünftigen Vorstandsmitglieder Organisationsalternativen in Abhängigkeit von deren Qualifikationen und Neigungen entwickelt werden. ${ }^{900}$ So erfordert der Vorstand einer Finanzholding, die primär Finanzierungsaufgaben für ihre ansonsten selbständig agierenden Tochtergesellschaften wahrnimmt, sowohl eine andere Organisation als auch andere Qualifikationen der Vorstandsmitglieder als der Vorstand einer Führungsholding, in der sich die Holdingvorstände selbst als Manager und nicht als Finanziers bzw. Portfoliospezialisten sehen. ${ }^{901}$ Die jeweiligen Alternativen scheinen jedoch nur dann realisierbar zu sein, wenn sowohl organisatorische als auch personale Handlungsoptionen bestehen. Im Ergebnis führt die Alternativenanalyse zu unterschiedlichen Handlungsmöglichkeiten der Unternehmung, von denen eine auszuwählen ist.

\footnotetext{
896 Vgl. Servatius, Vorstandskontrolle, 1995, S. 225.

897 Vgl. Chini, Aufsichtsrats-Informationssysteme, 1988, S. 102.

898 Vgl. Hahn, Führungskräfteplanung, 1999, S. 627.

899 Vgl. Chini, Aufsichtsrats-Informationssysteme, 1988, S. 102; Hinterhuber, Führungskräfteentwicklung, 1999, S. 660.

900 Vgl. Hahn, Führungskräfteplanung, 1999, S. 633.

901 Vgl. Theisen, Konzern, 2000, S. 179f.; mit Beispielen aus der Unternehmungspraxis Endres, Organisation, 1999, S. 45ff., wobei die ehemalige VEBA AG als Finanzholding bezeichnet werden kann und die Deutsche Bank AG als (virtuelle) Führungsholding, da hier der Konzernvorstand nicht nur Finanzierungsaufgaben, sondern auch strategische Aufgaben wahrnimmt.
} 


\section{d) Prognose, Bewertung und Entscheidung}

Die entwickelten Handlungsalternativen werden in der Prognose- und Bewertungsphase hinsichtlich ihrer erwarteten Auswirkungen überprüft und einer integrierten Bewertung unterzogen, die dem gesamten Aufsichtsrat vom Vorstandsausschuß zur Verabschiedung vorzulegen ist. Hierzu ist zunächst die Eignung potentieller interner und externer Vorstandsmitglieder zu ermitteln. Dies erfolgt weitgehend eigenverantwortlich durch den Vorstandsausschuß. ${ }^{902} \mathrm{Um}$ zu verhindern, daß der Vorstandsausschuß seine Verantwortung an einen Personalberater delegiert, sollte der Berater nur unterstützend hinzugezogen werden. Dies ist insbesondere unter dem Gesichtspunkt der Sorgfaltspflichtverletzung von Bedeutung, die auch einer nachträglichen gerichtlichen Kontrolle zugänglich sein muß. ${ }^{903}$ Mittels eines Profilvergleichs wird das Anforderungsprofil der Stelle mit dem Fähigkeitsprofil des Bewerbers verglichen. ${ }^{904}$ Während sich das Anforderungsprofil der Stelle aus der Aufgabenanalyse für den Vorstand ergibt, können zur Ermittlung des Fähigkeitsprofils verschiedene Methoden eingesetzt werden: ${ }^{905}$

1. Analyse der schriftlichen Bewerbungsunterlagen;

2. Führung eines Gesprächs (Interviews), wobei sowohl Einzel- als auch Gruppengespräche sinnvoll sein können;

3. Präsentation des Kandidaten vor dem Vorstandsausschuß;

4. Analyse und Überprüfung von Referenzen.

Zur Beurteilung unternehmungsinterner Kandidaten kann der Vorstandsausschuß zusätzlich auf bereits vorhandene Informationen zugreifen. Er sollte diese aber auf jeden Fall aktualisieren und gegebenenfalls spezifizieren. Der mit der Vorauswahl beauftragte Vorstandsausschuß sollte abschließend für jeden Kandidaten ein Eignungsprofil erstellen, das die relative Eignung jedes Kandidaten für die Anforderungen der Stelle ausdrückt.

Nachdem der Vorstandsausschuß geeignete Kandidaten ermittelt hat, sollte der Auswahlvorschlag überprüft werden. Hierzu wird ein zweistufiges Hearing vorgeschlagen, mit dem die Kandidaten zunächst dem Aufsichtsrat vorgestellt

902 Vgl. Chini, Aufsichtsrats-Informationssysteme, 1988, S. 103.

903 Vgl. Gollnick, Beurteilung, 1997, S. 70f.

904 Vgl. Gollnick, Beurteilung, 1997, S. 67ff.; von unterschiedlichen Anforderungsprofilen geht auch Hengst, Management Audit, 1997, S. 200, aus.

905 Vgl. Chini, Aufsichtsrats-Informationssysteme, 1988, S. 103, der unzutreffend von Eignungsprofil spricht, sowie Mag, Personalplanung, 1998, S. 95. 
werden: ${ }^{906}$ Auf der ersten Stufe erhält der Vorstandskandidat Informationen über die Unternehmung, auf deren Grundlage er dem Aufsichtsratsplenum innerhalb eines vorher zu bestimmenden Zeitraums seine Vision der zukünftigen Entwicklung der Unternehmung vorstellt. Die sich anschließende Diskussion mit dem Kandidaten gibt gleichzeitig Gelegenheit, nochmals über die Ziele der Unternehmung nachzudenken. Wenn das Plenum mit der Vorauswahl einverstanden ist, sollte das Hearing unter Teilnahme des Gesamtvorstands wiederholt werden. Dies ermöglicht die Hinzuziehung weiteren Sachverstands und gibt sowohl dem Kandidaten als auch den Vorstandsmitgliedern die Gelegenheit zum gegenseitigen Kennenlernen.

Die anschließende Bewertung der Handlungsalternativen sollte aufgrund ihrer Multikriteriellität mit Hilfe einer Nutzwertanalyse erfolgen (vgl. Abb. 26) ${ }^{907} \mathrm{Da}$ die Realisierung organisatorischer Handlungsalternativen nur dann zielführend ist, wenn die erforderlichen Vorstandsmitglieder verfügbar sind, ist die Beschaffung geeigneter Vorstandsmitglieder als Conditio sine qua non der verschiedenen Organisationsalternativen zu berücksichtigen. Das hier gewählte Beispiel zeigt, daß die organisatorisch optimale Organisationsalternative II vom Aufsichtsrat nicht gewählt werden kann, da die hierfür erforderlichen Vorstandsmitglieder weder vorhanden sind noch beschafft werden können.

Die abschließende Entscheidung für Alternative III umfaßt sowohl die Entscheidung über die Bestellung eines Vorstandsmitglieds als auch die hiermit verbundene Organisationsalternative. ${ }^{908}$ Sie erfolgt durch den gesamten Aufsichtsrat. Er hat hierbei auch die Empfehlungen des Strategieausschusses zur Strategischen Planung der Unternehmung zu berücksichtigen.

906 Vgl. zum Hearing Chini, Aufsichtsrats-Informationssysteme, 1988, S. 104.

907 Vgl. Hahn, Führungskräfteplanung, 1999, S. 636f., sowie grundlegend Mag, Unternehmungsplanung, 1995, S. $91 \mathrm{ff}$.

$908 \mathrm{Zu}$ Ursachen möglicher Fehlentscheidungen bei der Besetzung von Vorstandspositionen aus informationsökonomischer Perspektive Kräkel, Topmanagement, 1998, S. 580, der eine der Ursachen in Problemen kollektiver Entscheidungen im Aufsichtsrat sieht (S. 584). 


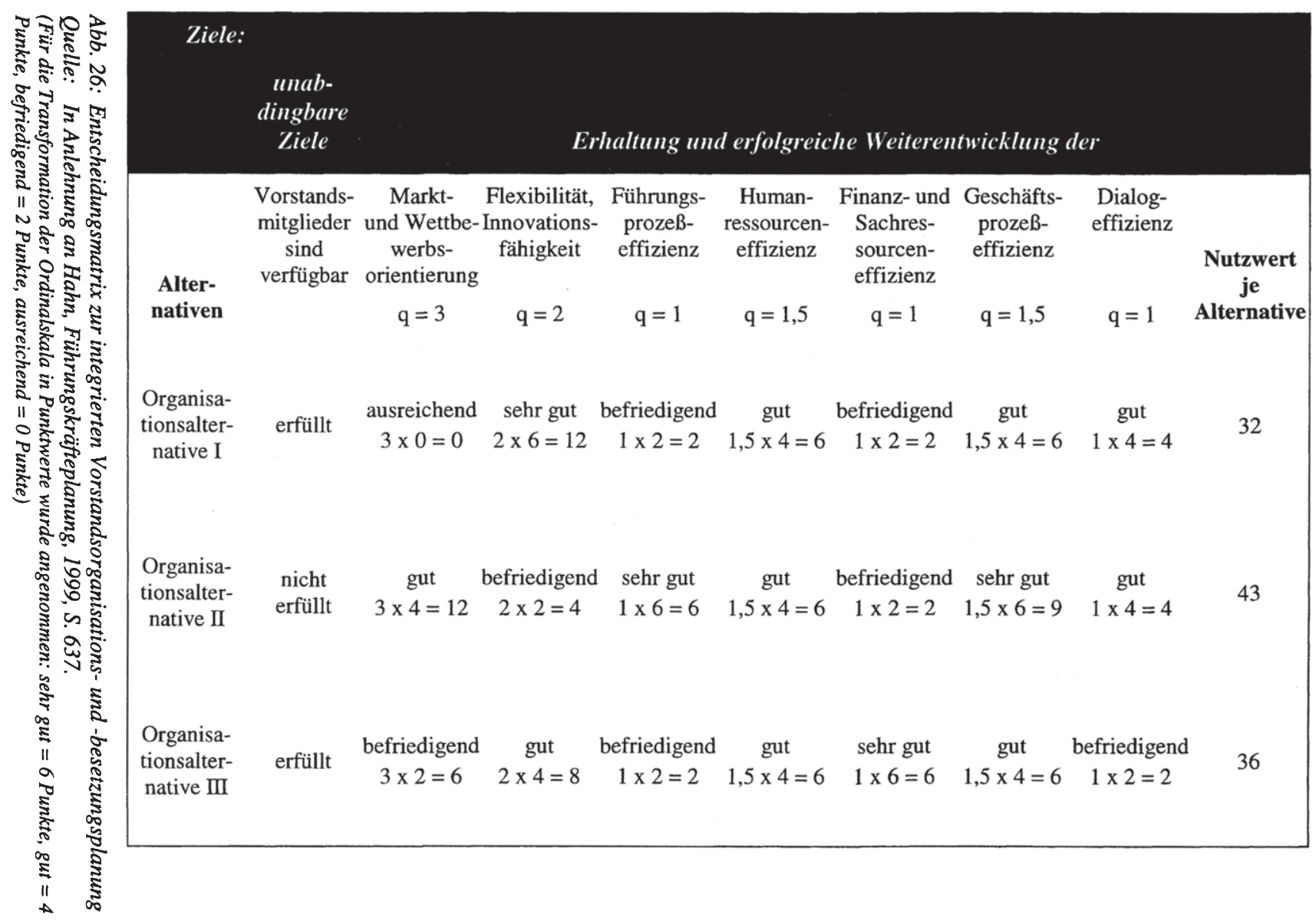


Ebenso wie bei der erstmaligen Bestellung eines Vorstandsmitglieds sollte auch im Rahmen der Wiederbestellung ein erneuter Profilvergleich durchgeführt werden. ${ }^{909}$ Dies bietet Anlaß zur Überprüfung der Stellengestaltung, der Anforderungsprofile der Vorstandsstellen sowie der Fähigkeitsprofile der Vorstandsmitglieder. Hierzu kann der Aufsichtsrat unter anderem auf Informationen aus seiner Kontrolltätigkeit zurückgreifen, die ihm eine Leistungsbeurteilung des Vorstandsmitglieds ermöglichen. Der Aufsichtsrat sollte sich bei seiner Wiederbestellungsentscheidung einerseits von einer gewissen Kontinuität im Vorstand leiten lassen, ${ }^{910}$ andererseits darf die Wiederbestellung nicht zu einem bloßen Routineakt degenerieren, sondern muß ebenso wie die erstmalige Bestellung an den Zielen der Unternehmung und den geplanten Strategien ausgerichtet werden. Daneben sollte eine Wiederbestellung durch den Aufsichtsrat in solchen Fällen unterbleiben, in denen eine weniger gravierende Pflichtverletzung des Vorstandsmitglieds erfolgt ist, auf deren Grundlage keine Abberufung möglich war. ${ }^{911}$ Dieses Drohpotential kann ausreichend sein, um Pflichtverletzungen zu verhindern.

Die Abberufung eines Vorstandsmitglieds erfolgt im Rahmen der Abberufungsplanung. Da sie de jure nur aus wichtigem Grund möglich ist, beispielsweise wegen „Verfehlungen“ des Betroffenen hinsichtlich Verhalten oder Strategie der Unternehmung, ist eine längerfristige Vorbereitung nicht wahrscheinlich. OESTERLE weist darauf hin, daß die Ablösung eines Spitzenmanagers mit zunehmender Amtszeit seltener wird. ${ }^{912}$ Er begründet dies mit einer zunehmenden Unangreifbarkeit erfolgreicher Manager. Der Vorstandsausschuß muß daher bei der Beurteilung der Vorstandsmitglieder die zukünftigen Erfolgsprognosen berücksichtigen und sich weniger von den Erfolgen des Vorstandsmitglieds in der Vergangenheit leiten lassen. Dabei kann die Funktionsfähigkeit des externen und des internen Marktes für Führungskräfte aus Sicht der Principal AgentTheorie handlungskontrollierend wirken: Sollte ein Vorstandsmitglied nicht im Interesse der Aktionäre handeln, schafft dies eine Diskussionsgrundlage für potentielle Nachfolgediskussionen, die um so realistischer sind, je funktionsfähiger der relevante Führungskräftearbeitsmarkt ist. ${ }^{913}$ Dies setzt jedoch gleichzeitig die tatsächliche Wahrnehmung der Besetzungshoheit des Aufsichtsrats über den Vorstand voraus.

\footnotetext{
909 Vgl. Gollnick, Beurteilung, 1997, S. 71.

910 Zustimmend Oesterle, Führungswechsel, 1999, S. 303.

911 Vgl. Raiser, Kapitalgesellschaften, 1992, S. 113.

912 Vgl. Oesterle, Führungswechsel, 1999, S. 302f.

913 Vgl. Picot/Dietl/Franck, Organisation, 1997, S. 196f
} 


\section{G. Professionalisierung}

Die in diesem Kapitel isoliert dargestellten Elemente Planungsobjekt, Planungsorganisation, Planungssubjekt, Planungsinstrument und Planungsprozeß bilden in ihrer Gesamtheit das Planungssystem des Aufsichtsrats. Es wurde gezeigt, wie die einzelnen Elemente ausgestaltet sein sollten, um eine ordnungsgemäße Funktionswahrnehmung von Aufsichtsräten zu gewährleisten. Die Elemente zeichnen sich durch vielfältige Interdependenzen aus, die die Durchführung des Planungsprozesses beeinflussen. Dabei läßt es die exemplarische Betrachtung des Aufsichtsratsplanungsprozesses „Vorstandsbesetzungs- und Organisationsplanung" fraglich erscheinen, ob die hier entwickelte Konzeption eines Aufsichtsratsplanungssystems vor dem Hintergrund des bisher vorzufindenden Verständnisses des Aufsichtsratsmandats umsetzbar ist. Während Aufsichtsratsmitglieder selbst ihr Mandat überwiegend als Ehrenamt betrachten, geht die juristische Sichtweise von einer nebenamtlichen Aufgabenwahrnehmung aus. ${ }^{914}$ Dieser nebenamtliche Charakter wird gleichzeitig als Begründung herangezogen, um nur begrenzte Anforderungen an die Aufgaben(erfüllung) zu stellen. MERTENS spricht in diesem Zusammenhang von normativ vorgegebenen institutionellen Kapazitätsgrenzen des Aufsichtsrats. ${ }^{915}$

Diese Sichtweise ist bereits in der Vergangenheit kritisiert worden, so daß Forderungen nach einer Professionalisierung der Aufsichtsratstätigkeit erhoben wurden. ${ }^{916} \mathrm{Da}$ sich noch kein einheitliches Begriffsverständnis entwickelt hat, ist zunächst danach zu fragen, was bisher unter der Professionalisierung verstanden wurde. Für LUTTER bedeutet Professionalität die Sicherung der Kontinuität der Aufsichtsratsarbeit, die regelmäßige, kurzfristige Information der Aufsichtsratsmitglieder in gleichbleibender, also vergleichbarer Form, Zugriff auf die Hilfsmittel der Gesellschaft, eine angemessene Vergütung, aber auch eine bei Pflichtverletzungen drohende Haftung. ${ }^{917}$ Auch LÜCK geht von einem sehr umfassenden Professionalisierungsverständnis aus, wenn er hierzu personelle Anforderungen an Aufsichtsratsmitglieder, organisatorische Regelungen der Auf-

\footnotetext{
914 Vgl. Lutter, Professionalisierung, 1995, S. 1133.

915 Vgl. Mertens, Kölner Kommentar, 1996, Anm. 7 der Vorbemerkungen zu § 95 AktG; aus betriebswirtschaftlicher Perspektive zustimmend Römer, Aufsichtsrat, 1990, S. 127.

916 Vgl. insbesondere Lutter, Aufsichtsrat, 1994, S. 177; Lutter, Professionalisierung, 1995, S. 11331134; Lutter, Aufsichtsratstätigkeit, 1995, S. 307-309; auch Lück, Professionalisierung, 1998, S. 39, sowie m.w.N. Möllers, Professionalisierung, 1995, S. 1725-1735; Oesterle, Führungswechsel, 1999, S. 310; mit ähnlichen Forderungen für amerikanische Gesellschaften Conger/Finegold/Lawler, Performance, 1998, S. 140.

917 Vgl. Lutter, Professionalisierung, 1995, S. 1133; ähnlich Lutter, Aufsichtsratstätigkeit, 1995, S. 307-309.
} 
sichtsratstätigkeit, die Informationsversorgung des Aufsichtsrats, seine Zusammenarbeit mit dem Abschlußprüfer und seine Berichterstattung gegenüber der Hauptversammlung zählt. ${ }^{918}$ Er fordert darüber hinaus eine stärkere Verantwortungsübernahme der Aufsichtsratsmitglieder und einen Wandel ihrer bisherigen Mentalität bei der Wahrnehmung des Mandats. Von einem weniger umfassenden Professionalisierungsverständnis geht dagegen MöLLERS aus, wenn er die Informationsversorgung und die Ausschußbildung in den Vordergrund stellt. ${ }^{919}$ Er unterscheidet zusätzlich einen zweiten, engeren Professionalisierungsbegriff, unter dem er die Frage nach dem Aufsichtsratsmandat als Beruf stellt; hierunter faßt er den erforderlichen zeitlichen Einsatz und die Vergütung.

Es zeigt sich, daß zur Professionalisierung teils unterschiedliche, teilweise aber auch identische Aspekte gezählt werden, wobei jedoch Abweichungen bei der jeweiligen Gewichtung bestehen. Inhaltlich bilden die Forderungen zur Professionalisierung der Aufsichtsratstätigkeit eine Teilmenge des umfassenderen Aufsichtsratsplanungssystems; hinzu kommen die später zu behandelnden Fragen der Vergütung und der Haftung. Daher soll unter Professionalisierung des Aufsichtsrats die Implementierung des hier konzipierten Aufsichtsratsplanungssystems verstanden werden. Diese Professionalisierung im weiteren Sinne beinhaltet die unternehmungsindividuelle Ausgestaltung der einzelnen Systemelemente. Die Individualisierung bezieht sich jedoch nicht auf die Entscheidung, ob ein bestimmtes Element in der hier vorgeschlagenen Form eingeführt wird, sondern lediglich darauf, in welcher konkreten Ausgestaltung dies erfolgt.

Die Professionalisierung des Aufsichtsrats erweitert seine normativ vorgegebenen institutionellen Kapazitätsgrenzen. Sie beinhaltet jedoch nicht nur das Potential zu einer umfassenden Interpretation der Aufsichtsratstätigkeit. Indem den Aufsichtsratsmitgliedern geeignete institutionelle Vorkehrungen für eine professionelle Aufgabenwahrnehmung aufgezeigt werden, entfällt gleichzeitig die Exkulpationsmöglichkeit für ihre bisher oftmals zu beobachtende unbefriedigende Aufgabenwahrnehmung.

Professionalisierung kann auch in einem personenbezogenen Sinn verwendet werden. Diese Professionalisierung im engeren Sinne erfordert erstens die Konkretisierung der bei der Analyse des Planungssubjekts entwickelten umfassenden Anforderungen an Aufsichtsratsmitglieder, von denen neben den Fähigkeiten sowie der Unabhängigkeit insbesondere dem zeitlichen Einsatz eine besondere Bedeutung beizumessen ist:

\footnotetext{
918 Vgl. Lück, Professionalisierung, 1998, S. 39.

919 Vgl. Möllers, Professionalisierung, 1995, S. 1725, 1728-1732.
} 
„Ein Gremium von 20 Leuten, die 89 Tage lang ganz andere Dinge machen, kann nicht am 90. Tag der wirklich kritische Partner und Kontrolleur eines hochprofessionellen Vorstands sein: er muß selbst und aus sich heraus Professionalität gewinnen, um den VorstandsProfessionals gewachsen zu sein.“920

Daher ist als Ausdruck einer personenbezogenen Professionalisierung eine berufsmäßige, sprich hauptberufliche Aufgabenwahrnehmung durch die Mandatsträger anzustreben.${ }^{921}$ Dies bedeutet nicht, daß jedes Aufsichtsratsmitglied nur in einem Aufsichtsrat vertreten sein sollte. Es sollte jedoch der Ausnahmefall sein, daß Aufsichtsratsmitglieder einen Hauptberuf, beispielsweise als Vorstandsmitglied, ausüben, der ihre zeitliche Kapazität vollständig in Anspruch nimmt. ${ }^{922}$ Dabei ist jedoch zu berücksichtigen, daß abhängig von der organisatorischen Ausgestaltung des Aufsichtsrats nicht jedes einzelne Mitglied diesen Anforderungen genügen muß. Dies führt jedoch im Umkehrschluß dazu, daß bestimmte Aufgaben nur dann wahrgenommen werden sollten, wenn das betreffende Aufsichtsratsmitglied dem Professionalitätsanspruch hinsichtlich seiner zeitlichen Verfügbarkeit und auch seiner Fähigkeiten entspricht.

Um die Unabhängigkeit dieser Aufsichtsratsprofis zu gewährleisten und um den Beruf „Aufsichtsrat“ für geeignete Kandidaten attraktiv zu machen, ist zweitens eine Anpassung ihres Anreizsystems vorzunehmen, die ebenfalls zur Professionalisierung im engeren Sinne gezählt werden soll. Hierzu zählt neben der Gewährung materieller Anreize auch eine berufsmäßige Haftung. ${ }^{923}$ Beide Aspekte sind Gegenstand des nachfolgenden Kapitels, in dem Möglichkeiten zur Verhaltensbeeinflussung von Aufsichtsratsmitgliedern aufgezeigt werden. Dabei soll bereits jetzt betont werden, daß trotz der isolierten Darstellung des Aufsichtsratsplanungssystems und des Anreizsystems nur beide Systeme gleichzei-

920 Lutter, Professionalisierung, 1995, S. 1133.

921 Dies fordert Lutter, Aufsichtsrat, 1994, S. 176f., beispielsweise für den Aufsichtsratsvorsitzenden; zustimmend wohl auch Potthoff/Trescher, Aufsichtsratsmitglied, 1999, S. 145, sowie Hutzschenreuter, Unternehmensverfassung, 1997, S. 186f., der mit der hiermit verbundenen höheren Informationsverarbeitungskapazität des Aufsichtsratsvorsitzenden jedoch aufsichtsratsinterne Informationsasymmetrien begründet; ablehnend dagegen m.w.N. Möllers, Professionalisierung, 1995, S. 1732.

922 Vgl. Lutter, Professionalisierung, 1995, S. 1133, der in diesem Kontext von Dilettantismus spricht. Hierbei ist zu berücksichtigen, daß die Wahrnehmung konzerninterner Aufsichtsratsmandate sehr wohl zum Aufgabenfeld eines Vorstandsmitglieds der Mutterunternehmung gehört; dies wird vom Gesetzgeber bei der Ermittlung der Höchstzahl der Aufsichtsratsmandate entsprechend berücksichtigt, da bis zu fünf Konzernmandate nicht angerechnet werden ( $\$ 100 \mathrm{Abs} .2 \mathrm{AktG})$. Ludwig, Aufsichtsräte, 1997, S. 202, spricht sich gegen eine Begrenzung konzerninterner Mandate aus, da diese aus Sicht der Konzernsteuerung sinnvoll seien.

923 Vgl. Lutter, Professionalisierung, 1995, S. 1134, sowie Lutter, Aufsichtsratstätigkeit, 1995, S. 309. 
tig als Ausdruck einer umfassenden Professionalisierung eingeführt werden sollten. Insbesondere ist zu verhindern, daß Aufsichtsratsmitglieder auch dann eine Verbesserung ihres Anreizsystems erfahren, wenn sie nicht gleichzeitig den erhöhten Anforderungen an die Funktionswahrnehmung gerecht werden. 


\section{Verhaltensbeeinflussung von Aufsichtsratsmitgliedern}

\section{A. Verhaltensdeterminanten}

Die ökonomische Analyse der Spitzenverfassung von Publikumsaktiengesellschaften hat ergeben, daß eine zielorientierte Aufgabenwahrnehmung durch Aufsichtsräte die Existenz und die Nutzung eines Aufsichtsratsplanungssystems voraussetzt. Dieses Planungssystem wurde in Kapitel III entwickelt und in seinen einzelnen Facetten dargestellt. Für die Aufsichtsratsmitglieder führt seine Einrichtung und Nutzung zwar zu einer weiteren Aufgabenanreicherung, ${ }^{924}$ es konnte jedoch gezeigt werden, daß die Funktionserweiterung aus betriebswirtschaftlicher Perspektive für die ordnungsgemäße Wahrnehmung der Überwachungsaufgabe im Interesse der Aktionäre notwendig und sinnvoll ist.

Es kann bereits für die bisherige Ausgangssituation festgestellt werden, daß für die Überwachungslücke durch Aufsichtsräte neben den Systemmängeln Verhaltensdefizite bei der Aufgabenwahrnehmung mitverantwortlich sind. ${ }^{925}$ Daher sollte gerade vor dem Hintergrund einer zwischen Aktionären und Aufsichtsrat bestehenden Agency-Beziehung von Aufsichtsräten nicht erwartet werden, daß sie die vorgeschlagenen Gestaltungsempfehlungen ohne eine entsprechende Verhaltensbeeinflussung umsetzen. Die notwendige Verhaltensbeeinflussung scheint aus zwei unterschiedlichen Richtungen erfolgversprechend zu sein: Erstens kann versucht werden, über die Gestaltung des Anreizsystems von Aufsichtsräten das Verhalten der Aufsichtsratsmitglieder zu beeinflussen. ${ }^{926}$ Hierzu müssen zunächst wirksame Verhaltensdeterminanten analysiert werden. ${ }^{927}$ Zweitens sind unterstützende Sanktionsmaßnahmen zu überlegen, die im Falle pflichtwidrigen oder unerwünschten Verhaltens zu einer Bestrafung des Aufsichtsratsmitglieds führen. ${ }^{928}$ Hierbei handelt es sich um negative Anreize (Abreize), die zum Anreizsystem im weiteren Sinne gezählt werden können.

924 Ebenfalls mit dem KonTraG gewachsene Anforderungen feststellend Feddersen, Aufsichtsrat, 2000, S. 386; zustimmend Mäger, Vergütung, 1999, S. 1389.

Vgl. Bernhardt, Aufsichtsrat, 1995, S. 315, der die Ursache von Aufsichtsratsmängeln in einem falsch verstandenen Rollenverständnis als Ehren-Amt und nicht als Arbeits-Amt sieht, sowie Lutter, Aufsichtsratstätigkeit, 1995, S. 295, der Bequemlichkeit, Unterbezahlung, Überbeschäftigung, mangelnde Professionalität und Ritualisierung als ganz normale menschliche und keine rechtlichen Mängel in der Arbeit von Aufsichtsräten feststellt.

Vgl. zur Anreizsystemgestaltung Pellens/Crasselt/Rockholtz, Entlohnungssysteme, 1998, S. 13f.; Picot/Neuburger, Agency Theorie, 1995, Sp. 19f.

Vgl. Becker, Anreizsysteme, 1995, Sp. 36.

Vgl. Dubs, Sanktionen, 1995, Sp. 1868f.; Potthoff/Trescher, Aufsichtsratsmitglied, 1999, S. 327. 
Eine beabsichtigte Verhaltensbeeinflussung von Aufsichtsratsmitgliedern setzt die Kenntnis ihrer Teilnahme- und Leistungsdeterminanten voraus. Sind diese Determinanten bekannt, können über die Gewährung geeigneter Anreize positive Teilnahme- und Leistungsentscheidungen bewirkt werden. ${ }^{929}$ Mit der AnreizBeitrags-Theorie liegt ein grundlegendes Verhaltensmodell zur Erklärung von Teilnahme- und Leistungsentscheidungen vor. ${ }^{930}$ Sie besagt, daß von einer Person immer dann eine positive Entscheidung zu erwarten ist, wenn alle subjektiv von ihr erwarteten Belohnungen (Anreiznutzen) die individuellen Kosten für die Beiträge (Beitragsnutzen) mindestens kompensieren. Die grundsätzliche Einsatz- und Leistungsbereitschaft (Motivation) der Person hängt wesentlich von ihrer Motivstruktur ab. Neben einer intrinsischen Motivation, bei der ein Verhalten um seiner selbst willen angestrebt wird, ist bei der Anreizgewährung primär die extrinsische Motivation zu berücksichtigen, die in einem instrumentellen Charakter zur angestrebten Belohnung steht. Während materielle extrinsische Motive auf die Erreichung monetär faßbarer Belohnungen ausgerichtet sind, werden mit immateriellen extrinsischen Motiven monetär nicht meßbare Ziele angestrebt. Darüber hinaus muß die Person davon ausgehen, eine erwartete Leistung erbringen und mit ihrer Leistung die angestrebte Belohnung erhalten zu können. Die individuelle Leistung der Person ist unter diesen Voraussetzungen abhängig von ihren Fähigkeiten, ihrer Motivation und der Wirkung der gewährten Anreize: ${ }^{931}$

\section{Leistung $=\mathrm{f}($ Fähigkeiten, Motivation, Anreize)}

In der Vergangenheit war sowohl die Erreichung einer positiven Teilnahme- als auch Leistungsentscheidung von qualifizierten Aufsichtsratsmitgliedern problematisch, ${ }^{932}$ so daß die bestmögliche Ausrichtung des Verhaltens auf die Ziele der Unternehmung und die Interessen der Anteilseigner nicht immer gewährleistet war. Die Ursache hierfür liegt in dem Umstand begründet, daß die Aufsichtsratsmitglieder als Bestandteil des Beziehungsgeflechts der Unternehmung ausgewählt werden, ${ }^{933}$ wodurch die Gefahr besteht, daß sie eher die Interessen

929 Vgl. Mag, Personalplanung, 1998, S. 123.

930 Vgl. Becker, Anreizsysteme, 1995, Sp. 36f., sowie mit Bezug auf wertorientierte Vergütungssysteme Achleitner/Wichels, Entlohnungssysteme, 2000, S. 7; grundlegend Cyert/ March, Theory, 1963; March/Simon, Organizations, 1958.

931 Vgl. Mag, Personalplanung, 1998, S. 127.

932 Vgl. Kienbaum, Vergütungsstudie, 2000, S. 59; Mäger, Vergütung, 1999, S. 200; bestätigend Pohle; vgl. hierzu o.V., Verhaltenskodex, 2001, S. 17.

933 Dies bestätigt eine empirische Untersuchung von Jansch, Publikumsgesellschaften, 1998, S. 182ff.; Bernhardt/Witt, Unternehmensleitung, 1999, S. 838, FN 42, bemängeln die weitgehende Abwesenheit von Eigentümern in den Aufsichtsräten von Publikumsaktiengesellschaften. 
ihrer Bezugsgruppe vertreten und weniger die der überwachten Unternehmung und ihrer Aktionäre. ${ }^{934}$ Dies läßt die These begründet erscheinen, daß die von der Unternehmung gewährten Anreize für die Mandatsträger nur von untergeordneter Bedeutung sind, so daß ihre individuelle Leistung zu gering ausfällt. ${ }^{935}$ Für LENZ/OsTROwSKI führt gerade die verzerrte Anreizstruktur der Aufsichtsratsmitglieder zu ihrer mangelnden Bereitschaft, die ihnen übertragenen Aufgaben effizient im Sinne der Aktionäre durchzuführen. ${ }^{936}$ Als Folge käme es zu Funktionsdefiziten im Überwachungssystem der Unternehmung. ${ }^{937}$ Die notwendige Professionalität der Aufsichtsratsmitglieder wird sich solange nicht einstellen, wie die hierfür erforderlichen Fähigkeiten sowie der notwendige zeitliche Einsatz nicht entsprechend vergütet werden. ${ }^{938}$

Zur unternehmungszielorientierten Verhaltensbeeinflussung der Aufsichtsratsmitglieder sowie zur Erfüllung der gestiegenen Anforderungen im Unternehmungsführungsproze $B$ scheint es erforderlich zu sein, geeignete Anreizsysteme zu implementieren. ${ }^{939}$ Die Entwicklung eines solchen Anreizsystems, bei dem eine Beschränkung auf materielle Anreize erfolgt, findet in drei Schritten statt: Zunächst wird der bestehende juristische Gestaltungsrahmen aufgezeigt. Hieran schließt sich eine kritische Analyse der Anreizwirkungen von in der Praxis vorfindbaren Vergütungsformen und -strukturen an. Sie bilden die Ausgangsbasis zur Formulierung von Anforderungen an ein unternehmungszielorientiertes Anreizsystem für Aufsichtsratsmitglieder sowie zur Darstellung anreizkompatibler Gestaltungsformen. Anschließend sind verschiedene unterstützende Sanktionsmechanismen vorzustellen, die zusätzlich ein unternehmungszielorientiertes Verhalten der Aufsichtsratsmitglieder bewirken sollen.

934 Auf diese Gefahr hinweisend auch Scheffler, Rollenverständnis, 2000, S. 433; bestätigend Jansch, Publikumsgesellschaften, 1998, S. 185. Um eine größere Transparenz herzustellen, hat der Gesetzgeber mit dem KonTraG das Aktiengesetz dahingehend geändert, da $B$ in der Tagesordnung der Hauptversammlung im Rahmen des Vorschlags zur Wahl von Aufsichtsratsmitgliedern deren ausgeübter Beruf einschließlich der betreffenden Unternehmung enthalten sein muß; vgl. Begründung aus dem Regierungsentwurf zu § 124 AktG in Ernst/Seibert/Stuckert, KonTraG, 1998, S. 59.

935 Vgl. Dufey/Hommel/Riemer-Hommel, Corporate Governance, 1998, S. 54, sowie Ludwig, Aufsichtsräte, 1997, S. 152f., die insbesondere die gewährten materiellen Anreize als untauglich zur Bewirkung positiver Leistungsentscheidungen der Aufsichtsratsmitglieder ansieht; anderer Ansicht auf der Grundlage einer empirischen Untersuchung Schmid, Aufsichtsratsvergütung, 1997, S. 80.

936 Vgl. Lenz/Ostrowski, Kontrolle, 1997, S. 1524.

937 In diesem Zusammenhang wirft Pohle der Spitzenverfassung deutscher Unternehmungen mangelnde Effizienz vor; vgl. o.V., Verhaltenskodex, 2001, S. 17.

Vgl. Oltmanns, Aufsichtsräte, 2000, S. $215 f$.

Vgl. Mäger, Vergütung, 1999, S. 1389, 1394. 


\section{B. Gestaltung des materiellen Anreizsystems}

\section{Gestaltungsmöglichkeiten}

Den Aufsichtsratsmitgliedern kann für ihre Tätigkeit eine Vergütung gewährt werden ( $\$ 113$ Abs. 1 S. 1 AktG) ${ }^{940}$ wodurch die Entgeltlichkeit der Überwachungstätigkeit unterstrichen wird. ${ }^{941}$ Dieser Grundsatz ist eine wesentliche Voraussetzung für ein verantwortungsbewußtes Engagement der Aufsichtsratsmitglieder. Er ist jedoch lediglich Ausdruck einer betriebswirtschaftlichen Notwendigkeit und nicht einer juristischen Zwangsläufigkeit, da ein Vergütungsanspruch nur durch die Satzung oder einen Hauptversammlungsbeschluß begründet wird, nicht jedoch per Gesetz ( $\S 113$ Abs. 1 S. 2 AktG) ${ }^{942}$ Hierdurch liegt die Entscheidung, ob und in welcher Höhe den Aufsichtsratsmitgliedern eine Vergütung gewährt wird, allein bei den Aktionären, so daß eine Art „Selbstbedienung" der Aufsichtsratsmitglieder ausgeschlossen werden kann. ${ }^{943}$ Durch die Notwendigkeit einer ordnungsgemäßen Beschlußfassung und die hiermit verbundene detaillierte Offenlegung von Einzelheiten des Beschlußvorschlags ( $\S$ 124 AktG) wird darüber hinaus Transparenz über die Vergütung von Aufsichtsräten geschaffen.

Erfolgt die Festsetzung der Vergütung in der Satzung, ist sie solange verbindlich, bis sie durch einen Hauptversammlungsbeschluß aufgehoben oder geändert wird. ${ }^{944}$ Die Satzung muß die für jedes einzelne Aufsichtsratsmitglied vorgesehenen Zahlungen enthalten. ${ }^{945}$ Soll die Übernahme einzelner Aufsichtsratsstellen (beispielsweise Aufsichtsratsvorsitzender, Stellvertreter oder Ausschußmitglied) zu einem höheren Vergütungsanspruch führen, ist auch dies explizit in die Satzung aufzunehmen. Alternativ kann die Satzung einen Gesamtbetrag für den Aufsichtsrat vorsehen, der bei Fehlen einer quotisierenden Regelung gleichmäBig verteilt wird. Eine differenzierte Zuteilung durch den Aufsichtsrat muß explizit in der Satzung vorgesehen sein, indem sie die Verteilung dem Aufsichtsrat

940 Vgl. Mertens, Kölner Kommentar, 1996, Anm. 3 zu § 113 AktG; Semler, Vergütung, 1999, S. 678.

941 Vgl. Theisen, Vergütungen, 1999, S. 1665; diese Notwendigkeit bezweifelnd Steinitzer, Aktiengesellschaft, 1908, S. 151; zur Vergütung von Aufsichtsratsmitgliedern aus juristischer Perspektive beispielsweise Berger, Aktiengesellschaft, 2000, S. 19-89. Vgl. Mertens, Kölner Kommentar, 1996, Anm. 25 zu § 113 AktG; Semler, Vergütung, 1999, S. 678f.

Vgl. Mertens, Kölner Kommentar, 1996, Anm. 3 zu § 113 AktG; Semler, Vergütung, 1999, S. 681.

Vgl. Mertens, Kölner Kommentar, 1996, Anm. 25 zu § 113 AktG; Semler, Vergütung, 1999, S. 679. 
überträgt. ${ }^{946}$ Von juristischer Seite wird hierzu vorgeschlagen, daß die Satzung einen Verteilungsmodus enthalten sollte, da der Aufsichtsrat ansonsten einen Teil der Vergütungshoheit übertragen bekäme und die Gefahr einer willkürlichen Benachteiligung einzelner Mitglieder bestehen könne. ${ }^{947}$

Eine Änderung der Satzungsbestimmungen oder die Festsetzung einer Vergütung durch einen Hauptversammlungsbeschluß bedarf der einfachen Hauptversammlungsstimmenmehrheit. ${ }^{948}$ Mit einem solchen Beschluß kann die Hauptversammlung auch über die in der Satzung festgelegten Beträge hinaus Vergütungen an einzelne oder an alle Aufsichtsratsmitglieder beschließen. ${ }^{949}$ Eine Vergütungsdifferenzierung ist jedoch grundsätzlich nur aufgabenabhängig möglich, d.h. beispielsweise für den Aufsichtsratsvorsitzenden, seine Stellvertreter oder Ausschußmitglieder. ${ }^{950}$ Dieses Differenzierungsmerkmal entspricht einer anforderungsgerechten Entlohnung, bei der die Leistung einer Person durch die Anforderungshöhe der von ihr ausgeübten Tätigkeit ausgedrückt wird. ${ }^{951}$ Die Leistungsmessung erfolgt auf Grundlage der Messung des Arbeitsinputs. Die gesetzlichen Rahmenbedingungen lassen somit eine outputorientierte Entlohnung, bei der die Leistung in Abhängigkeit vom Arbeitsergebnis gemessen wird, nicht zu. Wenn SEMLER eine unterschiedliche, leistungsabhängige Vergütung von Aufsichtsratsmitgliedern als mit dem geltenden Recht nicht vereinbar ablehnt, ${ }^{952}$ kann hiermit nur eine am individuellen Ergebnis orientierte leistungsgerechte Vergütung gemeint sein. Eine an den Anforderungen orientierte leistungsabhängige Vergütung ist dagegen zulässig. In diesem Sinne ist wohl auch THEISEN zu verstehen, wenn er im Kontext der Anforderungen an ein Aufsichtsratsmandat eine verstärkt leistungsorientierte Vergütungskomponente fordert. ${ }^{953}$ Ausgeschlossen ist ebenfalls eine höhere Vergütung in Abhängigkeit von einer

\footnotetext{
946 Nachdrücklich zustimmend Theisen, Vergütungen, 1999, S. 1670.

947 Vgl. Mertens, Kölner Kommentar, 1996, Anm. 30 zu § 113 AktG; Semler, Vergütung, 1999, S. $680 f$.

Vgl. Mertens, Kölner Kommentar, 1996, Anm. 25 zu § 113 AktG; Semler, Vergütung, 1999, S. $681 f$.

Vgl. Semler, Vergütung, 1999, S. 679; abweichend jedoch Mertens, Kölner Kommentar, 1996, Anm. $28 \mathrm{zu} \S 113$ AktG.

950 Vgl. Mertens, Kölner Kommentar, 1996, Anm. 9 zu § 113 AktG, der dies mit Bezug auf den Grundsatz der Gleichbehandlung aller Aufsichtsratsmitglieder fordert; auch Semler, Vergütung, 1999, S. 681; zustimmend Theisen, Vergütungen, 1999, S. 1669, der mit Verweis auf den Gleichbehandlungsgrundsatz nur sehr enge Grenzen einer Zusatzvergütung sieht.

951 Vgl. zu Entlohnungsprinzipien m.w.N. Mag, Personalplanung, 1998, S. 128.

952 Vgl. Semler, Vergütung, 1999, S. 686.

953 Vgl. Theisen, Vergütungen, 1999, S. 1665.
} 
persönlichen Qualifikation oder aufgrund eines höheren „Marktwertes“ ${ }^{954}{ }^{95}$ Diese beiden Kriterien entsprächen einer qualifikations- bzw. arbeitsmarktgerechten Entlohnung. Der Aufsichtsrat selbst darf jedoch keine Sondervergütungen für einzelne Aufsichtsratsmitglieder beschließen. ${ }^{955}$

Für den Aufsichtsrat kommen grundsätzlich die gleichen Vergütungsbestandteile wie für den Vorstand ( $§ 87 \mathrm{Abs} .1 \mathrm{AktG}$ ) in Betracht. ${ }^{956}$ Es können feste Vergütungen, variable Vergütungen, Aufwandsentschädigungen und Sachleistungen unterschieden werden. ${ }^{957}$ Die feste Vergütung soll als angemessene Entschädigung für die Aufsichtsratstätigkeit den erforderlichen Zeiteinsatz vergüten. ${ }^{958}$ Sie kann durch eine variable Vergütung ergänzt werden, die am wirtschaftlichen Erfolg der Unternehmung orientiert sein sollte. ${ }^{959}$ Im Gesetz wird ein Sonderfall geregelt, der dem Aufsichtsrat eine Beteiligung am Jahresgewinn gewährt ( 1113 Abs. 3 AktG). Als Bemessungsgrundlage dient der Bilanzgewinn, vermindert um $4 \%$ der auf den Nennbetrag des Aktienkapitals geleisteten Einlagen. Daneben ist auch eine dividendenabhängige Tantieme möglich. $\mathrm{Zu}$ den Aufwandsentschädigungen zählen Sitzungsgelder oder pauschalierte Aufwandsentschädigungen, als Sachleistungen sind beispielsweise Dienstwagen, Dienstwohnungen oder Deputate möglich. ${ }^{960}$

Die Vergütung der Aufsichtsratsmitglieder muß in einem angemessenen Verhältnis zu den Aufgaben des Aufsichtsrats und zur Lage der Gesellschaft stehen ( 1113 Abs. 1 S. 3 AktG). Was als angemessen zu bezeichnen ist, kann jedoch kaum ermittelt werden. ${ }^{961}$ Die konkrete Festlegung der Vergütungshöhe ist unter anderem abhängig von der Branche, der Unternehmungsgröße und -struktur sowie den Anforderungen, die das Amt an das Aufsichtsratsmitglied stellt. Sie ist im Grundsatz gesetzlich nicht begrenzt, so daß insbesondere vor dem Hintergrund ständig wachsender Anforderungen an die Tätigkeit der Aufsichtsratsmit-

\footnotetext{
954 Dies im Widerspruch zu den in Fußnote 950 genannten Autoren als strittig erachtend Potthoff/Trescher, Aufsichtsratsmitglied, 1999, S. 134, mit Verweis auf Lutter/Krieger, Rechte, 1993, S. 224f.

955 Vgl. Peltzer, Vergütung, 1997, S. 381.

956 Vgl. Mertens, Kölner Kommentar, 1996, Anm. 11 zu § 113 AktG.

957 Vgl. Semler, Vergütung, 1999, S. 682.

958 Zustimmend Theisen, Vergütungen, 1999, S. 1667.

959 Vgl. Semler, Vergütung, 1999, S. $682 \mathrm{f}$.

960 Mit weiteren Beispielen Mertens, Kölner Kommentar, 1996, Anm. 11 zu § 113 AktG.

961 Vgl. Semler, Vergütung, 1999, S. 684f.
} 
glieder für die Übernahme von Sonderfunktionen auch Vergütungen ähnlich der von Vorstandsmitgliedern begründbar sind. ${ }^{962}$

\section{Analyse empirischer Befunde}

Nachdem die Gestaltungsmöglichkeiten für Anreizsysteme von Aufsichtsräten aufgezeigt wurden, wird in diesem Abschnitt die in der Unternehmungspraxis vorzufindende Vergütungspraxis für Aufsichtsräte analysiert. Die Grundlage hierfür bilden drei in ihrer Vorgehensweise stark divergierende empirische Untersuchungen. Als erstes handelt es sich um eine Analyse von 125 Geschäftsberichten aus den Jahren 1989 bis 1993 von KNOLL/KNOESEL/Probst. ${ }^{963}$ Daneben wird eine Auswertung von THEISEN berücksichtigt, der die im Zeitraum vom 1.1.1999 bis 31.5.1999 im Bundesanzeiger veröffentlichten Tagesordnungen auf vergütungsrelevante Satzungsänderungen hin untersuchte. ${ }^{964}$ Schließlich fließen die Ergebnisse der 23. Auflage der KIENBAUM-Vergütungsstudie 1998/99 in die Analyse mit ein. ${ }^{965}$ Die Untersuchungsergebnisse werden nach der Höhe der Vergütung, ihrer Struktur sowie der Differenzierung gegliedert. Diese Kriterien bieten Anknüpfungspunkte für die angestrebte Entwicklung von Gestaltungsempfehlungen einer unternehmungszielorientierten Vergütung von Aufsichtsratsmitgliedern.

Die Höhe der Aufsichtsratsvergütung weist eine verhältnismäßig breite Streuung auf. ${ }^{966}$ Im Durchschnitt aller in der KIENBAUM-Studie erfaßten Gesellschaften beträgt sie $22.500 \mathrm{DM}$ pro Aufsichtsratsmitglied ${ }^{967}$ Damit liegt sie nur ca. $50 \%$ über dem Niveau von 1968 (14.400 DM). ${ }^{968}$ Unter der Berücksichtigung der in diesem Zeitraum erfolgten Verdreifachung der Lebenshaltungskosten bedeutet dies real einen drastischen Rückgang. Für die hier vorliegende Untersuchung von Publikumsaktiengesellschaften ist ein Betrag für die DAX-

962 Vgl. Mertens, Kölner Kommentar, 1996, Anm. 12 zu § 113 AktG; eine Begrenzung der absoluten Höhe dagegen de jure sehend Theisen, Vergütungen, 1999, S. 1665.

963 Vgl. Knoll/Knoesel/Probst, Aufsichtsratsvergütung, 1997, S. 236-254; zur Datenbasis und zur Methodik S. $242 \mathrm{ff}$.

964 Vgl. Theisen, Vergütungen, 1999, S. 1665-1672; zur Datenbasis S. 1666. Vgl. Kienbaum, Vergütungsstudie, 2000; zur Datenbasis und zur Methodik S. 5f. Vgl. Kienbaum, Vergütungsstudie, 2000, S. 56ff. Zur Berechnungsmethodik vgl. Kienbaum, Vergütungsstudie, 2000, S. 6.

Theisen, Vergütungen, 1999, S. 1665, nennt mit Bezug auf eine Kienbaum-Studie für 1993 einen Betrag von 16.000 DM, so daß für den Zeitraum von 1968 bis 1993 ein ca. 11\%iger Anstieg zu verzeichnen war und von 1993 ein ca. 40\%iger; aufgrund der geringen Höhe der absoluten Beträge kann jedoch noch immer nicht von einer beachtenswerten Höhe der Aufsichtsratsvergütungen gesprochen werden. 
Gesellschaften sowie die DAX100-Gesellschaften aussagekräftiger. ${ }^{969}$ Er liegt mit durchschnittlich 104.100 DM pro Aufsichtsratsmitglied (DAX) bzw. 70.600 DM (DAX100) zwar deutlich höher als der Durchschnittswert aller Gesellschaften, doch auch hierbei kann noch immer nicht von einer nennenswerten Vergütung gesprochen werden. ${ }^{970}$ Sie beträgt für die DAX-Gesellschaften lediglich 5,4 $\%$ der durchschnittlichen pro-Kopf-Vergütung der Vorstandsmitglieder, für die DAX100-Gesellschaften sind dies sogar nur 4,6\%. Dies liegt nur unwesentlich über dem Wert von $3 \%$ für die Aufsichtsrats-/Vorstands-Vergütungsrelation aller in der KIENBAUM-Studie erfaßten Gesellschaften und deutlich unter dem für 1968 ermittelten Wert von $9 \%$ (vgl. Abb. 27). ${ }^{971}$

\begin{tabular}{|c|c|c|c|}
\hline 1968 & \multicolumn{3}{|c|}{1998} \\
\hline \multicolumn{2}{|c|}{ alle Gesellschaften } & DAX100 & $D A X$ \\
\hline $9 \%$ & $3 \%$ & $4,6 \%$ & $5,4 \%$ \\
\hline
\end{tabular}

Abb. 27: Verhältnis von durchschnittlicher Aufsichtsratsvergütung pro Kopf zur durchschnittlichen Vorstandsvergütung pro Kopf in \%

Quelle: In Anlehnung an Kienbaum, Vergütungsstudie, 2000, S. 56, 106f.

Zudem ist davon auszugehen, daß mit der Einführung von wertorientierten Vergütungssystemen, insbesondere von Stock Option-Programmen, für Vorstände eine weitere Entkoppelung von Vorstands- und Aufsichtsratsvergütungen erfolgen wird. ${ }^{972}$

Die Vergütungsstruktur zeichnet sich in der Praxis durch die vollständige Ausschöpfung des juristisch möglichen Spektrums aus. ${ }^{973}$ Sie setzt sich zumeist aus mehreren Bestandteilen zusammen:

969 Bei den DAX-Gesellschaften dürfte es sich ausnahmslos um Publikumsaktiengesellschaften handeln, für die MDAX ${ }^{\circledast}$-Gesellschaften gilt dies beispielsweise im Falle dominanter Großaktionäre nicht immer. Da es sich bei ihnen jedoch zumindest um Gesellschaften mittlerer Kapitalisierung handelt (vgl. Deutsche Börse, MDAX ${ }^{\circledast}, 1997$, S. 2), werden die entsprechenden Werte hier zum Vergleich mit berücksichtigt.

970 Vgl. Kienbaum, Vergütungsstudie, 2000, S. 106f.

971 Vgl. Kienbaum, Vergütungsstudie, 2000, S. 56; eine viel zu geringe Vergütung von Aufsichtsratsmitgliedern bemängelnd auch Lutter, Aufsichtsratstätigkeit, 1995, S. 303, sowie Theisen, Vergütungen, 1999, S. 1665, die sich jedoch beide auf einen Durchschnittsbetrag von 16.000 DM beziehen.

972 Vgl. Seibert, Stock Options, 1998, S. 42f., der aus diesem Grund die Herausnahme des Aufsichtsrats aus dem Kreis der Begünstigten des mit dem KonTraG neu gefaßten $\S 192$ Abs. 2 S. 3 AktG bedauert; ablehnend im Gesetzgebungsverfahren DAV, Stellungnahme, 1997, S. 163, 173, wobei Oltmanns, Aufsichtsräte, 2000, S. 217, mit einer Beschreibung der Herausnahme des Aufsichtsrats aus dem Begünstigtenkreis im Gesetzgebungsverfahren des KonTraG im Ergebnis zutreffend feststellt, daß dies diametral zur Professionalisierungsforderung des KonTraG steht.

973 Vgl. Kienbaum, Vergütungsstudie, 2000, S. 62; Theisen, Vergütungen, 1999, S. 1668. 
1. fixe Vergütungen als Einmalzahlungen,

2. variable Vergütungen in Abhängigkeit von der Dividende oder dem Gewinn gemäß § 113 Abs. 3 AktG, vereinzelt auch von weiteren Erfolgskriterien oder der tatsächlichen zeitlichen Inanspruchnahme,

3. Aufwandsentschädigungen für tatsächlich entstandene Ausgaben und als Sitzungsgeld sowie

4. Versicherungsprämien.

THEISEN konstatiert für sein Untersuchungssample ein deutliches Übergewicht der variablen im Vergleich zur fixen Vergütung. ${ }^{974}$ Dies ist jedoch vor dem Hintergrund der bisher üblichen Vergütungsbasis zum Teil mit der anhaltend guten konjunkturellen Lage der Unternehmungen in Verbindung mit dem zu geringen Mindestverzinsungsanspruch der Aktionäre von nur $4 \%$ auf den Nennbetrag ihres eingesetzten Kapitals zu erklären. ${ }^{975}$ Problematisch ist an den in der Praxis gewählten variablen Vergütungsformen die fehlende Berücksichtigung von Marktwerten. ${ }^{976}$ Über die Ausrichtung am Bilanzgewinn bzw. an der Dividende besteht realiter nur eine unzureichende Anreizkompatibilität hinsichtlich der finanziellen Ziele der Aktionäre.

Eine organinterne Differenzierung der Vergütung erfolgt üblicherweise nach den wahrgenommenen Funktionen ${ }^{977}$ Es ist üblich, dem Aufsichtsratsvorsitzenden das doppelte und seinen Stellvertretern das eineinhalbfache der Vergütung eines einfachen Aufsichtsratsmitglieds zu gewähren. Für Aufsichtsratsvorsitzende finden sich zum Teil noch höhere Multiplikatoren. ${ }^{978}$ Daneben ist zunehmend eine aufgabenspezifische Differenzierung zu beobachten, beispielsweise nach der Zugehörigkeit zu Ausschüssen. ${ }^{979}$ Hiermit sollen die gestiegenen Anforderungen und Verpflichtungen der Ausschußmitglieder kompensiert werden. Die aufgabenspezifische Differenzierung kann als Grundlage für eine anforderungsgerechte Vergütung von Aufsichtsratsmitgliedern zu einer Erhöhung der Anreizkompatibilität beitragen.

\footnotetext{
974 Vgl. Theisen, Vergütungen, 1999, S. 1667.

975 Diesen ,ungenügenden Vorwegabzug“ bedauernd auch Semler, Vergütung, 1999, S. 683.

Vgl. Knoll/Knoesel/Probst, Aufsichtsratsvergütung, 1997, S. 239f.; zur theoretisch möglichen Erhöhung der Anreizkompatibilität Laux/Liermann, Organisation, 1993, S. 541ff.; eine „sachgerechte Erfolgsziffer als Tantiemegrundlage“ vermissend auch Semler, Vergütung, 1999, S. 683.

Vgl. mit weiteren Beispielen Kienbaum, Vergütungsstudie, 2000, S. 61; Theisen, Vergütungen, 1999, S. 1668f.

978 Vgl. Kienbaum, Vergütungsstudie, 2000, S. 61, sowie Semler, Vergütung, 1999, S. 685.

979

Vgl. hierzu insbesondere die Übersicht bei Theisen, Vergütungen, 1999, S. 1669.
} 
Insgesamt ist festzustellen, daß in der bisherigen Vergütungspraxis von Aufsichtsräten zwei wesentliche Problembereiche bestehen: Zum einen ist die Vergütungshöhe vor dem Hintergrund der unternehmerischen Verantwortung und der Aufgaben der Aufsichtsratsmitglieder als viel zu gering zu erachten, um eine wirkliche Anreizwirkung zu entfalten. ${ }^{980}$ Für MERTENS ist die erwünschte Professionalisierung von Aufsichtsräten daher nur mit ihrer erheblich höheren Vergütung zu erreichen. ${ }^{981} \mathrm{Er}$ kann sich für vollberuflich tätige Aufsichtsratsvorsitzende sogar das Niveau eines Vorstandsmitglieds vorstellen. Im Umkehrschluß bedeutet dies, da $\beta$ von dem aktuellen Vergütungsniveau keine hinreichende Verhaltensbeeinflussung auf die Aufsichtsratsmitglieder ausgeht.

Daneben führt die mangelnde Berücksichtigung von Marktgrößen im Rahmen der bisher üblichen variablen Vergütungsbestandteile dazu, daß die gegenwärtige Vergütung insgesamt nur unzureichend auf die Erreichung finanzieller Ziele der Aktionäre ausgerichtet ist. ${ }^{982}$

Als Ursache für die geringe Höhe der Aufsichtsratsvergütung kann primär das fehlende persönliche Interesse einzelner Gruppen im Aufsichtsrat an einer Erhöhung genannt werden. ${ }^{983} \mathrm{Zu}$ diesen Gruppen zählen Führungskräfte anderer Unternehmungen, Beamte sowie Gewerkschaftsvertreter, die häufig große Teile oder die gesamte Aufsichtsratsvergütung abführen müssen. Dabei ist zu beachten, daß jede Gegenleistung für diese Abführung einen gravierenden Pflichtverstoß des Aufsichtsratsmitglieds bedeuten würde. ${ }^{984}$ Weitere Ursachen für die seit Jahren zu geringe Vergütung können in der notwendigen Transparenz für Vergütungserhöhungen und in der Tatsache gesehen werden, daß Aufsichtsratsvergütungen nur zur Hälfte steuerlich als Betriebsausgabe abziehbar sind ( $\$ 10 \mathrm{Nr}$. $4 \mathrm{KStG}){ }^{985}$ Insgesamt kann somit festgehalten werden, daß für das Verhalten der Aufsichtsratsmitglieder die Anreize aus ihrem primären oder Hauptbeschäf-

980 Vgl. Theisen, Vergütungen, 1999, S. 1671, der dieses Problem trotz der zum Teil bereits erfolgten Anpassungen sieht.

981 Vgl. Mertens, Kölner Kommentar, 1996, Anm. 12 zu § 113 AktG; zustimmend auch Theisen, Vergütungen, 1999, S. 1665.

982 Vgl. Knoll/Knoesel/Probst, Aufsichtsratsvergütung, 1997, S. 251.

983 Vgl. Kienbaum, Vergütungsstudie, 2000, S. 58f.; Knoll/Knoesel/Probst, Aufsichtsratsvergütung, 1997, S. 241f.; Semler, Vergütung, 1999, S. 679.

Vgl. Theisen, Vergütungen, 1999, S. 1672.

Dies als ,absurde Idee“ des Gesetzgebers bezeichnend bereits Lutter, Aufsichtsratstätigkeit, 1995, S. 303f., der hierin mittelbar die Aussage sieht, daß es sich hierbei zur Hälfte um ein Geschenk oder eine Dividende handele; die Verfassungsmäßigkeit dieser Regelung bestreitend Mertens, Kölner Kommentar, 1996, Anm. 39 zu § 113 AktG; ebenso DSW, Aufsichtsräte, 1995, S. 94; m.w.N. zur historischen Entwicklung ebenfalls kritisch Theisen, Vergütungen, 1999, S. 1671. 
tigungsverhältnis von weitaus größerer Bedeutung sind und somit die bisherige Vergütungspraxis nicht als unternehmungszielkonform bezeichnet werden kann. ${ }^{986}$

\section{Gestaltungsempfehlungen}

\section{a) Wertorientierte Vergütungssysteme}

Wie die Analyse der empirischen Untersuchungsergebnisse gezeigt hat, gewährleisten die in der Praxis gegenwärtig vorzufindenden Vergütungsformen von Aufsichtsräten aufgrund der unzureichenden motivationalen Wirkung keine Ausrichtung ihres Handelns an den Zielen der Unternehmung. Es ist daher nachfolgend zu überlegen, durch welche Vergütungsformen die fehlende Anreizkompatibilität hergestellt werden kann. ${ }^{987}$ Hierzu wird unter Rückgriff auf die Ergebnisse der agencytheoretischen Analyse zur Verringerung der zwischen den Anteilseignern und den Aufsichtsratsmitgliedern bestehenden Principal AgentProblematik und der hiermit verbundenen Erhöhung der Anreizkompatibilität vorgeschlagen, für Aufsichtsräte eine wertorientierte Vergütung vorzusehen. ${ }^{988}$ Es ist daher zu untersuchen, ob und gegebenenfalls wie die vor dem gleichen Problemhintergrund für Vorstandsmitglieder und weitere Führungskräfte diskutierten wertorientierten Vergütungssysteme auch für Aufsichtsratsmitglieder anwendbar sind. ${ }^{989}$

Mit der Einführung wertorientierter Vergütungssysteme wollen die Anreizgeber (Anteilseigner) über eine variable Vergütungskomponente das Handeln der Anreiznehmer (Aufsichtsratsmitglieder) auf die Verfolgung ihrer finanziellen Inte-

986 Vgl. Knoll/Knoesel/Probst, Aufsichtsratsvergütung, 1997, S. 251.

987 Zu den Möglichkeiten einer Erhöhung der Anreizkompatibilität im Rahmen der bestehenden Vergütungsformen vgl. Knoll/Knoesel/Probst, Aufsichtsratsvergütung, 1997, S. 240f., sowie Mäger, Vergütung, 1999, S. 1390ff.

Wie gezeigt wurde, hat sich das Aufsichtsratshandeln ausschließlich an der Verfolgung der Unternehmungsziele zu orientieren, so daß Überlegungen zur Ablehnung einer wertorientierten Vergütung mit der Begründung, der Aufsichtsrat sei auch weiteren Stakeholdern verpflichtet, abzulehnen sind; vgl. hierzu Oltmanns, Aufsichtsräte, 2000, S. 220, der im Ergebnis zustimmt; eine performanceorientierte Vergütung zur Verringerung der Agency-Problematik bereits fordernd Schmidt u.a., Corporate Governance, 1997, S. 119.

Vgl. m.w.N. Friedrichsen, Aktienoptionsprogramme, 2000, S. 195; den Einsatz von Stock Options als variablen, erfolgsabhängigen Vergütungsbestandteil für Aufsichtsratsmitglieder nachhaltig fordernd Claussen, Aktienrechtsreform, 1996, S. 487, sowie Claussen, Aktienoptionen, 1997, S. 1829, der den zu engen Bezieherkreis für Aktienoptionen des KonTraG bemängelt; auch Zimmer, Ausgabe, 1999, S. 1003; während die Grundsatzkommission Corporate Governance, Corporate Governance-Grundsätze, 2000, S. 240, eine Orientierung der Aufsichtsratsvergütung an der Unternehmenswertsteigerung verlangt, wird dies von dem Berliner Initiativkreis German Code of Corporate Governance, Code, 2000, S. 73, IV.7.3, explizit abgelehnt. 
ressen ausrichten und so die Principal Agent-Problematik vermindern. ${ }^{990}$ Wertorientierte Vergütungssysteme werden in aktienkursorientierte und kennzahlenorientierte Systeme unterschieden. Letztere sind an internen Kennzahlen ausgerichtet, denen eine hohe Korrelation zur tatsächlichen Wertsteigerung zugesprochen wird (vgl. Abb. 28). ${ }^{991}$

\begin{tabular}{|c|c|c|}
\hline \multicolumn{3}{|c|}{ Wertorientierte Vergütungssysteme } \\
\hline \multicolumn{2}{|c|}{ Aktienkursorientiert } & \multirow[b]{2}{*}{ Kennzahlenorientiert } \\
\hline $\begin{array}{l}\text { Echte Eigenkapital- } \\
\quad \text { instrumente }\end{array}$ & $\begin{array}{l}\text { Virtuelle Eigenkapital- } \\
\text { instrumente }\end{array}$ & \\
\hline $\begin{array}{l}\text { Aktienoptionen } \\
\text { (Stock Options) }\end{array}$ & $\begin{array}{l}\text { Wertsteigerungsrechte } \\
\text { (Stock Appreciation } \\
\text { Rights) }\end{array}$ & $\begin{array}{l}\text { Economic Value Added } \\
\text { (EVA) }\end{array}$ \\
\hline \multirow{2}{*}{$\begin{array}{l}\text { Bedingte Aktienüber- } \\
\text { lassung (Restricted } \\
\text { Stock) }\end{array}$} & $\begin{array}{l}\text { Phantomaktien (Phantom } \\
\text { Stock) }\end{array}$ & $\begin{array}{l}\text { Cash Flow Return on } \\
\text { Investment (CFROI) }\end{array}$ \\
\hline & & $\begin{array}{l}\text { Discounted Cash Flow } \\
\text { (DCF) }\end{array}$ \\
\hline
\end{tabular}

Abb. 28: Wertorientierte Vergütungssysteme

Quelle: In Anlehnung an Pellens/Crasselt/Rockholtz, Entlohnungssysteme, 1998, S. 12.

Hier werden ausschließlich aktienkursorientierte Systeme berücksichtigt, da diese im Gegensatz zu den kennzahlenorientierten Systemen einen unmittelbaren Bezug zum Aktienkurs aufweisen, so daß eine direkte Verbindung zwischen der Entlohnung und der Wertentwicklung besteht. ${ }^{992}$ Im Rahmen aktienkursorientierter Vergütungssysteme, die echte oder virtuelle Eigenkapitalinstrumente beinhalten und Aktien- oder Optionscharakter besitzen, werden verschiedene Varianten unterschieden: Mit Aktienoptionen erhalten die Begünstigten das Recht, Aktien der Unternehmung innerhalb einer bestimmten Frist zu einem vorher festgesetzten Preis zu beziehen. ${ }^{993}$ Die Ausübung ist in der Regel erst

990 Vgl. mit Bezug auf das Management Pellens/Crasselt/Rockholtz, Entlohnungssysteme, 1998, S. 13f., sowie Achleitner/Wichels, Entlohnungssysteme, 2000, S. 2f., 10f.; zur Steuerungsfunktion von Anreizsystemen Becker, Anreizsysteme, 1995, Sp. 39, sowie mit Bezug auf die AgencyProblematik Picot/Neuburger, Agency Theorie, 1995, Sp. 19f.; mit einem internationalen Vergleich Schwalbach, Performance, 1999, S. 114-118; Wenger/Knoll/Kaserer, Stock options, 1999, S. 35; Zimmer, Ausgabe, 1999, S. 999.

991 Vgl. Pellens/Crasselt/Rockholtz, Entlohnungssysteme, 1998, S. 11ff.; auch Achleitner/Wichels, Entlohnungssysteme, 2000, S. 2, 7-10; zu einem kritischen Vergleich der Kennzahlen im Rahmen einer wertorientierten Unternehmungssteuerung Pfaff/Bärtl, Konzepte, 1999, S. 85-115.

Vgl. Achleitner/Wichels, Entlohnungssysteme, 2000, S. 2, 9; zu Problemen bei der Ausgestaltung kennzahlenorientierter Entlohnungssysteme Pellens/Crasselt/Rockholtz, Entlohnungssysteme, 1998, S. $18 f$.

993 Vgl. zu einer Übersicht verschiedener Optionsmodelle Steiner/Bruns, Wertpapiermanagement, 2000, S. 293-295. 
nach Ablauf einer Sperrfrist möglich und an bestimmte Bedingungen geknüpft. Bei der bedingten Aktienüberlassung übergibt die Unternehmung unter Verfügung bestimmter Einschränkungen eine bestimmte Anzahl an Aktien bzw. einen festgelegten Betrag in Aktien an einen Begünstigten. ${ }^{994}$

Im Gegensatz zu diesen echten Eigenkapitalinstrumenten, bei denen die Begünstigten eine Gesellschafterposition einnehmen, werden mit virtuellen Instrumenten die finanziellen Konsequenzen echter Instrumente auf Seiten des Begünstigten nachgebildet. ${ }^{995}$ Bei Wertsteigerungsrechten erfolgt eine Beteiligung der Begünstigten an der Wertsteigerung der Aktien, ohne daß sie das Recht auf den Erwerb der Aktien erhalten. Bei ihnen gelten ähnliche Bedingungen wie bei den Aktienoptionen. Dagegen sind Phantomaktien Bucheinheiten, deren Wert an die Aktienkursentwicklung gebunden ist. Ihr Gegenwert wird zu einem späteren Zeitpunkt ausgezahlt. Sowohl die echten als auch die virtuellen Eigenkapitalinstrumente sind aus juristischer Perspektive bereits de lege lata für Aufsichtsratsmitglieder grundsätzlich umsetzbar, wobei die Ausgabe nackter Optionen ( $\S$ 192 Abs. 2 Nr. 3 AktG) nicht zulässig ist. ${ }^{996}$

\section{b) Anforderungen und Konsequenzen}

Aufgrund der Heterogenität der Vergütungssysteme und der Vielzahl der Gestaltungsparameter werden nachfolgend nicht alle möglichen Ausprägungen diskutiert, sondern Anforderungskriterien ermittelt, die jedes Programm zur wertorientierten Vergütung von Aufsichtsratsmitgliedern erfüllen sollte. ${ }^{997}$ Anforderungen an die Gewährung wertorientierter Vergütungselemente lassen sich sowohl

994 Vgl. Gomez-Mejia/Paulin/Grabke, Executive Compensation, 1995, S. 559, mit weiteren Definitionen zu Long-term incentives; auch Pellens/Crasselt/Rockholtz, Entlohnungssysteme, 1998, S. 12; zu Belegschaftsaktienprogrammen umfassend Baus, Belegschaftsaktie, 1978.

995 Vgl. Pellens/Crasselt/Rockholtz, Entlohnungssysteme, 1998, S. 13.

996 Vgl. m.w.N. Friedrichsen, Aktienoptionsprogramme, 2000, S. 196ff.; Klahold, Aktienoptionen, 1999, S. 75; Mäger, Vergütung, 1999, S. 1392f.; Oltmanns, Aufsichtsräte, 2000, S. 224; mit Bezug auf Aktienoptionen gemäB § 192 Abs. 2 Nr. 1 AktG auch Zimmer, Ausgabe, 1999, S. 1000; Claussen/Bröcker, Corporate Governance-Grundsätze, 2000, S. 488, weisen in diesem Zusammenhang auf die in praxi vorzufindenden Ausweichhandlungen hin. Aufgrund der grundsätzlichen Zulässigkeit bleiben hier Fragen der konkreten Ausgestaltung unberücksichtigt; vgl. hierzu die in FN 997 genannten Quellen.

Vgl. für eine einführende Beschreibung der einzelnen Programme und ihrer Designparameter Kramarsch, Managementvergütung, 2000, S. 129-187; zur Ausgestaltung von Optionsplänen Winter, Optionspläne, 2000; auch Klahold, Aktienoptionen, 1999; Weber, Stock Options, 2000, S. 27-46; Weiß, Aktienoptionspläne, 1999, sowie die Beiträge bei Achleitner/Wollmert, Stock Options, 2000. 
aus betriebswirtschaftlicher als auch aus juristischer Perspektive ableiten. ${ }^{998} \mathrm{Da}$ sich diese zum Teil überschneiden, erfolgt eine integrierende Betrachtung.

Durch die gewählte Bezugsgröße muß gewährleistet sein, daß die Begünstigten nur dann eine höhere Vergütung erhalten, wenn auch die Ziele der Aktionäre in höherem Maße erfüllt worden sind (Zielgrößeneignung). ${ }^{999}$ Dies kann längerfristig für den Aktienkurs angenommen werden, so daß die Verwendung dieser Zielgröße bei aktienbasierten Programmen für Aufsichtsräte in Verbindung mit entsprechenden Haltefristen bzw. bei optionsbasierten Programmen mit geeigneten Sperrfristen geeignet scheint. ${ }^{1000}$ Optionsbasierte Programme sollten darüber hinaus indexiert sein. ${ }^{1001}$

Daneben muß die Bezugsgröße durch die Leistung der Begünstigten beeinflußbar sein (Beeinflußbarkeit). ${ }^{1002}$ Hiervon ist bei dem Aktienkurs zunächst nur für Führungskräfte der obersten Hierarchieebene, d.h. primär für Vorstandsmitglieder, auszugehen. Wie die Aufgabenanalyse des Aufsichtsrats gezeigt hat, unterscheidet sich seine Tätigkeit bereits heute zwar quantitativ, nicht aber qualitativ von der des Vorstands, so daß eine Beeinflußbarkeit des Aktienkurses auch durch Entscheidungen des Aufsichtsrats anzunehmen ist. ${ }^{1003}$ Dies gilt insbesondere im Falle einer Implementierung des hier vorgeschlagenen Planungssystems, das den Aufsichtsrat befähigt, aktiv auf die Unternehmungsführung und den Unternehmungserfolg Einfluß zu nehmen. ${ }^{1004}$

998 Vgl. aus betriebswirtschaftlicher Perspektive Pellens/Crasselt/Rockholtz, Entlohnungssysteme, 1998, S. 14ff., sowie aus juristischer Perspektive unter Beschränkung auf Stock Options Friedrichsen, Aktienoptionsprogramme, 2000, S. 197ff., und unter Einschluß virtueller Vergütungsformen Mäger, Vergütung, 1999, S. 1392f.; m.w.N. auch Achleitner/Wichels, Entlohnungssysteme, 2000, S. 3, 10f., für die die Erfüllung bestimmter Anforderungen die Grundvoraussetzung für die Wirksamkeit und Akzeptanz der Programme ist, sowie Riegler, Anreizsysteme, 2000, S. 159169; zu Defiziten realisierter Stock Option-Programme Wenger/Knoll/Kaserer, Stock options, 1999, S. 37f.

999 Vgl. Pellens/Crasselt/Rockholtz, Entlohnungssysteme, 1998, S. 14f.

1000 Vgl. hierzu kritisch Seibert, Stock Options, 1998, S. 47; zur Verwendung des Börsenkurses aus juristischer Perspektive im Ergebnis zustimmend Mäger, Vergütung, 1999, S. 1392.

1001 Vgl. Menichetti, Aktien-Optionsprogramme, 1996, S. 1690f.; Wenger, Aktienoptionsprogramme, 1998, S. 60ff.; Pellens/Crasselt/Rockholtz, Entlohnungssysteme, 1998, S. 15.

1002 Vgl. Ludwig, Aufsichtsräte, 1997, S. 153, die eine fehlende Beeinflußbarkeit bisheriger Bezugsgrößen variabler Aufsichtsratsentgelte bemängelt.

1003 Vgl. Mäger, Vergütung, 1999, S. 1393; zustimmend auch Claussen, Aktienrechtsreform, 1996, S. 487, sowie Friedrichsen, Aktienoptionsprogramme, 2000, S. 197f., 200; ablehnend dagegen DAV, Stellungnahme, 1997, S. 173; kritisch auch Theisen, Vergütungen, 1999, S. 1667.

1004 Schon jetzt aktive Gestaltungspotentiale konstatierend nachdrücklich Mäger, Vergütung, 1999, S. 1393; ebenso Friedrichsen, Aktienoptionsprogramme, 2000, S. 197f. 
Eng mit der Beeinflußbarkeit verbunden ist die erforderliche Manipulationsfreiheit. Auch für die Aufsichtsratsmitglieder ist davon auszugehen, daß sie aufgrund ihrer Insiderkenntnisse über kursbeeinflussende Manipulationsspielräume verfügen. ${ }^{1005}$ Dies schließt sie jedoch ebensowenig wie Vorstandsmitglieder grundsätzlich aus dem Bezieherkreis aus, so daß in den Programmen lediglich entsprechende Vorkehrungen, beispielsweise Ausübungsfenster bei Aktienoptionsprogrammen, vorzusehen sind. ${ }^{1006}$

Zur Gewährleistung der notwendigen Transparenz der Wirkungen wertorientierter Vergütungssysteme sowohl auf die Begünstigten als auch auf die Anteilseigner sind die Programme möglichst einfach auszugestalten und umfassende Informationen zur Verfügung zu stellen. ${ }^{1007}$ Daher ist zu fordern, daß über die Pflichtangaben zur Vergütung der Organmitglieder im Anhang hinaus ( $\$ 285$ Nr. 9 HGB) detaillierte Informationen über die Ausgestaltung des Programms publiziert werden. ${ }^{1008}$ Eine hohe Transparenz kann in Verbindung mit der notwendigen Beeinflußbarkeit durch die Begünstigten deren Akzeptanz erhöhen. Den Anteilseignern ist es zudem möglich, die Wirtschaftlichkeit der Programme besser beurteilen zu können.

Um zu verhindern, daß ein Teil der Vergütungskompetenzen für den Aufsichtsrat von der Hauptversammlung auf den Aufsichtsrat übergeht, muß die Entscheidung über das konkrete Vergütungssystem und alle relevanten Programmparameter durch die Anteilseigner in der Hauptversammlung erfolgen. ${ }^{1009}$ Daher kommt vor dem Hintergrund der Kompetenzen bezüglich der Aufsichtsratsver-

1005 Vgl. Claussen, Aktienoptionen, 1997, S. 1830.

1006 Vgl. Pellens/Crasselt/Rockholtz, Entlohnungssysteme, 1998, S. 16; Seibert, Stock Options, 1998, S. $43 \mathrm{f}$.

1007 Vgl. Pellens/Crasselt/Rockholtz, Entlohnungssysteme, 1998, S. 16f.; Wenger/Knoll/Kaserer, Stock options, 1999, S. 37f.

1008 Vgl. mit Bezug auf virtuelle Optionsprogramme Pellens/Crasselt, Bilanzierung, 2000, S. 169; Oltmanns, Aufsichtsräte, 2000, S. 222f., weist m.w.N. auf die deutlich strikteren Informationspflichten in den USA, Großbritannien und Frankreich hin; erste Ansätze qua Selbstverpflichtung bei der Deutsche Bank AG, Corporate Governance Grundsätze, 2001, S. 11.

1009 Vgl. Claussen, Aktienoptionen, 1997, S. 1830; Friedrichsen, Aktienoptionsprogramme, 2000, S. 201f.; mit einer Auflistung der von der Hauptversammlung zu regelnden Einzelheiten im Rahmen von Aktienoptionsprogrammen Lutter, Aktienoptionen, 1997, S. 6f.; Mäger, Vergütung, 1999, S. 1393; Semler, Vergütung, 1999, S. 680f.; Theisen, Vergütungen, 1999, S. 1670; Zimmer, Ausgabe, 1999, S. 1000; die Vergütungskompetenz der Hauptversammlung betonend auch LG München I, Anforderungen, 2000, S. 193; Oltmanns, Aufsichtsräte, 2000, S. 217f., zeigt, daß auch die Delegation der technischen Abwicklung nicht gegen das Verbot der Selbstalimentierung des Aufsichtsrats spricht; zum Informationsproblem der Aktionäre kritisch Knoll/Möller, AktienOptionspläne, 1999, S. 69-72. 
gütung der notwendigen Transparenz eine hohe Bedeutung zu. ${ }^{1010}$ FRIEDRICHSEN sieht die Gefahr, daß umfangreiche Detailregelungen eine Beschlußfassung in der Hauptversammlung verhindern. ${ }^{1011}$ Er schlägt daher für die Einführung von Aktienoptionsprogrammen gemäß §§ 192 Abs. 2 Nr. 1, 221 AktG de lege ferenda die Einrichtung von aus dem anglo-amerikanischen Bereich bekannten Compensation Committees vor, deren Hauptzweck in der Festlegung der Vergütung der obersten Führungskräfte einer Unternehmung besteht. ${ }^{1012}$ Eine im Rahmen der gesetzlichen Möglichkeiten realisierbare Alternative bietet dagegen die Verwendung von virtuellen Aktienoptionsprogrammen (Wertsteigerungsrechte), da sie im Vergleich zu echten Aktienoptionen verhältnismäßig transparent sind. ${ }^{1013}$ Wie die Verwendung dieses Modelltyps bei der Siemens AG zeigt, ist eine Entscheidung hierüber im Rahmen der Hauptversammlung möglich. ${ }^{1014}$ Zudem wird der Einsatz virtueller Programme im Vergleich zu echten Aktienoptionen insbesondere vor dem Hintergrund unterschiedlicher wirtschaftlicher Konsequenzen für die Aktionäre positiver beurteilt. ${ }^{1015}$

Mit der hier vorgeschlagenen Einführung von wertorientierten Vergütungssystemen für Aufsichtsratsmitglieder sollte nicht nur eine Abkehr von den bisherigen variablen Bezugsgrößen erfolgen, sie muß auch zu der notwendigen Erhöhung der Vergütung von Aufsichtsräten führen. ${ }^{1016}$ Dabei darf nicht die lineare Erhöhung des Vergütungsniveaus für alle Aufsichtsratsmitglieder im Vordergrund stehen, sondern die enge Verbindung zu ihren individuellen Leistungen. ${ }^{1017}$ Aufgrund der Unzulässigkeit einer outputorientierten Differenzierung

1010 Vgl. hierzu mit Bezug auf Stock Options Friedrichsen, Aktienoptionsprogramme, 2000, S. 200ff., sowie Mäger, Vergütung, 1999, S. 1393.

1011 Vgl. Friedrichsen, Aktienoptionsprogramme, 2000, S. 202f.; anderer Auffassung dagegen Oltmanns, Aufsichtsräte, 2000, S. 218f., sowie Weiß, Aktienoptionspläne, 1999, S. $208 f$.

1012 Vgl. mit Bezug auf die anglo-amerikanischen Verhältnisse sowie kritisch zur Festlegung aller Bedingungen des Aktienoptionsplans Seibert, Stock Options, 1998, S. 39f., 43; kritisch zur Übertragung des Compensation Committee-Modells auf Deutschland Claussen, Aktienoptionen, 1997, S. 1830.

1013 Vgl. hierzu Kramarsch, Managementvergütung, 2000, S. 136f.; zu steuerlichen Nachteilen im Vergleich zur reinen Aktienvergütung Mäger, Vergütung, 1999, S. 1393; zu einer finanzwirtschaftlichen Analyse virtueller Aktienoptionsprogramme Pellens/Crasselt, Bilanzierung, 2000, S. 155-160.

1014 Vgl. Einladung zur Hauptversammlung der Siemens AG am 18. Februar 1999; auch Theisen, Vergütungen, 1999, S. 1671.

1015 Vgl. m.w.N. Pellens/Crasselt, Bilanzierung, 2000, S. 159f.

1016 Vgl. Claussen, Aktienrechtsreform, 1996, S. 487, unter Bezug auf eine entsprechende Erhöhungsforderung bei Lutter, Aufsichtsratstätigkeit, 1995, S. 297, 304; zustimmend auch Mäger, Vergütung, 1999, S. 1390.

1017 Vgl. Begründung aus dem Regierungsentwurf zu § 100 AktG in Ernst/Seibert/Stuckert, KonTraG, 1998, S. 54f.; Seibert, Stock Options, 1998, S. 43; Semler, Vergütung, 1999, S. 685f.; dies fordert 
wird hier vorgeschlagen, eine verstärkte Verteilung der Gesamtvergütung in Abhängigkeit von den wahrgenommenen Aufgaben vorzunehmen. Dies ermöglicht sowohl eine entsprechend hohe Vergütung hauptberuflicher Aufsichtsratsvorsitzender als auch eine herausgehobene Vergütung von Mitgliedern arbeitsintensiver Ausschüsse. Ebenso wie für den Aufsichtsratsvorsitzenden als Einzelperson eine höhere aufgabenabhängige Vergütung gewährt wird, sollten auch alle weiteren Aufsichtsratsmitglieder, die besondere Aufgaben im Rahmen der Ressortierung des Aufsichtsrats übernehmen, mit der gleichen Begründung eine höhere Verguitung erhalten. ${ }^{1018}$ Hierbei handelt es sich nicht um eine nur in engen Grenzen mögliche Zusatzvergütung, sondern um eine Erweiterung der aufgabenabhängigen Differenzierung. ${ }^{1019}$ Diese Verteilungsregeln können zusammen mit der Entscheidung über die Ausgestaltung des Vergütungsprogramms durch die Hauptversammlung festgelegt werden.

Von nachrangiger Bedeutung sind die Konsequenzen einer wertorientierten Vergütung für das allgemeine Vergütungsniveau aller Aufsichtsratsmitglieder. Dies sollte eher über eine Anhebung der fixen Bestandteile der Vergütung erfolgen, durch die sicherzustellen ist, daß die Aufsichtsratsmitglieder unabhängig von der Erfolgslage für den erforderlichen Arbeitseinsatz vergütet werden. ${ }^{1020}$

Insgesamt kann festgestellt werden, daß über die unternehmungsindividuelle Ausgestaltung wertorientierter Vergütungssysteme für Aufsichtsratsmitglieder umfassende Potentiale für ihre notwendige Verhaltensbeeinflussung bestehen. Einer Erhöhung der Anreizkompatibilität steht dabei auch die steuerliche Diskriminierung aufgrund einer Zahlung der Hälfte der Vergütung aus versteuertem Einkommen grundsätzlich nicht entgegen. ${ }^{1021}$ Ziel muß es sein, die Unabhängigkeit der Aufsichtsratsmitglieder und insbesondere des Aufsichtsratsvorsitzenden zu erhöhen, ${ }^{1022}$ so daß die notwendige Professionalisierung der Aufsichtsratstä-

auch Oltmanns, Aufsichtsräte, 2000, S. 226, ohne jedoch zu sagen, wie diese Differenzierung nicht nur für den Aufsichtsratsvorsitzenden, sondern auch für weitere Aufsichtsratsmitglieder erfolgen könnte.

1018 Vgl. Semler, Vergütung, 1999, S. 681; Theisen, Vergütungen, 1999, S. 1669, der diese nur für den Fall ablehnt, daß der Aufsichtsrat über solche Vergütungen entscheidet.

1019 Vgl. nochmals Theisen, Vergütungen, 1999, S. 1669, der zu Recht auf die umfassend zu definierende Aufsichtsratstätigkeit hinweist, wodurch nur sehr begrenzt Zusatzvergütungen möglich sind; zustimmend auch Semler, Vergütung, 1999, S. 693.

1020 Vgl. Theisen, Vergütungen, 1999, S. 1667; kritisch zu einer alleinigen Erhöhung der Festvergütung Mäger, Vergütung, 1999, S. 1390f.

1021 Vgl. hierzu Knoll/Knoesel/Probst, Aufsichtsratsvergütung, 1997, S. 242, FN 39; anderer Auffassung Oltmanns, Aufsichtsräte, 2000, S. 217.

${ }^{1022}$ Vgl. Bernhardt, Aufsichtsrat, 1995, S. 315. 
tigkeit durch die Professionalisierung der Vergütung insbesondere vor dem Hintergrund einer wünschenswerten Internationalisierung der Aufsichtsräte unterstützt wird. ${ }^{1023}$ Um jedoch zu verhindern, daß die Anhebung der Vergütung ohne eine entsprechende Leistungs- und Qualitätssteigerung erfolgt, sind unterstützende Sanktionsmechanismen zu berücksichtigen.

\section{Unterstiitzende Sanktionsmechanismen}

\section{Haftung}

Eine Verhaltensbeeinflussung von Aufsichtsratsmitgliedern erfolgt nicht nur durch ihr Anreizsystem; daneben existieren weitere Mechanismen, die entweder über die Entfaltung eines Drohpotentials unerwünschte Verhaltensweisen verhindern sollen oder die Wirkung der Anreize unterstützen. ${ }^{1024}$ So bieten unternehmungsextern kommunizierte Regeln für Aufsichtsräte einerseits gerichtlich überprüfbare Verhaltensvorgaben, andererseits stellen potentiell positive Kapitalmarktreaktionen auf eine offene und umfassende Kommunikation einen Anreiz für einen wertorientiert vergüteten Aufsichtsrat dar. Die enge Verbindung von positiven Anreizen und negativer Sanktionsandrohung betont auch THEISEN, für den zu den Mindestvoraussetzungen einer betriebswirtschaftlich befriedigenden Funktionsweise des deutschen Aufsichtsratsmodells neben einer angemessenen, anforderungsgerechten Vergütung und einer fachlichen Mindestqualifikation auch die gerichtliche Konkretisierung und Durchsetzung der bestehenden gesetzlichen Haftung der Aufsichtsratsmitglieder zählt. ${ }^{1025}$

„Alle Änderungsvorschläge sind ja gut und schön, aber so richtig ans

Laufen bringt man die Leute nur mit dem Haftungsrisiko.“1026

Das Drohpotential der Haftung soll als „Fleet in being“ verhindern, daß die rechtliche Sollvorstellung der Aufsichtsratstätigkeit und das rechtstatsächliche Handeln auseinanderfallen. ${ }^{1027}$ Doch trotz der mit dem KonTraG vereinfachten Durchsetzung von Ersatzansprüchen kritisiert THÜMMEL, daß die scharfen Haftungstatbestände weiterhin einem schwachen Durchsetzungsmechanismus ge-

${ }^{1023}$ Vgl. Mäger, Vergütung, 1999, S. 1389; mit Bezug auf die notwendige Internationalisierung Oltmanns, Aufsichtsräte, 2000, S. 221.

1024 Vgl. zur Sanktionierung von Aufsichtsratsmitgliedern aus juristischer Perspektive Scholz, Sanktionen, 1999, S. 697-720.

1025 Vgl. Theisen, Reform, 1999, S. 247.

1026 Seibert, Aufsichtsrats-Reform, 1994, S. 353.

1027 Vgl. zum Abweichen der Aufsichtsratspraxis von den gesetzlichen Sollvorstellungen Theisen, Reform, 1999, S. 206ff.; nachdrücklich eine verschärfte Haftung fordernd beispielsweise Deckert, Ausschüsse, 1996, S. 994. 
genüberstehen, so daß den Aufsichtsratsmitgliedern nur durch den Insolvenzverwalter ernsthafte Konsequenzen drohen. ${ }^{1028}$

Die Ursache dieses mangelhaften Haftungsrisikos sieht THEISEN in dem Fehlen eines Pflichtenkatalogs, der die Aufsichtsratstätigkeit geeignet konkretisiert. ${ }^{1029}$ Solche anerkannten und überprüfbaren Verhaltensregeln für Aufsichtsräte sind eine Voraussetzung für die Identifikation von haftungsbegründendem Fehlverhalten. Diese Funktion könnten sowohl Grundsätze ordnungsmäßiger Überwachung als auch die in letzter Zeit entwickelten Corporate GovernanceGrundsätze übernehmen.

\section{Verhaltensregeln}

\section{a) Grundsätze ordnungsmäßiger Überwachung}

In der Vergangenheit wurde immer wieder die Entwicklung von Grundsätzen ordnungsmäßiger Überwachung durch den Aufsichtsrat ${ }^{1030}$ bzw. von Grundsätzen ordnungsmäßiger Aufsichtsratstätigkeit ${ }^{1031}$ gefordert; nachfolgend wird allgemein von Grundsätzen ordnungsmäßiger Überwachung ${ }^{1032}$ gesprochen. Sie sollen die de jure abgeleiteten Überwachungs-, Treue- und Verschwiegenheitspflichten der Aufsichtsratsmitglieder, die unter anderem eine Pflicht zur aktiven Mitarbeit, zur Gestaltung einer zweckentsprechenden Organisation oder zur Verfolgung der Unternehmungsziele beinhalten, ergänzen und konkretisieren. ${ }^{1033}$

Die Aufnahme der Gestaltungsvorschläge zum Aufsichtsratsplanungssystem bzw. einzelner Bestandteile in diese Grundsätze würde zu ihrer weiteren Konkretisierung beitragen. Dies gilt insbesondere für das in dieser Arbeit entwickelte Anforderungsprofil für Aufsichtsratsmitglieder, das zum einen die Grundlage

1028 Vgl. Thümmel, Aufsichtsratshaftung, 1999, S. 888; zustimmend m.w.N. Theisen, Reform, 1999, S. 249; zur Reformnotwendigkeit bereits Lutter, Aufsichtsratstätigkeit, 1995, S. 304ff., sowie Schmidt u.a., Corporate Governance, 1997, S. 116f., 140; zur Aktionärsklage beispielsweise Krieger, Aktionärsklage, 1999, S. 343-363, sowie Ulmer, Aktionärsklage, 1999, S. 290-342; Änderungen könnten sich jedoch aus den Forderungen der Regierungskommission „Corporate Governance“, Unternehmensführung, 2001, Rdz. 71, ergeben, die Erleichterungen bei der Klagedurchsetzung vorsieht.

1029 Vgl. Theisen, Reform, 1999, S. 245.

$1030 \mathrm{Vgl}$. Scheffler, Grundsätze, 1995, S. 207-212.

1031 Vgl. Arbeitskreis „Externe und interne Überwachung“ der Schmalenbach-Gesellschaft/Deutsche Gesellschaft für Betriebswirtschaft e. V., Grundsätze, 1995, S. 1-4.

1032 Vgl. Potthoff, Prüfung, 1961, S. 576ff.; Potthoff, Kodex, 1995, S. 163-164; umfassend Theisen, Unternehmungsführung, 1987; Theisen, Grundsätze, 1995, S. 193-203; Theisen, Überwachung, 1996, S. 75-106.

1033 Vgl. zu diesen und weiteren Pflichten nochmals Abschnitt II.B.2.c)(5). 
für eine angemessene Vergütung bildet, zum anderen zur Ermittlung von pflichtwidrigem Handeln herangezogen werden sollte. ${ }^{1034}$ Da das Anforderungsprofil für eine Aufsichtsratsstelle die bei dem Stelleninhaber notwendigen Fähigkeiten konkretisiert, kann das Fehlen entsprechender Fähigkeiten bei der Übernahme eines Aufsichtsratsmandats oder eines bestimmten Aufgabengebietes im Rahmen der Gremienarbeit aufgrund eines Übernahmeverschuldens haftungsbegründend sein, da hierdurch das Eignungsprinzip verletzt würde. ${ }^{1035}$ So ließe es sich präventiv vermeiden, daß die fehlende Eignung von Aufsichtsratsmitgliedern zu einer Vernachlässigung der Überwachungsaufgaben führt. Diese Situation beschreibt MöLLERS beispielsweise für die Gruppe der Arbeitnehmervertreter im Aufsichtsrat, die aufgrund ihrer fehlenden Fachkenntnis den Prüfungsbericht des Abschlußprüfers oftmals nicht zur Kenntnis nehmen würden. ${ }^{1036}$ Seine Schlußfolgerung hieraus ist jedoch zu kritisieren, da er von der in praxi häufig fehlenden Qualifikation darauf schließt, daß diese dann auch rechtlich nicht verlangt werden dürfe. Hier ist ihm nachhaltig zu widersprechen, da dies faktisch zu einer Aushöhlung der Funktionsfähigkeit des Aufsichtsorgans beitragen würde.

Unter teilweisem Rückgriff auf die Grundsätze ordnungsmäßiger Unternehmungsführung, die neben den Grundsätzen ordnungsmäßiger Überwachung auch solche zur Leitung sowie zur Abschlußprüfung enthalten, sind in letzter Zeit sogenannte Corporate Governance-Grundsätze formuliert worden, die ebenfalls Beschreibungen einer zweckentsprechenden Aufsichtsratstätigkeit beinhalten. ${ }^{1037}$ Insofern sind die Corporate Governance-Grundsätze als modifizierte, erweiterte und mit einem aktuellen Schlagwort versehene Weiterentwicklung zu verstehen.

\section{b) Corporate Governance-Grundsätze}

In Deutschland sind zwei konkurrierende Corporate GovernanceGrundsatzkataloge entwickelt worden, die eine länderspezifische Konkretisie-

1034 Vgl. zustimmend zu beiden Funktionen Feddersen, Aufsichtsrat, 2000, S. 389f.

1035 Vgl. zur Haftung aus Übernahmeverschulden Semler, Kompetenzen, 1999, S. 50; mit Bezug auf Ausschüsse Deckert, Ausschüsse, 1996, S. 992f.; zur Diskussion um die Bedeutung des Anforderungsprofils Bleicher/Leberl/Paul, Unternehmungsverfassung, 1989, S. 78f., sowie m.w.N. Theisen, Reform, 1999, S. 248.

1036 Vgl. Möllers, Professionalisierung, 1995, S. 1733.

1037 Vgl. zu den Grundsätzen ordnungsmäßiger Unternehmungsführung von Werder, Grundsätze, 1996; Berliner Initiativkreis German Code of Corporate Governance, Code, 2000, S. 65, IV.1.1., mit einem expliziten Verweis auf die Grundsätze ordnungsmäßiger Überwachung, sowie S. 65, IV.2.3, mit einem Verweis auf die Grundsätze ordnungsmäßiger Unternehmungsleitung; zu den Grundsätzen ordnungsmäßiger Unternehmungsführung auch Grundsatzkommission Corporate Governance, Corporate Governance-Grundsätze, 2000, S. 239. 
rung internationaler Kodizes darstellen. ${ }^{1038}$ Sie umfassen sowohl kapitalmarktals auch vor allem gesellschaftsrechtliche Empfehlungen zur Verbesserung der Corporate Governance von Unternehmungen, wobei dieser Begriff üblicherweise weiter gefaßt wird als in der vorliegenden Arbeit. ${ }^{1039}$ Als wesentlichen Bestandteil umschließen diese Best Practices immer auch Gestaltungsempfehlungen für den Aufsichtsrat und seine Zusammenarbeit mit dem Vorstand sowie mit dem Abschlußprüfer der Gesellschaft. ${ }^{1040}$ Vor allem internationale Kapitalanleger sind ein wichtiger Adressat dieser Grundsatzkataloge, da das deutsche, dualistische System der Unternehmungsleitung und -kontrolle sowie insbesondere die unternehmerische Mitbestimmung international unüblich und wenig akzeptiert sind. ${ }^{1041}$

Da die inhaltlichen Vorschläge der Corporate Governance-Grundsatzkataloge bereits bei der Entwicklung des Aufsichtsratsplanungssystems berücksichtigt wurden, soll an dieser Stelle auf eine Beschreibung der Einzelregelungen verzichtet werden. ${ }^{1042}$ Statt dessen ist danach zu fragen, welche Bedeutung das Aufsichtsratsplanungssystem für die Corporate Governance-Grundsätze gewinnen könnte und auf welchem Wege solche Verhaltensbeschreibungen für den Aufsichtsrat Verbindlichkeit erlangen.

1038 Vgl. zu den konkurrierenden Kodizes Grundsatzkommission Corporate Governance, Corporate Governance-Grundsätze, 2000, S. 238-241, sowie Berliner Initiativkreis German Code of Corporate Governance, Code, 2000, S. 29-85; weiterhin Claussen/Bröcker, Corporate GovernanceGrundsätze, 2000, S. 481-491, die auf die Gefahr der Überregulierung hinweisen (S. 486); Hopt, Grundsätze, 2000, S. 779-818; Peltzer/von Werder, Corporate Governance, 2001, S. 1-15; Schneider, Corporate Governance-Grundsätze, 2000, S. 2413-2417, der jedoch kritisiert, daß der Berliner Initiativkreis German Code of Corporate Governance die Rolle des Aufsichtsrats im Prozeß der Unternehmungsführung zu stark zurücknimmt, was international kritisch gesehen würde (S. 2414, insbesondere der Verweis in FN 16); Schneider/Strenger, Corporate Governance, 2000, S. 106-113; Sünner, Corporate Governance, 2000, S. 492-498; Volk, Corporate GovernanceKonzepte, 2001, S. 412-416; mit internationaler Perspektive beispielsweise Böckli, Corporate Governance, 2000, S. 133-152, sowie von Werder, Governance-Debatte, 2000, S. 8ff.; zu den OECD Principles OECD, Principles, 1999, S. 1-25, sowie Seibert, Principles, 1999, S. 337-350; eine internationale Übersicht findet sich unter der URL http://www.ecgn.org/ecgn/codes.htm, sowie bei Hopt/Wymeersch, corporate governance, 1997, M-1ff.

1039 Zu möglichen Begriffsausprägungen Schneider, Corporate Governance-Grundsätze, 2000, S. 2413.

1040 Vgl. Grundsatzkommission Corporate Governance, Corporate Governance-Grundsätze, 2000, S. 240f.; Berliner Initiativkreis German Code of Corporate Governance, Code, 2000, S. 65ff., IV.

1041 Mit einem Vergleich Dufey/Hommel/Riemer-Hommel, Corporate Governance, 1998, S. 52ff.; o.V., Unternehmensverfassung, 2001, S. 23; vgl. auch Claussen/Bröcker, Corporate GovernanceGrundsätze, 2000, S. 485; mit einem expliziten Aufklärungsanspruch auch Bundesverband der Deutschen Industrie e.V./PwC Deutsche Revision AG, Corporate Governance, 2001.

1042 Vgl. hierzu die in FN 1038 aufgeführte Literatur. 
Das hier entwickelte Planungssystem für Aufsichtsräte kann als Versuch aufgefaßt werden, für bestimmte Teilbereiche der aufsichtsratsbezogenen Governance-Regelungen die vorliegenden Governance-Grundsätze zu ergänzen bzw. aus planungstheoretischer Perspektive zu systematisieren. Dies kann an dem Beispiel des in dieser Arbeit entwickelten Anforderungsprofils für Aufsichtsratsmitglieder verdeutlicht werden. CLAUSSEN/BRÖCKER bemängeln das Fehlen eines solchen Anforderungsprofils in den Corporate Governance-Grundsätzen. ${ }^{1043}$ Über die Aufnahme der hier entwickelten Vorschläge könnte diese Lücke geschlossen werden und ebenso wie im Rahmen der Grundsätze ordnungsmäßiger Überwachung ein Beitrag zu einer weiteren Konkretisierung erfolgen.

Die Corporate Governance-Grundsätze können auf verschiedene Weise für den Aufsichtsrat verbindlich werden und damit Sanktionspotential entfalten: durch die Aufnahme in Verträge mit den Organmitgliedern oder in die Geschäftsordnung, durch eine Selbstverpflichtung der Organmitglieder, als Zugangskriterien einzelner Börsen ${ }^{1044}$ für einzelne Marktsegmente oder Indizes sowie am nachhaltigsten durch die Einforderung durch (insbesondere institutionelle) Anleger. ${ }^{1045}$ Dies könnte durch das Prinzip des „Comply or explain“ unterstützt werden, mit dem Unternehmungen gesetzlich verpflichtet werden, einen Corporate Governance-Kodex anzuerkennen oder eventuelle Abweichungen öffentlich zu erläutern. ${ }^{1046}$ Corporate Governance-Grundsätze verhindern so eine weitere Verrechtlichung und tragen zu der notwendigen Deregulierung bei. ${ }^{1047}$

Über die Aufnahme der Regelungen zum Planungssystem in die GovernanceGrundsätze würde die Implementierung eines solchen Subsystems in der gleichen Weise wie die Governance-Grundsätze für die hierunter fallenden Gesellschaften verbindlich werden. Die Durchsetzung und Ausgestaltung solcher Grundsätze wird dabei nachhaltig vom Verhalten der Kapitalanleger abhängen,

1043 Vgl. Claussen/Bröcker, Corporate Governance-Grundsätze, 2000, S. 490.

1044 Vgl. Schneider, Corporate Governance-Grundsätze, 2000, S. 2415f., der darauf hinweist, daß die Anerkennung des „Combined Code" der Londoner Börse Zulassungsvoraussetzung für an der Londoner Börse notierte Unternehmungen ist, im Ergebnis aber eine Übertragung auf Deutschland nicht für sinnvoll erachtet und statt dessen die Aufnahme einer Unternehmung in einen Börsenindex hiervon abhängig machen will.

1045 Vgl. Berliner Initiativkreis German Code of Corporate Governance, Code, 2000, S. 35.

1046 Vgl. mit entsprechenden Plänen die Pressemitteilung Nr. 304/01 der Bundesregierung auf Grundlage der Vorschläge der Regierungskommission „Corporate Governance“, Unternehmensführung, 2001, Rdz. D1.4.

1047 Gegen eine weitere Regulierung durch den Gesetzgeber auch Dreher, Organisation, 1996, S. 38; Potthoff/Trescher, Aufsichtsratsmitglied, 1999, S. 154; Holzer/Makowski, Corporate Governance, 1997, S. 692. 
deren (Nicht-)Anlageentscheidung von der Anerkennung solcher Grundsätze durch die Unternehmungen beeinflußt wird. ${ }^{1048}$

\section{Aktionärskontrolle}

\section{a) Exit}

Als letzter Bereich der Verhaltensbeeinflussung von Aufsichtsratsmitgliedern ist die Einflußnahme durch (potentielle) Aktionäre zu nennen. ${ }^{1049}$ Aktionäre nehmen neben der Rolle des Investors insbesondere die eines Gesellschafters ein. ${ }^{1050}$ Als Investor treffen sie am Kapitalmarkt eine (Nicht-)Anlageentscheidung, als Gesellschafter üben sie eine Überwachungsfunktion aus, um die Rentabilität der getätigten Investition sicherzustellen. Wenn die Unternehmungsleitung ihre Interessen nicht in ausreichendem Maße berücksichtigt, steht ihnen entweder die Möglichkeit zum Verkauf ihrer Aktien offen (Exit) oder sie können versuchen, ihre Interessen über ihr Stimmrecht in der Hauptversammlung wahrzunehmen (Voice). ${ }^{1051}$

Der (internationale) Kapitalmarkt gewinnt auch in Deutschland für Publikumsaktiengesellschaften zunehmend an Bedeutung, wohingegen die bisher dominante Rolle der Universalbanken als Eigen- und Fremdkapitalgeber der Unternehmungen rückläufig ist. ${ }^{1052}$ Ihren Platz nehmen verstärkt institutionelle Investoren ein, zu denen insbesondere große Pensions- bzw. Investmentfonds zählen. ${ }^{1053}$ Diese Bedeutungszunahme geht mit einer verstärkten Akzeptanz des Shareholder Value als generellem Unternehmungsziel einher. ${ }^{1054}$

1048 Vgl. Claussen/Bröcker, Corporate Governance-Grundsätze, 2000, S. 481; Pellens/Hillebrandt/Ulmer, Umseztung, 2001, S. 1250, kommen jedoch auf Grundlage einer empirischen Untersuchung der DAX 100-Unternehmungen zu dem Ergebnis, da $B$ die Implementierung von Corporate Governance-Standards noch in den Kinderschuhen stecke.

1049 Vgl. mit einer Beschreibung der deutschen Situation beispielsweise Schmidt u.a., Corporate Governance, 1997, S. 121-139, unter Einschluß von Reformvorschlägen.

1050 Vgl. Knoll/Möller, Aktien-Optionspläne, 1999, S. 69.

1051 Vgl. grundlegend zur Unterscheidung von Exit und Voice Hirschman, Exit, 1970.

1052 Vgl. Dufey/Hommel/Riemer-Hommel, Corporate Governance, 1998, S. 57ff.; Schröder/Schrader, corporate governance, 1998, S. 33.

1053 Vgl. zur zunehmenden Bedeutung institutioneller Investoren auch in Deutschland Matthes, Corporate-Governance-System, 2000, S. 34-38, 53, sowie Schilling, Aufsichtsräte, 1999, S. 35; zur aktiven Rolle institutioneller Investoren (Shareholder Activism) innerhalb der Corporate Governance beispielsweise Romano, Shareholder Activism, 2000.

1054 Vgl. Dufey/Hommel/Riemer-Hommel, Corporate Governance, 1998, S. 47, die fordern, daß jegliche Systemreform Hindernisse, die eine Maximierung des Shareholder Value verhindern, abzubauen hat. 
Die Berücksichtigung der Kapitalmarktinteressen kann, wie WITT zeigt, zu einer Kostensenkung und damit zu einer Steigerung der Wettbewerbsfähigkeit der Unternehmungen führen. ${ }^{1055} \mathrm{Er}$ unterstellt, daß in einem Systemwettbewerb zwischen (US-amerikanischem) Boardsystem und deutschem Aufsichtsratssystem dasjenige Corporate Governance-System Vorteile besitzt, dessen Unternehmungen niedrigere Gesamtkosten der folgenden drei Kostenarten haben:

1. Kosten des Eigenkapitals,

2. Kosten des dispositiven Faktors (Management) und

3. Kosten der objektbezogenen Arbeit.

Die hier im Vordergrund stehenden Managementkosten (Kosten der Anreizsysteme der Unternehmungsleitung) bzw. die Kosten des Eigenkapitals zeigen in beiden Governance-Modellen unterschiedliche Ausprägungen. ${ }^{1056}$ Während im US-amerikanischen Boardsystem geringere Eigenkapitalkosten ${ }^{1057}$ bestünden, seien dagegen die Managementkosten aufgrund der Stock Option-Programme deutlich höher. ${ }^{1058}$ Im deutschen System fielen aufgrund der höheren Renditeforderung der Kapitalgeber höhere Eigenkapitalkosten bei gleichzeitig geringeren Managementkosten an, die sich jedoch aufgrund der Anpassung an internationale Vergütungsstandards zukünftig erhöhen würden. Da große, international tätige Publikumsaktiengesellschaften auf dem globalen Kapitalmarkt im Wettbewerb um Eigenkapital stünden, geht insbesondere von den Eigenkapitalkosten ein starker Druck zur Vereinheitlichung und damit hin zu aktionärsorientierten Governance-Strukturen aus. Dies kann damit erklärt werden, daß aktionärsorientierte Governance-Strukturen zu einer Verminderung der Agency-Problematik

1055 Vgl. Witt, Corporate Governance-Strukturen, 1999, S. 8f., unter Hinweis auf weitere relevante Systemmerkmale wie beispielsweise Kapitalmarktregelungen; der bei Witt als Corporate Governance (S. 2) bezeichnete Regelungsbereich ist weitgehend identisch mit der hier betrachteten Spitzenverfassung von Unternehmungen.

1056 Auf eine Betrachtung der Arbeitskosten sei hier verzichtet, sie verschlechtern jedoch die Kostensituation deutscher Unternehmungen zusätzlich; vgl. hierzu Schröder, Arbeitskosten, 2000, S. 80; Schröder, Lohnstückkosten, 2000, S. 92, unter zusätzlicher Berücksichtigung der Produktivität; zu den Arbeitskostennachteilen durch die Mitbestimmung auf Unternehmungsebene Schmid/Seger, Arbeitnehmermitbestimmung, 1998, S. 453-473.

1057 Die These der in den Vereinigten Staaten im Vergleich zu Deutschland geringeren Eigenkapitalkosten stützt eine Studie von Uhlir/Steiner, Wertpapieranalyse, 2001, S. 165f., die für Deutschland höhere Aktienrenditen feststellt; dies ist ein Anzeichen für höhere Risikoprämien, die somit zu höheren Eigenkapitalkosten in Deutschland führen.

1058 Vgl. Witt, Corporate Governance-Strukturen, 2000, S. 163; Kienbaum, Vergütungsstudie, 2000, S. 39, mit Beispielen exorbitanter Gehälter amerikanischer Manager. 
beitragen und hierdurch zu geringeren Renditeforderungen der Eigenkapitalgeber führen. ${ }^{1059}$

„Current market trends however force each country to reoptimize the allocation of control rights along one dimension, firm value."“1060

Einen Beitrag kann das vorliegende Aufsichtsratsplanungssystem leisten, da mit dessen Einführung und seiner Kommunikation am Kapitalmarkt Kostenersparnisse bei den Eigenkapitalkosten für die Unternehmungen zu erwarten sind. ${ }^{1061}$ Der hiermit verbundene Anstieg des Unternehmungswertes wirkt sich unmittelbar über das wertorientierte Anreizsystem des Aufsichtsrats auf dessen Vergütung aus, so daß über die Aufnahme des Aufsichtsratsplanungssystems in am Kapitalmarkt akzeptierte Corporate Governance-Grundsätze eine Unterstützung der Anreizwirkung einer wertorientierten Vergütung des Aufsichtsrats erreicht werden kann. Trotz der hiermit verbundenen Erhöhung der Managementkosten ist nicht davon auszugehen, daß dies die potentielle Verminderung der Eigenkapitalkosten (über-)kompensiert.

Zusätzlich zu der Verhaltensbeeinflussung über die wertorientierte Vergütungskomponente kann bei einem funktionsfähigen Kapitalmarkt der Markt für Unternehmungskontrolle disziplinierend auf das Management wirken. ${ }^{1062}$ Diese Verhaltensbeeinflussung geht von dem Arbeitsplatzrisiko aus, dem das Management einer Unternehmung im Falle einer Übernahme durch eine andere Unternehmung oder zumindest bei einer hinreichend großen Änderung der Mehrheitsverhältnisse in der Hauptversammlung unterliegt. ${ }^{1063}$ Die Gefahr besteht immer dann, wenn aufgrund ausbleibender Unternehmungserfolge der Aktienkurs der Unternehmung sinkt und hierdurch potentielle Aufkäufer angelockt werden. Obwohl üblicherweise die Entlassung der Vorstandsmitglieder im Mittelpunkt solcher Überlegungen steht, gilt dies in den hier betrachteten Publikumsaktiengesellschaften in gleichem Maße auch für die Aufsichtsratsmitglie-

1059 Vgl. Feddersen, Aufsichtsrat, 2000, S. 396, der die Bedeutung der Corporate Governance einer Unternehmung im weltweiten Wettbewerb um Eigenkapital betont; auch schon Jaschke, Überwachungsfunktion, 1989, S. 39.

1060 Dufey/Hommel/Riemer-Hommel, Corporate Governance, 1998, S. 61.

1061 So betont Potthoff, Corporate Governance, 2000, S. 2, daß Investoren bereit sind, entsprechende Prämien zu bezahlen.

1062 Vgl. grundlegend Manne, Mergers, 1965, S. 110-120; Grossman/Hart, Takeover, 1980, S. 42-64; Franks/Mayer, takeovers, 1996, S. 163-181; Becht, Blockholders, 1997, S. 49-61, sowie die Beiträge in Romano, Foundations, 1993, S. 229-300; mit einem ausführlichen Überblick Preuschl, Unternehmensübernahmen, 1997, sowie Flassak, Unternehmenskontrolle, 1995.

1063 Vgl. Wenger, Unternehmenskontrolle, 1995, Sp. 1410; Schmidt u.a., Corporate Governance, 1997, S. 121. 
der, da im Falle einer Änderung der Mehrheitsverhältnisse in der Hauptversammlung davon auszugehen ist, daß auch die Aufsichtsratsmitglieder ausgetauscht werden. ${ }^{1064}$ Die Konsequenzen des Mandatsverlustes sind um so gravierender, je wesentlicher die den Aufsichtsratsmitgliedern gewährten monetären Anreize sind. Der Markt für Unternehmungskontrolle übt somit eine Kontrollfunktion aus und führt hierdurch zu einer Verringerung der Agency-Kosten. ${ }^{1065}$ Seine praktische Bedeutung ist in Deutschland bisher noch eher gering. ${ }^{1066}$ Aufgrund der zunehmenden Internationalisierung der Kapitalmärkte ist jedoch zu erwarten, daß die potentielle Bedrohung von Vorständen und Aufsichtsräten deutscher Unternehmungen durch eine feindliche Übernahme an Bedeutung gewinnen wird und zu einer Verringerung der Agency-Kosten zwischen Aktionären und den Organmitgliedern beiträgt. ${ }^{1067}$

\section{b) Voice}

Als letzter Punkt ist die Wahrnehmung ihrer Rechte durch die Aktionäre der Unternehmung in der Hauptversammlung zu nennen. ${ }^{1068}$ Sie können hierdurch sanktionierend auf die Aufsichtsratsmitglieder einwirken. ${ }^{1069}$ Dies beinhaltet neben der Verweigerung einer Entlastung des Aufsichtsratsmitglieds insbesondere die Wahrnehmung ihrer Personalkompetenz durch die Hauptversammlung. Deren aktive Wahrnehmung bis hin zur Abberufung von Aufsichtsratsmitgliedern würde eine ernsthafte Bedrohung der Reputation der Aufsichtsratsmitglieder bedeuten und so ebenfalls zu einer Verringerung der Principal Agent-Problematik

1064 Ein Hilfsmittel zur Verhinderung der Abwahl von Aufsichtsratsmitgliedern ist die Erhöhung des hierfür notwendigen Quorums; vgl. mit einer solchen Maßnahme mg technologies AG, Tagesordnung zur Hauptversammlung 2001, S. 7, sowie Koenen/Lipinski, MG-Aktionär, 2001, S. 20.

1065 Vgl. Hart, Corporate Governance, 1995, S. 684; Möllers, Kapitalmarkttauglichkeit, 1999, S. 433f., stellt fest, daß auch der Gesetzgeber die Kontrollfunktion der Kapitalmärkte ausdrücklich anerkennt.

1066 Vgl. Böhmer, Corporate Governance, 1999, S. 7; Schmidt u.a., Corporate Governance, 1997, S. 140.

1067 Vgl. Dorfs, Jahrtausend, 1999, S. 16.

${ }^{1068} \mathrm{Zu}$ Problemen von Aktionären bei der Wahrnehmung ihrer Rechte insbesondere in den Hauptversammlungen von Publikumsaktiengesellschaften beispielsweise Schmidt u.a., Corporate Governance, 1997, S. 137-140, sowie Sünner, Corporate Governance, 2000, S. 493; der Gesetzgeber hat mit dem KonTraG die Möglichkeit für eine eigene Geschäftsordnung der Hauptversammlung geschaffen ( $\S 121$ Abs. 1 AktG), um hiermit eine wirksamere Durchführung von Hauptversammlungen zu gewährleisten; vgl. hierzu Bachmann, Geschäftsordnung, 1999, S. 210-215; vgl. darüber hinaus zu weiteren Vereinfachungen durch die Durchführung virtueller Hauptversammlungen Hasselbach/Schumacher, Hauptversammlung, 2000, S. 258-286, sowie Spindler, Internet, 2000, S. 420-445; zu verschiedenen Möglichkeiten der Geltendmachung von Aktionärsrechten Schiessl, Aktienrecht, 1999, S. 444-449.

1069 Die „Schlüsselfunktion der Hauptversammlung“ betont bereits Bernhardt, Aufsichtsrat, 1995, S. 319. 
beitragen. ${ }^{1070}$ In diesem Zusammenhang würde ein direkter Aktienbesitz von Aufsichtsratsmitgliedern nicht nur Signalwirkung entfalten, ${ }^{1071}$ sondern zusätzlich die Gefahr der Hidden Intention weiter vermindern. Hierzu wäre jedoch eine größere Publizität des Aktienbesitzes von Organmitgliedern notwendig. ${ }^{1072}$

Diese Publizität steht in enger Verbindung mit der immer wieder erhobenen Forderung nach einer Verbesserung und Intensivierung der Berichterstattung des Aufsichtsrats gegenüber der Hauptversammlung über seine Überwachungsaktivitäten und -organisation, indem er über die unzureichenden gesetzlichen Mindestberichtsanforderungen hinausgeht. ${ }^{1073}$ Eine Abkehr von den bisher üblichen testathaften Formulierungen steht jedoch noch aus. LUTTER/KRIEGER betonen die Notwendigkeit, daß die einmal pro Jahr stattfindende Pflichtinformation nicht formelhaft erfolgen soll, sondern die Informationsbedürfnisse der Hauptversammlung zu berücksichtigen hat. ${ }^{1074}$ Hierzu kann der Aufsichtsrat jederzeit weitere, über die Mindesterfordernisse hinausgehende Informationen an die Hauptversammlung geben. Dies gilt entsprechend für den Bericht des Aufsichtsrats im Geschäftsbericht. ${ }^{1075}$

Zusammenfassend kann festgehalten werden, daß vielfältige Mechanismen für eine wirksame Verhaltensbeeinflussung der Aufsichtsratsmitglieder bestehen. Zur Vermeidung eines regulatorischen Übermaßes sollte der mit der Entwicklung von Corporate Governance-Grundsätzen eingeschlagene Weg einer marktlichen Verhaltensbeeinflussung weiter beschritten werden, um auch in der $\mathrm{Zu}$ kunft flexibel auf sich wandelnde Anforderungen reagieren zu können. ${ }^{1076}$

1070 Dies versuchte beispielsweise Wenger bei dem Aufsichtsratsvorsitzenden Kopper der DaimlerChrysler AG; vgl. o.V., Geldanlage, 2001, o.S.

1071 Vgl. Claussen, Aktienoptionen, 1997, S. 1830f.

1072 Vgl. Lückmann, Kontrolle, 2000, S. 16; eine entsprechende Regelung existiert lediglich für die am Neuen Markt notierten Unternehmungen; vgl. hierzu Gruppe Deutsche Börse, Regelwerk, S. 19, 4.1.14(2)4., die eine Aufschlüsselung des Akienbesitzes nach Aufsichtsratsmitgliedern vorsieht.

1073 Vgl. Möllers, Professionalisierung, 1995, S. 1734; mit einer empirischen Studie zur unzureichenden Berichterstattung des Aufsichtsrats Theisen, Berichterstattung, 1997, S. 105-115; die mit dem KonTraG erfolgte Verschärfung der Berichterstattung als unzureichend beklagend Theisen, Reform, 1999, S. 243; eine „erbarmungswürdige Kargheit“ des Aufsichtsratsberichts gegenüber der Hauptversammlung bemängeln Hommelhoff/Mattheus, Corporate Governance, 1998, S. 257; umfassend auch Portisch, Überwachung, 1997, S. 184-235, der darauf hinweist, daß über eine geeignet ausgestaltete Berichterstattung Informationsasymmetrien abgebaut und hieraus resultierende Agency-Probleme vermindert werden können (S. 234f.).

1074 Vgl. Lutter/Krieger, Rechte, 1993, S. 34.

1075 Vgl. mit einer entsprechenden Forderung beispielsweise DSW, Aufsichtsräte, 1995, S. $12 \mathrm{f}$.

1076 Vgl. Feddersen, Aufsichtsrat, 2000, S. 396; so beispielsweise explizit der Berliner Initiativkreis German Code of Corporate Governance, Code, 2000, S. 33. 
„Die weltweiten Chancen im Rahmen von Globalisierung, Deregulierung und Privatisierung sind [..] nicht nur eine Frage vorhandener Innovationen und Schlüsselkompetenzen, sondern zumindest auch abhängig von einer gesunden Führungsstruktur der Unternehmen. Corporate Governance ist damit auch ein für den globalen Wettbewerb wichtiger Faktor, dessen Optimierung anzustreben ist. “1077

Bei der Corporate Governance handelt es sich somit nicht nur um die Erfüllung gesetzlicher oder faktischer Regelungsstandards, sondern um einen kritischen Erfolgsfaktor für eine erfolgreiche Unternehmungsentwicklung. ${ }^{1078}$

1077 Feddersen, Aufsichtsrat, 2000, S. 396.

1078 Vgl. Dufey/Hommel/Riemer-Hommel, Corporate Governance, 1998, S. 46, die eine wettbewerbsfähige Corporate Governance als „core value driver" bezeichnen; zu Erfolgsfaktoren beispielsweise Henselek, Konfigurationsmanagement, 2000, S. 466-469; kritisch zur Umsetzung der Corporate Governance im Sinne einer Aktionärsorientierung in Deutschland Pellens, Corporate Governance, 2001, S. 4; der besonderen Bedeutung eines Corporate Governance-Kodex sieht sich auch die Bundesregierung verpflichtet, die eine Expertenkommission mit der Entwicklung eines Richlinienkataloges beauftragen wird, vgl. Pressemitteilung Nr. 304/01 der Bundesregierung. 


\section{Schlußbetrachtung}

Ausgangspunkt der vorliegenden Arbeit war die Forderung nach einer Funktionserweiterung der Tätigkeit von Aufsichtsräten in Publikumsaktiengesellschaften zur Erhöhung der Effektivität der Unternehmungsführung. Abschließend werden die wesentlichen Ergebnisse der Untersuchung thesenartig zusammengefaßt:

Der Gesetzgeber weist in Aktiengesellschaften der Hauptversammlung, dem Aufsichtsrat und dem Vorstand unterschiedliche Aufgaben im Unternehmungsführungsproze $B \mathrm{zu}$. Er sieht eine arbeitsteilige Aufgabenbearbeitung vor, mit der dem Aufsichtsrat als Überwachungsorgan nicht nur Kontroll- und Mitentscheidungsaufgaben, sondern auch originäre unternehmerische Entscheidungsaufgaben zukommen. Bei der Erfüllung ihrer Aufgaben sind alle Aufsichtsratsmitglieder gleichermaßen zur Verfolgung der Unternehmungsziele verpflichtet. Mit dem Shareholder Value-Ansatz liegt eine unternehmerische Zielkonzeption vor, die sowohl zu einer Interessenharmonisierung der Aktionäre untereinander als auch der übrigen Stakeholder führt und daher als ausschließlicher Handlungsmaßstab verwendet werden sollte.

Aus der Perspektive institutionenökonomischer Ansätze handelt es sich bei der mit der Ausgestaltung der Spitzenverfassung erfolgenden Verfügungsrechtsdelegation von den Aktionären auf angestellte Manager um ein effizientes Arrangement, das jedoch aufgrund von Interessendivergenzen und Informationsasymmetrien die Verfolgung der Unternehmungsziele im Interesse der Aktionäre nicht immer gewährleistet. Dieses Problem wird durch die zweistufige Agency-Beziehung verschärft, innerhalb derer nicht nur Leitungsrechte an den Vorstand, sondern zusätzlich Überwachungsrechte einschließlich der Personalkompetenz über das Leitungsorgan an den Aufsichtsrat delegiert werden. Zudem handelt es sich bei den Aufsichtsratsmitgliedern in Publikumsaktiengesellschaften in der Regel nicht um Aktionäre, sondern ebenfalls um angestellte Manager anderer Unternehmungen und weitere Interessenvertreter.

Sowohl die Analyse der juristisch determinierten Aufgaben des Aufsichtsrats als auch die agencytheoretische Analyse zeigen, daß die Aufsichtsratsmitglieder bei der Wahrnehmung ihrer Aufgaben auf umfassende zukunftsgerichtete Informationen angewiesen sind. Die systematische und vom Vorstand unabhängige Gewinnung von Informationen setzt eine eigenständige Planung durch den Aufsichtsrat voraus, mit der er insbesondere seine unternehmerischen Entscheidungen vorbereitet und gleichzeitig die Informationsasymmetrie zum Vorstand abbaut. Daher war es Ziel der Untersuchung, für den Aufsichtsrat ein Planungs- 
system zu konzipieren, das aus verschiedenen interdependenten Elementen besteht.

$\mathrm{Zu}$ den Planungsobjekten des Aufsichtsrats zählen neben den generellen Unternehmungszielen insbesondere die organisatorische Gestaltung des Vorstandsorgans und die hiermit eng verbundene Wahrnehmung seiner Personalkompetenz gegenüber den Vorstandsmitgliedern einschließlich der Gestaltung ihres Anreizsystems. Dies verlangt sowohl die regelmäßige Beurteilung der Vorstandsmitglieder als auch eine funktionsfähige Nachfolgeplanung, um die Besetzungsentscheidungen sachgerecht vorbereiten zu können und jederzeit über geeignete Handlungsalternativen zu verfügen. Daneben hat der Aufsichtsrat eine Organisationsplanung zur Gestaltung seiner eigenen Aufgabenerfüllung durchzuführen.

Die Planungsorganisation des Aufsichtsrats ist aufgrund der Komplexität und des Umfangs seiner Aufgaben arbeitsteilig auszugestalten. Plenumssitzungen sollten nur der finalen Entscheidung dienen. Im Rahmen seiner Primärorganisation erfolgt eine Ressortierung des Aufsichtsrats, mit der einzelnen Aufsichtsratsmitgliedern bestimmte Verantwortungsbereiche übertragen werden. Dem Aufsichtsratsvorsitzenden kommen primär koordinierende Tätigkeiten zu. Zur Erfüllung der Planungsaufgaben sind spezielle Ausschüsse einzurichten, die die Entscheidungen des Plenums vorbereiten. Für den Abschluß der Vorstandsverträge sowie für eilbedürftige Entscheidungen im Rahmen zustimmungspflichtiger Geschäfte wird auch die Entscheidungskompetenz vom Plenum an den zuständigen Ausschuß delegiert. Bei der Gestaltung der Kommunikationswege ist ein direkter Zugriff auf unternehmungsinterne Ressourcen vorzusehen. Schließlich hat der Aufsichtsrat innerhalb seiner organisatorischen Aufgaben eine Nachfolgeplanung durchzuführen, um der Hauptversammlung geeignete stellenbezogene Wahlvorschläge unterbreiten zu können.

Damit die arbeitsteilige, laufende Aufgabenerfüllung möglich ist, sind an die einzelnen Aufsichtsratsmitglieder (Planungssubjekte) hohe Anforderungen zu stellen. Hierzu zählen auf Basis der juristischen Mindestanforderungen sowohl personenbezogene Anforderungen wie die Verschwiegenheit, die Eigenverantwortlichkeit, die Unabhängigkeit und die Gewissenhaftigkeit als auch managementbezogene Anforderungen (technische, soziale und konzeptionelle Kompetenz). Zur Durchführung seiner Planungsaufgaben greift der Aufsichtsrat auf personelle unternehmungsinterne und -externe Ressourcen $\mathrm{zu}$.

Zur instrumentellen Unterstützung ist für den Aufsichtsrat ein computergestütztes Aufsichtsratsunterstützungssystem einzuführen, dessen Grundlagen hier 
erarbeitet wurden. Dieses ermöglicht ihm zum einen den unmittelbaren Zugriff auf alle relevanten unternehmungsinternen und -externen Informationen, zum anderen bietet es organisatorische Potentiale zur Unterstützung seiner dezentralen Aufgabenerfüllung. Hierdurch können die Aufsichtsratsmitglieder ihre Tätigkeit im Rahmen der bestehenden Restriktionen erfüllen. Zudem erfolgt eine Entkopplung ihrer Arbeit von den standardisierten und ritualisierten Sitzungsterminen, so daß eine permanente, dezentrale und zeitlich flexible Funktionswahrnehmung möglich ist. Dies ist gleichzeitig die wesentliche Voraussetzung für eine effiziente Durchführung der Planungsprozesse und die anzustrebende Intensivierung der Aufsichtsratstätigkeit.

Mit der Einführung des Aufsichtsratsplanungssystems findet eine Professionalisierung der Aufsichtsratstätigkeit auf der institutionalen Ebene statt (Professionalisierung im weiteren Sinne). Daneben erfordert sie auf der personalen Ebene professionelle Aufsichtsratsmitglieder (Professionalisierung im engeren Sinne), die ihre Arbeitszeit auf die Überwachung weniger Unternehmungen verwenden. Da die notwendige Professionalisierung nicht von heute auf morgen realisiert werden kann, sollte eine evolutorische Entwicklung des Planungssystems angestrebt werden mit dem Ziel, ein umfassendes Aufsichtsratsplanungssystem zu implementieren.

Die mit der Einführung des Aufsichtsratsplanungssystems notwendigerweise verbundene Intensivierung der Tätigkeit der Aufsichtsratsmitglieder ist nur dann zu erwarten, wenn eine entsprechende Verhaltensbeeinflussung erfolgt. Die Aufsichtsratsmitglieder müssen eine ihren Aufgaben und ihrer Rolle im Unternehmungsführungsproze $\beta$ adäquate, die Professionalisierung unterstützende Vergütung erhalten. Daher sollte unter Ausnutzung der vorhandenen umfassenden juristischen Gestaltungsspielräume von der bisher wenig unternehmungszielkonformen Vergütungspraxis abgewichen und den Aufsichtsratsmitgliedern eine wertorientierte Vergütung gewährt werden. Dies würde zusätzlich zu einem Abbau der Agency-Problematik beitragen. Hierbei ist darauf zu achten, daß der höheren Vergütung auch eine umfassende Sachkompetenz, ein großer zeitlicher Einsatz und ein nachhaltiges Commitment zur Erreichung der Unternehmungsziele entsprechen muß. Dies kann durch eine stellenbezogene Differenzierung der Vergütung unterstützt werden.

Neben der Vergütung ist eine zielorientierte Verhaltensbeeinflussung der Aufsichtsratsmitglieder von der Durchsetzung möglicher Haftungsansprüche sowie der Vorgabe von juristisch durchsetzbaren Verhaltensregeln (Grundsätze ordnungsmäßiger Überwachung, Corporate Governance-Grundsätze) zu erwarten. Insbesondere die Einhaltung international anerkannter Corporate Governan- 
ce-Grundsätze ist zudem ein wichtiger Wettbewerbsfaktor auf den internationalen Kapitalmärkten. Dabei kommt institutionellen Investoren eine herausragende Bedeutung zu. Sie beeinflussen die Unternehmungsleitungen nicht nur über ihre Anlageentscheidungen, sondern auch über die Wahrnehmung ihrer Gesellschafterrechte.

Das hier entwickelte Aufsichtsratsplanungssystem kann in Verbindung mit dem wertorientierten Anreizsystem für Aufsichtsratsmitglieder zu der auch international geforderten Verbesserung der Corporate Governance deutscher Publikumsaktiengesellschaften beitragen. Inwieweit bestehende Potentiale durch die Übernahme aller oder einzelner Systembestandteile genutzt werden, hat die Aufsichtsratspraxis zu zeigen. Trotz der geforderten Revitalisierung des Aufsichtsrats in der Unternehmungsführung ist jedoch an seiner eigenständigen Existenz festzuhalten, da nur so eine unabhängige Überwachung des Vorstands im Interesse der Aktionäre gewährleistet werden kann. 


\section{Literaturverzeichnis}

Abel, Christine / Joachim Becker: Trends in der Vergütung: Zunehmende Internationalisierung und Erfolgsorientierung, in: Personal, 52 (2000), S. 388-392

Achleitner, Ann-Kristin / Daniel Wichels: Stock Option-Pläne als Vergütungsbestandteil wertorientierter Entlohnungssysteme, in: Ann-Kristin Achleitner / Peter Wollmert (Hrsg.): Stock Options, Stuttgart 2000, S. 125

Achleitner, Ann-Kristin / Peter Wollmert (Hrsg.): Stock Options, Stuttgart 2000

Achleitner, Paul: Sozio-politische Strategien multinationaler Unternehmungen: ein Ansatz gezielten Umweltmanagements, Bern 1985

Albach, Horst: Strategische Unternehmensplanung und Aufsichtsrat, in: ZGR, 26 (1997), S. 32-40

Albach, Horst / Klaus Brockhoff (Schriftleitung): Betriebswirtschaftslehre und Rechtsentwicklung, (ZfB-Ergänzungsheft 4, 67. Jg.), Wiesbaden 1997

Albach, Horst u.a. (Hrsg.): Die Theorie der Unternehmung in Forschung und Praxis, Berlin u.a. 1999

Alchian, Armen A. (Hrsg.): Economic Forces at Work, Indianapolis, 1977

Alchian, Armen A.: Some Economics of Property Rights, in: Armen A. Alchian (Hrsg.): Economic Forces at Work, Indianapolis, 1977, S. 127-149

Alchian, Armen A. / Harold Demsetz: Production, Information Cost, and Economic Organization, in: The American Economic Review, 62 (1972), S. 777-795

Arbeitskreis „Externe und interne Überwachung der Unternehmung“ der Schmalenbach-Gesellschaft/Deutsche Gesellschaft für Betriebswirtschaft e.V.: Grundsätze ordnungsmäßiger Aufsichtsratstätigkeit - ein Diskussionspapier, in: DB, 48 (1995), S. 1-4

Arbeitskreis „Externe und interne Überwachung der Unternehmung“ der Schmalenbach-Gesellschaft für Betriebswirtschaft e.V.: Prüfungsausschüsse in deutschen Aktiengesellschaften, in: DB, 53 (2000), S. 22812285

Arbeitskreis Krähe der Schmalenbach-Gesellschaft: Die Organisation der Geschäftsführung. Leitungsorganisation, 2. Auflage, Opladen 1971 
Arrow, Kenneth J.: The Role of Securities in the Optimal Allocation of Riskbearing, in: Review of Economic Studies, 31 (1964), S. 91-96

Arrow, Kenneth J.: Essays in the Theory of Risk-bearing, Amsterdam 1970

Assmann, Heinz-Dieter: Kommentierungen zum Aktiengesetz, in: Klaus J. Hopt (Hrsg.): Großkommentar zum Aktiengesetz, AktG, 4. Auflage, Berlin u.a. 1992

Assmann, Heinz-Dieter (Hrsg.): Wirtschafts- und Medienrecht in der offenen Demokratie, Heidelberg 1997

Auge-Dickhut, Stefanie: Der Aufsichtsrat als Intermediär: Delegation von Kontrollaufgaben bei asymmetrischer Informationsverteilung, Wiesbaden 1999

Bachmann, Gregor: Die Geschäftsordnung der Hauptversammlung, in: AG, 44 (1999), S. 210-215

Backes-Gellner, Uschi / Linus Geil: Managerverguitung und Unternehmenserfolg - Stand der theoretischen und empirischen Forschung, in: WISU, 26 (1997), S. 468-475

Baetge, Jörg: Gesellschafterorientierung als Voraussetzung für Kundenund Marktorientierung, in: Manfred Bruhn, Hartwig Steffenhagen (Hrsg.): Marktorientierte Unternehmensführung: Reflexionen - Denkanstöße Perspektiven, Wiesbaden 1997, S. 103-117

Baetge, Jörg / Andreas Jerschensky: Frühwarnsysteme als Instrument eines effizienten Risikomanagement und -Controlling, in: Controlling, 11 (1999), S. 171-176

Bales, Robert F.: Bessere Konferenz-Führung, in: Edward C. Bursk / Dan H. Fenn (Hrsg.): Unternehmerstrategie, Essen 1957, S. 230-239

Ballwieser, Wolfgang / Reinhard H. Schmidt: Unternehmensverfassung, Unternehmensziele und Finanztheorie, in: Kurt Bohr / Jochen Drukarczyk / Hans-Jürgen Drumm / Gerhard Scherrer (Hrsg.): Unternehmungsverfassung als Problem der Betriebswirtschaftslehre, Berlin, Bielefeld, München 1981, S. 645-682

Baltzer, Uwe: Krisenerkennung durch den Aufsichtsrat, Frankfurt am Main 1983

Bamberg, Günter / Klaus Spremann (Hrsg.): Agency theory, Information, and Incentives, Berlin u.a. 1987

Bamberger, Ingolf (Hrsg.): Strategische Unternehmensberatung, 2. Auflage, Wiesbaden 2000 
Bassen, Alexander u.a.: Die Analyse von Unternehmen mit der ,Scorecard for German Corporate Governance“, in: Finanz Betrieb, 2 (2000), S. 693698

Baums, Theodor: Aktienoptionen für Vorstandsmitglieder, Arbeitspapier 1996

Baus, Joseph: Die Belegschaftsaktie im Lichte der betrieblichen Personalpolitik, Bochum 1978

Bea, Franz Xaver / Steffen Scheurer: Die Kontrollfunktion des Aufsichtsrats, in: DB, 47 (1994), S. 2145-2152

Becht, Marco: Strong Blockholders, Weak Owners and the Need for European Mandatory Disclosure, European Corporate Governance Network, Executive Report, 1997

Becker, Fred G.: Anreizsysteme als Führungsinstrumente, in: Alfred Kieser / Gerhard Reber / Rolf Wunderer (Hrsg.): Handwörterbuch der Führung, 2. Auflage, Stuttgart 1995, Sp. 34-45

Becker, Fred G.: Erfolgs- und leistungsbezogene strategischorientierte Anreizsysteme, in: Marktforschung \& Management, 41 (1997), S. 112-119

Becker, Thomas: Informationsorientierte Überwachungskonzepte zur Kontrolle von Vorständen, Stuttgart 1993

Behme, Wolfgang / Harry Mucksch: Das Data Warehouse-Konzept als Basis einer unternehmensweiten Informationslogistik, in: Harry Mucksch / Wolfgang Behme (Hrsg.): Das Data Warehouse-Konzept, 3. Auflage, Wiesbaden 1998, S. 33-100

Behme, Wolfgang / Harry Mucksch: Die Notwendigkeit einer entscheidungsorientierten Informationsversorgung, in: Harry Mucksch / Wolfgang Behme (Hrsg.): Das Data Warehouse-Konzept, 3. Auflage, Wiesbaden 1998, S. 3-31

Berens, Wolfgang / Werner Delfmann: Quantitative Planung, 2. Auflage, Stuttgart 1995

Berger, Konrad: Die Kosten der Aufsichtsratstätigkeit in der Aktiengesellschaft, Frankfurt am Main u.a. 2000

Berhold, Marvin: A theory of linear profit sharing incentives, in: Quarterly Journal of Economics, 85 (1971), S. 460-482

Berle, Adolf A. / Gardiner C. Means: The modern corporation and private property, New York 1932 
Berle, Adolf A. / Gardiner C. Means: The modern corporation and private property, 2. Auflage, New York 1968

Berliner Initiativkreis German Code of Corporate Governance: German Code of Corporate Governance, in: Axel v. Werder (Hrsg.): German Code of Corporate Governance (GCCG), Stuttgart 2000, S. 29-85

Bernhardt, Wolfgang: Aufsichtsrat - die schönste Nebensache der Welt?, in: ZHR, Bd. 159 (1995), S. 310-321

Bernhardt, Wolfgang: Diskussionsbeitrag zum Panel Qualitätsmessung von Aufsichtsräten im Rahmen der 62. Wissenschaftlichen Jahrestagung des Verbandes der Hochschullehrer für Betriebswirtschaft e.V. am 16. Juni 2000 in Berlin

Bernhardt, Wolfgang: Hohe Anforderungen an die fachliche und persönliche Kompetenz, in: Handelsblatt, 20.11.2000, Nr. 224, S. 59

Bernhardt, Wolfgang / Peter Witt: Stock Options und Shareholder Value, in: ZfB, 67 (1997), S. 85-101

Bernhardt, Wolfgang / Peter Witt: Unternehmensleitung im Spannungsfeld zwischen Ressortverteilung und Gesamtverantwortung, in: ZfB, 69 (1999), S. 825-845

Berthel, Jürgen: Betriebliche Informationssysteme, Stuttgart 1975

Betriebswirtschaftlicher Ausschuß des Zentralverbandes Elektrotechnik- und Elektronikindustrie (ZVEI) e.V. Frankfurt am Main: ZVEIKennzahlensystem: ein Instrument zur Unternehmenssteuerung, 4. Auflage, Mindelheim 1989

BGH, Urteil vom 15.11.1982 - II ZR 27/82 (OLG Frankfurt): Zur Zulässigkeit der externen Beratung eines Aufsichtsratsmitglieds, in: ZIP, 4 (1983), S. 55-57

Bialdiga, Kirsten: Thyssen-Krupp: Fauler Kompromiss, in: http://www.ftd.de/thyssenkrupp (12.03.2001)

Bischoff, Jörg: Das Shareholder-Value-Konzept: Darstellung, Probleme, Handhabungsmöglichkeiten, Wiesbaden 1994

Bischof, Stefan / Peter Oser: Zweifelsfragen zur Teilnahmepflicht des Abschlußprüfers an der Bilanzsitzung des Aufsichtsrats, in: Wpg, 51 (1998), S. 539-543

Bitz, Michael u.a. (Hrsg.): Vahlens Kompendium der Betriebswirtschaftslehre, Bd. 2, 4. Auflage, München 1999 
Black, Stanley W. / Mathias Moersch (Hrsg.): Competition and Convergence in Financial Markets, Amsterdam u.a. 1998

Bleicher, Knut: Probleme und Entwicklungstendenzen der Organisationspolitik industrieller Großunternehmungen, in: Dietger Hahn (Hrsg.): Führungsprobleme industrieller Unternehmungen, Berlin, New York 1980, S. 75-108

Bleicher, Knut: Organisation: Strategien - Strukturen - Kulturen, 2. Auflage, Wiesbaden 1991

Bleicher, Knut: Das Konzept integriertes Management: Visionen, Missionen, Programme, 5. Auflage, Frankfurt am Main, New York 1999

Bleicher, Knut / Bibi Hahn: Das Management-Potential - Dynamik-Faktor der Unternehmungsentwicklung, in: Ulrich Krystek / Jörg Link (Hrsg.): Führungskräfte und Führungserfolg: Neue Herausforderungen für das strategische Management, Wiesbaden 1995, S. 416-446

Bleicher, Knut / Diethard Leberl / Herbert Paul: Unternehmungsverfassung und Spitzenorganisation, Wiesbaden 1989

Bleicher, Knut / Diethelm Leberl: Der Aufsichtsrat im Wandel, Gütersloh 1987

Bleicher, Knut u.a.: Unternehmungsverfassung und Spitzenorganisation, in: zfo, 53 (1984), S. 21-29

Böckli, Peter: Corporate Governance auf Schnellstrassen und Holzwegen, in: Der Schweizer Treuhänder, 74 (2000), S. 133-152

Böhmer, Ekkehart: Corporate Governance in Germany: Institutional background and empirical results, Arbeitspapier Nr. 78, Humboldt Universität, Berlin 1999

Bohr, Kurt / Jochen Drukarczyk / Hans-Jürgen Drumm / Gerhard Scherrer (Hrsg.): Unternehmungsverfassung als Problem der Betriebswirtschaftslehre. Berlin, Bielefeld, München 1981

Boujong, Karlheinz: Rechtliche Mindestanforderungen an eine ordnungsgemäße Vorstandskontrolle und -beratung, in: AG, 40 (1995), S. 203-207

Bredow, Günther: Mustervereinbarung zu Aktienoptionsplänen für das Management und leitende Angestellte (Stock Option Plans), in: DStR, 36 (1998), S. 380-382

Breuer, Wolfgang: Die Marktwertmaximierung als finanzwirtschaftliche Entscheidungsregel, in: WiSt, 26. (1997), S. 222-226 
Bruhn, Manfred, Hartwig Steffenhagen (Hrsg.): Marktorientierte Unternehmensführung: Reflexionen - Denkanstösse - Perspektiven, Wiesbaden 1997

Bughin, Jacques / Thomas E. Copeland: The virtuous cycle of shareholder value creation, in: The McKinsey Quarterly, o.J. (1997), S. 156-167

Bühner, Rolf (Hrsg.): Der Shareholder-Value-Report : Erfahrungen, Ergebnisse, Entwicklungen, Landsberg/Lech 1994

Bühner, Rolf / Anja Tuschke: Zur Kritik am Shareholder Value - eine ökonomische Analyse -, in: BFuP, 49 (1997), S. 499-516

Bundespresseamt: Pressemitteilung Nr. 301/00, in: http://www.bundesregierung.de/dokumente/Pressemitteilung/ix_11430.htm (6.10.2000)

Bundespresseamt: Pressemitteilung Nr. 321/00, in: http://www.bundesregierung.de/dokumente/Pressemitteilung/ix_11871.htm (6.10.2000)

Bundespresseamt: Pressemitteilung Nr. 304/01, in: http://www.bundesregierung.de/dokumente/Pressemitteilung/ix_48099_1400.htm (10.7.2001)

Bundesverband der Deutschen Industrie e.V. / PwC Deutsche Revision AG (Hrsg.): Corporate Governance in Deutschland, BDI-Drucksache Nr. 322, Berlin 2001

Bundesverfassungsgerichts, Urteil des vom 1.3.1979 - 1 BvR 532/77, 533/77, 419/78 und BvL 21/78: Vereinbarkeit der erweiterten Mitbestimmung der Arbeitnehmer nach dem MitbestG 1976 mit den Grundrechten der mitbestimmten Gesellschaften, der Anteilseigner und der Koalition der Arbeitgeber, in: DB, 32 (1979), S. 593-605

Bursk, Edward C. / Dan H. Fenn (Hrsg.): Unternehmerstrategie, Essen 1957

Busse von Colbe, Walther: Was ist und was bedeutet Shareholder Value aus betriebswirtschaftlicher Sicht?, in: ZGR, 26 (1997), S. 271-290

Busse von Colbe, Walther / Bernhard Pellens: Lexikon des Rechnungswesens, 4. Auflage, München, Wien 1998

Busse von Colbe, Walther / Gert Laßmann: Betriebswirtschaftstheorie, Bd. 1: Grundlagen, Produktions- und Kostentheorie, 5. Auflage, Berlin u.a. 1991

Cassier, Siegfried C.: Wer bestimmt die Geschäftspolitik der Großunternehmer?, Frankfurt am Main 1962

Centre National de la Recherche Scientifique (Hrsg.): La Décision. Agrégation et dynamique des ordres de préférence, Paris 1969

Chamoni, Peter / Peter Gluchowski: Analytische Informationssysteme, 2. Auflage, Berlin u.a. 1999 
Chamoni, Peter / Peter Gluchowski: Analytische Informationssysteme Einordnung und Überblick, in: Peter Chamoni / Peter Gluchowski: Analytische Informationssysteme, 2. Auflage, Berlin u.a. 1999, S. 4-25

Chini, Leo W.: Aufsichtsrats-Informationssystem, Wiesbaden 1988

Chmielewicz, Klaus: Unternehmungsverfassung, Gremien der, in: Erwin Grochla (Hrsg.): Handwörterbuch der Organisation, 2. Auflage, Stuttgart 1980, Sp. 2272-2282

Chmielewicz, Klaus: Führung und Überwachung der deutschen Unternehmung, in: Wolfram Fischer (Hrsg.): Währungsreform und Soziale Marktwirtschaft: Erfahrungen und Perspektiven nach 40 Jahren (Jahrestagung des Vereins für Socialpolitik, Gesellschaft für Wirtschafts- und Sozialwissenschaften; 1988) Schriften des Vereins für Socialpolitik, Neue Folge Bd. 190, Berlin 1989, S. 567-579

Chmielewicz, Klaus: Unternehmungsleitung, Organisation der, in: Erich Frese (Hrsg.): Handwörterbuch der Organisation, 3. Auflage, Stuttgart 1992, Sp. 2464-2480

Chmielewicz, Klaus: Unternehmensverfassung, in: Waldemar Wittmann u.a. (Hrsg.): Handwörterbuch der Betriebswirtschaft, 5. Auflage, Stuttgart 1993, Sp. 4399-4417

Chmielewicz, Klaus / Marcell Schweitzer (Hrsg.): Handwörterbuch des Rechnungswesens, 3. Auflage, Stuttgart 1993

Chmielewicz, Klaus u.a.: Die Mitbestimmung im Vorstand und Aufsichtsrat, in: DBW, 37 (1977), S. 105-145

Chwolka, Anne: Der Aufsichtsrat als Überwachungsorgan - eine überflüssige Institution?, in: Horst Albach u.a. (Hrsg.): Die Theorie der Unternehmung in Forschung und Praxis, Berlin u.a. 1999, S. 627-649

Claussen, Carsten P.: Aktienrechtsreform 1997, in: AG, 41 (1996), S. 481494

Claussen, Carsten P.: Aktienoptionen - eine Bereicherung des Kapitalmarktrechts, in: WM, 51 (1997), S. 1825-1836

Claussen, Carsten P.: Wie ändert das KonTraG das Aktiengesetz?, in: DB, 51 (1998), S. 177-186

Claussen, Carsten P. / Norbert Bröcker: Corporate GovernanceGrundsätze in Deutschland - nützliche Orientierungshilfe oder regulatorisches Übermaß?, in: AG, 45 (2000), S. 481-491 
Claussen, Carsten P. / Oswald Hahn / Willy Kraus (Hrsg.): Umbruch und Wandel: Herausforderungen zur Jahrhundertwende, München, Wien 1997

Coase, Ronald: The Nature of the Firm, in: Economica, 4 (1937), S. 386-405

Coase, Ronald: The Problem of Social Cost, in: Journal of Law and Economics, 3 (1960), S. 1-44

Coenenberg, Adolf G. / Alexander Reinhart / Jochen Schmitz: Audit Committees - Ein Instrument zur Unternehmensüberwachung?, in: DB, 50 (1997), S. 989-1000

Coenenberg, Adolf G. / Klaus v. Wysocki (Hrsg.): Handwörterbuch der Revision, 2. Auflage, Stuttgart 1992

Conger, Jay A. / David Finegold / Edward E. Lawler III: Appraising Boardroom Performance, in: Harvard Business Review, 76 (1998), S. 136-148

Cornell, Bradford / Alan C. Shapiro: Corporate Stakeholders and Corporate Finance, in: Financial Management, 16 (1987), S. 5-14

Corsten, Hans / Michael Reiß (Hrsg.): Handbuch Unternehmungsführung, Wiesbaden 1995

Cyert, Richard M. / James G. March: A Behavioral Theory of the Firm, Englewood Cliffs, N.J. 1963

DAV, Stellungnahme zum Referentenentwurf zur Änderung des Aktiengesetzes („KonTraG“), in: ZIP, 18 (1997), S. 163-174

Davis, T. C.: How the Dupont organization appraises its performance, Financial Management Series Number 94, Reprint of the edition New York 1950, New York 1967

Debreu, Gérard: Theory of Value: An Axiomatic Analysis of Economic Equilibrium, New Haven u.a. 1959

Deckert, Martina: Effektive Überwachung der AG-Geschäftsführung durch Ausschüsse des Aufsichtsrats, in: ZIP, 17 (1996), S. 985-994

Deckert, Martina: Organschaftliche und vertragliche Beratungspflichten des Aufsichtsratsmitglieds, in: AG, 42 (1997), S. 109-114

Demsetz, Harold: Toward a Theory of Property Rights, in: American Economic Review, 57 (1967), S. 347-359

Déminor: Déminor rates 300 European companies based on Corporate Governance standards, Pressemitteilung vom 12.12.2000

Deutsche Bank AG: Unternehmensprofil, in: http://public.deutschebank.de/deuba/db/navigate.nsf/Frameset/TSPR-

45NKAX?OpenDocument (5.2.2001) 
Deutsche Bank AG: Corporate Governance-Grundsätze, Pressemitteilung vom 29.03.2001

Deutsche Börse: MDAX ${ }^{\circledR}$, Stand 1. September 1997, in: http://www. exchange.de/INTERNET/EXCHANGE/index.htm (29.12.2000)

Deutscher Bundestag: Drucksache 13/9716 vom 29.01.1998

Deutsche Vereinigung für Finanzanalyse und Asset Management: Scorecard for German Corporate Governance ${ }^{\odot}$, in: http://www.dvfa.de/ pdf/scorecard.pdf (13.4.2001), Stand 2000

Dirrigl, Hans: Wertorientierung und Konvergenz in der Unternehmensrechnung, in: BfuP, 50 (1998), S. 540-579

Dittmar, Carsten: Wissen sichtbar machen, in: Computerwoche extra: Business Intelligence, Heft Nr. 4 vom 16. Juni 2000, S. 14-17

Dorfs, Joachim: Feindlich ins neue Jahrtausend, in: Handelsblatt, 31.12.1999, Nr. 255, S. 16

Dörner, Dietrich / Dieter Menold / Norbert Pfitzer (Hrsg.): Reform des Aktienrechts, Rechnungslegung und Prüfung, Stuttgart 1999

Dose, Stefan: Die Rechtsstellung der Vorstandsmitglieder einer Aktiengesellschaft, 3. Auflage, Köln 1975

Dreher, Meinrad: Das Ermessen des Aufsichtsrats, in: ZHR, 158 (1994), S. 614-645

Dreher, Meinrad: Die Organisation des Aufsichtsrats, in: Dieter Feddersen / Peter Hommelhoff / Uwe H. Schneider (Hrsg.): Corporate Governance, Köln 1996, S. 33-60

Dreyer, Jörg-Detlev: Entwicklung und Beurteilung Aufsichtsratsorientierter Informationskonzeptionen, Schwarzenbeck 1980

Drukarczyk, Jochen: Theorie und Politik der Finanzierung, 2. Auflage, München 1993

Drukarczyk, Jochen / Lothar Müller-Hagedorn (Hrsg.): Betriebswirtschaftslehre: Eine Einführung in die Theorie der Unternehmung, Bd. 2, Wiesbaden 1978

DSW (Hrsg.): Aufsichtsräte in Deutschland, Düsseldorf 1995

Dubs, Rolf: Sanktionen als Führungsinstrumente, in: Alfred Kieser / Gerhard Reber / Rolf Wunderer (Hrsg.): Handwörterbuch der Führung, 2. Auflage, Stuttgart 1995, Sp. 1868-1873

Dufey, Gunter / Ulrich Hommel: Der Shareholder-Value-Ansatz: U.S.Amerikanischer Kulturimport oder Diktat des globalen Marktes?, in: 
Johann Engelhard (Hrsg.): Interkulturelles Management, Wiesbaden 1997, S. 183-211

Dufey, Gunter/ Ulrich Hommel / Petra Riemer-Hommel: Corporate Governance: European vs. U.S. Perpectives in a Global Capital Market, in: Christian Scholz / Joachim Zentes (Hrsg.): Strategisches EuroManagement, Bd. 2, Stuttgart 1998, S. 45-65

DWS: Verbesserte Unternehmensaufsicht in Deutschland - Empfehlungen der DWS zu Corporate Governance, o.O., o.J.

Edenfeld, Stefan / Sebastian Neufang: Die Haftung der Arbeitnehmervertreter im Aufsichtsrat, in: AG, 44 (1999), S. 49-57

Eisenhardt, Kathleen M.: Agency Theory: An Assessment and Review, in: Academy of Management Review, 14 (1989), S. 52-74

Elschen, Rainer: Agency-Theorie, in: DBW, 48 (1988), S. 248-250

Elschen, Rainer: Gegenstand und Anwendungsmöglichkeiten der AgencyTheorie, in: ZfbF, 43 (1991), S. 1002-1012

Elschen, Rainer: Shareholder Value und Agency-Theorie - Anreiz- und Kontrollsysteme für Zielsetzungen der Anteilseigner, in: BFuP, 43 (1991), S. $209-219$

Endres, Michael: Organisation der Unternehmensleitung aus Sicht der Praxis, in: ZHR, Bd. 163 (1999), S. 441-460

Entwurf eines Gesetzes zur Verbesserung von Transparenz und Beschränkung von Machtkonzentration der deutschen Wirtschaft (Transparenzund Wettbewerbsgesetz), Bundestagsdrucksache 13/367 vom 30.1.1995

Erlei, Matthias / Martin Leschke / Dirk Sauerland: Neue Institutionenökonomik, Stuttgart 1999

Ernst, Christoph / Ulrich Seibert / Fritz Stuckert: KonTrag, KapAEG, StückAG, EuroEG, Düsseldorf 1998

Evers, Heinz: Variable Vergütung für Führungskräfte: Wertorientierung als Herausforderung, in: Bernhard Pellens (Hrsg.): Unternehmenswertorientierte Entlohnungssysteme, Stuttgart 1998, S. 53-67

Ewert, Ralf / Eberhard Feess / Martin Nell: Prüfungsqualität, Dritthaftung und Versicherung, in: BFuP, 52 (2000), S. 572-593

Fama, Eugene F.: Agency Problems and the Theory of the Firm, in: Journal of Political Economy, 88 (1980), S. 288-307

Fama, Eugene F. / Michael C. Jensen: Separation of Ownership and Control, in: Journal of Law and Economics, 26 (1983), S. 301-325 
Feddersen, Dieter: Nochmals - Die Pflichten des Vorstands zur Unternehmensplanung, in: ZGR, 22 (1993), S. 114-117

Feddersen, Dieter: Neue gesetzliche Anforderungen an den Aufsichtsrat, in: AG, 45 (2000), S. 385-396

Feddersen, Dieter / Peter Hommelhoff / Uwe H. Schneider (Hrsg.): Corporate Governance, Köln 1996

Fernández-Araóz, Claudio: Die Führungsposition richtig besetzen - eine Kunst, in: Harvard Business Manager, 22 (2000), S. 56-72

Ferris, Gerald R. / Sherman D. Rosen / Darold T. Barnum (Hrsg.): Handbook of Human Resource Management, Cambridge 1995

Fischer, Guido: Lehren der Vergangenheit für die Zukunft, in: Personal, 27 (1975), S. 293

Fischer, Wolfram (Hrsg.): Währungsreform und Soziale Marktwirtschaft: Erfahrungen und Perspektiven nach 40 Jahren (Jahrestagung des Vereins für Socialpolitik, Gesellschaft für Wirtschafts- und Sozialwissenschaften; 1988) Schriften des Vereins für Socialpolitik, Neue Folge Bd. 190, Berlin 1989

Fisher, Irving: The Theory of Interest, New York 1930

Flassak, Hansjörg: Der Markt für Unternehmenskontrolle - Eine ökonomische Analyse vor dem Hintergrund des deutschen Gesellschaftsrechts, Köln 1995

Fonk, Hans-Joachim: Personalentscheidungen des Aufsichtsrats, in: Johannes Semler (Hrsg.): Arbeitshandbuch für Aufsichtsratsmitglieder, München 1999, S. 437-599, 788-822

Förschle, Gerhart / Martin Glaum / Udo Mandler: Gesetz zur Kontrolle und Transparenz im Unternehmensbereich: Umfrage unter Führungskräften börsennotierter Unternehmungen, in: DB, 51 (1998), S. 889-895

Franks, Julian / Colin Mayer: Hostile takeovers and the correction of managerial failure, in: Journal of Financial Economics, 40 (1996), S. 163-181

Freeman, Robert Edward: Strategic management: a stakeholder approach, Boston u.a. 1984

Frese, Erich: Unternehmensführung, Landsberg am Lech 1986

Frese, Erich (Hrsg.): Handwörterbuch der Organisation, 3. Auflage, Stuttgart 1992 
Frese, Erich: Führung, Organisation und Unternehmensverfassung, in: Waldemar Wittmann u.a. (Hrsg.): Handwörterbuch der Betriebswirtschaft, 5. Auflage, Stuttgart 1993, Sp. 1284-1299

Frese, Erich: Unternehmungsverfassung aus organisationstheoretischer Sicht, Teil I, in: WISU, 22 (1993), S. 933-937

Frese, Erich: Unternehmungsverfassung aus organisationstheoretischer Sicht, Teil II, in: WISU, 22 (1993), S. 1011-1016

Frese, Erich: Grundlagen der Organisation, 8. Auflage, Wiesbaden 2000

Fresenius Medical Care AG, Geschäftsbericht 1999

Friedrichsen, Sönke: Aktienoptionsprogramme für Führungskräfte, Köln u.a. 2000

Fritsch, Ulrich / Gerhard Liener / Reinhart Schmidt (Hrsg.): Die deutsche Aktie, Stuttgart 1993

Frühauf, Martin: Geschäftsleitung in der Unternehmenspraxis, in: ZGR, 27 (1998), S. 407-418

Gabriel, Roland: Strategische Bedeutung der analytischen Informationssysteme, in: Peter Chamoni / Peter Gluchowski: Analytische Informationssysteme, 2. Auflage, Berlin u.a. 1999, S. 418-426

Gabriel, Roland / Peter Gluchowski: Management Support Systeme, Teil I, in: WiSt, 26 (1997), S. 308-313

Gabriel, Roland / Peter Gluchowski: Management Support Systeme, Teil II, in: WiSt, 26 (1997), S. 422-427

Gabriel, Roland / Peter Gluchowski: Management Support Systeme, Teil III, in: WiSt, 26 (1997), S. 535-540

Gabriel, Roland / Peter Chamoni / Peter Gluchowski: Data Warehouse und OLAP - Analyseorientierte Informationssysteme für das Management, in: ZfbF, 52 (2000), S. 74-93

Gadow, Wilhelm: Großkommentar zum Aktiengesetz, Berlin 1961

Gaertringen, Christian Hiller von / Andreas Wildhagen: Total überfordert, in: Wirtschaftswoche, 53 (1999), Nr. 48, S. 76-77

Gassen, Joachim: Datenbankgestützte Rechnungslegungspublizität, Frankfurt am Main u.a. 2000

Gawrisch, Volker: Ermessensentscheidungen des Aufsichtsrates und ihre gerichtliche Kontrolle, Frankfurt am Main u.a. 2000 
Gebhardt, Günther / Bernhard Pellens (Hrsg.): Rechnungswesen und Kapitalmarkt, Düsseldorf, Frankfurt am Main 1999 (ZfbF Sonderheft 41)

Gedenk, Karen: Agency-Theorie und die Steuerung von Geschäftsführern, in: DBW, 58 (1998), S. 22-35

Gerke, Wolfgang: Agency-Theorie, in: Wolfgang Gerke / Manfred Steiner (Hrsg.): Handwörterbuch des Bank- und Finanzwesens, 2. Auflage, Stuttgart 1995, Sp. 17-26

Gerke, Wolfgang / Manfred Steiner (Hrsg.): Handwörterbuch des Bank- und Finanzwesens, 2. Auflage, Stuttgart 1995

Gerum, Elmar: Führungsorganisation, Eigentümerstruktur und Unternehmensstrategie, in: DBW, 55 (1995), S. 359-379

Gerum, Elmar: Manager- und Eigentümerführung, in: Alfred Kieser / Gerhard Reber / Rolf Wunderer (Hrsg.): Handwörterbuch der Führung, 2. Auflage, Stuttgart 1995, Sp. 1457-1468

Gerum, Elmar: Mitbestimmung und Corporate Governance, Gütersloh 1998

Gerum, Elmar: Organisation der Unternehmensführung im internationalen Vergleich, in: Horst Glaser / Ernst F. Schröder / Axel v. Werder (Hrsg.): Organisation im Wandel der Märkte, Wiesbaden 1998, S. 135-153

Geßler, Ernst u.a. (Hrsg.): Kommentar zum Aktiengesetz, Bd. 2, München 1973

Girnghuber, Gudrun: Das US-amerikanische Audit Committee als Instrument zur Vermeidung von Defiziten bei der Überwachungstätigkeit deutscher Aufsichtsräte, Frankfurt am Main 1998

Glaser, Horst / Ernst F. Schröder / Axel v. Werder (Hrsg.): Organisation im Wandel der Märkte, Wiesbaden 1998

Gluchowski, Peter / Roland Gabriel / Peter Chamoni: Management Support Systeme, Berlin u.a. 1997

Gollnick, Jörg: Die Beurteilung der Vorstandsleistung durch den Aufsichtsrat: eine vergleichende Untersuchung zum deutschen und USamerikanischem Recht, Frankfurt am Main u.a. 1997

Gomez, Peter: Shareholder Value, in: Wolfgang Gerke / Manfred Steiner (Hrsg.): Handwörterbuch des Bank- und Finanzwesens, 2. Auflage, Stuttgart 1995, Sp. 1720-1728

Gomez-Mejia, Luis R. / George Paulin / Arden Grabke: Executive Compensation: Research and Practical Implications, in: Gerald R. Ferris / 
Sherman D. Rosen / Darold T. Barnum (Hrsg.): Handbook of Human Resource Management, Cambridge 1995, S. 548-569

Götz, Heinrich: Zustimmungsvorbehalte des Aufsichtsrats der Aktiengesellschaft, in: ZGR, 19 (1990), S. 633-656

Götz, Heinrich: Die Überwachung der Aktiengesellschaft im Lichte jüngerer Unternehmenskrisen, in: AG, 40 (1995), S. 337-353

Grochla, Erwin: Einführung in die Organisationstheorie, Stuttgart 1978

Grochla, Erwin (Hrsg.): Handwörterbuch der Organisation, 2. Auflage, Stuttgart 1980

Grochla, Erwin: Betrieb, Betriebswirtschaft und Unternehmung, in: Waldemar Wittmann u.a. (Hrsg.): Handwörterbuch der Betriebswirtschaft, 5. Auflage, Stuttgart 1993, Sp. 374-390

Groh, Manfred: Shareholder Value und Aktienrecht, in: DB, 53 (2000), S. 2153-2158

Grossman, Sanford J. / Oliver D. Hart: Takeover bids, the free-rider problem, and the theory of the corporation, in: The Bell Journal of Economics, 11 (1980), S. 42-64

Großmann, Adolf: Unternehmensziele im Aktienrecht, Köln u.a. 1980

Grundsatzkommission Corporate Governance: Corporate Governance Grundsätze ('Code of Best Practice') für börsennotierte Gesellschaften, in: DB, 53 (2000), S. 238-241

Grundsatzkommission Corporate Governance: Corporate Governance Grundsätze ('Code of Best Practice') für börsennotierte Gesellschaften, in: http://www.corgov.de/download/code0700d.pdf (11.4.2001)

Grünewald, Hans-Günter: Informationssysteme für die Planung, in: Norbert Szyperski / Udo Winand (Hrsg.): Handwörterbuch der Planung, Stuttgart 1989, Sp. 692-708

Gruppe Deutsche Börse: Regelwerk Neuer Markt (Stand 1.3.2001), in: http://nm.deutsche-boerse.com/INTERNET/NM/nm4.nsf/WebMaskenfomeln/7A772C916105BE6541256A01005E93F2/\$FILE/Regelwerk0103-01dt.pdf (7.4.2001)

Günther, Thomas: Unternehmenswertorientiertes Controlling, München 1997

Gutenberg, Erich: Einführung in die Betriebswirtschaftslehre, Wiesbaden 1958 
Gutenberg, Erich: Unternehmensführung - Organisation und Entscheidung, Wiesbaden 1962

Gutenberg, Erich: Funktionswandel des Aufsichtsrats, in: ZfB, 40 (1970), Ergänzungsheft, S. 1-10

Gutenberg, Erich: Grundlagen der Betriebswirtschaftslehre, Bd. 1, Die Produktion, 24. Auflage, Berlin, Heidelberg, New York 1983

Hahn, Dietger (Hrsg.): Führungsprobleme industrieller Unternehmungen, Berlin, New York 1980

Hahn, Dietger: PuK - Planung und Kontrolle, 4. Auflage, Wiesbaden 1994

Hahn, Dietger: Konzepte strategischer Führung, in: ZfB, 68 (1998), S. 563579

Hahn, Dietger: Integrierte Organisations- und Führungskräfteplanung im Rahmen der strategischen Unternehmungsplanung, in: Dietger Hahn / Bernard Taylor (Hrsg.): Strategische Unternehmungsplanung - Strategische Unternehmungsführung, 8. Auflage, Heidelberg 1999, S. 617-640

Hahn, Dietger: Planungs- und Kontrollsysteme als Gegenstand strategischer Planung, in: Dietger Hahn / Bernard Taylor (Hrsg.): Strategische Unternehmungsplanung - Strategische Unternehmungsführung, 8. Auflage, Heidelberg 1999, S. 664-681

Hahn, Dietger: Stand und Entwicklungstendenzen der strategischen Planung, in: Dietger Hahn / Bernard Taylor (Hrsg.): Strategische Unternehmungsplanung - Strategische Unternehmungsführung, 8. Auflage, Heidelberg 1999, S. 1-27

Hahn, Dietger: Strategische Planung und Mitbestimmung, in: Dietger Hahn / Bernard Taylor (Hrsg.): Strategische Unternehmungsplanung - Strategische Unternehmungsführung, 8. Auflage, Heidelberg 1999, S. 780-803

Hahn, Dietger: Strategische Unternehmungsführung - Grundkonzept, in: Dietger Hahn / Bernard Taylor (Hrsg.): Strategische Unternehmungsplanung - Strategische Unternehmungsführung, 8. Auflage, Heidelberg 1999, S. $28-50$

Hahn, Dietger: Unternehmungsziele im Wandel, in: Dietger Hahn / Bernard Taylor (Hrsg.): Strategische Unternehmungsplanung - Strategische Unternehmungsführung, 8. Auflage, Heidelberg 1999, S. 303-323

Hahn, Dietger / Bernard Taylor (Hrsg.): Strategische Unternehmungsplanung - Strategische Unternehmungsführung, 8. Auflage, Heidelberg 1999 
Hahn, Friedrich von: Commentar zum Allgemeinen Deutschen Handelsgesetzbuch, Erster Bd., zweite vermehrte und verbesserte Auflage, Braunschweig 1871

Hakelmacher, Sebastian: Die Falken-Parabel, Lengwil 1999

Hakelmacher, Sebastian: Die korpulente Gouvernante II - Corporate Governance mit Aussicht auf KonTraG II -, in: Die Wirtschaftsprüfung, 54 (2001), S. 177-187

Hammann, Peter / Jörg Freiling (Hrsg.): Die Ressourcen- und Kompetenzperspektive des Strategischen Managements, Wiesbaden 2000

Hammer, Richard M.: Unternehmensplanung, 7. Auflage, München, Wien 1998

Hansen, Herbert: AG-Report - Zum Jahresende 2000: 10582 Aktiengesellschaften, in: AG, 46 (2001), R 67-68

Hansen, Klaus P.: Kultur und Kulturwissenschaft, 2. Auflage, Tübingen u.a. 2000

Harris, Milton / Artur Raviv: Some Results on Incentive Contracts with Applications to Education and Employment, Health Insurance, and Law Enforcement, in: American Economic Review, 68 (1978), S. 20-30

Hart, Oliver D.: Corporate Governance: Some Theory and Implications, in: The Economic Journal, 105 (1995), S. 678-689

Hartmann-Wendels, Thomas: Kontrollrechte der Gesellschafter, in: Wolfgang Gerke / Manfred Steiner (Hrsg.): Handwörterbuch des Bank- und Finanzwesens, 2. Auflage, Stuttgart 1995, Sp. 1214-1223

Hasselbach, Kai / Stephan Schumacher: Hauptversammlung im Internet, in: ZGR, 29 (2000), S. 258-286

Hauschildt, Jürgen: Entscheidungsziele, Tübingen 1977

Hax, Karl: Die Aufgaben des Aufsichtsrates der Aktiengesellschaft unter dem Einfluß der qualifizierten Mitbestimmung, in: Kurt Nemitz / Richard Becker (Hrsg.): Mitbestimmung und Wirtschaftspolitik, Köln 1967, S. 195-213

Heermann, Peter W.: Interessenkonflikte von Bankenvertretern in Aufsichtsräten bei (geplanten) Unternehmensübernahmen, in: Wertpapiermitteilungen, 51 (1997), S. 1689-1728

Hefermehl, Wolfgang: Kommentierungen zum Aktiengesetz, in: Ernst Geßler u.a. (Hrsg.): Kommentar zum Aktiengesetz, Bd. 2, München 1973 
Heinen, Edmund: Einführung in die Betriebswirtschaftslehre, 9. Auflage, Wiesbaden 1985

Hengst, Walter: Chancen realisieren durch Management Audit, in: Interne Revision, 32 (1997), S. 198-207

Henn, Günter: Handbuch des Aktienrechts, 6. Auflage, Heidelberg 1998

Henselek, Hilmar F.: Das Management von Unternehmungskonfigurationen, Wiesbaden 1996

Henselek, Hilmar F.: Konfigurationseigenschaft als strategische Ressource Konfigurationsmanagement als Metakompetenz, in: Peter Hammann / Jörg Freiling (Hrsg.): Die Ressourcen- und Kompetenzperspektive des Strategischen Managements, Wiesbaden 2000, S. 465-489

Henselek, Hilmar F. / Wolfgang Mag / Peter Ruhwedel: Die informationelle Unterstützung der Personalplanung durch SAP ${ }^{\circledR} \mathrm{R} / 3^{\circledR} \mathrm{HR}^{\circledR}$, in: Personal, 53 (2001), S. 122-127

Henze, Hartwig: Leitungsverantwortung des Vorstands - Überwachungspflicht des Aufsichtsrats, in: BB, 55 (2000), S. 209-216

Henze, Hartwig: Entscheidungen und Kompetenzen der Organe in der AG: Vorgaben der höchstrichterlichen Rechtsprechung, in: BB, 56 (2001), S. 53-61

Hess, Glen E.: Corporate Governance - zum Stand der Diskussion in den Vereinigten Staaten, in: Dieter Feddersen / Peter Hommelhoff / Uwe H. Schneider (Hrsg.): Corporate Governance, Köln 1996, S. 9-24

Hess, Thomas: Die Agency-Theorie als Gestaltungshilfe für Führungsinformationssysteme, in: WISU, 28 (1999), S. 1503-1509

Heymann, H.-Helmut / Lothar J. Seiwert / Manuel R. Theisen: Mitbestimmungsmanagement, Frankfurt am Main 1983

Hillebrandt, Franca: Entflechtung der Deutschland AG durch Steuerfreiheit von Veräußerungserlösen? in: DBW, 61 (2001), S. 711-726

Hinterhuber, Hans H.: Planung der Führungskräfteentwicklung als Gegenstand der strategischen Unternehmungsplanung, in: Dietger Hahn / Bernard Taylor (Hrsg.): Strategische Unternehmungsplanung - Strategische Unternehmungsführung, 8. Auflage, Heidelberg 1999, S. 641-663

Hirschmann, Albert O.: Exit, Voice, and Loyalty, Cambridge 1970

Hoff, Helmut: Die Gestaltung von Entscheidungsprozessen in betrieblichen Gremien, Frankfurt am Main u.a. 1986 
Hoffmann, Friedrich: Aufbauorganisation, in: Erich Frese (Hrsg.): Handwörterbuch der Organisation, 3. Auflage, Stuttgart 1992, Sp. 208-221

Hoffmann-Becking, Michael: Der Aufsichtsrat im Konzern, in: ZHR, 159 (1995), S. 325-345

Hoffmann-Becking, Michael: Zur rechtlichen Organisation der Zusammenarbeit im Vorstand der AG, in: ZGR, 27 (1998),S. 497-519

Hofmann, Rolf: Aufsichtsrat, Bochum 1996

Holmström, Bengt: Moral hazard and observability, in: Bell Journal of Economics, 10 (1979), S. 74-91

Holzer, H. Peter / Andreas Makowski: Corporate Governance, in: DB, 50 (1997), S. 688-692

Homburg, Christian: Quantitative Betriebswirtschaftslehre, 2. Auflage, Wiesbaden 1998

Hommel, Ulrich / Petra Riemer-Hommel: Die Evolution der nationalen Unternehmensüberwachungssysteme: Der Einfluß von Regulierung und internationalem Wettbewerb aufgezeigt am Beispiel der Vereinigten Staaten und der Bundesrepublik Deutschland, in: Michael Kutschker (Hrsg.): Management verteilter Kompetenzen in multinationalen Unternehmen, Wiesbaden 1999, S. 149-176

Hommelhoff, Peter: Der aktienrechtliche Organstreit, in: ZHR, 143 (1979), S. $288-316$

Hommelhoff, Peter: Störungen im Recht der Aufsichtsrats-Überwachung: Regelungsvorschläge an den Gesetzgeber, in: Arnold Picot (Hrsg.): Corporate Governance, Stuttgart 1995,S. 1-28

Hommelhoff, Peter: Corporate Governance: Vertragen sich die deutsche Unternehmensverfassung und das Shareholder Value-Prinzip?, in: Horst Albach / Klaus Brockhoff (Schriftleitung): Betriebswirtschaftslehre und Rechtsentwicklung, (ZfB-Ergänzungsheft 4, 67. Jg.), Wiesbaden 1997, S. $17-20$

Hommelhoff, Peter / Daniela Mattheus: Corporate Governance nach dem KonTraG, in: AG, 43 (1998), S. 249-259

Hopt, Klaus J. (Hrsg.): Großkommentar zum Aktiengesetz, AktG, 4. Auflage, Berlin u.a. 1992

Hopt, Klaus J.: Gemeinsame Grundsätze der Corporate Governance in Europa?, in: ZGR, 29 (2000), S. 779-818 
Hopt, Klaus J. / Eddy Wymeersch (Hrsg.): Comparative corporate governance, Berlin, New York 1997

Horváth, Péter: Balanced Scorecard: Wie Sie Strategien erfolgreich umsetzen, in: Gablers Magazin, 20 (1998), Heft 4,S. 22-25

Hübner, Ulrich: Managerhaftung: Rechtsgrundlagen und Risikopotentiale einer persönlichen Inanspruchnahme der Unternehmensleiter von Kapitalgesellschaften, München 1992

Hüffer, Uwe: Aktiengesetz, 4. Auflage, München 1999

Hungenberg, Harald: Anreizsysteme für Führungskräfte - Theoretische Grundlagen und praktische Ausgestaltungsmöglichkeiten, in: Dietger Hahn / Bernard Taylor (Hrsg.): Strategische Unternehmungsplanung Strategische Unternehmungsführung, 8. Auflage, Heidelberg 1999, S. 720-735

Hutzschenreuter, Thomas: Unternehmensverfassung und Führungssystem, Wiesbaden 1998

Iwd-Mitteilungen Nr. 38: Deutschland AG steht noch, in: Iwd-Mitteilungen Nr. 38, 26 (2000), S. 6-7

Jaeger, Carsten: Aufsichtsratsausschüsse ohne Arbeitnehmervertreter?, in: ZIP, 16 (1995), S. 1735-1739

Jansch, Torsten Adam: Die Rolle der Aktionäre in Publikumsgesellschaften, Wiesbaden 1999

Jaschke, Thomas: Die betriebswirtschaftliche Überwachungsfunktion aktienrechtlicher Aufsichtsräte, Köln 1989

Jensen, Michael C.: Organization Theory and Methodology, in: Accounting Review, 58 (1983), S. 319-339

Jensen, Michael C. / William H. Meckling: Theory of the firm: Managerial behavior, agency costs and ownership structure, in: Journal of Financial Economics, 3 (1976), S. 305-360

Kaiser, Bernd-Ulrich: Unternehmensinformation mit SAP ${ }^{\circledR}$-EIS, 4. Auflage, Braunschweig, Wiesbaden 1999

Kallmeyer, Harald: Pflichten des Vorstands der Aktiengesellschaft zur Unternehmensplanung, in: ZGR, 22 (1993), S. 104-113

Kaplan, Robert S. / David P. Norton: Balanced scorecard: Strategien erfolgreich umsetzen, Stuttgart 1997

Katz, Robert L.: Skills of an effective administrator, in: Harvard Business Review, Nr. 5, 52 (1974), S. 90-102 
Kau, Wolfgang M. / Klaus Kukat: Haftung von Vorstands- und Aufsichtsratsmitgliedern bei Pflichtverletzungen nach dem Aktiengesetz, in: BB, 55 (2000), S. 1045-1050

Kesten, Ulrike: Informale Organisation und Mitarbeiter-Lebenszyklus, Wiesbaden 1998

Kienbaum Management Consultants GmbH: Vergütungsstudie 1998/99 Vorstands- und Aufsichtsratsmitglieder, 23. Auflage, Gummersbach 2000

Kienbaum und Partner GmbH: Aufsichtsratsstudie 1998, Gummersbach 1997

Kieser, Alfred / Gerhard Reber / Rolf Wunderer (Hrsg.): Handwörterbuch der Führung, 2. Auflage, Stuttgart 1995

Kirchner, Christian: Szenarien einer feindlichen Unternehmensübernahme: Alternative rechtliche Regelungen im Anwendungstest, in: BB, 55 (2000), S. $105-113$

Kirsch, Werner / Natascha Eckert: Die Strategieberatung im Lichte einer evolutionären Theorie der strategischen Führung, in: Ingolf Bamberger (Hrsg.): Strategische Unternehmensberatung, 2. Auflage, Wiesbaden 2000, S. 265-310

Kistner, Klaus-Peter / Reinhart Schmidt (Hrsg.): Unternehmensdynamik, Wiesbaden 1991

Kittner, Michael / Roland Köstler / Ulrich Zachert: Aufsichtsratspraxis, 5. Auflage, Köln 1995

Klahold, Christoph: Aktienoptionen als Vergütungselement, Frankfurt am Main u.a. 1999

Knoll, Leonhard: Entlohnung deutscher Top-Manager, in: Der Betriebswirt, 38 (1997), S. 22-25

Knoll, Leonhard / Hans Peter Möller: Die Entscheidung der Aktionäre über Aktien-Optionspläne: Notwendigkeit der Vermittlung relevanter Informationen, in: Zeitschrift für Bankrecht und Bankwirtschaft, 11 (1999), S. 69-72

Knoll, Leonhard / Jochen Knoesel / Uwe Probst: Aufsichtsratsvergütungen in Deutschland: Empirische Befunde, in: ZfbF, 49 (1997), S. 236-254

Koch, Wolfgang: Das Unternehmensinteresse als Verhaltensmaßstab der Aufsichtsratsmitglieder im mitbestimmten Aufsichtsrat einer Aktiengesellschaft, Frankfurt am Main, Bern, New York 1983 
Koenen, Jens / Gregory, Lipinski,: MG-Aktionär Happel will Kajo Neukirchen stürzen, in: Handelsblatt, 28.3.2001, Nr. 62, S. 20

Köhler, Horst: Die Effizienz betrieblicher Gruppenentscheidungen, Bochum 1975

Kommission Mitbestimmung: Mitbestimmung und neue Unternehmenskulturen - Bilanz und Perspektiven, herausgegeben von der Bertelsmann Stiftung und der Hans-Böckler-Stiftung, Gütersloh 1998

Kopper, Hilmar: Vorwort, in: Johannes Semler (Hrsg.): Arbeitshandbuch für Aufsichtsratsmitglieder, München 1999, S. VII-IX

Korndörfer, Wolfgang: Unternehmensführungslehre, 9. Auflage, Wiesbaden 1999

Kosiol, Erich: Organisation der Unternehmung, Wiesbaden 1962

Kosiol, Erich: Organisation der Unternehmung, 2. Auflage, Wiesbaden 1976

Kräkel, Matthias: Informationsökonomische Erklärungsansätze für Fehlbesetzungen im Topmanagement, in: WiSt, 27 (1998), S. 580-584

Kräkel, Matthias: Organisation und Management, Tübingen 1999

Krallmann, Hermann / Bodo Rieger: Vom Decision Support System (DSS) zum Executive Support System (ESS), in: Handwörterbuch der modernen Datenverarbeitung, 24 (1987), Heft 138, S. 28-38

Kramarsch, Michael H.: Aktienbasierte Managementvergütung, Stuttgart 2000

Krieger, Gerd: Aktionärsklage zur Kontrolle des Vorstands- und Aufsichtsratshandelns, in: ZHR, Bd. 163 (1999), S. 343-363

Kromschröder, Bernhard / Wolfgang Lück für den Arbeitskreis „Externe und interne Überwachung" der Schmalenbach-Gesellschaft für Betriebswirtschaft e.V.: Grundsätze risikoorientierter Unternehmensüberwachung, in: DB, 51 (1998), S. 1573-1576

Kropff, Bruno: Aktiengesetz, Düsseldorf 1965

Kropff, Bruno: Mitwirkung des Aufsichtsrats bei einzelnen Maßnahmen der Geschäftsführung, in: Johannes Semler (Hrsg.): Arbeitshandbuch für Aufsichtsratsmitglieder, München 1999, S. 351-436, 785-787

Krüger, Burkhard: D\&O - echte Absicherung oder nur ein Modetrend?, in: eco, Heft 3, 1 (1999), S. 54-56

Krüger, Wilfried: Organisation der Unternehmung, 3. Auflage, Stuttgart, Berlin, Köln 1994 
Kruse, Michael: Organisation des Entscheidungsprozesses im Aufsichtsrat der Aktiengesellschaft, Frankfurt am Main 1972

Krystek, Ulrich / Jörg Link (Hrsg.): Führungskräfte und Führungserfolg: Neue Herausforderungen für das strategische Management, Wiesbaden 1995

Kuhl, Karin / Johann-Peter Nickel: Risikomanagement im Unternehmen Stellt das KonTraG neue Anforderungen an die Unternehmen?, in: DB, 52 (1999), S. 133-135

Kuhn, Alfred: Unternehmensführung, 2. Auflage, München 1990

Kürsten, Wolfgang: „Shareholder Value“ - Grundelemente und Schieflagen einer politökonomischen Diskussion aus finanzierungstheoretischer Sicht, ZfB, 70 (2000), S. 359-381

Kurzwelly, Jens-Peter: Die Rechtsprechung des Bundesgerichtshofes zur Aktiengesellschaft in den Jahren 1998 und 1999, in: AG, 45 (2000), S. 337342

Küting, Karlheinz / Günther Langenbucher (Hrsg.): Internationale Rechnungslegung, Stuttgart 1999

Kutschker, Michael (Hrsg.): Management verteilter Kompetenzen in multinationalen Unternehmen, Wiesbaden 1999

Langenbucher, Günther / Ulf Blaum: Audit Committees - Ein Weg zur Überwindung der Überwachungskrise?, in: DB, 47 (1994), S. 2197-2206

Langner, Sabine / Michael F. Bursee: Aktienoptionsprogramme als wertorientierte Vergütungskomponente - Ergebnisse einer Befragung institutioneller Kapitalmarktteilnehmer, in: Personal, 52 (2000), S. 530-534

Laux, Helmut / Felix Liermann: Grundlagen der Organisation, 4. Auflage, Berlin u.a. 1997

Leavitt, Harold J.: Applied Organizational Change in Industry: Structural, Technological and Humanistic Approaches, in: James G. March (Hrsg.): Handbook of Organization, Chicago 1965, S. 1144-1170

Lehmann, Karl: Das Recht der Aktiengesellschaften, Bd. 1, Neudruck der Ausgabe Berlin 1898, Aalen 1964

Lehmann, Karl: Die geschichtliche Entwicklung des Aktienrechts bis zum Code de Commerce, Reprographischer Nachdruck der Ausgabe Berlin 1895, Frankfurt/Main 1968 
Lenz, Hansrudi / Markus Ostrowski: Kontrolle und Transparenz im Unternehmensbereich durch die Institution Abschlußprüfung, in: BB, 52 (1997), S. 1523-1529

Levinthal, Daniel: A Survey of Agency Models of Organizations, in: Journal of Economic Behavior and Organization, 9 (1988), S. 153-188

LG München I, Urteil vom 7.12.2000 - 5HK O 14047/00, Anforderungen an einen Beschluss über die Aufsichtsratsvergütung mit der Möglichkeit der Zeichnung von Wandelanleihen, § 113 Abs. 1 AktG, in: DB, 54 (2001), S. 193

Link, Jörg: Organisation der strategischen Unternehmungsplanung, in: Dietger Hahn / Bernard Taylor (Hrsg.): Strategische Unternehmungsplanung - Strategische Unternehmungsführung, 8. Auflage, Heidelberg 1999, S. 804-829

Lorsch, Jay W. / Rakesh Khurana: Changing Leaders: The Board's Role in CEO Succesion, in: Harvard Business Review, 77 (1999), No. 3, S. 96105

Lorson, Peter: Shareholder Value-Ansätze, in: DB, 52 (1999), S. 13291339

Lück, Wolfgang: Audit Committees - Prüfungsausschüsse zur Sicherung und Verbesserung der Unternehmensüberwachung in deutschen Unternehmen, in: DB, 52 (1999), S. 441-443

Lück, Wolfgang: Die Professionalisierung des Aufsichtsrats ist das Gebot der Stunde, in: FAZ, 23.11.1998, Nr. 272, S. 39

Lück, Wolfgang: Elemente eines Risikomanagementsystems - Die Notwendigkeit eines Risikomanagementsystems durch den Entwurf eines Gesetzes zur Kontrolle und Transparenz im Unternehmensbereich (KonTraG), in: DB, 51 (1998), S. 8-14

Lück, Wolfgang: Internes Überwachungssystem (IÜS), in: WPKMitteilungen, 37 (1998), S. 182-188

Lück, Wolfgang: Plädoyer für ein Audit Committee, in: Karlheinz Küting / Günther Langenbucher (Hrsg.): Internationale Rechnungslegung, Stuttgart 1999, S. 507-523

Lückmann, Reinhard: Schärfere Regeln für die Führung und Kontrolle von Unternehmen, in: Handelsblatt, 29./30.12.2000, Nr. 251, S. 16

Ludwig, Susanne: Leitungsgremien und Aufsichtsräte im Konzern, Wiesbaden 1997 
Luhmann, Niklas: Zweckbegriff und Systemrationalität, Tübingen 1968

Lukarsch, Michael: Marktwertorientierte Überwachung der Unternehmensplanung durch den Aufsichtsrat, Frankfurt am Main u.a. 1998

Lutter, Marcus: Unternehmensplanung und Aufsichtsrat, in: Klaus-Peter Kistner / Reinhart Schmidt (Hrsg.): Unternehmensdynamik, Wiesbaden 1991, S. 345-360

Lutter, Marcus: Aufsichtsrat, Prüfungsbefugnisse, in: Adolf G. Coenenberg / Klaus v. Wysocki (Hrsg.): Handwörterbuch der Revision, 2. Auflage, Stuttgart 1992, Sp. 95-106

Lutter, Marcus: Das neue „Gesetz für kleine Aktiengesellschaften und zur Deregulierung des Aktienrechts“, in: AG, 39 (1994), S. 429-447

Lutter, Marcus: Der Aufsichtsrat: Konstruktionsfehler, Inkompetenz seiner Mitglieder oder normales Risiko?, in: AG, 39 (1994), S. 176-177

Lutter, Marcus: Das dualistische System der Unternehmensverwaltung, in: Eberhard Scheffler (Hrsg.): Corporate Governance, SzU, Bd. 56, Wiesbaden 1995, S. 5-26

Lutter, Marcus: Defizite für eine effiziente Aufsichtsratstätigkeit und gesetzliche Möglichkeiten der Verbesserung, in: ZHR, 159 (1995), S. 287309

Lutter, Marcus: Professionalisierung der Aufsichtsräte, in: NJW, 48 (1995), S. 1133-1134

Lutter, Marcus: Aktienoptionen für Führungskräfte - de lege lata und de lege ferenda, in: ZIP, 18 (1997), S. 1-9

Lutter, Marcus: Diskussionsbeitrag zum Panel Qualitätsmessung von Aufsichtsräten im Rahmen der 62. Wissenschaftlichen Jahrestagung des Verbandes der Hochschullehrer für Betriebswirtschaft e.V. am 16. Juni 2000

Lutter, Marcus / Gerd Krieger: Rechte und Pflichten des Aufsichtsrats, 3. Auflage, Freiburg im Breisgau 1993

Lutter, Marcus / Gerd Krieger: Hilfspersonen von Aufsichtsratsmitgliedern, in: DB, 48 (1995), S. 257-260

Macharzina, Klaus: Unternehmensführung, 3. Auflage, Wiesbaden 1999

Mag, Wolfgang: Die quantitative Erfassung der Kommunikationsstruktur und ihre Bedeutung für die Gestaltung der Unternehmensorganisation, in: ZfbF, 22 (1970), S. 22-49

Mag, Wolfgang: Mehrfachziele, Zielbeziehungen und Zielkonfliktlösungen, in: WiSt, 5 (1976), S. 49-55 
Mag, Wolfgang: Die Unternehmung als Organisation, in: Jochen Drukarczyk / Lothar Müller-Hagedorn (Hrsg.): Betriebswirtschaftslehre: Eine Einführung in die Theorie der Unternehmung, Bd. 2, Wiesbaden 1978, S. 246-308

Mag, Wolfgang: Kommunikation, in: Erwin Grochla (Hrsg.): Handwörterbuch der Organisation, 2. Auflage, Stuttgart 1980, Sp. 1031-1040

Mag, Wolfgang: Grundzüge der Entscheidungstheorie, München 1990

Mag, Wolfgang: Zeitprobleme in der Unternehmungsplanung, in: WISU, 19 (1990), S. 694-699

Mag, Wolfgang: Ausschüsse, in: Erich Frese (Hrsg.): Handwörterbuch der Organisation, 3. Auflage, Stuttgart 1992, Sp. 252-262

Mag, Wolfgang: Die Funktionserweiterung der Unternehmensführung, in: WiSt, 21 (1992), S. 60-64

Mag, Wolfgang: Unternehmungsplanung, München 1995

Mag, Wolfgang: Personalplanung, Kontrolle und Mitbestimmung, Bochum 1996

Mag, Wolfgang: Einführung in die betriebliche Personalplanung, 2. Auflage, München 1998

Mag, Wolfgang: Planung und Kontrolle, in: Michael Bitz u.a. (Hrsg.): Vahlens Kompendium der Betriebswirtschaftslehre, Bd. 2, 4. Auflage, München 1999, S. 1-66

Mag, Wolfgang: Zur Verbindung von Organisations- und Personalplanung, in: Gerd Rainer Wagner (Hrsg.): Unternehmungsführung, Ethik und Umwelt, Wiesbaden 1999, S. 375-401

Mag, Wolfgang / Hilmar Henselek / Peter Ruhwedel: Betriebliche Personalplanung, Beitrag Nr. 27 des Lehrstuhls für Theoretische Betriebswirtschaftslehre (Planung und Organisation), Ruhr-Universität Bochum, Bochum 2000

Mäger, Stefan: Vergütung des Aufsichtsrats - welchen Spielraum gibt das Aktienrecht?, in: BB, 54 (1999), S. 1389-1394

Malik, Fredmund: Wirksame Unternehmensaufsicht, Frankfurt am Main 1997

Malik, Fredmund: Immer wieder Kritik an den Aufsichtsorganen, in: Gablers Magazin, 12 (1998), S. 26-28

Manne, Henry G.: Mergers and the Market for Corporate Control, in: Journal of Political Economy, 73 (1965), S. 110-120 
March, James G. (Hrsg.): Handbook of Organization, Chicago 1965

March, James G. / Herbert A. Simon: Organizations, New York 1958

Marten, Kai-Uwe: Empirische Analyse des Prüferwechsels im Kontext der Agency- und Signalling-Theorie, in: ZfB, 65 (1995), S. 703-727

Martens, Klaus-Peter: Der Aufsichtsrat im Konzern, in: ZHR, 159 (1995), S. 567-592

Martens, Knuth: Managementüberwachung durch den Aufsichtsrat: Ein Beitrag zur Corporate Governance-Diskussion aus agencytheoretischer Sicht, Köln 2000

Martin, Thomas A.: Der Aufsichtsrat als Träger des strategischen Controlling?, in: controller magazin, 15 (1990), S. 21-25

Matschke, Manfred Jürgen / Thomas Schildbach (Hrsg.): Unternehmensberatung und Wirtschaftsprüfung, Stuttgart 1998

Matthes, Jürgen: Das deutsche Corporate-Governance-System, Köln 2000

McKinsey \& Company, Investor Opinion June 2000, in: http://www.mckinsey.com/features/investor_opinion/investor_opinion.pdf (30.12.2000)

Meffert, Heribert / Klaus Backhaus (Hrsg.): Stock Options und Shareholder Value, Dokumentation des 33. Münsteraner Führungsgesprächs vom 9./10. Oktober 1997, Dokumentationspapier Nr. 116, Wissenschaftliche Gesellschaft für Marketing und Unternehmensführung e.V., Münster 1998

Meier, Marco / Peter Mertens: Integration von Internetdaten in Führungsinformationssysteme, in: WISU, 29 (2000), S. 353-360

Meinhövel, Harald: Defizite der Principal-Agent-Theorie, Lohmar, Köln 1999

Menichetti, Marco: Aktien-Optionsprogramme für das Top-Management, in: DB, 49 (1996), S. 1688-1692

Mertens, Hans-Joachim: Buchbesprechung zu: Manuel René Theisen: Grundsätze einer ordnungsgemäßen Informationsversorgung des Aufsichtsrats, Stuttgart 1991, in: AG, 37 (1992), S. 208

Mertens, Hans-Joachim: Kommentierungen zum Aktiengesetz, in: Wolfgang Zöllner (Hrsg.): Kölner Kommentar zum Aktiengesetz, 2. Auflage, Bd. 2, Köln u.a. 1996

Meyer-Landrut, Joachim: Großkommentar zum Aktiengesetz, 3. Auflage, Berlin u.a. 1970

Meyer-Lohmann, Jochen: Der Aufsichtsrat der deutschen Aktiengesellschaft und seine neue Rolle im Prozess der Unternehmensführung, Dissertation 
der Universität St. Gallen, Hochschule für Wirtschafts-, Rechts- und Sozialwissenschaften, 1997

mg technologies AG: Tagesordnung zur Hauptversammlung 2001, in: http://www.mg-ag.de/imperia/md/content/aktionaers_ info/28.pdf (28. März 2001)

Mikus, Barbara: Zur Integration des Risikomanagements in den Führungsprozeß, in: ZfP, 10 (1999), S. 85-110

Möller, Hanspeter / Franz Schmidt (Hrsg.): Rechnungswesen als Instrument für Führungsentscheidungen, Stuttgart 1998

Möllers, Thomas M. J.: Professionalisierung des Aufsichtsrats, in: ZIP, 16 (1995), S. 1725-1735

Möllers, Thomas M. J.: Kapitalmarkttauglichkeit des deutschen Gesellschaftsrechts, in: AG, 44 (1999), S. 433-442

Möllmann, Sybilla: Executive Information System: Navigationsinstrumente zur Unternehmensführung, in: zfo, 61 (1992), S. 366-367

Monks, Robert A. G. / Nell Minow: Watching the Watchers: Corporate Governance for the $21^{\text {st }}$ Century, Cambridge 1996

Monopolkommission: Ordnungspolitische Leitlinien für ein funktionsfähiges Finanzsystem, Baden-Baden 1998

Mucksch, Harry / Wolfgang Behme (Hrsg.): Das Data Warehouse-Konzept, 3. Auflage, Wiesbaden 1998

Mülbert, Peter O.: Shareholder Value aus rechtlicher Sicht, in: ZGR, 26 (1997), S. 129-172

Müller, Jochen: Datenbeschaffung für das Data Warehouse, in: Peter Chamoni / Peter Gluchowski: Analytische Informationssysteme, 2. Auflage, Berlin u.a. 1999, S. 95-117

Müller, Jochen: Transformation operativer Daten zum Nutzung im Data Warehouse, Wiesbaden 2000

Müller, Jochen / Carsten Schöne: Modellierung und Implementierung einer Balanced-Scorecard-Anwendung mit einem mehrdimensionalen Datenbanksystem, Arbeitsbericht des Lehrstuhls für Wirtschaftsinformatik, Ruhr-Universität Bochum, 2. Auflage, Bochum 2001

Mutter, Stefan: Unternehmerische Entscheidungen und Haftung des Aufsichtsrats der Aktiengesellschaft, Köln 1994

Nagel, Bernhard: Deutsches und europäisches Gesellschaftsrecht, München 2000 
Nassauer, Frank: Corporate Governance und die Internationalisierung von Unternehmungen, Frankfurt am Main u.a. 2000

Nemitz, Kurt / Richard Becker (Hrsg.): Mitbestimmung und Wirtschaftspolitik, Köln 1967

Neus, Werner: Ökonomische Agency-Theorie und Kapitalmarktgleichgewicht, Wiesbaden 1989

Neus, Werner: Einführung in die Betriebswirtschaftslehre aus institutionenökonomischer Sicht, Tübingen 1998

Niedenhoff, Horst-Udo: Mitbestimmung in der Bundesrepublik Deutschland, 12. Auflage, Köln 2000

Niehus, Rudolf: Reform des Audit Committees gemäß den USBörsenbestimmungen, in: DB, 52 (1999), S. 1765-1769

Nölting, Andreas: Druck von draußen, in: Managermagazin, 90 (2000), S. 132-137

North, Douglas C.: Institutions, Institutional Change and Economic Performance, Cambridge 1990

o.V.: Die Zeit der unbeweglichen Aufsichtsräte ist vorbei, in: Handelsblatt, 31.10./1.11.1997, Nr. 210, S. 18

o.V.: Ende einer Ära, in: Managermagazin, 28 (1998), Nr. 10, S. 14-17

o.V.: Wirtschaft setzt auf einen Verhaltenskodex, in: FAZ, 20.1.2001, Nr. 17 , S. 19

o.V.: „Die deutsche Unternehmensverfassung ist ein Standortnachteil“", in: FAZ, 12.2.2001, Nr. 36, S. 23

o.V.: Menschen und Märkte - Karel van Miert soll Friedhelm Gieske im RWE-Aufsichtsrat ablösen, in: http://www.welt.de/ daten/2001/04/02/0402wi244738.htx (2.4.2001)

o.V.: Geldanlage: Der Aufmüpfigen Zähmung, in: http://www.ftd.de/ bm/ga/FTDJZ59E7LC.html (7.4.2001)

OECD: OECD Principles of Corporate Governance, in: http://www. oecd.org/daf/governance/principles.pdf (6.2.2001), 1999, S. 1-25

Oesterle, Michael-Jörg: Führungswechsel im Top-Management, Wiesbaden 1999

Oltmanns, Michael: Stock Options für Aufsichtsräte?, in: Ann-Kristin Achleitner / Peter Wollmert (Hrsg.): Stock Options, Stuttgart 2000, S. 213231 
Ott, Claus / Hans-Bernd Schäfer (Hrsg.): Ökonomische Analyse des Unternehmensrechts, Heidelberg 1993

Pape, Ulrich: Theoretische Grundlagen und praktische Umsetzung wertorientierter Unternehmensführung, in: BB, 55 (2000), S. 711-717

Parkin, Michael / A.R. Nobay: Current Economic Problems, London, 1975

Passow, Richard: Die Entstehung des Aufsichtsrats der Aktiengesellschaft, in: ZHR, Bd. 64 (1909), S. 27-57

Peemöller, Volker H.: Der Aufsichtsrat in der Krise, in: Karl Albrecht Schachschneider (Hrsg.): Wirtschaft, Gesellschaft und Staat im Umbruch, Berlin 1995, S. 183-201

Pellens, Bernhard (Hrsg.): Unternehmenswertorientierte Entlohnungssysteme, Stuttgart 1998

Pellens, Bernhard: Corporate Governance - Ein Schlagwort oder bewegt sich tatsächlich etwas?, in: DBW, 61 (2001), S. 1-4

Pellens, Bernhard / Franca Hillebrandt: Vorzugsaktien vor dem Hintergrund der Corporate Governance-Diskussion, in: AG, 46 (2001), S. 57-67

Pellens, Bernhard / Nils Crasselt: Bilanzierung virtueller Optionsprogramme (Stock Appreciation Rights) aus finanzwirtschaftlicher Sicht, in: AnnKristin Achleitner / Peter Wollmert (Hrsg.): Stock Options, Stuttgart 2000, S. 153-171

Pellens, Bernhard / Nils Crasselt / Carsten Rockholtz: Wertorientierte Entlohnungssysteme für Führungskräfte - Anforderungen und empirische Evidenz, in: Bernhard Pellens (Hrsg.): Unternehmenswertorientierte Entlohnungssysteme, Stuttgart 1998, S. 1-28

Pellens, Bernhard / Franca Hillebrandt / Claude Tomaszewski: Value Reporting: Eine empirische Analyse der DAX-Unternehmen, in: Alfred Wagenhofer / Gerhard Hrebicek (Hrsg.): Wertorientiertes Management, Stuttgart 2000, S. 177-207

Pellens, Bernhard / Franca Hillebrandt / Björn Ulmer: Umsetzung von Corporate Governance-Richtlinien in der Praxis, in: BB, 56 (2001), S. 12431250

Pellens, Bernhard / Carsten Rockholtz / Marc Stienemann: Marktwertorientiertes Konzerncontrolling in Deutschland, in: DB, 50 (1997), S. 19331939

Peltzer, Martin: Die Vergütung des Aufsichtsrats im Gesellschafts- und Steuerrecht, in: Carsten P. Claussen / Oswald Hahn / Willy Kraus (Hrsg.): 
Umbruch und Wandel: Herausforderungen zur Jahrhundertwende, München, Wien 1997

Peltzer, Martin / Axel von Werder: Der „German Code of Corporate Governance (GCCG)“ des Berliner Initiativkreises, in: AG, 46 (2001), S. 115

Perlitz, Manfred: Frühwarnsysteme, in: Klaus Chmielewicz / Marcell Schweitzer (Hrsg.): Handwörterbuch des Rechnungswesens, 3. Auflage, Stuttgart 1993, Sp. 679-688

Petersen, Thomas: Optimale Anreizsysteme, Wiesbaden 1989

Peus, Egon A.: Der Aufsichtsratsvorsitzende - seine Rechtsstellung nach dem Aktiengesetz und dem Mitbestimmungsgesetz 1976, Köln 1983

Pfaff, Dieter / Oliver Bärtl: Wertorientierte Unternehmenssteuerung - Ein kritischer Vergleich ausgewählter Konzepte, in: Günther Gebhardt / Bernhard Pellens (Hrsg.): Rechnungswesen und Kapitalmarkt, Düsseldorf, Frankfurt am Main 1999 (ZfbF Sonderheft 41), S. 85-115

Picot, Arnold (Hrsg.): Corporate Governance, Stuttgart 1995

Picot, Arnold: Verfügungsrechtstheorie, Transaktionskosten und Führung, in: Alfred Kieser / Gerhard Reber / Rolf Wunderer (Hrsg.): Handwörterbuch der Führung, 2. Auflage, Stuttgart 1995, Sp. 2106-2113

Picot, Arnold / Egon Franck: Die Planung der Unternehmensressource Information (I), in: WISU, 17 (1988), S. 544-549

Picot, Arnold / Egon Franck: Die Planung der Unternehmensressource Information (II), in: WISU, 17 (1988), S. 608-614

Picot, Arnold / Matthias Maier: Informationssysteme, computergestützte, in: Erich Frese (Hrsg.): Handwörterbuch der Organisation, 3. Auflage, Stuttgart 1992, Sp. 923-936

Picot, Arnold / Rahild Neuburger: Agency Theorie und Führung, in: Alfred Kieser, / Gerhard Reber / Rolf Wunderer (Hrsg.): Handwörterbuch der Führung, 2. Auflage, Stuttgart 1995, Sp. 14-21

Picot, Arnold / Helmut Dietl / Egon Franck: Organisation: eine ökonomische Perspektive, Stuttgart 1997

Picot, Arnold / Ralf Reichwald / Rolf T. Wigand: Die grenzenlose Unternehmung, 4. Auflage, Wiesbaden 2001

Pigout, Arthur Cecil: The economics of welfare, 4. Auflage, London 1932 (Nachdruck London 1962) 
Portisch, Wolfgang: Überwachung und Berichterstattung des Aufsichtsrats im Stakeholder-Agency-Modell, Frankfurt am Main u.a. 1997

Posner, Richard A.: Economic Analysis of Law, Fourth Edition, Boston, Toronto, London 1992

Posner, Richard A.: Economic Analysis of Law, Fifth Edition, New York 1998

Potthoff, Erich: Prüfung und Überwachung der Geschäftsführung, in: ZfhF, 13 (1961), S. 563-580

Potthoff, Erich: Ein Kodex für den Aufsichtsrat!, in: DB, 48 (1995), S. 163164

Potthoff, Erich: Board-System versus duales System der Unternehmensverwaltung - Vor - und Nachteile, in: BFuP, 48 (1996), S. 253-268

Potthoff, Erich: Risikomanagement ein Gegenstand der Abschlußprüfung?, in: DB, 47 (1997), S. I

Potthoff, Erich: Aufsichtsrat in der Aktiengesellschaft als betriebswirtschaftliches Subsystem, in: Manfred Jürgen Matschke / Thomas Schildbach (Hrsg.): Unternehmensberatung und Wirtschaftsprüfung, Stuttgart 1998, S. $127-142$

Potthoff, Erich: Der Ruck im Aufsichtsrat, in: DB, 51 (1998), S. I

Potthoff, Erich: Shareholder Value auf dem Prüfstand, in: DB, 48 (1998), S. I

Potthoff, Erich: Wandlungen der Aufsichtsratstätigkeit im Wandel der Weltwirtschaft, in: Horst Glaser / Ernst F. Schröder / Axel v. Werder (Hrsg.): Organisation im Wandel der Märkte, Wiesbaden 1998, S. 317342

Potthoff, Erich / Karl Trescher: Das Aufsichtsratsmitglied, 4. Auflage, Stuttgart 1999

Potthoff, Volker: Redeentwurf zur Verleihung der Déminor Corporate Governance Awards am 12.12.2000, S. 1-4

Pratt, John W. / Richard J. Zeckhauser (Hrsg.): Principal and Agents: The Structure of Business, Boston 1985

Quick, Reiner: Zivilrechtliche Verantwortlichkeit europäischer und amerikanischer Abschlußprüfer, in: BFuP, 52 (2000),S. 525-548

Raiser, Thomas: Recht der Kapitalgesellschaften, 2. Auflage, München 1992 
Rappaport, Alfred: Creating shareholder value, 10. Printing, New York 1986

Rathenau, Walther: Vom Aktienwesen, Berlin 1918

Rechenberg, Wolf-Georg Freiherr von: Zustimmungsvorbehalte des Aufsichtsrats für die Unternehmensplanung, in: BB, 45 (1990), S. 13561363

Redel, Wolfgang: Führungsgremien, in: Alfred Kieser / Gerhard Reber / Rolf Wunderer (Hrsg.): Handwörterbuch der Führung, 2. Auflage, Stuttgart 1995, Sp. 706-720

Regierungskommission „Corporate Governance“: Unternehmensführung Unternehmenskontrolle - Modernisierung des Aktienrechts, in: http://www.ovs.de/corporate_governance.htm (10.7.2001)

Reif-Mosel, Ane-Kristin: Computergestützte Kooperation im Büro, Frankfurt am Main u.a. 2000

Reischauer, Claudia: Über die Eitelkeit, in: Wirtschaftswoche, 53 (1999), Nr. 6, S. 76-78

Reiß, Michael / Hans Corsten: Schnittstellenfokussierte Unternehmungsführung, in: Hans Corsten / Michael Reiß (Hrsg.): Handbuch Unternehmungsführung, Wiesbaden 1995, S. 5-18

Rellermeyer, Klaus: Aufsichtsratsausschüsse, Köln u.a. 1986

Richter, Frank: Konzeption eines marktwertorientierten Steuerungs- und Monitoringsystems, Frankfurt am Main u.a. 1996

Richter, Rudolf / Eirik Furubotn: Neue Institutionenökonomik - Eine Einführung und kritische Würdigung, Tübingen 1996

Ridder-Aab, Christa-Maria: Die moderne Aktiengesellschaft im Lichte der Theorie der Eigentumsrechte, Frankfurt am Main, New York 1980

Riedel, Donata: Sommer ordnet Telekom-Vorstand neu, in: Handelsblatt, 12.4.2001, Nr. 73, S. 73

Riegler, Christian: Anreizsysteme und wertorientiertes Management, in: Alfred Wagenhofer / Gerhard Hrebicek (Hrsg.): Wertorientiertes Management, Stuttgart 2000, S. 145-176

Romano, Roberta: Foundations of Corporate Law, New York, Oxford 1993

Romano, Roberta: Less is More: Making Shareholder Activism a Valuable Mechanism of Corporate Governance, Yale Law School and National Bureau of Economic Research, Draft: May 14, 2000 
Römer, Gerhard: Aufsichtsrat und Controlling, in: controller magazin, 15 (1990), S. 126-130

Rosen, Rüdiger von: Aktienorientierte Vergütungssysteme, Vortrag auf dem Kapitalmarkt-Kolloquium der Ludwig-Maximilians-Universtität München, o. J.

Ross, Stephen A.: The Economic Theory of Agency: The Principal's Problem, in: American Economic Review, Papers and Proceedings, 63 (1973), S. 134-139

Rother, Franz / Andreas Wildhagen / Manfred Fischer: Schweres Versagen, in: Wirtschaftswoche, 52 (1998), Nr. 33, S. 44-45

Rürup, Lebrecht: Möglichkeiten verbesserter Kontrolle und Beratung der Geschäftsführung durch den Aufsichtsrat mit Hilfe des Wirtschaftsprüfers, in: AG, 40 (1995), S. 219-223

Rust, Walter / Udo Ulbert: Die kleine Aktiengesellschaft, Freiburg im Breisgau 1995

Salzberger, Wolfgang: Die Überwachung des Risikomanagements durch den Aufsichtsrat, in: DBW, 60 (2000), S. 756-773

SAP AG, SAP ${ }^{\circledR}$ Strategic Enterprise Management ${ }^{\mathrm{TM}}, 2000$

Sarrazin, Jürgen: Die besonderen Aufgaben des Aufsichtsratsvorsitzenden, in: Eberhard Scheffler (Hrsg.): Corporate Governance, SzU, Bd. 56, Wiesbaden 1995, S. 125-146

Schachschneider, Karl Albrecht (Hrsg.): Wirtschaft, Gesellschaft und Staat im Umbruch, Berlin 1995

Scharfenkamp, Norbert: Organisatorische Gestaltung und wirtschaftlicher Erfolg, Berlin, New York 1987

Schätzle, Rainer J.: Unternehmenswert langfristig steigern, in: Gablers Magazin, 12 (1998), S. 50-53

Scheffler, Eberhard: Der Aufsichtsrat - nützlich oder überflüssig?, in: ZGR, 22 (1993), S. 63-76

Scheffler, Eberhard: Die Überwachungsaufgabe des Aufsichtsrats im Konzern, in: DB, 47 (1994), S. 793-799

Scheffler, Eberhard: Betriebswirtschaftliche Überlegungen zur Entwicklung von Grundsätzen ordnungsmäßiger Überwachung der Geschäftsführung durch den Aufsichtsrat, in: AG, 40 (1995), S. 207-212

Scheffler, Eberhard (Hrsg.): Corporate Governance, SzU, Bd. 56, Wiesbaden 1995 
Scheffler, Eberhard: Zum Rollenverständnis der Aufsichtsräte, in: DB, 53 (2000), S. 433-437

Scherer, Andreas Georg: Können die „Grundsätze ordnungsmäßiger Unternehmungsleitung (GoU)“ zu einer Verbesserung der Managementpraxis beitragen? - Kritische Bemerkungen zu den Vorschlägen von Axel von Werder, in: BFuP, 52 (2000), S. 84-98

Schering AG, Geschäftsbericht 1999

Schewe, Gerhard / Jörn Littkemann / Per Olof Beckermeier: Interne Kontrollsysteme - Verhaltenswirkungen und organisatorische Gestaltung, in: WISU, 28 (1999), S. 1483-1488

Schiessl, Maximilian: Ist das deutsche Aktienrecht kapitalmarkttauglich?, in: AG, 44 (1999), S. 442-452

Schildbach, Thomas: Entscheidung, in: Michael Bitz u.a.: Vahlens Kompendium der Betriebswirtschaftslehre, Bd. 2, 4. Auflage, München 1999, S. $65-105$

Schilling, Florian: Der internationale Druck auf die deutschen Aufsichtsräte verstärkt sich, in: FAZ, 10.5.1999, Nr. 107, S. 35

Schmalenbach, Eugen: Die Überwachungspflicht des Aufsichtsrats, in: ZfhF, 5 (1910/11), S. 271-283

Schmid, Frank A.: Vorstandsbezüge, Aufsichtsratsvergütung und Aktionärsstruktur, in: ZfB, 67 (1997), S. 67-83

Schmid, Frank A. / Frank Seger: Arbeitnehmermitbestimmung, Allokation von Entscheidungsrechten und Shareholder Value, in: ZfB, 68 (1998), S. 453-473

Schmid, Uwe: Das Anspruchsgruppen-Konzept, in: WISU, 26 (1997), S. 633-635

Schmidt, Hartmut u.a.: Corporate Governance in Germany, Baden-Baden 1997

Schmidt, Karsten: Gesellschaftsrecht, 3. Auflage, Köln u.a. 1997

Schmidt, Monika: „Stock Options“ aus rechtlicher Sicht, in: Personal, 50 (1998), S. 76-79

Schmidt, Ralf-Bodo: Wirtschaftslehre der Unternehmung, 2. Auflage, Stuttgart 1977

Schmidt, Reinhard H.: Agency Costs are not a „Flop“!, in Günter Bamberg / Klaus Spremann (Hrsg.): Agency theory, Information, and Incentives, Berlin u.a. 1987, S. 495-509 
Schmidt, Reinhard H.: Corporate Governance: The Role of Other Constituencies, Paper Presented at the Conference on Workable Corporate Governance: Cross-Border Perspectives, held in Paris, March 17-19, 1997

Schmidt, Reinhard / Eva Terberger: Grundzüge der Investitions- und Finanzierungspolitik, 4. Auflage, Wiesbaden 1997

Schmidt, Reinhard H. / Gerald Spindler: Shareholder Value zwischen Ökonomie und Recht, in: Heinz-Dieter Assmann (Hrsg.): Wirtschafts- und Medienrecht in der offenen Demokratie, Heidelberg 1997, S. 514-555

Schmidt, Reinhart: Das Shareholder Value-Konzept, in: Ulrich Fritsch / Gerhard Liener / Reinhart Schmidt (Hrsg.): Die deutsche Aktie, Stuttgart 1993, S. 277-296

Schneider, Dieter: Agency Costs and Transaction Costs: Flops in the Principal-Agent-Theory of Financial Markets, in: Günter Bamberg / Klaus Spremann (Hrsg.): Agency theory, Information, and Incentives, Berlin u.a. 1987 , S. $481-494$

Schneider, Dieter: Betriebswirtschaftslehre, Bd. 1: Grundlagen, 2. Auflage, München, Wien 1995

Schneider, Dieter: Informations- und Entscheidungstheorie, München, Wien 1995

Schneider, Dieter: Betriebswirtschaftslehre, Bd. 3: Theorie der Unternehmung, München, Wien 1997

Schneider, Uwe H.: Auf dem Weg zum Pensionskassenkorporatismus?, in: AG, 35 (1990), S. 317-326

Schneider, Uwe H.: Kapitalmarktorientierte Corporate GovernanceGrundsätze, in: DB, 53 (2000), S. 2413-2417

Schneider, Uwe H. / Christian Strenger: Die „Corporate GovernanceGrundsätze“ der Grundsatzkommission Corporate Governance (German Panel on Corporate Governance), in: AG, 45 (2000), S. 106-113

Schoemaker, Paul J. / J. Edward Russo: Managers Last: Die richtige Entscheidung, in: Harvard Business Manager, 16 (1994), S. 94-106

Scholz, Christian / Joachim Zentes (Hrsg.): Strategisches Euro-Management, Bd. 2, Stuttgart 1998

Scholz, Wolfgang: Sanktionen gegen Mitglieder des Aufsichtsrats, in: Johannes Semler (Hrsg.): Arbeitshandbuch für Aufsichtsratsmitglieder, München 1999, S. 697-720 
Schreyögg, Georg: Zum Verhältnis von Planung und Kontrolle, in: WiSt, 23 (1994), S. 345-351

Schreyögg, Georg: Organisation, 3. Auflage, Wiesbaden 1999

Schreyögg, Georg / Horst Steinmann: Zur Trennung von Eigentum und Verfügungsgewalt, in: $\mathrm{ZfB}, 51$ (1981), S. 533-558

Schreyögg, Georg / Horst Steinmann: Strategic Control. A new perspective, in: Academy of Management Review, 12 (1987), S. 91-103

Schröder, Christoph: Industrielle Arbeitskosten im internationalen Vergleich, in: iw-trends, Heft 3, 27 (2000), S. 92-108

Schröder, Christoph: Produktivität und Lohnstückkosten im internationalen Vergleich, in: iw-trends, Heft 3, 27 (2000), S. 77-91

Schröder, Ernst F.: Modernes Unternehmens-Controlling, Ludwigshafen (Rhein) 2000

Schröder, Ulrich / Alexander Schrader: The changing role of banks and corporate governance in Germany: evolution towards the market?, in: Stanley W. Black / Mathias Moersch (Hrsg.): Competition and Convergence in Financial Markets, Amsterdam u.a. 1998, S. 17-34

Schulte-Florian, Gabriele: Determinanten der Karriere, München, Mering 1999

Schulte-Zurhausen, Manfred: Organisation, 2. Auflage, München 1999

Schwalbach, Joachim: Der Zusammenhang von Kompensation und Performance im internationalen Vergleich, in: Personal, 51 (1999), S. 114-118

Schwalbach, Joachim / Ulrike Graßhoff: Managervergütung und Unternehmenserfolg, in: ZfB, 67 (1997), S. 203-217

Seibert, Ulrich: Aufsichtsrats-Reform in der 13. Wahlperiode, in: Zeitschrift für Bankrecht und Bankwirtschaft, 6 (1994), S. 349-353

Seibert, Ulrich: Stock Options für Führungskräfte - zur Regelung im Kontrolle- und Transparenzgesetz (KonTraG), in: Bernhard Pellens (Hrsg.): Unternehmenswertorientierte Entlohnungssysteme, Stuttgart 1998, S. 2952

Seibert, Ulrich: OECD Principles of Corporate Governance - Grundsätze der Unternehmensführung und -kontrolle für die Welt, in : AG, 44 (1999), S. 337-350

Seidel, Eberhard: Gremienorganisation, in: Erich Frese (Hrsg.): Handwörterbuch der Organisation, 3. Auflage, Stuttgart 1992, Sp. 714-724 
Semler, Johannes: Leitung und Überwachung der Aktiengesellschaft, 2. Auflage, Köln u.a. 1996

Semler, Johannes (Hrsg.): Arbeitshandbuch für Aufsichtsratsmitglieder, München 1999

Semler, Johannes: Die Kompetenzen des Aufsichtsrats, in: Johannes Semler (Hrsg.): Arbeitshandbuch für Aufsichtsratsmitglieder, München 1999, S. $1-66$

Semler, Johannes: Vorschlags- und Wahlverfahren, Entsenden und Ausscheiden, in: Johannes Semler (Hrsg.): Arbeitshandbuch für Aufsichtsratsmitglieder, München 1999, S. 67-88

Servatius, Bernhard: Ordnungsgemäße Vorstandskontrolle und vorbereitende Personalauswahl durch den Aufsichtsratsvorsitzenden, in: AG, 40 (1995), S. 223-225

Shavell, Steven: Risk sharing and incentives in the principal and agent relationship, in: Bell Journal of Economics, 10 (1979), S. 55-73

Shleifer, Andrei / Robert W. Vishny: A Survey of Corporate Governance, in: The Journal of Finance, 52, (1997), S. 737-783

Siebel, Ulf: Arbeit von Ausschüssen, in: Johannes Semler (Hrsg.): Arbeitshandbuch für Aufsichtsratsmitglieder, München 1999, S. 287-349

Siebel, Ulf: Selbstorganisation und Konstituierung des ersten Aufsichtsrats, in: Johannes Semler (Hrsg.): Arbeitshandbuch für Aufsichtsratsmitglieder, München 1999, S. 89-117, S. 772-780

Siegert, Theo: Shareholder-Value als Lenkungsinstrument, in: ZfbF, 47 (1995), S. 580-607

Siemens AG: Einladung zur Hauptversammlung der Siemens AG am 18. Februar 1999, in: http://www.siemens.de/de2/html/investors/documents/ inv1999_d.pdf (6. Oktober 2000)

Sonnenschein, Olfa / Dietmar Schön / Dirk Nölken: Moderne Informationsund Kommunikationstechnologien für die strategische Planung, in: Controlling, 11 (1999), S. 185-192

Speckbacher, Gerhard: Das Shareholder Value-Konzept im Lichte der Corporate Governance-Debatte, in: WiSt, 27 (1998), S. 95-97

Spindler, Gerald: Internet und Corporate Governance - ein neuer virtueller (T)Raum?, in: ZGR, 29 (2000), S. 420-445

Spremann, Klaus: Asymmetrische Information, in: ZfB, 60 (1990), S. 561586 
Staehle, Wolfgang: Management, 8. Auflage, 1999

Steiner, Peter / Helmut Uhlir: Wertpapieranalyse, 4. Auflage, Stuttgart 2000

Steinitzer, Erwin: Ökonomische Theorie der Aktiengesellschaft, Leipzig 1908

Steinmann, Horst / Elmar Gerum: Die Unternehmung als Koalition, in: WiSt, 7 (1978), S. 469-476

Steinmann, Horst / Georg Schreyögg: Management, 5. Auflage, Wiesbaden 2000

Stiglitz, Joseph E.: Information and Economic Analysis, in: Michael Parkin / A.R. Nobay: Current Economic Problems, London, 1975, S. 27-52

Streim, Hannes: Meinungsspiegel: Haftung des Wirtschaftsprüfers im Lichte nationaler und internationaler Entwicklungen, in: BFuP, 52 (2000), S. 607-619

Streubel, Frauke: Organisatorische Gestaltung und Informationsmanagement in der lernenden Unternehmung, Frankfurt am Main u.a. 2000

Sünner, Eckart: Effizienz von Unternehmensorganen als Grundsatz der Corporate Governance, in: AG, 45 (2000), S. 492-498

Szyperski, Norbert / Udo Winand: Grundbegriffe der Unternehmungsplanung, Stuttgart 1980

Szyperski, Norbert / Udo Winand (Hrsg.): Handwörterbuch der Planung, Stuttgart 1989

Theisen, Manuel René: Überwachung der Unternehmungsführung, Stuttgart 1987

Theisen, Manuel René: Überwachung der Geschäftsführung, in: Waldemar Wittmann (Hrsg.): Handwörterbuch der Betriebswirtschaft, 5. Auflage, Stuttgart 1993, Sp. 4219-4231

Theisen, Manuel René: Grundsätze ordnungsmäßiger Kontrolle und Beratung der Geschäftsführung durch den Aufsichtsrat, in: AG, 40 (1995), S. 193-203

Theisen, Manuel René: Zwischenbilanz zur Diskussion über das Verhältnis zwischen Aufsichtsrat und Abschlußprüfer, in: WPK-Mitteilungen, 34 (1995), S. 185-195

Theisen, Manuel René: Grundsätze einer ordnungsmäßigen Information des Aufsichtsrats, 2. Auflage, Stuttgart 1996

Theisen, Manuel René: Grundsätze ordnungsmäßiger Überwachung (GoÜ) - Problem, Systematik und erste inhaltliche Vorschläge, in: Axel von 
Werder (Hrsg.): Grundsätze ordnungsmäßiger Unternehmungsführung (GoF) für die Unternehmungsleitung (GoU), Überwachung (GoÜ) und Abschlußprüfung (GoA), Düsseldorf, Frankfurt am Main 1996, S. 75-106

Theisen, Manuel René: Die Berichterstattung des Aufsichtsrats, in: DB, 50 (1997), S. 105-115

Theisen, Manuel René: Aufsichtsrats-Informationssysteme - Ein Instrument zur Verbesserung der Überwachungsleistung?, in: Hanspeter Möller / Franz Schmidt (Hrsg.): Rechnungswesen als Instrument für Führungsentscheidungen, Stuttgart 1998, S. 75-90

Theisen, Manuel René: Aufsichtsratsprobleme globaler Konzernunternehmungen, in: Horst Glaser / Ernst F. Schröder / Axel v. Werder (Hrsg.): Organisation im Wandel der Märkte, Wiesbaden 1998, S. 441-460

Theisen, Manuel René: Vergabe und Konkretisierung des WPPrüfungsauftrags durch den Aufsichtsrat, in: DB, 52 (1999), S. 341-346

Theisen, Manuel René: Zur Reform des Aufsichtsrats - Eine betriebswirtschaftliche Bestandsanalyse und Perspektive, in: Dietrich Dörner / Dieter Menold / Norbert Pfitzer (Hrsg.): Reform des Aktienrechts, Rechnungslegung und Prüfung, Stuttgart 1999, S. 203-251

Theisen, Manuel René: Zusammensetzung und Struktur der Vergütungen für den Aufsichtsrat nach dem KonTraG, in DB, 52 (1999), S. 1665-1672

Theisen, Manuel René: Der Konzern, 2. Auflage, Stuttgart 2000

Thom, Norbert: Kontrolle, in: Klaus Chmielewicz / Marcell Schweitzer (Hrsg.): Handwörterbuch des Rechnungswesens, 3. Auflage 1993, Sp. 1140-1145

Thümmel, Roderich: Aufsichtsräte in der Pflicht? - Die Aufsichtsratshaftung gewinnt Konturen, in: DB, 52 (1999), S. 885-888

Thümmel, Roderich: Aufsichtsräte in Unternehmen der öffentlichen Hand professionell genug?, in: DB, 52 (1999), S. 1891-1893

ThyssenKrupp AG: Satzung in der Fassung vom 8. Juni 2000, in: http://www.thyssenkrupp.de/download/Satzung_dt.pdf (04.02.01)

Trenkle, Thomas: Organisation der Vorstandsentscheidung: Eine empirische Analyse, Frankfurt am Main, Bern, New York 1983

Ulmer, Peter: Die Aktionärsklage als Instrument zur Kontrolle des Vorstands- und Aufsichtsratshandelns, in: ZHR, 163 (1999), S. 290-342

Ulrich, Hans: Betriebswirtschaftliche Organisationslehre, Bern 1949 
Ulrich, Hans: Die Unternehmung als produktives soziales System, 2. Auflage, Stuttgart 1970

Vancil, R. F. / P. Lorange: Strategic Planning in Diversified Companies, in: Dietger Hahn / Bernard Taylor (Hrsg.): Strategische Unternehmungsplanung - Strategische Unternehmungsführung, 8. Auflage, Heidelberg 1999 , S. 830-843

Vetschera, Rudolf: Informationssysteme der Unternehmensführung, Berlin u.a. 1995

Vogel, Wolfgang: Aktienrecht und Aktienwirklichkeit - Organisation und Aufgabenteilung von Vorstand und Aufsichtsrat - Eine empirische Untersuchung deutscher Aktiengesellschaften, Baden-Baden 1980

Vogler, Matthias / Martin Gundert: Einführung von Risikomanagementsystemen - Hinweise zur praktischen Gestaltung, in: DB, 51 (1998), S. 2377 2383

Volk, Gerrit: Deutsche Corporate Governance-Konzepte, in: DStR, 39 (2001), S. 412-416

Voß, Stefan / Kai Gutenschwager: Informationsmanagement, Berlin u.a. 2001

Wagenhofer, Alfred / Gerhard Hrebicek (Hrsg.): Wertorientiertes Management, Stuttgart 2000

Wagner, Franz W.: Shareholder Value: Eine neue Runde im Konflikt zwischen Kapitalmarkt und Unternehmensinteresse, in: BfuP, 49 (1997), S. 473-498

Wagner, Gerd Rainer (Hrsg.): Unternehmungsführung, Ethik und Umwelt, Wiesbaden 1999

Wagner, Jürgen: Aufsichtsgremien im Gesellschaftsrecht, Stuttgart u.a. 1998

Weber, Max: Formen und Ausgestaltungsmöglichkeiten von Stock Options in der internationalen Praxis, in: Ann-Kristin Achleitner / Peter Wollmert (Hrsg.): Stock Options, Stuttgart 2000

Weiß, Daniel M.: Aktienoptionspläne für Führungskräfte, Stuttgart 1999

Weizsäcker, Carl Christian von: Alle Macht den Aktionären, in: FAZ, 27.6.1998, Nr. 146, S. 15

Wellkamp, Ludger: Vorstand, Aufsichtsrat und Aktionär, 2. Auflage, Bonn 2000 
Wenger, Ekkehard: Markt für Unternehmenskontrolle, in: Wolfgang Gerke / Manfred Steiner (Hrsg.): Handwörterbuch des Bank- und Finanzwesens, 2. Auflage, Stuttgart 1995, Sp. 1409-1419

Wenger, Ekkehard: Die Organisation des Aufsichtsrats als Problem der politischen Ökonomie, in: Wirtschaftsdienst, 76 (1996), S. 175-180

Wenger, Ekkehard: Aktienoptionsprogramme für Manager aus der Sicht des Aktionärs, in: Heribert Meffert / Klaus Backhaus (Hrsg.): Stock Options und Shareholder Value, Dokumentation des 33. Münsteraner Führungsgesprächs vom 9./10. Oktober 1997, Dokumentationspapier Nr. 116, Wissenschaftliche Gesellschaft für Marketing und Unternehmensführung e.V., Münster 1998, S. 51-69

Wenger, Ekkehard / Leonhard Knoll / Christoph Kaserer: Stock options, in: WiSt, 28 (1999), S. 35-38

Werder, Axel von: Organisation der Unternehmungsleitung und Haftung des Top-Managements, in: DB, 40 (1987), S. 2265-2273

Werder, Axel von: Unternehmungsführung und Argumentationsrationalität, Stuttgart 1994

Werder, Axel von: Management: Mythos oder regelgeleitete Kunst des Möglichen?, in: DB, 48 (1995), S. 2177-2183

Werder, Axel von (Hrsg.): Grundsätze ordnungsmäßiger Unternehmungsführung (GoF) für die Unternehmungsleitung (GoU), Überwachung (GoÜ) und Abschlußprüfung (GoA), Düsseldorf, Frankfurt am Main 1996

Werder, Axel von: Grundsätze ordnungsmäßiger Unternehmungsleitung (GoU) - Bedeutung und erste Konkretisierung von Leitlinien für das TopManagement, in: Axel von Werder (Hrsg.): Grundsätze ordnungsmäßiger Unternehmungsführung (GoF) für die Unternehmungsleitung (GoU), Überwachung (GoÜ) und Abschlußprüfung (GoA), Düsseldorf, Frankfurt am Main 1996, S. 27-71

Werder, Axel von: Vorstandsentscheidungen auf der Grundlage „sämtlicher relevanter Informationen"?, in: ZfB, 67 (1997), S. 901-922

Werder, Axel von: Shareholder Value-Ansatz als (einzige) Richtschnur des Vorstandshandelns?, in: ZGR, 27 (1998), S. 69-91

Werder, Axel von: Grundsätze ordnungsmäßiger Unternehmensleitung in der Arbeit des Aufsichtsrats, in: DB, 52 (1999), S. 2221-2224

Werder, Axel von: Der German Code of Corporate Governance im Kontext der internationalen Governance-Debatte: Umfeld, Funktionen und in- 
haltliche Ausrichtung des GCCG, in: Axel von Werder (Hrsg.): German Code of Corporate Governance (GCCG), Stuttgart 2000, S. 1-27

Werder, Axel von (Hrsg.): German Code of Corporate Governance (GCCG), Stuttgart 2000

Werder, Axel von u.a.: Grundsätze ordnungsmäßiger Unternehmensleitung (GoU) im Urteil der Praxis, in: DB, 51 (1998), S. 1193-1198

Werther Jr., William B. / Jeffrey L. Kerr: Strengthening corporate governance through board-level consultants, in: Journal of Organizational Change Management, No. 3, 8 (1995), S. 63-74

Whitley, Richard: On the Nature of managerial tasks and skills, in: Journal of Management Studies, 26 (1989)

Wild, Jürgen: Organisation und Hierarchie, in: zfo, 42 (1973), S. 45-54

Wild, Jürgen: Grundlagen der Unternehmungsplanung, 4. Auflage, Opladen 1982

Wilhelm, Jochen: Marktwertmaximierung - Ein didaktisch einfacher $\mathrm{Zu}$ gang zu einem Grundproblem der Investitions- und Finanzierungstheorie, in: ZfB, 53 (1983), S. 516-534

Williamson, Oliver E.: The Economics of Discretionary Behavior: Managerial Objectives in a Theory of the Firm, Englewood Cliffs (N.J.) 1964

Williamson, Oliver E.: Markets and Hierarchies, New York u.a. 1975

Williamson, Oliver E.: Transaction-cost economics: The governance of contractual relations, in: Journal of Law and Economics, Vol. 22 (1979), S. 3-61

Wilson, Robert: The Structure of Incentives for Decentralization under Uncertainty, in: Centre National de la Recherche Scientifique (Hrsg.): La Décision. Agrégation et dynamique des ordres de préférence, Paris 1969, S. 387-307

Winter, Stefan: Optionspläne als Instrument wertorientierter Managementvergütung, Frankfurt am Main u.a. 2000

Witt, Peter: Der Wandel der Corporate Governance-Strukturen deutscher Aktiengesellschaften - Beitrag für den 23. Workshop der Kommission "Organisation" im Verband der Hochschullehrer für Betriebswirtschaft e.V. "Change Management in Theorie und Praxis" vom 26. bis zum 27. Februar 1999 in Zürich

Witt, Peter: Corporate Governance im Wandel, in: zfo, 69 (2000), S. 159163 
Wittmann, Waldemar: Unternehmung und unvollkommene Information, Köln, Opladen 1959

Wittmann, Waldemar u.a. (Hrsg.): Handwörterbuch der Betriebswirtschaft, 5. Auflage, Stuttgart 1993

Wöhe, Günther: Einführung in die Allgemeine Betriebswirtschaftslehre, 17. Auflage, München 1990

Zimmer, Daniel: Das Gesetz zur Kontrolle und Transparenz im Unternehmensbereich, in: Neue Juristische Wochenschrift, 51 (1998), S. 35213534

Zimmer, Lutz: Die Ausgabe von Optionsrechten an Mitglieder des Aufsichtsrats und externe Berater, in: DB, 52 (1999), S. 999-1003

Zimmermann, Gebhard / André Wortmann: Der Shareholder-Value-Ansatz als Institution zur Kontrolle der Führung von Publikumsgesellschaften, in: DB, 54 (2001), S. 289-294

Zöllner, Wolfgang (Hrsg.): Kölner Kommentar zum Aktiengesetz, 2. Auflage, Bd. 2, Köln u.a. 1996

Zünd, Andre: Revisionslehre, Zürich 1982 


\section{Verzeichnis der Rechtsquellen}

AktG: Aktiengesetz vom 6. September 1965, BGB1. I, S. 1089

Aktienrechtsnovelle 1870: Gesetz betreffend die Kommanditgesellschaften auf Aktien und die Aktiengesellschaften vom 11. Juni 1870, BGBl. Des Norddeutschen Bundes 1870, S. 375

BetrVG: Betriebsverfassungsgesetz 1952 vom 11. Oktober 1952, BGBI. I, S. 681

GG: Grundgesetz für die Bundesrepublik Deutschland vom 23. Mai 1949, BGB1. I, S. 1

HGB: Handelsgesetzbuch vom 10. Mai 1897, RGB1. 1897, S. 219

KonTraG: Gesetz zur Kontrolle und Transparenz im Unternehmensbereich vom 27. April 1998, BGB1. I, 1998, S. 786

KStG: Körperschaftsteuergesetz vom 22. Februar 1996, BGB1. I, S. 341

Mitbestimmungsergänzungsgesetz 1956: Gesetz zur Ergänzung des Gesetzes über die Mitbestimmung der Arbeitnehmer in den Aufsichtsräten und Vorständen der Unternehmen des Bergbaus und der Eisen und Stahl erzeugenden Industrie vom 7. August 1956, BGB1. I, S. 707

Mitbestimmungsgesetz 1976 (MitbestG): Gesetz über die Mitbestimmung der Arbeitnehmer vom 4. Mai 1976, BGBI. I, S. 1153

Montan-Mitbestimmungsgesetz 1951: Gesetz über die Mitbestimmung der Arbeitnehmer in den Aufsichtsräten und Vorständen der Unternehmen des Bergbaus und der Eisen und Stahl erzeugenden Industrie vom 21. Mai 1951, BGBl. I, S. 347 


\title{
Bochumer Beitrăge zur Unternehmungsführung und Unternehmensforschung
}

\author{
Herausgegeben vom Direktorium des Instituts \\ für Unternehmungsführung und Unternehmensforschung \\ der Ruhr-Universităt Bochum
}

Band 1 Busse von Colbe, Walther/Mattessich, Richard (Hrsg.): Der Computer im Dienste der Unternehmungsführung (1968)

Band 2 Busse von Colbe, Walther/Meyer-Dohm, Peter (Hrsg.): Unternehmerische Planung und Entscheidung (1969)

Band 3 Anthony, Robert N.: Harvard-Fälle aus der Praxis des betrieblichen Rechnungswesens. Herausgegeben von Richard V. Mattessich unter Mitarbeit von Klaus Herrnberger und Wolf Lange (1969)

Band 4 Mattessich, Richard: Die wissenschaftlichen Grundlagen des Rechnungswesens (1970)

Band 5 Schweim, Joachim: Integrierte Unternehmungsplanung (1969)

Band 6 Busse von Colbe, Walther (Hrsg.): Das Rechnungswesen als Instrument der Unternehmungsführung (1969)

Band 7 Domsch, Michel: Simultane Personal- und Investitionsplanung im Produktionsbereich (1970)

Band 8 Leunig, Manfred: Die Bilanzierung von Beteiligungen. Eine bilanztheoretische Untersuchung (1970)

Band 9 Franke, Reimund: Betriebsmodelle. Rechensystem für Zwecke der kurztristigen Planung, Kontrolle und Kalkulation (1972)

Band 10 Wittenbrink, Hartwig: Kurzfristige Erfolgsplanung und Erfolgskontrolle mit Betriebsmodellen (1975)

Band 11 Lutter, Marcus (Hrsg.): Recht und Steuer der internationalen Unternehmensverbindungen (1972)

Band 12 Niebling, Helmut: Kurztristige Finanzrechnung auf der Grundlage von Kosten- und Erlösmodellen (1973)

Band 13 Perlitz, Manfred: Die Prognose des Unternehmenswachstums aus Jahresabschlüssen deutscher Aktiengesellschaften (1973)

Band 14 Niggemann, Walter: Optimale Informationsprozesse in betriebswirtschaftlichen Entscheidungssituationen (1973)

Band 15 Reichardt, Harald: Der aktienrechtliche Abhängigkeitsbericht unter ökonomischen Aspekten (1974)

Band 16 Backhaus, Klaus: Direktvertrieb in der Investitionsgüterindustrie - Eine Marketing-Entscheiung (1974)

Band 17 Plinke, Wulff: Kapitalsteuerung in Filialbanken (1975)

Band 18 Steffen, Rainer: Produktionsplanung bei Fließbandfertigung (1977)

Band 19 Kolb, Jürgen: Industrielle Erlösrechnung - Grundlagen und Anwendungen (1978)

Band 20 Busse von Colbe, Walther/Lutter, Marcus (Hrsg.): Wirtschaftsprüfung heute: Entwicklung oder Reform? (1977)

Band 21 Uphues, Peter: Unternehmerische Anpassung in der Rezession (1979) 
Band 22 Gebhardt, Günther: Insolvenzprognosen aus aktienrechtlichen Jahresabschlüssen (1980)

Band 23 Domsch, Michel: Systemgestūtzte Personalarbeit (1980)

Band 24 Schmied, Volker: Alternativen der Arbeitsgestaltung und ihre Bewertung (1982)

Band 25 Wäscher, Gerhard: Innerbetriebliche Standortplanung bei einfacher und mehrfacher Zielsetzung (1982)

Band 26 Weber, Martin: Entscheidungen bei Mehrfachzielen - Verfahren zur Unterstützung von Individual- und Gruppenentscheidungen (1983)

Band 27 Kroesen, Alfred: Instandhaltungsplanung und Betriebsplankostenrechnung (1983)

Band 28 Plinke, Wulf: Erlōsplanung im industriellen Anlagengeschäft (1985)

Band 29 Chamoni, Peter: Simulation störanfälliger Systeme (1986)

Band 30 Arning, Andreas: Die wirtschaftliche Bewertung der Zentrenfertigung - Dargestellt am Beispiel einer Fertigungsinsel (1987)

Band 31 Gebhardt, Günther: Finanzielle Planung und Kontrolle bei internationaler Unternehmenstãtigkeit

Band 32 Markiewicz, Michael: Ersatzteildisposition im Maschinenbau - Betriebswirtschaftliche Methoden der Planung und Übenwachung (1988)

Band 33 Pellens, Bernd: Der Informationswert von Konzernabschlüssen - Eine empirische Untersuchung deutscher Börsengesellschaften (1989)

Band 34 Mrotzek, Rüdiger: Bewertung direkter Auslandsinvestitionen mit Hilfe betrieblicher Investitionskalküle (1989)

Band 35 Deppe, Joachim: Quality Circle und Lernstatt - Ein integrativer Ansatz (1989, 3. Auflage 1993)

Band 36 Rademacher, Michael: Arbeitszeitverkürzung und -flexibilisierung - Formen und betriebliche Auswirkungen (1990)

Band 37 Kaiser, Klaus: Kosten- und Leistungsrechung bei automatisierter Produktion (1991, 2. Auflage 1993)

Band 38 Müller, Hermann: Industrielle Abfallbewältigung - Entscheidungsprobleme aus betriebswirtschaftlicher Sicht (1991)

Band 39 Schörner, Peter: Gesetzliches Insiderhandelsverbot - Eine ordnungspolitische Analyse (1991)

Band 40 Bentler, Martin: Grundsätze ordnungsmäßiger Bilanzierung für die Equitymethode (1991)

Band 41 Brüggerhoff, Jürgen: Management von Desinvestitionen (1992)

Band 42 Bröker, Erich W.: Erfolgsrechnung im industriellen Anlagengeschäft - Ein dynamischer Ansatz auf Zahlungsbasis - (1993)

Band 43 Frankenberg, Peter: Transnationale Analyse US-amerikanischer und deutscher Jahresabschlüsse - Eine theoretische und empirische Untersuchung (1993)

Band 44 Kleinaltenkamp, Michael: Standardisierung und Marktprozeß - Entwicklungen und Auswirkungen im CIM-Bereich (1993)

Band 45 Pellens, Bernhard: Aktionärsschutz im Konzern - Empirische und theoretische Analyse der Reformvorschläge der Konzernverfassung (1994)

Band 46 Reckenfelderbäumer, Martin: Marketing-Accounting im Dienstleistungsbereich - Konzeption eines prozeßkostengestützten Instrumentariums (1995) 
Band 47 Knittel, Friedrich: Technikgestützte Kommunikation und Kooperation im Büro. Entwicklungshindernisse - Einsatzstrategien - Gestaltungskonzepte (1995)

Band 48 Riezler, Stephan: Lebenszyklusrechnung - Instrument des Controlling strategischer Projekte (1996)

Band 49 Schulte, Jöm: Rechnungslegung und Aktienkursentwicklung - Erklärung und Prognose von Aktienrenditen durch Einzel- und Konzernabschlußdaten (1996)

Band 50 Muhr, Martin: Zeitsparmodelle in der Industrie - Grundlagen und betriebswirtschaftliche Bedeutung mehrjähriger Arbeitszeitkonten (1996)

Band 51 Brotte, Jörg: US-amerikanische und deutsche Geschăftsberichte. Notwendigkeit, Regulierung und Praxis jahresabschlußergänzender Informationen (1997)

Band 52 Gersch, Martin: Vernetzte Geschäftsbeziehungen. Die Nutzung von EDI als Instrument des Geschäftsbeziehungsmanagement (1998)

Band 53 Wăhrisch, Michael: Kostenrechnungspraxis in der deutschen Industrie. Eine empirische Studie (1998)

Band 54 Völkner, Peer: Modellbasierte Planung von Geschäftsprozeßabläufen (1998)

Band 55 Fülbier, Rolf Uwe: Regulierung der Ad-hoc-Publizităt. Ein Beitrag zur ökonomischen Analyse des Rechts (1998)

\section{Band 1 - 55 erschienen beim Gabler Verlag Wiesbaden}

Band 56 Ane-Kristin Reif-Mosel: Computergestützte Kooperation im Büro. Gestaltung unter Berūcksichtigung der Elemente Aufgabe, Struktur, Technik und Personal (2000)

Band 57 Claude Tomaszewski: Bewertung strategischer Flexibilität beim Unternehmenserwerb. Der Wertbeitrag von Realoptionen (2000)

Band 58 Thomas Erler: Business Objects als Gestaltungskonzept strategischer Informationssystemplanung (2000)

Band 59 Joachim Gassen: Datenbankgestützte Rechnungslegungspublizität. Ein Beitrag zur Evolution der Rechnungslegung (2000)

Band 60 Frauke Streubel: Organisatorische Gestaltung und Informationsmanagement in der lernenden Unternehmung. Bausteine eines Managementkonzeptes organisationalen Lernens (2000)

Band 61 Andreas von der Gathen: Marken in Jahresabschluß und Lagebericht (2001)

Band 62 Lars Otterpohl: Koordination in nichtlinearen dynamischen Systemen (2002)

Band 63 Ralf Schremper: Aktienrückkauf und Kapitalmarkt. Eine theoretische und empirische Analyse deutscher Aktienrückkaufprogramme (2002)

Band 64 Peter Ruhwedel: Aufsichtsratsplanungssysteme. Theoretische Grundlagen und praktische Ausgestaltung in Publikumsaktiengesellschaften (2002) 
Peter Ruhwedel - 978-3-631-75491-7 Downloaded from PubFactory at 01/11/2019 04:17:06AM 


\section{Marco Albers}

\section{Corporate Governance in Aktiengesellschaften}

\section{Entscheidungsprozess und Wirkungsanalyse zum Gesetz zur Kontrolle und Transparenz im Unternehmensbereich (KonTraG)}

Frankfurt/M., Berlin, Bern, Bruxelles, New York, Oxford, Wien, 2002. 334 S., 16 Tab., 6 Graf.

Europäische Hochschulschriften: Reihe 5, Volks- und Betriebswirtschaft. Bd. 2844

ISBN 3-631-38480-7 · €50.10*

Angeheizt durch spektakuläre Unternehmensschieflagen steht die deutsche Corporate Governance seit vielen Jahren im öffentlichen Diskurs. Das 1998 in Kraft getretene Kontroll- und Transparenzgesetz stellt somit das Ergebnis einer mehrjährigen teilweise äußerst kontrovers geführten Diskussion dar. Der Verfasser analysiert die Thematik, in der die Reform des Aufsichtsrates einen bedeutenden Teilaspekt bildet, durch Umsetzung eines politikwissenschaftlichen Analysemodells auf die aktuelle wirtschaftspolitische Maßnahme. Durch eine detailgenaue Rekonstruktion des Entscheidungsverlaufs des KonTraG wird u. a. der Einfluss von Interessengruppen offengelegt sowie das Gesetzgebungsverfahren und dessen Ergebnis in seinen Auswirkungen präzise analysiert. Die anschließende Untersuchung der Praxisfälle der Metallgesellschaft und der Hypo Vereinsbank gibt der Arbeit darüber hinaus eine empirische Fundierung und veranschaulicht realitätsnah die Möglichkeiten und Grenzen der Neuregelungen. Im Schlussteil wird des Weiteren ein Resümee im Hinblick auf die künftige Bearbeitung des Politikfeldes der Corporate Governance geliefert.

Frankfurt/M - Berlin - Bern · Bruxelles · New York · Oxford · Wien

Auslieferung: Verlag Peter Lang AG

Jupiterstr. 15, CH-3000 Bern 15

Telefax (004131) 9402131

*inklusive der in Deutschland gültigen Mehrwertsteuer

Preisänderungen vorbehalten

Homepage http://wuw.peterlang.de 
Peter Ruhwedel - 978-3-631-75491-7 Downloaded from PubFactory at 01/11/2019 04:17:06AM 